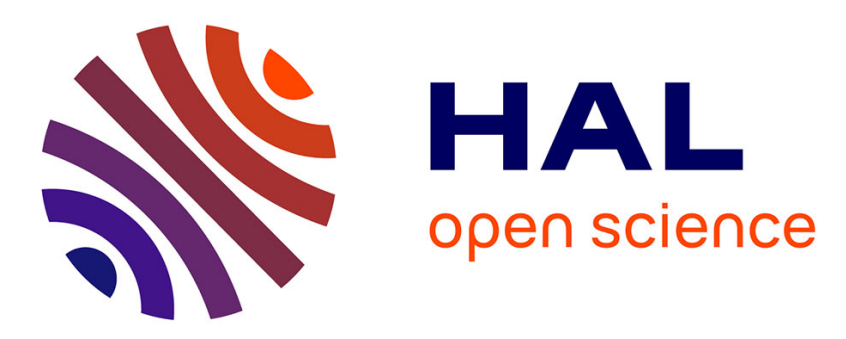

\title{
Mutations démographiques et sociales du Viet Nam contemporain
}

María Eugenia Zavala de Cosío, Myriam de Loenzien, Ngoc Luu Bich

\section{To cite this version:}

María Eugenia Zavala de Cosío, Myriam de Loenzien, Ngoc Luu Bich (Dir.). Mutations démographiques et sociales du Viet Nam contemporain. Presses universitaires de Paris Nanterre, 2016, 10.4000/books.pupo.4384. halshs- 01352821

\section{HAL Id: halshs-01352821 https://shs.hal.science/halshs-01352821}

Submitted on 9 Aug 2016

HAL is a multi-disciplinary open access archive for the deposit and dissemination of scientific research documents, whether they are published or not. The documents may come from teaching and research institutions in France or abroad, or from public or private research centers.
L'archive ouverte pluridisciplinaire HAL, est destinée au dépôt et à la diffusion de documents scientifiques de niveau recherche, publiés ou non, émanant des établissements d'enseignement et de recherche français ou étrangers, des laboratoires publics ou privés. 
MUTATIONS DEMOGRAPHIQUES ET SOCIALES DU VIET NAM CONTEMPORAIN

Coordonné par

COSIO ZAVALA MARIA E., DE LOENZIEN MYRIAM, LUU BICH NGOC 



\section{Sommaire}

Présentation - COSIO-ZAVALA Maria E., LOENZIEN Myriam de

Présentation des auteurs

Spécifications cartographiques

p. 13

Introduction- BELANGER Danièle, OUDIN Xavier

p. 15

$\underline{\text { Structure de la population et hétérogénéité }}$

Chapitre 1- «Structure de population: opportunités et défis » LUU Bich Ngoc, NGUYEN Thi Thiêng

Chapitre 2- «Deux perspectives régionales sur les déséquilibres de sexe à la naissance au Viêt Nam » BECQUET Valentine

Chapitre 3- «Exploring the heterogeneity of informal household business in Vietnam: from macro dynamics to micro characteristics and functioning" LE Thi Thuy Linh, RAZAFINDRAKOTO Mireille, ROUBAUD François

Enjeux sanitaires et sociaux

Chapitre 4- «La dimension familiale du dépistage VIH/sida au Viêt nam » LOENZIEN Myriam de

Chapitre 5- « Aides reçues et besoins d'aide des enfants séropositifs et de leurs familles » NGUYEN Thi Thiêng, BUI Thi Hanh, LERT France

Chapitre 6- « Jeunesse, genre et sexualité au Viêt Nam » SCORNET Catherine

Migration, urbanisation et développement

Chapitre 7- «Migrations internes au Viêt Nam: tendances et caractéristiques » NGUYEN Thi Thieng, LUU Bich Ngoc

Chapitre 8- «L'environnement urbain : Perception des habitants de Hanoi et Hô Chi Minh Ville » LE Thi Huong, NGUYEN Thi Thiêng, GUBRY Patrick

Chapitre 9- "Are non-farm jobs better for rural workers? A panel data analysis of earnings gaps in Vietnam" NGUYEN Huu Chi

Conclusion- « La population au centre des transitions en cours au Viêt Nam » LOENZIEN Myriam de 



\title{
Présentation
}

\author{
Maria E. COSIO ZAVALA (Université Paris Ouest Nanterre) \\ Myriam de LOENZIEN (IRD-CEPED)
}

Cet ouvrage est issu des activités scientifiques menées dans le cadre d'une collaboration entre des chercheurs et enseignants-chercheurs français et vietnamiens, au sein d'un vaste programme de recherche et de formation. Intitulé Projet Arcus ${ }^{1}$ Viêt Nam, lequel impliquait en France les Régions Ile-de-France et Midi-Pyrénées. Pluridisciplinaire, il comprenait cinq sous-projets : mathématiques et STIC, matériaux et nanosciences, énergies renouvelables, eau-environnement-océanographie, sciences humaines et sociales. Pour la Région Ile-de-France, les enseignants et chercheurs de l'Université Paris Ouest Nanterre La Défense assuraient la coordination des activités avec ceux de l'Ecole Normale Supérieure de Cachan, de l'Université Paris 13 Nord et de l'Université Paris 11 Sud. Ce programme a démarré en février 2011 et s'est terminé en octobre 2014.

Dans chaque sous-projet, une collaboration intense était établie entre les partenaires français et vietnamiens, sur des thématiques choisies en commun. La production scientifique a reposé sur des colloques, des séminaires, des cours, des publications, des thèses de doctorat, que sous-tendaient des échanges de chercheurs et d'étudiants entre la France et le Viêt Nam, rendus possibles par un financement ${ }^{2}$.

L'ouvrage que nous présentons est issu de différentes activités franco-vietnamiennes sur l'étude de la population, menées au sein du sous-projet "Sciences Humaines et Sociales ", sous la responsabilité de Maria Eugenia COSIO ZAVALA, Professeure à l'Université Paris Ouest Nanterre La Défense et de Myriam de LOENZIEN, chercheure à l'Institut de Recherche pour le Développement. Ces activités ont été effectuées en partenariat avec l'Institut d'Etudes sur la Population et la Société (IPSS) de l'Université Nationale d'Economie à Hanoi, dont la directrice, Luu Bich Ngoc est docteure en Démographie de l'Université de Paris Ouest Nanterre La Défense. A également participé une autre chercheure de cet institut, Nguyen Thi Thieng, également titulaire d'un doctorat réalisé en France. En France, les activités se sont déroulées au sein du Centre de Recherches Populations et Sociétés (CERPOS) de l'Université Paris Ouest Nanterre la Défense, lequel est rattaché depuis janvier 2014 à l'UMR 7217 du CNRS CRESPAA-GTM. Un partenariat a été mis en place avec le Centre Population et Développement (CEPED) de l'Université Paris Descartes, l'IRD et l'INED.

Le projet était centré sur une thématique intitulée : «La population du Viêt Nam au $21^{\mathrm{e}}$ siècle : enjeux sociodémographiques, sanitaires et environnementaux ». En effet, les multiples et profondes évolutions qu'a traversées le Viêt Nam au cours des dernières décennies suscitaient de nombreuses interrogations autour de questions peu analysées jusqu'ici. L'objectif était de contribuer à une meilleure connaissance scientifique des enjeux sociodémographiques actuels, sur la base des données des recensements et d'enquêtes. Parallèlement, il s'agissait d'opérer un transfert de compétences dans le domaine des études de population à travers la formation d'enseignants-chercheurs et d'étudiants à des méthodologies spécifiques et à la démographie, l'organisation

\footnotetext{
1 ARCUS (Actions en Régions de Coopération Universitaire et Scientifique) est un programme du Ministère français des Affaires Etrangères et du Ministère français de l'Enseignement Supérieur et de la recherche. Il vise à faire émerger, consolider et structurer des partenariats en matière de formation et de recherche dans des pays considérés comme stratégiques.

${ }^{2}$ Celui-ci était à parité entre le Ministère des Affaires Etrangères et les Régions.
} 
d'échanges scientifiques entre chercheurs français et vietnamiens et la réalisation de séminaires communs. Ces efforts devaient aboutir enfin à la valorisation des résultats obtenus à travers la rédaction de publications et l'élaboration de recommandations en matière de politiques de population.

De nombreuses missions en France et au Viêt Nam ont été réalisées, ce qui a permis une étroite collaboration entre les démographes français et vietnamiens. Des étudiants en doctorat des universités françaises ont participé au projet ainsi que des étudiants vietnamiens de niveau master et doctorat, lesquels ont suivi avec de jeunes professionnels des cours visant à une maîtrise d'outils utilisés pour l'étude des populations, comme l'analyse démographique, les méthodes biographiques, les approches qualitatives et l'analyse spatiale.

Au terme de ces échanges très riches, il nous est apparu indispensable qu'un ouvrage de synthèse traite des évolutions démographiques récentes de la population du Viêt Nam, et évoque des questions nouvelles encore peu étudiées. Celles-ci concernent de multiples phénomènes. Citons entre autres :

- les changements des structures démographiques, avec notamment un vieillissement accéléré,

- la baisse de la croissance de la population avec la fin de la transition démographique,

- la lutte contre certains problèmes de santé comme le VIH/sida,

- les difficultés en matière d'environnement en partie dues à l'urbanisation et l'industrialisation,

- les mouvements migratoires dont le contrôle se relâche progressivement,

- la santé de la reproduction, la sexualité, la fécondité et les relations de genre qui ont pris une expression particulière marquée par un déficit de naissances de filles,

- les difficultés d'emploi qui donnent lieu à des arbitrages des travailleurs et groupes familiaux,

- les mutations du système économique caractérisé par un large secteur informel.

Ces différents phénomènes donnent lieu à des clivages et remodèlent les liens de solidarité, mettent en évidence les stratégies ; ils se posent différemment aux niveaux individuels, du ménage ou à une échelle plus large.

Outre les auteurs des chapitres de ce livre, au nombre d'une quinzaine, cet ouvrage a bénéficié des multiples échanges développés dans un cadre pluridisciplinaire. Nous remercions en particulier Sarah Bales (Université Nationale de Singapour), Véronique Dupont (IRD), Sylvie Fanchette (IRD-CEPED), Christophe Guilmoto (IRD-CEPED), France Lert (INSERM) et Jacques Véron (INED) pour leurs commentaires et relectures. Cet ouvrage explore donc quelques tendances récentes de l'évolution de la population du Viêt Nam et leurs enjeux en termes démographiques, géographiques, sociopolitiques, économiques et méthodologiques. Il présente une façon d'approfondir l'apport de sources telles que le dernier recensement de 2009 et plusieurs enquêtes, de mobiliser des outils spécifiques et de montrer la nécessité de développer une approche interdisciplinaire pour la compréhension des phénomènes de population. Il constitue l'aboutissement de notre intention d'appréhender de façon large la population du Viêt Nam, en mobilisant diverses approches et disciplines des sciences sociales. A travers chacune d'elles sont abordés de multiples aspects, tels que les disparités spatiales, sociodémographiques et économiques après 30 années de politique du Renouveau (Doi moi). Car si cette politique a connu un succès indéniable sur le plan de la croissance économique et de l'amélioration des conditions de vie dans leur ensemble, ces progrès 
remarquables se sont accompagnés de la montée de fortes inégalités, suscitant de nouveaux enjeux au seuil du $21^{\mathrm{e}}$ siècle. 



\section{Présentation des auteurs}

Valentine BECQUET est docteure en démographie et chercheure associée au Centre Population et Développement (CEPED) de l'Université Paris Descartes et de l'Institut de Recherche pour le Développement (IRD). Sa thèse réalisée au CEPED ainsi qu'à l'Institut National d'Etudes Démographiques (INED) portait sur les déséquilibres du rapport de masculinité à la naissance au Viêt-Nam, et reposait à la fois sur des constats statistiques et une compréhension sociologique des rapports de genre. Valentine BECQUET a passé 15 mois (2012-2013) au Viêt-Nam pour mettre en place trois enquêtes qualitatives, afin d'observer les pratiques reproductives et les intentions de fécondité des couples, ainsi que la préférence de genre.

Danièle BELANGER est professeure au Département de Géographie de l'Université Laval à Québec (Canada). Entre 1997 et 2012, elle était professeure au Département de Sociologie de l'Université Western Ontario, à London (Ontario, Canada) et titulaire de la Chaire de recherche du Canada sur la population, le genre et le développement. Au Vietnam, elle a participé à de nombreux projets de recherche sur la famille, le genre, la santé de la reproduction et les migrations. Depuis 2005, elle travaille sur les trajectoires migratoires de migrants à statut précaire en Asie et au sein des Amériques. Ses publications récentes portent sur les travailleurs migrants temporaires au Canada et en Asie de l'Est, sur les migrants d'Amérique centrale en 'transit' au Mexique et sur les épouses migrantes en Asie.

BUI Thi Hanh est chercheure à l'Institut d'Etude Populations et Sociétés (IPSS) de l'Université Nationale d'Economie à Hanoi. Elle a participé au sein de cet institut à diverses études qualitatives et quantitatives en collaboration avec des organisations internationales et des institutions non gouvernementales. Ses sujets de recherche comprennent la migration et l'urbanisation, la population et la santé de la reproduction, le $\mathrm{VIH} /$ sida, le changement climatique ainsi que l'évaluation des politiques et leur mise en œuvre.

Maria Eugenia Cosio Zavala est Professeur de démographie, invitée à El Colegio de México et Professeur émérite de l'Université Paris Ouest Nanterre La Défense. Elle a dirigé le Centre de Recherche et de Documentation sur l'Amérique latine (CNRSCREDAL - Université Sorbonne nouvelle) à Paris entre 2000 et 2008. Elle est Docteur d'Etat ès Lettres et Sciences humaines en Démographie de l'Université René DescartesSorbonne. Ses recherches portent sur les migrations, la fécondité, le genre et les politiques de population.

Patrick GUBRY est chercheur en démographie à l'Institut de recherche pour le développement (IRD). Il a été affecté au Cameroun et au Viêt Nam. Il a travaillé à la réalisation du premier recensement national de la population au Cameroun en 1976. Il a eu l'occasion de créer un Département de recherche démographique avec des partenaires camerounais. Ses recherches ont porté sur la migration rurale-urbaine, la migration de retour, l'urbanisation, les mobilités intra-urbaines, l'emploi, la pauvreté, les questions population et environnement, et portent actuellement sur la «population flottante » à Hanoi et Hô Chi Minh Ville. 
LE Thi Huong est diplômée de l'Université de Hô Chi Minh Ville où elle a étudié la géographie. Elle a travaillé dans plusieurs institutions de recherche, notamment l'Institut d'études du développement de Hô Chi Minh Ville (HIDS). Elle a mené des recherches dans les domaines de la population, du vieillissement, du travail des enfants, du niveau de vie, de l'urbanisation, des migrations, de l'environnement urbain, en partenariat international. Elle a contribué à l'établissement du schéma directeur d'Hô Chi Minh Ville. Elle a rejoint le Centre de consultation pour les affaires relatives à l'Organisation Mondiale du Commerce en tant que coordinatrice. Elle dispense actuellement des consultances dans le cadre du Centre de consultance-information-formation en gestion économique et commerciale (CIT).

LE Thi Thuy Linh est doctorante en économie DIAL, unité mixte de recherche entre l'Institut de Recherche pour le Développement (IRD) et l'Université Paris-Dauphine. Elle est diplômée du Master 2 Politique Publiques et Développement de l'Ecole d'Economie de Paris (PSE), et de l'Ecole Supérieure de Commerce Extérieur du Viêt Nam. Ses recherches portent sur l'économie informelle dans les pays en développement, particulièrement au Viêt Nam. Elle s'intéresse aussi aux questions d'inégalité, d'éducation et de bonheur.

France LERT, chercheuse à l'Inserm, a abordé au cours de sa carrière différentes facettes de l'organisation de santé publique, le dispositif français de lutte contre la tuberculose, la sécurité et les conditions au travail, la politique à l'adresse des usagers de drogue, l'infection VIH/sida depuis les années 80. Dans les années 2000, elle a participé à de larges études évaluant l'impact des politiques de santé et mettant à l'épreuve des nouvelles stratégies de dépistage du VIH. France Lert a été responsable au sein de l'action coordonnée de l'ANRS «Comportement et prévention » et du groupe de travail sur le dépistage. Elle a dirigé de 2005 à 2014, l'équipe Epidémiologie des déterminants professionnels et sociaux de la santé au sein du Centre de recherche et santé des populations de l'Inserm (U1018) à Villejuif.

Myriam de LOENZIEN est démographe, chargée de recherche à l'Institut de Recherche pour le Développement (IRD) habilitée à diriger des recherches. Elle travaille au Centre Population et Développement (CEPED) et enseigne à l'université Paris Descartes. Dans le cadre d'affectations au Cameroun (1991-1993, 1994-1999) puis au Viêt Nam (20022008), elle a mené des programmes de recherche sur la santé de la reproduction. Elle a publié en 2014 un ouvrage intitulé «Famille et société au prisme du VIH/sida : l'exemple du Viêt Nam » et mène actuellement des recherches sur la famille, la santé, le handicap et la vulnérabilité en Asie du Sud-Est et en Afrique subsaharienne.

LuU Bich Ngoc est directrice de l'Institut Populations et Sociétés (IPSS) de l'Université Nationale d'Economie à Hanoi. Elle travaille sur le lien entre population et développement avec un intérêt particulier pour les politiques de population et de santé de la reproduction, la migration et l'urbanisation, l'éducation, le système de soins, les relations de genre, la prévention et la prise en charge sociale des personnes séropositives $\mathrm{VIH} /$ sida. Elle a été consultante auprès de nombreuses organisations internationales. Elle est titulaire d'un master en Population et Education de l'Université Nationale d'Economie, d'un master en Santé Publique de l'Université de Copenhague et d'un doctorat de démographie de l'université Paris Ouest Nanterre la Défense. 
NGuYen Huu Chi est Docteur en sciences économiques de l'Université Paris Nord et enseignant-chercheur de la faculté de Statistique de l'Université Nationale d'Economie au Viêt Nam. Il occupe actuellement un poste d'Attaché Temporaire d'Enseignement et de Recherche à l'Unité de Formation et de Recherche de Sciences Economiques et de Gestion de l'Université Paris Nord. Ses travaux de recherche portent sur les questions liées au marché du travail, l'économie informelle, la migration et les écarts de revenu dans les pays en développement.

NGuYEN Thi Thiêng a été directrice-adjointe de l'Institut d'Etudes Populations et Sociétés (IPSS) de l'Université Nationale d'Economie à Hanoi. Elle a enseigné les statistiques et les sciences de la population. Elle a réalisé des projets de recherche pour diverses institutions publiques et participé à des programmes de recherche communs entre le Viêt Nam et la France. Elle conduit régulièrement des consultances pour des institutions internationales et des organisations non gouvernementales sur des sujets tels que la migration et l'urbanisation, la population et la santé génésique, le VIH/sida, la planification familiale, le genre et le développement, la prévention de l'alcoolisme.

Xavier OUDIN est chercheur en économie à l'Institut de recherche pour le développement (IRD), unité de recherche DIAL. Diplômé de l'Institut d'Etudes Politiques de Paris et docteur en économie, il a travaillé en Afrique de l'Ouest (Cote d'Ivoire, Niger) puis en Asie du sud-est (Thaïlande, Viêt Nam) sur le marché du travail. Il s'intéresse notamment aux effets de la transition démographique sur le marché du travail. Il travaille actuellement à Hanoi sur un programme concernant la lutte contre la pauvreté, en particulier sur le secteur informel ainsi que la perception de la gouvernance par les ménages. Il est également coordinateur scientifique du projet européen Nopoor.

Mireille RAZAFINDRAKOTO est directrice de recherche à l'IRD et membre de l'Unité mixte de recherche DIAL. Elle est diplômée de l'ENSAE (ENSAE/CESD, Paris) et docteur en économie (École des hautes études en sciences sociales, EHESS). Ses recherches sur l'économie du développement portent plus particulièrement sur le marché du travail, la pauvreté et les inégalités, ainsi que sur les questions de gouvernance. Elle a été en poste à Madagascar (1994-1999) et au Viêt Nam (20062011) dans le cadre de programmes de recherche conduits par l'équipe DIAL-IRD (elle a assuré la coordination scientifique de ce dernier programme). Elle a acquis une expérience substantielle dans la conception et l'analyse d'enquêtes sur l'emploi et le secteur informel dans le cadre de ces programmes.

François ROUBAUD est économiste, directeur de recherche à l'IRD et membre de l'unité mixte de recherche DIAL. Il est diplômé de l'ENSAE et docteur en économie (université Paris Nanterre). Dans le domaine statistique, il a été l'un des pionniers de la conduite d'enquêtes mixtes (ménages/entreprises) et le concepteur des enquêtes 1-2-3 visant à mesurer le secteur informel. Il est aussi l'un des promoteurs des modules sur la gouvernance et la démocratie greffés sur les enquêtes officielles. Ses recherches sur l'économie du développement portent plus particulièrement sur le marché du travail et l'économie informelle, la gouvernance et l'économie politique des politiques de développement. Il a été affecté dans plusieurs pays dans le cadre de programmes de recherche de longue durée.

Catherine SCORNET, socio-démographe, est maître de conférences au département de sociologie d'Aix-Marseille Université, et chercheur au Laboratoire Population - 
Environnement - Développement (LPED/UMR 151 IRD - AMU). Elle s'intéresse aux liens entre politiques, changement social et mutations sociodémographiques et familiales. Elle a vécu quatre années au Viêt Nam, et a travaillé en collaboration avec le Comité National des Sciences Sociales et Humaines du Viêt-Nam sur les politiques de régulation des naissances et programmes de population. Elle a soutenu à La Sorbonne un doctorat de démographie intitulé «Fécondité et politique dans le delta du fleuve Rouge ». Ses objets de recherches actuels portent sur la sexualité des jeunes Vietnamiens. 


\section{Spécifications cartographiques}

Pour une bonne compréhension du texte, la figure ci-dessous (figure 1) propose une représentation des régions censitaires du Viêt Nam, avec mention des cinq principales villes-provinces du pays (noms soulignés) et des provinces où ont eu lieu les enquêtes (voir Tableau 1 ci-dessous pour la situation des provinces dans chaque région).

Carte 1- Régions du Viêt Nam, principales provinces et lieux des enquêtes

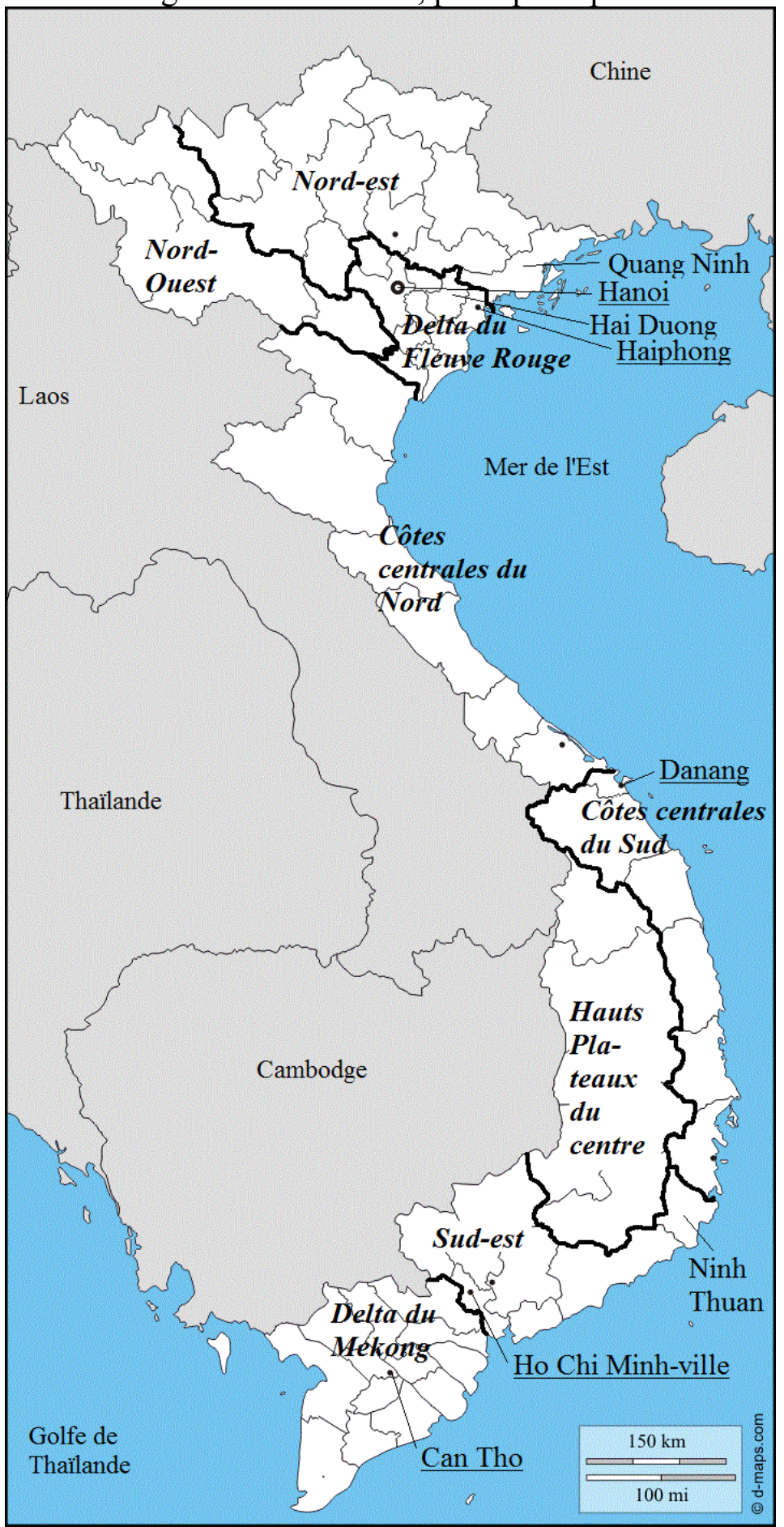

Source : M. de Loenzien, à partir du fond cartographique en ligne sur le site http://d-maps.com/m/asia/vietnam/vietnam39.gif, le 01022016 
Tableau 1- Répartition des provinces selon la région

\begin{tabular}{cccc}
$\begin{array}{c}\text { Delta du Fleuve } \\
\text { Rouge }\end{array}$ & Nord-Est & Nord-Ouest & $\begin{array}{c}\text { Côtes centrales } \\
\text { du nord }\end{array}$ \\
\hline Bac Ninh & Bac Giang & Dien Bien & Ha Tinh \\
Ha Nam & Bac Kan & Hoa Binh & Nghe An \\
Hai Duong & Cao Bang & Lai Chau & Quang Binh \\
Hung Yen & Ha Giang & Son La & Quang Tri \\
Nam Dinh & Lang Son & & Thanh Hoa \\
Ninh Binh & Lao Cai & & \\
Thai Binh & Phu Tho Thien Hue \\
Vinh Phuc & Quang Ninh & & \\
Hanoi & Thai Nguyen & & \\
Haiphong & Tuyen Quang & & \\
& Yen Bai & &
\end{tabular}

\begin{tabular}{cccc}
$\begin{array}{c}\text { Côtes centrales du } \\
\text { Sud }\end{array}$ & $\begin{array}{c}\text { Hauts Plateaux du } \\
\text { Centre }\end{array}$ & Sud-est & $\begin{array}{c}\text { Delta du } \\
\text { Mekong }\end{array}$ \\
\hline Binh Dinh & Dak Lak & Ba Ria Vung Tau & An Giang \\
Khanh Hoa & Dak Nong & Binh Duong & Bac Lieu \\
Phu Yen & Gia Lai & Binh Phuoc & Ben Tre \\
Quang Nam & Kon Tum & Dong Nai & Ca Mau \\
Quang Ngai & Lam Dong & Tay Ninh & Dong Thap \\
Binh Thuan & & Ho Chi Minh ville & Hau Giang \\
Ninh Thuan & & Kien Giang \\
Da Nang & & Long An \\
& & Soc Trang \\
& & Tien Giang \\
& & Tra Vinh \\
& & Vinh Long \\
& & Can Tho
\end{tabular}

Certains textes font références à l'ancien découpage du pays en 6 régions. La correspondance avec le découpage actuel est donnée dans le tableau ci-dessous (Tableau B).

Tableau 2- Correspondance entre l'ancienne et la nouvelle classification par régions Classification actuelle Classification précédente en 8 régions en 6 régions

\begin{tabular}{cc} 
en 8 regions & en 6 regions \\
\hline Delta du Fleuve Rouge & Delta du Fleuve Rouge \\
\hline Nord-est, Nord-Ouest & Montagnes du Nord \\
\hline $\begin{array}{c}\text { Côtes centrales du Nord, } \\
\text { Côtes centrales du Sud }\end{array}$ & Côtes centrales du Nord et du Sud \\
\hline Hauts Plateaux du Centre & Hauts Plateaux du Centre \\
\hline Sud-est & Sud-est \\
\hline Delta du Mekong & Delta du Mekong
\end{tabular}




\title{
Introduction
}

\author{
Danièle BeLANGER, (Université Laval, Québec, Canada) \\ Xavier OUDIN, (IRD-DIAL)
}

Il y a près de trente ans, le Viêt Nam initiait sa transition vers une économie mixte avec l'adoption de la politique du Doi moi ou Renouveau. Trente années sont une période équivalente à celle écoulée entre les années 1954 et 1985. Cette étape de l'histoire du Viêt Nam a été le théâtre de grands bouleversements historiques dont l'indépendance et la partition du pays en 1954, la réforme agraire et la collectivisation des biens de production et de l'agriculture au Nord, la guerre entre le Viêt Nam et les États-Unis, qui prendra fin en 1975, avec la réunification du pays, puis l'invasion du Cambodge et la guerre avec la Chine en 1979.

Alors que les générations précédentes avaient été marquées par la colonisation, les conflits armés et les difficultés économiques généralisées, la génération qui a aujourd'hui trente ans a grandi dans un pays qui a connu une croissance économique extraordinaire, une amélioration considérable du niveau de vie, une ouverture sur le monde, une entrée dans une économie mondialisée, une transition démographique accélérée et une insertion économique dans des réseaux régionaux et internationaux. S'ils ont été à la fois les témoins, les artisans et les bénéficiaires d'avancées remarquables, les jeunes de trente ans vivent au sein d'une société qui fait face à de nouveaux défis. C'est l'univers social, démographique et économique de cette génération charnière que décrivent et analysent les neuf chapitres de cet ouvrage. Au fil des résultats de recherches, nous prenons la mesure des succès, des questions, des défis, des incertitudes et des obstacles qui marquent aujourd'hui la société vietnamienne. Organisés autour de trois grands thèmes - structure et hétérogénéité de la population, enjeux sanitaires et sociaux et migrations, urbanisation et développement - les neuf chapitres de cet ouvrage brossent un tableau du chemin parcouru au cours des dernières décennies et rendent compte de phénomènes nouveaux ou émergents, dont certains sont encore peu étudiés.

\section{Sources et qualité des statistiques sociales}

Les analyses présentées dans cet ouvrage tirent profit des multiples enquêtes, à la portée des chercheurs tant vietnamiens qu'étrangers, qui ont vu le jour au fil des années 1990 et 2000 et qui touchent de nombreuses thématiques. Les chapitres de cet ouvrage offrent principalement des analyses qui couvrent la dernière décennie, et, dans certains cas, présentent des comparaisons avec les années 1990 et 1980.

En 1989, le Viêt Nam réalisait un recensement de population qui demeure aujourd'hui une référence majeure. S'ensuivront dans les années 1990, des enquêtes de fécondité et sur les niveaux de vie (VLSS 92-92 et 97-98). En 1999, à la suite d'un nouveau recensement, de nombreuses enquêtes sont effectuées et offrent désormais des données sur de multiples sujets pour l'étude de la population et de la société. Dans les années 2000, le système statistique national s'étoffe avec des enquêtes niveaux de vie tous les deux ans, des enquêtes emploi devenues permanentes (mensuelles), des enquêtes agricoles et recensements industriels réguliers, etc.

Les chapitres présentés ici font ainsi usage des recensements de 1999 et de 2009 et de diverses enquêtes nationales pour l'analyse des tendances démographiques, sociales et économiques. Plusieurs auteurs ont recours aux données des enquêtes du Bureau national des statistiques (General Statistics Office, GSO) dont les Enquêtes sur les 
changements démographiques et la planification familiale (Luu Bich Ngoc), l'Enquête emploi (Luu Bich Ngoc, Le Thi Thuy Linh et al,), l'Enquête sur les entreprises familiales et le secteur informel (2007 et 2009, en collaboration avec l'Institut de Recherche pour le Développement, IRD) et l'Enquête sur les migrations de 2004 (Nguyen Thi Thiêng et Luu Bich Ngoc). Les enquêtes ménages sur les niveaux de vie (Vietnam Households Living Standards Surveys ou VHLSS, en plusieurs vagues dont 2002, 2004 et 2006) sont citées par Luu Bich Ngoc alors que Nguyen Huu Chi (chapitre 9) utilise un panel extrait de ces enquêtes pour des analyses originales économétriques sur les revenus par secteur (agricole et non agricole) et les types d'emploi (salariés et travailleurs indépendants, secteur formel et informel). Catherine Scornet cite des résultats des enquêtes SAVY (Survey Assessment of Vietnamese Youth, financées par un consortium de partenaires) de 2003 et de 2010 effectuées auprès des jeunes.

Toutefois, après 25 ans de production de données statistiques, c'est la question de la qualité des données qui s'impose et qui, dans le futur, devrait davantage interpeller les analystes. Pincus et Sender publiaient en 2008 un article qui relevait le manque d'autocritique chez les chercheurs ayant recours aux Enquêtes Ménage sur les niveaux de vie dans le cadre d'analyses sur la pauvreté. La méthode d'échantillonnage de ces enquêtes, qui constituent la principale source pour le suivi des taux de pauvreté, n'inclut pas une vaste population mobile non enregistrée sur son lieu de résidence. Selon les auteurs, cette situation mène à une sous-estimation non négligeable de la pauvreté urbaine (Pincus et Sender, 2008) ${ }^{3}$. La question de l'échantillonnage et de sa représentativité, de la qualité des opérations de collecte et des conclusions que 1'on peut tirer des résultats sont au cœur de leur critique. A partir d'une enquête la plus exhaustive possible sur une petite aire géographique, ils montrent à quel point les enquêtes VHLSS sont biaisées en faveur de certaines catégories de ménages et mettent en doute leur représentativité statistique. Le développement économique du Viêt Nam et la réussite du pays dans de nombreux domaines ne doivent pas empêcher les chercheurs de garder un œil critique sur les données mises à leur disposition. Les vérifications des données, que ce soit leur exhaustivité, la validité des échantillons ou la qualité de la collecte pourraient se développer davantage. L'étude des inégalités, de la pauvreté ou de l'exclusion et de la marginalisation requièrent des données fiables, une garantie encore fragile dans le cas du Viêt Nam.

Au-delà des problèmes d'échantillonnage et d'exhaustivité, la mesure de la pauvreté et des inégalités s'avère particulièrement délicate. Elle est effectuée à partir des enquêtes VHLSS, mais celles-ci ne mesurent pas correctement les revenus. On a recours à un expédient, à un " proxy » qui est le niveau de dépenses des ménages, qu'on pense être un peu plus réaliste. Même avec cette mesure, il est très difficile de savoir si les inégalités augmentent au Viêt Nam et il faut rester prudent sur les diverses mesures de la pauvreté.

Les informations sur les revenus peuvent cependant être utilisées de façon relative pour comparer le niveau de vie des uns et des autres, avec néanmoins une limitation concernant les revenus non salariaux. Il est en effet probable que la sous-estimation des revenus est plus importante pour les revenus non salariaux qui concernent les trois quarts de la population active. Cela conduit à sous-estimer les revenus du secteur informel et les revenus de l'agriculture. Cependant, l'étude d'un panel sur trois vagues, comme le propose Nguyen Huu Chi, apporte une certaine robustesse aux analyses sur des données fragiles. Son analyse économétrique des différentiels de revenus fournit

\footnotetext{
${ }^{3}$ Voir aussi Gubry Patrick, Lê Thi Huong et Nguyên Thi Thiêng, 2011.
} 
ainsi des résultats fins quant aux trajectoires de sortie de la pauvreté pour les paysans pauvres.

En complément des enquêtes nationales, de nombreux chercheurs ont réalisé euxmêmes des enquêtes de terrain sur des sujets spécifiques et à portée régionale ou locale, contribuant ainsi à l'essor et à la diffusion de statistiques sociales sur une multitude de thématiques au cours des dernières années. Les collaborations entre chercheurs vietnamiens et étrangers ont favorisé la production de données originales quantitatives et qualitatives tandis que le système statistique national prenait de l'ampleur, encouragé par la Banque Mondiale et d'autres donneurs. Ainsi, dans cet ouvrage, nous retrouvons des analyses novatrices basées sur des données d'enquêtes de terrain qualitatives de Myriam de Loenzien sur le SIDA, de Catherine Scornet sur la sexualité, de Valentine Becquet sur le désir d'avoir un fils, de Nguyen Thi Thiêng, Bui Thi Hanh et France Lert sur les aides reçues par les familles avec un enfant séropositif, de Lê Thi Huong et coll. sur les questions d'environnement urbain. Ces données ponctuelles viennent enrichir les analyses au niveau national proposées par d'autres chapitres.

Enfin, les études présentées signalent aussi des lacunes en matière de données. Elles soulignent notamment des besoins urgents en données rigoureuses et exhaustives sur la santé reproductive et la sexualité des hommes en général et des femmes non mariées, sur l'avortement, sur les migrants internes non enregistrés dans la localité où ils ont migré, sur le secteur informel, sur les questions de santé, sur les populations marginalisées et vulnérables, sur les parcours d'emploi, sur l'environnement et sur le vieillissement démographique.

\section{Une transition démographique rapide}

C'est en premier lieu sur les changements survenus en matière démographique ces dernières décennies, et particulièrement depuis quelques années que ce livre met l'accent. La transition démographique, avec une chute de la fécondité spectaculaire en trente ans, comme le rappellent plusieurs des auteurs, a été très rapide ${ }^{4}$.

Le premier chapitre, de Luu Bich Ngoc, propose un bilan des opportunités et défis que présente la structure de la population du Viêt Nam des années actuelles. Les données des recensements de 1989, 1999 et 2009 montrent bien l'amélioration des conditions de vie, les taux très élevés d'alphabétisation, la chute de la fécondité et l'augmentation des migrations internes. Depuis 2007, le Viêt Nam est entré dans une période de dividende démographique avec moins de 50 individus d'âge non actif pour 100 d'âge actif (15 à 64 ans). Cette partie est documentée à partir de projections et analyses originales établies par l'IPSS (Institute of Population and Social Studies) auquel appartient l'auteure, et qui n'étaient pas disponibles pour le public non vietnamophone.

S'il est un enjeu important relatif à la population, c'est bien celui de tirer parti du dividende démographique comme l'ont fait d'autres pays d'Asie de l'Est avant le Viêt Nam. Toutefois, sans une augmentation de la qualité de la main-d'œuvre, le Viêt Nam pourra difficilement bénéficier des avantages du dividende démographique. Or, en 2009, les trois quarts de la population active étaient sans qualification. Le chapitre souligne également à quel point la question du vieillissement est au cœur des enjeux démographiques et requiert une attention accrue. L'auteure note également la diminution des inégalités entre hommes et femmes en matière d'éducation, d'emploi et de mortalité 5 .

\footnotetext{
${ }^{4}$ L'indice synthétique de fécondité est passé de 6,8 (1965-1969) à 2,5 enfants par femme (1995-1999). Il est en dessous de 2,0 en 2009 (voir chapitre 2).

${ }^{5}$ Voir aussi Bélanger, Danièle, Nguyen Thi Ngo Lan et Nguyen Thi Thuy Oanh. 2011
} 
Depuis le milieu des années 2000, le Viêt Nam attire l'attention par l'augmentation du rapport de masculinité à la naissance, ce qui montre une augmentation de la sélection des grossesses en fonction du sexe du fœetus (Guilmoto, 2012). Cette tendance est étudiée dans le chapitre 2 de manière fine par Valentine Becquet. A partir d'une analyse comparative de deux provinces, elle souligne d'abord les différences régionales en matière de comportement quant à la sélection du sexe des enfants. Les probabilités d'agrandissement des familles selon le sexe des enfants antérieurs s'avèrent très différentes dans les provinces de Hai Duong, près de Hanoi, et Ninh Thuan dans le sud (voir Carte 1 en tête d'ouvrage). Elle explique ces spécificités de manière qualitative en montrant bien comment la structure de la parenté est liée à la préférence pour les garçons. La structure de parenté patrilocale et patrilinéaire de la province Hai Duong entraîne un recours beaucoup plus fréquent à la sélection selon le sexe que dans la province de Ninh Thuan, qui tend à avoir une structure de parenté matrilinéaire. Cette analyse originale montre à quel point l'étude des causes sous-jacentes, bien illustrée ici par une analyse régionale comparative, contribue à une meilleure compréhension du phénomène.

Les témoignages recueillis par Valentine Becquet et d'autres travaux sur le sujet font allusion à la construction sociale d'une maîtrise " scientifique » de la fécondité qui non seulement permet le contrôle du nombre d'enfants et de l'espacement entre les naissances, mais également celui du sexe de l'enfant. La commercialisation de moyens de contrôle du sexe des enfants et la construction sociale de la femme moderne et scientifique qui réussit à produire l'enfant du sexe désiré au moment désiré - dès la première naissance par exemple- sont des facteurs peu étudiés dans les analyses des causes de l'augmentation du rapport de masculinité à la naissance. Les méthodes qui permettraient de contrôler le sexe des enfants à concevoir prolifèrent au Viêt Nam et inspirent la possibilité d'une maîtrise de cet aspect de la fécondité. Les livres populaires, les médicaments traditionnels, les diètes, les prescriptions sur le moment idéal de la conception, le recours fréquent à l'échographie et l'avortement donnent lieu à une économie et une science de la production d'un enfant mâle. Or, ce développement de la pseudoscience de la production d'enfants du sexe désiré, principalement destinée aux femmes dans le but d'avoir un fils, entraîne une pression sociale considérable bien audelà de la pression traditionnelle des belles-mères sur leurs belles-filles, ou du mari sur son épouse. Ainsi, le désir d'avoir au moins un garçon n'est plus seulement une tradition, mais également un objectif témoignant de l'appartenance à une ère moderne et scientifique.

La question du genre est également abordée par Catherine Scornet dans son chapitre sur la sexualité chez les jeunes (chapitre 6). L'auteure cherche à voir dans quelle mesure nous assistons à des «transformations des représentations, des attitudes et des pratiques sexuelles dans le contexte d'une transformation rapide de la société vietnamienne ». Sa conclusion montre, d'une part, une rupture entre la reproduction et la sexualité et l'apparition d'expériences sexuelles plus diversifiées et, d'autre part, la continuité de représentations et de pratiques inégalitaires entre jeunes hommes et jeunes femmes. Dans ce contexte, Catherine Scornet souligne la vulnérabilité des jeunes femmes, qui demeurent sujettes aux grossesses non désirées, en général suivies d'avortement, et à la peur d'être perçues comme « des filles faciles ».

Catherine Scornet décrit également la politisation de la sexualité, c'est-à-dire l'irruption de la sexualité dans le débat public. Elle explique que « la politisation de la sphère privée et de l'intimité » est l'une des conséquences du Doi moi, mais aussi de l'influence des organisations internationales. "La sexualité est devenue un objet politique légitime en lien avec la santé ». Mais dans le même temps, on assiste à 
l'extension du domaine privé ou intime. Le Parti ne s'immisce plus dans les affaires privées, par exemple pour les autorisations de mariage. Les Vietnamiens n'ont plus à subir de pression politique pour leurs agissements dans la sphère privée et une plus grande tolérance est de mise concernant les pratiques sexuelles. On est passé d'un cadre normatif imposé à un élargissement des libertés individuelles. Ce paradoxe est une illustration des évolutions sociales en cours et des profonds changements qui affectent la société vietnamienne. Le politique évolue, même si le système reste remarquablement stable.

\section{Opinions et perception des individus et des ménages}

Dans ce cadre, une évolution majeure est l'importance croissante donnée à l'opinion des citoyens et à leur participation aux affaires publiques, notamment au niveau local. Cela est rappelé dans nombre de directives du Parti, et est inscrit dans la loi. Et pour les chercheurs, la connaissance de l'opinion et de la perception des citoyens sur les thèmes étudiés fait partie de la démarche de recherche en sciences sociales.

La multiplication des enquêtes permet certes d'accumuler une information factuelle d'une grande richesse. Mais ces enquêtes sont aussi le média d'une expression d'opinions et de mesure de la perception de questions diverses par la population. Les questions « subjectives » sont maintenant fréquentes dans les enquêtes. S'il y avait bien eu une première enquête sur les valeurs en 1991, c'est surtout depuis le début des années 2000 que les questions portant sur l'opinion ou la perception des individus se multiplient, avec un certain nombre d'enquêtes plus particulièrement spécialisées dans ce domaine. La montée en puissance de certains thèmes de recherche, comme la mesure de la gouvernance et la lutte contre la corruption ont certainement favorisé ce type d'enquêtes. Ainsi, la grande enquête annuelle PAPI est-elle principalement basée sur la perception de la qualité des services publics ${ }^{6}$. Des enquêtes sur les valeurs (World Values Survey), sur la corruption (Transparency International), ont déjà été menées à plusieurs reprises.

L'opinion n'est pas seulement quelque chose de subjectif. Elle est objet de recherche et d'analyse en soi. Elle est également, du point de vue gouvernemental, le moyen de mesurer l'approbation par la population des politiques mises en œuvre, de connaitre les besoins ou les récriminations des citoyens afin d'améliorer l'efficacité de l'action publique. Les résultats d'enquêtes sont utilisés par le gouvernement pour identifier les provinces ou les services déficients ou au contraire performants. L'enquête PAPI citée plus haut est ainsi devenue un indicateur quasiment officiel de la qualité des administrations provinciales. Les enquêtes sont également le principal outil de connaissance de la corruption, grâce notamment aux enquêtes auprès des entreprises (VCCI, Vietnamese Chamber of Commerce and Industry) ou à de nombreuses enquêtes ponctuelles souvent financées par des sources gouvernementales en association avec des donneurs. Grâce à ces travaux, on a une meilleure connaissance de la perception de la corruption par les ménages, des administrations considérées comme les plus corrompues, à défaut de mesure de la corruption elle-même.

Plusieurs chapitres de ce livre s'appuient sur des opinions de personnes collectées au cours d'enquêtes. Myriam de Loenzien (chapitre 4) recueille ainsi les opinions de personnes séropositives ou de leur entourage, ce qui lui permet d'expliquer certains comportements concernant le recours aux tests de dépistage du sida. L'étude de Lê Thi Huong, Nguyên Thi Thiêng et Patrick Gubry (chapitre 8) repose presque entièrement sur une étude des opinions des citoyens de Hanoi et de Ho Chi Minh Ville. C'est à partir

\footnotetext{
${ }^{6}$ PAPI : Provincial Governance and Public Administration Performance Index (CECODES, Front de la Patrie, PNUD) .
} 
d'une enquête originale réalisée en 2007 que ces auteurs étudient la perception des problèmes environnementaux des habitants de ces deux villes, donnant ainsi la parole à la population sur des questions qui ne font pas souvent l'objet d'un débat public. Sur ce thème de l'environnement du quartier, les citoyens sont volontiers critiques (ce qui n'est pas le cas dans d'autres enquêtes d'opinions sur les problèmes du pays), le nombre de réponses défavorables l'emportant sur les réponses positives. Ils citent les nombreuses difficultés auxquelles ils sont confrontés: pollution sonore, olfactive, de l'air, inondations et problèmes de sécurité. Les problèmes d'évacuation des eaux usées, les problèmes de santé en lien avec la forte pollution de l'air et les craintes en matière de sécurité sont perçus comme étant en forte augmentation et requérant une attention urgente des pouvoirs publics. Les villes du Viêt Nam comptent parmi les plus polluées au monde et il ne fait pas de doute que la question de l'environnement deviendra de plus en plus une préoccupation majeure des habitants et que le débat public va se développer. Cette recherche montre déjà une prise de conscience des citoyens sur les questions liées à leur environnement.

Le chapitre de Le Thi Thuy Linh, Mireille Razafindrakoto et François Roubaud (chapitre 3) exploite de façon originale les réponses à des questions ouvertes en proposant une analyse quantitative textuelle des mots utilisés par les répondants. Avec pour objectif de décrire les caractéristiques et la diversité des situations des entrepreneurs dans le secteur informel, les auteurs mettent en évidence, outre l'importance de celui-ci au sein du marché du travail (un quart des emplois), sa forte hétérogénéité. Une analyse quantitative textuelle d'entretiens leur permet de dégager cinq types d'entreprises familiales: 'the impervious, the optimistic, the desolate, the precarious and the streteous'. Cet exercice de catégorisation, basé sur les termes utilisés par les enquêtés eux-mêmes pour parler de leur situation, montre l'étendue de la précarité au sein du secteur informel et souligne la nécessité de mettre en œuvre des politiques d'intervention et de soutien en fonction des modes de fonctionnement et des difficultés rencontrées par ces différents groupes. Ce chapitre innove au niveau méthodologique en exploitant des données textuelles issues de questions ouvertes traitées par des outils statistiques tels que l'analyse en composantes principales ou la constitution de « clusters ».

Donner la parole aux personnes concernées qui n'ont pas forcément les moyens de se faire entendre permet d'humaniser les thématiques étudiées. Les recherches présentées ici en offrent plusieurs exemples. A propos de la stigmatisation et de l'exclusion vécues par les séropositifs, le parent d'un enfant séropositif dit au chercheur " je souhaiterais que cette maladie soit une maladie normale, comme les autres maladies $»$. Cette citation résume bien la souffrance et les difficultés vécues par les adultes responsables d'enfants séropositifs dans une société où la stigmatisation envers le VIH demande encore à être combattue, en dépit d'améliorations au cours des dernières années. Catherine Scornet relate l'histoire d'une adolescente démunie devant sa propre grossesse dont elle ne savait rien. Cet exemple illustre les conséquences d'un manque encore criant d'information et d'éducation en matière de sexualité destinées aux jeunes célibataires. Myriam de Loenzien rapporte les propos d'un parent qui s'impose à son jeune fils afin que celui-ci aille se faire faire un test de dépistage du VIH dans un contexte et une période (milieu des années 2000) où le dépistage intervient surtout quand la maladie a déjà commencé à faire son œuvre. Le travail de Valentine Becquet comporte des témoignages éloquents quant au rôle de la pression sociale sur les jeunes femmes jugées sur leur capacité à concevoir un enfant mâle. 


\section{Précarité, vulnérabilité et pauvreté}

Ces derniers exemples mettent en exergue les conditions précaires d'existence d'une partie importante de la population. Certes, la pauvreté a beaucoup diminué au Viêt Nam, mais il reste de nombreuses personnes en situation de vulnérabilité. Les personnes vulnérables sont celles qui, même si elles ne sont pas (ou plus) pauvres, sont susceptibles de retomber dans la pauvreté, notamment en cas de choc économique. Ces chocs sont souvent des problèmes de santé dans la famille, des drames familiaux ou des revers de fortune.

La vulnérabilité est liée à la précarité de l'emploi. Comme nous l'avons vu, plus des trois quarts de la population active n'a aucune garantie concernant la stabilité de son emploi et de ses revenus. Tous les travailleurs non-salariés (et donc la quasi-totalité des agriculteurs) ainsi qu'une partie non négligeable des travailleurs salariés (ceux qui n'ont ni contrat ni sécurité sociale) sont dans une situation d'emploi précaire et, pour la plupart d'entre eux, vulnérables à un choc économique ou de santé.

La vulnérabilité de certains ménages en cas de problème de santé est illustrée dans le chapitre de Nguyen Thi Thiêng, Bui Thi Hanh et France Lert sur les adultes qui ont un enfant ayant le SIDA à leur charge. Le chapitre souligne le fossé entre les coûts remboursés par les assurances et ceux déboursés par les familles. Malgré l'élargissement des régimes d'assurances santé et des coûts pris en charge, les familles font face à des frais supplémentaires qui demandent, comme le signalent les auteurs, à « être caractérisés ». Ce chapitre traite de la marginalisation et de la stigmatisation dont sont victimes les familles ayant un enfant séropositif. Le gouvernement vietnamien a longtemps considéré certains groupes indésirables comme étant associés à des fléaux sociaux à éliminer. Les campagnes de propagande associaient le SIDA à la mort (jusqu'en 2003, voir chapitre de Myriam de Loenzien), l'utilisation de drogue et la prostitution à la criminalité. Depuis les années 2000, de nombreux projets du gouvernement et d'ONG ont tenté de réduire les préjugés et la stigmatisation à travers des formations et une meilleure information. Depuis quelques années, des politiques sociales basées sur la réduction des conséquences négatives (harm reduction approach), plutôt que la criminalisation, ont commencé à voir le jour, témoignant d'une amorce de changement d'idéologie politique. Malgré ces avancées, les auteurs montrent comment la peur qu'un enfant soit stigmatisé fait en sorte que les parents ou les adultes responsables de cet enfant tendent à soigneusement cacher la maladie. Cet isolement diminuait la possibilité de chercher un soutien psychologique. Le désarroi et la souffrance de ces adultes confrontés à la maladie de leur enfant montrent l'urgent besoin d'accroître les campagnes d'information et de sensibilisation concernant le SIDA et ses modes de transmission. Comme le soulignent les auteurs en conclusion, la question de la fréquentation scolaire des enfants séropositifs requiert une attention particulière dans un tel contexte.

Le chapitre de Myriam de Loenzien documente également la prévalence de la discrimination et de la stigmatisation à l'égard du VIH-Sida et aborde ces phénomènes comme de 'puissants freins au dépistage'. L'étude des processus de dépistage entre 2006 et 2008 est révélatrice de plusieurs aspects sociaux et sanitaires en lien avec la gestion et la construction sociale du VIH-Sida de ces années. Cette étude documente la faible proportion de personnes testées, le recours au test de dépistage à des fins de diagnostic plutôt que préventives, le non-respect de la confidentialité des consultations, et le rôle primordial de la famille dans le dépistage et la prise en charge de ceux affectés par la maladie. L'auteure souligne que son étude capte un moment spécifique de l'histoire du VIH/Sida, un moment qui précède la mise en place de normes standardisées internationales en matière de dépistage, de traitement et de prise en 
charge. Cet épisode rend compte des stratégies familiales déployées pour encourager le dépistage et organiser la prise en charge à une période de faible investissement et implication des pouvoirs publics. La politique de socialisation des coûts des soins, ou la responsabilité et responsabilisation de la famille est ici illustrée par un exemple sur le dépistage du sida.

Nguyen Thi Thieng et Luu Bich Ngoc étudient également la précarité de l'emploi chez les migrants (absence de contrat, non-accès à des assurances, etc.) et notent la méconnaissance qu'ont les migrants de leurs droits. Nombreux sont ceux qui ne demandent pas de certificat de résidence même s'ils pouvaient y avoir accès. Destiné au départ à contenir les migrations et les déplacements de population non encadrés (ce qui a fonctionné jusqu'au début des années 1990), le certificat de résidence est devenu de fait un obstacle à l'intégration et à la sortie de la précarité pour nombre de migrants.

L'emploi et les trajectoires de sortie de la pauvreté ('pathways out of poverty') sont traités dans le chapitre de Nguyen Huu Chi «Are non-farm jobs better for rural workers? A Panel Data Analysis of Earning Gaps in Vietnam ». L'auteur montre qu'en dépit de la présomption d'une réponse positive à cette question, les résultats scientifiques sont loin d'être clairs et univoques. A partir d'analyses sur les enquêtes sur les niveaux de vie (VLSS 2002-2004-2006) Nguyen Huu Chi évalue les écarts de revenus entre les emplois agricoles et non agricoles (toutes choses étant égales par ailleurs) afin d'étudier si l'emploi non agricole peut favoriser la 'sortie de la pauvreté'. Ses conclusions montrent des résultats différenciés selon les quintiles de revenus et selon le genre (hommes ou femmes). De manière générale, les travailleurs ruraux ayant un emploi non agricole tendent à avoir de meilleurs revenus que ceux qui ont un emploi agricole. Toutefois, cette association est particulièrement robuste au sein des quintiles de revenus inférieurs et moins significative pour les quintiles supérieurs. De plus, les femmes retirent systématiquement moins d'avantages que les hommes de l'occupation d'un emploi non agricole. En conclusion, l'auteur relève la nécessité d'étudier la question des réseaux pour mieux comprendre les relations entre emplois, revenus, et les trajectoires de mobilité socioéconomiques permettant de sortir de la pauvreté. En effet, la recherche sur les relations entre emploi, opportunités et pauvreté ne peuvent progresser sans l'inclusion du rôle des réseaux et de la corruption, deux réalités incontournables quant à l'insertion sur le marché du travail ${ }^{7}$.

Des avancées importantes ont été accomplies depuis quelques années concernant la couverture des soins de santé par les régimes d'assurance maladie. Mais, dans le même temps, qu'il s'agisse de l'accès aux soins de santé ou à l'éducation, les ménages sont appelés à débourser des sommes élevées, notamment s'ils recherchent des prestations de qualité, ce qui soulève des questions importantes quant aux effets bénéfiques de l'extension de la sécurité sociale. L'introduction des politiques de "socialisation » ( $x a$ hoi hoa), selon lesquelles la société et les familles doivent contribuer aux coûts économiques et sociaux de l'éducation et de la santé, laissent place à une zone grise peu reconnue et peu étudiée (London, 2004).

\section{Diversité régionale}

Les analyses présentées dans cet ouvrage montrent de façon éloquente à quel point l'étude du Viêt Nam doit prendre en compte les différences régionales. Qu'il s'agisse de différences nord-sud, entre les milieux ruraux et urbains, entre les différentes régions administratives, entre les villes de Hanoi et de Ho Chi Minh ville, entre les zones habitées par différents groupes ethniques, les résultats des études et des analyses de ce

\footnotetext{
${ }^{7}$ Voir aussi : Tran Thi Tuyet, 2014.
} 
livre illustrent bien que le Viêt Nam est un pays de contrastes, d'inégalités, et de différences.

En premier lieu, ces différences régionales sont un facteur explicatif des migrations à l'intérieur du pays. Le chapitre de Nguyen Thi Thieng et Luu Bich Ngoc montre toute la complexité de la réalité démographique migratoire interne. La première partie, basée sur le recensement de 1999 et de 2009, souligne la forte augmentation des migrations telle que mesurée par la question sur le lieu de résidence 5 ans avant le recensement. Même si elles sous-estiment la mobilité interne, ces données indiquent une augmentation de $30 \%$ de la population de migrants internes entre 1999 et 2009. Le recensement de 2009 mesurait une population totale de migrants de 6725000 , soit $8 \%$ de la population totale du pays (davantage pour la population adulte). Le pouvoir d'attraction des régions a été modifié en une décennie: les hauts plateaux du Centre sont devenus moins attrayants tandis que les régions industrielles du Sud-est ou du delta du fleuve rouge le sont davantage. Ce sont les provinces les plus industrialisées qui attirent le plus de migrants, ce qui tend à prouver que les flux migratoires sont principalement liés à des emplois dans l'industrie. Les données montrent aussi que les femmes sont plus nombreuses à migrer que les hommes (109 femmes migrantes pour 100 hommes migrants en 2009). A partir d'enquêtes spécifiques, dont celle de Patrick Gubry relatée dans le chapitre 8 , les auteures documentent les multiples raisons à la fois économiques, environnementales et celles en lien avec les études et la famille, qui poussent les gens à la mobilité interne. Elles analysent des difficultés rencontrées par les migrants. L'enregistrement sur le lieu de résidence, auquel sont rattachés les droits et l'accès aux services, demeure un obstacle majeur. En dépit de l'assouplissement en 2007 du contrôle de résidence et de l'existence de quatre types d'enregistrement de résidence, dont une résidence temporaire à court terme (enregistrement de type KT4), un nombre important de migrants demeurent non enregistrés. Les enquêtes nationales excluent les ménages non officiellement enregistrés ainsi que les migrants vivant dans des résidences collectives, comme les dortoirs des usines. Or, comme nous l'avons vu, ces exclusions biaisent les échantillons et donc les analyses sur la relation entre migrations et pauvreté.

C'est aussi un contraste saisissant entre deux provinces du Viêt Nam, l'une au nord dans le delta du fleuve Rouge, l'autre au centre-sud, avec des peuplements différents qui sous-tend l'analyse de Valentine Becquet sur la préférence pour les garçons à la naissance (voir Carte 1 en tête d'ouvrage). En raison d'histoires de peuplement différentes (il y a une forte minorité Cham dans la province du centre), on constate des comportements opposés concernant la sélection des fœus en fonction du sexe.

Le contraste est moins marqué dans l'étude sur la perception des problèmes d'environnement par les habitants de Hanoi et d'Ho Chi Minh ville. Certes, les réponses diffèrent dans les deux villes, mais cela est dû à des différences dans l'environnement des quartiers. Par exemple, il y a une plus grande sensibilité aux inondations à Ho Chi Minh ville.

En revanche, les contrastes entre milieu urbain et rural peuvent être relevés dans plusieurs chapitres de ce livre, à des titres divers. L'accès aux soins, surtout de façon anonyme pour des malades du sida ne se pose pas du tout dans les mêmes termes pour les habitants des campagnes et ceux des villes. Sur les différences de niveau de vie entre milieu rural et milieu urbain, on relèvera surtout l'analyse de Nguyen Huu Chi concernant la sortie de la pauvreté des travailleurs ruraux (et agricoles) qui se dirigent vers des emplois non agricoles et (pas toujours) urbains.

Les diversités géographiques ne sont pas le sujet des différentes recherches proposées dans cet ouvrage, mais elles sont omniprésentes et enrichissent les analyses. 
«Dynamique de la population et transformations de la société dans le Viêt Nam contemporain » propose un tour d'horizon sur plusieurs thématiques pertinentes et actuelles. Utilisant des méthodes d'investigations diversifiées, qualitatives ou quantitatives, et le plus souvent des données de première main collectées à l'initiative des chercheurs eux-mêmes, cet ouvrage au titre modeste aborde les thèmes de l'emploi, de la santé, de la sexualité, des jeunes, du vieillissement, des migrations, des inégalités hommes femmes et de l'environnement qui sont au cœur des enjeux sociaux et démographiques du Viêt Nam contemporain. Cet ouvrage présente des résultats inédits et éclairants tout en dressant un ordre du jour pour la recherche à venir.

\section{Bibliographie}

Belanger Danièle, Nguyen Thi Ngo Lan et Nguyen Thi Thuy Oanh, «Vers la réduction des disparités entre les genres au Viêt Nam: une analyse des recensements de 1989, 1999 et 2009» in Rapport publié par l'Observatoire de l'espace démographique et statistique francophone, ODSEF, Québec, Canada, Université Laval, 2012, p. 48.

CECODES, VFF-CRT et UNDP, "The Viet Nam Governance and Public Administration Performance Index (PAPI) 2012", in Measuring Citizens' Experiences. A Joint Policy Research Paper by Centre for Community Support and Development Studies (CECODES), Centre for Research and Training of the Viet Nam Fatherland Front (VFF-CRT), and United Nations Development Programme (UNDP), Hanoi, Viet Nam, 2013, p. 126 [En ligne, consulté le 21 février 2015].

GUBRY Patrick, LE Thi Huong et NGUYEN Thi Thiêng, "L'urbanisation au Viêt Nam : que sait-on de la "population flottante" ? » in Séminaire "Population, santé publique et développement au Viêt Nam après 25 ans de Renouveau »in Hanoi, IPSS, ARCUS, 10 octobre 2011.

Guilmoto, Christophe, "Son preference, sex selection, and kinship in Vietnam » in Population and Development Review, 38 (1), 2012, p. 31-54.

LONDON Jonathan, "Rethinking Vietnam's mass education and health systems" in Rethinking Vietnam, MCCARGo Duncan (dir.), London, Routledge Curzon, 2004, p. 127-142.

Pincus Jonathan et SENDER John, "Quantifying Poverty in Vietnam : Who Counts?" in Journal of Vietnamese Studies, Vol. 3, No. 1, 2008, p. 108-150.

TRAN Thi Tuyet, Graduate Employability in Vietnam : A Loose Relationship Between Higher Education and Employment Market, Anchor Academic Publishing, 2014, p. 188. 


\title{
Structure de la population : opportunités et défis
}

\author{
Luu Bich Ngoc (IPSS UNE)
}

Les résultats des recensements de la population et du logement effectués de 1979 à 2009 montrent que le «paysage démographique » a changé rapidement. Si en 1979, le pays ne comprenait que 52,742 millions d'habitants, en 2009 il en comptait 85,789, c'est-à-dire 32 de plus. Parallèlement, la structure de la population a aussi fortement changé, notamment en ce qui concerne la répartition par âge. Le pays est en phase de dividende démographique. Toutefois, la population vietnamienne doit également faire face à une série de défis. Il s'agit de disparités et de déséquilibres en matière de niveau de vie, d'indicateurs démographiques, d'accès à l'éducation et de santé. Ce chapitre analyse ces disparités, déséquilibres et défis. Il met l'accent notamment sur le processus de vieillissement en cours.

\section{I- Disparités, déséquilibre et défis}

Selon les résultats du recensement de la population et du logement de 2009, le Viêt Nam a accompli certaines performances dans le domaine de l'économie et la démographie. À la suite de la politique du Renouveau, avec le développement économique, le niveau de vie n'a cessé de s'améliorer. Après 50 ans de mise en œuvre de la politique de réduction du taux de natalité, l'indice synthétique de fécondité a atteint le niveau de remplacement. Le taux de mortalité, autrefois élevé, a été fortement réduit (il était de 4,6 pour mille en 2009).

\section{$\underline{\text { I.1- Conditions et niveau de vie }}$}

Selon les rapports sur le développement humain des Nations Unies, le PIB par habitant du Viêt Nam a nettement augmenté, de 2070 USD en 2001 à 2953 USD en 2009 (en parité de pouvoir d'achat). Le pays est classé à la $138^{\mathrm{e}}$ place sur le plan mondial (UNDP, $2003 ; 2011$ ). En 2010, les résultats de l'enquête sur le niveau de vie des habitants montrent que le revenu mensuel moyen par habitant était de 1211000 dongs (environ 60 USD), soit 52,8\% de plus que le revenu moyen en 2008, correspondant à une hausse moyenne annuelle de 23,6\% (Office général des Statistiques, 2011). Auparavant, de 2006 à 2008, les revenus mensuels moyens par habitant ont connu une augmentation de $25 \%$, bien plus forte que les hausses annuelles de 16,6\% de 2002 à 2004 et de 14,6\% de 2004 à 2006 (Office général des Statistiques, 2009). Le faible taux d'accès à l'eau potable et aux toilettes équipées d'une chasse d'eau constitue la lacune la plus marquante. Selon les résultats du recensement de la population et du logement de 2009, dans l'ensemble du pays, 25,2 \% des ménages n'avaient ni l'eau courante ni accès à un puits. Ce taux était seulement de $6,1 \%$ en milieu urbain tandis qu'il atteignait 33,6\% en milieu rural. Au total, 46,0\% des ménages n'avaient pas de toilettes équipées d'une chasse d'eau. En milieu rural, ce taux s'élève à $61,0 \%$ versus $12,2 \%$ en milieu urbain (Office général des Statistiques, 2010a : 128). Cette disparité est aussi observée entre provinces. Les localités ayant une forte proportion (plus de $90 \%$ ) de ménages ayant l'accès à l'eau courante sont celles situées dans le delta du fleuve Rouge, le Sud-est et les côtes centrales. Quant aux toilettes équipées d'une chasse d'eau, les localités comportant plus de $90 \%$ des ménages en disposant sont situées dans les villes relevant du pouvoir central ainsi que certaines provinces à forte urbanisation et industrialisation au Sud-est telles que Binh Duong, Dông Nai et Vung Tau (voir Carte 1 et Tableau 1 dans les spécifications cartographiques en tête d'ouvrage). 


\section{$\underline{\text { I.2- Accès à l'éducation }}$}

$\mathrm{Au}$ total, 93,5\% de la population vietnamienne $(95,8 \%$ des hommes et $91,4 \%$ des femmes) savent lire et écrire (Figure 1). Preuve de performance de l'éducation nationale, le taux d'alphabétisation du Viêt Nam est nettement supérieur à celui des pays voisins et d'autres pays au même revenu par habitant (Laos 72,7 \%, Cambodge 77,6 \%, Bhoutan 52,8 \%, Inde $62,8 \%$, Pakistan 55,5\%) (UNDP, 2011).

Figure 1- Taux d'alphabétisation selon l'année et le sexe

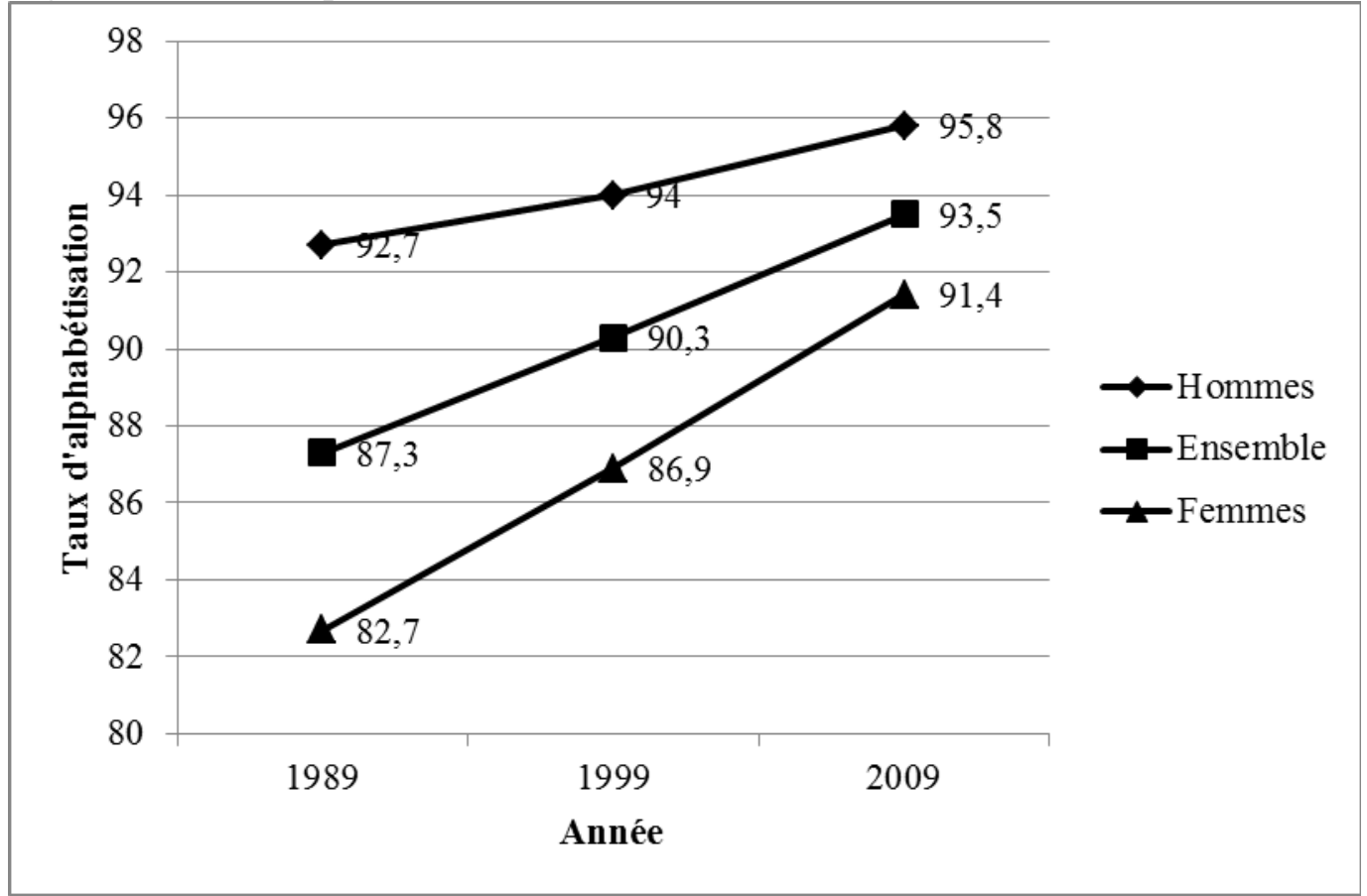

Source: Office général des Statistiques, 2011c, p. 24

L'augmentation du taux d'alphabétisation durant la période 1989-2009 a été beaucoup plus forte chez les femmes que chez les hommes (8,7 points versus 3,1 points). L'écart s'est donc réduit. Toutefois, les inégalités selon le sexe se situent à différents niveaux selon la région et la province (Figure 2). Elle s'élève à 11,6 points dans les régions montagneuses du Nord et 10,7 points dans les Hauts Plateaux du Centre (Office général des Statistiques, 2010a : 93). Certaines provinces ont presque atteint l'égalité, comme Binh Duong, Ho Chi Minh-Ville, Ca Mau (la différence des taux d'alphabétisation entre les hommes et les femmes n'est que de 1,4 point). Cette inégalité est plus accentuée dans certaines provinces montagneuses du Nord : la différence est de 20,9 points à Ha Giang, 22,9 points à Son La, 25,9 points à Diên Biên, 29,2 points à Lai Chau).

Le taux d'alphabétisation varie selon la province (Figure 3). Par ailleurs, il reste plus faible dans les régions rurales que dans les régions urbaines. Cette disparité atteint 2 à 3 points dans quatre des six régions (delta du fleuve Rouge, Centre-nord et Côtes centrales, sud-est, delta du Mékong). Si dans certaines provinces, la disparité n'existe presque plus entre les régions rurales et urbaines (Binh Duong, Bac Liêu, Ca Mau, Hung Yên), elle reste très importante dans d'autres localités $(30,4$ points à Ha Giang, 32,7 points à Diên Biên et 36,0 points à Lai Chau). 
Figure 2- Taux d'analphabétisme selon la province en 2009

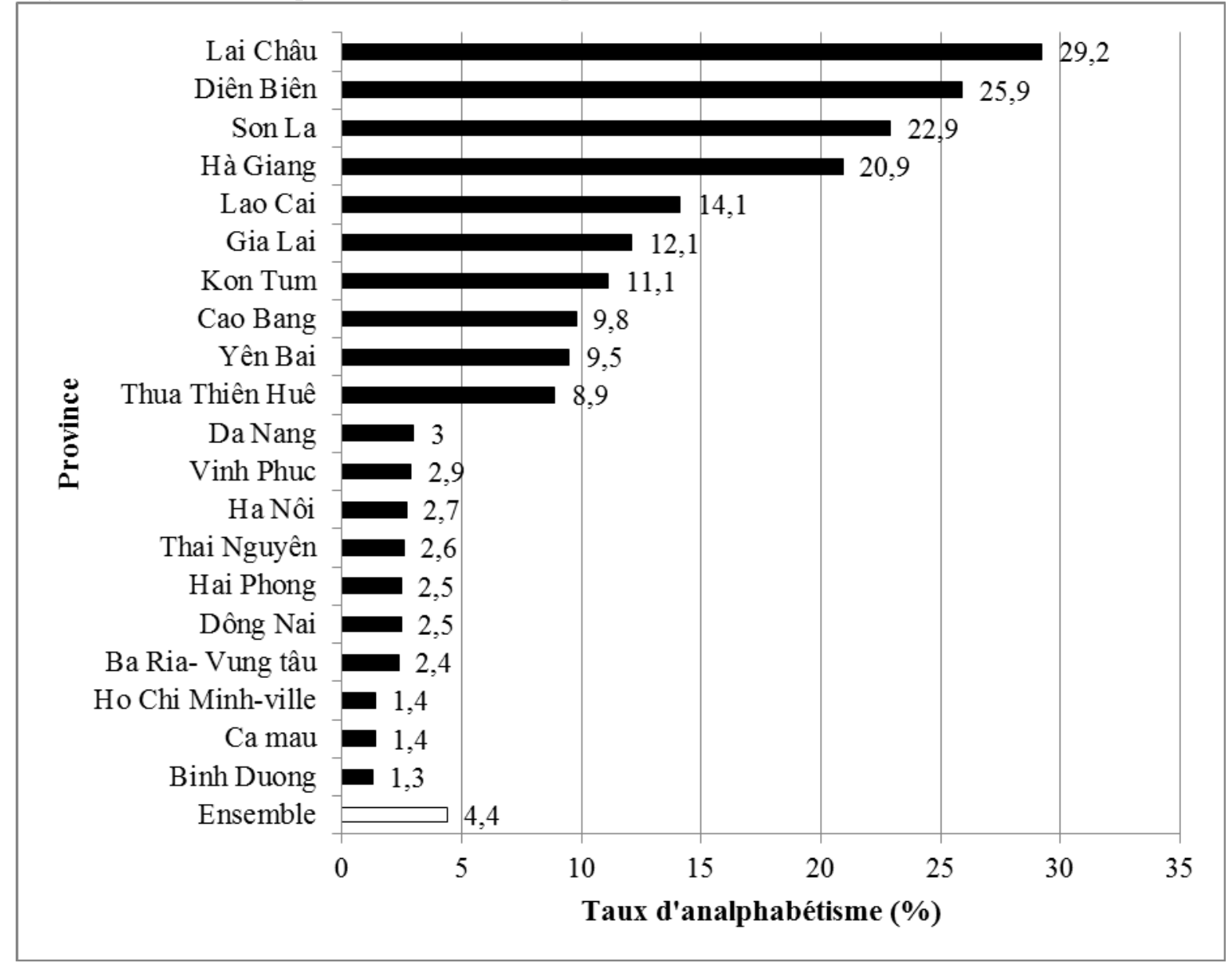

Source : Office général des Statistiques, 2011c, p. 30

Le taux d'alphabétisation de l'ethnie majoritaire Kinh, ainsi que celui des ethnies Tay et Muong atteint plus de $90 \%$ (Figure 4), avec une faible disparité entre hommes et femmes (2 à 3 points). Ce taux chez les Thaïs (quatrième groupe ethnique en nombre d'habitants) est de $79,8 \%$, avec toutefois une disparité entre hommes et femmes s'élevant à 17,9 points (respectivement 90,9\% et 73,0\%). Viennent ensuite les Khmers (sixième groupe ethnique en nombre d'habitants) avec un taux d'alphabétisation de 73,5\%, la disparité entre hommes et femmes étant de 10,2 points $(80,9 \%$ contre $70,7 \%)$. Il est à noter que le taux d'alphabétisation chez les Mong (cinquième groupe ethnique en nombre d'habitants) est de $37,7 \%$ avec une forte disparité entre hommes et femmes (Office général des Statistiques, 2010a : 171). 
Figure 3- Taux d'analphabétisme (\%) selon la province en 2009

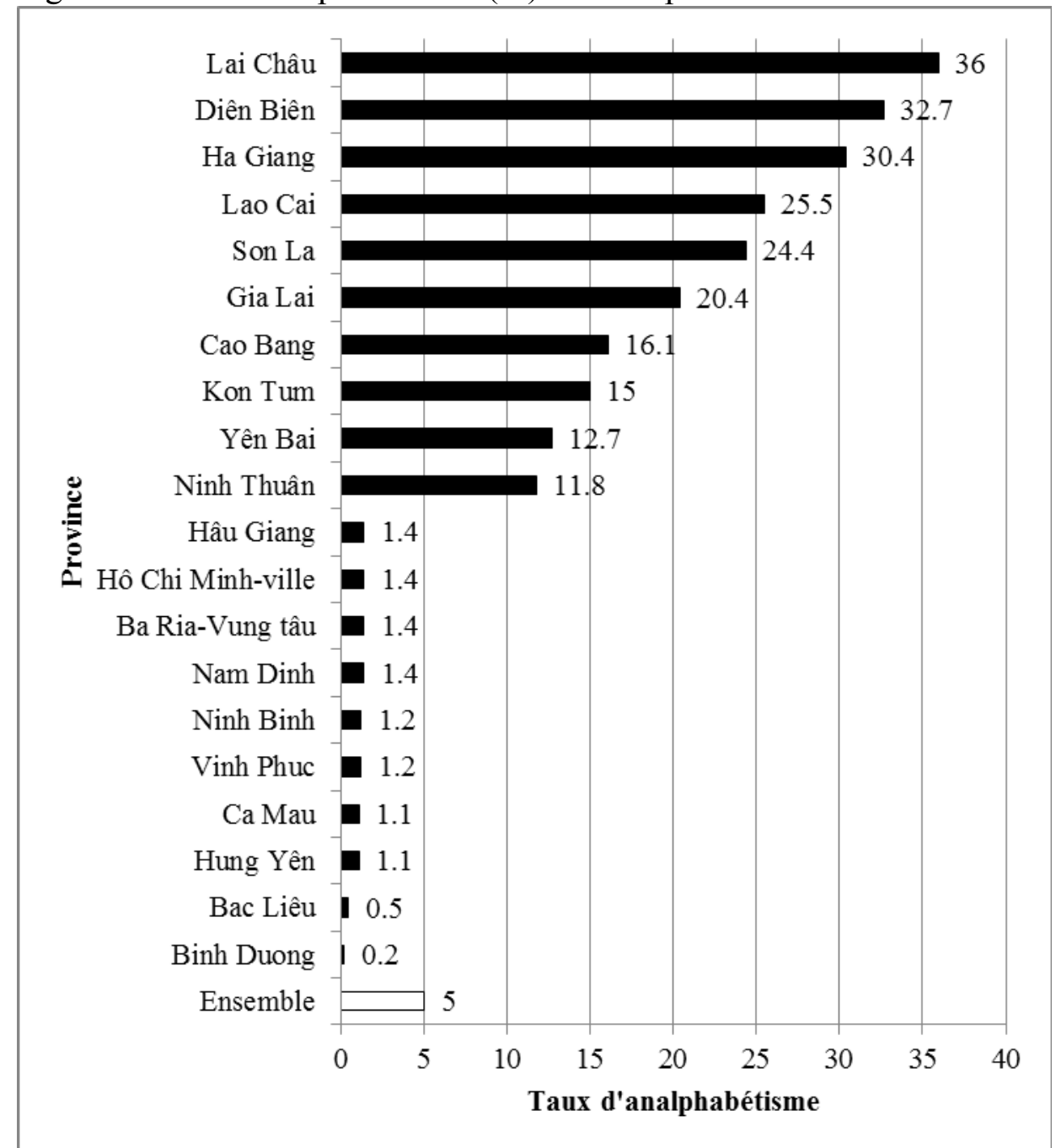

Source : Office général des Statistiques, 2011c, p. 30

Figure 2- Taux d'alphabétisation (\%) selon le groupe ethnique en 2009

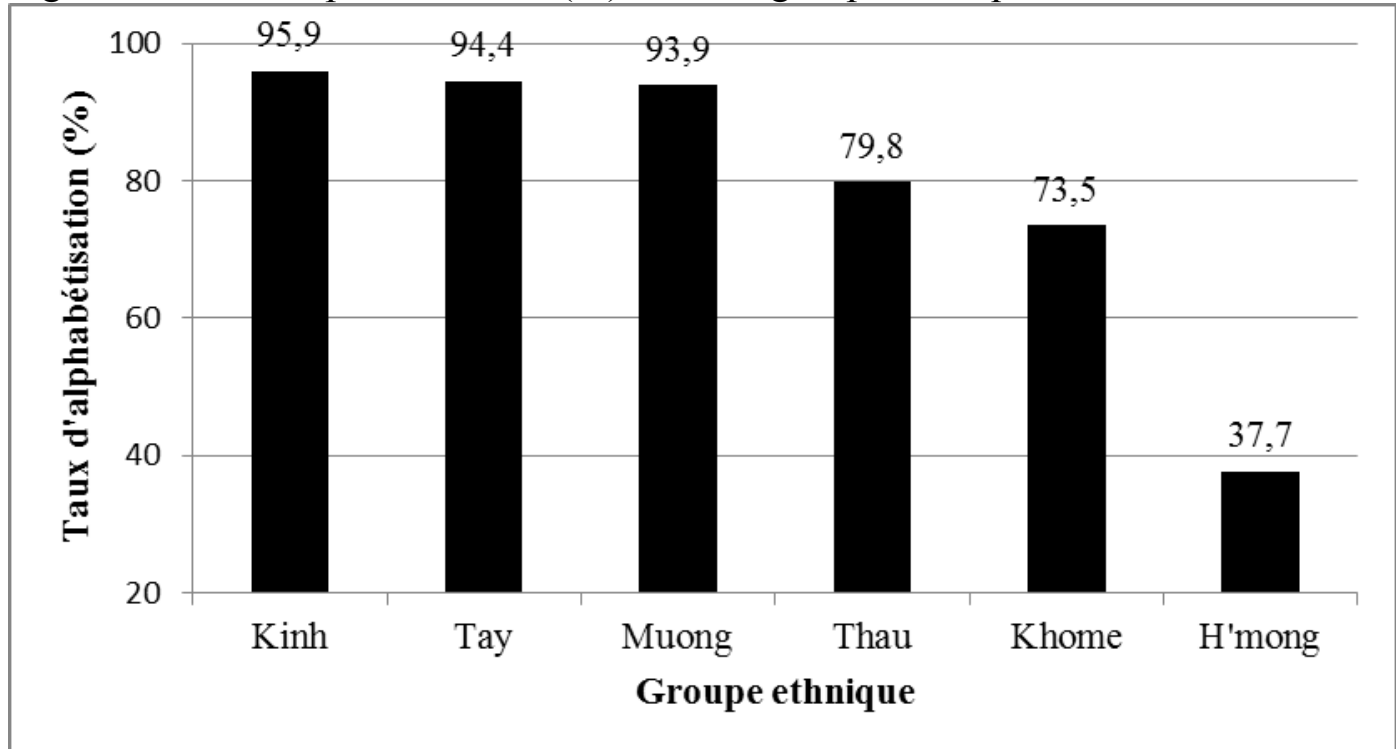

Source : Office général des Statistiques, 2011c, p. 26 


\section{I.3- Mariage précoce}

Selon les résultats des recensements, la proportion de personnes mariées parmi celles âgées de 15 ans et plus est assez élevée (67\% des hommes et $64 \%$ des femmes en 2009). L'âge moyen au premier mariage est de 24,5 ans (26,2 ans pour les hommes et 22,8 ans pour les femmes). C'est au Sud-est que cet âge est le plus élevé (27,4 ans pour les hommes et 24,2 ans pour les femmes), suivi du Centre Nord et des Côtes centrales (respectivement 26,8 ans et 23,0 ans). Le Nord-Ouest est la région où cet âge est le plus bas (respectivement 24,2 ans et 21,3 ans), suivi des Hauts-Plateaux du Centre (respectivement 25,2 ans et 21,8 ans) (Office général des Statistiques, 2010a : 49). De même, le taux de mariage précoce (entre 15 et 19 ans) est très élevé dans les régions montagneuses du Nord et les Hauts-Plateaux du Centre où la proportion de personnes appartenant à des minorités ethniques est élevée. Dans les régions montagneuses du Nord, 6,4\% des hommes et 15,9\% femmes âgés de 15 à 19 ans sont mariés. Dans les Hauts-Plateaux du Centre, ces taux sont respectivement de 3,3\% et 12,1\% (Office général des Statistiques, 2010a : 51). Dans certaines provinces de ces régions, plus de $20 \%$ des femmes et plus de $10 \%$ des hommes de cette tranche d'âge sont déjà mariés.

\section{I.4- Fécondité}

Après 50 années de mise en œuvre de la politique de planification familiale, le taux de natalité a fortement chuté. Les résultats des recensements montrent que l'indice synthétique de fécondité de six enfants par femme en 1961 a été ramené à 2,09 en 2009, niveau inférieur au taux de remplacement. Selon le recensement de 1989, en moyenne, chaque femme en âge de procréer (15-49 ans) avait 3,8 enfants, mais ce taux n'était que de 2,3 enfants au recensement de 1999, et 2,03 enfants au recensement de 2009. Les statistiques démographiques annuelles montrent que le niveau de remplacement (2,1 enfants par femmes) a été atteint en 2006. Toutefois, il existe des écarts importants entre provinces et entre groupes ethniques.

Le nombre d'enfants par femme reste élevé en milieu rural, notamment dans les régions montagneuses du Nord-ouest, du Centre Nord et des Hauts-Plateaux du Centre (indice synthétique de fécondité respectivement de 2,26, 2,30 et 2,83 enfants par femme). Les provinces ayant un taux de fécondité élevé sont également celles ayant une forte proportion d'illettrés dans la population féminine. Parmi les 63 provinces du pays, certaines localités ont connu une forte baisse. À Ho Chi Minh-ville l'indice synthétique de fécondité a atteint 1,5 enfant par femme, tandis qu'il reste très élevé ailleurs (3,1 à Ha Giang, 2,9 à Gia Lai). Les ethnies Kinh, Tay, Muong et Khmer ont atteint le niveau de remplacement (environ 2,0 enfants par femme), tandis que ceux d'autres groupes ethniques restent à des niveaux très élevés, notamment les Thaïs (2,3 enfants par femme), les Mong (4,9 enfants par femme) (Office général des Statistiques, 2010a : 171).

Le modèle reproductif des femmes vietnamiennes connaît une mutation non seulement en ce qui concerne le nombre d'enfants, mais aussi l'âge à la maternité. En 1999, le taux de fécondité le plus élevé était celui du groupe des 20-24 ans (158\%o), mais en 2009, le maximum était atteint par le groupe des 25-29 ans (133\%o). Ainsi, par rapport à 1999, en 2009 les Vietnamiennes avaient moins d'enfants et à un âge plus avancé (Office général des Statistiques, 2010a : 57). 


\section{I.5- Rapport de masculinité à la naissance}

L'un des risques pour la structure de la population est le déséquilibre du rapport de masculinité à la naissance. Ce déséquilibre est susceptible d'accroître les tensions sur le marché matrimonial, de favoriser une hausse de l'âge au mariage et la recherche d'une épouse à l'étranger. Les expériences qu'ont vécues la Chine, Taïwan et la République de Corée ont montré qu'un rapport de masculinité à la naissance dépassant 113 engendre de graves conséquences sociales à long terme. Au Viêt Nam, ce rapport n'a cessé d'augmenter ces dernières années pour atteindre un niveau inquiétant. Contrairement aux pays voisins où le choix du sexe du fœtus s'effectue pour l'ensemble des naissances suivantes, cette sélection se fait dès la première naissance (Guilmoto, 2011).

De 105 lors du recensement de 1979, 106 lors du recensement de 1989, 107 lors du recensement de 1999, le rapport de masculinité à la naissance a atteint 111 lors du recensement de 2009. Il est particulièrement élevé dans certaines provinces telles que Hung Yên (131), Hai Duong (120), Bac Ninh (119), Nam Dinh (116), Haiphong (115), toutes dans le delta du Fleuve Rouge. Le delta du fleuve Rouge a le niveau le plus haut pour les 0-4 ans (de 115 à 125 selon les provinces). Cet indicateur est de 110 dans les régions montagneuses du Nord, le Centre Nord, les Côtes centrales, le Sud-est et le delta du Mékong (voir Carte 1 en tête d'ouvrage). Il est de 105 dans les Hauts-Plateaux du Centre (Figure 3).

Figure 3- Rapport de masculinité à la naissance selon la région en 2009

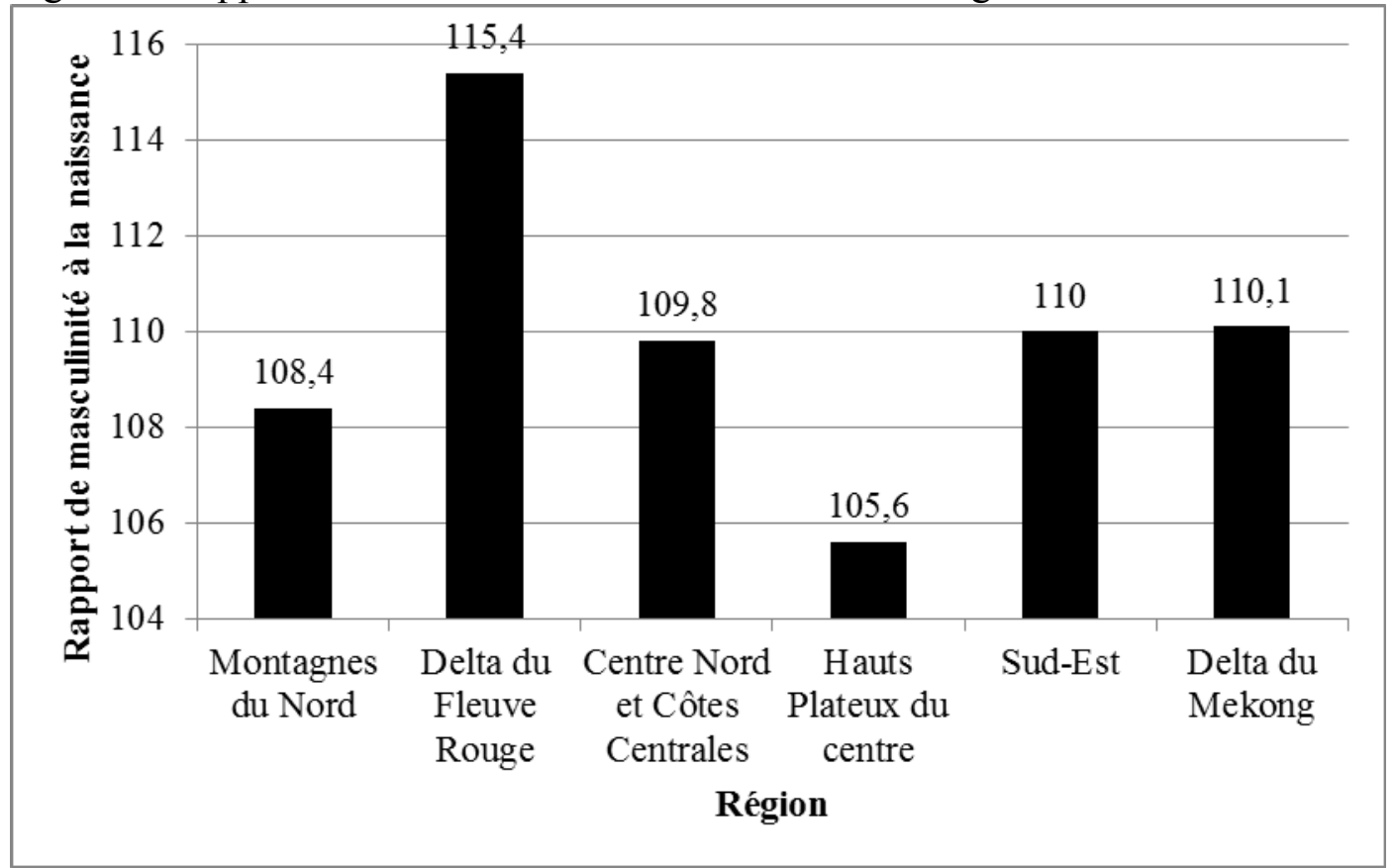

Source : Office général des Statistiques, 2011b, p. 19

Le rapport de masculinité des moins de 14 ans a fortement augmenté. Le recensement de 2009 a révélé un rapport de masculinité des 15-24 ans, supérieur à celui de 1999. Cela montre que le choix du sexe de l'enfant à la naissance a pu apparaître dès les années 1990 quand a commencé la mise en œuvre de la politique de planification familiale visant à limiter la taille de la famille à un ou deux enfants (Résolution du $\mathrm{IV}^{\mathrm{e}}$ plénum du comité central du Parti communiste vietnamien, VII ${ }^{\mathrm{e}}$ exercice, 1993). Il est à noter que le déséquilibre du taux de masculinité à la naissance survient chez les groupes ayant un niveau de vie aisé. En effet, ce taux est élevé chez les plus riches, les riches et la classe moyenne. Ces trois groupes 
représentent jusqu'à $60 \%$ de la population. Ce taux atteint 113 dans la classe moyenne (Figure 6).

Figure 4- Rapport de masculinité à la naissance selon le niveau socio-économique en 2009

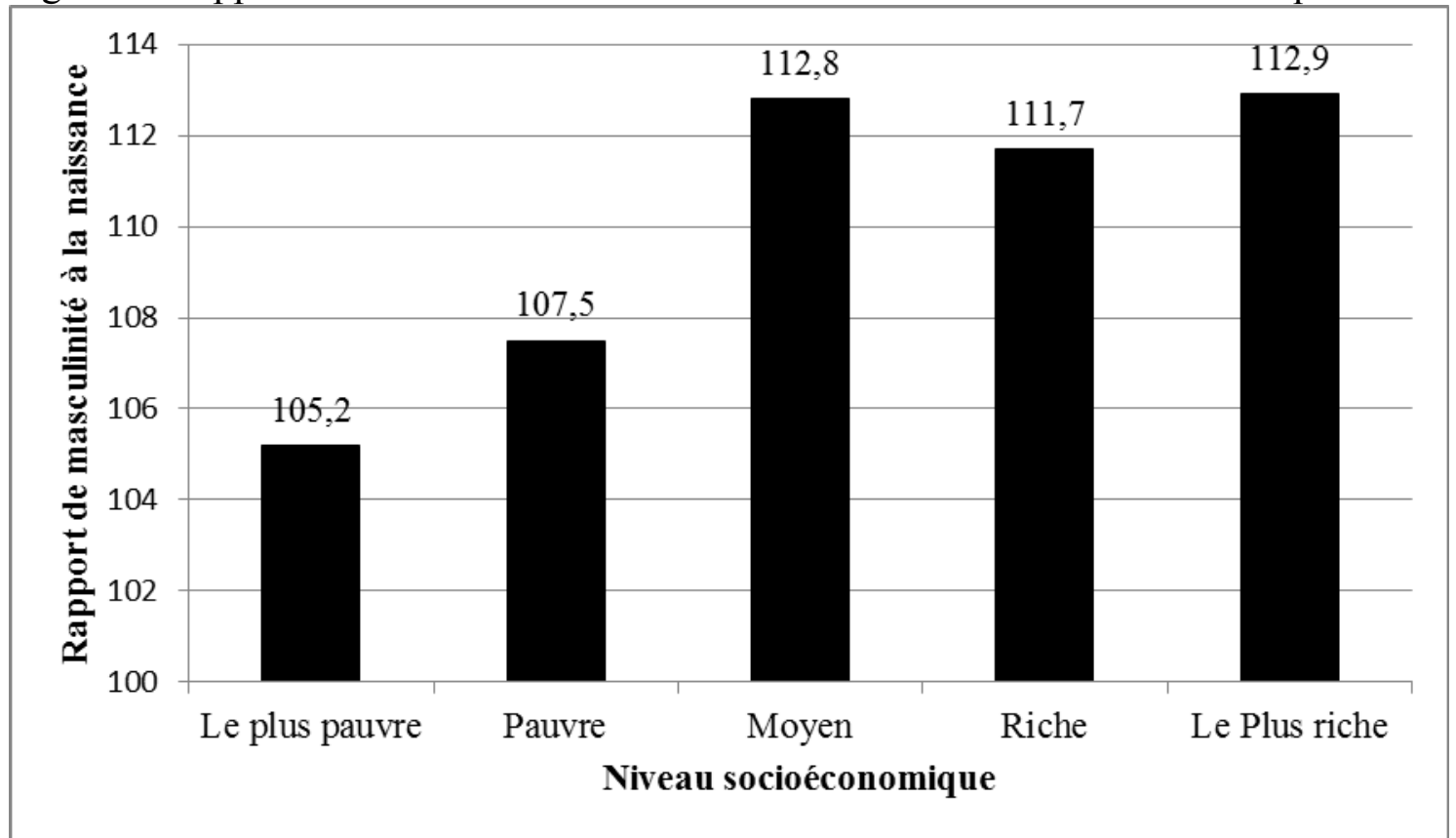

Source : Office général des Statistiques, 2011b, p. 30

\section{I.6- Migration interne}

Selon les résultats du recensement de 2009, l'effectif des migrants internes ${ }^{8}$ (ceux dont le lieu de résidence n'était pas le même cinq ans auparavant) avait doublé par rapport à la période 1994-1999. En effet, de 2004 à 2009, près de 7 millions de personnes ont migré, avec une forte hausse chez les jeunes. Cette augmentation suit toutefois des tendances différentes selon les régions.

En ce qui concerne l'émigration, le Centre Nord et les Côtes centrales occupent la première place avec plus de 775000 émigrés en 5 ans (2004-2009), ce qui correspond à une multiplication par un facteur de plus de 1,8 par rapport à la période 1994-1999. Le delta du Mékong est en deuxième position avec 734000 émigrés, soit une multiplication par près de 3,5. Il s'agit de la hausse la plus importante. Le delta du Mékong est d'ailleurs la région ayant le plus fort taux d'émigration du pays. Les émigrés du Centre-nord, des Côtes centrales et du delta du Mékong représentaient $48 \%$ de ceux de l'ensemble du pays durant la période 19941999 et 64 \% durant la période 2004-2009. Autrement dit, le Centre septentrional, les Côtes centrales et le delta du Mékong constituent les principales régions d'émigration (Figure 5). Le nombre d'émigrés des Hauts-Plateaux du Centre, bien que le plus faible de toutes les régions a aussi doublé. Il est à signaler une «inversion» des courants migratoires dans les HautsPlateaux du Centre. Région principale d'immigration après la réunification du pays, ils connaissent de nos jours une forte émigration. Les régions montagneuses du Nord connaissent également une forte tendance à l'émigration, multipliée par plus de 1,5 entre les deux derniers recensements. Le nombre d'émigrés du delta du fleuve Rouge et du Sud-est reste assez élevé, mais n'augmente pas.

$8 \quad$ Une personne est considérée comme migrante si ses lieux de résidence au moment du recensement et cinq ans auparavant ne se situent pas dans la même commune » (unité administrative la plus petite) (Comité central de pilotage du Recensement de la population et du logement de 2009, Hanoi, 2010). 
Les provinces méridionales sont la destination de la majorité des émigrés. Le Sud-est, les Hauts-Plateaux du Centre et le delta du Mékong attirent à eux seuls $80 \%$ des émigrés durant la période 2004-2009 (voir Carte 1 en tête d'ouvrage). Le Sud-est est l'unique région caractérisée par un solde migratoire positif (Figure 5). C'est la première région d'immigration selon trois critères : le nombre de personnes, le taux de croissance et le taux d'immigration. En 5 ans (2004-2009), 1635000 personnes ont migré vers cette région, soit trois fois plus que durant la période 1994-1999. Le taux d'immigration est de 127 pour mille. Il est trois fois plus élevé que celui des Hauts-Plateaux du Centre, huit fois plus que celui le Delta du fleuve Rouge et 32 fois plus que celui du Delta du Mékong. La population du Sud-est ne représente que $16,3 \%$ de la population nationale, mais ses entreprises représentent $37 \%$ de celles du pays (chiffres calculés au 31 décembre 2007) et la région a attiré jusqu'à $44 \%$ des investissements directs étrangers en 20 années (1988-2008). Il est à noter que si durant les 10 premières années (1988-1998), seulement 43\% des migrants ont choisi le Sud-est comme destination, durant les dix dernières années (1998-2008), ce taux s'est élevé à 70\%. En particulier, 99,7 \% des émigrés du Delta du Mékong ont choisi le Sud-est (Nguyên 2010).

Figure 5- Effectif d'émigrés et d'immigrés selon la région en 2009

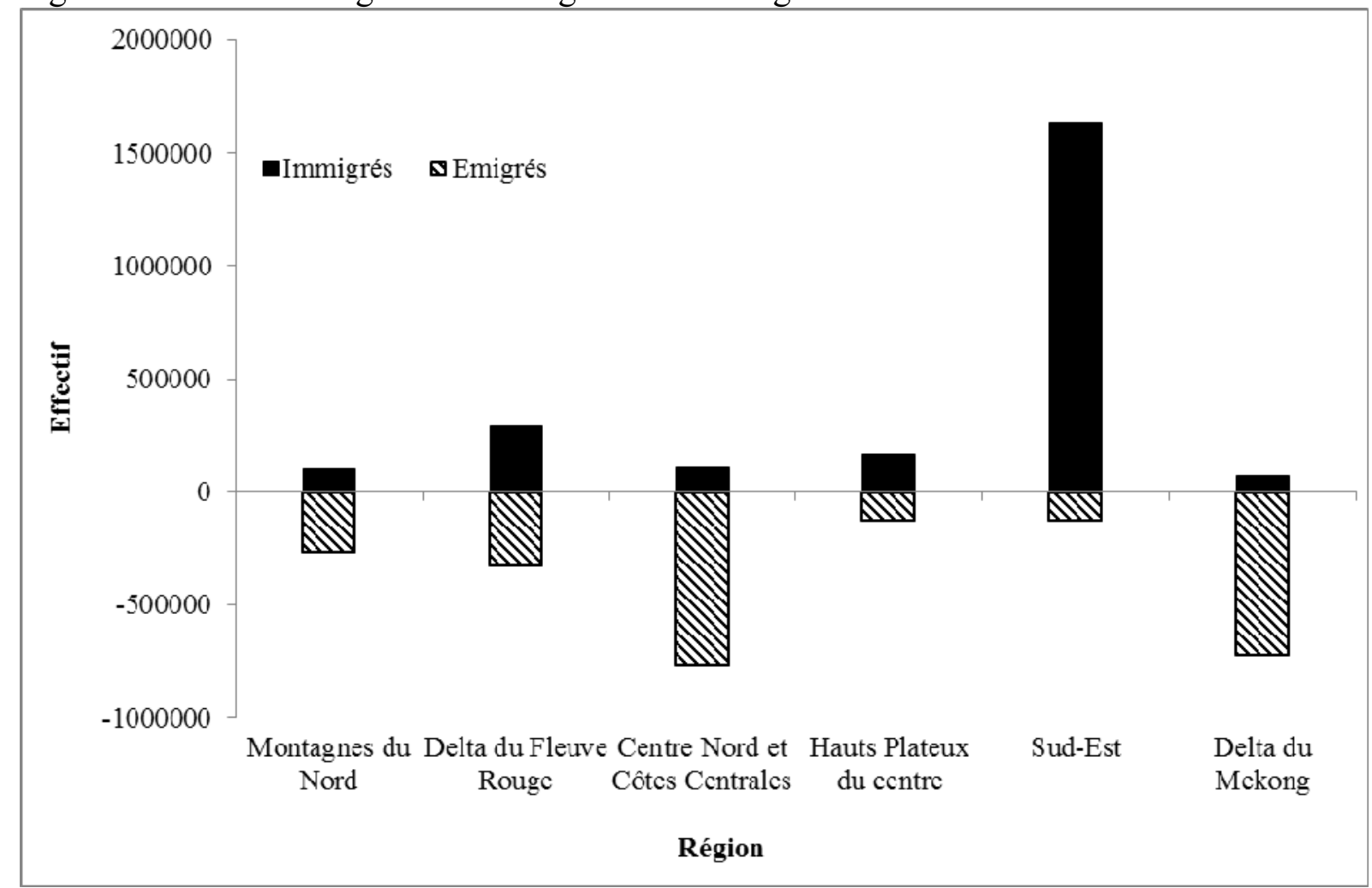

Source: Office général des Statistiques, 2011d, p. 32

\section{I.7- Mortalité infantile}

Le Viêt Nam a connu un grand succès dans la réduction de la mortalité des enfants de moins d'un an. Le taux de mortalité infantile est passé de 44, 4\%o en 1990 à 16,0\%o en 2009. Cependant, il existe une grande disparité entre les milieux ainsi qu'entre les provinces. En effet, le taux de mortalité infantile en milieu rural atteint le double de celui du milieu urbain $(18,7 \%$ contre $9,4 \%$ ). Dans de nombreuses provinces peuplées par des minorités ethniques, il atteint même 30,0\%o à 44,0\%. Dans le delta du fleuve Rouge, le Sud-est, le delta du Mékong, il est inférieur à la moyenne nationale (respectivement 12,4\%, 10,0\%o et 13,3\%o). Par contre, dans le Centre Nord, les côtes centrales, les régions montagneuses du Nord et le 
delta du Mékong, il reste élevé (respectivement 17,2 \%o, 24,5 \%o et 27,3\%o) (Office général des Statistiques, 2010a : 69). Parmi les 63 provinces, Lai Chau, Kon Tum et Ha Giang ont les taux de mortalité infantile les plus élevés (respectivement 44,7 \%o, 38,2 \%o et 37,5\%o). Ho Chi Minh-ville, Can Tho et Dong Nai sont les trois localités ayant les taux les plus faibles (respectivement 8,9\%, 9,6\%o et 9,8\%o). En ce qui concerne les groupes ethniques, les Kinh et les Kho Me ont des taux de mortalité infantile faibles $(12,9 \%$ et $18,0 \%$ o) tandis que ce taux reste élevé chez les Thai, Tay et Muong (27,1\%o, 23,4 \%o, 22,2 \%o). Il est de 45,5\%o chez les Mong et 32,0\% dans les autres groupes (Office général des Statistiques, 2010a : 149, 171).

\section{I.8- Handicap}

Au total, 7,8\% de la population, soit 6,1 millions de personnes âgées de 5 ans et plus ont au moins un handicap (auditif, visuel, moteur ou cérébral). Ce taux est plus élevé chez les femmes que chez les hommes $(8,5 \%$ versus $7,1 \%)$ et dans les régions rurales que dans les régions urbaines $(8,4 \%$ versus $6,5 \%)$. Il est particulièrement élevé chez le groupe âgé de plus de 50 ans, conséquence de la guerre et de l'âge (Office général des Statistiques, 2010a : 168). Le Centre Nord et les Côtes centrales ont les taux les plus élevés $(9,7 \%)$, suivis du delta du Fleuve Rouge $(8,1 \%)$. Ces régions ont été gravement ravagées par les bombes et les mines durant la guerre de 1965 à 1975. Par ailleurs, ce taux est de 8,0\% dans les provinces montagneuses du Nord, 7,2 \% dans le delta du Mékong, 6,7 \% dans les Hauts-Plateaux du Centre et 5,7 \% au Sud-est (Office général des Statistiques, 2010a : 168).

\section{II- Dividende démographique et vieillissement de la population}

Le Viêt Nam est en phase de dividende démographique. Dans 43 des 63 provinces, le taux de dépendance est de moins de 50 inactifs pour 100 actifs (structure " en or » de la population). Cette période a commencé en 2007 et pourrait durer 40 à 45 ans. Cette partie analyse les changements de la structure par âge de la population au cours des trois dernières décennies et met en évidence les tendances pour les trois décennies à venir.

\section{$\underline{\text { II.1- Taux de dépendance et « structure en or » de la population }}$}

Le taux de dépendance ${ }^{9}$ a baissé de 89,5 à $46,4 \%$, soit une division par deux en 30 ans (1979-2009). En 2006, il n'était que de 49,9\%. C'est à cette date que commence donc le phénomène d'opportunité ou dividende démographique appelé «structure en or ». Celui-ci intervient quand la structure de la population selon l'âge est bénéfique au développement économique, en raison d'un effectif de la population active équivalent au double de celui des dépendants. Il s'est accentué au cours des années suivantes (Tableau 1). Seul le taux de dépendance des personnes âgées a tendance à augmenter faiblement.

La Division de la Population des Nations Unies prévoit qu'en Asie, la période de la structure en or de la population durera environ 30 ans pour l'Indonésie, la Malaisie, les Philippines ; 35 ans pour Singapour et la Thaïlande ; 40 ans pour la Chine, le Japon et la République de Corée. Le développement du Japon, de la République de Corée et de Singapour, tout comme la croissance rapide des nouveaux pays industrialisés sont étroitement liés à la période de la «structure en or» de la population (United Nations, 2009). Selon les

$9 \quad$ Le taux de dépendance désigne le nombre de personnes d'âge inactif (moins de 15 ans pour les jeunes et plus de 65 ans pour les personnes âgées) pour une personne d'âge actif (15 à 64 ans). 
prévisions de l'ONU de $2008^{10}$, la «structure en or » de la population du Viêt Nam commencerait en 2008 et se terminerait en 2035, soit après une durée de 27 ans. De son côté, le Département de la population et du planning familial du Viêt Nam prévoyait qu'elle durerait 33 ans (2005 à 2038) selon le premier plan (prévision basse) et 46 ans (2005 à 2051) selon le deuxième plan (prévision haute). Toutefois, ces deux prévisions sont antérieures à la publication des résultats du recensement de 2009. Elles ont été proposées sur la base d'un indice synthétique de fécondité réduit à 1,1 enfant par femme comme le taux actuel de la République de Corée dans un cas, stable jusqu'en 2060 dans l'autre. Ces hypothèses semblent ne pas correspondre à la réalité des changements de la fécondité au Viêt Nam (United Nations, 2009).

Tableau 1- Taux de dépendance (\%) selon l'année de 1979 à 2012

\begin{tabular}{lrrrrrrr} 
Taux de dépendance & 1979 & 1989 & 1999 & 2006 & 2009 & 2011 & 2012 \\
\hline Des jeunes & 80,6 & 69,1 & 55,1 & 39,4 & 36,6 & 34,9 & 34,6 \\
Des personnes âgées & 8,9 & 8,2 & 9,6 & 10,5 & 9,8 & 10,1 & 10,3 \\
\hline Global & 89,5 & 77,3 & 64,7 & 49,9 & 46,4 & 45,0 & 44,9 \\
\hline
\end{tabular}

Source: Office général des Statistiques, 2000; Office général des Statistiques, 2007; Office général des Statistiques, 2010; Office général des Statistiques, 2013.

Après la publication des résultats du recensement de la population et du logement de 2009, l'Office général des Statistiques (2010) a publié des «Prévisions sur la population du Viêt Nam pour la période 2009-2049 ». Trois hypothèses ont été avancées, correspondant aux trois plans prévisionnels. La plus haute prévoit un indice synthétique de fécondité (ISF) de 2,15 enfants par femme en 2049. En réalité, il est difficile que ce plan se matérialise, car plus le niveau de développement s'élève, plus le taux de natalité baisse. Le Viêt Nam était encore en voie de développement lorsque l'ISF a atteint 2,03 enfants par femme en 2009. Cette hypothèse est donc peu probable.

Les prévisions du Fonds des Nations Unies pour la population et du groupe d'experts de l'Institut d'Étude sur la Population et la Société se fondent sur les résultats du recensement de 2009. Elles reposent sur des hypothèses plus convaincantes selon lesquelles l'ISF moyen serait respectivement de 1,8, de 1,6 ou de 1,4 enfant par femme selon différentes hypothèses (haute, moyenne, basse). Pour établir le calendrier de cette fenêtre d'opportunité, le logiciel Spectrum 3.1 a été utilisé. Dans ce modèle qui prend comme population de base celle du recensement de 2009 , le taux brut de natalité est de $17,8 \%$ ot les taux de fécondité par âge suivent un modèle spécifique à l'Asie, on ne prend pas en compte la migration internationale, la table de mortalité est celle du modèle «Custom » et l'espérance de vie en 2059 est de 78 ans pour les hommes et 82 ans pour les femmes. Enfin, la proportion de la population urbaine en 2059 est de $70 \%$. Selon ces prévisions, la «structure en or » de la population durerait 39 ans avec un ISF de 1,8 enfant par femme ; 42 ans avec un ISF de 1,6 enfant par femme ; 45 ans avec un ISF de 1,4 enfant par femme (Tableau 2).

Tableau 2- Paramètres de la «structure en or» de la population selon les prévisions de l'IPSS (Université Nationale d'Économie)

\begin{tabular}{lccc} 
Hypothèse & Haute & Moyenne & Basse \\
\hline Indice synthétique de fécondité & 1,8 & 1,6 & 1,4 \\
Année de début & 2006 & 2006 & 2006 \\
Année de fin & 2044 & 2047 & 2050 \\
Durée en années & 39 & 42 & 45 \\
\hline
\end{tabular}

Source : Nguyên et coll. 2009. 
Les prévisions montrent que 2016 serait l'année où le taux de dépendance serait le plus faible, à 43\% (Tableau 3). Cette année-là, la population serait de 92,2 à 92,4 millions d'habitants.

Tableau 3- Taux de dépendance et nombre d'habitants selon les dates de la «structure en or » de la population

\begin{tabular}{ccccccc} 
Année & \multicolumn{2}{c}{ Hypothèse haute } & \multicolumn{2}{c}{ Hypothèse moyenne } & \multicolumn{2}{c}{ Hypothèse basse } \\
& $\begin{array}{c}\text { Taux de } \\
\text { dépendance }\end{array}$ & $\begin{array}{c}\text { Habitants } \\
\text { (millions) }\end{array}$ & $\begin{array}{c}\text { Taux de } \\
\text { dépendance }\end{array}$ & $\begin{array}{c}\text { Habitants } \\
\text { (millions) }\end{array}$ & $\begin{array}{c}\text { Taux de } \\
\text { dépendance }\end{array}$ & $\begin{array}{c}\text { Habitants } \\
\text { (millions) }\end{array}$ \\
\hline 2015 & 43,37 & 91,49 & 43,27 & 91,42 & 43,16 & 91,35 \\
2016 & 43,35 & 92,43 & 43,23 & 92,34 & 43,08 & 92,25 \\
2017 & 43,41 & 93,35 & 43,25 & 93,24 & 43,08 & 93,12 \\
\hline
\end{tabular}

Source : Nguyên et coll. 2009.

En 2009, 43 des 63 provinces sont entrées dans la période de dividende démographique avec un taux de dépendance de moins de $50 \%$. Les 20 autres provinces entreront dans cette période au cours des années suivantes. Ainsi, le dividende démographique intervient à des périodes très différentes selon les provinces, avec un écart de 10 à 15 ans. Cette disparité est source de difficultés dans l'élaboration des politiques de développement.

\section{$\underline{\text { II.2- Population d'âge actif et population en activité }}$}

Le Code du travail stipule que l'âge actif va de 15 à 60 ans pour un homme et de 15 à 55 ans pour une femme. Les résultats des recensements montrent qu'au cours des 30 dernières années, la proportion de la population d'âge actif a connu une augmentation rapide dans les populations masculine et féminine (Tableau 4). $(\%)$

Tableau 4- Répartition de la population selon les groupes d'âge et le sexe de 1979 à 2009

\begin{tabular}{cccccccccc} 
& \multicolumn{4}{c}{ Population masculine } & \multicolumn{5}{c}{ Population féminine } \\
Année & $0-14$ & $15-59$ & $60+$ & Total & Année & $0-14$ & $15-54$ & $55+$ & Total \\
\hline 1979 & 45,0 & 49,0 & 6,0 & 100,0 & 1979 & 40,3 & 50,3 & 9,4 & 100,0 \\
1989 & 41,3 & 53,5 & 6,2 & 100,0 & 1989 & 36,9 & 51,8 & 11,3 & 100,0 \\
1999 & 34,6 & 59,0 & 6,7 & 100,0 & 1999 & 31,7 & 56,7 & 11,9 & 100,0 \\
2009 & 26,4 & 66,2 & 7,4 & 100,0 & 2009 & 23,7 & 62,1 & 14,2 & 100,0
\end{tabular}

Source: Office général des Statistiques, 2010; Office général des Statistiques, 2000.

En effet, en 2009 , la population active représentait $66,2 \%$, de la population masculine, soit 17,2 points de plus qu'en 1979 . Elle était de $62,1 \%$ de la population féminine en 2009 , soit une hausse de 11,8 points par rapport à 1979. Au cours des dix dernières années, en moyenne, chaque année, 1200000 personnes entraient dans cette catégorie tandis que seulement 500000 en sortaient. Cependant, toutes les personnes d'âge actif ne sont pas en situation d'emploi, comme le montrent les résultats de l'enquête sur les changements en matière de population et de main d'œuvre. La proportion des femmes atteignant l'âge de la retraite (55 à 59 ans selon le Code du travail) toujours en emploi est nettement supérieure à celle des femmes de 15 à 19 ans.

Dans les régions rurales où est concentrée la majorité de la main d'œuvre du pays, chez les hommes comme chez les femmes, le groupe des 60-64 ans a des taux d'activité plus élevés que les 15-19 ans (Tableau 5). Cette situation est observée en milieu urbain pour les hommes, mais elle est un peu différente pour les femmes : le taux d'activité des femmes âgées de 60 à 64 ans est de 20,7\% tandis qu'il est de 23,5\% dans le groupe des 15-19 ans. Dans l'ensemble, l'âge de la participation aux activités professionnelles est souvent plus élevé que l'âge actif défini par la législation. Dans le processus d'amendement du Code du travail en 2012, 
l'Assemblée nationale a discuté de la prolongation de l'activité au-delà de l'âge fixé par la législation. Toutefois, la réglementation selon laquelle l'âge de la retraite est de 60 ans chez les hommes et de 55 ans chez les femmes a été maintenue.

Tableau 5- Part (\%) de la population en activité selon le groupe d'âge et le sexe en 2006

\begin{tabular}{lrrrr} 
Groupe d'âge & \multicolumn{2}{c}{ Urbain } & \multicolumn{2}{c}{ Rural } \\
& Homme & \multicolumn{1}{c}{ Femme } & Homme & \multicolumn{1}{c}{ Femme } \\
\hline $15-19$ & 28,3 & 23,5 & 41,7 & 40,0 \\
$20-24$ & 70,6 & 61,4 & 88,6 & 80,1 \\
$25-29$ & 94,9 & 79,7 & 97,6 & 85,4 \\
$30-34$ & 97,3 & 78,2 & 98,5 & 87,5 \\
$35-39$ & 97,1 & 76,4 & 98,5 & 87,9 \\
$40-44$ & 96,7 & 76,8 & 98,1 & 88,4 \\
$45-49$ & 92,4 & 72,0 & 96,8 & 85,5 \\
$50-54$ & 83,0 & 56,2 & 92,2 & 76,1 \\
$55-59$ & 62,9 & 32,3 & 83,0 & 61,1 \\
$60-64$ & 33,6 & 20,7 & 65,5 & 43,8 \\
$65+$ & 13,8 & 6,8 & 27,9 & 13,8 \\
\hline Total & 74,0 & 56,7 & 79,8 & 67,5 \\
\hline
\end{tabular}

Source : Office général des Statistiques, 2007.

En 2011, la répartition de la main-d'œuvre âgée de 15 ans et plus comprenait 6,5\% d'hommes âgés de plus de 60 ans et $13 \%$ de femmes âgées de plus de 55 ans. Ces proportions étaient respectivement de $4,5 \%$ et $9,4 \%$ dans les régions urbaines, $7,4 \%$ et $14,6 \%$ dans les régions rurales. Il est à noter que parmi les femmes de 15 ans et plus en milieu rural, celles âgées de plus de 55 ans sont les plus nombreuses (Tableau 6).

Tableau 6- Part (\%) de la population en activité selon le sexe et selon le milieu de résidence en 2011

\begin{tabular}{lrrrrrr}
\hline Groupe d'âge & \multicolumn{2}{c}{ Milieu urbain } & \multicolumn{2}{c}{ Milieu rural } & \multicolumn{2}{c}{ Ensemble } \\
& Homme & \multicolumn{1}{c}{ Femme } & Homme & Femme & Homme & Femme \\
\hline $15-19$ & 3,7 & 3,2 & 7,7 & 6,4 & 6,5 & 5,5 \\
$20-24$ & 9,1 & 10,1 & 11,6 & 10,0 & 10,9 & 10,1 \\
$25-29$ & 13,9 & 14,7 & 12,4 & 12,2 & 12,9 & 12,9 \\
$30-34$ & 13,2 & 13,9 & 11,8 & 12,2 & 12,2 & 12,7 \\
$35-39$ & 13,5 & 13,7 & 12,2 & 12,0 & 12,6 & 12,5 \\
$40-44$ & 13,1 & 12,8 & 11,3 & 11,7 & 11,9 & 12,0 \\
$45-49$ & 12,4 & 12,0 & 10,8 & 11,6 & 11,3 & 11,7 \\
$50-54$ & 10,4 & 10,2 & 8,6 & 9,4 & 9,1 & 9,6 \\
$55-59$ & 6,1 & 5,0 & 6,2 & 6,4 & 6,1 & 6,0 \\
$60-64$ & 2,4 & 2,3 & 3,4 & 3,9 & 3,1 & 3,4 \\
$65+$ & 2,1 & 2,1 & 4,0 & 4,3 & 3,4 & 3,6 \\
\hline Total & 100,0 & 100,0 & 100,0 & 100,0 & 100,0 & 100,0 \\
\hline
\end{tabular}

Source : Office général des Statistiques, 2012.

II.3- Changements des proportions des groupes d'âge: évolution récente et perspectives

\section{Evolution récente}

Le tableau 7 décrit la structure de la population selon les groupes d'âge quinquennaux, d'après les recensements de 1979, 1989, 1999 et 2009. Il montre des évolutions notables. La proportion des individus âgés de 15 à 29 ans a peu changé au cours des 30 dernières années. En particulier, la proportion des 20-24 ans n'a pratiquement pas changé. Les groupes d'âge ayant connu les plus fortes baisses sont les enfants de moins de 14 ans, avec une réduction allant jusqu'à $40 \%$ dans certains groupes par rapport à 1979. Au cours des 30 dernières 
années, leur part est passée de $45 \%$ en 1979 à $26 \%$ en 2009. Cette forte baisse correspond aux faibles effectifs des générations nées de 1995 à 2009. En particulier, la part des enfants âgés de 5 à 9 ans qui représentaient $14 \%$ de la population en 1979 n'en représentait plus que $8 \%$ en 2009 , soit une réduction de moitié. La Résolution du IV ${ }^{\mathrm{e}}$ Plénum du comité central du Parti communiste vietnamien dans son $\mathrm{VII}^{\mathrm{e}}$ exercice a proposé une politique de population et de planification familiale. Celle-ci a été mise en œuvre avec succès. Elle a entraîné une réduction rapide du taux de natalité et du nombre d'enfants. La forte baisse de la part des enfants âgés de moins de 14 ans a créé des opportunités de prévention de la malnutrition et d'amélioration de l'éducation tant pour les garçons que pour les filles. Le nombre d'élèves à l'école primaire et au collège a fortement baissé.

Tableau 7- Proportion (\%) de la population selon le groupe d'âge et l'année, variation (ratio) de 1979 à 2009

\begin{tabular}{lrrrrrr}
$\begin{array}{l}\text { Groupe } \\
\text { d'âge }\end{array}$ & 1979 & 1989 & 1999 & 2009 & $\begin{array}{r}2009 / \\
1979\end{array}$ & Evolution 1979-2009 \\
\hline $0-4$ & 14,6 & 14,0 & 9,5 & 8,5 & 0,58 & \\
$5-9$ & 14,6 & 13,3 & 12,0 & 8,0 & 0,55 & Forte baisse \\
$10-14$ & 13,4 & 11,7 & 12,0 & 8,5 & 0,64 & \\
\hline $15-19$ & 11,4 & 10,5 & 10,8 & 10,2 & 0,89 & \\
$20-24$ & 9,3 & 9,5 & 8,9 & 9,2 & 0,99 & Peu de changements \\
$25-29$ & 7,1 & 8,8 & 8,5 & 8,9 & 1,26 & \\
\hline $30-34$ & 4,7 & 7,3 & 7,9 & 7,9 & 1,68 & \\
$35-39$ & 4,0 & 5,1 & 7,3 & 7,6 & 1,88 & \\
$40-44$ & 3,8 & 3,4 & 5,9 & 7,0 & 1,84 & Forte hausse \\
$45-49$ & 4,0 & 3,1 & 4,1 & 6,4 & 1,60 & \\
$50-54$ & 3,3 & 2,9 & 2,8 & 5,3 & 1,62 & \\
\hline $55-59$ & 3,0 & 3,0 & 2,4 & 3,4 & 1,14 & \\
$60-64$ & 2,3 & 2,4 & 2,3 & 2,3 & 1,02 & Peu de changements \\
$65-69$ & 1,9 & 1,9 & 2,2 & 1,9 & 0,98 & \\
\hline $70-74$ & 1,3 & 1,2 & 1,6 & 1,7 & 1,27 & \\
$75-79$ & 0,9 & 0,8 & 1,1 & 1,4 & 1,59 & Forte hausse \\
$80-84$ & 0,4 & 0,4 & 0,6 & 0,9 & 2,32 & \\
$85+$ & 0,2 & 0,3 & 0,4 & 0,8 & 4,69 & \\
\hline Total & 100,0 & 100,0 & 100,0 & 100,0 & 100,00 & \\
\hline
\end{tabular}

Source: Office général des Statistiques, 2010a; Office général des Statistiques, 2000.

Les groupes d'âge dont la proportion a fortement augmenté sont ceux de 30 à 54 ans ainsi que 75 ans et plus. En 2009, la proportion de ces groupes d'âge a augmenté de plus de $60 \%$ par rapport à 1999. En particulier, la part des 85 ans et plus a plus que quadruplé, passant $0,16 \%$ en 1979 à $0,75 \%$ en 2009 . La forte hausse des 30-54 ans a créé un avantage, car elle correspond à une augmentation de la main d'œuvre potentielle. La proportion des individus âgés de 55 à 74 ans a peu changé. En 1975, ces personnes avaient entre 20 et 40 ans. Elles ont été les plus influencées par la guerre (décès ou santé fragilisée). Ainsi, bien que les membres de ces groupes d'âge soient nés durant des périodes où le taux de natalité était élevé, en 2009 leur part est peu importante. La part des personnes âgées (plus de 60 ans) n'a pas beaucoup augmenté ( $6 \%$ en $1979 ; 7,4 \%$ en 2009), mais elle devrait connaître une hausse considérable à l'avenir pour atteindre $15 \%$ en 2029. Ainsi, le processus de vieillissement de la population vietnamienne a bel et bien commencé. Il s'accélère. En 1999, dix provinces avaient un indice de vieillissement ${ }^{11}$ de plus de 30 . En 2009, cet effectif était de 45 provinces. Parmi ces provinces, 13 avaient un indice supérieur à 43. Le rapport de la population de 60 ans et plus à celle de moins de 15 ans est passé de 18,2 en 1989 à 24,3 en 1999 et 35,7 en 2009. Il est

\footnotetext{
${ }^{11} \mathrm{Il}$ s'agit du rapport de la population de 60 ans et plus à celle de moins de 15 ans.
} 
nettement plus élevé que la moyenne de l'Asie du Sud-est (30) (Office général des Statistiques, 2010a : 43). En 2030, les deux rapports seront égaux. Le premier équivaudra à 1,5 fois le deuxième en 2047, puis il en représentera le double en 2060.

\section{Perspectives}

Les perspectives réalisées par les chercheurs de l'Institut d'Étude sur la Population et la Société de l'Université nationale de l'Économie prévoient plusieurs types d'évolutions pour la période de la fenêtre d'opportunité démographique. Tout d'abord, la population continuerait d'augmenter jusqu'en 2049 (environ 106 millions d'habitants avec l'hypothèse d'un ISF de 1,6). Ce n'est qu'après qu'elle connaîtrait une baisse légère (Nguyên et coll. 2009). Ces perspectives prévoient que le nombre d'enfants va diminuer, tant en effectifs absolus que sur le plan relatif. Si, en 1999, au Viêt Nam, le groupe des enfants âgés de 0 à 14 ans représentait un tiers de la population $(33,1 \%)$, il n'était que d'un quart en $2009(25,0 \%)$, un cinquième en 2029 (environ 20\%) et un septième de la population en 2059 (13\%-17\% selon les différentes hypothèses relatives au taux de natalité) (Figure 8).

Figure 6- Part des 0-14 ans dans la population vietnamienne pour la période 1999-2059 $(\%)$

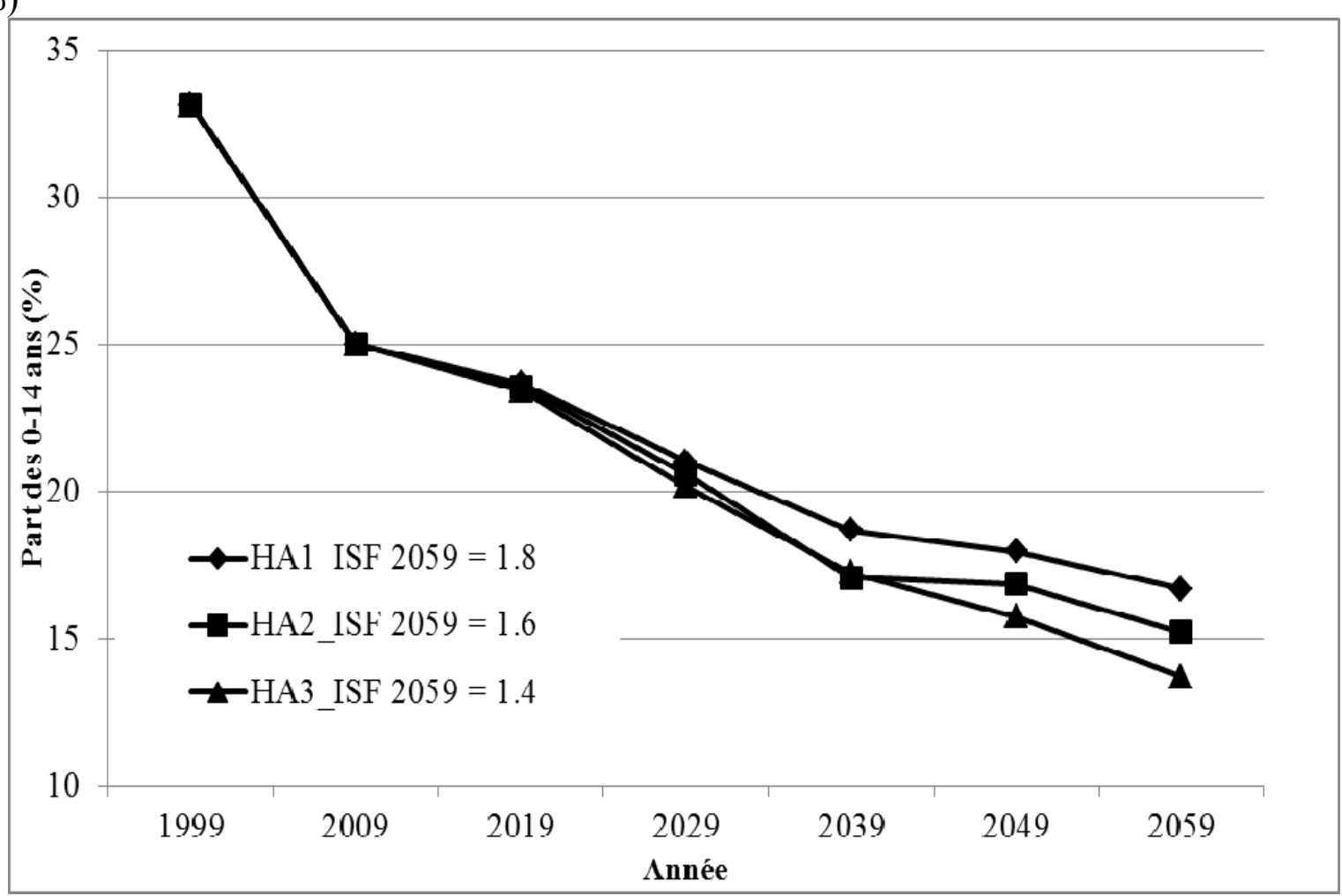

Source : Nguyên et coll. 2009.

Les prévisions montrent aussi que le nombre de jeunes mineurs (personnes âgées de moins de 18 ans) connaîtra une chute importante. En 2009, les jeunes mineurs représentaient 16,06 millions d'habitants. Avec un ISF de 1,6 enfant par femme en 2059, ce groupe serait réduit à 11,69 millions en 2059 (Tableau 8 en annexe). Par ailleurs, ces perspectives prévoient que la main d'œuvre augmentera rapidement. Selon ces prévisions, la part de la population en activité connaîtrait une hausse graduelle pour atteindre son plus haut niveau en 2039. Elle aurait ensuite tendance à baisser légèrement au cours des années suivantes (Tableau 9 en annexe). Malgré la baisse de la proportion des femmes en âge de procréer, leur nombre continuerait d'augmenter jusqu'en 2029 (Tableau 10 en annexe). Selon les prévisions du groupe d'experts de l'Institut d'Étude sur la Population et la Société, de 1999 à 2059, l'âge 
médian de la population vietnamienne va presque doubler (passant de 23 à 43 ans) (Tableau 11 en annexe) (Nguyên et coll. 2009). Le pourcentage des personnes âgées de plus de 65 ans au Viêt Nam augmentera rapidement dans les décennies prochaines. C'est la conséquence d'une baisse rapide du taux de natalité des décennies précédentes. Par rapport à 2009, le pourcentage des personnes âgées aura doublé en 2029 (13,6\% - 13,9\%) et quadruplé en 2059 (20,2\% - 21,8\%) (Figure 9). L'effectif des personnes âgées augmentera également. Si en 2009 le Viêt Nam comptait 7,67 millions de personnes âgées de plus de 60 ans, ce nombre s'élèverait à 15 voire 16 millions en 2029 et plus de 28 millions en 2059 (Tableau 12 en annexe).

Figure 7- Part des personnes âgées de 65 ans et plus dans la population de 1999 à 2059 selon l'hypothèse relative à l'évolution de la fécondité

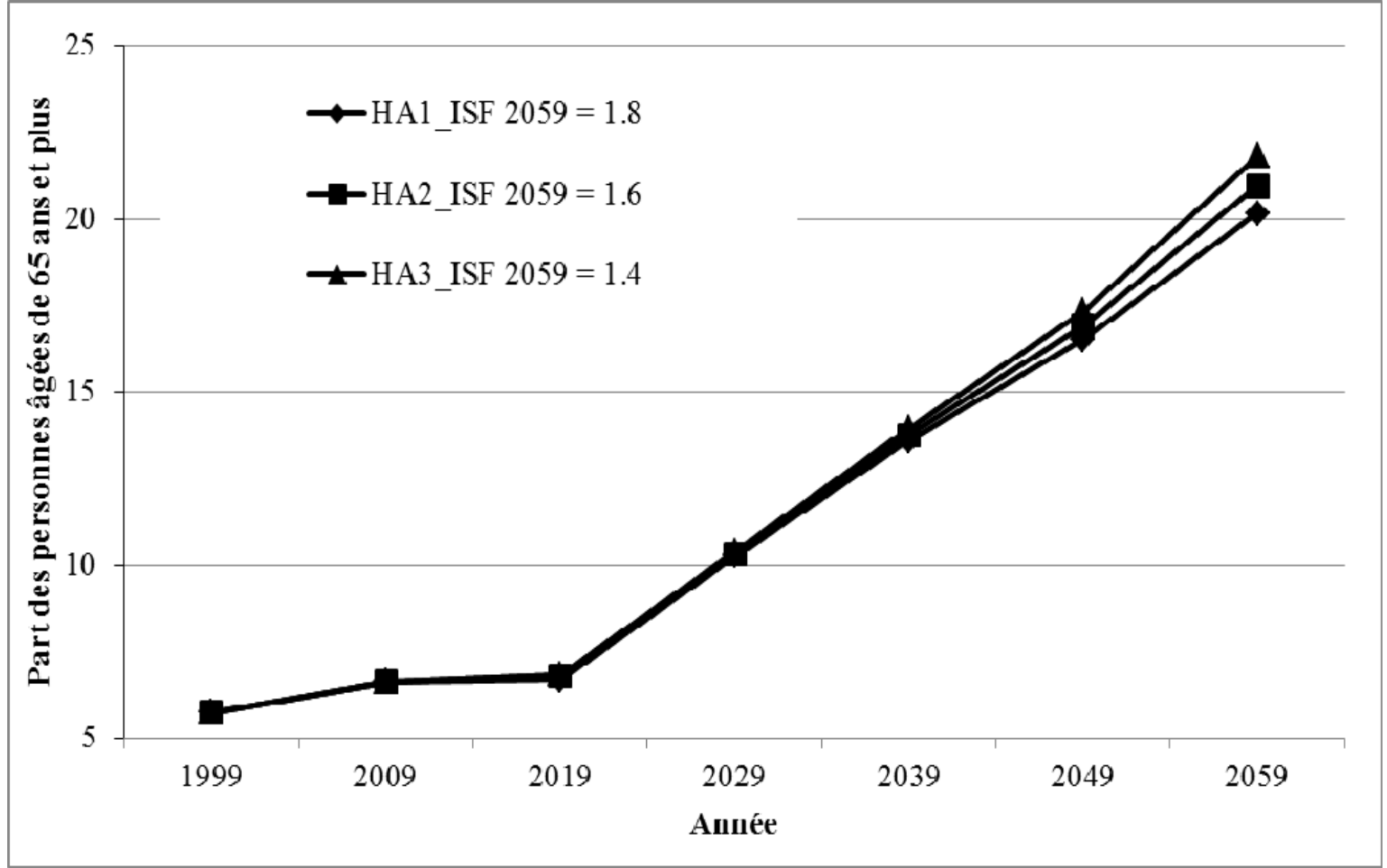

Source : Nguyên et coll. 2009.

Ces changements de la structure par âge de la population ont créé une fenêtre d'opportunité dite "structure en or» de la population ou "dividende démographique ». Cela pose le problème du système de sécurité sociale qui n'est pas préparé.

\section{II.5- Opportunités et défis de la «structure en or » de la population}

Selon les estimations de Nguyên Thi Minh (2014), le changement de la structure par âge de la population a contribué à hauteur de $14,5 \%$ à la croissance du PIB durant la période 20042009. La principale raison réside dans l'augmentation rapide de la population d'âge actif et du taux d'activité. Cela montre qu'à un certain degré, le Viêt Nam a su profiter de l'avantage offert par le dividende démographique. Toutefois, la capacité à profiter de cet avantage est bien modeste par rapport à d'autres pays d'Asie de l'Est où cette contribution a été de $30 \%$ (Giang Thanh Long, 2010). Au Viêt Nam, l'avantage de la «structure en or » de la population n'est manifeste qu'en termes quantitatifs. Or son impact dépend beaucoup de la qualité de la main d'œuvre et de la capacité de l'économie à l'utiliser avec efficacité. 
Le Viêt Nam bénéficie d'une main-d'œuvre abondante, mais manque de gestionnaires et de travailleurs qualifiés, lesquels, en 2009 , ne représentent que respectivement $1,0 \%$ et $4,6 \%$ de la main d'œuvre vietnamienne. Toujours en 2009 , 75,3\% de la population active occupée est sans qualification. Une différence subsiste entre les taux de participation à la population active des hommes et des femmes. Malgré une amélioration certaine du taux de participation des femmes, ce taux est toujours inférieur à celui des hommes de 5 à 10 points. De plus, le salaire des femmes ne représente que $85 \%$ de celui des hommes. Le chômage connaît une évolution complexe, avec un taux moyen de 4,5\% durant la période 1999-2009. Selon les résultats du recensement de 2009, les jeunes âgés de 15 à 29 ans représentent jusqu'à 64,9\% des 1,3 million de chômeurs en 2009. Et 17,4\% des membres du groupe des plus jeunes travailleurs (de 15 à 19 ans) sont également au chômage (Office général des Statistiques, 2010).

L'apparition de la «structure en or » de la population est synonyme d'une forte chute du nombre des enfants âgés de 0 à 4 ans et de 5 à 9 ans à l'avenir. Le nombre des élèves de l'école primaire et du collège baissera également. Cette tendance créera des conditions favorables à l'amélioration de la qualité de l'enseignement à l'école primaire comme au collège, car le rapport des élèves sur les enseignants et l'effectif des élèves dans chaque classe connaîtront une baisse. Parallèlement, l'augmentation de la proportion de la population d'âge actif et la restructuration économique avec un accent sur l'industrie et les services dans les années à venir créeront un besoin important de formation professionnelle. Cela constituera une opportunité, mais exercera aussi une pression à l'élargissement de l'accès à l'éducation et à la formation au niveau supérieur ainsi qu'à l'amélioration de leur qualité.

La réduction de l'effectif des enfants permet de réduire les frais, de rehausser la qualité des soins de santé, de réduire le taux de malnutrition tout comme le taux de mortalité à la naissance et au cours de la première année de vie. Ces facteurs se répercuteront de façon positive sur la qualité de la population à venir. Avec une population d'âge actif en augmentation, une bonne santé physique et mentale permettra à la société d'économiser des frais médicaux. Toutefois, la forte hausse de la population active exercera une pression sur les soins de santé reproductive, dans un contexte où ce besoin sera croissant. L'augmentation continue de l'effectif des femmes en âge de procréer montre que le Viêt Nam devra toujours faire face à des besoins croissants de planification familiale et d'approvisionnement en services de contraception au cours des 20 années à venir, sur les plans quantitatif et qualitatif. Or, il est difficile d'améliorer la qualité des services de planification familiale quand la pression quantitative est toujours très présente. Le vieillissement de la population pose également le problème du système de sécurité sociale qui n'est pas préparé.

\section{Conclusion}

La structure de la population vietnamienne a connu et connait toujours une forte mutation avec la réduction de la proportion d'enfants et l'augmentation de la part de la population d'âge actif. Le Viêt Nam est entré dans la période de «structure en or » de la population ou du dividende démographique en 2006. Cette période durera de 40 à 45 ans selon la vitesse de réduction du taux de natalité. Durant cette période, les effectifs de main d'œuvre augmenteront fortement pour atteindre jusqu'à 71 ou 72 millions de personnes en 2039, soit 11 millions de plus qu'aujourd'hui. La baisse de la proportion des femmes en âge de procréer et la poursuite de l'augmentation de leurs effectifs jusqu'en 2029 suggèrent que la planification familiale et l'approvisionnement en moyens de contraception continueront de subir de fortes pressions. 
La «structure en or» de la population n'est vraiment bénéfique que quand cette maind'œuvre abondante est valorisée. De plus, la baisse de la proportion d'enfants offre l'occasion d'améliorer la qualité de l'éducation à leur intention. Or, parallèlement à la réduction de leur effectif et au développement économique, le taux de malnutrition persiste dans les régions en difficulté tandis que l'obésité a tendance à augmenter chez les enfants dans les régions urbaines.

Le Viêt Nam devrait donc promulguer des politiques permettant de profiter des opportunités et avantages apportés, tout en surmontant les défis que représente cette structure. Une attention particulière devrait être accordée aux domaines de l'éducation, la formation, la création d'emplois et du bien-être pour les personnes âgées. La tendance au vieillissement de la population va être explosive de 2019 à 2029 et se poursuivra pendant les années suivantes. Afin de faire face à ce vieillissement et de ne pas tomber dans la situation " d'être vieux avant d'être riche ", le Viêt Nam devra éduquer ses citoyens à la conscience de s'occuper de leur vieillesse dès leur jeunesse ; planifier une communication adéquate à une population vieillie ; élaborer et mettre en œuvre des politiques nationales en termes de bien-être social. Les défis nés du changement démographique rapide nécessitent des politiques d'intervention tant au niveau central qu'au niveau local. Ils doivent être pris en compte dans les politiques de développement, des soins de la santé, de l'éducation et de la sécurité sociale. En ce qui concerne les politiques de développement, l'élaboration de ces politiques pour les régions doit prendre en compte les avantages du dividende démographique de ces localités.

\section{Remerciements}

Cette recherche a bénéficié d'un financement de la Fondation Nationale pour le Développement de la Science et la Technologie du Viêt Nam (Nafosted) relatif au protocole II6.2-2012.01.

\section{Bibliographie}

GIANG Thanh Long, Profiter des avantages de la structure " d'or " de la population au Viêt Nam : Opportunités, défis et recommandations de politiques, Hanoi, UNFPA, 2010, 68 p.

Guilmoto, Christophe, Pham NGuYen Bang, « Préférence pour les garçons et déséquilibre du sex-ratio à la naissance au Viêt Nam », Communication présentée au Colloque sur la Population, santé publique et développement au Vietnam après 25 ans de Renouveau, Programme ARCUS France-Vietnam, Université Nationale d'Économie, CERPOS, CEPED, Hanoi, 10 octobre 2011, 40 diapositives.

NGUYEN Thi Minh et coll. «La démographie en période de croissance économique et le piège du revenu moyen au Viêt Nam », in Le revenu moyen au Viêt Nam, conférence du Comité Central sur l'Économie et du Comité central d'Éducation ["Nhân khẩu học trong tăng trưởng kinh tế và vấn đề bẫy thu nhập trung bình tại Việt nam". Hội thảo quốc gia về "Bẫy thu nhập trung bình ở Việt Nam", Ban Kinh tế trung ương và Ban Tuyên giáo trung ương], 2014, Hanoi, 24 p.

NGUYEN Dinh $\mathrm{Cu}$, "Changement démographique et croissance économique ", in Revue Sociologie, $\mathrm{n}^{\circ} 1 / 117,2012$, p. 11-16.

NGUYEN Dinh $\mathrm{Cu}$, "Migration et développement", in Colloque sur les questions de population et de développement selon le Recensement de Population et de l'Habitat en 
2009, Haiphong, UNFPA, Université nationale d'Économie, 11-12 décembre 2010, 87 diapositives.

Nguyen Dinh $\mathrm{Cu}$, Ha Tuan Anh, "Profiter de la structure d'or de la population pour promouvoir la croissance économique au Viêt Nam », in Actes du Colloque Modèle de croissance économique du Viêt Nam : État des lieux et choix pour la période 2011-2020, Hanoi, Commission économique de l'Assemblée nationale et Université nationale d'Économie, 2010, p. 133-152.

NguYen Dinh $\mathrm{Cu}$, Ha Tuan Anh, «Prévision de la période de la structure d'or de la population au Viêt Nam ", in Actes du Colloque national sur le changement de la structure de la population, Hanoi, Université nationale d'Économie, 2009, 39 diapositives.

OfFICE GENERAL DES STATISTIQUES, Enquête sur les changements démographiques, les ressources humaines et la planification familiale, Hanoi, Éditions Thống kê, 2007, 267 p. [Tổng cục Thống kê, Điều tra biến động Dân số, nguồn lao động và kế hoạch hoá gia đình, Hà Nội ,Nhà xuất bản Thống kê, 2007, 267 trang.]

OFFICE GENERAL DES STATISTIQUES, Enquête sur les changements démographiques et la planification familiale au ler avril 2011, Hanoi, Éditions Thống kê, 2011, 325 p. [Tổng cục Thống kê, Điều tra biến động Dân số và kế hoạch hoá gia đình ngày 1.4.2011. Hà Nội, Nhà xuất bản Thống kê, 2011, 325 trang.]

OfFICE GENERAL DES STATISTIQUES, Enquête sur les changements démographiques et la planification familiale au ler avril 2012, Hanoi, Éditions Thống kê, 2013, 314 p. [Tổng cục Thống kê, Điều tra biến động Dân số và kế hoạch hoá gia đình ngày 1.4.2012, Hà nội, Nhà xuất bản Thống kê, 2013, 314 trang.]

OfFice General Des StatistiQues, L'éducation au Vietnam: Analyse des indicateurs clés, Hanoi, Viêt Nam, Éditions Thông kê, 2011c, 178 p. [Tổng cục Thống kê, Giáo dục ở Việt Nam: Phân tích các chỉ số chủ yếu,Hà Nội, Việt Nam, Nhà xuất bản Thống kê, 2011c, 178 trang.]

OFFICE GENERAL DES STATISTIQUES, Migration et urbanisation au Vietnam: situation actuelle, tendances et différences, Hanoi, Viêt Nam, Éditions Thông kê, 2011d, 144 p. [Tổng cục Thống kê, Di cư và đô thị hoá ở Việt Nam: Thực trạng, xu hướng và những khác biệt, Hà Nội, Việt Nam,Nhà xuất bản Thống kê, 2011d,144 trang.]

OFFICE GENERAL DES STATISTIQUES, Natalité, mortalité au Vietnam: situation actuelle, tendances et différences, Hanoi, Viêt Nam, Éditions Thông kê, 2011f, 276 p. [Tổng cục Thống kê, Mức sinh, mức chết ở Việt Nam: Thực trạng, xu hướng và những khác biệt, Hà Nội, Việt Nam, Nhà xuất bản Thống kê, 2011f, 276 trang.]

OfFICE GENERAL DES StATISTIQUes, Résultats de l'Enquête sur le travail et l'emploi au Viêt Nam en 2011, Hanoi, Éditions Thống kê, 2012, 348 p. [Tổng cục Thống kê, Kết quả điều tra Lao động và việc làm ở Việt Nam năm 2011, Hà Nội, Nhà xuất bản Thống kê, 2012, 348 trang.]

OfFiCE GENERAL Des STATISTIQUes, Rapport de masculinité à la naissance au Viêt Nam: nouveaux résultats sur l'état, les tendances et les différences, Hanoi, Viêt Nam, Éditions Thông kê, 2011b, 72 p. [Tổng cục Thống kê , Tỷ số giới tính khi sinh ở Việt Nam: Các bằng chứng mới về thực trạng, xu hướng và những khác biệt. Hà Nội, Việt Nam, Nhà xuất bản Thống kê, 2011b, 72 trang.]

Office General Des StatistiQues, Recensement de la population et du logement du Viêt Nam en 2009 : Résultats principaux, Hanoi, Éditions Thống kê, 2010b, 896 p. [Tổng cục 
Thống kê, Tổng điều tra Dân số và Nhà ở Việt Nam năm 2009 : Các kết quả chủ yếu, Hà Nội, Nhà xuất bản Thống kê, 2010b, 896 trang.]

OfFICE GENERAL DES STATISTIQUeS, Recensement de la population et du logement du ler avril 1999: Résultats principaux, Hanoi, Éditions Thống kê, 2000, 465 p. [Tổng cục Thống kê , Tổng điều tra Dân số và Nhà ở ngày 1.4.1999: Các kết quả chủ yếu, Hà Nội, Nhà xuất bản Thống kê, 2000, 465 trang.]

OfFICE GENERAL Des StATISTIQUeS, Recensement de la population et du logement du ler avril 2009 : Résultats principaux, Hanoi, Éditions Thống kê, 2010, 506 p. [Tổng cục Thống kê . Tổng điều tra Dân số và Nhà ở ngày 1.4.2009 : Các kết quả chủ yếu. Hà Nội, Nhà xuất bản Thống kê, 2010, 506 trang.]

OfFice General Des StATISTIQUes, Recensement de la population et du logement du Viêt Nam en 2009: Résultats principaux, Hanoi, Éditions Thông kê, 2010a, 490 p. [Tổng cục Thống kê . Tổng điều tra Dân số và Nhà ở Việt Nam năm 2009 : Các kết quả chủ yếu. Hà Nội,Nhà xuất bản Thống kê, 2010a, 490 trang.]

OfFice General des StATISTIQues, Résultats de l'Enquête sur le niveau de vie des ménages en 2008, Hanoi, Éditions Thống kê, 2009, 561 p. [Tổng cục Thống kê. Kết quả điều tra mức sống hộ gia đình năm 2008, Hà Nội, Nhà xuất bản Thống Kê, 2009, 561 trang.]

OfFICE GENERAL DES STATISTIQUes, Résultats de l'Enquête sur le niveau de vie des ménages en 2010, Hanoi, Viêt Nam, Éditions Thống kê, 2011a, 711 p. [Tổng cục Thống kê, Kết quả điều tra mức sống hộ gia đình năm 2010, Hà Nội,Việt Nam, Nhà xuất bản Thống kê, 2011a ,711 trang.]

OfFICE GENERAL DES STATISTIQUeS, Structure par âge, sexe et état matrimonial de la population du Viêt Nam, Hanoi, Viêt Nam, Éditions Thông kê, 2011e, 164 p. [Tổng cục Thống kê, Cấu trúc Tuổi, Giới tính và Tình trạng hôn nhân của dân số Việt Nam, Hà Nội, Việt Nam, Nhà xuất bản Thống kê, 2011e,164 trang.]

UNDP, Human Development Report - Millenium Development Goals: A compact among nation to end human poverty, Oxford, Oxford University Press, 2003, 368 p.

UNDP, Human Development Report - Sustainability and Equity: A Better Future for All, Oxford, Oxford University Press, 2011, 185 p.

United Nations, POPUlation Division, World Population Prospects: The 2008 Revision, New York, 2009 http://esa.un.org/wpp [Consulté le 14 octobre 2009].

\section{Annexes}

Tableau 8- Effectif (en millions) des jeunes (moins de 18 ans) pour la période 2009-2059 selon l'hypothèse relative au niveau de fécondité pour 2059

\begin{tabular}{ccccccc} 
ISF & 2009 & 2019 & 2029 & 2039 & 2049 & 2059 \\
\hline 1.8 & 16,06 & 14,08 & 15,23 & 13,82 & 13,21 & 12,88 \\
1.6 & 16,06 & 14,08 & 15,07 & 13,38 & 12,46 & 11,69 \\
1.4 & 16,06 & 14,08 & 14,89 & 12,95 & 11,71 & 10,53 \\
\hline
\end{tabular}

Source : NGUYEN et coll. 2009. 
Tableau 9- Effectif (en millions) de la main d'œuvre pour la période 2009-2059 selon l'hypothèse relative au niveau de fécondité pour 2059

\begin{tabular}{lllllll} 
ISF & 2009 & 2019 & 2029 & 2039 & 2049 & 2059 \\
\hline 1.8 & 58,65 & 66,13 & 70,18 & 72,20 & 71,23 & 68,33 \\
1.6 & 58,65 & 66,13 & 70,14 & 71,84 & 70,30 & 66,43 \\
1.4 & 58,65 & 66,13 & 70,09 & 71,49 & 69,39 & 64,55 \\
\hline
\end{tabular}

Source : NGUYEN et coll. 2009.

Tableau 10- Effectif (en millions) de la population féminine en âge de procréer (15-49 ans) pour la période 2009-2059 selon l'hypothèse relative au niveau de fécondité pour 2059

Source : NGUYEN et coll. 2009.

\begin{tabular}{cccccccc} 
ISF & 1999 & 2009 & 2019 & 2029 & 2039 & 2049 & 2059 \\
\hline 1.8 & 20,79 & 24,53 & 25,29 & 25,67 & 24,94 & 23,57 & 23,17 \\
1.6 & 20,79 & 24,53 & 25,29 & 25,65 & 24,77 & 23,13 & 22,26 \\
1.4 & 20,79 & 24,53 & 25,29 & 25,63 & 24,60 & 22,69 & 21,36 \\
\hline
\end{tabular}

Tableau 11- Âge médian (en années) de la population de 1999 à 2059 selon l'hypothèse relative au niveau de fécondité pour 2059

\begin{tabular}{cccccccc} 
ISF & 1999 & 2009 & 2019 & 2029 & 2039 & 2049 & 2059 \\
\hline 1.8 & 23 & 28 & 31 & 35 & 38 & 40 & 42 \\
1.6 & 23 & 28 & 31 & 36 & 38 & 40 & 43 \\
1.4 & 23 & 28 & 32 & 36 & 39 & 41 & 44 \\
\hline
\end{tabular}

Source : NGUYEN et coll. 2009.

Tableau 12- Effectifs (en millions) et pourcentages de personnes âgées de 60 ans et plus de 1999 à 2059 (perspectives) selon l'hypothèse relative au niveau de fécondité pour 2059

\begin{tabular}{lllllllll} 
ISF & $\begin{array}{l}\text { Population de } 60 \\
\text { ans et plus }\end{array}$ & 1999 & 2009 & 2019 & 2029 & 2039 & 2049 & 2059 \\
\hline 1.8 & Effectif & 6,14 & 7,67 & 10,52 & 15,17 & 20,38 & 24,89 & 28,33 \\
& $\%$ & 8,05 & 8,94 & 11,06 & 14,85 & 19,12 & 22,89 & 26,18 \\
\hline 1.6 & Effectif & 6,14 & 7,67 & 10,52 & 15,70 & 20,38 & 24,89 & 28,33 \\
& $\%$ & 8,05 & 8,94 & 11,08 & 15,46 & 19,36 & 23,44 & 27,22 \\
\hline 1.4 & Effectif & 6,14 & 7,67 & 10,52 & 15,70 & 20,38 & 24,89 & 28,33 \\
& $\%$ & 8,05 & 8,94 & 11,10 & 15,56 & 19,62 & 24,01 & 28,31 \\
\hline
\end{tabular}

Source : NGUYEN et coll. 2009. 


\title{
Deux perspectives régionales sur les déséquilibres de sexe à la naissance au Viêt Nam
}

\author{
Valentine Becquet (UPD CEPED)
}

Différents pays dans le monde, de l'Albanie à la Chine, sont affectés par des déséquilibres du rapport de masculinité à la naissance (Attané et coll. 2005 ; Attané et coll. 2007 ; Duthé et coll. 2012). Le Viêt Nam est de ceux parmi lesquels le phénomène est le plus récent, mais la hausse que l'on mesure depuis 2006 est également l'une des plus rapides. En effet, le rapport de masculinité à la naissance s'établit aujourd'hui à plus de 112 naissances de garçons pour 100 naissances de filles, et excède 120 dans certaines provinces ${ }^{12}$.

Si en Chine et en Inde, le déséquilibre du rapport de masculinité à la naissance est aggravé par la surmortalité infanto-juvénile des petites filles (Coale et coll. 1994, Bedi 2008), il n'y a pas de discrimination postnatale au Viêt Nam. En effet, il n'existe aucun témoignage d'infanticide au cours des siècles passés, et la surmortalité des petites filles, causée ailleurs par la négligence de soins, semble minime : elle est de 0,7 pour 1000 filles âgées de moins de 5 ans, pour chaque année de la période $2005-2009^{13}$. Comparé à la surmortalité estimée des petites filles en Chine (99,5 pour 1000) et en Inde $(261,8$ pour 1000), le chiffre du Viêt Nam est négligeable (Guilmoto 2012b). D'autre part, si Mark Banens (2000) dénote 10 à 20\% de sous-déclaration des naissances féminines en Cochinchine au début du $20^{\mathrm{e}}$ siècle, il ne semble plus exister aujourd'hui de discrimination envers les filles lors de la déclaration à l'état civil. Selon l'enquête MICS $^{14}$ menée par UNICEF en 2005, il y aurait en réalité un pourcentage légèrement plus important de filles que de garçons déclarés à l'âge de 5 ans (UNICEF 2005). De plus, si la sous-déclaration des naissances féminines était un phénomène important au Viêt Nam, elle aurait influé sur le rapport de masculinité bien avant 2006. Par conséquent, en l'absence de discrimination postnatale envers les petites filles, la hausse du rapport de masculinité à la naissance est exclusivement liée à la sélection sexuelle prénatale, c'est-à-dire l'avortement des fœetus féminins.

Selon les données issues du recensement vietnamien de 2009, le rapport de masculinité est plus élevé pour les naissances des couples plus riches et plus éduqués, qui agissent comme des groupes «pionniers » dans un mécanisme d'innovation sociale (l'adoption de la sélection sexuelle prénatale). Ainsi, le rapport de masculinité à la naissance est normal chez les couples appartenant au quintile le plus pauvre $(105,2)$, il augmente légèrement chez les couples du deuxième quintile $(107,5)$ mais il apparaît réellement déséquilibré pour les naissances des trois quintiles les plus riches - il varie

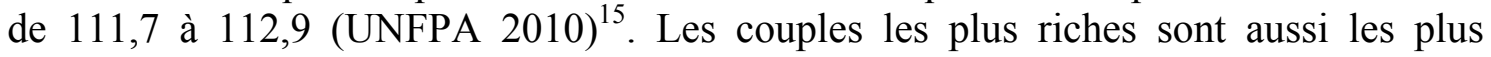

\footnotetext{
${ }^{12}$ Source: Office général des Statistiques, chiffres donnés pour l'année 2012.

${ }^{13}$ Comme le montrent les chiffres de l'Enquête Démographique et de Santé de 2001-2002, la mortalité infanto-juvénile est en réalité plus élevée chez les garçons vietnamiens car, en raison de facteurs biologiques, les garçons en bas âge sont plus vulnérables. C'est pourquoi la surmortalité est ici définie comme l'excès de morts chez les petites filles âgées de moins de 5 ans, comparé au ratio moyen mortalité masculine/mortalité féminine, lorsqu'il n'y a pas de discrimination de genre (environ 123 garçons pour 100 filles âgées de moins de cinq ans, dans les pays ayant la même espérance de vie à la naissance que le Viêt Nam) (National Committee for Population, Family and Children 2003).

${ }^{14}$ Multiple Indicator Cluster Survey.

${ }^{15}$ L'indicateur synthétique du niveau de vie du ménage a été calculé à partir de multiples variables : possession de biens (moto, air conditionné, etc.), d'équipements (eau courante, toilettes, etc.) et matériaux de construction de la maison (toit, murs).
} 
éduqués, ils ont donc un meilleur accès à l'information sur la sélection sexuelle moderne.

Cet article se propose d'examiner les facteurs qui ont mené à l'émergence de ce phénomène récent au Viêt Nam, puis d'analyser les disparités régionales qui apparaissent dans les statistiques de la masculinité des naissances, à travers l'étude de deux provinces, Hai Duong et Ninh Thuậân, qui ont été l'objet d'enquêtes qualitatives en octobre 2012 et en janvier 2013.

La province de Hai Duong comporte 1700000 habitants, elle est située dans une région fortement peuplée du nord, le Delta du Fleuve Rouge (voir Carte 1 en tête d'ouvrage), région qui comprend les rapports de masculinité à la naissance les plus déséquilibrés du pays ; celui de Hai Duong était de 120,2 naissances de garçons pour 100 naissances de filles en 2009, c'est pourquoi elle fait partie des 18 provinces où le Comité national pour la Population et la Planification familiale a mis en place les premiers projets de lutte contre la discrimination sexuelle prénatale en $2009 .{ }^{16} \mathrm{C}$ 'est l'une des provinces les plus industrialisées du Viêt Nam, qui englobe également de grandes zones rurales de riziculture et de fruiticulture - le district de Thanh Hà, où s'est déroulée une partie de l'enquête, est notamment spécialisé dans la culture des goyaves et des litchis. Hai Duong est une province relativement riche ; le taux de pauvreté, issu de l'enquête sur les niveaux de vie des ménages ${ }^{17}$, et basé sur la ligne de pauvreté fixée par le gouvernement, ${ }^{18}$ est de $10,8 \%$ en 2010 . De plus, le niveau d'éducation est élevé, puisque selon les données du recensement de 2009 , 64\% de la population a atteint la dernière année du collège (neuvième année). Cette province comporte une écrasante majorité de Kinh $(99,6 \%)$, et témoigne d'une très forte préférence pour les garçons ${ }^{19}$, liée à des structures patriarcales. L'indice synthétique de fécondité se situe en dessous du seuil de renouvellement des naissances, avec 1,99 enfant par femme en 2009.

La province de Ninh Thuân, elle, compte seulement 570000 habitants (voir Carte 1 en tête d'ouvrage). Si la majorité de sa population est d'origine Kinh aujourd'hui $(76,5 \%)$, elle comporte toujours une forte proportion de Cham $(11,9 \%)$ et de Raglay $(10,4 \%)$, deux minorités ethniques ayant des structures de parenté relativement similaires (Nakamura 2008, Kiernan 2010). Située sur la côte centrale du sud, c'est une province essentiellement rurale $(73 \%$ de la population vit en zone rurale en 2009) et agricole, avec de grandes zones de riziculture, mais également un secteur de pêche très développé. Ninh Thuân est une province plus pauvre que celle de Hai Duong - le taux de pauvreté était évalué à $19 \%$ en 2010 contre 10,8\% à Hai Duong -, avec un niveau d'éducation beaucoup plus bas. En effet, les chiffres du recensement de 2009 montrent que seuls $21 \%$ des individus ont terminé le collège (contre $64 \%$ des individus à Hai Duong). D'autre part, la fécondité est plus élevée que la moyenne nationale : l'indice synthétique de fécondité s'élève à 2,4 enfants par femme en 2009, avec un rapport de masculinité à la naissance évalué à 110,8 cette même année. Ici, le projet de prévention mis en place par le Comité national pour la Population et la Planification familiale date

${ }^{16}$ Ces projets consistent principalement en la diffusion de campagnes de sensibilisation sur la problématique de la masculinité des naissances, et de prévention visant à améliorer le statut des petites filles.

${ }^{17}$ Vietnam Household Living Standards Survey 2010.

${ }^{18}$ Cette « ligne de pauvreté » est déterminée à 400000 VND (soit environ 20 dollars US) par personne et par mois en zone rurale, et 500000 VND (environ 25 dollars US) par personne et par mois en zone urbaine. Les ménages ayant un revenu inférieur sont considérés comme pauvres.

19 Il semble exister peu de différences entre les zones rurales et urbaines en termes de préférence pour les garçons : la probabilité pour qu'un couple sans fils ait un troisième enfant est 5,7 fois supérieure à celle d'un couple ayant au moins un fils en zone urbaine, et 5,4 fois supérieure en zone rurale. De même, les témoignages recueillis diffèrent peu entre les districts urbain et rural. 
seulement de 2012, les habitants semblaient donc encore peu au courant de la problématique de la masculinité des naissances au moment de l'enquête (janvier 2013).

\section{I- Les trois facteurs de la masculinité des naissances}

Le phénomène de la masculinité des naissances, conséquence de la discrimination sexuelle prénatale, résulte de la combinaison de trois facteurs. En effet, comme le démontre Christophe Z. Guilmoto (2009), les couples qui ont recours aux avortements sexo-sélectifs doivent être disposés à le faire (facteur de demande), parce qu'ils ont une préférence pour les garçons liée à différents arguments sociaux, culturels et économiques, mais ils doivent aussi en avoir la possibilité (facteur d'accessibilité), grâce à la légalité du recours à l'avortement provoqué et le développement de nouvelles technologies telles que les appareils échographes. Enfin, il existe un facteur de pression, exercée par le faible niveau de fécondité, qui augmente la probabilité de n'avoir que des filles. Avant d'aller plus avant dans l'analyse du phénomène à proprement parler, nous allons détailler ces trois facteurs dans le contexte particulier du Viêt Nam.

\section{I.1- Le contexte de faible fécondité}

Selon les données de l'Office Général des Statistiques, l'indice synthétique de fécondité (ISF) du Viêt Nam a atteint le seuil de remplacement des générations $(2,1)$ en 2005, puis est tombé sous ce niveau dans les années suivantes; il était de 1,99 en 2011. Ce bas niveau de fécondité a été atteint remarquablement rapidement, l'ISF passant en 30 ans de 6,8 enfants par femme (1965-1969) à 2,5 enfants par femme (1995-1999). Ainsi, le gouvernement de la République démocratique du Viêt Nam (au nord), alarmé par les résultats du recensement de 1960 montrant un accroissement très rapide de la population, a mis en place une première politique de contrôle des naissances dès 1963 (Décret 99/TTg du 16 octobre 1963), incitant les familles à n'avoir que trois enfants et à espacer les naissances (Scornet 2009). Ensuite, dans les années 70, il a fortement encouragé l'utilisation de la contraception, plus particulièrement du dispositif intrautérin, et de l'avortement, légalisé en 1954, afin de contrôler les naissances. Au contraire, la République du Viêt Nam, au sud du pays, a entretenu une politique à tendance nataliste (Scornet 2000).

Le pays s'est réunifié en 1975, et en 1984 a été créé le Comité national pour la Population et la Planification familiale; la politique de limitation à un ou deux enfants par couple (một hoặc hai con) a été mise en place par le Décret 162/HDBT du 18 octobre 1988, alors que l'ISF était de 4 enfants par femme. Le gouvernement a ainsi durci la politique de 1963, en poursuivant le même objectif: limiter l'accroissement naturel de la population afin d'améliorer le niveau de vie des Vietnamiens. Cependant, la limitation ne concernait pas les minorités ethniques situées en majorité dans les Régions montagneuses du nord et les Hauts Plateaux du centre, autorisées à avoir trois enfants.

Comme l'explique Catherine Scornet (2009), la politique des deux enfants, à travers les activités de planification familiale, a été implantée de manière décentralisée, du niveau provincial au niveau communal, favorisant son succès et le déclin rapide de la fécondité. À travers différents outils de communication, posters, médias de masse, performances théâtrales ou chansons, le gouvernement a élaboré dans les années 90 une véritable propagande vantant les mérites de la famille idéale, "petite, en bonne santé, heureuse et prospère ». Dès cette époque, l'égalité entre les garçons et les filles est 
également soulignée dans la stratégie de communication : "pour que le public réalise et accepte que chaque enfant mérite d'être aimé, quel que soit son sexe. La chose la plus importante n'est pas d'avoir un fils ou une fille ou plusieurs enfants, mais la manière dont l'enfant ou les enfants sont éduqués et grandissent $\|^{20}$.

Plusieurs mesures sont instaurées : les couples - particulièrement les fonctionnaires, les militaires et les membres du Parti communiste - dépassant le nombre d'enfants autorisés doivent payer une taxe foncière plus élevée, et l'État vietnamien fournit gratuitement des moyens de contraception modernes, et des services de santé permettant la pose d'un dispositif intra-utérin ou le recours à l'avortement. De fait, le taux d'utilisation de la contraception moderne est passé de 53,2\% en 1988 à 57,9\% en 1998 et $68,6 \%$ en $2011^{21}$. Près de la moitié des femmes utilisant un moyen de contraception ont un dispositif intra-utérin. Il est cependant important de noter que cette politique a varié dans sa mise en place, car si le non-respect de la prescription entraînait automatiquement le paiement d'une amende dans certaines provinces, les couples ayant deux filles étaient tacitement autorisés à concevoir un troisième enfant dans d'autres provinces (Scornet 2000). Quelques années plus tard, en 2003, avant même que la surmasculinité des naissances au Viêt Nam ne devienne statistiquement significative, mais alors que les experts soupçonnent le développement imminent du phénomène (Croll 2000, Bélanger 2002), l'Assemblée nationale vietnamienne a acté une Ordonnance de Population, interdisant la sélection du sexe du fœtus (article 7), et stipulant que les couples étaient autorisés à avoir autant d'enfants qu'ils le désiraient (article 10). Ce dernier article a soulevé une controverse sur un éventuel accroissement de la population en cas d'abandon de la prescription à deux enfants par couple, et l'Assemblée nationale amenda l'Ordonnance en 2008, en réintroduisant l'obligation pour les couples de limiter les naissances à un ou deux enfants. Cependant, cette révision ne comprend pas de mesures punitives pour les couples ne respectant pas cette prescription.

Aujourd'hui, avoir deux enfants est devenu la norme, en particulier chez les Kinh, l'ethnie majoritaire du Viêt Nam. En effet, beaucoup de couples préfèrent avoir peu d'enfants pour pouvoir leur fournir une meilleure éducation, dans un pays où l'école coûte très cher (Papin et coll. 2010).

«La nouvelle idéologie a été inculquée par des campagnes de propagande de style socialiste, et elle est devenue une préférence intériorisée $»^{22}$ (Bélanger et Khuât 2009).

S'il n'y a plus d'amendes pour les couples ayant plus de deux enfants, comme le montrent différents témoignages recueillis à Hai Duong comme à Ninh Thuân, les parents ayant plus de deux enfants sont cités en public et désignés lors des réunions de village, en signe de désapprobation. D'autre part, le gouvernement vietnamien a mis en place le concept de "famille cultivée » (gia đình văn hóa), un certificat délivré aux familles considérées comme heureuses, bien éduquées et n'ayant pas plus de deux enfants - un concept calqué sur celui de "village cultivé », et qui vise à définir un comportement « convenable » tout en renforçant le contrôle social de la communauté.

\footnotetext{
20 "To make audiences realize and accept the view that either of the two is also one's born child and deserves one's love. The most important thing is not to have a son or a daughter or many children, but how the child/children are brought up and grow up" Article 3 de la "Strategy for information education : Communication of population and family planning 1992-2000»du Comité national pour la Population et la Planification familiale. Cité par Scornet (2009).

${ }^{21}$ Ces chiffres sont issus respectivement de l'Enquête de Démographie et de Santé de 1988 et des enquêtes annuelles du Comité national sur la population et la planification familiale de 1998 et 2011.

22 "The new ideology was instilled by socialist-style propaganda campaigns, and became an internalized preference".
} 
Dans ce contexte de faible fécondité, la probabilité de ne pas avoir de fils est relativement élevée : $24 \%$ des couples ayant deux enfants n'auront que des filles, contre $6 \%$ des couples avec quatre enfants, et $1 \%$ des couples avec six enfants. Ce risque de demeurer sans descendance masculine pousserait donc certains à avoir recours à la sélection sexuelle prénatale. Et si cette norme des deux enfants est intériorisée, une partie des couples interrogés pendant les enquêtes de terrain effectuées à Hai Duong et Ninh Thuân, ainsi que certains cadres politiques, blâment pourtant la politique de contrôle des naissances, qui empêche les couples d'avoir un troisième enfant lorsqu'ils n'ont que deux filles et désirent un fils. Même s'ils expliquent dans le même temps qu'ils n'ont pas les moyens financiers d'élever un enfant supplémentaire, ils sembleraient préférer avoir le choix hypothétique d'ajuster leur fécondité à leur désir qualitatif de famille idéale.

I.2- L'accès aux nouvelles technologies et la lutte contre la sélection sexuelle prénatale

Comme nous l'avons vu précédemment, l'avortement a été légalisé en 1954, sans jamais être controversé (Bélanger et Khuât 2009). Il était utilisé comme un moyen de limiter les naissances, au même titre que la contraception moderne, l'État veillant à ce que les femmes y aient accès sur demande dans les institutions médicales accréditées par le Ministère de la Santé. ${ }^{23}$ Puis, avec le Renouveau (Đổi Mới) et la privatisation du système de santé au début des années 90, les praticiens privés ont également pu pratiquer des avortements. Comme l'explique Goodkind (1994), les données montrent une augmentation rapide des taux d'avortements au début des années 1990, peu après la mise en place de la politique de limitation des naissances. Henshaw et coll., dans leur article de 1999 sur l'avortement dans le monde, précisent que le nombre moyen d'avortements en 1996 au Viêt Nam était de 2,5 avortements par femme en âge de reproduction, et que cette même année, 43,7 grossesses sur 100 se terminaient par un avortement, ce qui était l'un des ratios les plus élevés des pays les moins développés.

L'avortement est légal au Viêt Nam au premier comme au second trimestre de la grossesse. Cependant, les données du Ministère de la Santé montrent que $77 \%$ des avortements étaient pratiqués à moins de huit semaines de grossesse en 2005 - ces avortements sont alors qualifiés de "régulation menstruelle» (Pham et coll., 2011). Selon les directives du Ministère de la Santé, les avortements pratiqués au second trimestre de grossesse doivent l'être dans les hôpitaux provinciaux et nationaux exclusivement, car ils requièrent des médecins formés à la technique utilisée, dite de dilatation et évacuation. Il semble cependant que ces avortements tardifs soient également pratiqués dans les structures privées, qui sont plus chères, mais également moins contrôlées (Bélanger et Khuât 2009).

D'autre part, l'accès aux échographies s'est généralisé au Viêt Nam dans les années 2000 , ce qui a probablement été le déclencheur du recours à la sélection sexuelle prénatale, en permettant de déterminer le sexe du fœtus avant la naissance. Ainsi, selon les données de l'École de Médecine de Hanoi, à la fin de l'année 2001, des services d'échographie, publics et privés, étaient accessibles dans la quasi-totalité des districts du pays, qu'ils soient urbains ou ruraux (Bélanger et Khuât 2009). De plus, Tine Gammeltoft (2007) décrit dans une étude à petite échelle effectuée dans la ville de Hanoi en 2007 que les femmes avaient recours à l'échographie en moyenne huit fois par grossesse. Grâce à la privatisation du système de santé, le Viêt Nam a pu importer plus

\footnotetext{
${ }^{23}$ Selon l'article 44 de la loi sur la protection de la santé publique, datée du 30 juin 1989.
} 
facilement des appareils échographiques, mais également commencer à les fabriquer, ce qui a permis de faire baisser le prix des échographies. Ainsi, Christophe Z. Guilmoto et coll. (2009) expliquent, à partir des données du Ministère de la Santé, que le nombre d'échographies est passé de 1 million en 1998 à 3,7 millions en 2002 et 10,8 millions en 2007.

Le pourcentage de femmes ayant connaissance du sexe du fœtus pendant leur grossesse grâce aux échographies a ainsi augmenté dans tout le pays durant la dernière décennie, à la fois dans les zones urbaines et rurales. Bang Pham et coll. (2011) expliquent que si cette connaissance était au départ plus répandue dans les zones urbaines que rurales ( $83,1 \%$ contre $56,6 \%$ en 2006), l'augmentation était cependant plus rapide dans les zones rurales. Ainsi, selon les données décrites dans l'enquête annuelle de l'Office général des statistiques ${ }^{24}$ en 2012 , le pourcentage de femmes connaissant le sexe de leur fœtus est passé de $83,1 \%$ en 2006 à 86,7\% en 2012 dans les zones urbaines, et de 56,6\% en 2006 à 78,9\% en 2012 dans les zones rurales; $99 \%$ de ces femmes déclarent connaître le sexe grâce à une échographie, dont l'accès s'est ainsi généralisé dans l'ensemble du pays. Le propriétaire d'une clinique privée à Hai Duong raconte ainsi qu'une majorité d'échographies est pratiquée autour du troisième mois de grossesse, et que les femmes viennent souvent plusieurs fois autour de cette date afin de s'assurer du sexe du fœtus, ce dont témoigne également le recours répété à l'échographie décrit par Gammeltoft.

La légalité de l'avortement au premier comme au second trimestre de grossesse, et l'accès généralisé aux échographies, dans un contexte de faible fécondité et de préférence traditionnelle pour les garçons, ont permis le recours par certains couples à la sélection sexuelle prénatale, ce qui a pour conséquence un déséquilibre du rapport de masculinité à la naissance de plus en plus marqué. Ces avortements sélectifs ne sont pas nécessairement des avortements tardifs, puisque selon Efrat et coll. (1999), la précision dans la détermination du sexe du fotus augmente de $70.3 \%$ à 11 semaines de grossesse, à $98,7 \%$ à 12 semaines et $100 \%$ à 13 semaines de grossesse.

Comme nous l'avons expliqué précédemment, l'Assemblée nationale vietnamienne a mis en place une Ordonnance de Population en 2003 afin de prohiber l'identification du sexe du fœtus lors des échographies, et l'avortement sélectif. Ce document légal inclut un ensemble d'amendes et charges criminelles pour ceux qui violent la loi (Article 38), et le décret d'exécution de cette loi a été promulgué en octobre 2006 par le premier ministre (Bélanger et Khuât 2009). La loi sur l'égalité de genre ${ }^{25}$ date de cette même année, et stipule à son tour l'interdiction de «choisir le sexe du fœetus sous quelque forme que ce soit», mais également «d'inciter ou de forcer quelqu'un à avorter en raison du sexe du fotus ». Cependant, il est extrêmement difficile de faire respecter cette prohibition, affichée sur les murs des hôpitaux et des cliniques privées, car elle dépend de la coopération des médecins. Si l'ensemble des praticiens de santé rencontrés le nie, il semble pourtant que la majorité des femmes connaisse aujourd'hui le sexe du fœtus. Ainsi, l'intégralité des femmes interrogées pendant les enquêtes qualitatives ayant des enfants de moins de 10 ans, à Ninh Thuân comme à Hai Duong, en zone urbaine comme en zone rurale - ainsi que les clients rencontrés dans les salles d'attente des cliniques privées-, a confié avoir eu connaissance du sexe du fœtus grâce aux échographies, car les praticiens de santé privés le révèlent sans hésitation.

\footnotetext{
${ }^{24}$ Population Change and Family Planning Survey.

${ }^{25}$ Loi numéro 73/2006/QH11 du 29 novembre 2006.
} 


\section{I.3- La préférence pour les garçons}

Les nombreuses études effectuées sur la préférence pour les garçons se situent principalement dans la région du delta du fleuve Rouge, marquée par un système patrilinéaire et patrilocal fort dans l'ethnie majoritaire Kinh $(85,7 \%$ de la population en 2009), en partie similaire au modèle culturel chinois (Haughton et coll. 1995, Bélanger 2002 , 2006). Ces valeurs patriarcales, issues du confucianisme, sont enracinées dans la culture vietnamienne, et continuent de façonner les perceptions de genre, à la fois chez les hommes et chez les femmes. La colonisation chinoise, qui a commencé en 111 av. J.-C. et s'est étendue jusqu'à la moitié du Xe siècle, a eu un impact profond sur la culture et les traditions des familles vietnamiennes. Selon l'idéologie confucéenne, les femmes sont dépendantes des hommes tout au long de leur vie. Elles doivent pratiquer les «quatre vertus» confucéennes (Tú Đúrc), exclusivement féminines, à savoir l'habileté au travail ménager, la beauté, la fidélité et la passivité, ainsi que les trois dépendances (Tam Tòng) au père, au mari puis au fils aîné après le décès du mari. Les femmes s'efforcent de légitimer leur statut en donnant naissance à un fils, qui est le seul habilité à rendre le culte aux ancêtres, garantissant ainsi la cohésion de la famille et la stabilité de la société vietnamienne (Scornet 2000, Bélanger 2006, Institute for Social Development Studies 2007, Bélanger et Barbieri 2009). Comme le montre le dicton traditionnel «Nhất nam viết hũu, thập nũu viết vô » (qui signifie : si tu as un fils alors tu as un descendant ; mais tu ne peux pas en dire autant, même en ayant dix filles), seul un fils peut assurer la continuité de la lignée familiale. De plus, engendrer un fils confirme la réputation d'un homme dans la communauté, ainsi que sa masculinité.

Il faut cependant noter que le confucianisme tel qu'il est adopté au Viêt Nam se distingue du modèle chinois ; l'étude du code légal mis en place sous la dynastie Lê (1428-1788) révèle que si les femmes avaient un statut inférieur aux hommes, l'héritage était cependant partagé entre garçons et filles, et ces dernières pouvaient pratiquer le culte des ancêtres en l'absence d'un fils. De plus, la corésidence des fils mariés avec leurs parents était moins systématique qu'en Chine; il n'était pas rare que les couples mariés s'établissent dans le village du mari, mais de manière autonome. En réalité, si les valeurs confucéennes furent adoptées par les membres de la classe aisée dans l'espoir d'améliorer leur statut social en se conformant à l'idéologie d'Etat, la majorité du peuple conserva durant cette période ses valeurs et ses pratiques traditionnelles, fondées sur un système de parenté bilatéral (Haines 1984, Yu 2008). Ce n'est en réalité que plus tard, sous la dynastie Nguyên (1802-1945), que le confucianisme fut renforcé. En outre, une part importante de l'ethnie Kinh actuelle, dans le sud du Viêt Nam, est composée de descendants de populations d'origines ethniques différentes, y compris de groupes qui ont longtemps été connus pour avoir des règles de parenté et des modèles de résidence bilatéraux comme ailleurs en Asie du Sud-est (Liljestrom et coll. 1991, Bélanger 2000). En effet, l'expansion progressive des Vietnamiens (et des Chinois) dans les territoires Khmer et Cham, connue sous le nom de « Marche vers le sud» (Nam tiến), ne date que du $17^{\mathrm{e}}$ siècle.

Ce dernier facteur, la préférence de genre, est aussi le plus ancien - avant que la faible fécondité n'exerce une pression sur les couples, et que les nouvelles technologies permettent le recours aux avortements sexo-sélectifs, les couples continuaient de concevoir des enfants jusqu'à ce qu'ils aient atteint le nombre désiré de fils (Guilmoto 2012a). Ce phénomène peut être étudié grâce aux probabilités d'agrandissement, un indicateur que nous allons analyser un peu plus loin. D'autre part, c'est l'intensité de la préférence pour les fils, liée aux systèmes de parenté patrilinéaires ou mixtes, qui est le principal élément expliquant les disparités régionales que nous pouvons observer dans 
le graphique 1, le niveau de fécondité étant relativement faible dans l'ensemble des provinces vietnamiennes, et l'accès aux nouvelles technologies se développant rapidement dans l'ensemble du pays. Cette étude se propose donc de comparer deux provinces, afin d'analyser les différences dans la préférence pour les garçons. Si le système de parenté dans la province de Hai Duong apparaît fortement patriarcal, justifiant la nécessité de concevoir un fils qui accomplira le culte des ancêtres et continuera la lignée familiale, nous allons démontrer que la situation à Ninh Thuân est différente, car cette province comporte l'un des plus forts pourcentages de Cham dans le pays, un groupe ethnique ayant un système de parenté matrilinéaire.

Graphique 1. Rapport de masculinité à la naissance selon la région du lieu de résidence de la mère

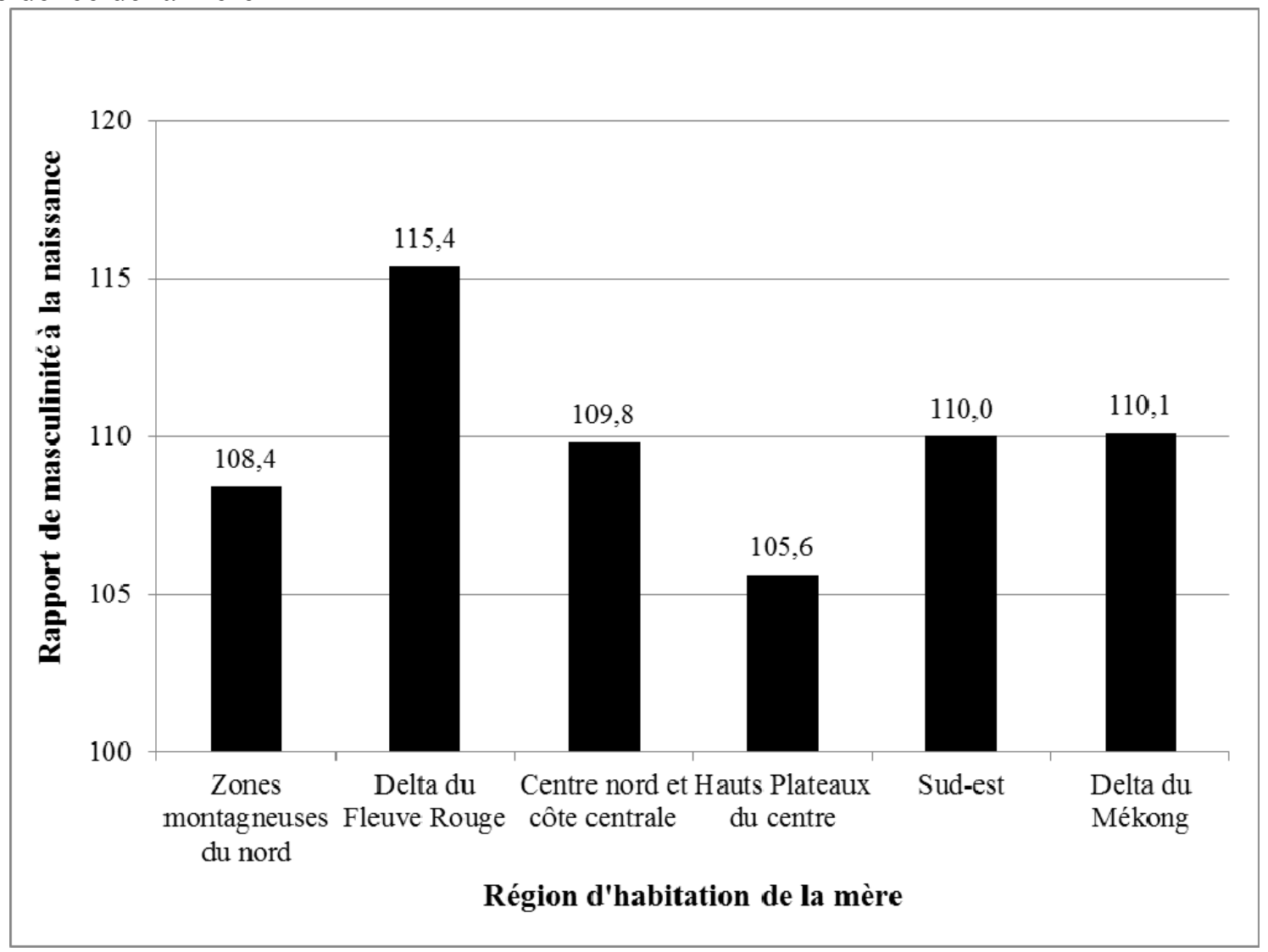

Source : recensement de 2009

\section{II- Données et méthodologie}

\section{$\underline{\text { II.1- Source quantitative }}$}

Les statistiques présentées dans cette étude proviennent de l'analyse du Recensement général de la population et de l'habitat du Viêt Nam de 2009. Il porte sur 85,8 millions de personnes réparties dans 22,6 millions de ménages, mais les données (brutes) analysées sont issues du « questionnaire long », restreint à $15 \%$ de la population. Cet échantillon représente une base de données de 14177590 personnes (3 692042 ménages), dont quatre millions de femmes âgées de 15 à 49 ans. Le rapport de masculinité à la naissance est calculé à partir des 247603 naissances ayant eu lieu dans les 12 mois précédant le recensement. 


\section{II.2- Enquête sur les pratiques reproductives et la préférence de genre}

Les enquêtes qualitatives se sont déroulées, en octobre 2012 et janvier 2013, dans deux provinces différentes, sélectionnées en raison de leurs particularités, et de leurs niveaux différents du rapport de masculinité à la naissance. La première enquête a eu lieu à Hai Duong, dans le Delta du Fleuve Rouge au nord du Viêt Nam, une province ayant l'un des rapports de masculinité à la naissance les plus déséquilibrés du pays $\left(120,2\right.$ en 2009) ${ }^{26}$. La seconde s'est déroulée à Ninh Thuân, située sur la côte centrale du sud, car c'est l'une des provinces ayant le plus haut pourcentage de villages Cham - une minorité ethnique dont le système de parenté est matrilinéaire -, où le déséquilibre du rapport de masculinité à la naissance est moindre (110,8 en 2009). ${ }^{27}$

Dans chaque province, l'enquête a été mise en place dans deux districts différents, l'un urbain et l'autre rural - Viêt Hòa et Thanh Hà dans la province de Hai Duong, et ville de Phan Rang et Ninh Hai dans la province de Ninh Thuân -, afin d'élargir les catégories socioéconomiques des personnes incluses dans l'échantillon.

\section{II.2.1- Méthodologie de la collecte}

L'enquête repose sur des entretiens semi-directifs approfondis afin d'observer les pratiques reproductives et les intentions de fécondité des couples, ainsi que la préférence de genre et les différences entre garçon et fille au sein d'une fratrie. Les hommes et les femmes ont été interrogés séparément, à propos de leur profil socioéconomique, leur mariage, leur utilisation de la contraception et leur expérience de l'avortement, ainsi que sur des normes culturelles spécifiques (le culte des ancêtres, l'héritage et l'aide aux parents âgés), et leur connaissance de la problématique de la masculinité des naissances. Des entretiens collectifs exclusivement féminins ou masculins ont également été organisés afin d'analyser plus finement les rapports familiaux intergénérationnels, et la pression familiale et communautaire qui s'exerce autour du sexe de l'enfant à naître.

Les personnes interrogées ont été sélectionnées directement par les travailleurs des centres communaux de santé, en lien avec les Comités provinciaux pour la Population et la Planification familiale de Hai Duong et de Ninh Thuân, à partir de plusieurs caractéristiques que nous avions établies : âge, genre, nombre et sexe des enfants. Les entretiens semi-directifs individuels ont eu lieu au domicile des enquêtés, tandis que les entretiens collectifs, organisés avec six hommes ou six femmes, ont été enregistrés dans la salle du Comité populaire du village enquêté. Chaque personne interrogée a signé un accord après une présentation détaillée du contexte de l'enquête, et la confidentialité des témoignages recueillis a été préservée, en s'assurant de la seule présence de l'enquêtrice et de l'interprète lors des entretiens, notamment en raison de l'illégalité du recours à l'avortement sexo-sélectif ${ }^{28}$. Il n'a jamais été demandé par les autorités, locales comme nationales, de parcourir ni de vérifier les données recueillies.

\section{II.2.2- Les participants}

Les deux enquêtes ont des échantillons similaires regroupant des personnes ayant seulement des filles, interrogées séparément des personnes ayant un ou plusieurs fils. Huit entretiens individuels et quatre entretiens collectifs ont été effectués au total dans

\footnotetext{
${ }^{26}$ Source : données du recensement 2009 publiées par l'Office général des statistiques

27 Source : ibid.

${ }^{28}$ Une sous-estimation du recours à cette pratique par les personnes interrogées est toutefois vraisemblable.
} 
chaque province, avec autant d'hommes que de femmes interrogées. Dans la province de Hai Duong, l'intégralité des individus enquêtés était mariée et d'origine Kinh ; les seize femmes étaient âgées de 25 à 51 ans, et les seize hommes de 28 à 55 ans. L'échantillon à Ninh Thuân est plus varié : si tous les individus interrogés sont mariés, huit hommes de 30 à 43 ans et huit femmes de 33 à 41 ans sont Kinh, tandis que huit hommes de 28 à 44 ans et huit femmes de 25 à 44 ans sont Cham.

Nous avons également rencontré un(e) chef de village dans chaque district - deux femmes à Hai Duong et deux hommes à Ninh Thuân -, et quatre chefs de clan, qui étaient exclusivement des hommes. De plus, nous avons visité des centres communaux de santé afin de vérifier les registres de naissance, et interrogé des praticiens de santé au sein des hôpitaux de province et des cliniques privées. Enfin, nous avons organisé des réunions avec des organisations civiles telles que l'Union des Femmes ou l'Union des Paysans, avec le Comité populaire au niveau provincial et communal, ainsi que le Comité provincial pour la Population et la Planification familiale, afin de cerner les politiques et les campagnes locales mises en place pour lutter contre la sélection sexuelle prénatale.

\section{II.2.3- Méthodologie d'analyse}

Un des indicateurs de la préférence pour les garçons est la probabilité d'agrandissement à la parité $n$, c'est-à-dire la proportion de femmes avec $n$ naissances qui ont une naissance supplémentaire, selon le sexe des enfants précédents. Contrairement aux variables d'opinions sur le nombre idéal d'enfants par sexe, cet indicateur permet de mesurer la sur-fécondité des couples en l'absence de fils, et met ainsi en évidence la « préférence révélée » des couples.

La probabilité d'agrandissement est calculée à partir de la reconstruction des fratries: dans les données du recensement, les enfants et petits-enfants du chef de ménage sont identifiés en s'assurant des liens de parenté ; ainsi, les ménages où des cousins peuvent cohabiter sont éliminés (environ $6 \%$ de la population des fratries). Ensuite, seuls les enfants ayant entre 0 et 10 ans révolus sont gardés, afin de limiter les cas où un membre de la fratrie a déjà quitté le foyer. À partir de ces fratries, nous calculons la probabilité pour qu'un enfant ait un petit frère ou une petite sœur, selon son rang de naissance, son sexe et éventuellement celui de son ou ses aîné(s). Ce calcul peut ensuite être décliné selon différentes variables individuelles. ${ }^{29}$

Autres indicateurs de préférence pour les garçons, les rapports de masculinité selon le rang de naissance de l'enfant et la présence éventuelle d'un frère aîné sont calculés à partir du fichier de reconstruction des fratries utilisé pour les probabilités d'agrandissement. Ils concernent donc les enfants âgés de moins d'un an au moment du recensement, et non les naissances ayant eu lieu dans les 12 mois précédant le comptage. Cependant, la variation entre les deux indicateurs est minime, en raison du faible niveau de mortalité infantile au Viêt Nam (16 pour 1000 en 2009).

\section{III- Analyse comparative de deux provinces}

Les disparités régionales sont liées à l'intensité de la préférence pour les garçons, comme le montre l'analyse comparative de deux provinces : Hai Duong et Ninh Thuân.

\footnotetext{
${ }^{29}$ Notons que cette méthode est transversale et non longitudinale ; en effet, les calculs sont effectués sur des femmes qui n'ont pas forcément atteint leur descendance finale.
} 


\section{III.1- La sur-fécondité des couples en l'absence de garçons}

Le tableau 1 montre les probabilités d'agrandissement pour l'ensemble du Viêt Nam, selon le rang de naissance et la composition sexuelle de la fratrie.

Ainsi, au Viêt Nam, 30\% des couples ayant deux filles auront un troisième enfant, contre $15 \%$ des couples qui ont au moins un fils parmi leurs deux enfants. La probabilité qu'un couple ayant deux filles ait un troisième enfant est donc deux fois supérieure à celle d'un couple ayant au moins un fils. Cette préférence pour les garçons peut également se retrouver en comparant les rapports de masculinité selon le rang de naissance de l'enfant et la présence éventuelle d'un frère aîné (tableau 2). Nous pouvons voir que le fait de ne pas avoir de fils influe fortement sur le rapport de masculinité des enfants de moins d'un an, c'est-à-dire sur le recours des couples à la sélection sexuelle prénatale, et ce d'autant plus que le rang de naissance augmente.

Tableau 1. Probabilités d'agrandissement selon la taille de la fratrie et la présence antérieure d'un fils, enfants âgés de 0 à 10 ans révolus

\begin{tabular}{crrrrr}
\hline \multicolumn{1}{c}{ Taille de la } & \multicolumn{2}{c}{ Composition de la fratrie (\%) } & Total (\%) & Ratio (\%) & Effectif \\
fratrie & Sans garçon & Avec garçon(s) & & & \\
\hline $1 \rightarrow 2$ & 51 & 47 & 49 & 109 & 1061997 \\
$2 \rightarrow 3$ & 30 & 15 & 19 & 200 & 889389 \\
$3 \rightarrow 4$ & 33 & 19 & 22 & 174 & 315595 \\
$4 \rightarrow 5$ & 36 & 24 & 25 & 150 & 191798 \\
\hline Total & \multicolumn{7}{c}{} & & & & \\
\hline
\end{tabular}

Ratio = probabilité d'agrandissement dans une fratrie sans garçon/ probabilité d'agrandissement dans une fratrie avec garçon(s)

Source: calculs de l'auteure, à partir de l'échantillon de 15\% du recensement de 2009

Tableau 2. Rapports de masculinité des enfants âgés de moins d'un an, selon le rang de naissance et la présence d'un frère aîné

Viêt Nam

\begin{tabular}{ccrr}
\hline \multirow{2}{*}{$\begin{array}{c}\text { Rang de } \\
\text { naissance }\end{array}$} & \multicolumn{2}{c}{ Composition de la fratrie } & \\
\hline 2 & 108,8 & Avec garçon(s) & Effectif \\
3 & 125,1 & 106,2 & 88839 \\
4 & 133,9 & 106,4 & 28669 \\
Total & & 103,8 & 9430 \\
\hline
\end{tabular}

Source: calculs de l'auteure, à partir de l'échantillon de 15\% du recensement de 2009

Si nous comparons maintenant les probabilités d'agrandissement à Hai Duong et à Ninh Thuân (tableau 3), les résultats montrent que 25\% des femmes ayant eu deux filles à Hai Duong ont eu un troisième enfant, contre seulement $4 \%$ des femmes qui ont eu au moins un garçon. Cependant, à Ninh Thuận, la différence est beaucoup plus faible : $37 \%$ des femmes ayant eu deux filles ont eu un troisième enfant, contre $29 \%$ des femmes ayant eu au moins un fils. Ainsi, la probabilité pour qu'une femme sans fils ait un troisième enfant est plus de six fois supérieure à Hai Duong, et seulement 1,3 fois supérieure à Ninh Thuân. Ces variations de probabilités d'agrandissement démontrent différentes intensités de préférence pour les garçons, que nous allons illustrer avec les témoignages recueillis lors des enquêtes de terrain.

Les deux provinces étudiées ont un niveau de fécondité relativement faible, et un accès développé aux échographies et à la détermination prénatale du sexe du fœtus. Cependant, les données montrent différents niveaux de préférence pour les garçons. 
L'hypothèse que nous proposons ici est que ces disparités sont liées à des systèmes de parenté et des pratiques culturelles sous-jacentes, qui influencent finalement le rapport de masculinité à la naissance.

Tableau 3. Probabilités d'agrandissement selon la taille de la fratrie et la présence antérieure d'un fils dans les provinces de Hai Huong et Ninh Thuân, enfants âgés de 0 à 10 ans révolus

\begin{tabular}{|c|c|c|c|c|c|}
\hline \multicolumn{6}{|c|}{ Province de Hai Duong } \\
\hline \multirow{2}{*}{$\begin{array}{l}\text { Taille } \\
\text { de la } \\
\text { fratrie }\end{array}$} & \multicolumn{2}{|c|}{$\begin{array}{l}\text { Composition de la } \\
\text { fratrie }(\%)\end{array}$} & \multirow[t]{2}{*}{$\begin{array}{l}\text { Tota } \\
(\%) 1\end{array}$} & \multirow[t]{2}{*}{$\begin{array}{l}\text { Ratio } \\
(\%)\end{array}$} & \multirow[t]{2}{*}{ Effectif } \\
\hline & $\begin{array}{l}\text { Sans } \\
\text { garçon }\end{array}$ & $\begin{array}{c}\text { Avec } \\
\text { garçon(s) }\end{array}$ & & & \\
\hline $1 \rightarrow 2$ & 50 & 45 & 47 & 111 & 18454 \\
\hline $2 \rightarrow 3$ & 25 & 4 & 9 & 625 & 16972 \\
\hline $3 \rightarrow 4$ & 25 & 2 & 8 & 1250 & 3566 \\
\hline $4 \rightarrow 5$ & 24 & 2 & 7 & 1200 & 549 \\
\hline Total & & & & & 39541 \\
\hline \multicolumn{6}{|c|}{ Province de Ninh Thuân } \\
\hline \multirow{2}{*}{$\begin{array}{l}\text { Taille } \\
\text { de la } \\
\text { fratrie }\end{array}$} & \multicolumn{2}{|c|}{$\begin{array}{l}\text { Composition de la } \\
\text { fratrie }(\%)\end{array}$} & $\begin{array}{l}\text { Total } \\
(\%)\end{array}$ & $\begin{array}{r}\text { Ratio } \\
(\%)\end{array}$ & Effectif \\
\hline & $\begin{array}{c}\text { Sans } \\
\text { garçon }\end{array}$ & $\begin{array}{c}\text { Avec } \\
\text { garçon(s) }\end{array}$ & & & \\
\hline $1 \rightarrow 2$ & 53 & 54 & 53 & 98 & 9327 \\
\hline $2 \rightarrow 3$ & 37 & 29 & 31 & 128 & 8236 \\
\hline $3 \rightarrow 4$ & 36 & 29 & 30 & 124 & 4669 \\
\hline $4 \rightarrow 5$ & 35 & 27 & 28 & 130 & 4339 \\
\hline Total & & & & & 26571 \\
\hline
\end{tabular}

Ratio $=$ probabilité d'agrandissement dans une fratrie sans garçon/ probabilité d'agrandissement dans une fratrie avec garçon(s)

Source: calculs de l'auteure, à partir de l'échantillon de 15\% du recensement de 2009

\section{III.2- Province de Hai Duong}

Dans la province de Hai Duong, les couples qui n'ont que des filles sont moqués au sein de leur communauté et subissent une forte pression de la famille paternelle étendue. Les pères sans fils sont invités à s'asseoir à des tables différentes, qui correspondent à un statut moins élevé, et les femmes sont encouragées par leurs beaux-parents à avoir un troisième voire un quatrième enfant, et à essayer différentes méthodes afin de concevoir un fils. Ces méthodes se déclinent sous différentes formes : recours à un voyant ou à un calendrier chinois qui indique, selon l'âge de la mère, quand avoir des rapports sexuels afin de concevoir un enfant du sexe désiré, prise de médicaments traditionnels qui, mixés avec de l'alcool de riz, renforceraient les spermatozoïdes $\mathrm{Y}$, ou encore régimes alimentaires.

«Les femmes doivent être plus faibles que les hommes pour qu'un garçon puisse être conçu ». (Entretien collectif avec six femmes ayant des fils, district urbain de Việt Hòa, province de Hai Duong).

Deux éléments semblent être à l'origine de la préférence pour les garçons. D'une part, la transmission du nom de famille, et plus globalement la continuation de la lignée patrilinéaire explique le «besoin » d'un fils. En effet, une femme mariée n'appartient plus à son clan familial, mais à celui de son époux - elle n'est souvent pas inscrite dans le « livre du clan ». 
« Le clan familial dure moins longtemps avec des filles qu'avec des fils ». (Entretien collectif avec six hommes ayant des fils, district rural de Thanh Hà, province de Hai Duong).

Ainsi, seuls les fils peuvent hériter des terres de leurs parents ${ }^{30}$, et un couple sans descendant masculin sera considéré comme ayant "construit une maison par charité ». Il existe pourtant au Viêt Nam depuis 2000 une loi sur le mariage et la famille prescrivant que l'héritage soit distribué également entre frères et sœurs. Cependant, à Hai Duong, le fils aîné reçoit traditionnellement la majorité des biens, des terres cultivables ainsi que le terrain de résidence, tandis que le ou les cadets héritent du reste des terres. Cette pratique s'explique notamment par le culte des ancêtres, pris en charge par le fils aîné, qui reçoit alors les terres de ses parents sous forme de " compensation » (Bélanger et $\mathrm{Li}, 2009$ ). Ce culte est réglementé par différentes coutumes, telles que des dates précises pour les offrandes, au milieu du mois lunaire et lors des anniversaires de mort, des lieux dédiés au sein de chaque maison, mais également une "maison des ancêtres » transmise de fils aîné en fils aîné ${ }^{31}$. Comme l'explique Xin, âgé de 73 ans et chef de clan, il existe une hiérarchie des différentes " branches », et seuls les fils aînés sont en charge de rendre le culte à l'ensemble de la famille, tandis que les cadets s'occupent uniquement de leurs parents directs. Les travaux anthropologiques de Hy Van Luong, cités par Bélanger et Khuât (2009), montrent « une augmentation dans la pratique de ces rituels depuis le début des années 90 , qui a renforcé la nécessité d'avoir un fils ».

D'autre part, avoir un fils est l'assurance pour des parents d'un support économique dans le futur, dans un contexte où le versement de pension aux personnes âgées est quasi inexistant. Pourtant, les hommes et les femmes interrogés expliquent que leurs filles prennent soin d'eux - parfois mieux que leurs fils - lorsqu'ils sont malades ou âgés. Par exemple, Nhu et Thai, deux femmes âgées respectivement de 39 et 45 ans, ont migré 3 et 5 ans pour travailler à Taïwan, et aider leurs parents financièrement - ce système de versements, effectués à la fois par les fils et les filles, est un support intergénérationnel qui se développe, et pallie partiellement l'absence de corésidence entre les parents âgés et leur fils (Barbieri 2009). S'il y a une préférence marquée pour les garçons, il n'y a pas « d'aversion » pour les filles : les couples désirent des enfants des deux sexes. Cependant, il est rare que des parents vivent avec leur fille et leur gendre, ce dernier ayant peur de perdre la face (UNFPA 2011). Ainsi, si l'on considère l'ensemble des ménages du Delta du Fleuve Rouge, 16,1\% des couples résident avec leur(s) enfant(s) marié(s), mais seulement 14,1\% de ces enfants sont des filles (données du recensement 2009).

Il est intéressant de noter que selon les données du recensement, le rapport de masculinité des naissances de rang 1 - qui constituent une part importante de l'ensemble des naissances, toutes parités confondues - est élevé, à 111,5 garçons pour 100 filles au niveau national (enquête annuelle de l'Office Général des Statistiques, 2012). Ce phénomène, unique en comparaison des autres pays connaissant un déséquilibre des naissances en faveur des garçons, est d'autant plus étonnant que le rapport de masculinité des naissances de rang 2 est moins élevé (110,1 en 2012). Différents couples interrogés ont ainsi expliqué qu'avoir un fils en premier est considéré

\footnotetext{
${ }^{30}$ En réalité, les citoyens vietnamiens ne possèdent pas de terre, mais un « droit d'utilisation de la terre » pour un nombre d'années défini. Ce droit, transmis des parents aux enfants, est réévalué périodiquement en fonction de la taille du ménage.

${ }^{31}$ Cependant, plusieurs personnes interrogées ont expliqué que dans leur famille, le fils aîné était parti s'installer avec sa femme dans un nouveau foyer. C'est alors le fils benjamin qui hérite de la maison des parents et prend la responsabilité du culte des ancêtres.
} 
comme une « assurance » pour les grands-parents d'avoir au moins un petit-fils, afin de continuer la lignée familiale et de pratiquer le culte des ancêtres, mais également pour les femmes.

«C'est l'attitude commune chez les femmes, avoir un fils la première fois est une sécurité ». (Entretien individuel avec la mère de deux garçons, 30 ans, district urbain de Việt Hòa, province de Hai Duong).

En réalité, plusieurs femmes interrogées se sentent obligées de mettre au monde un fils «pour» leur mari, et redoutent que celui-ci entretienne une relation extra-conjugale afin de "déposer un fils» et concevoir un héritier. Cette pression exercée sur les femmes provient également de la belle-famille.

«Ma sœur a deux filles, et son mari est le fils aîné. Son beau-père a été direct avec ma sœur, si elle ne pouvait pas avoir de garçon, il trouverait une autre femme pour son fils. » (Entretien collectif avec six femmes ayant seulement des filles, district urbain de Viêt Hòa, province de Hai Duong).

En effet, le sexe de l'enfant à naître est considéré comme la responsabilité de la femme, et celles qui n'ont pas de fils sont estimées être "incapables de donner naissance ». Ce témoignage se retrouve dans l'étude de Danièle Bélanger sur la préférence pour les garçons dans un village du nord Viêt Nam (Bélanger, 2002). Mais il semble que la pression soit encore plus grande du fait de l'accessibilité des échographies, et donc de la sélection sexuelle prénatale, les femmes ayant alors moins de raison de ne pas « réussir » à avoir un fils.

"A ma génération, les choses étaient plus simples ». (Entretien individuel avec la mère d'un garçon et d'une fille, 45 ans, district urbain de Viêt Hòa, province de Hai Duong).

Dans ce contexte, nombreux sont les exemples d'avortements sexo-sélectifs rapportés par les femmes interrogées.

« Près de chez moi, un couple avait trois filles et vient d'avoir un fils. Avant ce garçon, ma voisine a avorté d'une fille. Même si elle a 40 ans, elle a continué d'essayer jusqu'à ce qu'un fils naisse. Elle ne voulait pas continuer, mais elle a dû essayer à cause de ses beaux-parents, pas à cause de son mari». (Entretien individuel avec la mère de deux garçons, 35 ans, district rural de Thanh Hà, province de Hai Duong).

Si aucune expérience individuelle n'a été directement mentionnée, l'acte étant illégal, chaque femme et chaque homme interrogé à Hai Duong connaît une voisine, une sœur ou une cousine qui a eu recours à la sélection sexuelle prénatale.

« Ma cousine a deux filles de 11 et 6 ans. Cette année, elle a essayé d'avoir un fils, mais elle a dû attendre que le fotus ait 12 semaines pour connaître le sexe. Elle a fait une échographie, qui a montré que c'était une fille. Elle n'a pas cru au résultat. A la $14^{\mathrm{e}}$ semaine de grossesse, elle a vérifié encore et a trouvé le même résultat. Elle ne le croyait toujours pas. Pour être sûre, elle est allée voir une voyante qui lui a aussi dit que c'était une fille. A la fin, elle a décidé d'avorter. " (Entretien collectif avec six hommes ayant des fils, district rural de Thanh Hà, province de Hai Duong).

\section{III.3- Province de Ninh Thuân}

Contrairement à la province de Hai Duong, nous pouvons voir une différence marquée s'établir entre le milieu urbain et le milieu rural en termes de préférence pour les garçons (tableau 4). En effet, la probabilité pour qu'un couple sans fils ait un troisième enfant est presque deux fois supérieure à celle d'un couple ayant au moins un 
fils en zone urbaine. Ce rapport est seulement 1,15 en zone rurale. Ce résultat n'est pas étonnant: si les zones urbaines sont peuplées à 95\% de Vietnamiens Kinh, les zones rurales comptent $34 \%$ de Vietnamiens issus de minorités ethniques, soit des Cham et des Raglay, dont le système de parenté matrilinéaire n'induit pas de préférence pour les garçons $^{32}$. En effet, les données issues de l'enquête approfondie dans la province de Ninh Thuân montrent au contraire une préférence pour les filles chez les Cham. Cette minorité ethnique représente aujourd'hui seulement $11,9 \%$ de la population de la province, mais c'était, jusqu'au début du XIXe siècle, le groupe dominant ${ }^{33}$.

Tableau 4. Probabilités d'agrandissement selon la taille de la fratrie et la présence antérieure d'un fils dans la province de Ninh Thuân, en milieu urbain et rural, enfants âgés de 0 à 10 ans révolus

\begin{tabular}{|c|c|c|c|c|c|}
\hline \multicolumn{6}{|c|}{ Urbain } \\
\hline \multirow[t]{2}{*}{$\begin{array}{l}\text { Taille de } \\
\text { la fratrie }\end{array}$} & \multicolumn{2}{|c|}{$\begin{array}{c}\text { Composition de la } \\
\text { fratrie }(\%)\end{array}$} & \multirow[t]{2}{*}{$\begin{array}{c}\text { Total } \\
(\%)\end{array}$} & \multirow[t]{2}{*}{$\begin{array}{l}\text { Ratio } \\
(\%)\end{array}$} & \multirow[t]{2}{*}{ Effectif } \\
\hline & $\begin{array}{l}\text { Sans } \\
\text { garçon }\end{array}$ & $\begin{array}{c}\text { Avec } \\
\operatorname{garçon}(\mathrm{s})\end{array}$ & & & \\
\hline $1 \rightarrow 2$ & 48 & 47 & 47 & 102 & 2521 \\
\hline $2 \rightarrow 3$ & 31 & 16 & 19 & 194 & 2168 \\
\hline $3 \rightarrow 4$ & 32 & 19 & 21 & 169 & 827 \\
\hline $4 \rightarrow 5$ & 15 & 17 & 17 & 88 & 518 \\
\hline Total & & & & & 6034 \\
\hline \multicolumn{6}{|c|}{$\begin{array}{r}\text { Rural } \\
\end{array}$} \\
\hline \multirow[t]{2}{*}{$\begin{array}{l}\text { Taille de } \\
\text { la fratrie }\end{array}$} & \multicolumn{2}{|c|}{$\begin{array}{l}\text { Composition de la } \\
\text { fratrie }(\%)\end{array}$} & $\begin{array}{l}\text { Total } \\
(\%)\end{array}$ & $\begin{array}{l}\text { Ratio } \\
(\%)\end{array}$ & Effectif \\
\hline & $\begin{array}{l}\text { Sans } \\
\text { garçon }\end{array}$ & $\begin{array}{c}\text { Avec } \\
\text { garçon(s) }\end{array}$ & & & \\
\hline $1 \rightarrow 2$ & 55 & 56 & 56 & 98 & 6806 \\
\hline $2 \rightarrow 3$ & 39 & 34 & 35 & 115 & 6068 \\
\hline $3 \rightarrow 4$ & 37 & 31 & 32 & 119 & 3842 \\
\hline $4 \rightarrow 5$ & 40 & 29 & 30 & 138 & 3821 \\
\hline Total & & & & & 20537 \\
\hline
\end{tabular}

Ratio = probabilité d'agrandissement dans une fratrie sans garçon/ probabilité d'agrandissement dans une fratrie avec garçon(s)

Source: calculs de l'auteure, à partir de l'échantillon de 15\% du recensement de 2009

Dans le district de Ninh Hai, où les entretiens individuels et collectifs ont été effectués, la majorité des Cham pratique la religion Bani, qui combine des influences musulmanes, hindoues et bouddhistes. Dans ces villages, la plupart des jeunes mariés résident dans la famille de la femme jusqu'à ce qu'ils puissent construire une nouvelle maison. Comme nous l'ont expliqué les anciens, "la tradition ici est que la femme se marie à l'homme, et non le contraire ». Les parents de la future mariée apportent des offrandes à la famille de l'époux et font une proposition de mariage.

« Si la famille accepte, ils le ramènent à la maison pour célébrer le mariage, puis [l'époux] reste. Il devient un membre du clan familial de sa femme ».

\footnotetext{
${ }^{32}$ Malheureusement, les données désagrégées du recensement en notre possession ne permettent pas d'identifier précisément l'origine ethnique; nous savons seulement si les individus sont «Kinh » ou «d'autre origine ethnique ». Cependant, les données publiées officiellement par l'Office général des Statistiques montrent que les Vietnamiens non Kinh dans la province de Ninh Thuân sont très majoritairement Cham ou Raglay.

${ }^{33}$ La province de Ninh Thuận, dernier bastion du royaume Cham, s'appelait Panduranga, jusqu'à sa prise par les Vietnamiens en 1832 (Hardy et coll. 2009).
} 
(Entretien individuel avec un chef de clan âgé de 61 ans, père de quatre filles et trois fils, district rural de Ninh Hai, province de Ninh Thuân).

Ici, la plus jeune fille des familles Cham porte les mêmes responsabilités que le fils aîné dans les familles Kinh du Delta du Fleuve Rouge. Communément, la dernière fille et son mari résident chez ses parents pour prendre soin d'eux jusqu'à leur mort, puis ils héritent de la "maison principale», où l'autel des ancêtres se situe, pour prendre en charge le culte des ancêtres ${ }^{34}$. Dans ce cas, la dernière fille hérite de la plupart des biens familiaux : la maison, les animaux, les biens, et les terres cultivables.

Ainsi, nous avons trouvé dans cette zone des caractéristiques marquées de la préférence de genre, mais cette fois-ci biaisées envers l'autre sexe. Par exemple, il est considéré comme essentiel d'avoir au moins une fille, de préférence en premier. C'est ici aussi considéré comme une "assurance ", et cela réduit la pression sur le sexe des futurs enfants. Les pères et les mères interrogés n'ayant que des fils racontent subir les moqueries de leur entourage familial et amical pendant les fêtes. Cependant, beaucoup ont mentionné une solution à l'absence de descendante: l'adoption d'une fille généralement une nièce du clan maternel -, qui peut « hériter des biens et prendre soin [d'eux] ». Ces filles sont soit légalement adoptées et élevées par le couple, soit désignées dans le testament comme héritières de la maison principale pour rendre le culte aux ancêtres.

« J'ai beaucoup de nièces, donc pour l'instant je n'ai pas de plan concret, mais quand je serai vieux, je réfléchirai à qui je donnerai tout. » (Entretien individuel avec le père de trois fils, 44 ans, district rural de Ninh Hai, province de Ninh Thuân).

Cette solution n'a jamais été évoquée dans les autres villages d'enquête - aucun Kinh n'a mentionné l'éventualité d'adopter un garçon au sein du clan paternel. De plus, aucun recours à l'avortement sexo-sélectif n'a été énoncé durant les entretiens. L'étude du tableau 5 nous indique en effet que la préférence de genre, si elle est biaisée envers les filles, n'induit pas de comportement reproductif différencié en fonction du sexe chez les minorités ethniques de Ninh Thuân contrairement à ce que l'on observe chez les Kinh. Chez les minorités ethniques de Ninh Thuận, les probabilités pour un couple d'avoir un enfant supplémentaire sont égales quelle que soit la composition de la fratrie ; on note seulement un léger biais dans la probabilité d'avoir un cinquième enfant lorsque les quatre enfants précédents sont des filles.

Une autre particularité intéressante de cette société réside dans la terminologie utilisée pour désigner les grands-parents et les petits-enfants dans le langage vietnamien : "nội" signifie intérieur, domestique et "ngoại" signifie extérieur. Pour les Kinh, "ông bà nội" et "chaú nội" sont utilisés pour se référer respectivement aux grands-parents et petits-enfants de la branche paternelle. Mais chez les Cham, ces termes sont utilisés pour désigner les grands-parents et petits-enfants de la branche maternelle.

Ces signes clairs d'un système de parenté matrilinéaire sont toutefois contrebalancés par quelques pratiques patrilinéaires. Ainsi, le nom de famille est transmis de père en fils, les chefs de clan sont exclusivement des hommes et, si le culte des ancêtres est pratiqué pour les membres familiaux du clan maternel, c'est en réalité le mari qui est en charge des rituels.

Quant aux villages Kinh enquêtés dans la province de Ninh Thuận, ils témoignent d'un système de parenté patrilinéaire mais avec des pratiques bilatérales. Par exemple, l'héritage est partagé entre les filles et les garçons, et si le culte des ancêtres doit être

\footnotetext{
${ }^{34} \mathrm{Ce}$ culte est très simplifié en comparaison des pratiques des Kinh, il se fait seulement au moment de la nouvelle année, qui a lieu pendant le septième mois du calendrier lunaire.
} 
effectué de préférence par le fils, une fille et son mari peuvent également être en charge de ce culte sans que cela pose réellement problème. Ainsi, un chef de clan âgé de 82 ans et résidant dans la ville de Phan Rang nous a expliqué rendre le culte aux ancêtres aux parents de sa femme, puisque celle-ci n'a pas de frère. Cependant, la corésidence des enfants mariés avec les parents semble en majorité patrilocale.

Notons ici que 4 personnes sur les 16 individus Kinh interrogés à Ninh Thuân sont de religion catholique, et ont donc exprimé leur opposition fondamentale au recours à l'avortement, mais également une préférence de genre peu marquée, puisque chaque enfant peut hériter et pratiquer le culte des ancêtres (dans une version extrêmement simplifiée), quel que soit son sexe. Seul Cuong, un homme catholique âgé de 37 ans et père de 3 filles puis d'un fils, a expliqué avoir conçu quatre enfants afin d'avoir un fils qui pratique le culte des ancêtres.

Il existe donc globalement une préférence pour les garçons, pour les mêmes raisons que celles exprimées dans les villages Kinh du Delta du Fleuve Rouge, mais la pression familiale semble bien moins marquée; des arrangements sont trouvés en l'absence de descendance masculine.

Tableau 5. Probabilités d'agrandissement selon la taille de la fratrie et la présence antérieure d'un fils à Ninh Thuân dans les populations appartenant à une minorité ethnique et celles appartenant à l'ethnie Kinh, enfants âgés de 0 à 10 ans révolus Ninh Thuân (minorité ethnique)

\begin{tabular}{|c|c|c|c|c|c|}
\hline $\begin{array}{l}\text { Taille } \\
\text { de }\end{array}$ & \multicolumn{2}{|c|}{ Composition de la fratrie (\%) } & \multirow[t]{2}{*}{ Total (\%) } & \multirow[t]{2}{*}{ Ratio (\%) } & \multirow[t]{2}{*}{ Effectif } \\
\hline $\begin{array}{r}\text { la } \\
\text { fratrie }\end{array}$ & Sans garçon & Avec garçon(s) & & & \\
\hline $1 \rightarrow 2$ & 57 & 59 & 58 & 97 & 3191 \\
\hline $2 \rightarrow 3$ & 43 & 43 & 43 & 100 & 2811 \\
\hline $3 \rightarrow 4$ & 39 & 39 & 39 & 100 & 2091 \\
\hline $4 \rightarrow 5$ & 39 & 35 & 35 & 111 & 2552 \\
\hline Total & & & & & 10645 \\
\hline \multicolumn{6}{|c|}{ Ninh Thuân (ethnie Kinh) } \\
\hline de $\begin{array}{c}\text { Taille } \\
\end{array}$ & \multicolumn{2}{|c|}{ Composition de la fratrie $(\%)$} & Total $(\%)$ & Ratio (\%) & Effectif \\
\hline $\begin{array}{c}\text { la } \\
\text { fratrie }\end{array}$ & Sans garçon & Avec garçon(s) & & & \\
\hline $1 \rightarrow 2$ & 52 & 51 & 51 & 102 & 6136 \\
\hline $2 \rightarrow 3$ & 33 & 21 & 24 & 157 & 5425 \\
\hline $3 \rightarrow 4$ & 34 & 21 & 23 & 162 & 2578 \\
\hline $4 \rightarrow 5$ & 31 & 19 & 20 & 163 & 1787 \\
\hline
\end{tabular}

Ratio = probabilité d'agrandissement dans une fratrie sans garçon/ probabilité d'agrandissement dans une fratrie avec garçon(s)

Source: calculs de l'auteure, à partir de l'échantillon de 15\% du recensement de 2009

L'analyse des probabilités d'agrandissement chez les Kinh de la province le confirme : la probabilité des couples sans fils d'avoir un enfant supplémentaire est supérieure à celle des couples ayant un ou plusieurs garçons. Cependant, ces chiffres sont très largement inférieurs à ceux de Hai Duong, où la probabilité d'avoir un troisième enfant est plus de six fois supérieure pour un couple sans fils, alors qu'elle est seulement 1,5 fois supérieure chez les Kinh de Ninh Thuận.

La province de Ninh Thuân illustre donc une trajectoire différente de la masculinité des naissances au Viêt Nam. Les Cham émergent comme un groupe 
relativement important, et leur structure de parenté matrilinéaire tend à réduire le besoin en héritiers mâles. Les Vietnamiens Kinh de la province eux-mêmes rapportent certaines pratiques bilatérales - nous pouvons faire l'hypothèse que les mariages mixtes entre les Cham et les Kinh suite à l'expansion du territoire vietnamien au sud (Nam tiến) ont influé sur leur modèle de parenté. De plus, la présence de catholiques Kinh réduit la préférence pour les garçons, mesurée par les probabilités d'agrandissement, dans l'ensemble de la population. Si aucun cas d'avortement sexo-sélectif n'a été rapporté durant les entretiens, le rapport de masculinité à la naissance apparaît tout de même déséquilibré dans cette province, témoignant du recours à la sélection sexuelle prénatale d'une partie de la population.

\section{Conclusion}

Avec la généralisation de l'accès à l'échographie durant la dernière décennie, à Hai Duong comme à Ninh Thuận, la population peut recourir à la sélection sexuelle prénatale. Contrairement à certaines prénotions, la plupart des femmes en ruralité $(78,9 \%)$ ou peu éduquées $(73,3 \%)$ connaissaient le sexe de leur enfant à venir en $2012^{35}$. Les enquêtes qualitatives confirment cette tendance, puisque toutes les personnes interrogées ont eu recours aux échographies. De plus, si le faible niveau de fécondité constitue un facteur de pression, celui-ci n'est pas suffisant pour expliquer les écarts du rapport de masculinité dans les deux provinces étudiées. En effet, la probabilité de ne pas avoir de garçon, entre la province de Hai Duong avec un indice de fécondité à 2 enfants par femme, et Ninh Thuân avec 2,4 enfants par femme, varie seulement de quelques décimales. Il semble donc que le troisième facteur, celui de la préférence pour les garçons, soit décisif pour expliquer les différences du rapport de masculinité à la naissance entre les deux provinces. L'intensité de cette préférence pour les garçons, qui pousse certains couples à recourir à la sélection sexuelle prénatale, est principalement liée à des facteurs culturels comme le système de parenté ou la religion.

Ces deux provinces illustrent bien les disparités régionales qui existent au Viêt Nam en termes de préférence pour les garçons. Tandis que le Delta du Fleuve Rouge est marqué par un système de parenté patriarcal et patrilinéaire, dans lequel avoir un fils apparaît indispensable, la situation au sud du Viêt Nam apparaît plus complexe, avec une préférence pour les garçons moins marquée et un système de parenté à tendance bilatérale, notamment grâce à la présence de minorités ethniques. Un brassage culturel entre les Khmer et les Cham ayant des structures de parenté respectivement bilatérales et matrilinéaires, et la population Kinh traditionnellement patrilinéaire s'est opéré au cours des derniers siècles dans plusieurs régions du centre et du sud du pays.

$\mathrm{Au}$ Viêt Nam, les fortes disparités régionales du rapport de masculinité à la naissance sont en partie dues aux différences de niveau de fécondité et de richesse. Les changements démographiques et socioéconomiques en cours affectent les régions encore défavorisées ; par la diffusion de l'information, l'expansion des échographies et de la détermination sexuelle prénatale, ils entraînent une détérioration de leurs rapports de masculinité à la naissance. Ainsi, les ratios sont globalement en augmentation dans l'ensemble du pays depuis six ans. Cependant, il semble peu probable que certaines

\footnotetext{
${ }^{35}$ Selon l'enquête annuelle de l'Office Général des Statistiques portant sur l'ensemble du pays en 2012 , $78,9 \%$ des femmes en milieu rural (contre $86,7 \%$ en milieu urbain) et $73,3 \%$ des femmes n'ayant pas terminé l'école primaire (contre $83,2 \%$ des femmes ayant terminé le collège) ont eu connaissance du sexe du fœtus lors de leur dernière grossesse (Ministry of Planning and Investment et coll. 2012).
} 
provinces au sud du pays atteignent de hauts niveaux de déséquilibre (comme on peut le voir dans le delta du Fleuve Rouge), en raison des résistances à la patrilinéarité.

Le gouvernement vietnamien a mis en place différents projets pour lutter contre la sélection sexuelle prénatale, qui consistent principalement en la diffusion de campagnes de communication sur la prohibition des avortements sexo-sélectifs et les conséquences à venir du déséquilibre du rapport de masculinité à la naissance. Si la problématique semble moins connue dans les villages enquêtés de la province de Ninh Thuận, particulièrement chez les Cham, la totalité des personnes rencontrées à Hai Duong est consciente du problème, et connaît les conséquences possibles de ce déséquilibre, à travers l'exemple de la Chine.

En effet, en Chine et en Inde, le déséquilibre du rapport de masculinité à la naissance, aggravé par la négligence de soins envers les petites filles, entraîne une masculinisation de la population générale qui se fera ressentir mécaniquement dans les 50 ans à venir avec le vieillissement de la population (Croll 2000, Das Gupta et coll. 2003, Attané et coll. 2007). Lorsque ces générations déséquilibrées seront en âge de se marier, le surplus démographique d'hommes provoquera un goulot d'étranglement et conduira donc à un retard obligatoire de l'âge au mariage masculin et une croissance prévisible du volume du célibat définitif parmi les hommes - celui-ci étant encore traditionnellement très faible, à la différence de l'Europe. Le phénomène étant très récent au Viêt Nam, ce problème de marché matrimonial ne se posera toutefois pas avant la prochaine décennie.

En 2010, il était estimé que 245000 femmes environ manquaient au Viêt Nam, en grande partie à cause de la sélection sexuelle prénatale, bien que le rapport de masculinité de la population générale soit encore légèrement déséquilibré en faveur des femmes en raison des forts taux de mortalité masculine durant la guerre ${ }^{36}$ (Guilmoto 2012b). ${ }^{37}$ Nghiêm, dans le district de Viêt Hòa à Hai Duong, conclut ainsi : "Nous avons deux fils. Mes amis s'amusent souvent à me dire que dans le futur, mes deux fils devront se partager une femme ».

\section{Bibliographie}

AtTANÉ Isabelle, GuiLmoto Christophe Z. (éd.), Watering the neighbour's garden: The growing demographic female deficit in Asia, Paris, Cicred, 2007, 425 p.

ATTANÉ Isabelle, VERON Jacques, Gender discriminations among young children in Asia, All India Press, Pondicherry, IFP/Ceped, 2005, 314 p.

BANENS Mark, Vietnam: a reconstitution of its 20th Century Population History, Institute of Economic Research, Tokyo, Hitotsubashi University, 2000, 47 p.

BARBIERI Magali, « Doi Moi and the ederly: intergenerational support under the strain of reforms" in Reconfiguring Families in contemporary Vietnam, Magali Barbieri and Danièle Bélanger eds, Contemporary issues in Asia and the Pacific, Stanford University Press, 2009, p. 133-165.

\footnotetext{
${ }^{36}$ Selon les projections de population effectuées par les Nations Unies, ce sex ratio de la population générale va cependant s'inverser en faveur des hommes dans les cinq prochaines années, et ce en raison de la masculinité des naissances.

${ }^{37} \mathrm{Ce}$ calcul est le résultat de la comparaison entre la population féminine actuelle, et la population estimée si la distribution par âge était identique à celle du reste du monde, c'est-à-dire l'ensemble des pays auxquels ont été soustraits les pays indiquant un déséquilibre du rapport de masculinité à la naissance.
} 
BEDI Arjun Singh, Bare branches and drifting kites: tackling female infanticide and feticide in India, Institute of Social Studies Public Lecture Series $\mathrm{N}^{\circ} 5$, The Hague, The Netherlands, 2008, 33 p.

BÉLANGER Danièle, "Regional differences in household composition and family formation patterns in Vietnam », in Journal of Comparative Family Studies, $\mathrm{n}^{\circ}$ 2/31, 2000, p. 171-189.

BÉLANGER Danièle, "Son preference in a rural village in North Vietnam ", in Studies in Family Planning, $n^{\circ}$ 4/33 2002, p. 312-334.

BÉLANGER Danièle, «Indispensable Sons: Negotiating reproductive desires in rural Vietnam », in Gender, Place \& Culture, n 3/13, 2006, p. 251-265.

BÉLANGER Danièle, BARBIERI Magali «Introduction : state, families and the making of transitions in Vietnam » in Reconfiguring Families in contemporary Vietnam, Magali Barbieri and Danièle Bélanger ed., Contemporary issues in Asia and the Pacific, Stanford University Press, 2009, March, pp. 1-44.

BÉLANGER Danièle, KHUÂT Thi Hai Oanh, Second trimester abortions and sex-selection of children in Hanoi, Vietnam", in Population Studies, n² 2/63, 2009, p. 163-171.

BÉLANGER Danièle, Li Xu, « Agricultural Land, Gender and Kinship in Rural China and Vietnam: A Comparison of Two Villages », in Journal of Agrarian Change, $\mathrm{n}^{\circ} 2 / 9$, 2009, p. 204-230.

CoAle Ansley J., BANiSTer Judith, "Five Decades of Missing Females in China", in Demography, n 3/31, 1994, p. 459-479.

Croll Elisabeth, Endangered Daughters: Discrimination and Development in Asia, London, Routledge, 2000, $221 \mathrm{p}$.

Das Gupta Monica, Zhenghua Jiang, Li Bohua, XIE Zhenming, Chung Woojin, BaE Hwa-Ok, "Why is son preference so persistent in East and South Asia? A crosscountry study of China, India, and the Republic of Korea ", in The Journal of Development Studies, $\mathrm{n}^{\circ} 2 / 40,2003$, p. 153-187.

DuthÉ Géraldine, Mesle France, VAllin Jacques, BADURASHVILI Irena et KUYUMJYAN Karine, " High sex ratios at birth in the Caucasus. Modern technology to satisfy old desires », in Population and Development Review, $\mathrm{n}^{\circ} 3 / 38$, 2012, p. 487-501.

Efrat Zeev, AKInfEnWA Olayinka O., Nicolaides Kypros H. " First-trimester determination of fetal gender by ultrasound ", in Ultrasound in Obstetrics and Gynaecology, ${ }^{\circ}$ 5/13, 1999, p. 305-307.

Gammeltoft Tine, NguyÊN Hanh Thi Thuy, « The Commodification of Obstetric Ultrasound Scanning in Hanoi, Viet Nam », in Reproductive Health Matters, $\mathrm{n}^{\circ}$ 29/15, 2007, p. 163-171.

GoODKIND Daniel, « Abortion in Vietnam: measurements, puzzles and concerns », in Studies in Family Planning, ${ }^{\circ}$ 6/25, 1994, p. 343-352.

Guilmoto Christophe, "The Sex Ratio transition in Asia ", in Population and Development Review, $\mathrm{n}^{\circ}$ 35/3, 2009, p. 519-549.

Guilmoto Christophe, "Son preference, sex selection, and kinship in Vietnam », in Population and Development Review, $\mathrm{n}^{\circ}$ 1/38, 2012a, p. 31-54. 
Guilmoto Christophe, Sex Imbalances at birth. Trends, Differentials and Policy Implications, UNFPA, Bangkok, 2012b, 84 p.

Guilmoto Christophe, HoAng Xuyên, Ngo Van Toan, " Recent Increase in Sex Ratio at Birth in Viet Nam », in PLoS ONE, n ${ }^{\circ} 2 / 4,2009$, p. 1-7.

HAINES David, « Reflections of Kinship and Society under Vietnam's Lê Dynasty », in Journal of Southeast Asian Studies, ${ }^{\circ}$ 2/ 15, 1984, p. 307-314.

HARDY Andrew, CuCARZI Mauro, Zolese Patrizia, Champa and the archaeology of My Son (Vietnam), Singapour, NUS Press, 2009, p. 440.

HAUghton Jonathan, HaUghton Dominique, "Son preference in Vietnam », in Studies in Family Planning, n 6/ 26, 1995, p. 325-337.

HENSHAW Stanley, SINGH Susheela, HAAS Taylor, " The incidence of abortion worldwide », in International Family Planning Perspectives, vol. 25, Supplement, éditeur, 1999, p. S30-S38.

Institute for Social Development Studies, New «Common Sense »: FamilyPlanning Policy and Sex Ratio in Viet Nam, Findings from a qualitative study in Bắc Ninh, Hà Tây and Bình Định, 2007, 4th Asia Pacific Conference on Sexual and Reproductive Health, Hyderabad, 28 p.

KIERNAN Ben, "Chams », in The Encyclopeadia of Islam, Third Edition, Leiden, Kate Fleet, Gudrun Kramer, Denis Matringe, John Nawas, Everett Rowson (ed.), Brill, 2010, p. 173-180.

LiLjestrom Rita, Lai Tương (éd.), Sociological Studies on the Vietnamese Family, Hanoi, Social Sciences Publishing House, 1991, p.192.

Ministry of Planning And InVestment, General Statistics OfFice, The 1/4/2012 time-point Population Change and Family Planning Survey Major findings, Hanoi, General Statistics Office, 2012, 316 p.

NAKAmURA Rie, "The Cham Muslims in Ninh Thuân Province, Vietnam », in Islam at the Margins: The Muslims of Indochina, Center for Integrated Area Studies, discussion paper number 3, 2008, p. 7-23.

NAtional COMmittee FOR POPUlation, FAMILy AND ChILdREn, Vietnam Demographic and Health Survey 2002, Hanoi, September, 2003, General Statistics Office, ORC Macro, 124 p. + 94 p. appendices.

PAPIN Philippe, PAssicousset Laurent, Vivre avec les Vietnamiens, Paris, L'Archipel, 2010, p. 373.

Pham Nguyên Bang, Hall Wayne, Hill Peter Stewart, "Indirect evidence of the contribution of prenatal sex selection practices to the high sex ratio at birth in Vietnam", in Journal of Population Research, n4/28, 2011, p. 293-299.

SCORNET Catherine, «Un exemple de réduction de la fécondité sous contraintes: la région du delta du fleuve Rouge au Viêt Nam », in Population, $\mathrm{n}^{\circ} 2 / 55,2000$, p. $265-$ 300 .

SCORNET Catherine, "State and the Family", in Reconfiguring Families in Contemporary Vietnam, BARBIERI Magali, BELANGER Danièle (éd.), Stanford, Stanford University Press, (« Series on Contemporary Issues in Asia and the Pacific »), 2009, p. 47-74. 
UNFPA, Sex Ratio at Birth Imbalances in Viet Nam: Evidence from the 2009 Census, 2010, Hanoi, UNFPA Vietnam, 35 p.

UNFPA, Son Preference in Viet Nam: Ancient Desires, Advancing Technologies. Qualitative research report to better understand the rapidly rising sex ratio at birth in Viet Nam, 2011, Hanoi, UNFPA Viet Nam, 58 p.

UNICEF, The 'rights' start to life. A statistical analysis of birth registration, New York, UNICEF, 2005, p. 35.

YU Insun, « Myth and Reality: the Confucian influence on Northern Vietnamese society during the Le Dynasty (1428-1788) ", in Monde du Viêt Nam - Vietnam World, Hommage à Nguyên The Anh, MANTIENNE Frédéric \& TAYlOR Keith (éd.), Paris, Les Indes Savantes, 2008, p. 505-524. 


\title{
Exploring the heterogeneity of informal household businesses in Vietnam: from macro dynamics to micro characteristics and functioning
}

\author{
LE Thi Thuy Linh (PSE \& DIAL) \\ Mireille RAZAFINDRAKOTO (IRD DIAL) \\ François ROUBAUD (IRD DIAL)
}

Despite its economic weight, knowledge of the informal economy is extremely limited in Vietnam as it is in most developing countries. Researchers, whether Vietnamese or foreign, have paid little attention to the subject. This situation is due to a number of factors. First of all, the concept of what constitutes "informal" is vague with a multitude of definitions having been put forward by different authors. Second, measuring the informal economy is a difficult task since it operates on the fringes of the economy. Third, the informal economy is often neglected by the authorities as it does not pay (or pays little) taxes and is seen more as a nuisance (especially in the towns) and a mark of underdevelopment inevitably doomed to extinction by the country's economic growth. These elements explain why there has been no real significant effort to date to improve knowledge in this area. Our work sets out to amend this situation by providing accurate statistical data and in-depth analyses on the informal sector and informal employment in Vietnam. It draws on the results of several statistical surveys conducted with support from the authors and largely refers to different studies recently published on this subject (Cling et coll., 2010a \& 2012; Demenet et coll., 2010; Nguyen Huu Chi et coll., 2010; Razafindrakoto et coll., 2011; Razafindrakoto et coll., 2012; Nguyen Huu Chi et coll., 2013).

Prior to 2007, statistical data on the informal economy (in terms of labor, income and production) in Vietnam was scarce. Acknowledging this, the General Statistics Office (GSO) launched a joint research project with the French Institute of Research for Development (IRD-DIAL) in 2006. The prime objective was to set up a statistical system that would measure Vietnam's informal sector and informal employment in a comprehensive and sustainable way, and in-keeping with international recommendations.

First, an operational definition of both the informal sector and informal employment has been adopted. The informal sector is defined as all private unincorporated enterprises that produce at least some of their goods and services for sale or barter, are not registered (no business license) and are engaged in non-agricultural activities. Informal employment is defined as employment with no social security (especially social insurance). All employment in the informal sector is thus considered to be informal employment, as is part of the employment in the formal sector. In keeping with the ILO (2003), both the informal sector and informal employment are defined as belonging to the informal economy.

Second, in line with these definitions, data collection and analysis providing sound statistical indicators of the informal economy have been conducted, following the recommended two-phase (or mixed household/enterprise) survey methodology. The Labor Force Survey (LFS) has been redesigned to capture accurately employment in the informal sector and informal employment. The LFS has been implemented nationwide in 2007, 2009 and 2010. Additionally, a specific Household Business \& Informal Sector Survey (HB\&IS) was grafted on to the LFS and carried out by interviewing household business heads identified by the LFS in 2007 and 2009 in Hanoi and Ho Chi Minh City. In 2009, open-ended questions were added to capture more precisely the advantages and disadvantages that the heads of household business (HB) put forward regarding their activity. Thus the survey has information from household businesses which are representative of the informal sector in the two cities. 
This chapter aims at investigating the characteristics of the production units which operate in the informal sector in Vietnam. One of the main questions is to what extent all informal Household businesses (IHB) constitute a homogeneous group. In the economic literature, there exist three dominant schools of thought which characterize the informal sector: the Dualist, the Structuralist and the Legalist schools. The "dualist" approach is an extension of the work by Lewis (1954) and Harris and Todaro (1970). It is based on a dual labor market model where the informal sector is considered to be a residual component of this market totally unrelated to the formal economy. It is a subsistence economy that only exists because the formal economy is incapable of providing enough jobs. Unlike the dualist school, the "structuralist" approach focuses on the interdependencies between the informal and formal sectors (Moser, 1978; Portes et coll., 1989). Under this neo-Marxist approach, the informal sector is part of, but subordinate to the capitalist system; by providing formal firms with cheap labor and products, the informal sector increases the economy's flexibility and competitiveness. The "legalist" or "orthodox" approach considers that the informal sector is made up of micro-entrepreneurs who prefer to operate informally to evade the economic regulations (de Soto, 1989); this liberal school of thought is in sharp contrast to the other two in that the choice of informality is voluntary due to the exorbitant legalization costs associated with formal status and registration.

In fact, the informal sector presents a "multi-segmentation" phenomenon, whereby a number of very different categories of IHBs co-exist. In earlier work (Cling et coll., 2010) based only on quantitative data, we have identified three specific IHB groups. The first is the "Survivors" (about $40 \%$ of the total) which are the most precarious and insecure, and most of the heads of these IHBs have ended up in this business because they could not find a job elsewhere. The second is the "Resourceful" (51\% of the total), which are better off and most of the IHBs in this group were created for reason not related to labor market constraints. The third is the "Professionals" ( $10 \%$ of the total), which constitute the high-end group and for almost all of these IHBs, their creation stems from their head's desire to be their own boss.

Thanks to the richness of the data collected in Hanoi and Ho Chi Minh City in 2009, the objective of this chapter is to combine qualitative and quantitative approaches, using in particular open-ended questions, in order to explore further the heterogeneous nature of the informal sector.

The chapter is structured as follows. Section 1 gives an overview of the context, in particular the recent trends in the labour market in Vietnam and the weight of the informal sector. In order to explore the heterogeneous nature of the informal sector, Section 2 presents the methodology and some descriptive statistics derived from the open-ended questions. Section 3 discusses the results of the textual correspondence and cluster analysis.

\section{I- The labor market in Vietnam: the context}

This section presents the general trends in the labour market (in normal time) and analyses the extent of the impact of the international crisis in 2008 and 2009. Similar to other Asian countries, Vietnam has been affected by the international crisis and economic growth has slowed down. During 2004-07, the average annual growth rate was $8.5 \%$, which decreased to $6.5 \%$ in 2008 and $5.3 \%$ in 2009 . However, the impact has been relatively moderate compared to other countries in the region. Along with China, Vietnam has been one of the few Asian countries to not go into recession in 2009. Empirical evidence from the LFS suggests that the Vietnamese labour market reacted remarkably well during the crisis. 


\section{I.1- The flexibility of the labor market in Vietnam}

There was no increase in global unemployment rates (with a structurally low level of $2.0 \%$ in 2007 and $1.7 \%$ in 2009 , too small a change to be statistically significant). But while the global level was stable, a significant downward trend can be noticed in the youth unemployment rates from 2007 to 2010 (a decrease from $3.6 \%$ to $1.6 \%$ in urban areas for the age group 15-24 years).

While the main structures of the labor market remained unaffected overall, the principal variable of adjustment during the slowdown has been the working hours and the multiactivity. The economic slowdown resulted in a drop in the average number of hours worked (from 43.9 to 42.6 hours per week between 2007 and 2009) and by a rise in part-time employment (less than 35 hours per week): 13.2\% of workers were concerned by this in 2007 and $26.7 \%$ in 2009 . Paradoxically, this average evolution also went hand in hand with a lengthening of working hours for the most vulnerable part of the population, resulting in another form of "invisible" under-employment: the percentage of the labor force working more than 60 hours per week rose from $5.6 \%$ to $9.3 \%$ in two years. Finally, a very high rise in the number of workers with more than one job was observed: having more than one job constituted a strategy to compensate for the reduction in working hours by seeking an alternative source of income. The percentage of workers with more than one job thus rose from 18.2 in 2007 to 25.4 in 2009 and 27.3 in 2010.

Indeed, the flexibility of the labor market in Vietnam (both in the formal and informal sector) allows to mitigate the negative impact of the global crisis. The informal sector plays an important role particularly in absorbing the shocks at the macro level. However, at the individual level, the affected workers and households have fully endured the negative impact of the crisis (Cling et coll., 2010; Demenet et coll., 2010). More structurally, the predominance of informal sector jobs and informal employment, characterized by poor labor conditions, is an issue that needs to be addressed.

\section{I.2- A resilient job's distribution by institutional sector}

The distribution by institutional sectors shows that the informal sector represents $24 \%$ of the total employment in 2009 in Vietnam (Table 1). The number of jobs in this sector grew between 2007 and 2009 (500,000 new jobs, which corresponds to a 4.9\% increase). But in general, the overall structure did not change significantly with the crisis. Agricultural jobs, which represented $48 \%$ of the total employment in 2009, continued their declining pattern (2.3 percentage points compared to 2007), in line with previous trends. Public jobs also reduced their share (less than 10\% in 2009), also consistent with the previous trends. The informal sector and, more surprisingly, the private formal sector benefited from the shrinking share of the two above mentioned institutional sectors. The foreign direct investment (FDI) sector experienced the highest job growth with an increase of 52\% (mainly in rural areas), even if it still accounts for less than $3 \%$ of the labor force. Domestic enterprises follow, with one million additional jobs ( $40 \%$ and a 2 percentage point increase in the distribution), and finally the formal household business jobs, witnessing an increase of 100,000 new jobs in two years. 
Table 1- Employment by institutional sector and area, 2007 \& 2009 (\%)

Public

Foreign Enterprise

Domestic Enterprise

Formal HB

Informal sector

Agriculture

Total

\begin{tabular}{rr} 
Urban & Ru \\
\hline 23.8 & \\
3.4 & \\
11.6 & \\
16.9 & \\
31.5 & 20. \\
11.1 & 63. \\
100.0 & 100.
\end{tabular}

$2007 \quad 2009$

\begin{tabular}{ccccr} 
Rural & Total & Urban & Rural & Total \\
\hline 6.1 & 10.5 & 20.2 & 5.7 & 9.7
\end{tabular}

14.5

$\begin{array}{lllll}20.8 & 23.4 & 31.6 & 20.7 & 23.7\end{array}$

$\begin{array}{rrrrr}63.0 & 50.4 & 14.7 & 60.9 & 48.1\end{array}$

$100.0 \quad 100.0 \quad 100$

$100.0 \quad 100.0$

Sources: LFS, 2007 \& 2009, GSO. Total: Occupied population; authors' calculation.

\section{3- The informal economy will still be predominant in the following years}

At the national level, informal employment represented around $80 \%$ of the total jobs in 2006-2010 (table 2). The rate of informal employment varies a great deal among sectors, obviously higher in the informal sector and agriculture. But formal sectors are not spared $(9 \%$ of employment in the public sector and $38 \%$ in domestic enterprises, which are still relatively high even if a downward trend can be noticed).

Table 2- Informal employment in the main job by institutional sector, 2007 and 2010 Number Informal employment by institutional sector (\%) of jobs Public sector Foreign enterprise Domestic enterprise Formal Informal Agricul-

\begin{tabular}{cccccccc} 
& $(1,000)(\%)$ & & & HB & sector & ture \\
\hline 2007 & $37,70581.9$ & 12.3 & 17.2 & 52.9 & 48.0 & 100 & 99.0 \\
2009 & $38,28880.5$ & 12.6 & 12.9 & 48.0 & 51.6 & 100 & 98.6 \\
2010 & $39,53979.1$ & 9.2 & 11.4 & 38.0 & 52.5 & 100 & 98.5 \\
\hline
\end{tabular}

Note: In 2010, 79.1\% of the jobs (those declared as the main job by Vietnamese workers) are informal. When each institutional sector is considered, $11.4 \%$ of the jobs in foreign enterprise are informal. By definition, $100 \%$ of the jobs in the informal sector are informal.

Source: LFS 2007, 2009 \& 2010, GSO; authors’ calculations.

In the long term, it is expected that a country's development is accompanied by a progressive reduction of the weight of its informal sector, in conformity with the observation of the marginal weight of this sector in developed countries (La Porta et coll. 2008). However, this mechanism only works in the long term, as stated by Bacchetta et coll. (2009). Given the rapid rates of growth of the Vietnamese economy since the 1980s and the launch of Doi Moi, one would thus think that the informal sector's weight on the job market would have tended to diminish somewhat. But in spite of the expansion of the private formal sector, the informal sector will continue to grow in Vietnam, the consequence of rapid agrarian, urban and demographic transition. Forecasts for employment in 2015 (using pre-crisis trends) show that employment in the informal sector and its relative weight in total employment will continue to grow over the next few years (Cling et coll., 2010b). Regarding labor supply, firstly Vietnam is in a period of "demographic dividend" with a huge number of young people arriving on the labor market (more than one million per year) and the situation will last until 2015 (Table 3). At the same time, the growth of the formal private sector, though rapid, is not high enough to absorb all the new arrivals on the labor market (in particular given the progressive decline of agricultural employment, which represents nearly half of the total employment). Even if a return to strong growth scenario is envisaged for the Vietnamese economy, once the effects of the crisis are over, these forecasts suggest that the informal sector will continue to represent a considerable share of employment in the years to come. 
Table 3- Projections for the evolution of employment from 2007 to 2015: number and share by institutional sector

2007

\begin{tabular}{lrrrr} 
& \multicolumn{2}{c}{ (LFS adjusted) } & \multicolumn{2}{c}{ (Projections) } \\
Institutional sector & Number & Structure & Number & Structure \\
& $(1000)$ & $(\%)$ & $(1000)$ & $(\%)$ \\
\hline Public sector & 4,921 & 10.8 & 4,810 & 9.1 \\
Foreign Enterprise & 902 & 2.0 & 2,522 & 4.8 \\
Domestic Enterprise & 2,628 & 5.7 & 5,883 & 11.1 \\
Formal Household Business & 3,560 & 7.8 & 3,801 & 7.2 \\
Informal Household Business & 10,794 & 23.6 & 14,444 & 27.2 \\
Agriculture & 22,957 & 50.0 & 2,570 & 40.7 \\
\hline Total & 45,762 & 100.0 & 53,031 & 100.0 \\
\hline Unemployment & 1,043 & 2.2 & 1,209 & 2.2 \\
\hline Active population & 46,805 & 100.0 & 54,240 & 100.0 \\
\hline
\end{tabular}

Sources: LFS2007, GSO, RGPH1999-2009, GSO; Projection of population by age, United Nations, 2009. See Cling et coll., 2010 b.

I.4- Global characteristics of the informal sector compared to other institutional $\underline{\text { sectors }}$

Given the weight of the informal sector in terms of employment in Vietnam, the objective is to further investigate its characteristics. The data from the LFS provide some stylized facts (Table 4):

- The informal sector is characterized by low level of education and low incomes; precarious labor conditions; vulnerability of informal household businesses, which operate almost without capital and mostly without professional premises.

- The informal sector consists mainly of micro-businesses (self-employment) and is not strongly integrated into the rest of the economy. Purchases from and sales to the formal sector are marginal. The main supplier of the informal sector is the informal sector itself. Its main market is households and household businesses; sales to the formal sector and subcontracting are marginal and IHBs mainly compete with each other. ${ }^{38}$

Table 4- Characteristics of the workforce and of employment by institutional sector in Vietnam, 2009

Institutional sector

Public sector

Foreign enterprise

Domestic enterprise

Formal household business

\begin{tabular}{rrrrr}
$\begin{array}{c}\text { Structure } \\
(\%)\end{array}$ & $\begin{array}{c}\text { Migrant } \\
(\%)\end{array}$ & $\begin{array}{c}\text { Salaried } \\
\text { workers (\%) }\end{array}$ & $\begin{array}{c}\text { Average monthly } \\
\text { income }\end{array}$ & $\begin{array}{c}\text { Professional } \\
\text { premises (\%) }\end{array}$ \\
\hline 9.7 & 10.4 & 99.7 & 1964 & 96.4
\end{tabular}

Agriculture

Total

48.0

$2.9 \quad 32.1$

99.9

1735

2093

97.6

$\begin{array}{rr}7.7 & 16.0 \\ 7.8 & 8.4\end{array}$

93.6

1805

86.4

$\begin{array}{lll}7.8 & 5.6 & 26.7\end{array}$

1273

$\begin{array}{lll}100 & 6.3 & 33.6\end{array}$

703

Source: LFS2009, GSO; authors' calculations.

Figure 1 represents job satisfaction levels expressed by workers. There is a very clear hierarchy depending on the institutional sector, which consists of three main categories. The public sector is at the top of the ladder: nearly three-quarters $(72 \%)$ of employees in the public sector (civil servants or salaried workers in public or para-public enterprises) declare

\footnotetext{
${ }^{38}$ See Cling et coll. (2010a) for a more detailed presentation of informal household businesses in Vietnam.
} 
themselves to be satisfied or very satisfied with their job. Next are workers from the private formal sector, of which a little more than half (52\%) report being at least satisfied, without any significant difference between those who work in foreign, domestic or individual enterprises? Finally, workers in the informal sector and in agriculture are the most critical; the proportion of those declaring being satisfied is around one third, with the informal sector doing relatively better ( $38 \%$ and $29 \%$ respectively).

Figure 1- Job satisfaction levels by institutional sector in Vietnam, 2009

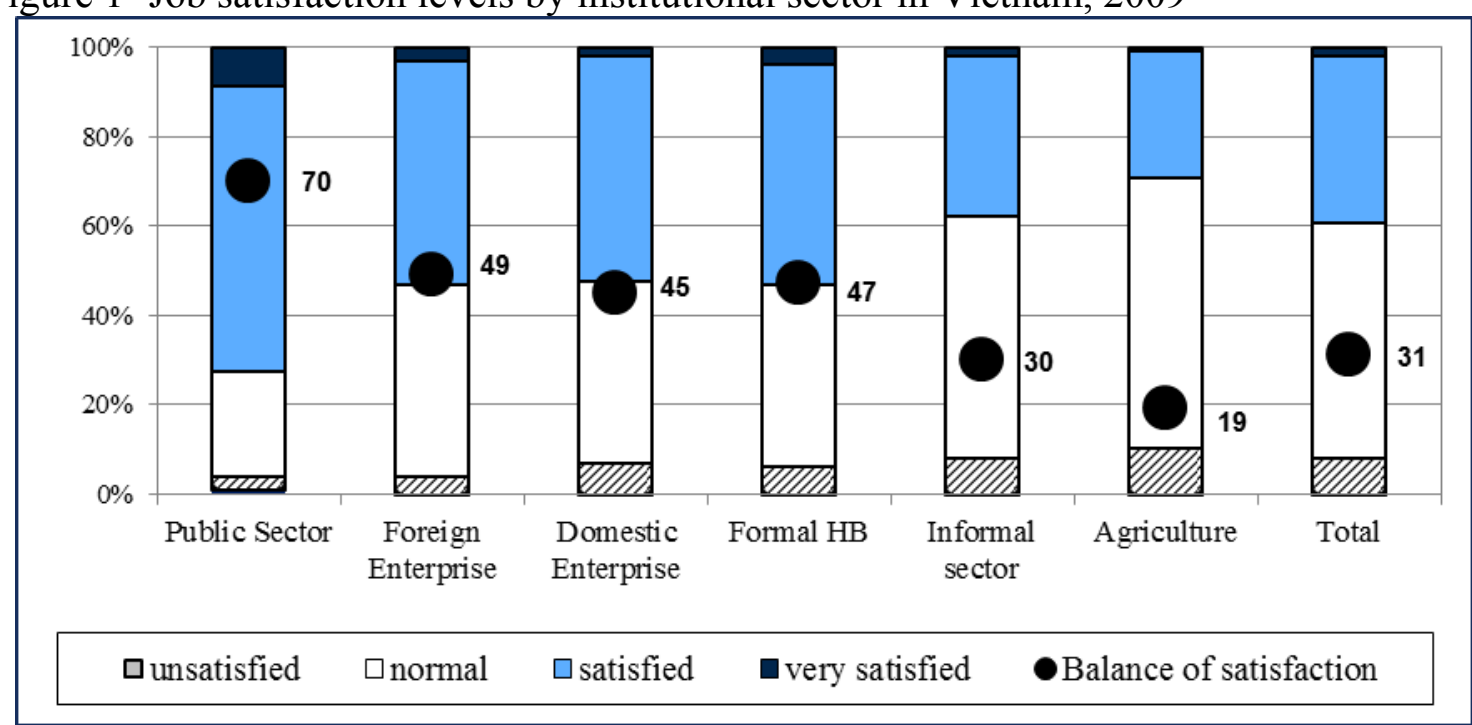

Note: The balance of satisfaction is defined as the difference between the proportion of respondents having expressed a positive opinion (satisfied \& very satisfied) and the proportion of respondents having expressed a negative opinion (unsatisfied \& very unsatisfied).

Source: LFS2009, GSO; authors' calculations.

\section{II- A quali-quanti approach to explore the heterogeneity of the informal sector in Vietnam}

The following sections aim at furthering the analysis and identifying more precisely the characteristics of the different types of HBs in the informal sector. The main finding is that the informal sector can be considered as a continuum of HBs in the multi-dimensional space. It is far from homogeneous, with different groups of HBs encountering different constraints and advantages as well as having different characteristics. It also appears to be more complex than the dual segmentation view.

\section{II.1- Methodology}

We employ a combination of quantitative and qualitative approaches (i.e., so-called qualiquanti approach). The quantitative approach is appreciated for its precision and ability to generalize, while being criticized for its restricted freedom and imposed a priori conceptions of researchers. Meanwhile, the qualitative approach often raises the problem of subjectivity and lack of generalizability. However, it gives the respondents the freedom to express their own experience, thus suggesting new insights (Hughes, 2012). When used together, these methods can be complementary. In this research, the interaction between these two approaches is reflected in both data collection and data analysis. First, a representative statistical survey with random sampling (i.e. a quantitative approach) permits to collect qualitative data through open-ended questions along with quantitative data. Second, 
qualitative data are quantified by a quantitative analytical tool (i.e., DTM-Vic software). Also, the correspondence and cluster analysis on texts is supplemented by categorical variables. Moreover, we use the findings from one type of study to check against the findings derived from the other type (i.e., "logic of triangulation" - Bryman, 2006).

The analyses are performed mainly in DTM-Vic version 5.6, a program developed by L. Lebart, which is devoted to the exploratory analysis of multi-dimensional data. The basic tools that are employed in this chapter are correspondence and clustering analysis.

Correspondence analysis $(\mathrm{CA})^{39}$ is a multivariate statistical technique that is conceptually similar to Principal Component Analysis (PCA). It involves a mathematical procedure that transforms a number of possibly correlated variables into a smaller number of uncorrelated variables called principal components. The components are created to account for maximal variation among the original variables, i.e., the first principal component accounts for as much of the variability in the original variables as possible, and each succeeding component accounts for as much of the remaining variability as possible (Lebart et coll., 2006).

Clustering is a division of data into groups of similar objects. Clusters are formed such that HBs in the same cluster are as similar to each other as possible, and that HBs in different clusters are as distinct as they can be (Wanner, 2004). Clustering can be performed on the principal components of PCA "to denoise the data" (Husson et coll., 2010). In DTM-Vic version 5.6, clustering is based on a hybrid method using both bottom-up hierarchical (agglomerative) and k-means clustering (Lebart et coll., 2006). ${ }^{40}$

According to Lebart and Mirkin (1993), CA and clustering are practically complements and it seems wiser to use both. They argue that CA could result in shrinkages and distortions due to both the projection onto the principal dimensions and the possible lack of robustness of the global fit (sensitivity to outliers). It is thus desirable to complement it with a classification performed in the whole space. Being derived in a much higher dimensional space allows them to provide the information that could have been obscured by the projection onto a low dimensional subspace. Moreover, it is much easier to describe a set of clusters than a continuous space.

Interestingly, these techniques can be applied to textual analysis. This extension requires a transformation of texts into numerical data. This step cross-tabulates a lexical table with columns being distinct words used and rows being HBs (i.e., "contingency table"). The cell value is thus the frequency with which a certain word appears in a response (Lebart et coll., 1998; Yelland, 2010). Correspondence and clustering analyses are then implemented just as with categorical data. In other words, results of these data analyses are based only on word frequency, and other aspects of the corpus (context, discursive aspect) are not taken into account in CA. However, our interpretation of results is done based on the semantic aspect of words. Categorical data (from closed question in the survey) are also considered in order to characterize each cluster. A v-test is computed in order to assess whether the percentage of respondents in a class holding a modality is significantly different from that of the whole sample or not.

Economic research employing these text mining techniques seems to be rare. It is even non-existent for Vietnamese texts. Until now, statistical programs such as DTM-Vic have not

\footnotetext{
${ }^{39}$ More precisely, we use multiple correspondence analysis (MCA) which permits to consider qualitative as well as quantitative data in the same procedure.

${ }^{40}$ Agglomerative clustering algorithm starts with one-point (singleton) clusters and recursively merges two most similar clusters. The similarity matrix is then updated to reflect the pairwise similarity between the new cluster and the remaining clusters. These steps are repeated until a single cluster remains. The output of this procedure is a hierarchical tree or "dendrogram." In K-means clustering method, however, $\mathrm{k}$ data points are randomly chosen as the initial centroids. All points are then assigned to their closest centroids and centroid of each newly assembled cluster is recomputed. This process is repeated until the centroids do not change.
} 
been able to work properly with Vietnamese. This is due to specific features of this language as explained below: ${ }^{41}$

- The Vietnamese alphabet contains special letters that are non-existent in the classical Latin alphabet such as ê, ă, â, ô, ơ, ư, đ.

- Vietnamese has six tones: "level," "hanging," "sharp," "asking," "tumbling," and "heavy." They are expressed by diacritic marks. Words with similar letters may have totally different meanings while marked by different tone marks. For example, "tôi" (I, myself) changes its semantic content to be "bad" (tồi), "dark" (tối) or "crime" (tội). Therefore, ignoring those marks in the analyses is impossible.

- Unlike English or French, where "word is a single distinct meaningful element of speech or writing, used with others (or sometimes alone) to form a sentence and typically shown with a space on either side when written or printed" as stated in the English Oxford dictionary, in Vietnamese and other Asian languages, whitespaces are not used to identify the word boundaries. Indeed, Vietnamese is monosyllabic in nature. Each "syllable" (i.e., "tiếng") tends to have its own meaning and is written with a space before and after. However, "Vietnamese words" are often made of two or more syllables. Thus, morphosyllables in Vietnamese are considered to have the status of morphemes (Tran et coll., 2007). Words made from two or more morphemes can only be recognized by context. DTM-Vic may consider morphemes as words, thus misunderstanding their implications. For example, in the sentence: "Gia đình tôi gặp khó khăn về tài chính" (My family faces financial difficulties), "tài” could imply "talent," "chính" separately means "main," but a combination of "tài chính" turns out to be "finance." In this context, it must be the whole "tài chính" as a single word to be meaningful.

Cutting-edge research in linguistics and informatics has developed various methods to tackle the issue of word segmentation in Vietnamese, such as Dinh et coll. (2001), Nguyen, Cam Tu et coll. (2006) and Nguyen, Thanh et coll. (2006). Yet, none of them are perfect. Moreover, the first two problems remain in DTM-Vic. Taking into account the sample size and the response length; we decided to code the texts manually in a way that addresses the aforementioned problems:

- The alphabet problem (i.e., "chữ cái") was solved by replacing special letters by a pair of Latin letters that imply no other meaning in Vietnamese: $\breve{a}=a w ; \hat{a}=a a ; ~ đ=d d ; ~ \hat{e}$

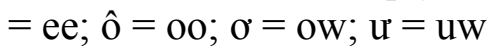

- The tone marks problem ("dấu") was tackled by the same logic: placing a specific Latin letter, which never ends a word in Vietnamese, for each tone: ' ("hanging") = f, " ("sharp") = $\mathrm{s}$, ("asking") $=\mathrm{r}, \sim$ ("tumbling") $=\mathrm{x}$, . ("heavy") $=\mathrm{j}$. The idea to deal with the two first problems comes from the Vietnamese typing rule with the English keyboard of Vietkey and Unikey software programs.

- Separate morphemes of a compound word are identified and linked with each other, making compound words continuous strings of pure Latin letters. E.g., "cạnh tranh" = canhjtranh (competition), "rảnh rỗi" = ranhrroix (free, idle)

\section{II.2- Descriptive statistics}

The textual data mobilized for the analysis are the precise answers provided by the head of HBs to two open-ended questions: on advantages and disadvantages they want to emphasize

\footnotetext{
${ }^{41}$ See also Pham et coll. (2008) for an overview of the Vietnamese language.
} 
upon regarding their activity. ${ }^{42}$ The qualitative data reveal copious information that not only directly answers the questions asked, but also provides explanations, inferences and attitudes of the HBs. One HB, for example, puts forward difficulty due to price increase, after explaining its causes and mechanisms: "Gold price increases, gasoline price goes up day by day so goods prices follow the trend." Another HB considers that their grocery store is impossible to sell because their main customers are manual workers, who are unemployed at the moment. Meanwhile, some HBs report very job-specific problems such as: "There was once a customer who ate our sweetened porridge and had a belly-ache, she laid the blame on us and scolded us loudly." This kind of information is hardly available in a quantitative survey.

Basic statistics resulting from numerical coding of texts are provided in Table 5. We report the number of responses (i.e. observations or head of HBs who have provided answers) to each question and count the total number of words and distinct words contained in the responses. We then remove all the words with frequency equal or less than two, as these words contribute little to the text corpus. Table 5 shows that the response rate of the "disadvantage" question is significantly higher than that of the "advantage" question (72.2\% versus $61.9 \%$ ). Non-responses appear to be random, as most of the key variables (i.e., HB size, value-added, educational attainment of HB heads) are very similar between the respondents and the non-respondents (Table 14 in appendix). Table 5 also indicates that the number of words and distinct words used by HBs to present their disadvantages is about $50 \%$ higher than for advantages. These figures, to some extent, reflect the low level of satisfaction of HBs in the informal sector. This result is consistent with the findings mentioned at the end of the first section.

Table 5- Numerical Coding of Texts: summary results

\begin{tabular}{lrr} 
& Disadvantages & Advantages \\
\hline Total number of responses & 505 & 433 \\
Total number of words* & 5,188 & 3,498 \\
Number of distinct words & 867 & 585 \\
Frequency threshold chosen & 2 & 2 \\
Kept words & 4,551 & 3,045 \\
Distinct kept words & 336 & 229 \\
\hline
\end{tabular}

* The same word might be counted many times as it can be used by different respondents.

Source: HB\&IS HCMC survey 2009, GSO-ISS / IRD-DIAL; authors' calculations.

A closer look at the responses gives us a list of the most frequent words used by HBs (Table 6). In terms of advantages, "home" matters the most. Two possible explanations can be put forward: either a HB owner has a premise at home and she saves rent, or she is happy working at home to take care of her family. The first explanation seems to be more plausible since "premise" and "location" also appear very frequently. "Stability" is also one of the main advantages revealed, which reflects the risk-aversion of these HBs.

As far as disadvantages are concerned, the HB\&IS survey already includes a close-ended question asking the main difficulties faced by the HBs (Table 7). A comparison of the results from these two types of questions could give an insight into the methodological problem regarding question choice (interesting examples are provided in Piau, 2004).

\footnotetext{
${ }^{42}$ The two open-ended questions were analyzed in parallel but separately to avoid misinterpretation (given the fact that the analysis presented is a first exploratory phase), but they could be combined in further steps of the analysis.
} 
Table 6- Most Frequent Words Used by Informal HBs

\begin{tabular}{lrlr} 
Disadvantages & Frequency & Advantages & Frequency \\
\hline Customers & 102 & Home & 78 \\
Prices & 79 & Customers & 67 \\
Capital & 56 & Stable & 57 \\
Location & 35 & Premise & 54 \\
Competition & 32 & Location & 50 \\
\hline
\end{tabular}

Source: HB\&IS HCMC survey 2009, GSO-ISS / IRD-DIAL; authors' calculations.

As suggested by the close-ended question (Table 7), "competition" is the most predominant difficulty encountered by the HBs. "Lack of customers" and "access to loan" come in the second and third place. Reasonably, these three issues also appear in the top five produced by textual analysis. However, the order is different. "Customers", instead of competition, becomes their primary concern. Interestingly, "prices" emerges as a major issue in the open-ended question, whereas it is non-existent in the close-ended question. This may be due to the fact that "prices" is not explicitly mentioned in the latter. It is possibly grafted into "inflation and exchange rate" category, which might appear as an abstract concept to informal HBs. Moreover, this category is positioned towards the end of the list, inducing the "primacy effect", i.e., when the question is long, respondents tend to choose the first categories (Schuman \& Presser, 1996).

Table 7- The Main Difficulties Faced by Informal HBs

\begin{tabular}{lc} 
The difficulty faced & Percentage \\
\hline Competition & 30.7 \\
Lack of customers & 14.7 \\
Access to loan & 14.2 \\
Premise, space & 12.7 \\
Cash flow & 6.5 \\
Machine, equipment & 5.4 \\
Material supply & 4.6 \\
Crime, theft, disorder & 3.4 \\
Inflation, exchange rate & 2.8 \\
Transportation & 2.3 \\
Other & 1.8 \\
Access to land & 0.8
\end{tabular}

Source: HB\&IS HCMC survey 2009, GSO-ISS / IRD-DIAL; authors' calculations.

III- The heterogeneous nature of the informal sector: Results of multivariate data analysis

\section{III.1- Correspondence Analysis on two open-ended questions}

\section{III.1.a- Overview}

There are two distinguished groups of variables involved in this analysis. Active variables, which are in this case the two open-ended questions, will contribute to the performance of $\mathrm{CA}$ and explain the factors derived from the analysis. In these questions, HBs were asked about the advantages and disadvantages in the course of operating their business. Such questions reveal the satisfaction and well-being of HBs in a much less restricted manner than close-ended questions, thus the analysis based on these textual data may provide richer and interesting results. CA is performed separately for each question to avoid the confusion if we 
mix "advantages" with "disadvantages." 43 Besides, supplementary variables, which are in this case categorical variables constructed from the quantitative data, are added into the analysis to provide more informative results.

The explanatory power of the principal components is reflected through their corresponding eigenvalues as well as percentage in the total variance. However, the larger the dimensionality of the original data, the lower the eigenvalues and percentages (Table 8$)^{44}$. Acknowledging the number of distinct words contained in "advantages" and "disadvantages," which is equivalent to the number of textual dimensions (229 and 336, respectively), the figures below are relatively large. The first factor of "advantages," for instance, accounts for nearly $2 \%$ of the total variance, whose explanatory power equals to that of four and a half variables in the original data set. ${ }^{45}$

Table 8- The First Five Eigenvalues of CA

\begin{tabular}{crrrr} 
& \multicolumn{2}{c}{ Advantages } & \multicolumn{2}{c}{ Disadvantages } \\
Factor & Eigenvalue & Percentage & Eigenvalue & Percentage \\
\hline 1 & 0.5848 & 1.96 & 0.5114 & 1.39 \\
2 & 0.5597 & 1.88 & 0.4925 & 1.34 \\
3 & 0.5311 & 1.78 & 0.4878 & 1.32 \\
4 & 0.5093 & 1.71 & 0.4578 & 1.24 \\
5 & 0.4940 & 1.66 & 0.4537 & 1.23 \\
\hline
\end{tabular}

Source: HB\&IS HCMC survey 2009, GSO-ISS / IRD-DIAL; authors' calculations

Hereafter we consider only the two first factors. ${ }^{46}$ The interpretation of principal axes is based on the words that have extreme contributions to their creation (Tables 9, 10 and 11). ${ }^{47}$

\section{III.1.b- Advantages}

The first factorial axis clearly differentiates "social" and "non-social" logics (Table 9 and Figure 2). On the negative side, informal HBs take advantage of their social networks such as local authority, friends and family. On the positive side, informal HBs find the advantages right from the nature of their business (i.e., "small" scale, no or little "tax" duty, "premise," "initiative," "time" and so on). The second factorial axis contrasts "maximizing" against "satisfying" logics (Table 9 and Figure 2). Some informal HBs, on the one hand, actively "make use of" as many opportunities as they can. This is demonstrated through words such as "try," "initiative" and economic advantages that they mention like "tax," "premise," "production," "expenses." On the other hand, other informal HBs seem to lower their ambition to achieve job satisfaction. These people choose less demanding jobs which are "easy," "light," "simple" and maybe appropriate with their "health" and "age," in order to earn a just "sufficient" and "acceptable" living.

\footnotetext{
${ }^{43}$ We performed also an analysis mixing "advantages" with "disadvantages" in a preliminary step but the results were very difficult to interpret and not convincing since the technique used refers mainly to words and not to sentences and the same word can be mentioned to stress an advantage or a disadvantage.

${ }^{44}$ The Kaiser-Gutman rule for CA indicates that we should keep the factorial axes whose eigenvalues greater than average eigenvalue. This is equivalent to eigenvalue-greater-than-one criterion for PCA (Guttman, 1954; Kaiser,

1960, 1970)

${ }_{45}$ Actually, any axis contributing more than $0.4 \%$ (1/229) could be considered for the analysis.

${ }^{46}$ Although the other axes are also interpretable (i.e. meaningful), we only focus on the two most important factorial axes, since the objective is to provide an illustration of the heterogeneous nature of the informal sector and the multidimensionality is taken into account in the cluster analysis.

${ }^{47}$ We eliminate several words that can be considered as "outliers", i.e., which do not belong to the systematic meaning of the axes.
} 
Table 9- Selective Points with Extreme Contributions to the First Two Principal Axes CA on "Advantages" of Informal HBs

Axis 1

Axis 2

\begin{tabular}{|c|c|c|c|c|c|}
\hline Words & Translation & Coordinate & Words & Translation & Coordinate \\
\hline Giúp đỡ & help & -11.85 & Giúp đỡ & Help & -15.66 \\
\hline Chính quyền & authority & -10.23 & Chính quyền & Authority & -14.85 \\
\hline Địa phương & locality & -8.46 & Thuế, & Tax & -10.50 \\
\hline Bạn bè & friends & -6.51 & Cố gắng & Try & -4.11 \\
\hline Gia đình & family & -5.72 & Kinh doanh & Business & -2.34 \\
\hline Quen biết & accquainted & -5.26 & Mặt bằng & Premise & -1.58 \\
\hline Cố gắng & Try & -4.47 & Tận dụng & make use of & -1.51 \\
\hline Thu nhập & income & -4.27 & Sản xuất & Production & -1.33 \\
\hline Quen & familar & -3.19 & Kinh phí & fund, expense & -1.26 \\
\hline Nguồn & sources & -3.01 & Chủ động & Initiative & -1.21 \\
\hline Thời gian & time & 1.80 & Tuổi & Age & 1.33 \\
\hline Chi phí & costs & 1.85 & Tạm & Acceptable & 1.49 \\
\hline Chủ động & initiative & 2.62 & Con & Children & 1.67 \\
\hline Địa điểm & location & 2.83 & Ổn định & Stable & 1.79 \\
\hline Nhà & home & 3.08 & Sức khỏe & Health & 1.86 \\
\hline Kinh doanh & business & 4.43 & Sống & Live & 1.91 \\
\hline Thuê & hire & 4.57 & Đủ & Sufficient & 1.99 \\
\hline Mặt bằng & premise & 5.15 & Thời gian & Simple & 2.04 \\
\hline Nhỏ & small & 6.19 & Nhẹ nhàng & Light & 2.05 \\
\hline Thuế & Tax & 12.82 & Dễ & Easy & 2.35 \\
\hline
\end{tabular}

Source: HB\&IS HCMC survey 2009, GSO-ISS / IRD-DIAL; authors' calculations

It is interesting to see the link between HBs' advantages with the motivation of heads of HBs to set up their business and to choose their business activity. Figure 2 suggests that HBs which were established as a voluntary choice (i.e., to get better income, to have higher profit than other activities) rather than as a constraint, either employment constraint (i.e., because the HB head did not find a job in another HB) or expertise constraint (i.e., because that is the profession that they know) are located towards the bottom right of the dimensional space formed by the two first principal axes. In other words, "voluntary HBs" are less dependent upon personal networks such as family, neighbors and friends in their business operation. They also take advantage of economic opportunities to develop their bus²iness.

\section{III.1.c- Disadvantages}

The first factorial axis distinguishes "entrepreneurship" from "personal" perspectives (Table 10, Figures 3 and 4). Those who are located towards the negative side of the axis are more likely to raise their concerns in an entrepreneurship perspective. These informal HBs stress more economic problems such as "capital," "finance," "scale" and "prices of materials." In contrast, those who are positioned towards the positive side of this axis tend to put forward their difficulties in a personal perspective. Their disadvantages, for instance, are the fact that they have to "get up" "early" in the "morning" to work, which is "strenuous" as far as they are concerned.

The second factorial axis separates out "internal" and "external" disadvantages: (i) those that are directly linked to the informal HBs themselves and that they can actively improve (e.g., "strenuous," "finance," "family" and "production"); and (ii) those that are external and almost cannot be changed by HB's own actions (e.g., "street," "petrol," "dust," "rain" and so on).

Given these interpretations, we could expect that those located in the bottom left of the plane view formed by the two first factorial axes (i.e., have negative values on both axes) would be the "high-end" informal HBs and vice versa. They could be more likely to operate on a larger scale, have a better economic performance and more stable working conditions. 
As a validation of this hypothesis, we project the significant quantitative modalities onto this plane (Figure 4). Impressively, we observe an evolution of informal HBs from the top right to the bottom left: scale is enlarged (from "size=1," "size=2" to "size3-6"), business turnover increases (from the first quintile to the fifth quintile), business premise is stabilized (from unstable premise to premise at home), their attitude toward their business becomes more optimistic (from thinking that their HB has no future to the reverse), and so on.

Table 10- Selective Points with Extreme Contributions on the First Two Principal Axes CA on "Disadvantages" of Informal HBs

\begin{tabular}{|c|c|c|c|c|c|}
\hline Word & $\begin{array}{c}\text { Axis } 1 \\
\text { Translation }\end{array}$ & Coordinate & Word & $\begin{array}{c}\text { Axis } 2 \\
\text { Translation }\end{array}$ & Coordinate \\
\hline Vốn & capital & -7.32 & Sớm & Early & -16.33 \\
\hline Tài chính & finance & -7.01 & Dậy & get up & -15.89 \\
\hline Vay & borrow & -4.97 & Thức & Awake & -13.14 \\
\hline Hỗ trợ & support & -4.88 & Sang & Morning & -10.72 \\
\hline Tay nghề & workmanship & -4.48 & Vất vả & strenuous & -6.23 \\
\hline Mở rộng & enlarge & -4.43 & Vốn & Capital & -5.69 \\
\hline Diện tích & area, surface & -4.26 & Tài chính & Finance & -3.76 \\
\hline ổn định & stable & -3.04 & Vay & Borrow & -3.06 \\
\hline Quy mô & scale & -3.02 & Gia đình & Family & -3.04 \\
\hline Máy móc & machines & -3.00 & Sản xuất & production & -2.94 \\
\hline Kinh tế & economy & -2.85 & Mở rông & Enlarge & -2.76 \\
\hline Mặt bằng & premise & -2.84 & Diện tích & area, surface & -2.53 \\
\hline Giải tỏa & land clearance & -2.66 & Già & Old & -2.4 \\
\hline Vật giá & prices of materials & -2.65 & Chồng & Husband & -2.04 \\
\hline Địa điểm & location & -2.52 & Con & Children & -1.82 \\
\hline Mất & lose, lost & 2.45 & Thị trường & Market & 2.07 \\
\hline Trung Quốc & China & 2.51 & Chất lượng & Quality & 2.20 \\
\hline Bụi & dust, dusty & 2.81 & Bụi & dust, dusty & 2.25 \\
\hline Xăng & petrol & 3.38 & Siêu thị & supermarket & 2.28 \\
\hline Xe ba bánh & tricycles & 3.41 & Nắng & Sunny & 2.57 \\
\hline Kẹt & (traffic) jams & 3.74 & Nước & Water & 2.57 \\
\hline Chất lượng & Quality & 3.85 & Mưa & Rain & 2.68 \\
\hline Cấm & Ban & 4.36 & Khách hàng & customers & 2.79 \\
\hline Vất vả & Strenuous & 4.37 & Dọn dẹp & tidy up & 2.83 \\
\hline Khách hàng & Customers & 4.54 & Buýt & Bus & 2.92 \\
\hline Đường & road, street & 4.94 & Kẹt & (traffic) jam & 3.53 \\
\hline Sang & Morning & 8.31 & Xăng & Petrol & 4.09 \\
\hline Thức & Awake & 9.40 & $\mathrm{Xe}$ & Vehicle & 4.65 \\
\hline Dậy & get up & 10.87 & Đường & street, road & 5.36 \\
\hline Sớm & Early & 11.46 & Giá & Prices & 6.64 \\
\hline
\end{tabular}

Source: HB\&IS HCMC survey 2009, GSO-ISS / IRD-DIAL; authors' calculations

A broader view on both informal and formal production units further elucidates the heterogeneity of the urban HBs (Table 11, Figures 5 and 6). It should be noted that the majority of the analyzed sample of non-farm HBs is informal (68\%) and that medium, large and formal enterprises are excluded from the HB\&IS Survey (Cling et coll., 2010a). For this reason, these results obtained here are very relevant to the informal sector.

The first factorial axis is mainly on disadvantages which affect personally each HB: It opposes those who face individual constraints (e.g., "get up" "early" in the "morning") to those who are more integrated into the market economy and put forward business and economic constraints (on the supply side) in terms of "capital", "premise," "production," "interest rate" and "machines", for examples.

The second factorial axis appears to be more on disadvantages faced globally by HBs: from economic and market-related difficulties (on the demand side) such as "prices," 
"competition," "market," and "taxes" to non-economic, non-market factors (e.g., "weather," "rain," "season," "flooded").

Table 11- Selective Points with Extreme Contributions on the First Two Principal Axes CA on "Disadvantages" for all HBs (Informal and formal)

\begin{tabular}{|c|c|c|c|c|c|}
\hline Word & $\begin{array}{l}\text { Axis } 1 \\
\text { Translation }\end{array}$ & Coordinate & Word & $\begin{array}{l}\text { Axis } 2 \\
\text { Translation }\end{array}$ & Coordinate \\
\hline Sớm & early & -13.10 & Giá & prices & -10.47 \\
\hline Dậy & get up & -12.57 & Cạnh tranh & competition & -7.22 \\
\hline Thức & awake & -10.44 & Gia công & processing & -6.5 \\
\hline Sang & morning & -9.74 & Đối thủ & competitors & -4.06 \\
\hline Khách & customers & -5.99 & Thị trường & markets & -3.31 \\
\hline $\mathrm{Xe}$ & vehicle & -5.69 & Lợi nhuận & profit & -3.15 \\
\hline Đường & street, road & -4.97 & Điện & electricity & -3.05 \\
\hline Vất vả & strenuous & -4.51 & Chi phí & costs & -2.85 \\
\hline Cấm & forbid & -4.06 & Tiêu thụ & consume & -2.69 \\
\hline Quản lý & manage & -3.67 & Dân cư & inhabitants & -2.67 \\
\hline Bụi & dust & -3.42 & Thuế & $\operatorname{tax}$ & -2.60 \\
\hline Xe ba bánh & tricycles & -3.23 & Tay nghề & workmanship & -2.51 \\
\hline Xăng & petrol & -3.16 & Sản phẩm & products & -2.45 \\
\hline Kẹt & (traffic) jam & -3.05 & Doanh thu & revenue & -2.39 \\
\hline L & edge & -2.52 & Chất lượng & quality & -2.29 \\
\hline Chật hẹp & narrow & 3.14 & Bụi & dust & 2.96 \\
\hline Quy mô & scale & 3.65 & Xe ba bánh & tricycles & 3.61 \\
\hline Máy móc & machines & 4.09 & Nước & water & 4.02 \\
\hline Lãi suất & interest rate & 4.10 & Cấm & forbid & 4.37 \\
\hline Địa điểm & location & 4.32 & Nắng & sunny & 4.6 \\
\hline Sản xuất & production & 4.44 & Dọn dẹp & tidy up & 5.26 \\
\hline Ngành nghề & industry & 4.50 & Vất vả & strenuous & 5.38 \\
\hline Diện tích & surface area & 4.7 & Sáng & morning & 5.67 \\
\hline Mặt bằng & premise & 5.42 & Ngập & flooded & 6.42 \\
\hline Di dời & move & 5.56 & Đường & street, road & 6.50 \\
\hline Vay & borrow & 6.99 & Mùa & season & 7.37 \\
\hline Mở rộng & enlarge & 7.31 & Dậy & get up & 8.98 \\
\hline Tài chính & finance & 8.40 & Sớm & Early & 9.42 \\
\hline Kinh doanh & business & 9.03 & Trời & Weather & 13.35 \\
\hline Vốn & capital & 10.81 & Mưa & Rain & 15.21 \\
\hline
\end{tabular}

Source: HB\&IS HCMC survey 2009, GSO-ISS / IRD-DIAL; authors' calculations

As a further attempt to visualize the heterogeneity of the HBs, we project the significant words and quantitative modalities onto the planes of the first two principal axes above. Figure 6 shows that from the top left of the figure, we have the "low-end," i.e., the most precarious, the poorest, the least educated, the less integrated into the economy; and at the bottom-right of the figure we have the "high-end" businesses, i.e., the biggest, the best in terms of performance, the more educated, the more embedded in the economy and so on. This figure is the clearest illustration of our argument that the informal sector is a continuum, rather than a homogeneous sector, or a dual segmented phenomenon. Business size, for instance, gradually grows from "size $=1$ " in the top left quarter to "size3-6" and then to "size $>6$ " in the far bottom right of the plane. School attendance of HB head, in the same manner, escalates from primary to secondary school, and finally high school. 
Figure 2- The Space of Informal HB Characteristics (Projection of Illustrative Variables on the First Factorial Plane of CA on Advantages)

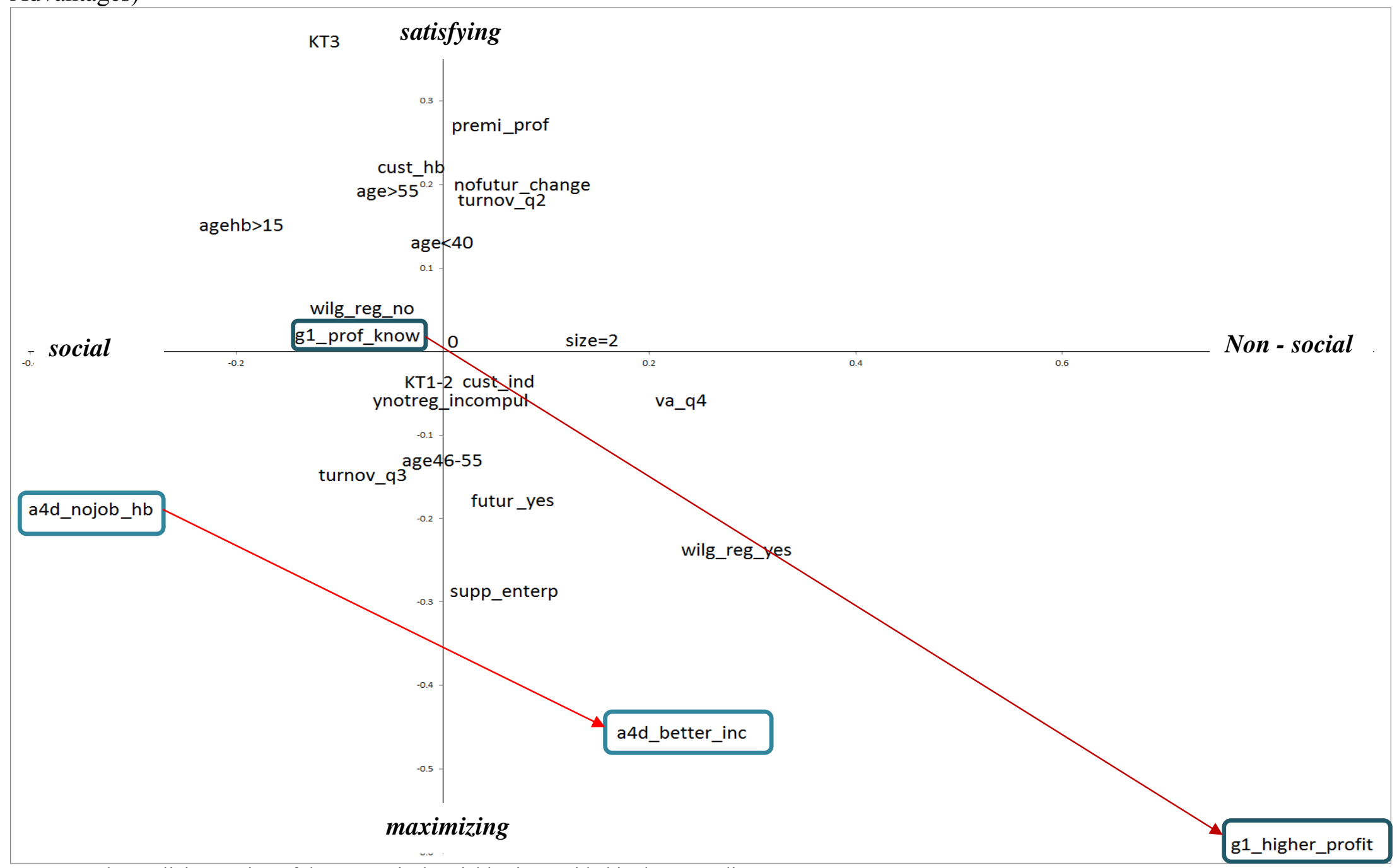

Note: The explicit meaning of the categorical variables is provided in the appendix

Source: HB\&IS HCMC survey 2009, GSO-ISS / IRD-DIAL; authors' calculations. 
Figure 3- The Space of Disadvantages Put Forward by Informal HBs (First Factorial Plane of CA)

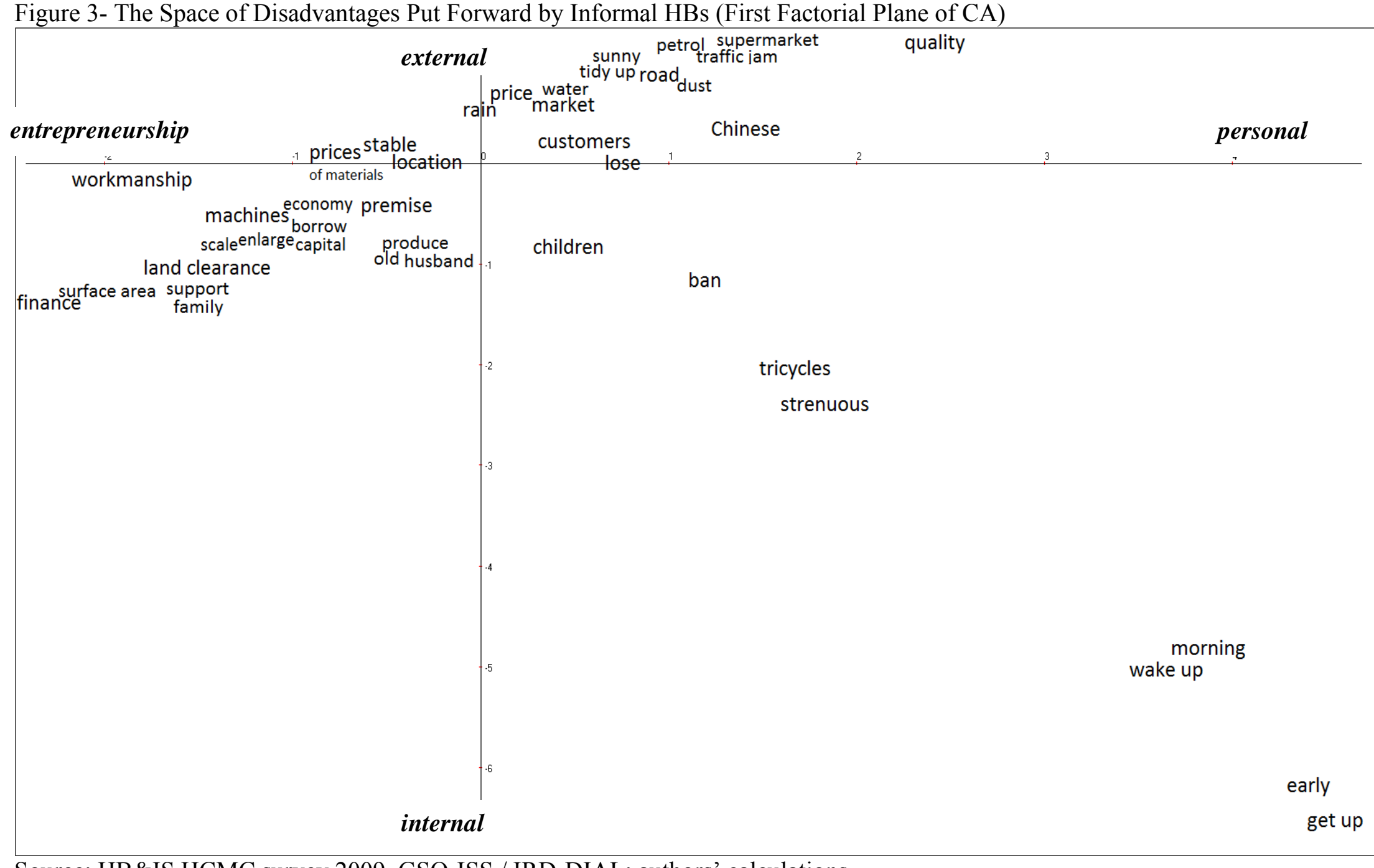

Source: HB\&IS HCMC survey 2009, GSO-ISS / IRD-DIAL; authors' calculations 
Figure 4- Space of Informal HB Characteristics (Projection of Illustrative Variables in the First Factorial Plane of CA on Disadvantages.

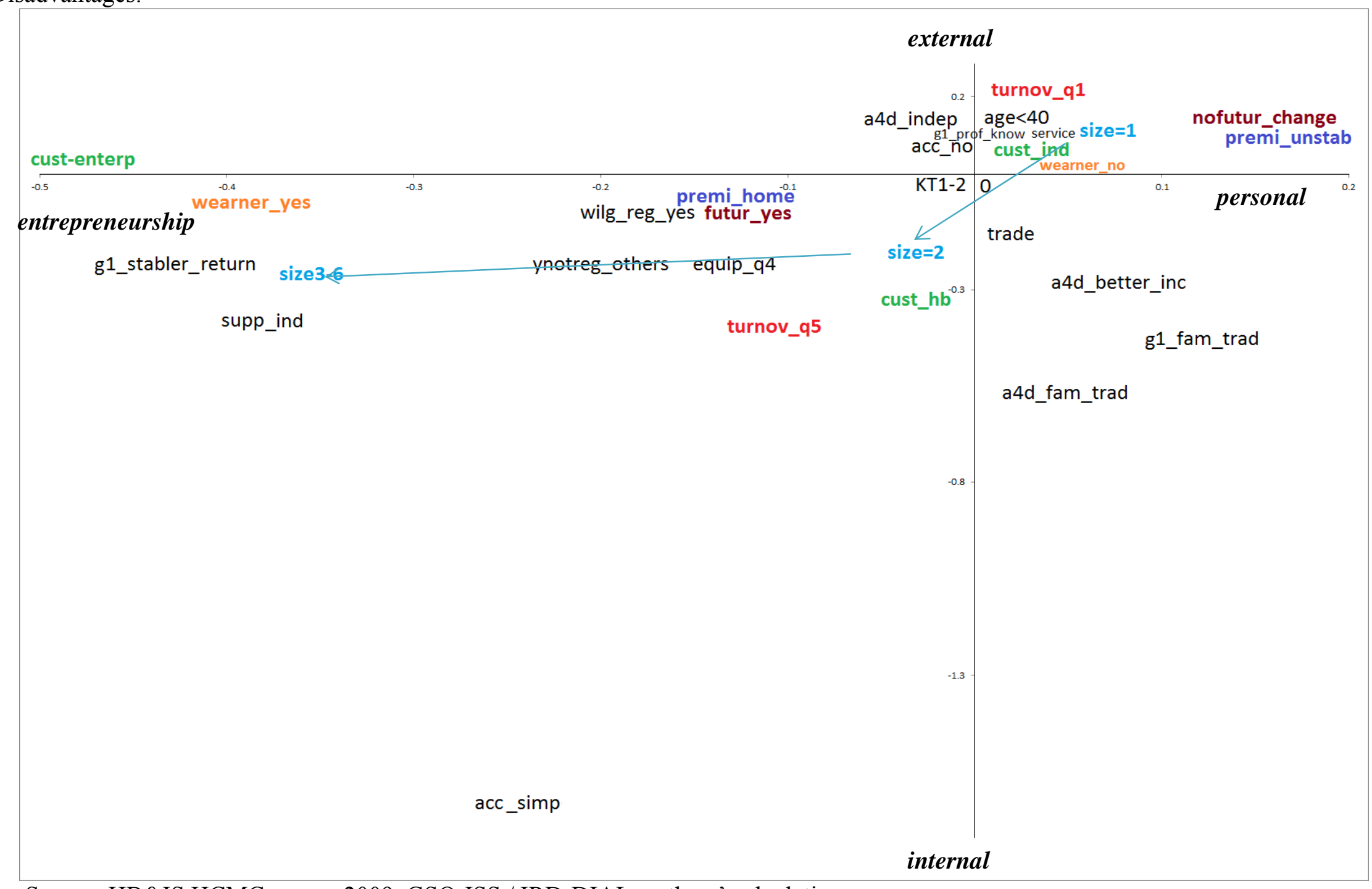

Source: HB\&IS HCMC survey 2009, GSO-ISS / IRD-DIAL; authors' calculations. 


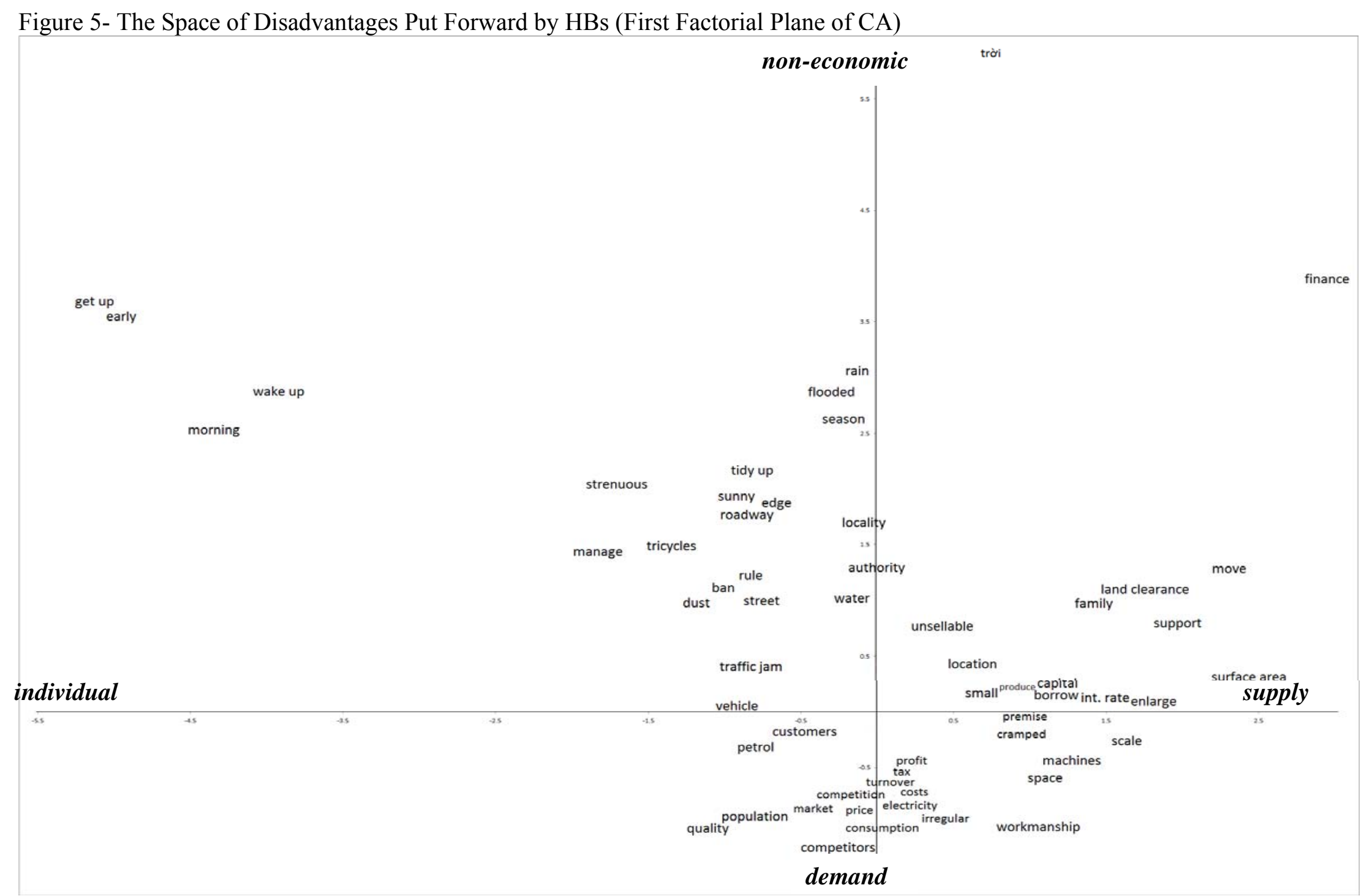

Source: HB\&IS HCMC survey 2009, GSO-ISS / IRD-DIAL; authors' calculations. 
Figure 6- The Space of HB Characteristics (Projection of Illustrative Variables in the First Factorial Plane of CA on Disadvantages)

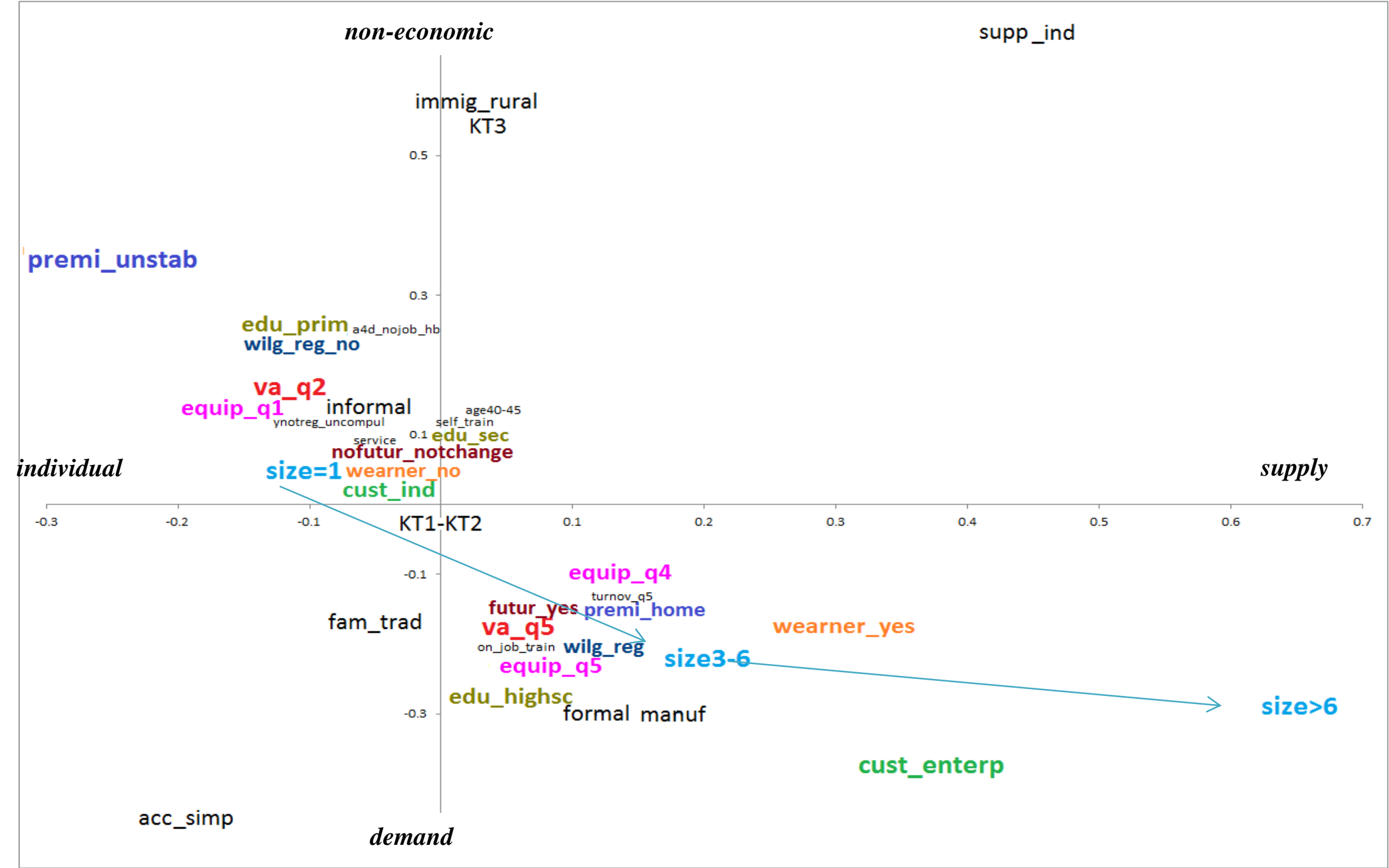

Note: The explicit meaning of the categorical variables is provided in the appendix.

Source: HB\&IS HCMC survey 2009, GSO-ISS / IRD-DIAL; authors' calculations 
By viewing these characteristics of HBs in parallel with their disadvantages (Figure 5), insightful policy lessons can be drawn: Depending on the types of HBs, the policy to support them is very different. Moving downward from the top left to the bottom right, government first of all should adopt anti-poverty policy for the households, then for the HBs which are not the most precarious but with limited performance, policies should focus more on infrastructure (e.g., roadways) and finally policies for the "professional" ought to be exercised on business environment (i.e., macro-economic management such as taxes, monetary policies to confine inflation, and so on).

\subsection{Cluster analysis}

Based on the dimensional space generated by CA, cluster analysis is then applied to obtain a typology of informal HBs. Lebart et coll. (2006) propose a test with the null hypothesis assuming that the average coordinate of a cluster on a factor is equal to zero, i.e., the profile of that cluster on this factor does not differ from the profile of the whole sample. If a cluster has a test absolute value greater than or equal to 1.65 , the null hypothesis is rejected at $5 \%$ level of significance. The number of clusters can be controlled by cutting the dendrogram at a certain height. In this analysis, five distinct clusters of informal HBs are identified, based on their disadvantages.

Table 12 shows that the profile of the clusters is significantly different from the global (i.e., their test absolute values are greater than 1.65), except the second cluster on the first factorial axis. The position of clusters in the factorial space in visualized in Figure 7. Each cluster is characterized by their representative responses and then depicted by significant quantitative categories (Table 13).

Table 12- Coordinates of Clusters in the Dimensional Space -Disadvantages of Informal HBs

\begin{tabular}{rrrrrr}
\multicolumn{2}{c}{ Clusters } & \multicolumn{2}{c}{ Coordinates } & \multicolumn{2}{c}{ Test-values } \\
$\mathrm{N}^{\circ}$ & Size & 1 & 2 & 1 & 2 \\
\hline 1 & 260 & -0.39 & -0.23 & -12.6 & -7.6 \\
2 & 103 & 0.08 & 0.45 & 1.35 & 7.23 \\
3 & 89 & 0.58 & 0.3 & 8.42 & 4.42 \\
4 & 42 & 0.43 & 0.57 & 4.05 & 5.54 \\
5 & 10 & 2.7 & -3.63 & 12.05 & -16.5 \\
\hline
\end{tabular}

Source: HB\&IS HCMC survey 2009, GSO-ISS / IRD-DIAL; authors' calculations

The first cluster covers "the impervious HBs," who are more likely to declare that "There is no difficulty" obstructing their business. More than half of the HBs in this cluster have their premise at home, which appears to be a major advantage as suggested by the previous section. This is the most populous among the five groups, with $260 \mathrm{HBs}$ being classified in this cluster.

The second cluster comprises "the optimistic HBs," who are more inclined to put forward their disadvantages in terms of prices, costs and profit. A substantial share of HBs in this group $(41 \%)$ thinks that there is a future for a business like theirs. HBs in this cluster are more prone to achieve very high economic performance (the fifth quintile of value added).

The third cluster is made up of "the desolate HBs," which includes the HBs who are more likely to face a lack of customers. They can be a tailor, who complains that people are increasingly buying cheap ready-made clothes in market-places; or a motorbike taxi driver, who has less and less customers because his vehicle is too old. Many of them have a turnover of the lowest quintile (38\%), compared to the average (27\%). 
Table 13- Categorical Characteristics of the Clusters - Cluster Analysis on Disadvantages of Informal HBs

\begin{tabular}{|c|c|c|c|c|c|c|}
\hline Characteristic categories & v.test & cla $/ \bmod$ & $\begin{array}{c}\text { Percentages } \\
\mathrm{mod} / \mathrm{cla}\end{array}$ & global & Weight & Prob. \\
\hline Cluster 1 & & & & 51.49 & 260 & \\
\hline Supplier_individual & 2.59 & 83.33 & 5.77 & 3.56 & 18 & 0.0048 \\
\hline Premise_home & 2.5 & 57.63 & 52.31 & 46.73 & 236 & 0.0062 \\
\hline Cluster 2 & & & & 20.4 & 103 & \\
\hline va_quin5 & 2.68 & 37.78 & 16.5 & 8.91 & 45 & 0.0037 \\
\hline Future_yes & 2.64 & 28.19 & 40.78 & 29.5 & 149 & 0.0041 \\
\hline Cluster 3 & & & & 17.62 & 89 & \\
\hline turnov_quin1 & 2.5 & 25.19 & 38.2 & 26.73 & 135 & 0.0061 \\
\hline Cluster 4 & & & & 8.32 & 42 & \\
\hline Premise_unstable & 4.98 & 16.33 & 76.19 & 38.81 & 196 & 0 \\
\hline Future no will chang & 2.45 & 15.31 & 35.71 & 19.41 & 98 & 0.0072 \\
\hline Cluster 5 & & & & 1.98 & 10 & \\
\hline Future_catb_4 & 2.87 & 50 & 20 & 0.79 & 4 & 0.0021 \\
\hline
\end{tabular}

Note: $\mathrm{Cla} / \mathrm{mod}$ indicates what share of all individuals with this category is found in this class. Mod/cla shows the percentage of all individuals in this cluster who have a certain modality.

See in the appendix for a detailed presentation of the categories

Source: HB\&IS HCMC survey 2009, GSO-ISS / IRD-DIAL; authors' calculations.

The fourth cluster is characterized by "the precarious HBs," who typically complain about slow consumption of their goods due to bad weather (i.e., rain) and urban police expelling street vendors. This is consistent with their characteristic modalities. A majority of HBs in this group $(76 \%)$ have unstable premises. These have among the most precarious working conditions. Predictably, they are nearly twice likely than the average to conceive that there is no future for their business, and to think of changing activity.

The fifth cluster covers "the strenuous HBs," who generally complain that they have to get up very early in the morning to work. Interestingly, up to half of these HBs keep silent about their future prospect, i.e., neither upset nor optimistic. There are only ten HBs classified into this cluster $^{48}$.

Overall, the results obtained by the clustering analysis do provide further insights into the continuum of HBs suggested by CA. This continuum is not as "smooth" as a usual continuous variable, since there is no absolute order of different clusters from the low-end to the high-end HBs. We could not tell, for example, which one among cluster 3 and cluster 4 is of lower end than the other. In fact, a HB has a variety of characteristics, some of them may be considered as "high-end," whereas others are "low-end." Therefore, it is hard (if not to say impossible), and unnecessary to have an absolute ranking of HBs in the continuum. In practice, government policies should be designed to target different groups of HBs with their own characteristics, which encounter specific types of disadvantages as we can identify in the factorial space.

\footnotetext{
${ }^{48}$ For this reason, we do not illustrate this cluster in the factorial plane.
} 
Figure 7- Cluster View of Informal HBs on the First Factorial Plane of CA on Disadvantages

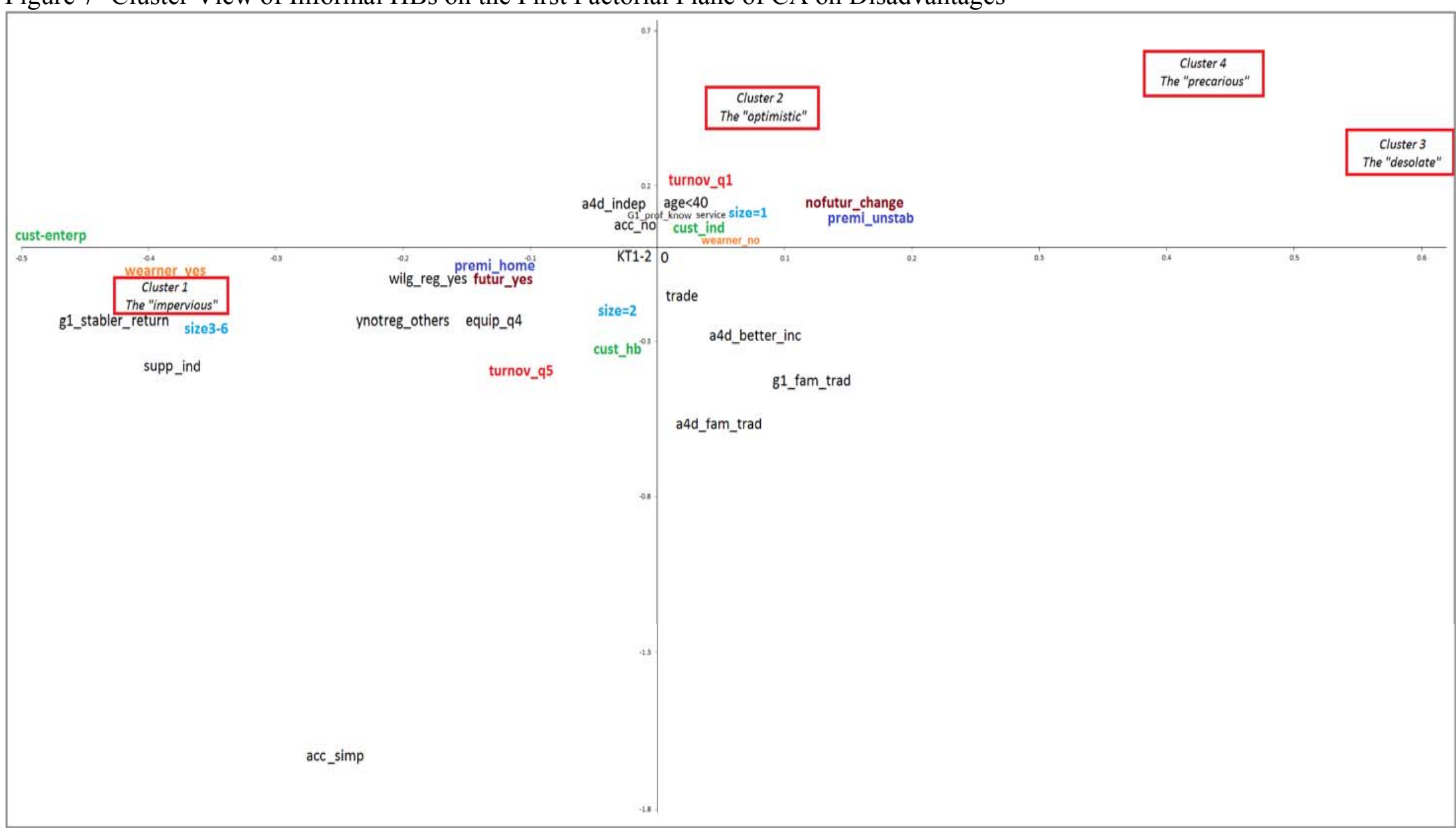

Note: The explicit meaning of the categorical variables is provided in the appendix

Source: HB\&IS HCMC survey 2009, GSO-ISS / IRD-DIAL; authors' calculations. 


\section{Conclusion}

As the informal sector is here to stay, and since there is a strong connection between the informal sector and urban poverty, public policies cannot ignore this sector. Nonetheless, the State's ambivalent and inconstant attitude to the informal sector constitutes a source of uncertainty that needs to be lifted if the productive effort of informal entrepreneurs is not to be constantly frustrated. In Vietnam, there are currently no policies targeting the informal sector.

To improve that situation, Vietnamese government should first of all understand the sector's features and its diversity. This is the aim of our analysis. Based on a unique quali-quanti analytical approach, we acutely point out the heterogeneity of this sector. Textual CA shows that HBs in the informal sector are located along a continuum generated in a multi-dimensional space of their disadvantages or advantages. HBs at the lower end of the continuum are generally poorer, smaller, more precarious and less integrated into the economy. Their owners are less educated and more pessimistic about their business future. These HBs often lower their ambition and choose less demanding work in order to achieve job satisfaction. Yet, low-end HBs still put forwards their constraints in a personal and passive perspective. Reversely, the higher-end HBs have better economic performance, bigger size, more stable working conditions and are more integrated in the economy. Their owners have higher educational attainment and are more optimistic about their business future. These HBs tend to stress their difficulties in an entrepreneurship perspective, for that they can grasp opportunities and actively improve their situation. Furthermore, we conduct a cluster analysis on disadvantages faced by HBs and obtain a typology with five categories of informal HBs, namely: "the impervious," "the optimistic," "the desolate," "the precarious" and "the strenuous."

Our research stands out for a number of originalities. Firstly, the analyses are based on representative textual data, which measure the direct utility of HBs in an open and unrestricted manner. Secondly, we employ exploratory analytical methods (i.e, CA and clustering) to understand the underlying structure of these data, which help limit the pre-conceived ideas of researchers. Last but not least, this is the first economic paper that treats properly the linguistic problems associated with Vietnamese texts, for which factor analysis can be applied.

Nonetheless, some limits still exist in our analysis. First, the codification process is performed manually, which would be impractical for larger data sets. This calls for the development of statistical packages that incorporate cutting-edge linguistics' and informatics' achievements in order to properly treat Vietnamese language in a systematic way. Second, textual analysis is sensitive to data cleaning, which necessarily depends on our subjective understanding of texts. CA, in particular, is sensitive to "outliers," whose identity could be controversial. Future research could accordingly conduct a number of sensitivity tests by modifying the list of words to be deleted (i.e., "stop-words"), grouping different sets of synonyms as single items and removing some potential "outlier" HBs. In addition, we could take advantage of the panel dimension of the data to further explore the dynamics of the informal sector. In addition, given the meaningfulness of the principal axes generated by textual $\mathrm{CA}$, it is possible to employ HBs' coordinates on these factors as continuous variables in further studies.

In conclusion, our analysis directly suggests that targeted policies should take into account the heterogeneity of the informal sector in Vietnam. A "one size fits all" scheme would not be appropriate as there is not one single reason for working in this sector, and different categories of informal HBs experience different kinds of difficulties as well as advantages. Instead, a policy package could include, for examples, 
anti-poverty policies for lower-end $\mathrm{HBs}$ and policies related to the business environment for professional and higher-end $\mathrm{HBs}$; or premise stabilization for "precarious HBs," vocational training for "desolate HBs" and inflation control for "optimistic HBs."

\section{References}

BACCHETTA Marc, ERnst Ekkehard, BustamAnTE Juana, Globalization and informal jobs in developing countries, Geneva, OIT et OMC, 2009, 187p.

BRYMAN Alan, «Integrating Quantitative and Qualitative Research: How is it done? », in Qualitative Research, 6(1), 2006, p. 97-113.

Cling Jean-Pierre, Nguyen Thi Thu Huyen, Nguyen Huu Chi, Phan Thi Ngoc Tram, RAZAFINDRAKOTO Mireille, RoubAUD François, The Informal Sector in Vietnam: A focus on Hanoi and Ho Chi Minh City, Hanoï. Editions The Gioi Editions, 2010a, 248 p.

Cling Jean-Pierre, RazAfindrakoto Mireille, Roubaud François, «Assessing the Potential Impact of the Global Crisis on the Labour Market and the Informal Sector in Vietnam », in Journal of Economics \& Development, N /38, june 2010b, p. 16-25

Cling Jean-Pierre, RAZAFINDRAKoto Mireille, and Roubaud François, «To be or not to be registered? Explanatory factors behind formalizing non-farm household businesses in Vietnam », in Journal of the Asia Pacific Economy, $n^{\circ} 4 / 17,2012$, p. $632-652$

De SOTO Hernando, The Other Path: The Invisible Revolution in the Third World, New York: Harper and Row, 1989, 271 p.

Demenet Axel, Nguyen Thi Thu Huyên, Razafindrakoto Mireille, Roubaud François, Dynamics of the informal sector in Hanoi and Ho Chi Minh City 20072009, GSO-IRD, UKaid, World Bank, December (available at http://www.worldbank.org/en/country/vietnam/research, reference DT $\mathrm{N}^{\circ} 6174$ 112/2010), 2010.

Dinh Dien, HoAng Kiem, NguYen Van Toan, « Vietnamese Word Segmentation », in Proceedings of the Sixth Natural Language Processing Pacific Rim Symposium (NLPRS2001), Tokyo, 2001, p. 749-756.

GUTTMAN Louis, "Some necessary conditions for common factor analysis », in Psychometrika, nº 19, 1954, p. 149-161.

HARRIS John, TODARO Michael, «Migration, unemployment, and development: A twosector analysis », in American Economic Review, n 1/60, 1970, p. 126-42.

HugHES Christina, Qualitative and Quantitative Approaches, URL: http://tinyurl.com/bmztxp8, 2012.

Husson François, Josse Julie, PAges Jérôme, «Principal Component Methods Hierarchical Clustering -Partitional Clustering: Why Would We Need to Choose for Visualizing Data ", in Technical Report-Agrocampus, Applied Mathematics Department, 2010, p. 1-17.

ILO, « Guidelines Concerning a Statistical Definition of Informal Employment », in Seventeenth International Conference of Labour Statisticians, ILO, Geneva, 2003. 
KAISER Henry, "The application of electronic computers to factor analysis », in Educational and Psychological Measurement, $\mathrm{n}^{\circ} 20,1960$, p. 141-151.

KAISER Henry, " A second generation Little Jiffy », in Psychometrika, n 35, 1970, p. 401-417.

LA PORTA Rafael, SHLEIFER Andrei, «The Unofficial Economy and Economic Development», Brookings Papers on Economic Activity, Economic Studies Program, The Brookings Institution, vol. 39(2 (Fall)), p. 275-363.

LEBArt Ludovic, Morineau Alain, PIRON Marie, Statistique Exploratoire Multidimensionnelle, Paris, Dunod, 2006, 480p.

LEBART Ludovic, MIRKIN Boris, "Correspondence Analysis and Classification », in Multivariate Analysis: Future Directions, CUADRAS Carlos María, RAO Calyampudi Radhakrishna (éd.), Amsterdam: North Holland, 1993, p. 341-357.

Lebart Ludovic, SAlem André, Berry Lisette, Exploring Textual Data, Dordrecht, Kluwer Academic Publisher, 1998, 222p.

LEWIS W. Arthur, " Economic development with unlimited supplies of labor », in Manchester School, n²/28, 1954, p. 139-191.

MOSER Caroline, " Informal sector or petty commodity production: dualism or independence in urban development ", in World Development $n^{\circ} 6,1978$, p. 10411064.

Nguyen Huu Chi, Nguyen Thi Thu Huyen, Razafindrakoto Mireille, Roubaud François, Vietnam labour market and informal economy in a time of crisis and recovery 2007-2009 ; Main findings of the Labour Force Surveys (LFS), Hanoi: GSO/IRD, UKaid, World Bank, December (consult the site : http://www.worldbank.org/en/country/vietnam/research, reference DT $\mathrm{N}^{\circ} 6175$ of 1/12/2010), 2010.

Nguyen Huu Chi, Nordman Christophe, Roubaud François, "Who suffers the penalty? A panel data analysis of earnings gaps in Vietnam ", in Journal of Development Studies, n²12/49, 2013, p. 1694-1710.

Nguyen Cam Tu, Nguyen Trung Kien, Phan Xuan Hieu, Nguyen Le Minh, HA Quang Thuy, " Vietnamese Word Segmentation with CRFs and SVMs: An Investigation ", in Proceedings of the 20th Pacific Asia Conference on Language, Information and Computation (PACLIC 2006), Paris: Organization for Economic Co-operation and Development, 2006.

Nguyen Thanh V., Tran Hoang K., Nguyen Thanh T. T., Nguyen Hung, « Word Segmentation for Vietnamese Text Categorization: An Online Corpus Approach », in the 4rd IEEE International Conference in Computer Science, 2006.

PHAM Giang, KOHNERT Kathryn, CARNEY Edward, «Corpora of Vietnamese Texts: Lexical effects of intended audience and publication place», Behavior research methods 40.1, 2008, p. 154-163.

PIAU Claire, Quelques expériences sur la formulation des questions d'enquête. CREDOC, Département Conditions de vie et Aspirations des Français, Cahier de recherche $\mathrm{n}^{\circ} 206,2004,65 \mathrm{p}$. 
Portes Alejandro, CAstells Manuel, Benton Lauren (éd.) The Informal economy: Studies in advanced and less developed countries, Baltimore MD, The John Hopkins University Press, 1989.

RAZAFIndRaKoto Mireille, Roubaud François, Nguyen Huu Chi, « Vietnam Labor Market: An Informal Sector Perspective »), in Vietnam Annual Economic Report 2011: The Economy at a Crossroad, NGUYÊN Duc Thành (éd.), Hanoi, Edition Tri Thuc, 2011, p. 223-258.

RAZAFINDRAKOTO Mireille, ROUBAUD François, WACHSBERgER Jean-Michel, «Travailler dans le secteur informel : choix ou contrainte? Une analyse de la satisfaction dans l'emploi au Vietnam », in L'économie informelle dans les pays en développement, Cling Jean-Pierre, LAgree Stéphane, RAZAFindraKoto Mireille, RouBAUD François (éd.), Paris, Edition AFD, «collection conférence et séminaire», 2012, p. 47-66.

SCHUMAN Howard, PRESSER Stanley, Question and answers in attitude surveys: Experiments on question form, wording and context, New York, Academic Press, 1996, 392 p.

Tran Q. Tri, Pham T. X. Thao, Ngo Q. Hung, Dinh Dien, Collier Nigel, «Named entity recognition in Vietnamese documents », in Progress in Informatics, $n^{\circ} 4,2007$, p. 5-13.

WANNER Leo, «Introduction to Clustering Techniques», International Union of Local Authorities, 2004, 32p. (URL : http://www.iula.upf.edu/materials/ 040701wanner. pdf.)

YELLAND Phillip, «An Introduction to Correspondence Analysis », in The Mathematica Journal, $\mathrm{n}^{\circ} 12,2010$, p. 1-23.

\section{Appendix}

Table 14- Mean Tests for the Non-responses to Open-ended Questions

\begin{tabular}{l|rrr|rrr} 
& $\begin{array}{c}\text { Disadvantages } \\
\text { Non- }\end{array}$ & & \multicolumn{1}{c}{$\begin{array}{c}\text { Non- } \\
\text { Advantages }\end{array}$} \\
\hline $\begin{array}{l}\text { Total size } \\
\text { Value- }\end{array}$ & 1.48 & 1.55 & -0.07 & 1.38 & 1.63 & $-0.25^{* *}$ \\
$\begin{array}{l}\text { added } \\
\begin{array}{l}\text { Educational } \\
\text { attainment }\end{array}\end{array}$ & 6507.52 & 5458.01 & 1049.509 & 5291.29 & 6030.65 & -739.36 \\
& 7.79 & 7.84 & -0.04 & 7.78 & 7.85 & -0.08
\end{tabular}

**: significant at $5 \%$ level

Source: HB\&IS HCMC survey 2009, GSO-ISS / IRD-DIAL; authors' calculations

Table 15- Categorical Variables Used in Correspondence Analysis

\begin{tabular}{llll}
\hline & Description & \multicolumn{1}{c}{ Modalities } & Abbreviation \\
& & Characteristics of HB & \\
\hline 1 & Branch of activity & Manufacturing & manuf \\
& & Trade & trade \\
& & Services & service \\
\hline 2 & Informality of HBs & Formal & formal \\
& & Informal & informal \\
\hline
\end{tabular}




\begin{tabular}{|c|c|c|c|}
\hline & Description & $\begin{array}{l}\text { Modalities } \\
\text { Characteristics of } \mathrm{HB}\end{array}$ & Abbreviation \\
\hline \multirow[t]{4}{*}{3} & Accounting method & Professional accounting & acc no \\
\hline & & Simplified accounting & acc_simp \\
\hline & & Personal record/ notes & acc_pers \\
\hline & & No accounts & acc no \\
\hline \multirow[t]{3}{*}{4} & Type of premise & Unstable premise & premi_unstab \\
\hline & & Premise at home & premi_home \\
\hline & & Professional premise & premi prof \\
\hline \multirow[t]{2}{*}{5} & Has wage earners or & No & wearner_no \\
\hline & not & Yes & wearner_yes \\
\hline \multirow[t]{4}{*}{6} & Total size of HB & 1 person & $\operatorname{size}=1$ \\
\hline & & 2 persons & size $=2$ \\
\hline & & 3 to 6 persons & size $3-6$ \\
\hline & & More than 6 persons & size $>6$ \\
\hline \multirow[t]{5}{*}{7} & Quintile of monthly & First quintile & turnov_q1 \\
\hline & turnover of $\mathrm{HB}$ & Second quintile & turnov_q2 \\
\hline & & Third quintile & turnov_q3 \\
\hline & & Fourth quintile & turnov_q4 \\
\hline & & Fifth quintile & turnov_q5 \\
\hline \multirow[t]{5}{*}{8} & Quintile of monthly & First quintile & va $q 1$ \\
\hline & value-added of $\mathrm{HB}$ & Second quintile & va_q2 \\
\hline & & Third quintile & va $\mathrm{q} 3$ \\
\hline & & Fourth quintile & va $\mathrm{q} 4$ \\
\hline & & Fifth quintile & va_q5 \\
\hline \multirow[t]{5}{*}{9} & Quintile of monthly & First quintile & equip_q1 \\
\hline & equipment costs of $\mathrm{HB}$ & Second quintile & equip_q2 \\
\hline & & Third quintile & equip_q3 \\
\hline & & Fourth quintile & equip_q4 \\
\hline & & Fifth quintile & equip_q5 \\
\hline \multirow[t]{4}{*}{10} & Main customer of $\mathrm{HB}$ & Public or para-public sector & cust_pub \\
\hline & & Enterprises & cust_enterp \\
\hline & & Household businesses & cust_hb \\
\hline & & Individuals & cust_ind \\
\hline \multirow[t]{4}{*}{11} & Main supplier of HB & Public or para-public sector & supp_pub \\
\hline & & Enterprises & supp_enterp \\
\hline & & Household businesses & supp_hb \\
\hline & & Individuals & supp ind \\
\hline \multirow[t]{4}{*}{12} & Age of $\mathrm{HBs}$ & Less than 3 years & Agehb $<3$ \\
\hline & & From 3 to 7 years & agehb3-7 \\
\hline & & From 8 to 15 years & agehb8-15 \\
\hline & & More than 15 years & agehb $>15$ \\
\hline \multirow[t]{2}{*}{13} & Gender of HB heads & Male & male \\
\hline & & Female & female \\
\hline \multirow[t]{4}{*}{14} & Age of $\mathrm{HB}$ & Less than 40 years old & Age $<40$ \\
\hline & head & From 40 to 45 years old & age $40-45$ \\
\hline & & From 46 to 55 years old & age46-55 \\
\hline & & More than 55 years old & age $>55$ \\
\hline \multirow[t]{4}{*}{15} & Educational level of & Primary school & edu_prim \\
\hline & HB head & Secondary school & edu_sec \\
\hline & & High school & edu_highsc \\
\hline & & University or more & edu_univ \\
\hline \multirow[t]{4}{*}{16} & Apprenticeship of HB & Technical school & tech_school \\
\hline & head & On-job training & on_job_train \\
\hline & & Self-trained & self_train \\
\hline & & Others & aprt others \\
\hline
\end{tabular}




\begin{tabular}{|c|c|c|c|}
\hline & Description & $\begin{array}{l}\text { Modalities } \\
\text { Characteristics of HB }\end{array}$ & Abbreviation \\
\hline 17 & $\begin{array}{c}\text { Immigration status of } \\
\text { HB head }\end{array}$ & $\begin{array}{l}\text { Has lived in this province/ city for at least } \\
10 \text { years } \\
\text { Has migrated from another city to this } \\
\text { province/ city for less than } 10 \text { years } \\
\text { Has migrated from a countryside to this } \\
\text { province/ city for less than } 10 \text { years }\end{array}$ & $\begin{array}{l}\text { immig_no } \\
\text { immig_city } \\
\text { immig_rural }\end{array}$ \\
\hline 18 & $\begin{array}{l}\text { Residence status of HB } \\
\text { heads }\end{array}$ & $\begin{array}{l}\text { Registered in the same province (KT1- } \\
\text { KT2) } \\
\text { Temporary registration for a period of } 6 \\
\text { months and more (KT3) } \\
\text { Temporary registration for a period of less } \\
\text { than } 6 \text { months (KT4) } \\
\text { No registration at the destination }\end{array}$ & $\begin{array}{l}\text { KT1-2 } \\
\text { KT3 } \\
\text { KT4 } \\
\text { res_no }\end{array}$ \\
\hline 19 & $\begin{array}{l}\text { Why you do not } \\
\text { register your HB? }\end{array}$ & $\begin{array}{l}\text { Not obligatory } \\
\text { Do not know if it has to be registered } \\
\text { Other reasons (e.g., too complicated } \\
\text { procedures, too expensive) }\end{array}$ & $\begin{array}{l}\text { ynotreg_incompul } \\
\text { ynotreg_dontknow } \\
\text { ynotreg_others }\end{array}$ \\
\hline 20 & $\begin{array}{l}\text { Reasons to set up or } \\
\text { manage this HB }\end{array}$ & $\begin{array}{l}\text { Did not find a salaried job in an enterprise } \\
\text { Did not find a salaried job in a HB } \\
\text { To earn higher income } \\
\text { To be independent } \\
\text { To follow family tradition } \\
\text { Others }\end{array}$ & $\begin{array}{l}\text { a4d_nojob_enterp } \\
\text { a4d_nojob_hb } \\
\text { a4d_better_inc } \\
\text { a4d_indep } \\
\text { a4d_fam_trad } \\
\text { a4d_others }\end{array}$ \\
\hline 21 & $\begin{array}{l}\text { The main reason to } \\
\text { choose this business } \\
\text { activity }\end{array}$ & $\begin{array}{l}\text { Family tradition } \\
\text { The profession that you know } \\
\text { Have higher profit than other activities } \\
\text { More stable returns than other products } \\
\text { Others }\end{array}$ & $\begin{array}{l}\text { g1_fam_trad } \\
\text { g1_prof_know } \\
\text { g1_higher_profit } \\
\text { g1_stabler_return }\end{array}$ \\
\hline 22 & $\begin{array}{c}\text { Willingness to register } \\
\text { your HB }\end{array}$ & $\begin{array}{l}\text { Yes } \\
\text { No } \\
\text { Do not know } \\
\text { Already registered }\end{array}$ & $\begin{array}{l}\text { wilg_reg_yes } \\
\text { wilg_reg_no } \\
\text { wilg_reg_dontknow } \\
\text { registered }\end{array}$ \\
\hline 23 & $\begin{array}{l}\text { Do you think there is a } \\
\text { future for a HB like } \\
\text { yours? }\end{array}$ & $\begin{array}{l}\text { Yes } \\
\text { No, and think of changing activity } \\
\text { No, but do not think of changing activity }\end{array}$ & $\begin{array}{l}\text { futur_yes } \\
\text { nofutur_change } \\
\text { nofutur_notchange }\end{array}$ \\
\hline
\end{tabular}

Source: HB\&IS HCMC survey 2009, GSO-ISS / IRD-DIAL 


\title{
La dimension familiale du dépistage VIH/sida
}

\author{
Myriam de Loenzien (IRD CEPED)
}

$\mathrm{Au}$ Viêt Nam, la famille constitue une unité de référence essentielle pour les individus. Conçue comme insérée dans la communauté et plus largement dans la société, elle fournit un modèle aux relations sociales. Celui-ci est d'ailleurs objectivé dans le langage. Traversée par des tensions dues à une recherche de cohésion, de fortes normes d'entraide, mais aussi des revendications liées à une montée plus récente d'individualisme, elle est le lieu d'expression de nombreux enjeux parmi lesquels la gestion de la santé et des questions qui lui sont liées. L'épidémie VIH/sida est caractérisée par une prévalence relativement faible $(0,5 \%$ des adultes de 15 à 49 ans depuis 2007) et une concentration des infections chez les utilisateurs de drogue par voie intraveineuse, les femmes qui font du commerce sexuel (Vu et coll., 2008) et les homosexuels (National Committee for AIDS, Drugs and Prostitution prevention and control, 2012). Dans ce contexte, la famille joue un rôle essentiel. Encouragées par les autorités centrales, ses interventions revêtent des formes multiples. Elles concernent la prévention et la prise en charge, mais aussi la gestion de la stigmatisation et la discrimination à l'égard des personnes atteintes et de leurs proches. En amont, le dépistage est déterminant. Ce chapitre s'intéresse aux modalités de sa réalisation auprès de la population générale. Il s'agit de savoir comment est prise la décision du test, par qui et de quelle façon elle est mise en œuvre. Pour chacune de ces étapes, nous explorons la place qu'occupe la famille. Pour cela, nous nous appuyons sur les données qualitatives et quantitatives d'une enquête cas-témoin menée dans la province de Quang Ninh en milieux rural et urbain (voir Carte 1 en tête d'ouvrage). Les résultats permettent de mieux situer les interventions de la famille dans les différentes étapes du dépistage, tout en tenant compte des contrastes entre les situations des hommes et des femmes.

\section{I- Une mobilisation croissante incluant la famille}

Les modalités de réalisation du dépistage conditionnent la prise en charge. Au Viêt Nam, celle-ci s'est développée rapidement au cours de ces dernières années. Ces évolutions se sont déroulées au moment où s'est produit un changement radical d'orientation de la lutte contre le VIH/sida, vers des approches plus empathiques et participatives marquées par un appel à la responsabilité individuelle et à la mobilisation des familles. Celles-ci sont en effet fortement sollicitées.

\section{$\underline{\text { I.1- Une réorientation tardive, rapide et radicale de la lutte contre le VIH/sida }}$}

La lutte contre le VIH/sida a connu au Viêt Nam des changements décisifs convergents avec l'évolution internationale, selon un calendrier relativement tardif. Dans ce pays, le premier cas de séropositivité déclarée date de 1990. En 1996, le Viêt Nam était le dernier en Asie avec la Chine à clore une phase de déni et de peur caractérisée par des mesures punitives. Il a alors adopté des réponses «ad hoc» consistant en l'adoption d'interventions plus nombreuses, bien que peu fondées sur des informations concrètes (Independent Commission on AIDS in Asia, 2008). Mais c'est surtout à partir du début des années 2000 que l'implication du gouvernement dans la lutte contre le VIH/sida a changé de façon significative (Independent Commission on 
AIDS in Asia, 2008). En effet, jusque fin 2003, le VIH était considéré et présenté par les pouvoirs publics comme un "fléau social » au même titre que la consommation de drogue ou la prostitution. La violence des images diffusée par le biais de panneaux d'affichage dans le cadre de cette campagne a durablement marqué les esprits (Loenzien, 2013; McNally, 2002). Ce rattachement à une politique déployée lors de la colonisation française (Proschan, 2002) a été abandonné en 2004 en raison de son caractère jugé nocif, notamment en matière de stigmatisation et de discrimination. On est donc passé d'une criminalisation des porteurs du VIH à la promotion de la santé et du bien-être. Cette nouvelle orientation correspond au modèle « humaniste » caractérisé par la prise en compte des droits de la personne dans la réponse au VIH/sida. Formalisée par Jonathan Mann (Global AIDS Policy Coalition, 1996), cette approche a dominé la lutte contre le VIH/sida dans de nombreux pays, dès les années 1980. Le Viêt Nam l'a donc adoptée près de deux décennies plus tard.

En 2006, le Viêt Nam était qualifié de peu avancé dans la phase suivante dite « informée », marquée par la mise en place de mesures fondées sur des données scientifiques. Il ne devançait que deux pays (Sri Lanka et Pakistan) sur les 14 que compte la région et n'avait pas entamé la phase de maturité, où le gouvernement est censé déployer les moyens nécessaires pour une réponse durable et complète (Independent Commission on AIDS in Asia, 2008). Celle-ci est néanmoins amorcée avec la loi sur le VIH/sida adoptée en juin 2006 et entrée en vigueur début 2007, laquelle prévoit de nouvelles aides en faveur des séropositifs, comme des soins plus accessibles et une plus grande protection des personnes, avec des garanties de confidentialité et d'anonymat. D'autres textes légaux relatifs à la santé et la population témoignent également de cette évolution : d'une intervention répressive vers une plus large mobilisation. C'est le cas notamment de la Stratégie sur la santé de la reproduction (2001-2010) et la Stratégie de lutte contre le VIH/sida pour 2010, avec une vision à 2020. Ainsi, l'évolution de la politique de lutte contre le VIH/sida au Viêt Nam fait écho à des évolutions qui surviennent plus largement, mais elle s'impose d'une façon plus directement normative dans le champ du VIH.

Ce développement de procédures standard de prise en charge s'est accompagné et a été favorisé par une internationalisation des ressources utilisées pour lutter contre l'épidémie. En effet, de 1996 à 2004, la part des dépenses consacrées à la lutte contre le VIH/sida est passée d'environ 20\% à $80 \%$ (Independent Commission on AIDS in Asia, 2008). Ce caractère international des ressources concerne tous les pays d'Asie, mais avec des parts de financement plus faibles pour la Thaïlande et la Chine (environ $25 \%$ et $30 \%$ respectivement) (Independent Commission on AIDS in Asia, 2008). Au Viêt Nam, il est perceptible dans le déploiement de vastes projets, tels que le PEPFAR (President's emergency plan for AIDS relief) des États-Unis, pour un montant de 506 millions de dollars de 2004 à 2011 (PEPFAR, 2010) ou les actions de la Banque Mondiale (35 millions de dollars de 2005 à 2012) (Nguyen, 2013).

L'appel à la mobilisation et à la participation de tous s'est par ailleurs traduit par un développement des associations et groupes d'aide aux séropositifs (Hoang, 2013).

\section{I.2- Développement du dépistage}

L'évolution de la lutte contre le VIH/sida est perceptible dans les mesures prises dans le domaine du dépistage. Au niveau mondial, le milieu des années 2000 a marqué un tournant, avec la diffusion par l'Organisation mondiale de la santé de nouvelles recommandations internationales (Organisation mondiale de la santé et ONUSIDA, 2007). Celles-ci ont consisté en la promotion du conseil et du dépistage à l'initiative du 
patient, mais aussi du soignant, afin d'améliorer l'accessibilité de la prévention, du traitement, des soins et du soutien. On est donc passé d'un dépistage du «tout volontaire » à des propositions plus systématiques de la part des professionnels, afin de faire reculer le diagnostic tardif et d'optimiser les bénéfices du traitement.

$\mathrm{Au}$ Viêt Nam, le mouvement a été différent, mais convergent avec cette tendance. La surveillance sentinelle avait commencé en 1994 dans 8 provinces, puis avait été étendue à 12 provinces en 1995 et 30 en 2001 (Hoang et coll., 2004). Jusqu'à cette date, le dépistage était réalisé au sein des centres de médecine préventive, sans séparation des tests volontaires par rapport aux autres. Le fort taux de prévalence obtenu à cette période (18\% pour 2000 personnes testées) montre le faible accès au dépistage des personnes considérées comme à faible risque. Cette même année a été établi le premier centre de dépistage volontaire, anonyme et gratuit à Hô Chi Minh-Ville. L'année suivante, cette mesure a été étendue à 18 provinces (Hoang et coll., 2004). Dès 2002, un centre de dépistage volontaire, anonyme et gratuit était mis en place à Hanoi (hôpital de Bach Mai). Progressivement, les services ont été établis dans les autres provinces. Ainsi, d'une approche relativement directive le pays est passé à une plus grande prise en compte de la volonté des individus. Cette évolution a pris la forme d'un accès plus personnalisé et confidentiel aux tests, avec le recueil du consentement mis en avant dans les directives nationales relatives au dépistage à partir de 2007 (Ministry of Health, Socialist Republic of Vietnam, 2007). Cependant, la stigmatisation et la discrimination subies par les personnes séropositives et leurs proches demeurent de puissants freins au dépistage (Pharris et coll., 2011).

Les tendances observées quant à la population séropositive reflètent l'évolution de l'épidémie, mais aussi celle du dépistage. Au cours des deux dernières décennies, la population dépistée positive a tendu à rajeunir et à se féminiser. De 1993 à 2008, la proportion de jeunes de 20 à 29 ans parmi les cas déclarés est passée d'environ $18 \%$ à plus de 50\%. En 2009, plus de 80\% avaient entre 20 et 39 ans (Socialist Republic of Vietnam, 2010). Le manque de précision des données rend difficiles leurs comparaisons, mais les tendances qui se dégagent des différentes sources sont similaires. Elles conduisent à un constat de rajeunissement de la population séropositive, ce qui peut correspondre à une plus forte proportion de jeunes atteints, mais aussi à un meilleur dépistage de cette population grâce à un meilleur accès, un niveau plus élevé de connaissances et une transformation des représentations de l'épidémie. Par ailleurs, la lente diffusion hétérosexuelle du VIH se traduit par une augmentation de la part des femmes dans la population atteinte. Cette tendance concerne plusieurs pays d'Asie.

Par rapport à l'Afrique subsaharienne où $60 \%$ des personnes séropositives sont des femmes, en Asie celles-ci représentaient 35\% des adultes séropositifs VIH en 2008, alors que cette proportion n'était que de $17 \%$ en 1990 (UNAIDS, 2010). Sur ce continent, plus de $90 \%$ des 1,7 million de femmes séropositives VIH ont été infectées par leur mari ou leur partenaire sexuel régulier (UNAIDS, 2010).

$\mathrm{Au}$ Viêt Nam, la part des femmes parmi les séropositifs déclarés a augmenté de $20 \%$ en 2005 à 24\% en 2007 (Ministry of Planning and Investment, 2008). Aujourd'hui, trois quarts des cas déclarés sont des hommes (Socialist Republic of Vietnam, 2010). Ces tendances sont à mettre en relation avec le développement du dépistage des femmes enceintes. En effet, au sein des actions de dépistage, celles visant à prévenir la transmission materno-infantile occupent une place particulière. Lancées à la fin des années 1990, leur objectif visait un dépistage volontaire de $60 \%$ des femmes enceintes pour la période 2006-2010. Cependant, des études ont révélé que celui-ci concernait surtout les hôpitaux centraux, ne s'accompagnait pas toujours d'une remise du résultat 
du test et ne reposait pas uniquement sur le volontariat (Khuat, 2007). De plus, une part importante de la population, notamment en milieu rural, échappe à ce dispositif (Nguyen et coll., 2010; Pharris et coll., 2011). Ainsi, en 2009, seul un quart des femmes enceintes étaient dépistées pour le VIH (United Nations Development Programme et Regional Centre in Bangkok, 2011).

Une autre population reste très peu présente dans les actions de dépistage et dans les messages relatifs à la lutte contre le VIH/sida: les homosexuels. Ceux-ci n'ont été inclus dans les sites sentinelles qu'en 2011, et seulement dans quelques provinces (Socialist Republic of Vietnam, 2010). De plus, les campagnes d'affichage dans l'espace public ne faisaient aucune mention des risques liés aux relations homosexuelles masculines, au moins jusqu'en 2008 (Loenzien, 2013). Or, la prévalence du VIH, au sein de cette population, varie de 5,2 à $16,3 \%$ en 2011 , avec de fortes variations selon les provinces, les taux les plus élevés étant atteints à Hô Chi Minh-Ville (Socialist Republic of Vietnam, 2010).

Ainsi, le dépistage qui a connu un développement rapide reste limité. Une des causes réside dans le caractère relativement restreint de l'accès au traitement. Incitatif majeur au dépistage, il restait encore restreint sur le plan géographique, les médicaments et infrastructures de prise en charge étant surtout disponibles dans les centres urbains. En 2010, l'effectif des séropositifs bénéficiant d'une prise en charge par antirétroviraux représentait environ la moitié de ceux qui en avaient besoin, soit près de 50000 séropositifs (UNAIDS, 2011) et une proportion relativement faible des cas estimés (de l'ordre d'une personne sur six) (Socialist Republic of Vietnam, 2010; UNAIDS, 2011; Viet Nam Commission for Population, Family and Children et coll., 2006)

\section{I.3- Rôle central de la famille}

Dans un contexte où la prise en charge des séropositifs n'est pas assumée de façon continue par des institutions sanitaires, la famille joue un rôle prépondérant. Ce lien a été mis en évidence dans divers contextes de prise en charge d'adultes séropositifs VIH. La corésidence des personnes séropositives et de leurs aidants est déterminante, comme l'ont montré les études réalisées dans diverses régions, notamment en Afrique subsaharienne (Miller et Murray, 1999; Moore et Henry, 2005; Zimmer et Dayton, 2003), en Thaïlande (Knodel, 2011), au Cambodge (Williams et coll., 2008) et en Chine (Li et coll., 2011, 2006). Au Viêt Nam, la famille représente un recours essentiel, mais ambivalent (Loenzien, 2009a). Elle permet l'accès aux soins et aux organisations de support, mais peut également constituer une barrière à certains contacts. Elle protège de la communauté, au sens d'entourage social formé par les voisins, mais aussi par les associations et groupes de solidarité, mais elle est aussi un lieu d'exposition à des attitudes de stigmatisation ou des comportements de discrimination. En effet, elle est susceptible de subir une stigmatisation quand la séropositivité d'un de ses membres est connue, mais peut à son tour exercer un rejet total ou partiel de son membre séropositif (Luu, 2010; UNDP et AusAID, 2005). Elle procure un soutien économique, mais contrôle le pouvoir de ceux qui dépendent d'elle. Elle ancre les individus dans des rôles, mais doit aussi accompagner l'évolution de ceux-ci et assumer les tensions qui surgissent en cas d'écueil majeur, par exemple lorsque la séropositivité d'un homme compromet la possibilité d'envisager de perpétuer la lignée familiale. Son rôle prépondérant dans la prise en charge, encouragé par les textes de loi, définit un lieu privilégié d'expression de la solidarité familiale, mais aussi du contrôle social.

Ce rôle central de la famille a été renforcé par les réformes du Renouveau (Dôi Moi) lancées pendant les années 1980. En effet, caractérisées par une ouverture internationale 
et le passage d'un système planifié à une économie socialiste de marché sous la direction de l'État (Beresford, 2008), celles-ci ont provoqué et accompagné une profonde mutation socio-économique et culturelle. Au cours de celle-ci, la famille a été placée au centre d'enjeux sociopolitiques et économiques importants (Bélanger et Barbiéri, 2009). La fin de la collectivisation en 1988 a consacré le ménage comme unité économique (Kleinen, 2001). Certains auteurs vont plus loin, en affirmant qu' « en augmentant la portée économique du ménage, la rénovation économique a revitalisé les liens de parenté et les réseaux de lignage et leur a créé des champs d'action politique et économique » (Werner et Bélanger, 2006). Cette mise en avant de l'économie du foyer a renforcé le poids social de la famille, mais aussi l'autorité patrilinéaire (Werner et Bélanger, 2006). La complexité des relations familiales tient à la coexistence de deux modèles opposés et complémentaires, dont l'importance relative varie selon la période (Luong 1989). Le premier «patrilinéaire » est orienté vers les hommes. Il est caractérisé par une hiérarchie de genre et une division du travail, qui reflètent une domination masculine marquée par des ressources destinées préférentiellement aux individus masculins dont dépend la continuité du lignage. Il est renforcé par la propriété terrienne et la doctrine confucéenne. Le deuxième modèle, «bilatéral " est caractérisé par le maintien du contact de l'épouse avec sa famille d'origine, auprès de laquelle elle accomplit les obligations rituelles. Il est favorisé par l'endogamie, qui entraîne souvent une proximité géographique des apparentés paternels et maternels, et il est considéré comme plus proche des principes du bouddhisme (Luong, 1989). Ces deux modèles coexistent au sein de chaque famille, avec une prééminence du premier. Celui-ci a cependant été affaibli depuis les années 1950 au profit du deuxième, en partie en raison des transformations de l'appareil étatique idéologique, politique et légal (Luong, 1989). Ce fonctionnement familial est susceptible d'influencer le mode de prise en charge des séropositifs, dans le contexte d'une population atteinte majoritairement masculine, où la prise en charge est en grande partie assumée par des femmes (Loenzien, 2010).

\section{II- Enquête cas-témoin}

L'enquête que nous avons menée a vocation à identifier le rôle et la place de la famille dans le processus de dépistage, de prévention du VIH et de prise en charge des séropositifs en comparant les milieux urbain et rural. Elle s'est déroulée dans la province de Quang Ninh.

\section{II.1- Une province assez fortement affectée à l'échelle du pays}

La province de Quang Ninh, où a eu lieu notre enquête, se situe au nord-est du Viêt Nam, à la frontière avec la Chine (voir Carte 1 en tête d'ouvrage). Elle a développé d'intenses activités touristiques dans la baie d'Halong, d'importantes exploitations minières et de nombreux liens commerciaux avec son voisin. Elle constitue une zone de transit à proximité du port de Haiphong et de la capitale politico-administrative Hanoi. Elle comptait un peu plus d'un million d'habitants en 2006, avec une densité de population de 179 personnes au $\mathrm{km} 2$, ce qui correspond à un niveau inférieur à la moyenne nationale. Près de la moitié de ses habitants vivent en milieu urbain (Central Population and Housing Census Steering Committee, 2010; General Statistical Office, National Institute of Hy et ORC Macro, 2006).

Sur le plan de l'épidémie VIH/sida, Quang Ninh est une des provinces les plus affectées. Fin 2002, parmi les 5954 cas de séropositivité détectés dans cette province, 
$92 \%$ étaient des hommes et $70 \%$ renvoyaient à l'utilisation de drogue par voie intraveineuse (UNDP et AusAID, 2005). Le milieu urbain regroupe davantage de cas, les connaissances de la population concernant le $\mathrm{VIH} /$ sida y sont plus répandues, les interventions de lutte plus nombreuses et les études menées plus fréquentes.

\section{$\underline{\text { II.2- Enquête auprès de ménages affectés et de ménages témoins }}$}

Nous avons mené une enquête cas-témoin en 2006 et 2008. En effet, dans un contexte où la prévalence VIH est très faible, celle-ci fournit des données apportant une connaissance des personnes atteintes et de leurs proches par comparaison avec des membres de ménage non atteints. La collecte s'est tenue dans la ville de Halong pour le milieu urbain (2006), puis dans la commune rurale de Dông Triêu (2008). Un même protocole a été utilisé.

Les ménages ont été identifiés par le biais d'une liste fournie par le Comité local de lutte contre le VIH/sida, émanation du comité national, lui-même sous la tutelle du Ministère de la Santé. Nous nous sommes adressés à tous les ménages qui nous ont été indiqués par ce biais. Afin de diversifier les types de ménages recrutés, nous nous sommes également adressés aux associations de lutte contre le VIH/sida. Enfin, à l'issue de l'enquête d'un ménage, nous avons demandé aux participants s'ils pouvaient nous mettre en contact avec des membres d'autres ménages affectés. Le processus de sélection des ménages se faisait par paire de ménages comportant un ménage cas et un ménage témoin. Les ménages cas sont ceux comprenant au moins une personne séropositive vivante. Le statut sérologique des individus correspond à celui qui a été déclaré. Chaque ménage cas, défini comme comportant au moins un adulte (se déclarant) séropositif, a été apparié à un ménage témoin sur la base de deux critères : des niveaux de vie similaires et des habitations distantes d'une dizaine de logements l'une de l'autre au sein d'un même ensemble résidentiel. Cette démarche était mise en œuvre par les enquêteurs avec l'aide de coordinateurs de l'enquête. Elle repose sur l'hypothèse d'un lien entre niveau économique du ménage et atteinte VIH, susceptible de faire du niveau de vie un facteur de confusion. Le niveau de vie du ménage a été évalué par les enquêteurs au moyen d'observations, puis contrôlé par la collecte

d'informations sur les matériaux de construction de l'habitation et la disposition d'équipements.

Au sein de chaque ménage enquêté, nous avons recensé tous les membres grâce à un questionnaire ménage, puis interrogé les personnes âgées de 15 à 59 ans à l'aide d'un questionnaire individuel. Le questionnaire ménage est du même type que celui qui est utilisé pour les enquêtes démographiques et de santé, comprenant un descriptif des caractéristiques du logement, les équipements et biens du ménage ainsi qu'une liste des membres avec leurs principales caractéristiques sociodémographiques indiquées de façon succincte. Le questionnaire individuel, dont la passation durait une demi-heure à trois quarts d'heure, comportait un recueil des principales caractéristiques sociodémographiques des personnes interrogées. Il comprenait en outre des questions sur les connaissances, attitudes et pratiques des individus en matière de transmission, prévention, dépistage, traitement et prise en charge de l'épidémie VIH. Il incluait également des questions relatives aux pratiques des personnes interrogées en matière de solidarité en cas de problème sanitaire, économique ou psychologique.

Les participants aux discussions de groupe ont été identifiés parmi les membres des ménages interrogés par questionnaire, mais aussi parmi d'autres habitants du site. Les discussions de groupe ont eu lieu de façon concomitante avec la passation des questionnaires. Elles portaient sur la vie quotidienne et les relations au sein de la 
famille, les problèmes de santé rencontrés, l'influence constatée ou potentielle de l'épidémie sur la vie et les relations interpersonnelles, les connaissances, attitudes et pratiques en matière de prévention de la transmission du VIH ainsi que les programmes et groupes de lutte contre l'épidémie et d'aide aux personnes séropositives. Dans chaque site, nous avons mené des discussions avec au moins deux groupes de parents de séropositifs et un groupe de personnes membres de fratries comprenant un séropositif. À Dông Triêu en outre, nous avons pu interroger un groupe d'épouses de séropositifs.

Les discussions avec la population générale se tenaient en général au domicile d'une des personnes interrogées. Chaque discussion d'une heure trente à deux heures était enregistrée sur magnétophone. Les entretiens ont été retranscrits en vietnamien et analysés à l'aide d'une approche thématique.

L'échantillon a été redressé pour assurer la qualité de l'appariement des ménages. L'analyse a été réalisée au moyen des logiciels d'analyse statistique SAS et SPSS.

\section{III- Résultats}

Dans quel contexte sont effectués les tests VIH ? Qui prend la décision de faire un dépistage et pour quelles raisons? Ces questions ont été abordées à travers les questionnaires et groupes de discussion que nous avons menés. Si ces entretiens de groupe permettent des discours sur l'ensemble des tests effectués, les aspects quantitatifs liés aux raisons du test et à son contexte de réalisation font référence principalement au dernier test, en vue de minimiser les problèmes de perte de mémoire.

\section{$\underline{\text { III.1- Des ménages diversement affectés }}$}

Au total, plus de la moitié des ménages cas interrogés à Halong sont issus de la liste fournie par le comité local de lutte contre le VIH/sida (60\%), ainsi que la quasi-totalité des ménages cas à Dông Triêu (98\%). Les associations de lutte contre le VIH/sida nous ont permis d'identifier une part non négligeable des ménages interrogés à Halong (12\%) mais cette voie a été inopérante à Dông Triêu. Enfin, plus d'un quart des ménages retenus à Halong $(28 \%)$ et une minorité à Dông Triêu $(2 \%)$ nous ont été présentés par des personnes qui avaient été précédemment interrogées. Les données utilisées sont issues de la passation de 398 questionnaires ménages (310 à Halong, 88 à Dông Triêu) correspondant à 977 questionnaires individuels (779 à Halong et 198 à Dông Triêu).

Dans les deux sites, la moitié des personnes interrogées vit dans un foyer témoin (51\%), l'autre se partage de façon relativement équilibrée entre personnes séronégatives et séropositives, les premières étant un peu plus nombreuses (27\% versus $23 \%)$. Dans les deux sites, les hommes constituent un peu moins de la moitié des membres recensés $(49 \%)$ et des personnes interrogées (48\%), proportions identiques à celles de l'enquête nationale Sida (General Statistical Office, National Institute of Hy et ORC Macro, 2006), sans différence significative selon le milieu de résidence.

Par ailleurs, 21 groupes de discussions sont pris en compte dans l'analyse des résultats, totalisant 129 participants. Parmi eux, 12 groupes de discussions ont été réalisés avec des membres de ménages témoins (dont 6 à Halong et 6 à Dông Triêu) et 9 groupes de discussions se sont déroulés avec des membres de ménages cas (dont 5 à Halong et 4 à Dông Triêu). 
Les trois types de population définis par le statut VIH individuel et du ménage sont distincts sur le plan sociodémographique. Si la population est plus jeune à Dông Triêu qu'à Halong, correspondant à une population plus jeune en milieu rural qu'en milieu urbain, les séropositifs sont plus jeunes que les autres membres de leurs ménages, euxmêmes plus âgés que les individus des ménages non affectés (tableau 1). De même, les personnes en union sont plus nombreuses dans la commune rurale de Dông Triêu, mais les ménages affectés comprennent une proportion plus forte de personnes veuves, divorcées ou séparées. De manière surprenante, la proportion de personnes en union est plus élevée dans les ménages affectés que dans ceux qui ne sont pas affectés à Dông Triêu. Cette différence s'explique en partie par une proportion moindre de célibataires. Ainsi, si les ménages affectés sont caractérisés dans les deux sites par une plus forte proportion de personnes hors union, c'est d'une part parce que la séropositivité est associée au célibat et aux ruptures d'union, d'autre part parce que ceux qui cohabitent avec les séropositifs sont également plus fréquemment hors union. C'est le cas notamment de parents âgés devenus veufs.

La proportion de personnes ayant au moins un enfant vivant est plus élevée à Dông Triêu (82\%) qu'à Halong (66\%). Cette divergence correspond à une situation très courante d'une fécondité plus forte en milieu rural. Elle s'explique en partie par la proportion de personnes en union : comme nous l'avons vu, celle-ci est plus élevée à Dông Triêu. Or, la quasi-totalité des personnes vivant en union (96\%) ou ayant rompu une union (92\%) a déclaré avoir au moins un enfant vivant, alors que ce n'est le cas de quasiment aucun célibataire (une seule personne parmi 263). À Halong, les séropositifs sont moins nombreux à avoir des enfants $(58 \%)$ que les membres séronégatifs de leurs ménages et les membres des ménages témoins (68\%). La différence n'est pas significative à Dông Triêu.

Tableau 1- Indicateurs sociodémographiques pour la population interrogée selon son statut VIH à Halong et Dông Triêu : âge moyen en années et \% de répondants dans chaque catégorie.

\begin{tabular}{|c|c|c|c|c|c|c|c|c|c|c|}
\hline & \multicolumn{5}{|c|}{ Halong } & \multicolumn{5}{|c|}{ Dông Triêu } \\
\hline & $\begin{array}{c}\text { Séropo- } \\
\text { sitif } \\
\text { ménage } \\
\text { affecté }\end{array}$ & $\begin{array}{l}\text { Séroné- } \\
\text { gatif } \\
\text { Ménage } \\
\text { affecté }\end{array}$ & $\begin{array}{c}\text { individu } \\
\text { ménage } \\
\text { non } \\
\text { affecté }\end{array}$ & $\begin{array}{c}\text { Ensem } \\
\text { ble }\end{array}$ & & $\begin{array}{l}\text { Séropo- } \\
\text { sitif } \\
\text { ménage } \\
\text { affecté }\end{array}$ & $\begin{array}{l}\text { Séroné- } \\
\text { gatif } \\
\text { Ménage } \\
\text { affecté }\end{array}$ & $\begin{array}{c}\text { individu } \\
\text { ménage } \\
\text { non } \\
\text { affecté }\end{array}$ & $\begin{array}{c}\text { Ensem } \\
\text { ble }\end{array}$ & \\
\hline $\begin{array}{l}\text { Age moyen } \\
\text { (années) }\end{array}$ & 30,6 & 39,5 & 36,1 & 35,8 & * & 31,7 & 41,1 & 33,5 & 35,1 & \\
\hline En union & 34 & 59 & 64 & 56 & & 63 & 85 & 71 & 73 & \\
\hline Célibataire & 34 & 29 & 29 & 30 & & 13 & 10 & 21 & 16 & \\
\hline & 32 & 12 & 7 & 14 & * & 25 & 6 & 7 & 11 & * \\
\hline À un e & 58 & 68 & 68 & 66 & * & 83 & 90 & 77 & 82 & $\mathrm{n}$ \\
\hline A été scolar. & 99 & 97 & 97 & 98 & - & 100 & 100 & 100 & 100 & - \\
\hline Actif(ve) & 61 & 70 & 66 & 66 & $\mathrm{n}$ & 79 & 69 & 75 & 74 & $\mathrm{n}$ \\
\hline Natif(ve) & 89 & 69 & 77 & 78 & & 85 & 79 & 92 & 87 & $\mathrm{n}$ \\
\hline Effectif & 170 & 205 & 393 & 768 & & 48 & 52 & 98 & 198 & \\
\hline
\end{tabular}

* : différence significative au seuil de $5 \%, \mathrm{n}$ : différence non significative, - : effectif insuffisant

Source : enquêtes FAVIHVIET 2006-2008 (questionnaires individuels)

Halong et Dông Triêu représentent deux zones géographiques peu éloignées (environ 60 kilomètres) très contrastées sur le plan économique. Le tourisme constitue la principale source de revenus de la ville de Halong, qui comprend une baie maritime 
classée au patrimoine mondial de l'UNESCO. Les autres secteurs prédominants incluent le commerce, les activités portuaires, l'industrie du charbon, la pêche, l'élaboration de matériaux de construction, la transformation d'articles aquatiques. L'économie de Dông Triêu est fondée sur l'agriculture notamment la culture de céréales, la foresterie et l'industrie, mais également l'extraction du charbon et la production de céramique. Les personnes d'âge actif sont présentes dans des proportions semblables dans les deux sites. La séropositivité et le fait d'appartenir à un ménage comportant une personne séropositive ne sont pas liés à des niveaux d'activité différents. Parmi les personnes interrogées, la proportion de personnes scolarisées est très élevée. Enfin, à Dông Triêu $(87 \%)$ et Halong $(78 \%)$ plus des trois quarts des personnes interrogées n'ont jamais changé de commune, signe d'une mobilité spatiale relativement faible.

\section{III.3- Une pratique du dépistage relativement peu répandue mais dotée d'une forte}

\section{intensité}

Lors des discussions de groupe avec des membres des ménages infectés, le dépistage est apparu comme peu répandu. Au sein de notre échantillon, la proportion de personnes qui ont effectué un test varie selon le statut VIH du ménage. À Dông Triêu, elle concerne la moitié des séronégatifs des ménages cas (44\%) et un quart des membres des ménages témoins (24\%). À Halong, quel que soit le ménage, c'est le cas de $18 \%$ des séronégatifs. Dans les deux sites, plus de la moitié des individus ayant fait un test l'ont effectué au cours des douze mois précédant l'enquête (55\% à Halong, 58\% à Dông Triêu), ce qui témoigne du développement relativement récent de cette pratique. S'il concerne une proportion relativement modeste d'individus, le dépistage se caractérise souvent, pour ceux qui l'effectuent, par la réalisation de tests multiples, dont le nombre croît avec la présence d'une personne séropositive dans le ménage et plus encore avec un résultat individuel positif (figure 1). À Halong, près de la moitié des séropositifs $(46 \%)$ ont effectué au moins trois tests, tandis que ce n'est le cas que d'un peu plus d'un tiers $(37 \%)$ des séronégatifs des ménages cas et d'un quart $(25 \%)$ des membres des ménages témoins. En discussion de groupe, la question du test a été débattue surtout en rapport à une expérience de résultat positif. Cependant, même en cas de séropositivité du conjoint, la décision de faire le dépistage n'est pas toujours prise. Ainsi, des épouses de séropositifs interrogées à Dông Triêu ont déclaré ne pas avoir effectué de test malgré les pressions familiales qu'elles ont subies. Celles qui l'ont fait affirment être séronégatives. Pour justifier cette absence, est évoqué le manque de temps ou de volonté. Cette absence de recours va à l'encontre de l'action d'associations qui assurent une information sur les modalités de réalisation du test, notamment l'association "Compassion» d'aide aux séropositifs et à leurs proches.

Les discussions de groupe permettent de mieux comprendre ces recours multiples et redondants aux infrastructures sanitaires. De nombreuses personnes effectuent des démarches successives, se rendant chaque fois dans une infrastructure de niveau supérieur, voire allant jusqu'à Hanoi pour être sûres du diagnostic. Plusieurs affirment avoir réalisé deux voire trois dépistages de suite dans des hôpitaux et centres différents, avant d'accepter leur séropositivité. Le manque de conseils pré et post tests, conjugué à la nécessité d'un délai pour obtenir le résultat, puis d'un nouveau prélèvement pour la confirmation d'une sérologie positive, jette le discrédit sur une procédure perçue par les patients et leurs familles comme non fiable et devant donc être renouvelée. Il aurait été utile d'obtenir la date à laquelle les séropositifs ont appris leur séropositivité. Il est rare cependant que celle-ci soit précisément connue. Son acceptation est l'aboutissement d'un processus relativement long difficile à dater pour des raisons de mémoire, et en 
raison de liens complexes entre processus de dépistage et perception d'éventuelles ruptures temporelles.

Figure 1- Nombre de tests de dépistages effectués selon le statut VIH à Halong parmi les personnes ayant effectué au moins un test

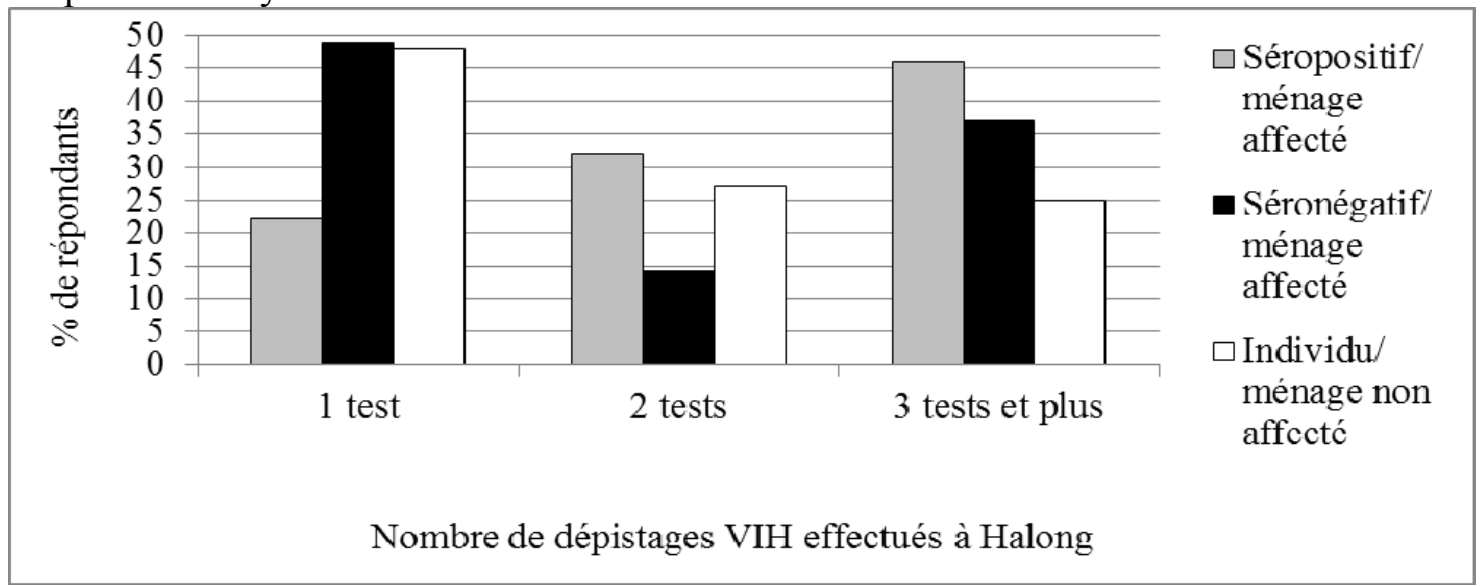

Source : enquêtes FAVIHVIET 2006 à Halong (questionnaires individuels)

\section{III.4- Les motivations du test : prédominance des problèmes sanitaires et familiaux}

Deux facteurs prédominent dans la réalisation ou au contraire la résistance au test : des enjeux sanitaires et des facteurs familiaux, étroitement imbriqués les uns aux autres. Le dépistage représente en effet la possibilité de concrétiser une menace très redoutée, exacerbée par les campagnes d'affichage associant l'épidémie à une condamnation morale (Loenzien, 2013; McNally, 2002). Devant la quasi-certitude d'une issue fatale, qui surviendrait au terme d'un processus de soins très coûteux en termes financiers, sociaux et familiaux, l'échéance du test est retardée voire sa réalisation évitée. Ce comportement vise entre autres à préserver les relations familiales :

«La grande sœur de mon mari dernièrement est décédée infectée du VIH, ainsi que son mari. Elle s'était mariée à un mineur et quand elle est revenue, elle est morte. Son mari est mort avant elle. Ils ont eu un fils. On a peur d'avoir un nouveau choc, qui rendrait l'enfant encore plus triste, alors ses grandsparents n'osent pas lui faire faire le test. Ils disent juste qu'il faut considérer que l'enfant est normal, rester proche de lui » (Discussion de groupe avec 8 épouses et mères membres de ménages témoins à Dông Triêu âgés de 26 à 42 ans).

Les membres des ménages non concernés nous ont fait part des mêmes comportements destinés à se protéger :

«Beaucoup de personnes savent que leur mari était malade et est mort, mais elles ne veulent pas faire de consultation, car elles ont peur qu'en y allant elles pensent à leur mari mort (...) Elles se disent : « tant pis, quand ça [la maladie] se déclarera, ça se déclarera et c'est tout» (Discussion de groupe avec 6 épouses et mères membres de ménages témoins à Dông Triêu âgés de 25 à 43 ans).

Les freins sont puissants. Ils contrecarrent les pressions familiales ou encore le discours normatif recueilli dans le cadre des discussions de groupe, selon lequel la méconnaissance de son statut sérologique constitue une entrave à la prévention. Aussi, franchir le pas implique souvent la confrontation à plusieurs types de difficultés, et les 
raisons du dépistage données par les personnes qui ont fait le test sont multiples. Les séropositifs en particulier sont nombreux à citer deux raisons ou plus (35\% à Halong, $38 \%$ à Dông Triêu) par rapport aux séronégatifs, en particulier ceux des ménages témoins ( $8 \%$ dans les deux sites).

Le motif le plus fréquent est la survenue de problèmes de santé. Il concerne plus d'un quart des individus (26\% à Halong, 30\% à Dông Triêu). À Halong, cette proportion est plus élevée pour les séropositifs $(32 \%)$ que pour les autres $(21 \%$ des membres des ménages témoins et $6 \%$ des séronégatifs des ménages cas, figure 2 ).

Figure 2- Raisons des tests de dépistages effectués selon le statut VIH individuel et du ménage à Halong et Dông Triêu parmi les personnes ayant effectué au moins un test (modalités non exclusives)

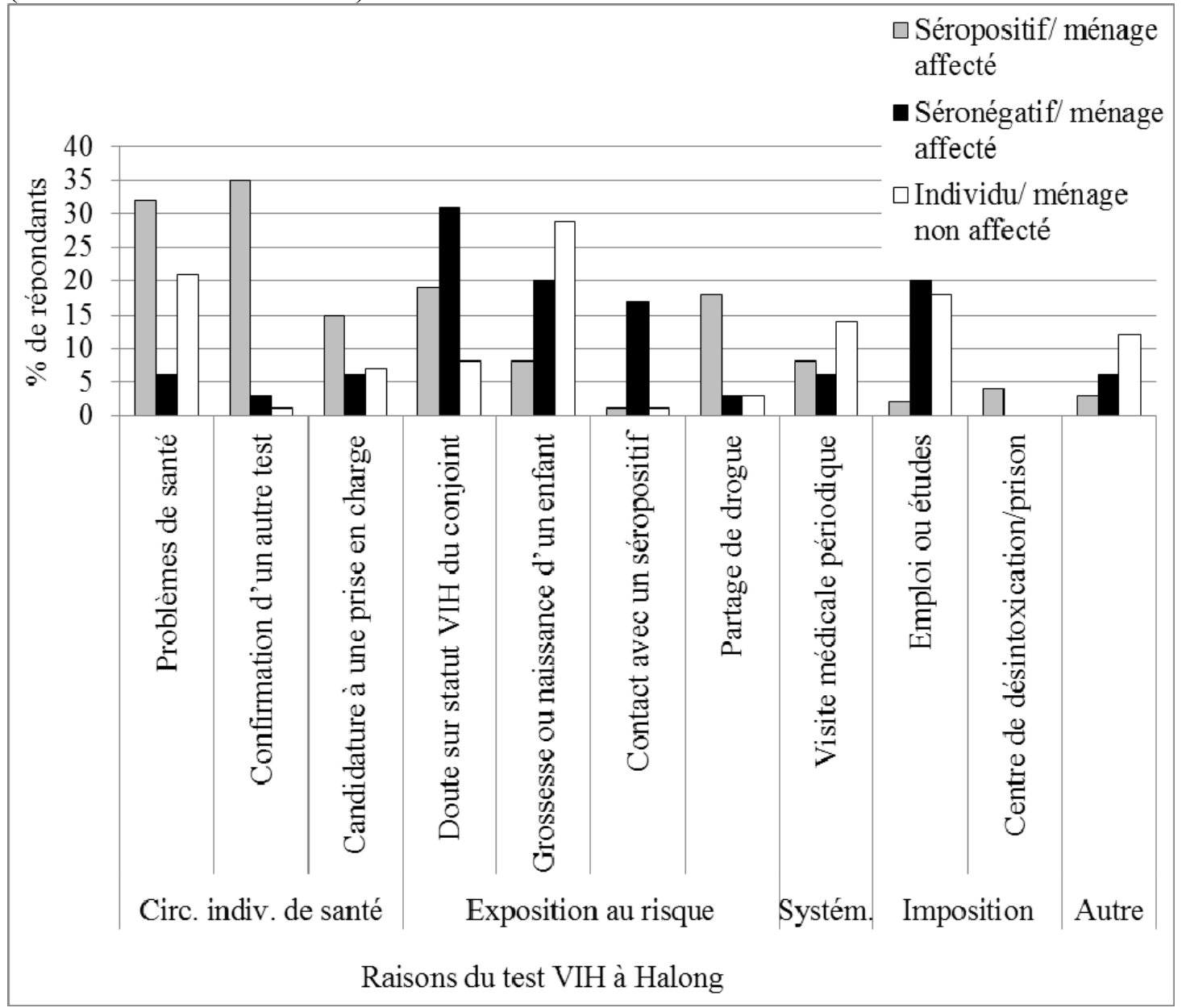

Raisons : Circonstances individuelles de santé, Exposition au risque, mesure systématique de santé, imposition non souhaitée par l'individu, autre

Source : enquêtes FAVIHVIET 2006 à Halong (questionnaires individuels)

La réalisation du test intervient parfois à un stade avancé de l'infection :

« Ce n'est que quand on découvre qu'on est malade qu'on va à l'hôpital faire une consultation et là c'est presque toujours le stade final» (discussion de groupe avec 6 mères de famille dans des ménages témoins à Halong, 38 à 52 ans).

Cette situation peut être liée à des contraintes familiales qui conduisent le patient à travailler jusqu'au dernier moment : 
« Il savait qu'il avait la maladie [VIH/sida] mais il continuait à travailler, car il était le support de la famille, il avait une épouse et un enfant. Il continuait et quand il a été malade il est rentré [chez lui] » (Discussion de groupe avec 6 pères de séropositifs à Halong âgés de 50 à 60 ans).

Pour les frères et sœurs de séropositifs, les indications incitant à demander un dépistage lors d'une consultation sont une fatigue, une perte de poids et le sentiment d'avoir des problèmes de santé. Une autre raison fréquemment donnée au test est la recherche de la confirmation d'un dépistage antérieur, mentionnée surtout par les séropositifs (35\% dans les deux sites). La recherche d'un accès à un programme de mise à disposition d'antirétroviraux (ARV) via une infrastructure sanitaire, ou la participation à une association d'aide aux séropositifs est aussi plus spécifique à cette population (15\% à Halong, $17 \%$ à Dông Triêu).

Le deuxième type de raison donné à la pratique du test concerne l'exposition au risque de transmission. Ainsi, les membres séronégatifs des ménages cas citent plus souvent que les autres le doute sur le statut VIH du conjoint (31\% à Halong, 30\% à Dông Triêu) ou leurs contacts quotidiens avec un séropositif (17\% à Halong). Les membres des ménages témoins sont plus nombreux à évoquer une grossesse (29\% à Halong, $21 \%$ à Dông Triêu) tandis que les séropositifs citent le partage de drogue. Parmi les autres réponses, des personnes indiquent le fait d'avoir subi un accident $(2 \%$ des personnes dans les deux sites) ou encore la préparation à un mariage, un don de sang, un voyage à l'étranger (États-Unis). Les consultations médicales périodiques concernent surtout les séronégatifs dans leur ensemble.

Enfin, l'imposition du test non souhaitée par l'individu a été mentionnée à l'occasion du l'entrée dans un cursus d'études ou d'emploi pour les séronégatifs principalement, ou encore lors de l'internement dans une prison, un camp de désintoxication ou de rééducation pour les séropositifs ( $4 \%$ des séropositifs à Halong).

\section{$\underline{\text { III.5- Un contexte hospitalier et familial }}$}

L'initiative du test n'est pas toujours individuelle. D'après les personnes que nous avons interrogées, l'initiative du test leur revient dans la moitié des cas (57\% à Halong, $47 \%$ à Dông Triêu). À Halong, cette proportion représente les deux tiers des séropositifs $(65 \%)$ et des séronégatifs des ménages cas $(60 \%)$ mais seulement un tiers des membres des ménages témoins (36\%). Cela peut s'expliquer, pour cette dernière population, en partie par le dépistage prénatal, les membres des ménages affectés devant faire face à des situations spécifiques liées à l'épidémie. Dans le cadre des discussions de groupe, plusieurs parents d'hommes séropositifs décédés ont parlé de la pression qu'ils exercent sur leur belle-fille et les enfants de celles-ci pour qu'ils aillent faire un dépistage VIH. La contrainte exercée peut être très forte pour les adolescents. Certains se sont vu imposer le dépistage par des membres de leur famille :

"Ầ ce moment-là, mes enfants étaient au collège. La famille a entendu dire que mon fils s'était injecté de la drogue avec quelques autres jeunes. On s'est mis en colère et on l'a emmené de force faire une consultation tout de suite » (Discussion de groupe avec 6 pères de séropositifs à Halong âgés de 50 à 60 ans).

La mobilisation de la famille peut être relativement large :

« Mon enfant, quand il était hospitalisé à l'hôpital provincial, s'est caché. Il ne voulait pas qu'on lui fasse un dépistage. Mais quand les deux familles - car c'est mon gendre - sont tombées d'accord pour l'emmener à l'hôpital Bach 
Mai [à Hanoi], on n'a pas eu besoin de son accord pour faire le dépistage. On l'a fait tout de suite » (Discussion de groupe avec 6 mères de séropositifs à Halong âgées de 50 à 70 ans).

Ces situations autoritaires vis-à-vis du patient sont survenues dans le cadre d'un soupçon de consommation de drogue. Elles visent entre autres à ce que la famille prévoie son implication dans la prise en charge du séropositif, dès le test de dépistage, malgré les réticences du patient. Dans ce contexte, une famille a effectué un dépistage à l'insu du jeune homme concerné :

« On lui a dit d'aller faire une consultation pour demander à travailler comme ouvrier. On l'a emmené faire une consultation pour tromper son attention et c'est comme ça qu'on a su [qu'il était séropositif]. Sinon il protestait toujours en disant qu'il ne l'est pas» (Discussion de groupe avec 6 parents de séropositifs à Dông Triêu âgés de 50 à 87 ans).

Ce test visait, dans le cas où le résultat aurait été négatif, à cloîtrer l'enfant pour qu'il se sèvre. Ces comportements reposent sur une conception que se font les proches du bien-être de la personne concernée et de l'ensemble de la famille. Dans ces circonstances, le test revêt une connotation familiale forte au détriment des besoins de l'individu.

Le lieu du dépistage est déterminant pour la définition du type de participation de la famille. Outre l'hôpital provincial, au moment de l'étude, trois lieux étaient disponibles à Halong pour la réalisation d'un test VIH : un centre de dépistage anonyme et gratuit situé dans le $5^{\mathrm{e}}$ arrondissement dans le quartier central de Trân Hung Dao, le centre de santé de la ville et le centre de médecine préventive. À Dông Triêu, des participants ont évoqué la mise à disposition de tests gratuits au centre de santé, d'un centre de conseil, d'un système gratuit de consultations VIH. Les personnes interrogées ont réalisé leur dernier test pour la plupart au sein d'un hôpital, tant à Halong (75\%) qu'à Dông Triêu $(92 \%)$, sans différence selon le statut VIH. Les personnes de Halong qui ont effectué leur test ailleurs mentionnent surtout un centre de dépistage volontaire (14\%) et le centre de médecine préventive (6\%). À Dông Triêu, elles citent aussi le centre de médecine préventive du chef-lieu de la province, Halong. D'autres ont évoqué la mise à disposition de tests gratuits au centre de santé. Dans le cadre des discussions de groupe, les personnes interrogées citent l'hôpital provincial de Halong, mais aussi l'hôpital vietnamo-suédois dans la ville d'Uông Bi.

Les habitants de Halong ont été testés dans leur ville pour la plupart d'entre eux (81\%), tandis que seul un peu plus du tiers de ceux de Dông Triêu (39\%) sont restés dans leur arrondissement. Près de la moitié sont allés à Halong. Hanoi a été cité davantage par les habitants de Halong (12\%) que par ceux de Dông Triêu (8\%). Le fait de partir dans une autre province est lié à un déficit d'équipements sur place, aux activités du patient au moment du dépistage, mais également au souhait d'éviter des problèmes de manque d'anonymat. À titre d'exemple, un jeune homme a profité de son séjour chez son oncle pour se rendre au centre de dépistage de Thai Nguyen, province relativement éloignée, située dans la région nord du pays. Il a eu ensuite recours à des infrastructures locales pour faire de nouveaux tests visant à confirmer le résultat du premier qui s'était avéré positif. La recherche de la confidentialité favorise la fréquentation d'infrastructures privées. Ainsi, d'après les groupes de discussion, une consultation dans une polyclinique à Halong évite la diffusion de l'information d'une éventuelle séropositivité, mais la réalisation du test nécessite le versement d'une somme de 50000 dôngs. 
Pour le test, les patients sont généralement accompagnés. C'est le cas de $71 \%$ des personnes testées à Halong et 64\% à Dông Triêu, sans différence selon le statut sérologique. Les aidants comprennent souvent au moins un membre de leur famille (67\% à Halong, 77\% à Dông Triêu). La prise en compte du type de personne concernée permet de distinguer quatre groupes (Figure 3).

Le premier est composé uniquement de personnes avec lesquelles le patient n'est pas apparenté. Elles peuvent être des membres d'infrastructures sanitaires, des amis, des collègues. Ce groupe non familial représente une proportion d'individus un peu plus élevée à Halong qu'à Dông Triêu. Le deuxième est le groupe parental. Il est constitué d'au moins un parent (père ou mère) ou un membre de la fratrie du patient, à l'exclusion de toute autre relation. Le troisième, groupe conjugal, inclut au moins une personne parmi le conjoint ou les enfants à l'exclusion de tout autre lien. Il correspond également à une part importante des aidants, surtout à Dông Triêu. Ces trois groupes occupent des places équivalentes en termes de nombre de personnes concernées. Un dernier groupe représente une part marginale. Il peut être mixte, avec au moins un membre du groupe parental et du groupe conjugal ou élargi avec au moins un apparenté plus éloigné qu'un parent, conjoint ou enfant. À Halong, le modèle parental est cité plutôt par des personnes séropositives (28\%) tandis que le modèle conjugal est plus souvent mentionné par les séronégatifs des ménages cas $(26 \%)$ et les membres des ménages témoins $(32 \%)$ (figure 3$)$. Le groupe non familial constitue le deuxième recours pour chacune de ces populations.

Figure 3- Accompagnement du patient pour le test de dépistage selon le statut VIH à Halong parmi les personnes ayant effectué au moins un test

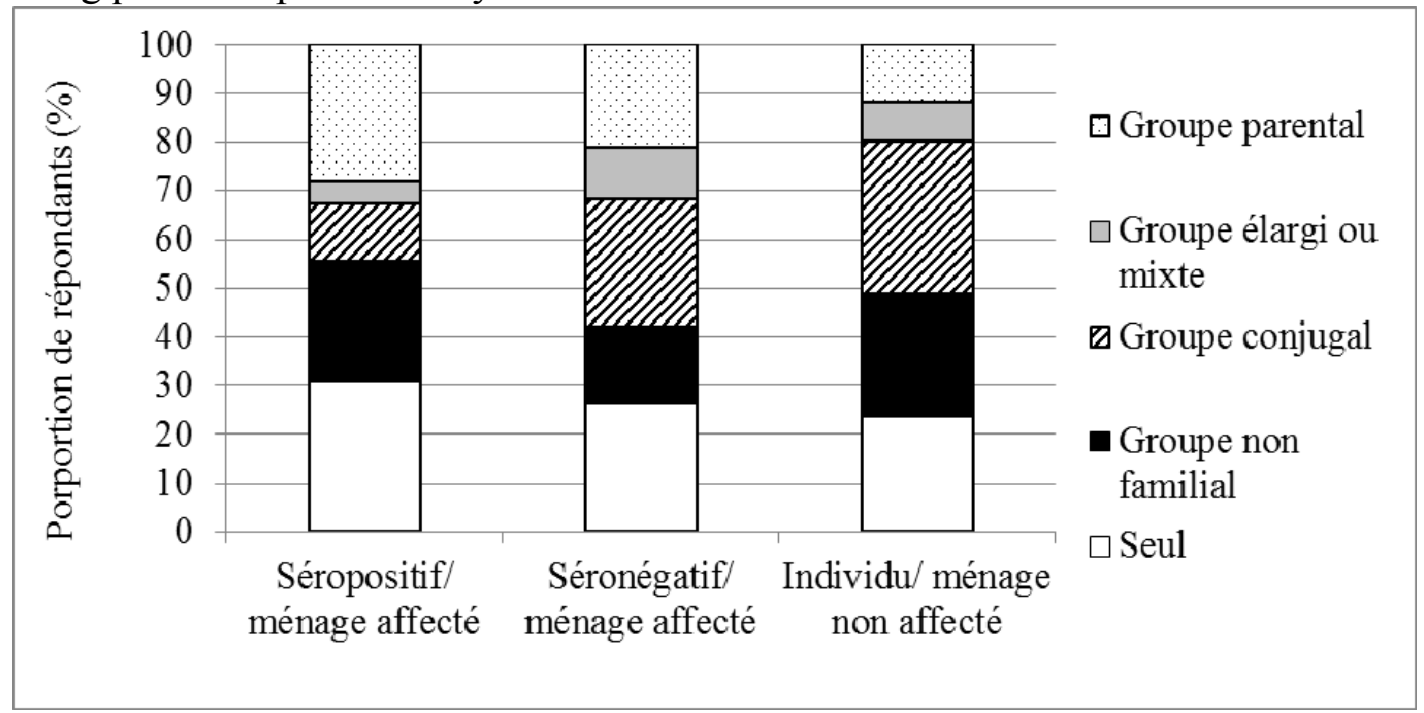

\section{Source : enquêtes FAVIHVIET 2006 à Halong (questionnaires individuels)}

La présence de la famille aux côtés du patient lors du dépistage s'explique en partie par la situation sociodémographique des personnes interrogées. En effet, la proportion d'individus hors union est relativement forte parmi les séropositifs, ainsi que la part des personnes qui cohabitent avec leurs parents. Globalement, les séropositifs, en proie à de problèmes sanitaires et sociaux en raison de leur atteinte VIH ou de comportements associés tels que l'injection de drogue, sont déjà en quelque sorte "sous tutelle » au moment du dépistage. 


\section{$\underline{\text { III.6- Hommes et femmes : deux utilisations du test }}$}

Étant donné les écarts observés en termes d'état matrimonial, d'activité, de fécondité entre hommes et femmes parmi les séropositifs et les séronégatifs des ménages cas, l'analyse de l'utilisation du test doit tenir compte du sexe de la personne interrogée. À Halong, la proportion d'individus qui ont fait un dépistage est plus élevée chez les hommes $(40 \%)$ que chez les femmes $(33 \%)$. Cette disparité est liée à la plus forte prévalence du VIH chez les premiers. Cependant, le nombre de tests effectués ne varie pas selon le sexe. Il en est de même pour l'ancienneté du dernier test, la personne qui en a eu l'initiative et son lieu de réalisation. Par contre, si hommes et femmes donnent le même nombre de motivations à ce test, celles-ci diffèrent. L'examen de la distribution de ces raisons données à la pratique du dépistage permet de saisir l'écart entre hommes et femmes (Figures 4.1, 4.2, 4.3).

Figure 4.1- Raisons du dernier test de dépistage effectué selon le statut VIH à Halong parmi les hommes et femmes ayant effectué au moins un test (modalités non exclusives) : personnes séropositives

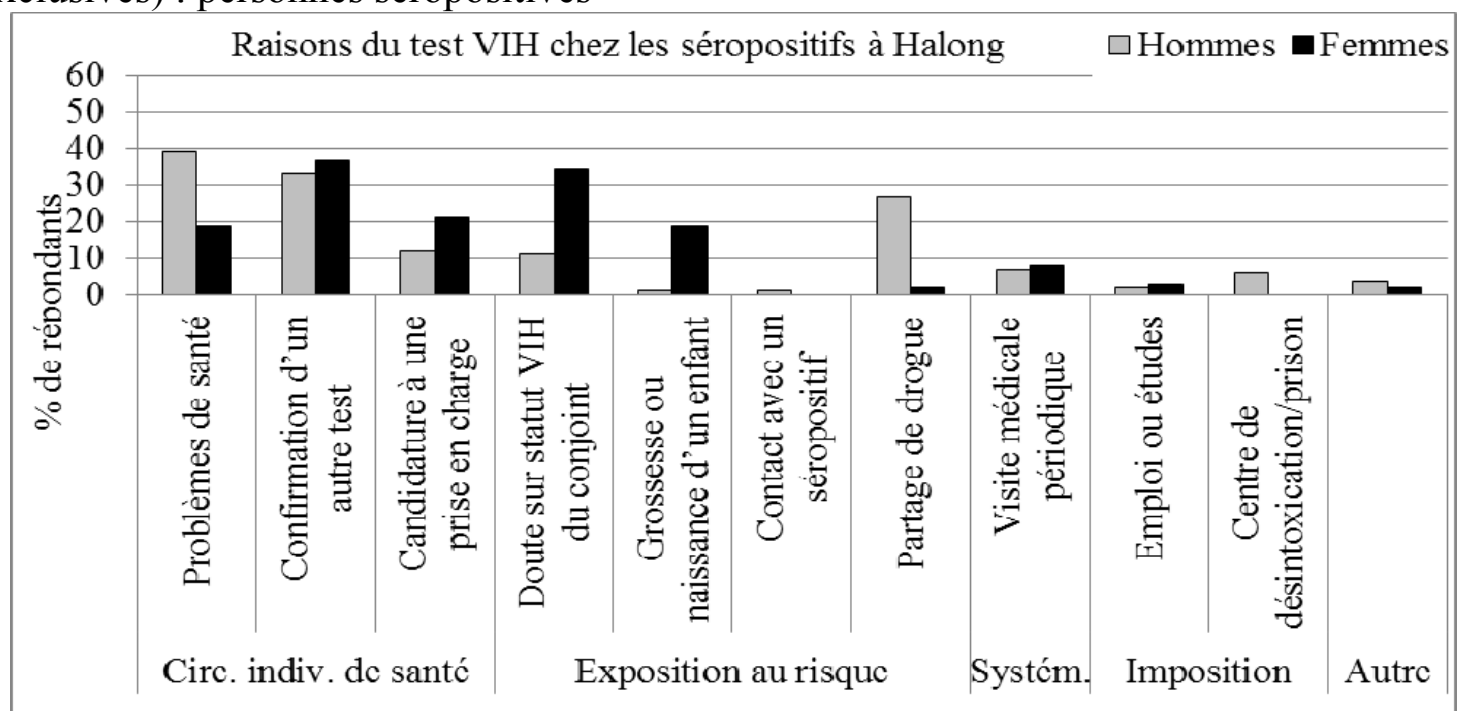

Raisons: Circonstances individuelles de santé, Exposition au risque, mesure systématique de santé, imposition non souhaitée par l'individu, autre

Source : enquêtes FAVIHVIET 2006 à Halong (questionnaires individuels)

À l'intérieur d'une même catégorie VIH, ces motivations diffèrent en effet selon le sexe. Les hommes citent plus souvent un problème de santé, notamment les séropositifs (39\% versus $19 \%)$ et les séronégatifs des ménages cas (18\% versus $0 \%)$. Ils citent plus que les femmes le partage de stupéfiant, notamment les séropositifs $(27 \%$ versus $2 \%)$ et les séronégatifs des ménages cas $(9 \%$ versus $0 \%)$. Parmi les membres des ménages témoins, les hommes mentionnent plus que les femmes l'accès à un emploi ou les études $(31 \%$ versus $9 \%)$. Un test réalisé à l'occasion d'un changement d'activité professionnelle, pour obtenir un poste ou pour leur service militaire, intervient lors du recrutement et de la libération par l'armée, moments de consultations médicales systématiques. À l'inverse, les femmes évoquent une grossesse ou la naissance d'un enfant, surtout au sein des ménages témoins ( $48 \%$ versus $0 \%)$. Cette raison a été donnée par quelques hommes dépistés après que leur épouse ait été diagnostiquée séropositive suite à une grossesse. Les femmes déclarent plus fréquemment que les hommes avoir fait le test en raison des doutes sur la séropositivité de leur conjoint, surtout chez les séronégatifs des ménages cas $(46 \%$ versus $0 \%)$ et en second lieu chez les séropositifs 
(34\% versus $11 \%$ ) avec un écart selon le sexe important. Les autres motivations ne marquent pas de clivage significatif selon le sexe.

Figure 4.2- Raisons du dernier test de dépistage effectué selon le statut VIH à Halong parmi les hommes et femmes ayant effectué au moins un test (modalités non exclusives) : personnes séronégatives membres de ménages affectés

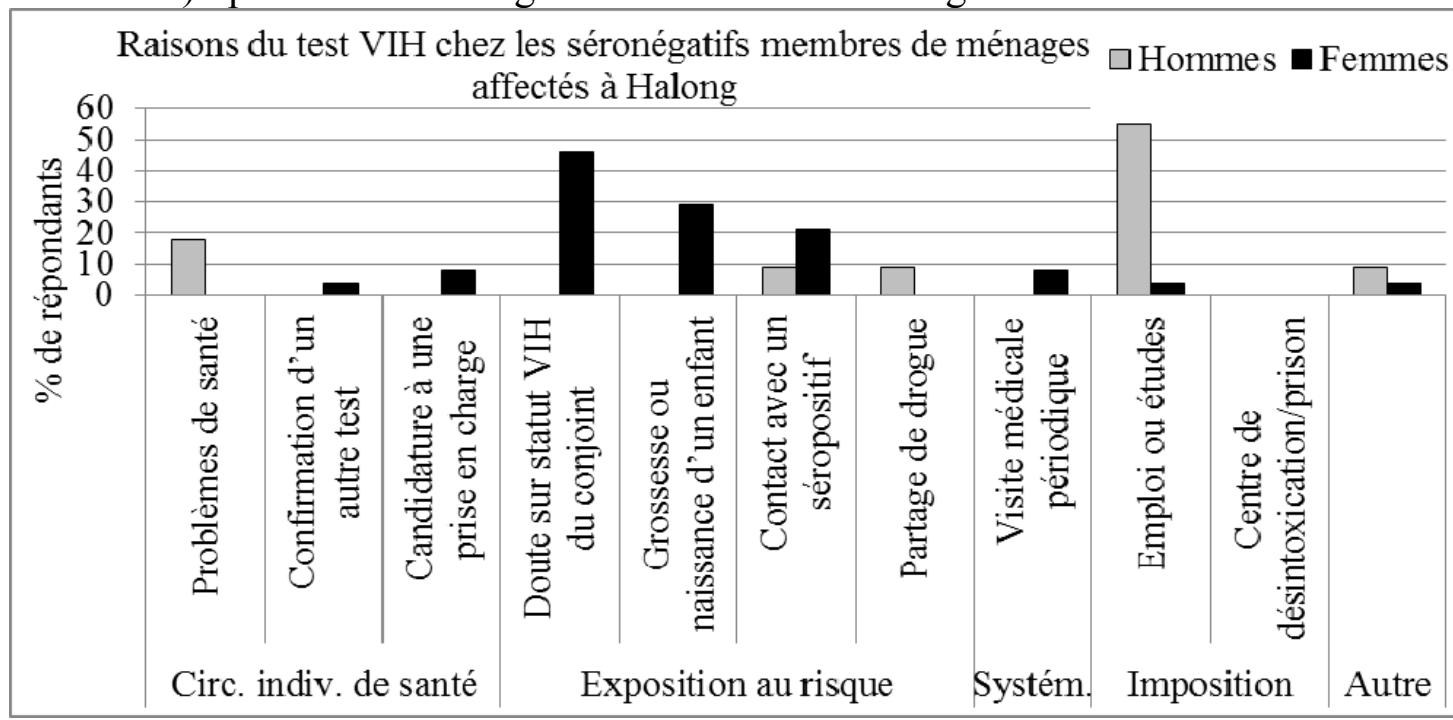

Raisons : Circonstances individuelles de santé, Exposition au risque, mesure systématique de santé, imposition non souhaitée par l'individu, autre

Source : enquêtes FAVIHVIET 2006 à Halong (questionnaires individuels)

Figure 4.3- Raisons du dernier test de dépistage effectué selon le statut VIH à Halong parmi les hommes et femmes ayant effectué au moins un test (modalités non exclusives) : membres de ménages témoins

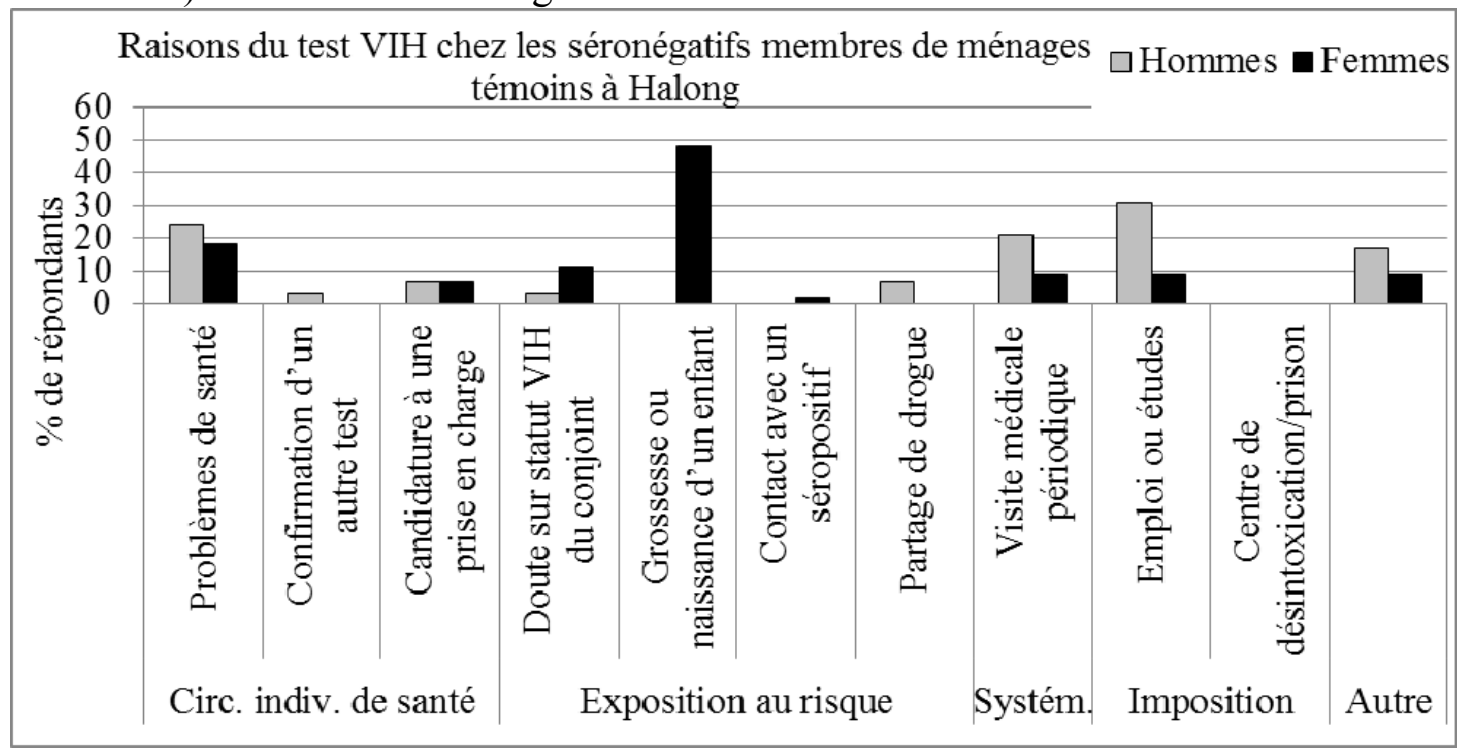

Raisons : Circonstances individuelles de santé, Exposition au risque, mesure systématique de santé, imposition non souhaitée par l'individu, autre

Source : enquêtes FAVIHVIET 2006 à Halong (questionnaires individuels)

Certaines raisons sont citées de façon équilibrée par les hommes et les femmes, notamment la confirmation d'un résultat antérieur pour les séropositifs ou une visite médicale périodique pour les membres des ménages témoins. Ces disparités ne changent pas les modalités de réalisation du test. En effet, la proportion de personnes 
accompagnées, le taux de présence de la famille et le type de personne accompagnant le patient ne varient pas de manière significative selon le sexe de la personne testée, une fois pris en compte le statut VIH.

\section{IV- Discussion}

L'utilisation de la méthode cas-témoin pour la constitution de l'échantillon ne vise pas une représentativité statistique. Cependant, quelques comparaisons peuvent être établies afin de situer les populations étudiées dans un environnement sociodémographique plus large. Sur le plan des pratiques de dépistage, les proportions de personnes testées dans les deux sites étudiés sont proches de celle de l'ensemble de la province de Quang Ninh : un quart des habitants en milieu urbain (24\%) et seulement un dixième (11\%) en milieu rural (General Statistical Office, National Institute of Hy et ORC Macro, 2006). En revanche elles sont élevées par rapport à l'ensemble du pays (5\% des adultes de 15 49 ans) selon l'enquête nationale Vietnam Population and AIDS Survey (VPAIS 2005). Les résultats d'enquêtes plus récentes donnent des résultats un peu plus élevés, mais toujours avec une différence importante entre milieu rural et milieu urbain. Ainsi, l'étude menée dans le district rural de Bavi au sein de l'observatoire de population donnait un pourcentage de personnes testées ajusté selon l'âge de 7,6\% en 2011 (Pharris et coll., 2011). Ce niveau s'explique en partie par la pauvreté et le caractère rural de la population étudiée, qui rend plus difficile l'accès aux infrastructures de dépistage. Des études réalisées en milieu urbain donnent des taux de dépistage plus élevés (Nguyen et coll., 2009). Certaines études préconisent un élargissement de l'accès au dépistage, en particulier en milieu rural (Nguyen et coll., 2007). Ces taux varient également selon la province, celle de Quang Ninh se situant à un niveau proche d'Hô Chi Minh-Ville et nettement plus faible que Hanoi (General Statistical Office, National Institute of Hy et ORC Macro, 2006).

La faible proportion de personnes testées est liée à l'orientation politique prise dans la lutte contre le VIH/sida, où l'accent a été mis, notamment dans le domaine du dépistage, sur des populations spécifiques considérées comme à fort risque de transmission du $\mathrm{VIH}$, telles que les personnes pratiquant une sexualité transactionnelle et celles qui s'injectent de la drogue par voie intraveineuse (Nguyen et coll., 2011). Or, ces populations sont fortement stigmatisées en partie en raison des campagnes de sensibilisation menées dans le cadre de la politique des fléaux sociaux à laquelle était rattachée la lutte contre le VIH/sida jusqu'en 2003. Pour ces raisons, on pourrait supposer que le fait de s'intéresser aux séropositifs au sein de leurs foyers pourrait entraîner une sous-représentation des contextes où les séropositifs sont isolés, ces derniers étant moins disponibles et probablement moins disposés à exposer leur situation. Cependant, à Halong quatre ménages (sur 170) étaient composés d'une personne séropositive seule ${ }^{49}$, ce qui montre que ces situations ont pu être prises en compte.

Notre étude met en évidence la pratique quasi exclusive du test à des fins diagnostiques plutôt que préventives. Cela pourrait être dû en partie à la stigmatisation qui limite les déclarations relatives à un soupçon de séropositivité. Ainsi, seules les personnes clairement concernées par une atteinte sont amenées à évoquer clairement leur pratique passée de test. Cependant, ces biais de déclaration n'expliquent

\footnotetext{
${ }^{49}$ Cette proportion de 2,4\% est supérieure à celle des ménages témoins comportant une seule personne au nombre de 7 sur 393 , soit $1,8 \%$ des ménages témoins.
} 
probablement pas à eux seuls ces résultats. La stigmatisation est également un frein au recours au test comme nous l'avons vu plus haut.

Les membres des foyers cas participant aux groupes de discussion font souvent partie de familles lourdement affectées par le VIH/sida. Certains pères ont eu plusieurs enfants séropositifs. Ceux-ci étaient parfois à la fois séropositifs et consommateurs de drogue. Beaucoup de mères sont engagées dans des associations de lutte contre le VIH/sida. Cette situation a probablement été favorisée par le mode de recrutement et de passation des discussions de groupe : nous avons travaillé avec des personnes acceptant de parler ouvertement des problèmes liés au VIH/sida interne à leur foyer, circonstances dans lesquelles le maintien de l'anonymat est presque impossible, étant donné le caractère retreint des communautés étudiées et la portée symbolique d'une atteinte par le VIH/sida. Par conséquent, les propos recueillis sont souvent ceux de personnes appartenant à des familles ayant une expérience relativement longue, une infection ancienne, donc des types de prise en charge qui font référence à des pratiques ne correspondant pas toujours aux préconisations actuelles, car l'évolution est rapide. Ceci n'exclut pas la participation de familles pour lesquelles la confrontation au VIH/sida est plus récente. Nous disposons ainsi d'un recul historique.

Près de deux ans séparent l'enquête effectuée à Halong en novembre 2006 de celle réalisée à Dông Triêu en août 2008. Étant donné la dynamique de l'épidémie et la mobilisation pour la lutte contre le $\mathrm{VIH} /$ sida, le contexte a évolué pendant cet intervalle. L'année 2006 a vu notamment le lancement de la loi relative à la lutte contre le VIH/sida. Ainsi, le compte-rendu des résultats tend à minimiser l'écart entre les deux milieux, puisque la décentralisation des soins a progressé entre les deux dates et le milieu rural, généralement défavorisé, bénéficie deux ans plus tard d'un dispositif institutionnel plus complet que précédemment. Cette situation est perceptible dans la prise en charge par antirétroviraux. Ceux qui y avaient accès paraissaient proportionnellement moins nombreux en 2006 à Halong qu'à Dông Triêu en 2008, ce qui présente une tendance inversée par rapport aux disparités habituelles entre milieux rural et urbain.

Il n'en est pas de même des associations de lutte contre le VIH/sida qui restaient en 2008 à Dông Triêu relativement peu présentes et peu visibles en comparaison avec Halong en 2006. Ainsi, l'analyse des deux sites doit tenir compte de l'évolution qui a eu lieu au cours de la période qui a séparé les deux enquêtes. Malgré cette contrainte, étant donné la mise en œuvre d'une méthodologie commune aux deux phases, le rapprochement des deux sites semble plus intéressant que leur étude disjointe. Ainsi, la plus forte fréquence observée à Dông Triêu pourrait s'expliquer par la période écoulée entre les deux collectes. Celle-ci pourrait avoir été marquée par un développement du dépistage, en particulier au sein des ménages cas.

Ce chapitre approfondit une période particulière de la lutte contre le VIH/sida. Elle permet de mieux connaître la phase d'adoption par le Viêt Nam de normes standardisées de prise en charge, par le biais de la mise en œuvre de programmes internationaux. La période d'observation concerne en effet les années 2006 à 2008. Elle est caractérisée par un certain retard du Viêt Nam dans l'accès au dépistage, comme le montrent les taux élevés de tests positifs, la place importante des symptômes dans les raisons du recours au test et la place de la maladie dans la démarche de dépistage.

L'étude permet d'approfondir les circonstances du dépistage, l'implication de la famille dans ce processus et les évolutions qui ont eu lieu. Elle montre l'influence de la famille dans le processus de décision relatif au dépistage ainsi que lors de sa réalisation. En effet, comme nous l'avons vu, certaines familles ont pu aller jusqu'à imposer un dépistage voire à se substituer à la personne testée pour lancer un test et en recevoir le 
résultat. Ces pratiques tendent à devenir plus rares avec l'adoption de règles internationales. En effet, depuis le début des années 2000, l'évolution des procédures se développe en faveur d'un plus grand respect de la confidentialité des tests. Ces changements conduisent progressivement à une modification de la place et du rôle de la famille qui, de pourvoyeuse essentielle de ressources aux séropositifs et agent de contrôle, prend davantage le rôle d'une accompagnatrice, mettant un peu plus en avant les valeurs de d'autonomie individuelle.

Les déclarations relatives à la personne qui a décidé de réaliser le dépistage sont spécifiques à la période étudiée. En effet, depuis la loi de 2006, le test VIH ne peut pas être imposé (article 8 alinéa 7, loi sur le VIH/sida 2006) à l'exception de quelques contextes spécifiques pour lesquels le test est gratuit. Cela intervient dans trois circonstances : pour une expertise légale, une enquête auprès de la population ou sur décision d'un tribunal populaire, pour les besoins de diagnostic et de prise en charge sur décision du Ministère de la Santé, pour la surveillance de certaines professions sur décision du gouvernement (article 28). En dehors de ces cas spécifiques, la loi stipule que le test est fondé sur le volontariat et suppose le consentement éclairé de la personne testée. Celle-ci doit être âgée d'au moins 16 ans ou obtenir l'accord de son parent ou tuteur (article 27). Le test ou le résultat d'un test ne peut pas être demandé par un employeur (article 14) ni un établissement éducatif (article 15). Les motivations relatives à l'employeur ou les études devraient donc ne plus être présentes dans les enquêtes postérieures. Le non-respect de ces procédures a cependant été observé dans certains services de consultation prénatale. Ainsi, dans un hôpital de la capitale Hanoi, moins de la moitié des femmes enceintes savaient qu'elles avaient été testées pour le VIH, et parmi elles une minorité recevait un conseil pré ou post-test (Nguyen et coll., 2009). Le dépistage au Viêt Nam a été longtemps accompagné de non-respect de la confidentialité des résultats du test, parfois communiqués à la famille ou à l'entourage du patient (Khuat, 2007). Ces pratiques, également observées dans le cadre de notre enquête, constituent un frein au dépistage.

Peu d'études ont été réalisées sur les différences entre hommes et femmes en matière de pratique du dépistage VIH dans la population générale. Une revue de la littérature réalisée sur la situation des femmes en regard de l'infection VIH/sida au Viêt Nam, portant sur des documents antérieurs à 2005, aboutit à la nécessité d'améliorer la sensibilisation et l'accès au dépistage des femmes non considérées à haut risque de transmission du VIH/sida, malgré la faible rentabilité économique d'une telle mesure (Nguyen et coll., 2008). Elle conclut également au besoin de la mise en place de procédures fiables et systématiques de dépistage dans les services prénataux (Nguyen et coll., 2008). Dans notre étude, les raisons du test sont cohérentes avec le fait que les femmes (séropositives ou non) ont davantage d'occasions que les hommes de faire le test avant que ne survienne un problème de santé grave lié au VIH. Les femmes sont entre autres soumises à un dépistage systématique pendant les visites prénatales. Cette différence entre hommes et femmes, conduisant à un accès plus précoce des femmes, intervient dans le contexte d'une épidémie où la population séropositive est majoritairement masculine, et où le recours au dépistage n'est pas toujours plus intense chez les femmes (Nguyen et coll., 2011). Les résultats de notre étude sont également cohérents avec ceux d'une étude plus récente portant sur la situation des femmes dans un contexte de VIH, montrant une plus forte activité économique de celles-ci contrairement aux hommes (United Nations Development Programme et Regional Centre in Bangkok, 2011).

L'implication de la famille dans la prévention de la transmission et la prise en charge des personnes séropositives pose le problème du caractère individuel et collectif des 
interventions et modèles de prise en charge. L'approche individualiste, mise en avant dans le cadre de la santé de la reproduction depuis les années 1980 avec la conférence de Mexico sur la population et le développement puis celle du Caire (Levy, 1984; Pelchat, 1995), a été reprise dans de nombreuses campagnes de lutte contre le VIH/sida. Elle comporte des limites dans un pays où les individus mettent en avant quatre niveaux sociologiques dans la vie quotidienne : l'individu, la maison ou famille, la communauté et l'ensemble de la société. Les représentations sont perceptibles dans certains messages diffusés après le détachement de la lutte contre le VIH/sida d'avec la politique des fléaux sociaux. En effet, ceux-ci mettent l'accent sur la nécessité pour la famille et la communauté d'aider les personnes séropositives. L'individu et la société sont impliqués dans la prévention tandis que la communauté intervient dans le support aux séropositifs, la famille étant mobilisée dans ces différents aspects. Par ailleurs, l'individualisme de la prise en charge se renforce. Les consultations ambulatoires à l'attention des séropositifs, au début organisées et tenues de façon collective, accentuent progressivement la personnalisation des soins et du suivi (Loenzien, 2009b).

\section{Conclusion}

Les résultats de notre étude ainsi que les données relatives à l'épidémie VIH fournissent de multiples signes du retard du Viêt Nam dans le domaine du dépistage au cours des années 2000. Le fort taux de prévalence des séropositifs est significatif du fait que les personnes qui viennent faire le test ont une santé déjà dégradée. Ce fait est confirmé par la place importante des symptômes dans les raisons du recours et la présence de maladies dans les démarches de dépistage telles qu'elles ont été décrites dans les entretiens. Pour remédier à ces insuffisances, le Viêt Nam a engagé des transformations rapides. Ainsi, dans un contexte de faible prévalence et d'épidémie concentrée, la lutte contre le VIH/sida a été marquée par une ouverture vers des approches plus participatives. Celles-ci ont été caractérisées par un appel à la famille et à la communauté pour l'aide aux séropositifs et la prévention de la transmission, ainsi qu'une interpellation des individus comme acteurs dont la volonté est prise en compte. Ces changements se sont produits dans un contexte de restructuration des infrastructures de santé. Celles-ci ont dû en effet faire face à une diminution des moyens financiers alloués et mettre en place des procédures de recouvrement des soins de plus en plus exigeantes. Dans le domaine du VIH/sida, les services de prise en charge ambulatoire se développent. Les changements récents confirment cette tendance, avec la fermeture des camps de rééducation pour les consommateurs de drogue et les travailleuses du sexe en 2011 ; le lancement de programmes de réduction des dommages prévoyant le recours à des produits de substitution tels que la méthadone depuis 2008 ; le développement des associations et groupes d'aides aux séropositifs. Les droits des patients, leur volonté et leur parole sont davantage pris en compte. Ces évolutions font progressivement passer le VIH/sida d'une crise sanitaire aigüe à un problème chronique multisectoriel. À terme, l'individualisation des traitements laisse entrevoir une baisse de l'investissement familial dans les procédures de dépistage, et plus largement dans la prise en charge des séropositifs. Le milieu des années 2000 est donc marqué par un investissement particulièrement intense de la famille par rapport aux périodes qui le précèdent, mais sans doute aussi à celles qui le suivent. 


\section{Bibliographie}

BÉLANGER Danièle, Barbiéri Magali, "Introduction: The State and families in the making of transitions", in Reconfiguring Families in Contemporary Vietnam, BÉLANGER Danièle et BARBIERI Magali (dir.), Stanford, Stanford University Press, 2009, 1-44.

BERESFORD Melanie, "Doi Moi in review: The challenges of building market socialism in Vietnam", in Journal of Contemporary Asia, vol. 38, n², London, Routledge 2008, p. 221-243.

Central Population and Housing Census Steering Committee, The 2009 Vietnam population and housing census: completed results, Hanoi, Statistical Publishing House, 2010, 893 p.

General Statistical Office, National Institute of Hy, ORC Macro, Vietnam Population and AIDS Indicator Survey 2005. Hanoi, Calverton, Maryland 2006, 94 p. + appendices.

Global AIDS Policy COALITION, AIDS in the world, II: global dimensions, social roots, and responses, New York, Oxford University Press, 1996, p. 648.

HOANG Tu-Anh, "Civil society organizations' roles in health development in Vietnam: HIV as a case study" in Global Public Health: An International Journal for Research, Policy and Practice, 8, 2013, pp. S92-S103.

Hoang, T.N., Nguyen, M.N., Tran, D.T., Chong, P.S., Kamb, M.L., LuU, C.M., Vietnam Ministry of Health - LIFE/GAP Office, Hanoi, Vietnam, CDC/Global AIDS Program - Vietnam, Hanoi, "Vietnam establishes a national program on Voluntary Counseling and Testing (VCT) for high-risk persons: Preliminary results",. in $X V$ International AIDS Conference, Bangkok, abstract n ${ }^{\circ} \mathrm{WePeE6810,2004,1} \mathrm{p}$.

INDEPENDENT COMMISSION ON AIDS IN ASIA, Redefining AIDS in Asia: crafting an effective response: report of the Commission on AIDS in Asia. Oxford University Press, New Delhi, 2008, 230 p. + appendices.

KhUAT Thi Hai Oanh, HIV/AIDS Policy in Vietnam. À Civil Society Perspective, Public Health Watch Monitoring reports, Open Society Institute, New York, 2007, 66 p.

KLEINEN John, «La comédie de l'État-parti. Le Viêt Nam depuis la réunification », in Raisons Politiques, $\mathrm{n}^{\circ}$ 3, 2001, p. 37.

KNODEL John, "The Changing Impact of the AIDS Epidemic on Older-Age Parents in the Era of ART: Evidence from Thailand", in Journal of Cross-Cultural Gerontology, n 1/27, mars 2011, p. 1-15.

LEVY Michel Louis, "La conférence de Mexico sur la population » in Population et Sociétés, n¹84, INED, Paris, 1984, pp. 1-4.

Li Li, Ji Guoping, Liang Li-Jung, Ding Yingying, TIAN Junru, XIAO Yongkang, “A multilevel intervention for HIV-affected families in China: Together for Empowerment Activities (TEA)", in Social Science and Medicine, $\mathrm{n}^{\circ} 8 / 73$, octobre 2011, p. 1214-1221.

Li Li, Wu Sheng, Wu Zunyou, Sun Stéphanie, CUI Haixia, JiA Manhong, "Understanding Family Support for People Living with HIV/AIDS in Yunnan, China", in AIDS and Behavior, n¹0, 2006, p. 509-517. 
LOENZIEN Myriam de, "Family: the cornerstone of the current fight against HIV/AIDS epidemic in Vietnam", in Reconfiguring Families in Contemporary Vietnam, Contemporary Issues in Asia and the Pacific, BARBIERI Magali et BÉLANGER Danièle (dir.), Stanford, Stanford university press, 2009a, p. 97-132.

LOENZIEN Myriam de, «Institutionnalisation d'un continuum de prise en charge des séropositifs VIH/sida à Hanoi (Viêt-Nam) », in Cahiers Santé vol. 19, n³, 2009b, p. $141-148$.

LOENZIEN Myriam de, «Relations de genre et participation de la famille à la prise en charge du VIH/sida: Etude socio-démographique auprès des premiers patients sous antirétroviraux à Hanoi (Viêt Nam) », in Famille et Santé, GENEVIEVE Cresson et Mohamed Mebtoul (dir.), Rennes, Presses de l'École des Hautes Études en Santé Publique, 2010. p. 127-142.

LOENZIEN Myriam de, "Knowing HIV/AIDS epidemic through billboards: awareness campaigns in Vietnam", Oral communication presented at the 2nd International HIV Social Sciences and Humanities Conference, Paris, 2013, 15 diapositives.

LuONG Hy Van, "Vietnamese Kinship: Structural Principles and the Socialist Transformation in Northern Vietnam", in Journal of Asian Studies, n 48, 1989, p. 741.

LUU Bich Ngoc, La famille vietnamienne face au VIH/sida: connaissances, stigmatisation et exclusion. Études de cas-témoin à Ha-Long, province de Quang Ninh, Viêt Nam, Thèse de Doctorat en Démographie, Université Paris Ouest Nanterre la Défense, CERPOS, Nanterre, 20 juillet 2010, 1 vol., 409 p. +69 p. annexes (dactyl.).

MCNAlly Stephen Peter, HIV in Contemporary Vietnam. An anthropology of development, Canberra, The Australian National University, 2002, 279 p.

MiLler Riva, Murray Durval, "The impact of HIV illness on parents and children, with particular reference to African families", in Journal of Family Therapy, $\mathrm{n}^{\circ} 21,1999$, p. 284-302.

Ministry of Health, Socialist RePublic of Vietnam, Voluntary HIV counseling and testing (VCT) guidelines, promulgated at the Decision $n^{\circ} 647 / \mathrm{QD}-\mathrm{BYT}$ dated on February 22, 2007 by the Ministry of Health, Hanoi, 2007, 10 p.

Ministry OF PlanNING AND INVESTMENT, Viet Nam continues to achieve the millennium development goals, Hanoi, Ministry of Planning and Investment, 2008, p. 84. + appendices.

MoOre Ami R., Henry Doug, "Experiences of older informal caregivers to people with HIV/AIDS in Lomé, Togo", in Ageing International, n³ 30, 2005, p. 147-166.

NATIONAL COMMITTEE FOR AIDS, DRUGS AND PROSTITUTION PREVENTION AND CONTROL, Vietnam AIDS response progress report 2012. Following up the 2011 political declaration on HIV/AIDS, Hanoi, 2012, 175 p.

NguYen Hai Thuong, BhUIYAN Shafi Ullah, NANTHAMONGKOlCHAI Sutham, "Determinants Related to the Utilization of Voluntary Counselling and HIV Testing Service in Vietnam", in Journal of Public Health, n 5, 2007, p. 64.

NGuYen Lan T.N., Christoffersen Sarah V., RAsch Vibeke, "Uptake of Prenatal HIV Testing in Hai Phong Province, Vietnam, in Asia Pacific", Journal of Public Health, $\mathrm{n}^{\circ} 22,2010$, p. 451-459. 
NGuYen Thi Mai, Implementation Status \& Results Vietnam HIV/AIDS Prevention (P082604) sequence 12 (Public disclosure copy No.ISR11485), The World Bank, Washington D.C., 2013, 6 p.

Nguyen Thi Thu Hong, Wolfe Mitchell I., Tran Tien Dat, McFarland Deborah A., Kamb Mary L., Nguyen Trong Thang, HoAng Nam Thai, Del Rio Carlos, "Utilization of HIV Voluntary Counseling and Testing in Vietnam: An Evaluation of 5 Years of Routine Program Data for National Response", in AIDS Education and Prevention, $\mathrm{n}^{\circ} 23,2011$, p. 30-48.

Nguyen Thu Anh, Oosterhoff Pauline, Hardon Anita, Tran Hien, Coutinho Roel A., WRIGHT Pamela, "A hidden HIV epidemic among women in Vietnam", in BMC Public Health, $\mathrm{n}^{\circ}$ 8, 2008, p. 37.

Nguyen Thu Anh, Oosterhoff Pauline, Pham Ngoc Yen, Wright Pamela, Hardon Anita, NguYen Tran Hien, COUTINHO Roel A., "Availability and accessibility of HIV counselling and testing services fo pregnant women in Hanoi, Vietnam", in Asian Journal of Medical Sciences, $\mathrm{n}^{\circ} 1,2009$, p. 1-11.

Organisation Mondiale De la SANTe, ONUSIDA, Guide du conseil et du dépistage du VIH à l'initiative du soignant dans les établissements de santé, Genève, 2007, 56 p.

Pelchat Yolande, «La Conférence internationale sur la population et le développement du Caire: un parti pris pour les femmes ?», in Recherches Féministes, n8, 1995, p.155.

PEPFAR, Partnership to fight HIV/AIDS in Vietnam, PEPFAR, 2010, 1 p. [En ligne, http://www.pepfar.gov/countries/vietnam, consulté le 7 novembre 2014]

Pharris Anastasia, Nguyen Thi Kim Chuc, Tishelman Carol, Brugha Ruairi, NguYen Phuong Hoa, ThORson Anna, "Expanding HIV Testing Efforts in Concentrated Epidemic Settings: A Population-Based Survey from Rural Vietnam”, in PLOS One 6, e16017, 2011. [En ligne, consulté le 8 novembre 2014]

Proschan Franck, "Syphilis, Opiomania, and Pederasty": Colonial Constructions of Vietnamese (and French) Social Diseases, in Journal of the History of Sexuality, 11, 2002, p. 610-636.

Socialist Republic of Vietnam, Vietnam Country Progress Report. Monitoring the Progress towards the Implementation of the Declaration of the UNGASS Commitment on HIV/AIDS, Hanoi, 2010, 43 p. +108 p. appendices.

UNAIDS, Annual report 2009, Genève, Suisse, 2010, 77 p. +5 p. appendices.

UNAIDS, UNAIDS Data Tables 2011, UNAIDS, Genève, Suisse, 2011, 9 p.

UNDP, AUSAID, Impact of HIV/AIDS on household vulnerability and poverty in Viet Nam, Hanoi, 2005,40 p. +32 p. appendices.

United Nations Development Programme, Regional Centre in Bangkok, The socio-economic impact of HIV at the household level in Asia. HIV, Health and Development Programme for Asia and the Pacific, UNDP Asia-Pacific Regional Centre, Bangkok, 2011, 41 p.

Viet Nam COMmission for Population, FAmily and Children, Ministry of Health, Population Reference Bureau, HIV/AIDS in Viet Nam, The current situation, the national response, the emerging challenges, Hanoi, 2006, $55 \mathrm{p}$. 
VU, Minh Quan, HIEN, Nguyen Tran, Go, Vivian F., The HIV epidemic in Vietnam: past, present, and opportunities, in: Public Health Aspects of HIV/AIDS in Low and Middle Income Countries, Springer, 2008. p. 457-479.

Werner Jane, BÉlanger Danièle, "Gender, Household, and State: Renovation (Dôi Moi) as Social Process in Viêt Nam", in Gender, Household, State : Dôi Moi in Viêt Nam, Southeast Asia Program, Werner Jane et BÉLANGer Danièle, New York, 2006, p. 29-47.

WILLIAMS Nathalie, KNODEL John, KIRY KIM Sovan, PUCH Sina, SAENGTIENCHAI Chanpen, "Overlooked potential: older-age parents in the era of ART" in AIDS Care, 20, 2008, p.1169-1176.

ZIMMER Zachary, DAYTON Julia, " The living arrangements of older adults in subsaharan Africa in a time of HIV/AIDS », Working paper, Population Council, New York, No. 169, 2003, 34 p. 


\title{
Aides reçues et besoins d'aide des enfants séropositifs et de leur famille
}

\author{
Nguyên Thị Thiêng (UNE IPSS) \\ Bùi Thi Hanh (UNE IPSS) \\ France Lert (INSERM CESP U1018) \\ et l'équipe du projet « Enfants séropositifs au Viêt Nam » ${ }^{50}$
}

Cet article a pour but de présenter les difficultés des enfants séropositifs et de leurs familles dans la vie quotidienne tant du point de vue matériel que psychologique et de décrire les besoins d'aide prioritaires pour les familles concernées.

\section{I- Contexte et objectifs de la recherche}

Depuis la mise en place de la prévention de la transmission périnatale, les enfants infectés à la naissance ou pendant l'allaitement naissent surtout dans les pays à ressources faibles ou moyennes. En 2009, le Ministère de la Santé estimait que le Viêt Nam comptait environ 4.720 enfants de moins de 15 ans porteurs de l'infection à VIH avec une projection à 5700 pour l'année 2012 (Département de prévention du VIH/sida, 2009). Parmi ces enfants, moins de 1.500 (31\%) étaient alors sous traitement antirétroviral (ART) (UNICEF Viêt Nam 2010) Depuis 2008, l'aide internationale a permis l'ouverture de centres de soins spécialisés pour le traitement des adultes et des enfants avec la gratuité des médicaments, des consultations et d'une partie des examens complémentaires. L'accent a été mis sur la mise en place des programmes de prévention et l'accès au traitement pour les femmes enceintes (OMS 2010). Pour les enfants jusqu'à 6 mois, le lait maternisé est aussi fourni aux familles. Certaines organisations internationales au Viêt Nam telles que CARE International, Pact et UNICEF apportent des aides sociales.

Dans la littérature, l'accent a été mis au premier chef sur le devenir des enfants nés de mère séropositive, en termes de morbidité et de mortalité (KIDS-ART-LINC Collaboration, 2008) et plus rarement en termes de développement (Newell 2003, Hazra 2010). Les problèmes de développement et de conditions de vie ont été abordés sous l'angle de la pauvreté ou des besoins des enfants orphelins (Schenk, 2009). Actuellement, les travaux de recherche sur les difficultés et les besoins d'aide des familles des enfants séropositifs ne sont pas nombreux (Steele 2007, Yanagisawa 2010). Nous n'avons pu collecter que très peu de documents en lien avec ce sujet au Viêt Nam. Selon le docteur Nguyễn Trong An, chef adjoint du Département de la protection et des soins de la santé des enfants (Ministère du Travail, des Invalides de guerre et des Affaires sociales), la moitié des enfants séropositifs vivent dans des familles pauvres, et quelques 2,1 \% dans des familles très pauvres (Trân 2011). Une étude menée dans la ville de Halong en 2007 sur les conditions de vie des enfants séropositifs ou vivant dans

\footnotetext{
${ }^{50}$ L'équipe était composée de Luu Bich Ngoc, Bùi Duc Kinh, Lê Thi My, Nguyên Thi Minh Châu, Ha Thuc Dung, Lê The Vung et Dao Quang Binh. Nous tenons à remercier l'ANRS pour son soutien financier. Nous tenons à exprimer notre reconnaissance aux médecins et infirmières des hôpitaux pédiatriques de Hanoi et de Hô Chi Minh-Ville ainsi qu'à ceux de la clinique An Hoa pour leur aide au quotidien pendant la collecte des données; enfin et surtout aux personnes qui s'occupent des enfants atteints par la maladie VIH et qui ont accepté les questionnaires et les entretiens dans une vie souvent très difficile.
} 
une famille dont un membre est atteint d'infection VIH met en évidence le manque de ressources pour la scolarité des enfants, puisque au Viêt Nam les dépenses scolaires comprennent non seulement les frais de scolarité selon la réglementation du Ministère de l'Éducation et de la Formation, mais aussi d'autres frais, telle que la participation à la construction de l'école ou encore des cotisations pour la caisse des parents par exemple. Les attitudes discriminatoires de la part de parents d'autres enfants empêchent certains enfants d'aller à l'école (Nguyên 2007). Ainsi, « nombre de ces enfants sont privés de droits à l'éducation et aux loisirs, obligés d'interrompre l'école et de travailler avant l'âge » (Nguyên 2005). Les besoins d'aide concernent, en priorité et avec le même poids pour chacun, l'éducation, l'alimentation, les soins médicaux et le traitement (Nguyên $2007)^{51}$.

L'étude portant sur le «Besoin d'intégration des enfants séropositifs au centre Mai Hòa, avril 2008 » de Pham Hang Hà et Pham Thi Lan Anh (2010) cherche à rassembler les besoins et souhaits exprimés par les enfants séropositifs. Les attentes sont de vivre en famille, d'aller à l'école, de jouer avec d'autres enfants de leur âge, d'être en bonne santé et de recevoir un traitement. D'autres travaux montrent que la stigmatisation et la discrimination à l'égard des séropositifs ont empêché leur accès aux soins et au traitement, avec des impacts directs sur la vie matérielle et psychologique des enfants et des familles concernées par la séropositivité. Certains orphelins doivent se débrouiller seuls pour gagner leur vie, notamment en ville avec des risques de marginalisation. Certains enfants séropositifs ne sont pas acceptés à l'école. D'autres sont abandonnés par les familles et confiés à des orphelinats spécialisés avec de bonnes conditions médicales, mais où ils sont alors exposés aux risques de traumatismes psychologiques et à des difficultés ultérieures d'intégration sociale (Nguyên 2005, Nguyên 2007). Ainsi, au Viêt Nam, bien qu'il existe peu de recherches sur les difficultés et besoins d'aide des enfants séropositifs et de leur famille, les résultats des travaux cités plus haut montrent que ces enfants et leur famille ont besoin d'aide sur le plan psychologique, dans l'éducation, dans les soins de la santé, dans la communication avec d'autres enfants et pour les besoins de base comme l'alimentation.

La recherche que nous ${ }^{52}$ avons réalisée avait pour objectif de décrire de façon précise la situation sociale des familles et leurs besoins afin de compléter les programmes de soins d'un volet social garantissant de bonnes conditions pour le développement de l'enfant. Elle s'intéressait à un large éventail des difficultés de la vie des familles concernées par le VIH telles que la stigmatisation, la discrimination dans la famille ou à l'école, l'influence des facteurs socio-économiques sur l'accès aux soins médicaux, notamment dans la phase précédant le diagnostic, les facteurs influençant l'adhésion au traitement et la régularité du suivi médical. L'identification de ces situations critiques est complétée par la perception et la hiérarchisation par les parents ou gardiens de leurs besoins d'aide pour améliorer la vie quotidienne et le développement de l'enfant.

Quelles sont les difficultés matérielles et psychologiques auxquelles doivent faire face les familles prenant soin des enfants séropositifs? Quelle est la difficulté majeure ? Quelles sont les priorités dans les besoins d'aide exprimés par les familles ? Afin de répondre à ces questions, nous nous basons sur les hypothèses suivantes :

\footnotetext{
${ }^{52}$ Cette recherche a été réalisée dans le cadre de la coopération entre l'Agence Nationale de Recherche sur le Sida et les hépatites (ANRS), l'hôpital pédiatrique 1, l'hôpital pédiatrique 2, le centre de soins An Hòa et l'hôpital pédiatrique central de Hanoi. L'Institut Population et Société de l'Université nationale d'Économie (IPSS NEU) et l'Institut du Développement durable du sud (SISD) de l'Académie des Sciences sociales du Viêt Nam sont les deux établissements chargés de mener la recherche sur le volet social.
} 
- $\quad$ La plupart des enfants séropositifs naissent dans des familles pauvres, avec des conditions de vie difficiles. Ainsi, ces besoins matériels sont les plus impératifs pour les familles.

- La stigmatisation constitue la principale barrière à l'accès aux soins de santé et à l'éducation. Pour permettre cet accès, les familles cherchent à échapper à la stigmatisation en choisissant de garder le secret sur la séropositivité des enfants et de les faire prendre en charge dans des établissements médicaux situés loin de leur lieu de résidence.

Les résultats mettent au premier plan les difficultés financières et les besoins psychologiques. Les familles expriment au premier chef un besoin d'aide financière puis des besoins de traitement. L'absence de relais pour prendre soin de l'enfant dans le quotidien, mais aussi dans l'avenir constitue une préoccupation majeure des familles. L'inquiétude et la tristesse concernent plus de la moitié des parents ou gardiens, et sont plus marquées chez les parents, surtout les mères.

\section{II- Méthode de recherche}

Cette recherche utilise des données quantitatives et qualitatives. Les premières permettent de décrire les difficultés et besoins d'aides essentiels et de les classer par ordre de priorité. Les secondes apportent un éclairage sur l'expérience et la détresse des familles face à la maladie.

L'enquête porte sur les paires enfant-parent ou gardien, puisque l'étude porte sur la santé et les besoins de l'enfant, mais aussi de la personne qui prend soin de lui au quotidien et plus largement de la famille dans laquelle il vit. L'accompagnant était invité à participer à l'étude si il ou elle s'occupait de l'enfant au quotidien ou au moins plusieurs fois par semaine. Pour respecter un aléa dans la construction de l'échantillon fixé à 700 a priori compte tenu de l'inconnue sur le taux de participation avant l'étude (hypothèse de $70 \%$ ) pour une population d'un millier d'enfants suivis dans les centres de l'étude, la participation était proposée aux accompagnants lors de leur venue pour la consultation des enfants dont l'infection VIH était confirmée. L'invitation à participer intervenait lors de la consultation correspondant aux dates anniversaires de la première consultation dans le centre (soit 12, 24, 36 mois, etc., avec un délai de plus ou moins deux mois). Les enfants récemment diagnostiqués étaient définis par un diagnostic de moins de 3 mois. Cette stratégie a permis de constituer une population d'étude qui respecte la structure d'ancienneté de la file active de chaque service. Finalement, les refus de participer ont été exceptionnels.

Les populations étudiées se distinguent selon plusieurs critères :

(1) Les sites sont situés à Hanoi et Hô Chi Minh-Ville (voir Carte 1 en tête d'ouvrage). Lors de l'enquête, les soins pédiatriques de l'infection VIH n'étaient accessibles que dans quelques grandes agglomérations du pays disposant d'un bon niveau de qualité de soins et la gratuité, obligeant une partie des familles affectées à effectuer des déplacements longs, coûteux et inconfortables, en particulier avec de très jeunes enfants.

(2) Le type de ménage combine la présence des parents au sein du foyer et d'autres adultes selon qu'il s'agit d'adultes jeunes ou d'adultes de plus de 60 ans. Les catégories distinguent les situations dans lesquelles l'enfant vit: 1) avec ses seuls parents ; 2) dans une famille d'adultes jeunes avec ou sans ses parents) ; 3) avec seulement des personnes de plus de 60 ans qui ne sont pas ses parents ; 4) dans un foyer multigénérationnel. 
(3) La charge assumée auprès de l'enfant définit trois catégories : 1) père ou mère ; 2) grand-mère ou grand-père du côté maternel ou paternel. L'analyse montre en effet qu'il n'y a pas d'association particulière selon que l'enfant est pris en charge du côté maternel ou paternel, comme on aurait pu le penser dans une culture de famille patrilinéaire. 3) Le gardien qui n'est ni un parent ni un grand-parent (très rarement des personnes sans lien de parenté).

(4) La notion de discrimination ou stigmatisation a été définie par au moins une réponse positive aux modalités évoquant un acte discriminatoire ou la crainte de la discrimination : par exemple un retard dans le diagnostic motivé par « la crainte qu'on sache que l'enfant est positif», une « expérience de « stigmatisation dans la famille » ou encore une non-fréquentation scolaire, car «l'enfant est stigmatisé par les enfants de la classe/les parents ». Le questionnaire comportait des questions sur les pratiques séparant les objets utilisés par l'enfant de ceux de la famille, pratiques qui auraient signalé des craintes de transmission ou des pratiques de mise à l'écart de l'enfant. Cependant, il apparait que pour les familles vietnamiennes, ces pratiques relèvent plutôt de l'hygiène courante pour les très jeunes enfants et il a été décidé de ne pas les retenir comme indicateur d'attitudes discriminatoires.

La passation des questionnaires et des entretiens avait lieu dans les services de soins dans une pièce fermée afin de préserver la confidentialité et l'intimité, compte tenu des émotions exprimées par les personnes interrogées se remémorant des moments difficiles. Les parents ou gardiens de l'enfant ont été interrogés sur les difficultés rencontrées pour prendre soin de l'enfant, les émotions ou difficultés psychologiques en lien avec la maladie de l'enfant, les aides reçues, les besoins supplémentaires persistants. Les besoins sont comparés par ville, par type de famille, selon le sexe du répondant, sa relation avec l'enfant séropositif et selon l'expérience de la stigmatisation.

\section{III- Résultats}

Les résultats concernent les caractéristiques des participants, les difficultés rencontrées et besoins exprimés ainsi que les aides reçues.

\section{III.1- Caractéristiques des répondants}

Au total, 699 personnes parents ou gardiens des enfants séropositifs ont participé à l'enquête par questionnaire : $605(86,6 \%)$ enfants étaient suivis depuis 12 mois ou davantage, et $94(13,4 \%)$ depuis moins de trois mois au moment de l'interview. Les enfants étaient âgés d'un peu plus de 6 ans (médiane 68 mois, intervalle interquartile 41-89 mois). Ils se répartissent en 499 enfants à Hô Chi Minh-Ville et 200 à Hanoi reflétant la taille des files actives respectives des hôpitaux de ces deux métropoles. Parmi les enfants de l'échantillon, 163 (23,4 \%) vivent sans leurs parents biologiques, $267(38,3 \%)$ vivent avec un seul de leurs parents, $268(38,4 \%)$ vivent avec leurs deux parents, ce qui est très faible pour des enfants aussi jeunes. Lorsque l'enfant vit sans ses parents ou seulement avec l'un des parents, il s'agit d'orphelins des deux parents dans $12 \%$ des cas, d'orphelins de père $(35,8 \%)$ ou de mère $(9,9 \%)$, pour les autres. Dans $17,8 \%$ des cas, le parent travaille loin du foyer et pour $15,4 \%$ le parent est absent sans donner de nouvelles.

Parmi les accompagnants, la majorité des répondants sont des femmes (79,5\%). Quant au lien avec l'enfant, les deux tiers des répondants (466) sont le père ou la mère, 
23,9\% (167) des grands-parents et seulement 9,4\% (66) sont d'autres membres de la famille (tante, oncle ou autre personne en charge de l'enfant).

III.2- Difficultés rencontrées en matière de soins et traitements des enfants séropositifs

\section{III.2.a- Types de difficultés}

Bien que le suivi médical de l'enfant et les médicaments soient fournis gratuitement, les difficultés concernant le financement des soins sont citées par près de la moitié des répondants. Il peut s'agir d'examens ou de médicaments non couverts par la gratuité du programme, de recours à des médecines alternatives ou de médicaments pour d'autres maladies. Près de $30 \%$ des répondants indiquent que la difficulté tient au fait que les dépenses pour élever l'enfant se sont accrues (figure 1).

Figure 1: Répondants citant chaque difficulté parmi ceux qui prennent soin des enfants vivant avec le VIH/Sida (\%) (Plusieurs réponses possibles)

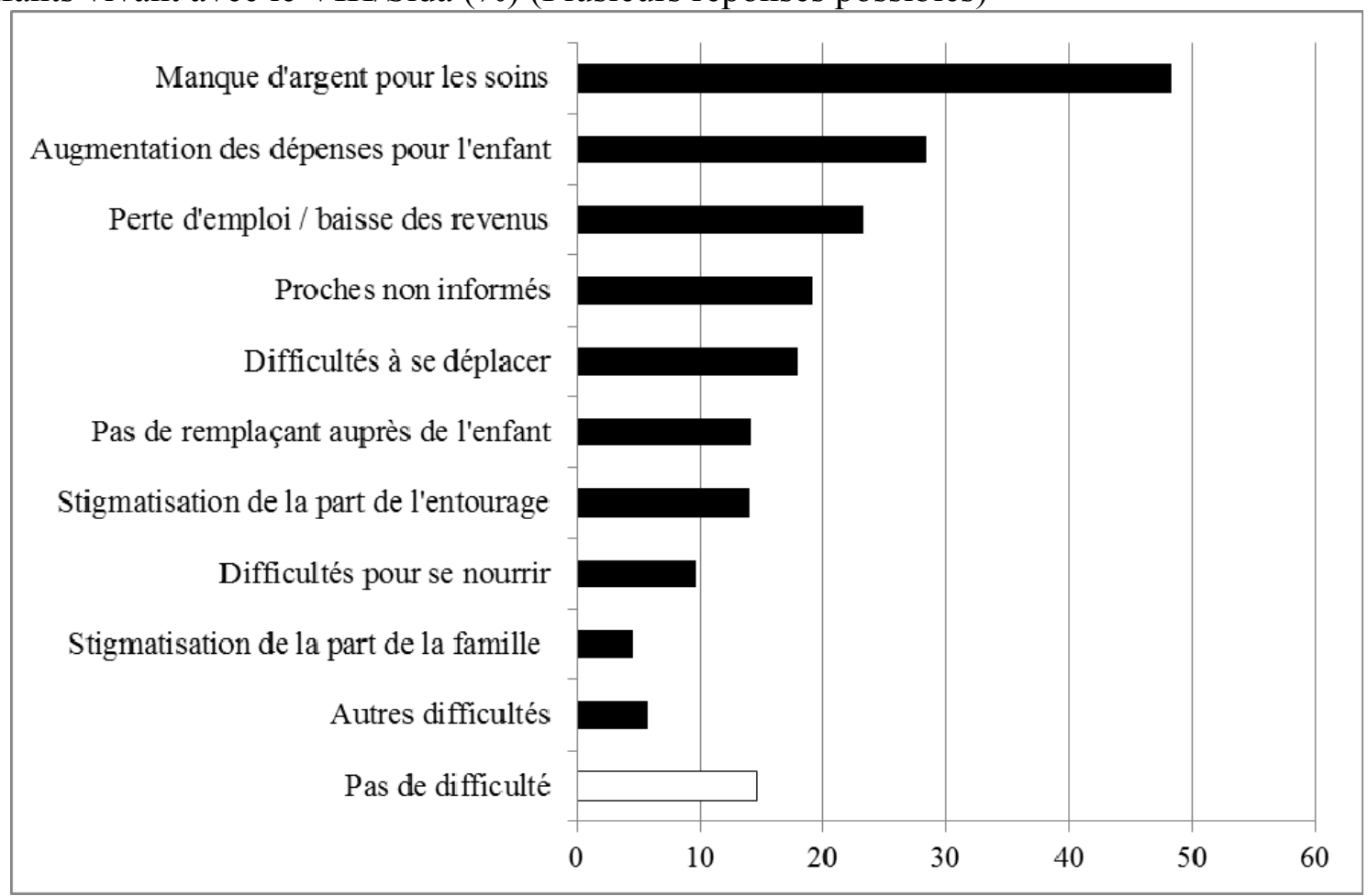

Source : enquête « Enfants séropositifs au Viêt Nam » 2009-2010

Un quart des répondants citent comme difficulté la perte d'emploi et une baisse des revenus. Environ $14 \%$ indiquent le fait de ne pas pouvoir être remplacé auprès de l'enfant séropositif. Presque autant de répondants évoquent les frais de transport pour venir en consultation. La stigmatisation est davantage évoquée par l'entourage que par la famille (respectivement 14,6\% et 5,0 \%). Les répondants de Hô Chi Minh-Ville ont plus de difficulté que ceux de Hanoi pour le paiement des soins $(55,1 \%$ contre $31,5 \%$, $\left.\mathrm{p}^{53}=0,000\right)$, en raison de la perte d'emploi ou d'une baisse des revenus $(30,6 \%$ contre $6,0 \%, p=0,000)$. Par contre, les familles de Hanoi rencontrent plus de difficultés

\footnotetext{
${ }^{53} \mathrm{p}$ correspond au paramètre utilisé pour l'interprétation des résultats du test statistique. Une valeur de ce paramètre inférieure à $5 \%$ peut être interprétée comme signe d'une différence significative entre les deux pourcentages cités.
} 
concernant l'augmentation des dépenses pour l'enfant $(34,0 \%$ contre $25,8 \%, \mathrm{p}=$ $0,029)$ et les frais de transport $(35,0 \%$ contre $10,9 \%, p=0,000)$.

«Quand mon enfant a eu plus de 18 mois, on lui a recommandé un traitement à l'Hôpital pédiatrique. Depuis, je le fais traiter ici, c'est mieux ainsi. Même si à Nam Dinh ou à Thai Binh, on peut le traiter, je ne le transférerai pas là-bas. Je le ferai traiter ici, parce que je voudrais que personne ne soit au courant. C'est pourquoi, quand je venais chercher les médicaments pour mon enfant et qu'on m'a dit qu'ils étaient disponibles également à Nam Dinh, j'ai répondu que je préférais les prendre à Hanoi. Si je prends les médicaments à Hanoi, personne à Nam Dinh ou à Thai Binh n'est au courant. Tout va bien ainsi. On fait semblant d'aller à Hanoi juste pour un voyage.» (Femme, 26 ans, veuve, mère, Hanoi, hôpital pédiatrique central).

"...Ici [l'hôpital pédiatrique 2] les médecins sont meilleurs. Ils accordent une grande attention à mon enfant... Je n'ose pas l'emmener dans un hôpital situé près de chez nous, de peur que les gens ne soient au courant. » (Femme, 33 ans,veuve, mère, Hô Chi Minh-Ville, hôpital pédiatrique 2).

À Hô Chi Minh-Ville, la proportion des répondants estimant que le soin des enfants séropositifs a entraîné la perte de leur emploi et/ou une baisse de revenu est trois fois supérieure à celle observée à Hanoi (cette différence est statistiquement significative). Les difficultés évoquées par les personnes prenant soin de l'enfant sont variables selon leurs caractéristiques : les parents citent davantage la perte d'emploi et la baisse des revenus $(26,9 \%$ pour les parents vs $17,2 \%$ pour les grands-parents et $20 \%$ pour les autres personnes, $\mathrm{p}=0,024)$; ceci peut être attribué,de la part de personnes en âge de travailler, à leur état de santé, souvent la séropositivité ou à la stigmatisation. Les grands-parents évoquent davantage le manque de ressources pour le traitement et l'achat des médicaments, beaucoup plus que les parents ou les autres membres de la parenté chargés de l'enfant $(58,7 \%$ contre $45,6 \%$ et $41,5 \%$ respectivement, $\mathrm{p}=0.008)$. Cela peut s'expliquer par les faibles revenus des personnes âgées. L'impossibilité d'être remplacé en cas de besoin est surtout mentionnée par les parents et les aidants âgés (de plus de 60 ans). Cette observation est corroborée par les entretiens :

«Pauvre enfant! Peut-on trouver un jour un médicament pour guérir cette maladie? Je suis vieux. Quand je mourrai, qui prendra soin de lui ?» (Homme, marié, 64 ans, grand-père maternel, Hanoi, hôpital pédiatrique central)

« Je lui ai dit : « Tu dois travailler bien à l'école pour pouvoir t'occuper de toi plus tard. Quand je quitterai ce monde, personne ne prendra soin de toi. Si tu es éduqué, tu pourras trouver du travail pour gagner ta vie. Vois-tu, la vie est dure quand on vend des billets de lotos ou qu'on ramasse des ordures. Il y a toujours des conflits entre les chiffonniers. » Voilà ce que je lui ai dit pour qu'il comprenne sa situation. » (Homme, 70 ans, marié, grand-père paternel, Hô Chi Minh-Ville, hôpital pédiatrique 1).

« J'aimerais être en forme pour pouvoir m'occuper de lui. Un jour, moi et mon mari, nous allons mourir. Si je pars avant lui, je lui dirai de placer le petit dans un centre de soin pour les enfants comme lui. Actuellement, des centaines d'enfants comme lui sont soignés dans ces centres. »(Femme, 64 ans, mariée, grand-mère paternelle, Hô Chi Minh-Ville, centre de An Hòa).

De façon générale et aussi bien à Hanoi qu'à Hô Chi Minh-Ville, les femmes expriment plus de difficultés que les hommes. 
En résumé, les difficultés rencontrées par les familles qui prennent soin d'un enfant séropositif sont principalement d'ordre économique (pas de moyens financiers pour le traitement et l'achat des médicaments; frais de soin en augmentation; perte d'emploi, réduction de revenus). Viennent ensuite la difficulté dans la fréquence et le coût des déplacements pour emmener l'enfant en consultation, puis l'absence d'une aide pour la garde de l'enfant, mais aussi l'inquiétude à plus long terme si la personne vient à disparaître. Les difficultés sont plus fortement exprimées par les femmes. Elles diffèrent en nature selon qu'il s'agit de parents ou de grands-parents. Les difficultés économiques sont plus marquées à Hô Chi Minh-Ville et les problèmes liés au déplacement ou l'isolement de la personne chargée de l'enfant davantage à Hanoi.

\section{III.2.b- Difficultés psychologiques}

Les questions portant sur les difficultés émotionnelles en lien avec la maladie de l'enfant ne reposent pas ici sur des échelles de mesure des troubles psychologiques adaptées au contexte culturel et langagier Vietnamien, mais sur des questions directes sur la fatigue, l'inquiétude, la tristesse, la colère et les conflits dans la famille et avec l'entourage plus éloigné ou le voisinage.

La figure 2 met en évidence la fréquence élevée de l'inquiétude $(79,4 \%)$ et de la tristesse $(78,7 \%)$, puis viennent la fatigue $(61,7 \%)$ et la colère $(39,1 \%)$. Peu de répondants rapportent des conflits, que ce soit avec la famille ou avec l'entourage. Les difficultés sont beaucoup plus marquées dans les familles qui viennent d'apprendre la maladie de l'enfant (diagnostic de moins de 3 mois) que parmi celles dont les enfants sont suivis depuis plus longtemps: tristesse $(92,6 \%$ pour les nouveaux cas versus $77,4 \%$ pour les cas suivis depuis plus d'un an); fatigue $(65,7 \%$ versus $61 \%)$, colère $(42,6 \%$ versus $38,6 \%)$. Les femmes expriment significativement plus de difficultés que les hommes pour la colère $(41,6 \%$ pour les femmes versus $29,3 \%$ pour les hommes), la fatigue $(65,5 \%$ versus $46,9 \%)$, l'inquiétude ( $82,4 \%$ versus $67,8 \%)$, la tristesse $(81,5 \%$ versus $67,8 \%)$. On ne note pas de différence entre les répondants de Hanoi et de Hô Chi Minh-Ville.

Les personnes chargées des enfants expriment un haut niveau de difficulté en particulier dans la suite immédiate de l'annonce de la maladie, et beaucoup plus les femmes, surtout les mères. Les parents sont les plus nombreux à exprimer la plupart des difficultés, viennent ensuite les grands-parents paternels et maternels puis les autres membres de la famille (Tableau 1). Plus de la moitié des répondants dans l'échantillon (385 personnes, 55,1\%) rapportent une expérience ou une crainte de discrimination liée à la séropositivité. Les difficultés psychologiques sont davantage rapportées par les personnes stigmatisées et discriminées que par les autres, qu'il s'agisse d'inquiétude $(84,7 \%$ versus $73,0 \%)$; de tristesse $(85,2 \%$ versus $70,7 \%)$; de fatigue $(68,3 \%$ versus $53,5 \%)$ ou de colère $(44,6 \%$ contre $32,2 \%)$. 
Figure 2: Répondants citant chaque difficulté psychologique parmi ceux qui prennent soin des enfants vivant avec le $\mathrm{VIH} /$ sida (\%) (Plusieurs réponses possibles)

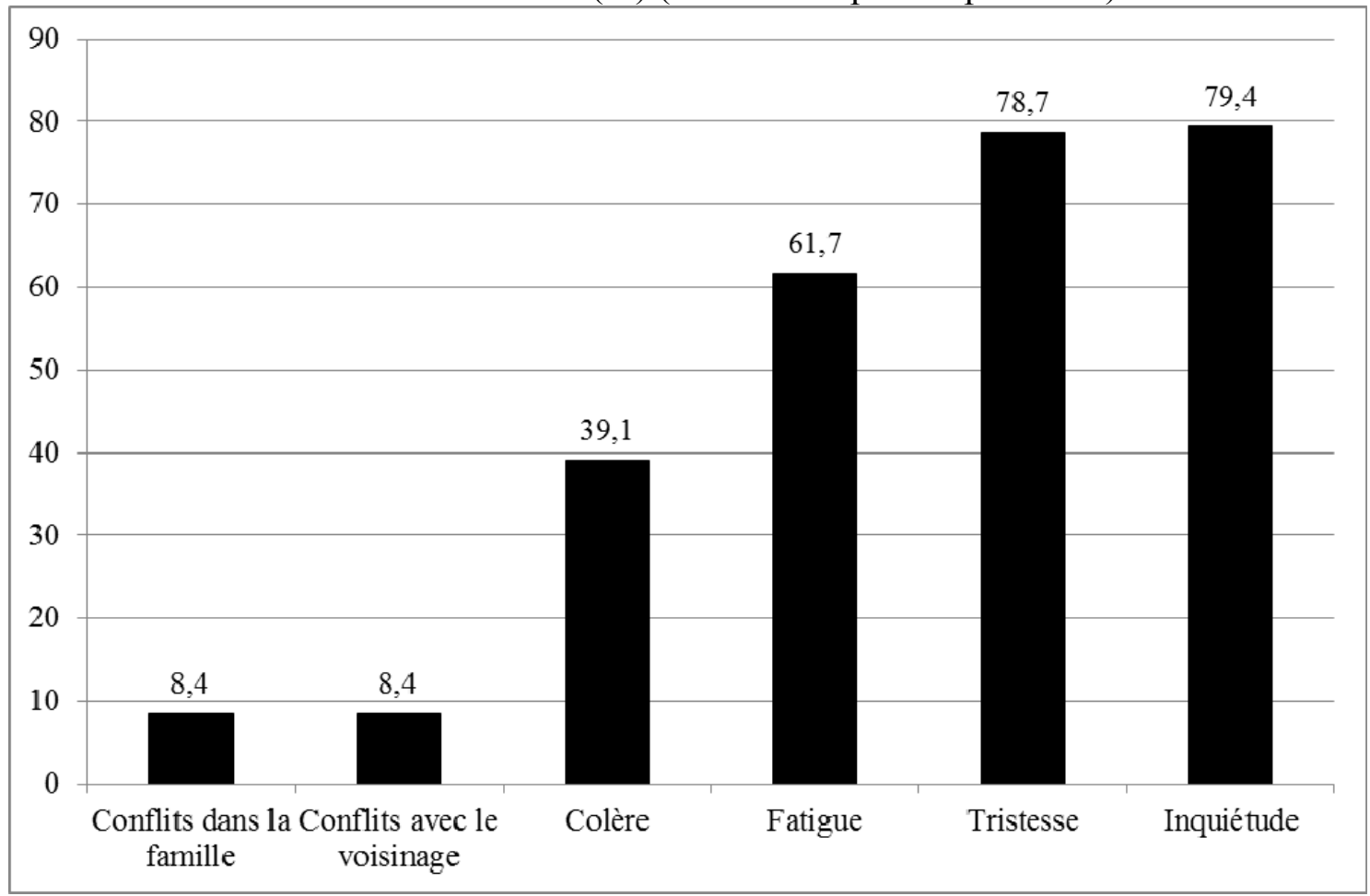

Note : Les réponses « beaucoup » ou « un peu » ont été groupées.

Source : enquête « Enfants séropositifs au Viêt Nam » 2009-2010

Tableau 1: Répondants exprimant des difficultés psychologiques parmi ceux qui prennent soin de l'enfant vivant avec le VIH/sida (\%)

\begin{tabular}{|c|c|c|c|c|c|c|}
\hline \multirow{3}{*}{ Fatigue } & & Parents & Grands-parents & Autres membres de la famille & Total & $\mathrm{p}$ \\
\hline & Beaucoup & 40,3 & 25,4 & 28,9 & 35,0 & 0,005 \\
\hline & Peu & 25,7 & 29,8 & 26,7 & 27,0 & \\
\hline & Pas du tout & 34,0 & 44,8 & 44,4 & 38,0 & \\
\hline \multirow[t]{3}{*}{ Inquiétude } & Beaucoup & 64,4 & 51,7 & 44,4 & 59,2 & 0,002 \\
\hline & Peu & 19,0 & 23,4 & 20,0 & 20,4 & \\
\hline & Pas du tout & 16,6 & 24,9 & 35,6 & 20,4 & \\
\hline \multirow[t]{4}{*}{ Tristesse } & Beaucoup & 57,4 & 48,8 & 28,9 & 52,9 & 0,000 \\
\hline & Peu & 25,5 & 26,8 & 26,7 & 26,0 & \\
\hline & Pas du tout & 17,1 & 24,4 & 44,4 & 21,1 & \\
\hline & Beaucoup & 14,8 & 4,4 & 8,9 & 11,3 & 0,003 \\
\hline \multirow[t]{2}{*}{ Colère } & Peu & 26,6 & 31,4 & 24,4 & 27,9 & \\
\hline & Pas du tout & 58,6 & 64,2 & 66,7 & 60,8 & \\
\hline
\end{tabular}

Source : enquête « Enfants séropositifs au Viêt Nam » 2009-2010

\section{$\underline{\text { III.3- Soutien et aides reçus }}$}

\section{III.3.a- Soutien psychologique}

La maladie de l'enfant, et pour les parents leur propre séropositivité sans doute, mettent les personnes qui le prennent en charge à l'épreuve sur le plan émotionnel, en particulier dans un contexte de stigmatisation de la maladie. Avoir des confidents, partager ses émotions est donc essentiel. Les répondants étaient donc interrogés sur les personnes avec lesquelles, elles pouvaient parler de leurs préoccupations. 
Environ un tiers des répondants (34\%) ont leur conjoint pour confident (Figure 3). Une proportion voisine a un confident parmi les autres membres de la famille. Ainsi, deux tiers des répondants partagent leurs émotions au sein de la famille, mais très peu ont plusieurs confidents. En revanche, ils sont peu nombreux à trouver du soutien en dehors de la famille (amis: 8,4\% ; personnes séropositives : 4,2 \%). Les professionnels de santé sont très rarement cités (médecins : $2,4 \%$, psychologues :1,4\%). Près d'une personne sur trois n'a aucun confident. Parmi elles, $20 \%$ disent ne pas vouloir se confier, et $10 \%$ n'ont personne avec qui partager ses sentiments. Cet isolement est plus fréquent à Hô Chi Minh-Ville qu'à Hanoi (22,2 \% versus 13,1\%).

Hommes et femmes répondent de façon très différente. Les hommes sont davantage soutenus par leur conjointe $(45,5 \%$ versus $30,8 \%$ ) alors que les femmes trouvent plus de soutien auprès d'autres membres de la famille $(34,7 \%$ versus $21,0 \%)$. Ceci tient en partie à la proportion plus élevée de femmes seules. De plus, quand l'enfant vit seul avec un de ses parents, plus d'une personne sur cinq (22\%) dit n'avoir aucun confident possible et $17 \%$ ne pas en vouloir, soit au total près de 4 personnes sur 10 totalement isolées sur le plan émotionnel. C'est le groupe des répondants des ménages qui ne comptent que des personnes âgées (plus de 60 ans) qui a le taux de partage des émotions au sein du couple le plus élevé $(54,5 \%)$. Même si leur rôle est peu important, c'est à Hanoi que les autres personnes séropositives sont le plus fréquemment citées $(7,5 \%$ contre 2,8 \% à Hô Chi Minh-Ville).

En bref, dans un contexte social où la stigmatisation et la discrimination à l'égard des séropositifs restent très fréquentes, le soutien psychologique est recherché dans le couple ou dans la famille avec un rôle très limité des amis, des associations de personnes atteintes ou des professionnels de santé. Près d'une personne sur trois est sans soutien.

Figure 3- Personnes auxquelles les répondants ont confié leurs difficultés psychologiques $(\%)$

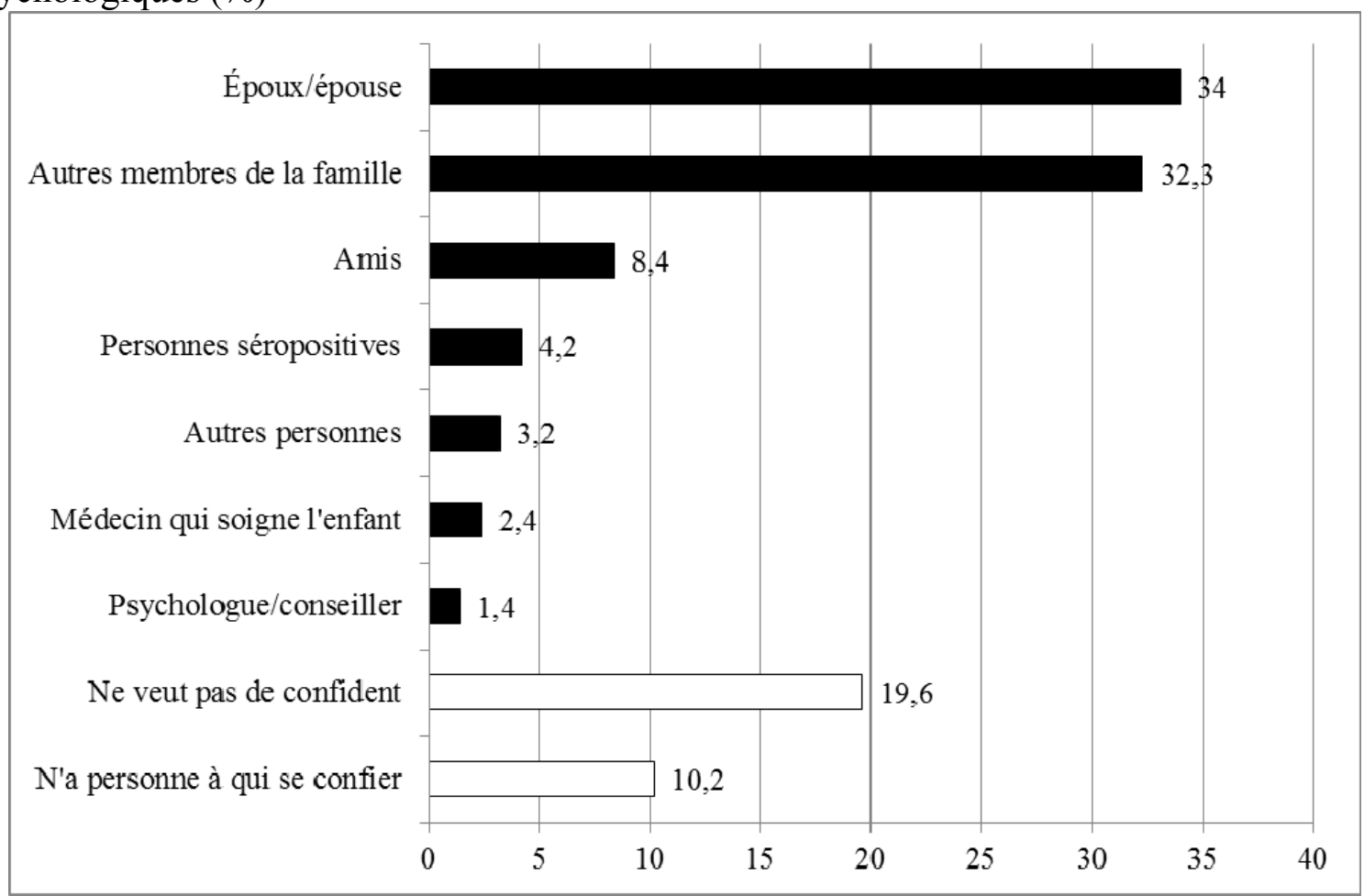

Source : enquête « Enfants séropositifs au Viêt Nam » 2009-2010 


\section{III.3.b-Aides reçues}

Les aides reçues, rapportées par les répondants ont trait d'une part aux besoins médicaux : médicaments $(77,8 \%)$, consultation médicale $(34 \%)$ et counseling ${ }^{54}$ $(14,2 \%)$, aide alimentaire $(71 \%)$ et riz $(28,9)$, les aides financières $(52,8 \%), 19,8 \%$ citent le soutien et l'aide pour s'occuper de l'enfant (Figure 4). Les aides concernant la scolarisation restent très limitées, sans doute en partie à cause de l'âge très jeune des enfants. De plus, les enseignants et les voisins ne savent pas que les enfants sont infectés par le VIH: sept répondants sur 10 pensent le voisinage n'est pas au courant et presque tous souhaitent continuer à garder le secret par crainte d'un rejet par l'école ou d'un ostracisme des voisins $(86,7 \%)$.

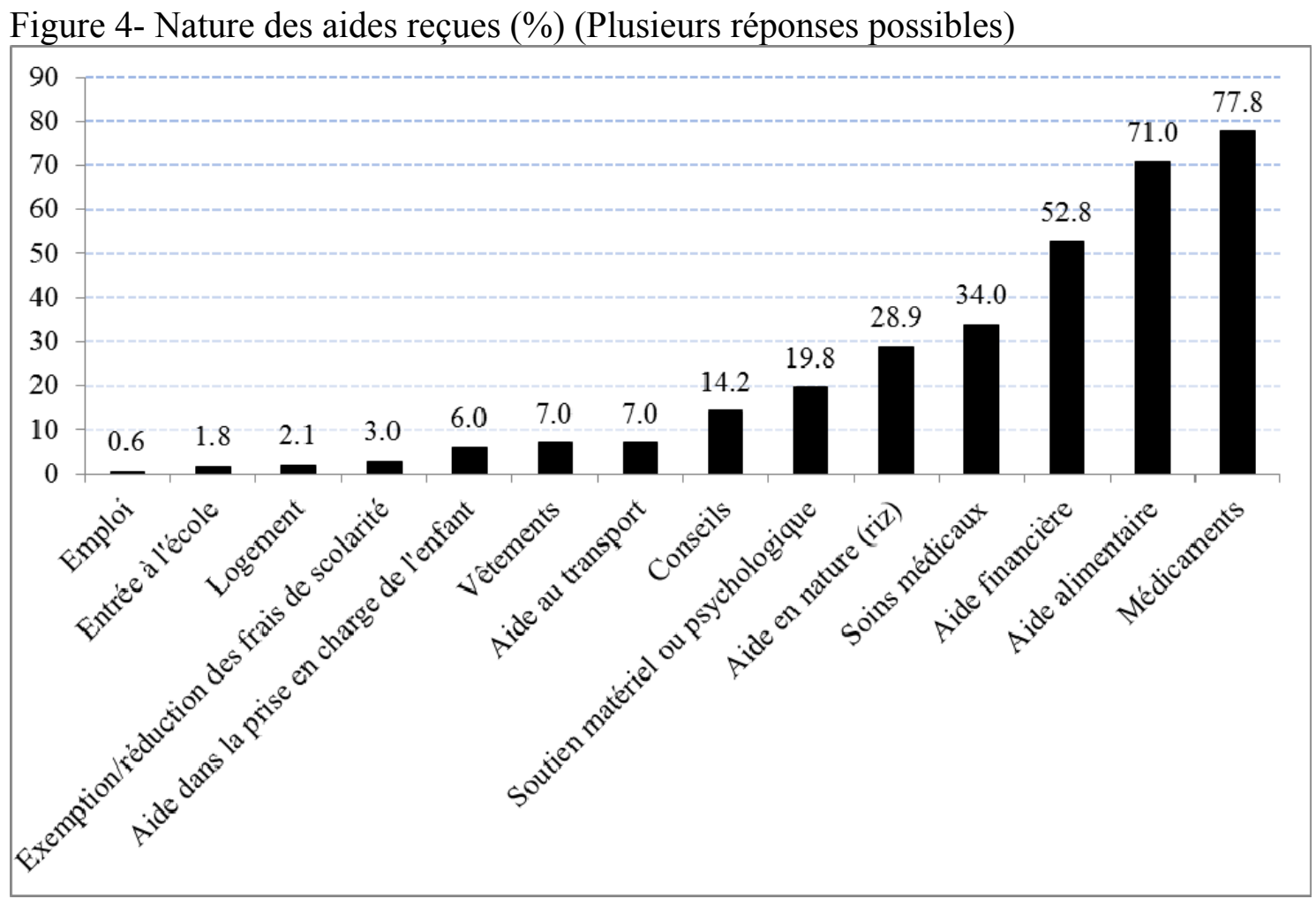

Source : enquête « Enfants séropositifs au Viêt Nam » 2009-2010

Les aides reçues diffèrent entre les familles de Hanoi et celles de Hô Chi MinhVille : les aides pour les médicaments et le soutien matériel ou psychologique sont plus fréquemment rapportées par les personnes de Hanoi (respectivement 84,3\%et $75,2 \%$ pour les médicaments à et Hô Chi Minh-Ville et 36,0\% contre 13,0\% pour le soutien) par contre l'ordre est inverse pour les aides alimentaires $(57,4 \%$ versus $76,6 \%)$,le counseling $(6,1 \%$ versus $17,6 \%)$ et le traitement $(5,1 \%$ versus $46,1 \%)$. Les aides reçues ne sont pas associées à la stigmatisation, les associations observées selon cette variable étant plutôt inverses de celles attendues, sans être significatives.

\footnotetext{
${ }^{54}$ Les recommandations internationales recommandent de proposer dans les services qui offrent les soins pour la maladie VIH aux adultes comme aux enfants des entretiens de counseling par un personnel spécialisé (travailleurs sociaux, psychologues, personnes spécialement formées, parfois des personnes atteintes) pour encourager les personnes atteintes dans leur prise en charge, offrir une éducation thérapeutique destinée à renforcer l'adhésion au traitement et apporter un soutien pour faire face à la stigmatisation de la maladie. Ce soutien doit être apporté d'une façon empathique, sans jugement, avec une attitude non prescriptive en référence à la notion de counseling. Cette fonction existe dans tous les programmes soutenus par l'aide internationale au Viêt Nam comme ailleurs. Nous employons ici le terme de counseling et de conseillers pour cette dimension de la prise en charge et son personnel.
} 
Encadré 1 : Cacher la séropositivité de l'enfant par crainte de stigmatisation

Question : Les gens sont-ils au courant de la séropositivité de votre petit-fils?

Réponse : Non. Je la cache depuis quelques années déjà.

Question : Pourquoi la cachez-vous?

Réponse : Parce que je crains qu'il soit stigmatisé. Si ses parents étaient encore en vie, ils pourraient l'emmener dans un lieu lointain pour éviter les rumeurs.

(Homme, 70 ans, marié, grand-père paternel, Hô Chi Minh-Ville, hôpital pédiatrique 1)

Question :Pourquoi est-ce que vous ne parlez pas de la séropositivité de votre petite aux voisins?

Réponse : Parce que si cela se savait, il y aurait de la stigmatisation de la part des autres.Nous le cachons tant que nous pouvons. On verra bien après.

Question :Pourquoi pensez-vous qu'il y aurait de la stigmatisation?

Réponse :Parce qu'en fait, les gens ne savent pas grand-chose de cette maladie. Dès qu'ils entendent le mot, ils se montrent antipathiques. Comme cette maladie est nouvelle, on ignore par quelle voie elle est propagée, comment la traiter.On n'entend parler que de la contamination. Et puis, comme on voit que dans le village, dans le quartier, de nombreuses personnes sont mortes d'une maladie nommée sida, on cherche à isoler les séropositifs.

(Homme, 40 ans, remarié, père, Hanoi, hôpital pédiatrique central)

Question :Voulez-vous que la séropositivité de votre enfant soit connue, mais que les gens ne vous stigmatisent pas, vous et votre enfant ?

Réponse : Si j'avais un vœu, je souhaiterais que cette maladie soit une maladie normale comme les autres maladies. Qu'on ne soit pas isolé, que les autres n'aient pas peur en nous voyant... Que la vie soit moins dure depuis la contamination jusqu'à la mort! Que nous ne soyons pas stigmatisés ni l'objet de la pitié des gens. Or, rien qu'à entendre le nom de la maladie, les gens ont peur.

Question : Pourquoi les gens ont-ils peur, à votre avis ?

Réponse:Parce qu'ils ont vu que d'autres gens avaient peur de cette maladie et cherchaient à rester l'écart des séropositifs. Je pense souvent: « Si maintenant, par malheur, j'étais contaminée par cette maladie, que se passerait-il ? Les autres auraient sans doute peur de moi ». C'est pour cette raison que je cache la séropositivité de mon enfant.

Question : Pourquoi ne participez-vous pas aux associations pour les gens vivant avec le VIH/SIDA dans votre localité (Nam Dinh) ?

Réponse : Parce que je voudrais que rien ne soit révélé. Je crains que les gens m'isolent. C'est ce que je crains le plus. Je n'ai jamais isolé mes amis. Je n'ai jamais vu les autres de manière désobligeante ou méprisante. Mais je crains qu'on me voie de cette manière. C'est pourquoi je ne participe à aucune activité. À tel point qu'en regardant la télévision, si l'on parle du $\mathrm{VIH}$, je passe tout de suite. Je ne regarde presque jamais d'émission sur ce sujet...

Question : Avez-vous vu une personne séropositive mise à l'écart par la communauté 


\begin{tabular}{|l|}
\hline là où vous vivez? \\
Réponse : Pas encore, mais quand mon mari est mort, on a pensé que moi aussi, \\
j'étais séropositive. Dans l'ensemble, on me voyait d'une autre façon, on \\
me traitait différemment. Je n'en suis pas sûre à $100 \%$, mais à $70 \%$ qu'on \\
nous traiterait, moi et mon enfant, de cette manière. J'en ai peur ! Après le \\
dépistage de la séropositivité de mon enfant et la mort de mon mari, \\
certaines familles ont interdit à leurs enfants de jouer avec le mien. Une \\
fois, mon enfant s'est rendu chez une famille voisine, mais il est revenu \\
immédiatement. Quand je lui ai demandé pourquoi, il a répondu : « Elle ne \\
me permet pas de jouer ». En entendant cette réponse, mes larmes ont \\
coulé à l'intérieur. J'ai peur. Je cache tout ce que je peux. \\
(Femme, 26 ans, veuve, mère, Hanoi, hôpital pédiatrique central)
\end{tabular}

Ainsi, les aides citées par les familles des enfants séropositifs sont les médicaments, la nourriture et des apports financiers.

\section{III.3.c-Importance accordée aux aides reçues}

Les répondants accordent une grande valeur aux aides reçues (Figure 5). Les entretiens montrent que même si les aides reçues ne sont pas très significatives en termes matériels, elles sont fortement valorisées et perçues comme un encouragement pour les familles concernées par le VIH.

Figure 5 : Répartition des répondants selon l'importance accordée aux aides reçues

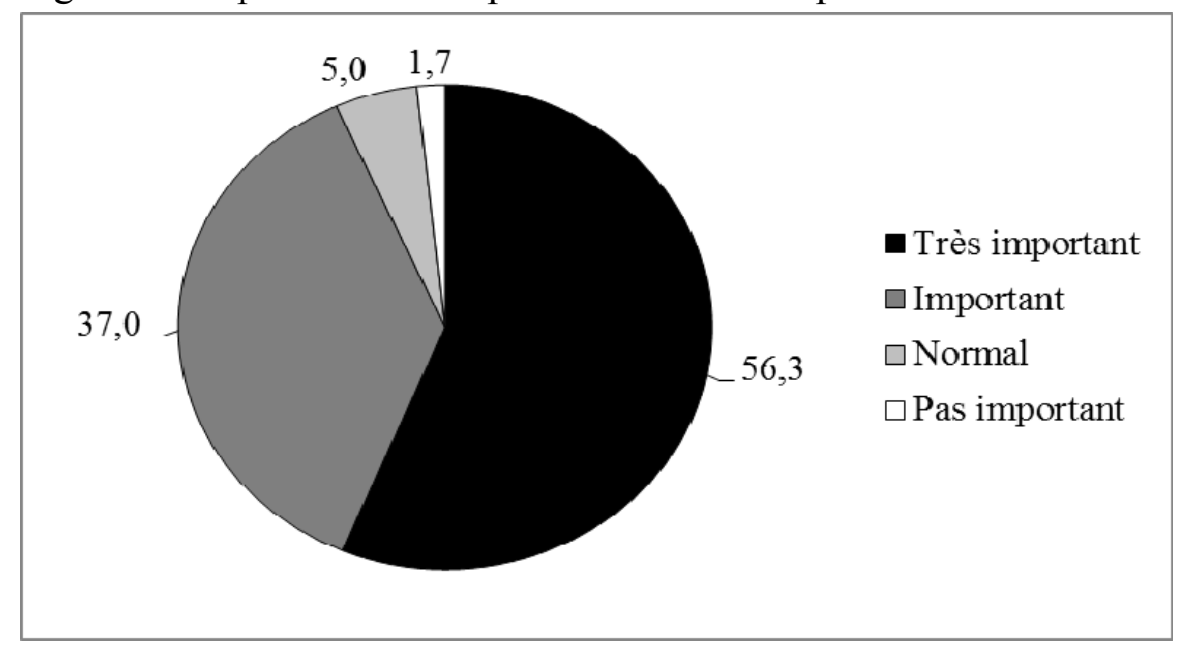

Source : enquête « Enfants séropositifs au Viêt Nam » 2009-2010

\section{Encadré 2 : Sens donné aux aides reçues}

Question : Avez-vous reçu des aides sous forme de nourriture, de lait ou d'argent ?

Réponse : Pas d'argent. Je n'ai reçu que des aides en nature. L'an dernier, pendant trois mois, j'ai reçu chaque mois un carton de nouilles instantanées, cinq kilos de riz, deux boîtes de médicaments et une bouteille d'huile.

Question : Pensez-vous que ces aides étaient significatives pour votre famille?

Réponse : Oui. Dans l'ensemble, pour nous, l'important, c'est l'attention qu'on nous accorde. Cet encouragement nous touche au cœur.

(Femme, 33 ans, veuve, mère, Hanoi, hôpital pédiatrique central)

«Chaque mois et à l'occasion de fêtes comme le Têt, le $1^{\text {er }}$ juin [fête des enfants] et lors d'autres fêtes, mon enfant reçoit des cadeaux du Fonds de protection des mères 
et des enfants, des autorités du quartier, de l'association des personnes séropositives ou du quartier. Ces cadeaux traduisent de la compassion des gens envers nous. Comme je travaille loin de chez nous, je ne rentre à la maison qu'une fois par mois. Chaque fois qu'on me voit, on me demande : «Comment va ta santé ?» ou encore «Prends-soin bien de toi! Tu dois encore t'occuper de ton enfant.» Voilà, on m'encourage comme ça, personne ne cherche à m'isoler. »

(Femme, 32 ans, mère, divorcée, Hanoi, hôpital pédiatrique central)

\section{III.3.d- Origine des aides reçues}

Les aides sont apportées d'abord par les services de santé où les enfants sont soignés $(84,4 \%)$, puis par la famille $(55,4 \%)$ et dans une moindre mesure par les organisations communautaires et les services sociaux (21,7\%) (Figure 6). Celles-ci se distribuent entre associations d'entraide des personnes atteintes $(41,4 \%)$, services locaux $(26,9 \%)$ et services pour les orphelins $(15,2 \%)$. Les organisations sociopolitiques sont rarement citées $(6,2 \%)$. Toutefois, les aides reçues par les familles de la part des autorités locales ne sont pas attribuées au titre de la séropositivité. Il s'agit d'aides sociales pour les pauvres et les orphelins relevant du droit commun. En effet, le plus souvent, les services locaux ne sont pas informés de la séropositivité de ces enfants. À Hanoi, l'aide vient plus fréquemment qu'à Hô Chi Minh-Ville des services sociaux et communautaires $(35,0 \%$ versus $16,4 \%)$, du voisinage $(12,5 \%$ versus $7,2 \%)$ et de l'école $(10,0 \%$ versus $2,8 \%$ ). Ces différences tiennent au fait qu'à Hanoi la révélation de la maladie de l'enfant est plus étendue $(41,0 \%$ versus $21,6 \%)$ et la stigmatisation moins sensible $(9,0 \%$ versus $24,3 \%)$.

Figure 6 : Personnes ou services à l'origine des aides reçues (\%) (plusieurs réponses possibles)

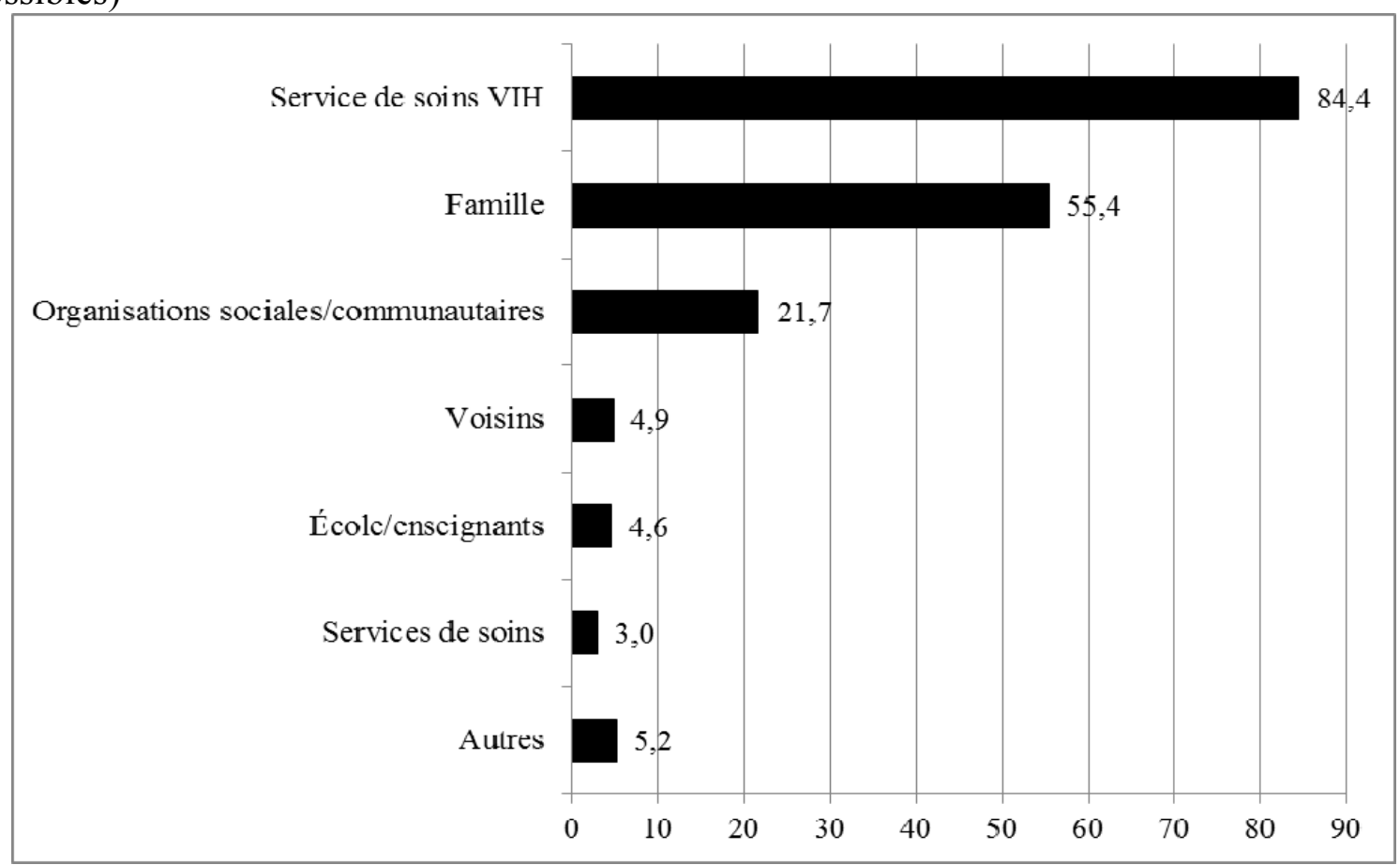

Source : enquête « Enfants séropositifs au Viêt Nam » 2009-2010

Les personnes qui rapportent une expérience ou une crainte de discrimination citent plus fréquemment les services de santé comme source d'aide. Il est possible que pour ces personnes, le service de soins avec médecins, infirmières et conseillers, soit mieux 
identifié comme une ressource $(87,5 \%$ versus $80,6 \%)$. De fait, aucun répondant ne dit se sentir stigmatisé ou craindre la stigmatisation dans le service où l'enfant est suivi. Logiquement, les répondants qui ne sont pas inclus dans le groupe marqué par la stigmatisation rapportent plus d'aide de la part des voisins $(10,7 \%$ versus $3,6 \%)$ et des organisations sociales $(24,5 \%$ versus $14,9 \%)$. Seuls $3,7 \%$ des répondants de l'échantillon estiment qu'ils ne reçoivent aucune aide, y compris de la part des autres membres de leur famille.

\section{Encadré 3 : Les enfants séropositifs ne font pas l'objet d'aides spécifiques \\ Question : Quelle est l'attitude du voisinage et de la famille envers l'enfant ? \\ Réponse: Ils lui accordent beaucoup d'affection. Ils ne sont pas au courant de sa séropositivité. Ils ont de la compassion envers nous, des grands-parents âgés qui prennent soin d'un petit enfant avec peu de moyens financiers. Les voisins lui ont donné des vêtements et même un pupitre dont leurs enfants n'avaient plus besoin. Je l'ai un peu réparé pour que le petit puisse l'utiliser. \\ (Homme, 70 ans, marié, grand-père paternel, Hô Chi Minh-Ville, Hôpital pédiatrique 1)}

Question : Avez-vous des difficultés pour payer les frais de scolarité de votre enfant?

Réponse : Non, parce que nous avons une allocation de scolarité.

Question: Pourquoi avez-vous une telle allocation?

Réponse : Parce que nous sommes classés ménage pauvre.

Question : Recevez-vous une allocation pour l'enfant séropositif?

Réponse : Non, parce que... mes parents ne sont pas au courant [de la séropositivité du petit]. Dans la famille, seul mon mari était au courant ainsi que son frère. Les autres ne le sont pas... Ils [voisins] s'en doutent, mais ne savent pas exactement la vérité.

(Femme, 33 ans, veuve, mère, Hanoi, Hôpital pédiatrique central)

Question : Le petit reçoit actuellement des aides du quartier, mais dans quel cadre?

Réponse : Le programme 67 pour les personnes pauvres. Ses parents n'ayant pas d'acte de mariage, le petit n'avait pas donc d'acte de naissance. Maintenant, comme ses parents sont morts, les autorités du quartier ont établi l'acte de naissance pour le petit afin qu'il puisse aller à l'école.

Question : Comment se sont déroulées les formalités?

Réponse : J'ai présenté notre situation aux autorités locales: nous sommes un ménage pauvre venu de la campagne ; nous ne sommes pas inscrits dans le registre civil d'ici. Les autorités ont envoyé les papiers à l'école, mais l'école n'avait pas accepté le petit. Ensuite, j'ai dû m'adresser au bureau de l'éducation de l'arrondissement. Avec l'intervention de ce bureau, le petit a pu aller à l'école.

(Femme, 64 ans, mariée, Grand-mère paternelle, Hô Chi Minh-Ville, centre médical An Hòa)

En bref, les familles des enfants séropositifs mentionnent en premier l'aide des services de soins qui traitent l'enfant, des membres de la famille et des organisations sociales et communautaires.Cependant en dehors de la famille, les aides semblent plus diversifiées et plus fréquentes pour les enfants de Hanoi que de Hô Chi Minh-Ville. L'expérience ou la crainte des discriminations semblent faire obstacle aux soutiens en dehors de la famille et des services de soins spécialisés. 


\section{III.4- Besoins d'aide supplémentaire}

Le questionnaire envisageait également les besoins d'aide non satisfaits ainsi que les personnes ou les services dont les familles attendaient un soutien.

À la question du besoin d'aide psychologique supplémentaire, 254 répondants $(36,3 \%)$ répondent qu'ils en ont besoin, sans différence selon le site, le sexe ou le lien de parenté avec l'enfant. Seul le critère de stigmatisation différencie les répondants $(41,1 \%$ pour les personnes rapportant des expériences de discrimination ou de stigmatisation versus 32,5\% parmi celles qui n'en rapportent pas). Les médecins en charge du suivi de l'enfant séropositif sont les premières personnes dont les répondants souhaitent recevoir un soutien psychologique, et ceci de façon indifférenciée selon les sites, le sexe du répondant ou le type de famille (Figure 7). Ce résultat témoigne de la confiance et des attentes envers ces services. Les conseillers viennent en deuxième position. Ils sont davantage cités par les personnes qui rapportent une crainte ou une expérience de stigmatisation $(71,0 \%$ versus $52,5 \%)$. Il est à souligner que les répondants stigmatisés au sein de la famille expriment de fortes attentes vis-à-vis des médecins et du personnel sanitaire $(71,0 \%$ versus $52,5 \%)$.

Figure 7 : Personnes ou services dont est souhaité un soutien psychologique (\%) (Plusieurs réponses possibles)

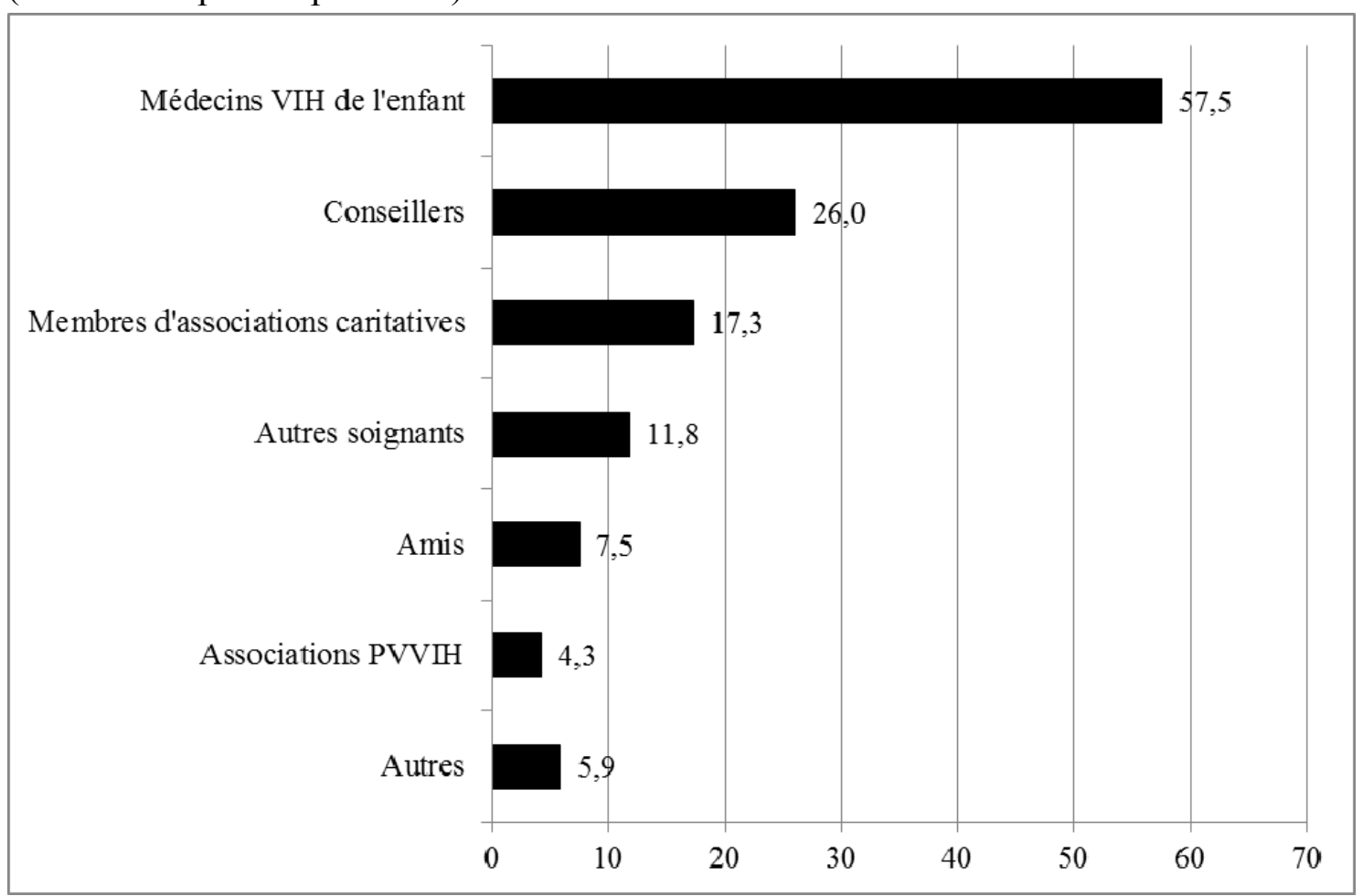

Source : enquête « Enfants séropositifs au Viêt Nam » 2009-2010

Les quatre autres types d'aide souhaitée les plus mentionnés par les répondants sont l'aide financière $(51,8 \%)$, les médicaments $(44,6 \%)$, la nourriture $(23,3 \%)$ et une aide aux déplacements $(18,5 \%)$ (Figure 8$)$. L'appel à un conseiller n'est évoqué que par $10,2 \%$ des répondants. Ce chiffre suggère que la fonction du conseiller est mal reconnue alors qu'en principe sa présence au sein des services spécialisés est dévolue à l'accompagnement et au soutien. Ainsi, placé dans une question spécifique, le besoin d'aide psychologique est plus souvent mentionné que lorsqu'il est placé dans une liste d'autres besoins, parmi lesquels les besoins matériels. Ceux-ci deviennent 
prépondérants pour ces familles. À l'époque de l'enquête, le revenu médian mensuel par personne était de 625000 dongs, montant, beaucoup plus bas que le revenu moyen qui était en 2008 de 995000 dongs par personne et par mois. On peut s'étonner que les médicaments viennent au second rang dans l'ordre de priorité alors qu'une grande partie des soins sont gratuits pour l'infection VIH dans les services spécialisés. Les entretiens éclairent en partie cette priorité. Il faut rappeler que les médicaments sont délivrés à l'hôpital une fois par mois. Les familles vivant loin de l'hôpital ont donc des difficultés pour s'y rendre en raison de la distance. De plus, les enfants scolarisés, certes encore peu nombreux, doivent aussi demander l'autorisation des enseignants pour s'absenter. Enfin, les familles s'inquiètent pour le long terme : le traitement ARV, aujourd'hui gratuit, indispensable à vie sera-t-il toujours disponible? S'il ne l'est plus, que deviendra l'enfant?

\section{Encadré 4 : Besoins de soutien par des personnes spécialisées}

Question : Avez-vous besoin d'un conseiller pour vous confier?

Réponse : Je veux bien. J'aimerais bien me confier, à condition qu'on ne me présente pas au public, qu'on ne prenne pas une photo de moi que l'on diffuse à la télévision ou sur Internet. Mais oui, je voudrais bien me confier pour être soulagée.

(Femme, 33 ans, mère, Hanoi, hôpital pédiatrique central)

Figure 8 : Besoin d'aide supplémentaire par rapport à l'aide existante (\%) (Plusieurs réponses possibles)

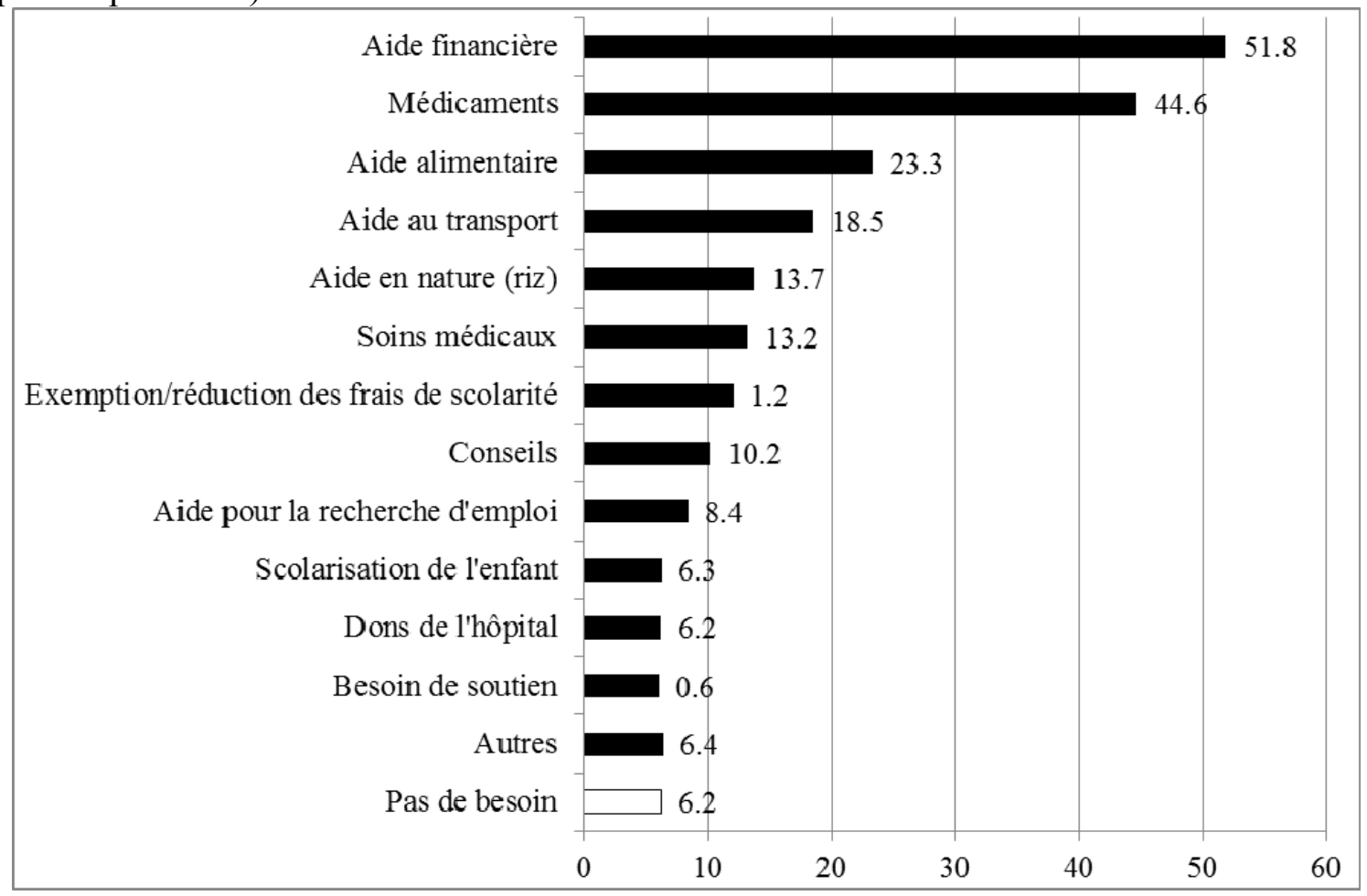

Source : enquête « Enfants séropositifs au Viêt Nam » 2009-2010 


\section{Encadré 5 : Besoin d'aide en médicaments}

Question : S'il y a un programme ou projet d'aide des enfants séropositifs, quelle aide souhaiteriez-vous le plus obtenir?

Réponse : Tout ce que je souhaite maintenant, c'est que mon petit-fils soit soigné gratuitement. Actuellement, chaque fois que je l'emmène à l'hôpital, cela nous coûte déjà quatre ou cinq cent mille dongs, sans compter les médicaments. Chaque ordonnance coûte de cinq cents à six cents mille dongs. C'est pourquoi, tout ce que je souhaite, c'est que mon petit-fils soit soigné gratuitement. D'autres aides, s'il y en a, tant mieux, mais d'autres personnes sont aussi en difficulté.

(Femme, 62 ans, grand-mère, Hanoi, Hôpital pédiatrique central)

« J'aimerais que les médicaments soient fournis à mon fils tous les deux ou trois mois. Pendant une certaine période, je ne sais pas pourquoi, nous devions aller à l'hôpital tous les huit jours. Mon fils était en plus malade. C'était très dur... Comme cette fois, mon fils doit faire des prélèvements, $\mathrm{j}$ 'aimerais que tous les examens soient faits en une seule journée. Cela nous épargnerait les multiples déplacements. Ce serait moins fatigant pour mon fils aussi. J'aimerais que les tests soient faits le matin, puis que dans l'après-midi, nous recevions les médicaments $\gg$.

(Femme, 31 ans, veuve, mère, Hanoi, Hôpital pédiatrique central)

« Tout ce que je souhaite, c'est que mon enfant soit traité. Je peux vivre dans la misère, pourvu que mon enfant ait des médicaments pour qu'il aille mieux. Toutes les aides sont bonnes, mais s'il fallait choisir, je choisirais les médicaments. S'il faut payer les médicaments, je les paierais, que puis-je faire ? S'il existait un remède pour cette maladie, bien qu'il soit cher, je vendrais notre maison pour que mon enfant ait ce remède, ensuite, je m'installerais à la campagne »

(Femme, 33 ans, veuve, mère, Hanoi, hôpital pédiatrique central)

«La plus grande difficulté est le déplacement. J'ai demandé au médecin de me délivrer les médicaments tous les deux mois, ce serait plus raisonnable. Je travaille dans le secteur public. C'est difficile de demander un jour de congé tous les mois. Ensuite, plus tard, quand il ira à l'école, si chaque mois, il doit demander à son institutrice d'être absent un jour, elle se doutera [de sa séropositivité]. En plus, chaque déplacement nous coûte un million de dongs. Si nous allons tous les deux mois à l'hôpital, nous pourrons économiser un million de dongs "

(Femme, 26 ans, mariée, mère, Hanoi, hôpital pédiatrique central)

Quand l'enfant vit avec ses seuls grands-parents, sans autre personne jeune dans le ménage, les besoins d'aide supplémentaires sont plus élevés : médicaments $(72,7 \%)$, aide financière $(54,5 \%)$, aide au transport $(36,0 \%)$, aide alimentaire $(36,0 \%)$ et riz $(18,0 \%)$ (Figure 9). 
Figure 9 : Nature des besoins d'aide selon le type de ménage (\%) (plusieurs réponses possibles)

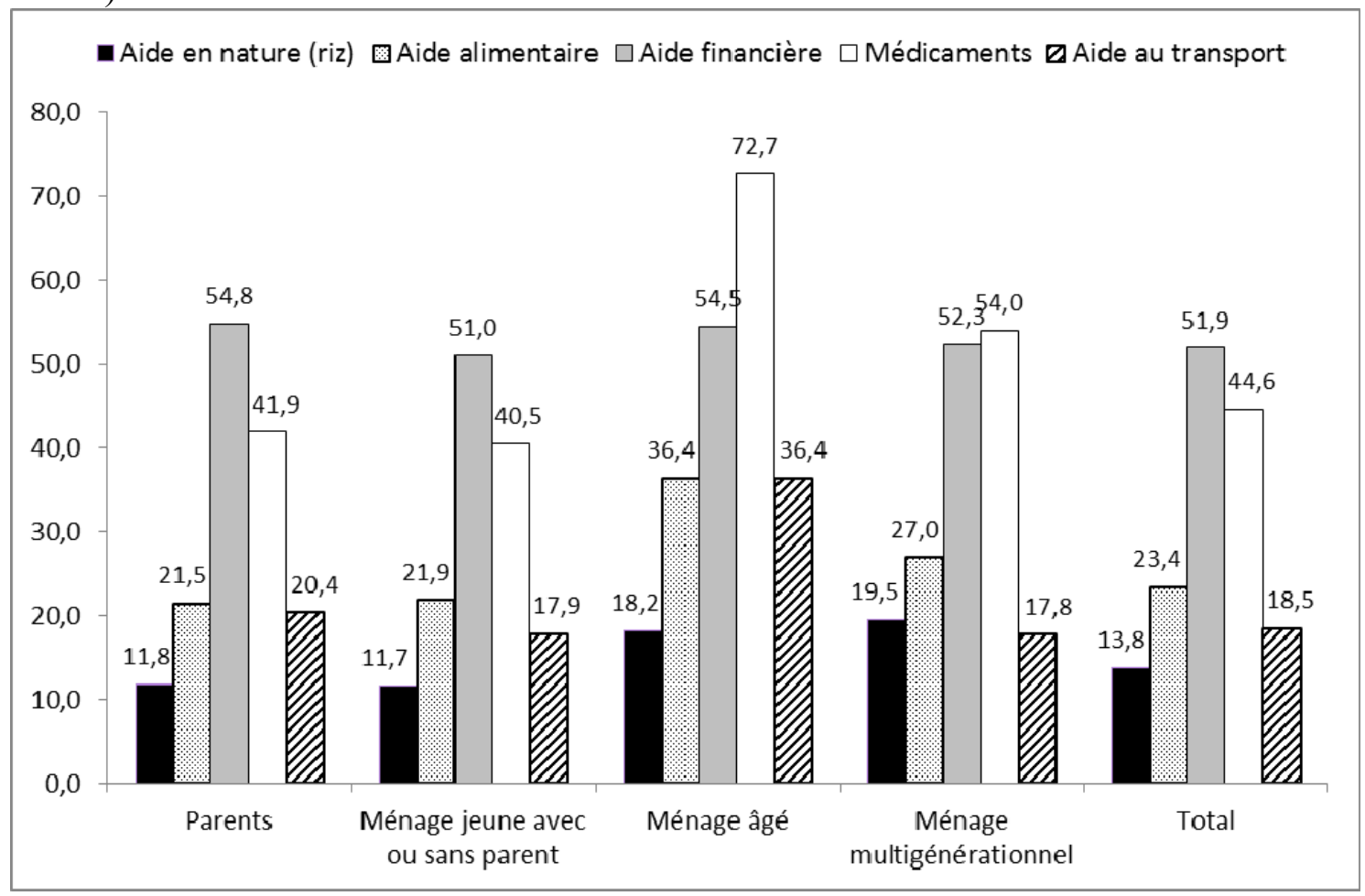

Source : enquête « Enfants séropositifs au Viêt Nam » 2009-2010

Dans l'ensemble, les besoins d'aide des familles en termes de médicaments, d'aliments, de transport, de counseling et de recherche d'un emploi sont plus élevés à Hanoi qu'à Hô Chi Minh-Ville (voir Figure 10). Les besoins matériels exprimés par les grands-parents apparaissent plus importants que ceux des adultes plus jeunes, parents ou oncles et tantes. C'est le cas pour les aides financières $(65,3 \%$ pour les grands-parents, $46,8 \%$ pour les parents et 53,0\%pour les autres membres de la famille) et pour les aides alimentaires (respectivement $21,0 \%, 11,8 \%$ et 9,1\%). Les parents des enfants ont un peu plus d'attente en termes d'aide dans la recherche d'emploi (10,3\% des parents versus 5,4\% des grands-parents et 3,0 \% des autres personnes).

«Je voudrais seulement qu'on m'aide à trouver un emploi stable. »

(Femme, 33 ans, veuve, mère, Hanoi, Hôpital pédiatrique central)

Placés parmi les autres besoins, ceux portant sur le soutien psychologique sont peu exprimés. Ils le sont surtout par les personnes qui rapportent des expériences ou des craintes de discrimination ( $13 \%$ contre $6,7 \%)$. Très peu demandent à être aidés pour s'occuper de l'enfant.

Ainsi, les besoins d'aide les plus pressants pour les enfants séropositifs qui sont traités à l'hôpital concernent l'argent, les médicaments, le traitement et les déplacements. Les priorités sont différentes selon les répondants. Les besoins de base et les médicaments sont plus importants pour les grands-parents, notamment quand ils vivent seuls avec l'enfant. Logiquement, les attentes d'aide dans la recherche d'emploi sont exprimées par les parents et les adultes jeunes. À la question sur le besoin d'aide psychologique, un tiers des participants expriment un besoin de counseling. Ce besoin supplémentaire est davantage le fait des personnes qui se sentent discriminées. 
Figure 10: Nature des besoins d'aide à Hanoi et à Hô Chi Minh-Ville (\%) (plusieurs réponses possibles)

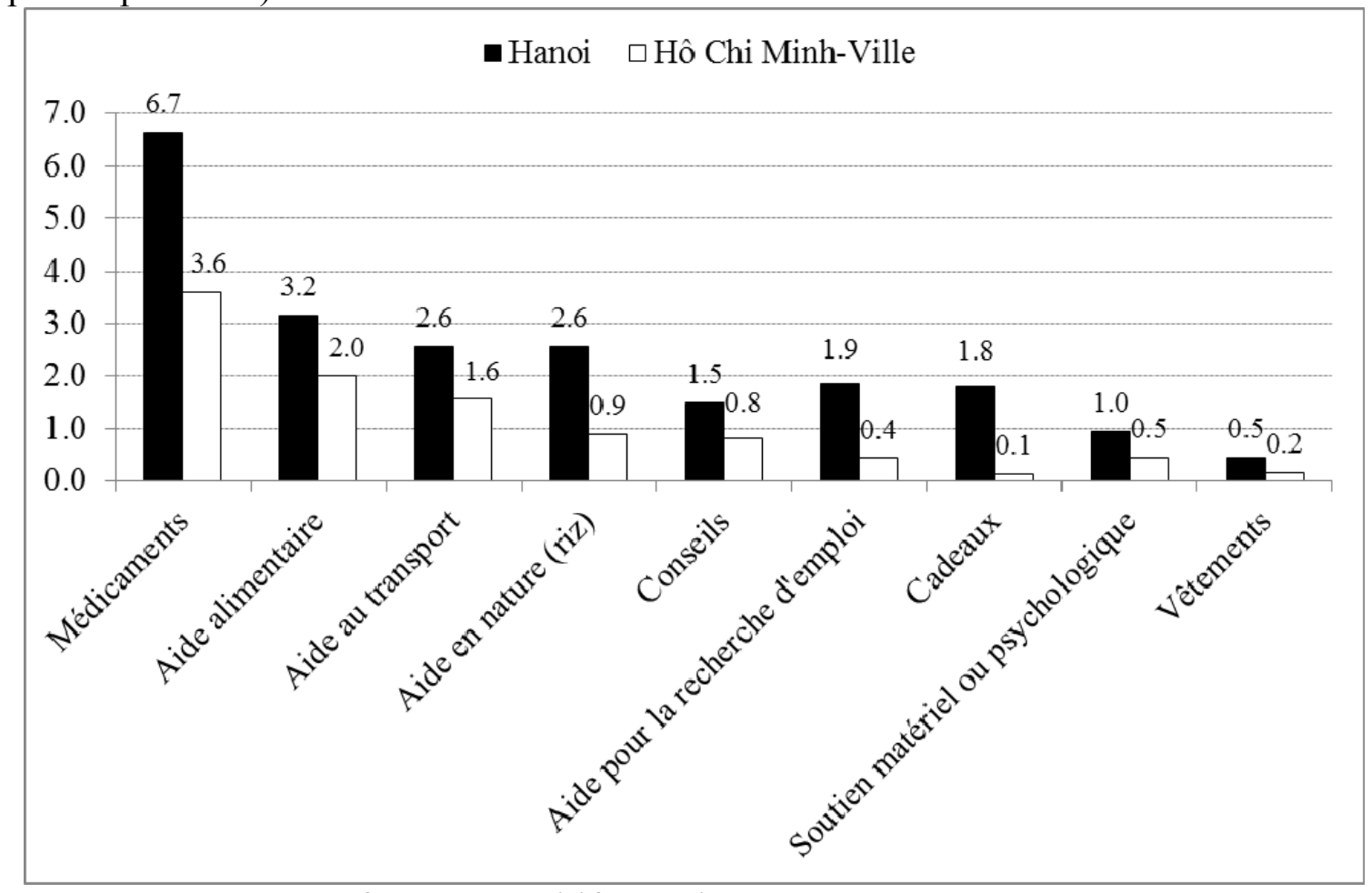

Source : enquête « Enfants séropositifs au Viêt Nam » 2009-2010

\section{IV- Discussion/Conclusion}

L'étude porte sur des enfants qui vivent au sein de leur famille. S'agissant d'enfants encore très jeunes, la proportion de ceux qui vivent avec leurs deux parents est très basse (moins de $40 \%$ ), en raison principalement du décès de l'un des parents. La maladie et l'absence d'un des parents sont les premières causes des difficultés rencontrées tant en termes matériels qu'en termes d'isolement. Les besoins des enfants placés dans des institutions n'ont pas été abordés dans cette étude, car ils ne relèvent pas de la problématique des besoins des familles. Quant aux conditions qui, dans le contexte d'une société dont la famille est le pilier, poussent à abandonner ou placer les enfants, qu'ils s'agissent d'enfants séropositifs ou de tout autre enfant, elles sont très difficiles à étudier.

Interroger la personne qui accompagne l'enfant en consultation, dès lors qu'elle s'occupe de lui de façon très régulière, répond à un choix pratique visant à éviter de rencontrer les familles à domicile parfois loin du lieu de soins et surtout parce que la question du VIH reste délicate, voire impossible, à aborder en dehors du lieu de soin. De ce fait, la personne interrogée n'est pas toujours le père ou la mère de l'enfant et aucune question n'a été posée sur la maladie VIH de la mère ou du père pour des raisons éthiques. Ces choix limitent sans doute la compréhension des difficultés rencontrées.

L'étude des besoins met en exergue :

(1) La priorité donnée à la sécurité des soins à l'enfant. En effet, sous diverses formes les médicaments, les soins, le traitement sont exprimés comme prioritaires. Dans le contexte du Viêt Nam, où les soins médicaux nécessitent en général une forte 
contribution financière des familles faute de couverture maladie universelle, la nécessité de soins spécialisés et d'un traitement permanent reste au premier plan malgré la gratuité d'une partie importante des soins et des traitements pour la maladie VIH. Pour des enfants dont la survie dépend des soins médicaux, l'inquiétude sur le maintien à long terme d'un tel système, les coûts complémentaires engendrés par les déplacements, les soins non couverts, voire les pertes d'activité contribuent à considérer ce besoin comme majeur. L'observation suggère aussi que les coûts de santé pour la prise en charge de la maladie VIH dépassent de beaucoup, pour certaines familles, ce qui est couvert par la gratuité. La nature de ces dépenses mériterait d'être caractérisée.

(2) L'importance des difficultés économiques, pour faire face à un large éventail de besoins fondamentaux dans la prise en charge des enfants. Les familles dans lesquelles vivent les enfants infectés par le VIH ont un niveau de vie plutôt inférieur au niveau moyen, en partie parce que les parents atteints par la maladie ne peuvent pas travailler ou sont décédés. Ces besoins, en partie couverts par des aides financières modestes ou des aides en nature, suscitent des attentes d'aide financière accrue notamment quand ce sont des grands-parents qui prennent en charge l'enfant. Les attentes à l'égard des services sociaux ou des aides caritatives sont cependant modestes, dans un contexte social où la famille continue à remplir une fonction sociale majeure, et où les formes d'assistance sont réservées aux situations extrêmes.

(3) Le poids psychologique de la maladie. Il est abordé ici par l'inquiétude, la tristesse, la fatigue,qui atteignent des niveaux très élevés chez la personne qui prend soin de l'enfant, en particulier dans la période qui suit le diagnostic ; niveau plus élevé chez le père et la mère, et de façon générale chez les femmes, mères ou grands-mères. Les confidents sont situés parmi les personnes les plus proches dans le cercle familial et rarement à l'extérieur; les organisations de personnes atteintes ne semblent pas ou très peu remplir ce rôle de confidents. De ce fait, la proportion de personnes qui n'ont pas ou ne veulent pas se confier est très élevée, situation aggravée quand la personne qui prend soin de l'enfant redoute ou subit la discrimination.

(4) La place encore réduite des problématiques autour de la scolarité. Les travaux antérieurs, portant sur les familles affectées par le VIH, ont montré l'acuité des enjeux autour de la scolarisation des enfants, recouvrant l'inscription elle-même, lorsque les démarches de déclaration de l'enfant ont été incomplètes, les dépenses de scolarité et le rejet par le milieu scolaire- malgré la loi- des enfants des familles affectées par le VIH. Cette préoccupation est peu présente dans cette étude, sans doute en raison de l'âge des enfants et du secret confiné dans le cadre familial sur la maladie de l'enfant. Les aides reçues, qu'elles soient matérielles ou psychologiques, importantes ou peu importantes, ont une forte valeur symbolique pour des personnes fragilisées directement ou indirectement par la maladie, et surtout par la crainte d'être mis à l'écart du fait de la maladie.

\section{Bibliographie}

DÉPARTEMENT DE LA PRÉVENTION DU VIH/SIDA, Estimation et prévision de la séropositivité du VIH/Sida au Viêt Nam pour la période 2007-2012, Hanoi, ministère de la Santé du Viêt Nam, 2009, p. 54. Link: http://pdf.usaid.gov/pdf_docs/Pnadx992.pdf. Consulté le 14 octobre 2014.

HAZRA Rohan, SIBERRY George, MOFENSON Lynne, «Growing up with HIV: children, adolescents, and young adults, the perinatally acquired HIV infection», in Annual Review of Medecine, $\mathrm{n}^{\circ}$ 61, 2010, p. 169-185. 
KIDS-ART-LINC COLLABORATION, «Low risk of death, but substantial programattrition, in Pediatric HIV treatment cohorts in Sub-Saharan Africa», in JAIDS Journal of Acquired Immune Deficiency Syndromes, , ${ }^{\circ} 5 / 49$, décembre 2008, p. 523-31.

Newell Marie-Louise, BorJa Mario-Cortina, PECKHAM Catherine, EUROPEAN COLLABORATIVE STUDY, Height, weight, and growth in children born to mothers with HIV-1 infection in Europe, in Pediatrics, $n^{\circ} 1 / 111$, janvier 2003, p. 52-60.

NGuYen Ngoc Linh, CENTRE DE MEdicine PREVEnTIVE DE LA PROVINCE DE LONG AN, Analyse de l'état des lieux et les besoins des enfants séropositifs ou influencés par le VIH/sida à la cité municipale de Tân An et dans le district de Duc Hoa, province de Long An, Études menées avec le concours de Save the Children de juillet 2005 à mai 2005. La recherche scientifique sur le VIH / sida sur la période 2000-2005, Journal of Medical Practice, $\mathrm{n}^{\circ} 528+529$, mai 11 2005, pages 270-273.

NGUYÊN Thi Thiêng, The HIV epidemic and the difficulties of children infected and affected by HIV/AIDS in Ha Long City, Quang Ninh Province, document présenté au Congrès international de Population de l'UISSP, Busan, 27-31 août 2013, 25 p.

NGUYÊN Thi Thiêng, Rapport sur l'état des lieux et les besoins des enfants séropositifs ou influences par le VIH/sida dans la ville de Halong, province de Quang Ninh. Rapport achevé en 2007 avec le concours de Care International au Viêt Nam, Hanoi, 2007, p. 62. (dact.)

Office des Statistiques, Statistical Yearbook of Viêt Nam 2009, Hanoi, Statistical Publishing House, 2009, p. 831.

OMS, Recommandations rapides sur l'utilisation des antirétroviraux pour traiter la femme enceinte et prévenir l'infection à VIH chez l'enfant, Suisse, 2010, http://www.who.int/hiv/pub/mtct/rapid_advice_mtct_fr.pdf: consulté le 14 octobre 2014.

Pham Hang Hà, Pham Thi Lan Anh, Faculté de la SAnté Publique - Université de Médecine et de Phamacie de Hô Chi Minh-Ville, «Besoin d'intégration des enfants séropositifs au centre Mai Hòa avril 2008», in Revue Médecine de Hô Chi Minh-Ville, ${ }^{\circ}$ 1/14, 2010, p. 211-217.

SCHENK Katie. «Community interventions providing care and support to orphans and vulnerable children: a review of evaluation evidence», in AIDS Care, Juillet 2009, 7/21, p. 918-42

Steele Ric G, Nelson Timothy D, Cole Brian P. «Psychosocial functioning of children with AIDS and HIV infection: review of the literature from a socioecological framework», in Journal of Developmental \& Behavioral Pediatrics, , $\mathrm{n}^{\circ} 1 / 28$, février 2007, p. 58-69.

TRAN Ngoc Thien, Les femmes et les enfants vivant avec le VIH/sida ne devraient pas être victimes de discrimination, http://www. bvss.org/day-lui-dai-dich/tin-hivaids/69ph-n-va-tr-em-nhim-hivaids-khong-ang-b-phan-bit-i-x.html, en ligne le ler août 2011, consulté le 9 décembre 2014, [TRẦ Ngọc Thiện, Phụ nữ và trẻ em nhiễm HIV/AIDS không đáng bị phân biệt đối xử, http://bvss.org/day-lui-dai-dich/tinhivaids/69-ph-n-va-tr-em-nhim -hivaids-khong-ang-b-phan-bit-i-x.html, cập nhật trên truy cập ngày truy cập ngày Thứ hai, 01 tháng 8 năm 2011] 
UNICEF VIÊT NAM, An analysis of the situation of children in Viêt Nam 2010, Hanoi, 2010, p. 312, http://www.un.org.vn/images/stories/pub_trans/SITAN_2010_vn.pdf, [En ligne, consulté le 08 janvier 2012] [UNICEF Viêt Nam, Báo cáo phân tích tình hình trẻ em tại Việt Nam 2010, Hà Nội, 312 trang, tr.128.]

YANAGISAWA Satoko, Poudel Krishna C, Jimba Masamine, " Sibling caregiving among children orphaned by AIDS: synthesis of recent studies for policy implications in Health Policy, 2-3/98, decembre 2010, p. 121-30. 


\title{
Jeunesse, genre et sexualité au Viêt Nam
}

\author{
Catherine Scornet (AMU LPED)
}

Jusqu'au début du $21^{\mathrm{e}}$ siècle, les recherches sur la sexualité au Viêt Nam étaient quasi inexistantes. Si les discussions relatives à la sexualité sont très présentes dans la vie quotidienne des Vietnamiennes et des Vietnamiens (au bureau, au marché, au restaurant populaire, au café, entre amis, entre collègues, etc., toutefois et pour reprendre le titre d'un ouvrage de deux sociologues vietnamiens

« La sexualité, il est facile d'en plaisanter, mais il est difficile d'en parler » (Khuât et coll. 2009).

Qu'est-ce qui a changé au Viêt Nam pour que la sexualité devienne un objet de débat et de discussion, et un objet de recherches?

\section{I- Les conditions d'émergence de la sexualité des jeunes au Viêt Nam}

Déjà dans les années 1960, mais surtout à l'apogée des programmes de limitation des naissances dans les années 1990, les préoccupations politiques et scientifiques en matière de population tournaient autour de la croissance démographique et des moyens de la juguler (Scornet, 2009). À l'orée du $21^{\mathrm{e}}$ siècle, on assiste à l'émergence des questions sexuelles dans le débat public, et en particulier de la sexualité des jeunes. Dans quel contexte les questions sexuelles ont-elles émergé au Viêt Nam ? Quelles en sont les conditions?

\section{$\underline{\text { I.1- La baisse de la fécondité comme condition d'émergence de la sexualité }}$}

La baisse de la fécondité est une des conditions sociale et démographique d'émergence de la sexualité et le résultat d'un changement des rapports de genre et de la sexualité. La tendance à limiter le nombre de ses enfants est un élément particulièrement important quant à ses conséquences sur la sexualité. L'apparition du contrôle des naissances signale "l'émergence d'un calcul dans le rapport des individus à la reproduction et à la vie, ainsi que dans les rapports entre les hommes et les femmes et contribue ainsi à faire sortir la sexualité et le genre de l'évidence et de la nature » (Bozon, 2002 : 26). Pour la première fois, les femmes ont eu la possibilité de dissocier sexualité et enchaînement des grossesses et des accouchements. C'est dans le contexte d'une différenciation progressive du sexe vis-à-vis des impératifs de la reproduction que peut émerger la sexualité. Les femmes ne sont plus cantonnées à un rôle de reproductrice - la baisse de la fécondité peut ainsi apparaître comme un facteur d'émancipation des femmes -, la sexualité n'a plus comme seule finalité la reproduction, elle peut aussi avoir une finalité de bien-être et de jouissance.

Le Viêt Nam illustre une situation de baisse rapide de la fécondité : en quarante ans, le comportement reproducteur des femmes vietnamiennes a été bouleversé. Ayant en moyenne 6,8 enfants sur la période 1964-1969, elles mettent au monde 2 enfants au début du XXIe siècle (en 2009, à la date du dernier recensement) (Tableau 1).

Certes, au Viêt Nam, la fécondité est une fécondité légitime. Dans la très grande majorité des cas, les naissances ont lieu dans le cadre du mariage. Le mariage est un phénomène quasi universel. En 2009, seuls 2,1\% des Vietnamiens et 5,6\% des Vietnamiennes de 45-49 ans sont célibataires (Tableau 2). La proportion des célibataires 
dans les jeunes âges ne cesse d'augmenter, quel que soit le sexe. Toutefois, même s'il augmente aussi, le célibat féminin est moins élevé que le célibat masculin pour les jeunes groupes d'âge observés au tableau 2. À 20-24 ans, en 1989, 42,5\% des Vietnamiennes sont célibataires (contre 62,4\% des hommes de leur âge), et en 2009, elles sont 50,8 \% à l'être (contre 75,6\% des hommes de leur âge).

Tableau 1- Évolution de l'indice synthétique de fécondité au Viêt Nam, 1959-2009 Période Indice synthétique de fécondité

\begin{tabular}{ll}
\hline $1959-1964$ & 6,4 \\
$1964-1969$ & 6,8 \\
$1969-1974$ & 5,9 \\
$1974-1979$ & 5,3 \\
$1979-1984$ & 4,7 \\
$1984-1989$ & 4,0 \\
$1989-1994$ & 3,3 \\
1999 & 2,3 \\
2009 & 2,0 \\
\hline
\end{tabular}

Sources: General Statistics Office 2001, 2010, Committee for Population, Family and Children 2003 p. 229.

Ce mouvement est dû à un âge moyen au mariage plus faible pour les femmes que pour les hommes et à un écart croissant de l'âge d'entrée en conjugalité selon le sexe : l'âge moyen au mariage chez les hommes a augmenté (passant de 24,5 ans en moyenne en 1989 à 26,2 ans en moyenne en 2009), alors que chez les femmes c'est un léger recul qui s'est opéré : de 23,2 ans en 1989 à 22,8 ans en 2009 (Tableau 3).

Tableau 2- Proportion de célibataires selon le sexe et l'âge, 1989, 1999 et 2009 Année

$$
\text { Hommes }
$$

Proportion de célibataires

\section{Femmes}

Proportion de célibataires

\begin{tabular}{lcccccc} 
& $15-19$ ans & $20-24$ ans & $45-49$ ans & $15-19$ ans & $20-24$ ans & $45-49$ ans \\
\hline 1989 & 95,5 & 62,4 & 1,4 & 88,6 & 42,5 & 3,3 \\
1999 & 97,5 & 69,6 & 1,5 & 90,7 & 45,7 & 5,8 \\
2009 & 97,8 & 75,6 & 2,1 & 91,5 & 50,8 & 5,6 \\
\hline
\end{tabular}

Sources : General Statistics Office 1991, 2001, 2010.

Le divorce est rare : en 2009, seulement $0,9 \%$ des hommes (de plus de 15 ans) sont divorcés ou séparés et 2,0\% des femmes (General Statistics Office 2010). Cette différence entre les sexes est fort probablement due au fait que les hommes vietnamiens ont plus d'opportunités de se remarier que les femmes, et sortent ainsi dans le recensement de la catégorie des « divorcés ». Le divorce touche certes plus les individus vivant en ville, mais l'écart est peu marqué entre les sexes : $1,2 \%$ des hommes urbains ( $0,8 \%$ des hommes ruraux), et 2,5\% des femmes urbaines (1,8\% des femmes rurales). Aujourd'hui les biographies conjugales restent peu fragmentées comme en témoigne le faible taux de divorce. Les ruptures conjugales sont liées ou ont été liées au décès du conjoint pendant les trente années de guerre qu'a connues la population vietnamienne au $\mathrm{XXe}$ siècle. On se marie pour la vie. Cependant, les nouvelles expériences sexuelles vécues pendant la jeunesse viennent rompre avec le modèle d'une vie sexuelle au cours d'une relation conjugale avec un partenaire unique toute la vie. 
Tableau 3- Évolution de l'âge moyen au premier mariage selon le sexe

\begin{tabular}{ccc} 
Année & Hommes & Femmes \\
\hline 1989 & 24,5 & 23,2 \\
1999 & 25,4 & 22,8 \\
2009 & 26,2 & 22,8 \\
\hline
\end{tabular}

Sources: General Statistical Office, 1991, 2001, 2010.

La progression rapide de la prévalence contraceptive depuis presque trente ans a enfin contribué à dissocier sexualité et fécondité : si en 1988, 53,2\% des femmes vietnamiennes utilisaient un moyen de contraception $(37,7 \%$ utilisent une méthode moderne) (NCPFP 1990), 76,2\% y ont recours en 2012 (66,6 \% utilisent une méthode moderne) (General Statistics Office 2012).

\section{I.2- Le sexe est politique}

L'autre condition d'émergence de la sexualité est une politique : ce qui change aujourd'hui c'est la politisation de la sexualité, la politisation de la sphère privée et de l'intimité. Les questions sexuelles émergent dans le débat public, et cette politisation s'est faite sous l'influence d'organisations internationales (FNUAP, PNUD, OMS) et d'organisations non gouvernementales, à la suite de la Conférence internationale sur la Population du Caire en 1994 et la Conférence mondiale sur les Femmes qui s'est tenue à Pékin en 1995 qui ont mis en avant le concept de santé de la reproduction. Ces conférences mettent en œuvre des mécanismes transnationaux qui aboutissent à des formes de législation supranationale à l'initiative des pays du nord avec des effets privilégiés dans les pays du Sud (Bozon, 2010). La sexualité est devenue au Viêt Nam un objet politique légitime en lien avec la santé sexuelle et reproductive alors que les questions sexuelles intimes étaient jusqu'alors cantonnées au non-dit familial. Ainsi, dans les années 1990, cette légitimité s'est manifestée par les premières enquêtes consacrées à la santé reproductive, mais la sexualité des jeunes n'était pas devenue un objet de recherche en tant que tel qui serait détaché de la reproduction et de la vie matrimoniale. La sexualité n'a été étudiée que dans la perspective du risque de santé qu'elle représente - grossesse, avortement, maladies sexuellement transmissibles telles que le Sida - (Gammeltoft 2002), et se concentre sur une perspective sanitaire de prévention. C'est surtout une recherche sur les problèmes sanitaires liés à la sexualité. Ainsi l'enquête sur les jeunes, SAVY 2 (Survey Assessement of Vietnamese Youth) menée en 2010, est une recherche incluse dans le projet de prévention du HIV/SIDA auprès des jeunes Vietnamiens, lancée en 2009 par l'Office général de la population et de la Planification familiale du Ministère de la Santé, et soutenu financièrement par la Banque asiatique de Développement (Ministry of Health et coll., 2012).

Par contraste, en Occident la politisation de l'intimité et de la vie sexuelle s'est faite autour de mouvements sociaux et féministes dans les années 1960 puis autour de mouvements homosexuels dans les années 1980. Ces mouvements revendiquaient une égalité de droit et une légalisation des pratiques contraceptive et abortive (loi Lucien Neuwirth en 1967, loi Simone Veil en 1975). Dans une revendication d'égalité de droit entre hommes et femmes et de maîtrise du corps féminin, les mouvements féministes ont participé à ces légalisations, alors qu'au Viêt Nam, la contraception et l'avortement ont été déjà largement promus dans le cadre d'une politique de limitation des naissances très incitative, voire coercitive, dont l'objectif initial et principal était de réguler la croissance démographique (Scornet, 2000). 


\section{II- La sexualité à l'épreuve de l'égalité : des représentations et des pratiques inégalitaires}

Au Viêt Nam comme ailleurs, les transformations de l'intimité se sont faites parallèlement à d'autres transformations dans le domaine de la famille, du couple, de la reproduction, de la politique et des rapports de genre (Giddens, 1992). Dans la revue Esprit, Maurice Godelier a écrit que «la sexualité humaine dissimule toujours en elle d'autres choses qu'elle-même " (Godelier, 2001). L'approche anthropologique développée ici (voir encadré 1) pose que ce qui se joue dans la sexualité est subordonné aux enjeux de la reproduction d'autres rapports sociaux, économiques et politiques.

\section{Encadré 1 : L’enquête}

L'enquête qualitative se fonde sur des entretiens (de jeunes et moins jeunes, personnel médical de cliniques privées pratiquant l'avortement, personnel de l'ONG Marie Stropes international, experts du FNUAP) menés en juillet et août 2011 à Hanoi ainsi que sur l'analyse de blogs, de sites internet traitant des relations sexuelles et amoureuses, d'émissions de radio telles que Cua so tinh yêu " La fenêtre de l'amour », de sites de rencontres amoureuses et amicales tels que Nôi.vn, et d'articles de presse soit féminine (ex: magazines 2! Dep, Tâp chi gia dinh) soit à destination des jeunes Vietnamiens (ex : Hoa hoc tro «Fleur d'écolier»). Depuis une dizaine d'années, la presse vietnamienne et divers sites internet se font écho de cette publicisation de la sexualité au travers de conseils en matière de sexualité aussi bien par le corps médical, que par des psychologues, des conseillers matrimoniaux, etc. Le travail présenté dans ce chapitre exploite une partie des entretiens : 25 entretiens individuels de femmes et d'hommes essentiellement jeunes, non mariés et interrogés sur leur vie sexuelle et amoureuse, les représentations de la sexualité, l'éducation sexuelle, les pratiques contraceptives, etc. Certains sont étudiants, d'autres sont déjà engagés dans une vie professionnelle. Les rencontres ont eu lieu dans les cafés qui bordent les universités, au Temple de la Littérature où de nombreux étudiants viennent faire leurs révisions, car « le lieu porte chance », le long du lac de l'Ouest, sur la rue Thanh Niên, où se promènent beaucoup de jeunes Vietnamiens. Mais afin de comprendre les changements sexuels, des personnes plus âgées et des parents ont aussi constitué le corpus. Trois entretiens de groupes auprès d'étudiants ont aussi été menés.

Une analyse des deux enquêtes quantitatives SAVY 1 et 2 a aussi servi de support au travail de recherches présenté ici.

La sexualité, en tant que produit de l'histoire, est une construction sociale autour de pratiques sexuelles, d'interactions entre partenaires, d'émotions, de représentations (Foucault, 1976). La complexité des évolutions de la sexualité tient au fait qu'elles doivent être interprétées en fonction des contextes sociaux, économiques, et politiques dans lesquels elles s'inscrivent. Ce travail de contextualisation vise à établir les relations entre des phénomènes sexuels et d'autres processus sociaux, en sachant que les limites de ce qui est considéré comme sexuel varient dans l'espace et dans le temps, d'une société à l'autre et au sein d'une même société.

Le champ de la sexualité reste marqué par des inégalités, notamment entre hommes et femmes, qui font écho à d'autres inégalités qui perdurent dans d'autres sphères sociales. On pourrait ainsi reprendre un mot d'ordre des mouvements féministes : «le privé est politique » à savoir que le privé façonne le politique dans la mesure où les règles ordonnant les rapports intimes entre les hommes et les femmes (attirance 
sexuelle, amour, vie quotidienne des couples) façonnent les interactions entre les sexes dans la sphère publique.

Dans le cadre de la politique du Đổi Mới - Renouveau - qui débute en 1986, un des enjeux majeurs de la dynamique de mutation du système politique est l'émergence d'espaces de débats publics, notamment au sein de l'Assemblée nationale qui devait devenir un réel lieu de débat (Salomon, 2004). La logique démocratique devrait s'appliquer partout, même dans le sexe, avec une double exigence: la liberté et l'égalité. Faisant suite à la politique du Renouveau, sommes-nous confrontés à de nouvelles règles du jeu et de la négociation sexuelle? Quelles sont ces transformations des représentations, des attitudes et des pratiques sexuelles dans le contexte d'une transformation rapide de la société vietnamienne ? Bien sûr, les idéaux et la réalité ne sont pas la même chose. Sommes-nous passés d'un contexte normatif rigide avec des normes sexuelles monolithiques, extérieures, dictées par la tradition, la morale, la religion, à une diversification des trajectoires affectives et conjugales et une individualisation croissante des normes ? Dès qu'il y a individualisation des pratiques, on observe des injonctions qui peuvent être tout à fait contradictoires.

\section{II.1- Les résistances}

Dans cette perspective, et pour reprendre Nathalie Bajos et Michel Bozon dans l'ouvrage qu'ils ont dirigé (2008), la vision différentialiste de la sexualité - qui situe l'origine de la différence homme/femme dans la nature - permet de légitimer des pratiques encore inégalitaires entre les sexes dans les autres sphères sociales. La thèse du déterminisme biologique très fréquente au Việt Nam à l'évocation du désir sexuel (le fait que les femmes vietnamiennes expriment peu ou pas leurs désirs sexuels, ou le fait de dire que les hommes ont des besoins sexuels plus fréquents que les femmes) permet de légitimer des pratiques inégalitaires qui sont socialement construites. Ainsi les rapports hommes femmes, et la différence construite de pouvoir entre eux, conduisent à des interactions sexuelles inégalitaires. Le nombre important d'avortements que vivent les Vietnamiennes (Committee for Population, Family and Children, 2003) soulève la question de la négociation contraceptive dans un contexte de gestion du risque de grossesse par des partenaires sexuels inégalitaires. C'est le cas par exemple dans la discussion éventuelle des partenaires autour d'une pratique contraceptive, ou dans l'absence de négociation, le fait que la femme demande ou non à son partenaire de mettre un préservatif, etc. Est-il possible, fréquent qu'une jeune fille vietnamienne demande à son partenaire de porter un préservatif lors du premier rapport sexuel et lors des rapports suivants? Nous verrons plus loin dans le chapitre qu'à peine la moitié des premiers rapports sexuels sont protégés (Ministry of Health et coll. 2012).

Un élément révélateur des résistances à traiter de la vie sexuelle, et en particulier de celle des femmes vietnamiennes, est l'exclusion des femmes non mariées, qu'elles soient célibataires, divorcées, séparées ou veuves, des enquêtes nationales sur la contraception. En raison du caractère incomplet des enregistrements à l'État civil au Viêt Nam, les données sur la fécondité sont recueillies au travers d'enquêtes sur échantillon (General Statistics Office 2011). La première enquête démographique et de santé (EDS) a été menée en 1988, elle a été suivie d'une enquête intercensitaire en 1994 puis d'une deuxième enquête démographique et de santé en 1997 et d'une troisième en 2002. Depuis 1988, les questions relatives à la contraception ne sont posées qu'aux femmes mariées. De plus, par les EDS, il n'est pas possible de connaître la fécondité illégitime. L'enquête sur les changements démographiques et la planification familiale (diêu tra biên dông dân sô va kê hoach hoa gia dinh), menée annuellement en avril par 
l'Office général des statistiques et dont les objectifs sont de recueillir des données sur les changements en matière de natalité, mortalité et migrations, et des informations de base sur la contraception et la santé reproductive des femmes, ne déroge pas à la règle : celle de 2012 n'interroge pas les femmes non mariées sur ces questions de contraception $^{55}$. Il n'existe donc pas d'information à l'échelle nationale sur les pratiques contraceptives des femmes célibataires, divorcées, séparées ou veuves. Ces fortes résistances subsistent dans la mesure où ces droits touchent directement aux rapports entre les hommes et les femmes. Dans l'échantillon des recensements de 1989 à 2009 auquel est adressé un questionnaire long, les questions sur la fécondité sont posées aux femmes de 15 à 49 ans, quel que soit leur statut matrimonial (il n'existe pas de filtre en fonction du statut matrimonial). Ces questions sont beaucoup moins détaillées que dans les EDS et non collectées auprès des hommes : le recensement a aussi ses limites et objectifs propres.

\section{II.2- Sexualité masculine juvénile versus sexualité féminine préconjugale}

Certes, les deux enquêtes SAVY (Survey Assessment of Vietnamese Youth de $2003^{56}$ et de $2010^{57}$ ) s'intéressent à la sexualité des jeunes qu'ils soient célibataires ou pas (l'échantillon de 2003 comprend 15,8 \% de jeunes, mariés, celui de 2010, 17,0 \%). En 2010, 9,5\% des jeunes interrogés (qu'ils soient mariés ou pas) disent avoir eu des relations sexuelles préconjugales. Ils étaient $7,6 \%$ en 2003. L'inégalité de genre est ici marquée, puisque la sexualité avant le mariage est presque trois fois plus importante chez les jeunes hommes $(13,6 \%)$ que chez les jeunes femmes $(5,2 \%)$.

Si l'on exclut les mariés, l'inégalité d'entrée en sexualité selon le genre est encore plus marquée. Parmi les jeunes célibataires de 14 à 25 ans, seulement 6,4\% déclarent avoir déjà eu des relations sexuelles; cette faible proportion cache des disparités de genre très marquées. Quel que soit l'âge et quelle que soit la zone de résidence, les hommes célibataires sont beaucoup plus nombreux en proportion à avoir des relations sexuelles que les femmes, comme le montre la figure 1 . Ainsi, près du tiers $(29,8 \%$ des hommes célibataires de 22-25 ans ont déjà eu des relations sexuelles, les femmes du même âge sont cinq fois moins nombreuses $(6,1 \%)$.

\footnotetext{
55 Un filtre est utilisé pour les questions sur les pratiques contraceptives. Seules les femmes mariées sont interrogées. Les femmes qui ne sont pas mariées, divorcées ou veuves ne sont pas interrogées sur leurs pratiques contraceptives. Toutefois les questions sur la fécondité sont posées à toutes les femmes de 15 à 49 ans.

56 L'enquête SAVY 1, menée en 2003 sous l'égide du Ministère de la santé, du Bureau général des statistiques, de l'Unicef et de l'OMS, est la première enquête quantitative représentative à l'échelle nationale de la jeunesse vietnamienne (Ministry of Health, GSO, 2005). Elle a interrogé 7584 jeunes de 14 à 25 ans vivant dans 42 provinces sur des sujets aussi différents que leurs relations avec leur famille, leur instruction, leur travail, leurs relations amicales, leur sexualité, la santé de la reproduction, le sida, la consommation de drogues et alcool, la violence... Une partie du questionnaire a été posée en face à face avec un enquêteur en dehors du domicile des jeunes (au Comité populaire ou à la maison de la culture de la commune) et une autre partie (notamment les questions sur les relations amoureuses et la sexualité) a été remplie par l'intéressé(e) de manière anonyme, et devait être retournée par boîte postale.

57 L'enquête SAVY 2 a été menée en 2010 auprès de 10044 jeunes de 14 à 25 ans, vivant dans les 63 provinces vietnamiennes (Ministry of Health, GSO, 2012).
} 
Figure 1- Proportion (en \%) des jeunes célibataires de 14 à 25 ans qui ont déjà eu des relations sexuelles, selon leur sexe ainsi que leur âge et leur lieu de résidence (SAVY 2, 2010)

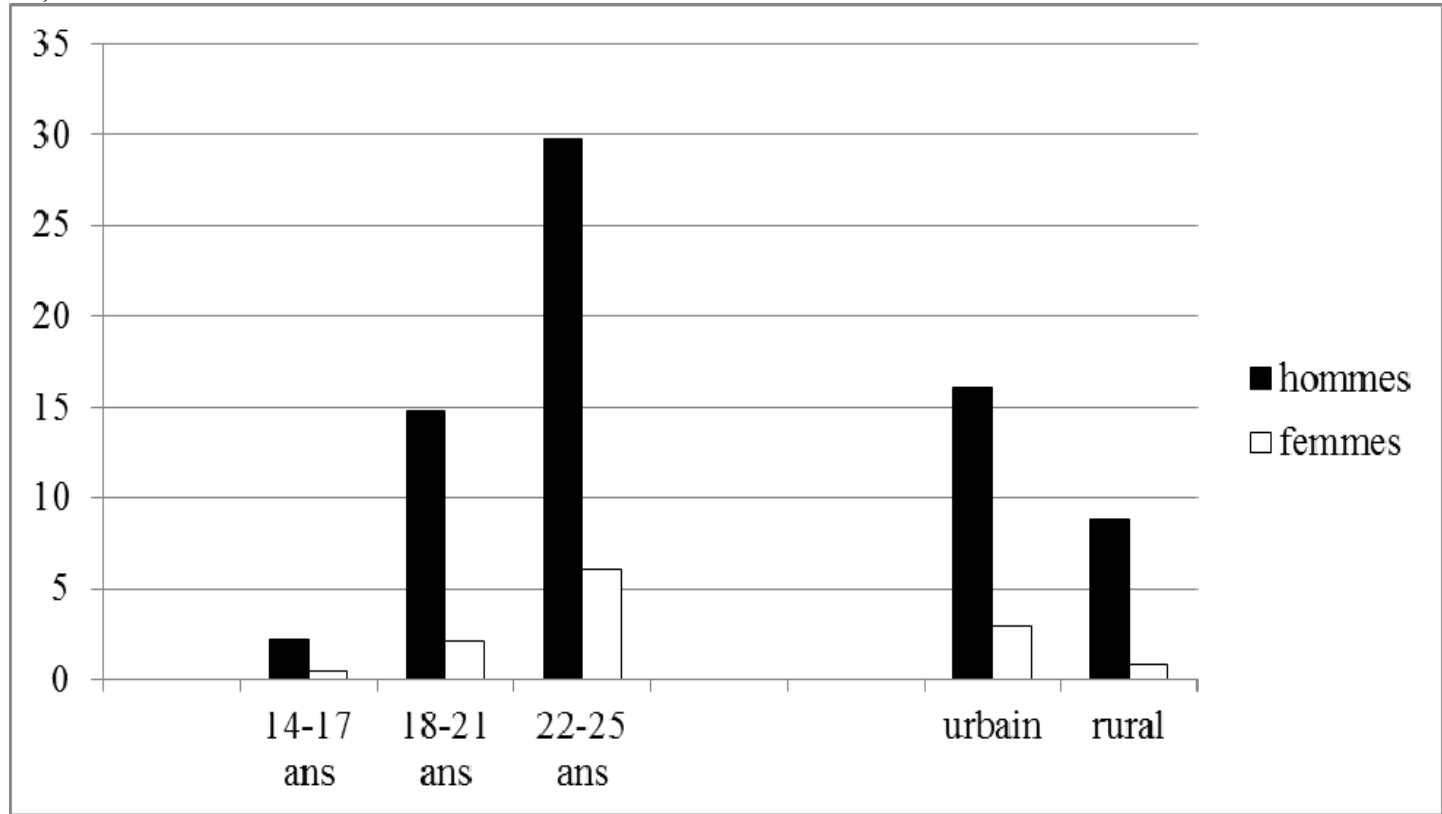

Source : Ministry of Health et coll. 2012.

La faible proportion de jeunes filles célibataires déclarant avoir des relations sexuelles est révélatrice certes d'une faible activité sexuelle des jeunes filles. Elle peut aussi être sous-évaluée par la non-déclaration des filles à avoir une activité sexuelle, ce non-dit autour de sa sexualité étant un indicateur d'une sexualité féminine stigmatisée.

L'âge moyen au premier rapport sexuel (jeunes mariés ou pas) a légèrement diminué entre les deux enquêtes (Tableau 4).

Tableau 4- Âge moyen au premier rapport sexuel des jeunes selon le sexe et l'année

\begin{tabular}{ccccc} 
Année & Enquête & Hommes & Femmes & Ensemble \\
\hline 2003 & SAVY 1 & 20,0 & 19,4 & 19,6 \\
2010 & SAVY 2 & 18,2 & 18,0 & 18,1 \\
\hline
\end{tabular}

Source : Ministry of Health et coll. 2005, 2012

Pour les sociologues John Gagnon et William Simon qui ont élaboré une théorie des « scripts sexuels » dans leur ouvrage Sexual Conduct. The Social Sources of Human Sexuality, paru en 1973, « toutes nos expériences sexuelles sont construites comme des scripts, c'est-à-dire qu'elles sont apprises, codifiées, inscrites dans la conscience, structurées, élaborées comme des récits » (cité par Bozon 2002 : 104). Ces expériences résultent de l'inculcation de règles et d'interdits, et d'une imprégnation de récits multiples. Un savoir-faire sexuel est ainsi acquis. Les individus apprennent à identifier, voire à produire des situations potentiellement sexuelles, des contextes d'un scénario sexuel avec acteurs, intrigue, cadre... susceptibles de créer une disponibilité sexuelle. Les scripts sexuels énoncent les scénarios d'une sexualité possible : dans la sexualité humaine, tout n'est pas possible à tout moment avec n'importe qui, en n'importe quelle circonstance. Les scénarios culturels disent le possible et ce qui ne doit pas être fait en matière sexuelle. Mais les individus s'approprient à leur façon des contenus culturels dont le rythme de transformation peut être très lent. Les acteurs, aux pouvoirs et aux ressources inégaux, ne partagent pas toujours le consensus qui existe en apparence au plan culturel. Cette réappropriation individuelle varie selon les contextes sociaux et 
historiques. Dans des contextes traditionnels (où l'individualisme est faible), le degré d'improvisation individuelle est faible. Avec l'évolution sociale et historique longue qui a produit l'individualisation, l'intimité et l'intériorité psychique, les scénarios culturels ont peu à peu perdu leur homogénéité, et les normes sexuelles sont devenues plus floues, moins figées. Les individus sont alors dans des situations d'adaptation mutuelle entre partenaires, et d'improvisation. Ainsi la virginité des jeunes filles au mariage au Viêt Nam était une valeur partagée et faisait consensus jusqu'aux années 1990 (Ghuman et col. 2006). Aujourd'hui certaines jeunes filles se détachent de cette norme. Qu'est-ce qui fait que l'on rompt avec un modèle partagé ? La mondialisation et la démocratisation des relations entre les hommes et les femmes sont-elles liées à la rupture de ce modèle ? Qui sont ceux et celles qui transgressent ce modèle ?

Dans le Viêt Nam contemporain, on peut faire la distinction entre une sexualité juvénile masculine - un temps à part et socialement accepté au cours duquel les garçons peuvent avoir des expériences multiples avec des femmes qui ne seront pas forcément leur épouse (des prostituées, des femmes plus âgées qu'eux) - et une sexualité préconjugale féminine - qui n'est pas encore réellement socialement acceptée, et qui est vécue comme une période préparatoire au mariage (Chamboredon 1985). La sexualité féminine n'a pas de sens en soi, c'est le couple qui lui donne un cadre légitime d'expression, et l'amour qui lui donne un sens. Ainsi Binh, 22 ans, étudiante en finances à l'Université nationale d'économie à Hanoi, souligne :

«Parce que je me respecte, je ne peux me donner qu'à celui que j'aime et celui qui deviendra mon mari. Pour les garçons, c'est différent. Ils ont plus de besoins que les filles, donc il est difficile pour eux de maîtriser leurs désirs ${ }^{58}$ (Tu, 32 ans, employée dans une agence de voyages).

Elle estime aussi que :

« L'important, c'est qu'on dédie notre virginité à l'homme qu'on aime, sinon, ce n'est pas acceptable. ${ }^{59}(\mathrm{Tu}, 32$ ans, employée dans une agence de voyages)

Tout comme Huong qui affirme :

« Pour les femmes, il faut une certaine émotion pour avoir des relations sexuelles. Sinon, ce n'est pas différent de la prostitution » (Huong, 25 ans, employée de banque).

D'après l'enquête SAVY de 2003, une majorité (92\%) des jeunes actuellement mariés qui disent avoir eu des relations sexuelles avant le mariage ont eu ces relations avec celui ou celle qui est devenu(e) leur conjoint(e). Le préjugé différentialiste en vertu duquel l'amour et la maternité sont la nécessaire finalité du destin féminin perdure (Gazalé 2012). Pour les jeunes filles vietnamiennes, la sexualité ne trouve sa place que dans le cadre d'un couple déjà formé, et dans une relation affective. Il faut que le couple préexiste à l'existence de relations sexuelles. Par conséquent, il y a une période de fréquentation chaste avant la sexualisation de la relation. L'existence de rapports sexuels est subordonnée à l'existence préalable du couple.

Celles qui contreviennent à cette règle peuvent en payer le prix. Un autre indicateur de l'absence d'une sexualité juvénile féminine, acceptée par le corps social, est le recours à la chirurgie reconstructrice de l'hymen (hyménoplastie). Celles qui enfreignent cette règle d'existence préalable d'un couple à la relation sexuelle (et/ou qui ont des relations sexuelles avec un autre homme que leur futur mari) peuvent être contraintes de recourir à la reconstitution de l'hymen. Ces opérations qui ont lieu dans

$58 \quad$ L'entretien a lieu le 6 août 2011 dans un bar pour étudiants près de l'université nationale d'économie à Hanoi.

$59 \quad$ L'entretien a lieu le 13 août 2011 
des cliniques ou centres médicaux privés (situés près de l'hôpital obstétrique de Hanoi, cliniques "spécialisées" situées dans une impasse près de l'Hôpital E dans la rue Hoàng Quôc Viêt (arrondissement de Câu Giây), centres esthétiques de l'arrondissement de Hai Bà Trung) ont un coût élevé : de 3-5 millions à 7-10 millions VND selon les cas (de 110 euros à 370 euros) ${ }^{60}$.

Des stratégies palliatives, moins onéreuses, sont aussi à l'œuvre : les jeunes filles ont recours à des artifices qui simulent l'hémorragie de la défloration en portant un hymen artificiel lors du premier rapport sexuel avec le partenaire à qui elles veulent faire preuve de leur virginité. Originaire de Chine ou du Japon, ce produit se vend à un prix qui varie de 300.000 à 10 millions VND (pour les produits japonais jugés de meilleure qualité et dont le liquide se rapproche le plus du sang humain), sur internet ou en vente directe mais discrète ${ }^{61}$ dans des échoppes de la rue Hàng Chiêu, surnommé le « quartier du plaisir ${ }^{62}$ à Hanoi. Les risques sanitaires (allergies au produit, blessures, mycoses, infections vaginales et risque de stérilité) sont importants.

C'est quand la sexualité des jeunes célibataires devient possible et qu'elle n'est plus liée à la mise en couple qu'une vision plus individualiste des relations sexuelles peut émerger plus massivement (Lagrange, 1998). En cela, hommes et femmes ne se placent pas sur un pied d'égalité. Les hommes vietnamiens répondent à un double modèle de la sexualité (une sexualité conjugale et une sexualité extra-conjugale) et bénéficient d'une représentation plus individualiste de la sexualité. Si certaines jeunes filles vietnamiennes ne sont plus tenues à rester " pures » jusqu'au mariage et gagnent peu à peu le droit à l'expérimentation sexuelle, elles n'ont en effet ni la même liberté d'action, ni le même droit à s'autoaffirmer à travers la sexualité que les garçons (Löwy, 2006). Les différences de comportements sexuels, surveillées par les figures d'autorité adultes (parents, voisins, enseignants, personnel médical, etc.), restent également maintenues par la pression collective des jeunes et le mécanisme des réputations sexuelles, notamment pour les jeunes filles, pour lesquelles une réputation sexuelle négative peut être établie. On dira d'un garçon aux multiples partenaires qu'il est Đào hoa (charmant), à la limite qu'il n'est pas sérieux "không nghiêm túc", qu'il n'est pas correct "không đứng đắn". Alors qu'une fille est appelée une phò, une femme qui change tout le temps de partenaire, une «dê $d \tilde{a} i$ »,

"Une femme facile, une femme qui peut avoir de l'intimité avec n'importe qui, comme: se tenir la main ou aller plus loin comme échanger des baisers, et même avoir des relations sexuelles.» (Thu, 20 ans, étudie la finance à l'université privée de Đông Đô).

Mais sa possibilité symétrique au masculin n'existe pas :

«On ne dit pas «dễ dãi» pour les garçons, parce qu'ils n'ont rien à perdre » (Thu, 20 ans, étudie la finance à l'université privée de Đông Đô).

Cette asymétrie découle de la domination masculine de la représentation de la sexualité.

Le manque d'information sexuelle auprès des jeunes contribue à maintenir dans l'ignorance du fonctionnement de leur corps une grande masse d'individus. Dans les enquêtes SAVY (2003 et 2010), les jeunes sont interrogés sur leur connaissance de la

60 Pour un euro $=27000$ dông au 20 mars 2013

61 Une étudiante en faculté d'économie raconte le 16 août 2011 qu'elle s'est rendue dans une de ces échoppes, et que la vendeuse lui a communiqué, contre une commission de 100000 dông, le numéro de téléphone d'une femme qui pourrait lui apporter le produit demandé dans la soirée. Le commerce de ce produit étant illégal au Viêt Nam, la discrétion s'impose.

Dans la rue Hang Chieu au cœur du quartier des 36 rues se vend, au milieu des produits cosmétiques, ce qu'on appelle par euphémisme les «do nguoi lon» («les choses pour les adultes ») : stimulants, jouets sexuels. 
période de fertilité de la femme au cours de son cycle menstruel. Seuls $13 \%$ des jeunes Vietnamiens donnent une bonne réponse (certes dans une plus grande proportion pour les jeunes femmes : $18 \%$ contre $7 \%$ des jeunes hommes). Le fait d'être une femme et de vivre en ville améliore la connaissance du cycle reproductif : $23 \%$ des jeunes femmes vivant en ville connaissent la période de fertilité (9\% des jeunes hommes urbains). La situation de cette jeune fille de 15 ans accompagnée de sa mère, toutes deux rencontrées dans une clinique privée en face de l'hôpital Bach Mai où elles consultaient pour un avortement, illustre aussi cette absence de connaissances sur la reproduction. L'adolescente n'avait pas compris qu'à la suite d'une relation sexuelle on pouvait être enceinte. Quand son ventre a commencé à s'arrondir, elle a cru qu'elle avait grossi, elle a alors commencé à faire de la gymnastique et à faire un régime. La jeune fille était à 5 mois de grossesse quand sa mère s'est rendu compte de la grossesse de sa fille.

Les premiers rapports sexuels au Viêt Nam, et en particulier le premier rapport, ne font pas l'objet, dans la majeure partie des cas, d'une préparation et d'une protection de la part des partenaires, anticipant les conséquences des rapports sexuels.

«Les jeunes Vietnamiens n'ont pas l'habitude de se préparer comme ça, de prévoir un moyen de contraception. Ils ne se préparent pas avant, mais après que ça s'est passé, on peut toujours résoudre tout de suite. ("chữa cháy": éteindre un incendie, tirer du danger, d'une mauvaise situation). Pour les filles que je connais, elles prennent souvent la pilule du lendemain après avoir eu des relations sexuelles. Elles ne prennent jamais la pilule avant d'en avoir. » (Quynh Anh, 19 ans, étudiante en gestion à l'Université Nationale d'Économie).

L'enquête SAVY 2 (2010) montre qu'à peine la moitié (46\%=185 jeunes) des jeunes ayant eu des relations sexuelles (396 jeunes sur 4168 interrogés), ont utilisé un préservatif lors du premier rapport sexuel.

«Si tout le monde découvre qu'on porte ces trucs-là avec soi (les préservatifs), on a honte. On a peur que les autres se moquent et qu'ils pensent qu'on est trop dépendants de la sexualité » (Hung, 24 ans, étudiant en médecine).

Si la sexualité juvénile pour les garçons, et préconjugale pour les filles, se répand dans la population urbaine vietnamienne, c'est souvent sans usage de la contraception. Chez les jeunes, on ne peut donc pas parler de séparation entre vie sexuelle procréative et vie sexuelle non procréative (pas encore de séparation de la sexualité et de la reproduction) comme en témoigne la forte résistance à ce que les jeunes filles vietnamiennes aient une sexualité protégée par la contraception. C'est une façon de nier l'accès des jeunes à la vie sexuelle :

«Les Vietnamiens disent souvent que si les parents parlent avec leurs enfants des moyens de contraception, ou des choses liées aux relations sexuelles, c'est inciter les enfants à en avoir. Pour les adultes, si on parle des choses qui se passent entre les garçons et les filles, c'est qu'on est mal éduqué ; et si une jeune fille connaît trop de choses sur la contraception, on dira d'elle qu'elle a déjà eu des relations sexuelles. Elle sera mal considérée » (Giang, 24 ans, jeune diplômée en interprétariat et traduction de l'Université nationale de Hanoi).

La contraception n'est utilisée que par les femmes, déjà mères, qui ont donc prouvé leur fertilité. La vie sexuelle est donc dans les premières années de la vie maritale associée à la procréation et à la fécondité. Sur l'ensemble des femmes qui utilisent un 
moyen de contraception, seules $0,8 \%$ n'ont pas d'enfant ${ }^{63}$. Avec un enfant, elles sont $22,3 \%$ à avoir recours à la contraception, elles sont $51,2 \%$ avec deux enfants et $25,7 \%$ avec trois enfants et plus (General Statistics Office 2011). La contraception n'est utilisée que par les femmes, déjà mères, qui ont donc prouvé leur fertilité. La vie sexuelle est donc dans les premières années de la vie maritale associée à la procréation et à la fécondité.

\section{Conclusion}

La préoccupation de mise en conformité de l'individu avec un idéal moral absolu (tel que la virginité des jeunes filles vietnamiennes) se heurte désormais à des expériences intimes (sexuelles, affectives et conjugales) plus diversifiées. Enfin, la transition s'opère depuis le $21^{\mathrm{e}}$ siècle entre une socialisation verticale à la sexualité fondée sur l'autorité (par l'inculcation de règles morales et de conduites, souvent édictées par les adultes, par les institutions d'encadrement de la jeunesse telles que l'école, l'union de la Jeunesse communiste, qui apparaissent comme des institutions de contrôle et d'encadrement des corps et des esprits qu'elles forment), et une socialisation horizontale, fondée sur des principes moins rigides, et transmise par les pairs - amis, frères et sœurs, jeunes internautes, étudiants qui partagent les mêmes cités universitaires - au travers de leurs expériences individuelles, et les moyens de communication tels que les journaux, internet. On observe donc au Viêt Nam une transition entre un modèle de contrôle familial et conjugal de la sexualité (qui s'exerce surtout et encore sur les adolescentes et les femmes mariées) à une représentation plus individualiste de la sexualité. Le Đổi Mới a ouvert la voie à une individualisation des normes sexuelles. Les transitions sexuelles sont à l'œuvre, dans un contexte de vulnérabilités plus marquées pour les jeunes filles.

\section{Bibliographie}

BAjos Nathalie et Bozon Michel (Dir.), Enquête sur la sexualité en France. Pratiques, genre et santé, Paris, La Découverte, 2008, 612 p.

Bozon Michel, Sociologie de la sexualité, Paris, Nathan («Collection 128), 2002, 128 p.

Bozon Michel, "Préface », in Les jeunes et la sexualité. Initiations, interdits, identités (XIXe-XXIe siècle), Blanchard Véronique, REvenIn Régis, Yvorel Jean-Jacques (éd.), Paris, Autrement («Collection Mutations/Sexe en tous genres »), 2010, 414 p.

CHAMBOREDON Jean-Claude, «Adolescence et post-adolescence : la juvénisation » in Adolescence terminée, adolescence interminable, ALLEON Anne-Marie, MORVAN Odile et LEBOVICI Serge (dir.), Paris, PUF, 1985, p. 13-28.

Committee for Population, FAmily And Children, Vietnam Demographic and Health Survey 2002, Hanoi, General Statistical Office, September 2003, 229 p.

Foucault Michel, Histoire de la sexualité. 1 : La volonté de savoir, Paris, Gallimard (« Collection Bibliothèque des Histoires »), 1976, 224 p.

63 Enquête annuelle ( $\mathrm{du} 1^{\mathrm{er}}$ avril 2011) sur les changements démographiques et la planification familiale (GSO, 2011). En 2011, la prévalence contraceptive (donc chez les femmes mariées de 15 à 49 ans) s'élève à $78,2 \%$, les méthodes modernes étant largement les plus utilisées à hauteur de $68,6 \%$. 
GadANT Monique, "Le Corps dominé des femmes, réflexions sur la valeur de la virginité (Algérie) », in L'Homme et la société, n 99/100 ("Femmes et sociétés »), 1991, p. 37-56.

GAMMELTOFT Tine, «Seeking trust and transcendence: sexual risk-taking among Vietnamese youth ", in Social Science \& Medicine, $\mathrm{n}^{\circ}$ 55, décembre 2002, p. 483496.

GAZALE Olivia, Je t'aime à la philo. Quand les philosophes parlent d'amour et de sexe, Paris Robert Laffont, 2012, 432 p.

General Statistical OfFice, Vietnam population census-1989, Detailed analysis of sample results, Hanoi, Statistical Publishing House, 1991, 168 p.

General Statistical Office, The 1999 Population and Housing Census, Hanoi, Statistical Publishing House, 2001, 646 p.

General Statistical Office, The 2009 Population and Housing Census, Hanoi, Statistical Publishing House, 2010, 893 p.

General Statistical Office, Ministry of Planning And InVestment, The 1/4/2011 Population Change and Family Planning Survey: Major Findings, Hanoi, Statistical Publishing House, 2011, 339 p.

General Statistical OfFice, Ministry OF Planning And InVESTMent, The 1/4/2012 Time-Point Population Change and Family Planning Survey: Major Findings, Hanoi, Statistical Publishing House, 2012, 334 p.

GHuman Shanon, Vu Manh Loi, Vu Tuan Huy et KNODEL John, «Continuity and Change in Premarital Sex in Vietnam», in International Family Planning Perspectives, $\mathrm{n}^{\circ}$ 32, December 2006, p. 166-174.

GIDDENS Anthony, The Transformation of Intimacy, Sexuality, Love and Eroticism in Modern Societies, Oxford, Polity Press, Blackwell Publishing Ltd., 2002, p. 216.

GODELIER Maurice, «La sexualité est toujours autre chose qu'elle-même », in Esprit, mars-avril 2001, p. 96-104.

KHuÂt Thu Hông, LE Bach Duong, NGuYÊn Ngoc Huong, Sexuality in Contemporary Vietnam. Easy to Joke about but Hard to Talk about, Hanoi, Knowledge Publishing House, 2009, 420 p.

LAGRANGE Hugues, "Le sexe apprivoisé ou l'invention du flirt» in Revue Française de Sociologie, n¹, 1998, p. 139-176.

Lowy Ilana, L'emprise du genre: masculinité, féminité, inégalité, Paris, La Dispute Collection (« Le Genre du monde »), 2006, 227 p.

Ministry of Health, General Statistics Office, Unicef, World Health OrganizATION, 2003 Survey Assessment of Vietnamese Youth, Hanoi, Interuniversity Consortium for Political and Social Research, University of Michigan, 2005, 112 p.

Ministry of Health, General Statistics Office, World Health Organization AND THE United NATiOns ChILDREN's Fund, 2010 Survey Assessment of Vietnamese Youth Round 2. (SAVY 2) Hanoi, Asian Development Bank, Viet Nam, 2012, $215 \mathrm{p}$. 
National Committee for Population and Family Planning (NCPFP), 1988 Viet Nam Demographic and Health Survey, Hanoi, National Committee for Population and Family Planning, 1990, 185 p.

SALOMON Mathieu, Les arcanes de la « démocratie socialiste » vietnamienne. Évolution des assemblées populaires et du système juridique depuis le lancement du Dôi Moi, Paris, Les Etudes du CERI, $\mathrm{n}^{\circ}$ 104, Centre d'Etudes et de Recherches Internationales, Sciences Po., 2004, 36 p.

SCORNET Catherine, «Un exemple de réduction de la baisse de la fécondité sous contraintes: la région du delta du fleuve Rouge au Viêt Nam », in Population, n⿳055/2, INED, Paris, 2000, p. 265-300.

SCORNET Catherine, "State and the Family», in Reconfiguring Families in Contemporary Vietnam, BARBIERI Magali, Belanger Danièle (éd.), Stanford, Stanford University Press, («Series on Contemporary Issues in Asia and the Pacific»), 2009, p. 47-74. 



\title{
Migration interne au Viêt Nam : tendances et caractéristiques
}

\author{
Nguyên Thi Thiêng (UNE IPSS) \\ Luu Bich Ngoc (UNE IPPS)
}

Depuis 1986, Le Viêt Nam a mis en œuvre la politique du Renouveau « dôi moi » comprenant la transition d'une économie planifiée à une économie de marché avec orientation socialiste. Par ailleurs, il a mis en place des politiques visant à attirer les investissements étrangers. Depuis lors, l'économie n'a cessé de croître. Selon le rapport sur la situation socio-économique de la décennie 2001-2010, le taux annuel moyen de croissance du produit intérieur brut a été de 7,26\%. Avec une telle croissance, le Viêt Nam se situe juste derrière la Chine et l'Inde, et devance la Corée du Sud, la Thaïlande, Singapour, l'Indonésie, la Malaisie et les Philippines (Office général des Statistiques, 2011). Depuis 2008, il est sorti du groupe des pays à faible revenu pour faire partie des pays à revenu faible moyen. Cependant, la croissance économique n'est pas homogène. Si le revenu de toutes les couches de la population ${ }^{64}$ a augmenté au cours des dernières années, cette croissance a été faible pour une partie de la population. Par conséquent, l'écart entre les pauvres et les riches est relativement élevé et a tendance à croître (Office général des Statistiques, 2011). De plus, les régions ${ }^{65}$ qui ont des conditions naturelles favorables et un développement des infrastructures attractifs pour les investissements étrangers ont des revenus plus élevés que les zones essentiellement agricoles. Elles ont augmenté leurs parcs industriels et créent des emplois. Le développement inégal entre les régions socioéconomiques a suscité une demande en travailleurs pour les régions qui ont développé des entreprises industrielles aux revenus élevés. Cela a entraîné des flux de migration rurale-urbaine. Ces flux deviennent maintenant de plus en plus puissants. Ils ont été favorisés par le développement des infrastructures de circulation de l'information, de communication et de transport au cours des cinq dernières années (Office général des Statistiques, 2011a).

Ce chapitre se concentre sur la recherche des tendances, causes et problèmes sociaux auxquels les migrants internes doivent faire face. Il utilise pour cela quatre sources de données. La première est constituée des recensements de la population de 1999 et 2009. La deuxième est l'Enquête nationale sur les migrations réalisée en 2004 dans 11 provinces appartenant à 5 zones au nord, au centre et au sud du pays ${ }^{66}$. La troisième est l'Enquête sur la migration, la pauvreté et l'environnement urbain dont la collecte a eu lieu en 2007 (Gubry et coll., 2007). Enfin, nous analysons les résultats d'une petite enquête réalisée par l'Institut d'Étude sur la Population et la Société de l'Université Nationale d'Économie en 2009 auprès de jeunes immigrés travaillant dans le secteur informel à Hanoi (Nguyen et al 2009). Ces sources sont complémentaires les unes des

\footnotetext{
${ }^{64} \mathrm{Au} 1^{\text {er }}$ avril 2009, selon le Recensement de la population et de l'habitat, la population était de 85789573 habitants avec un taux de croissance annuel moyen de 1,2\% pendant la période 1999-2009 (Office général des Statistiques, 2010). Entre les recensements de 2009 et 1999, la population du Viêt Nam a augmenté de 11,3\% (Office général des Statistiques, 2010).

${ }^{65}$ Le Viêt Nam, qui occupe une superficie de $331212 \mathrm{~km}^{2}$, est divisé en 6 zones géographiques et socioéconomiques: la région montagneuse au Nord (28,8\% de la surface totale du pays); le Delta du fleuve Rouge $(6,3 \%)$; le Centre septentrional et les côtes centrales $(29,0 \%)$; les Hauts Plateaux du Centre (16,5 $\%)$; le sud-est (7,1\%) et le delta du Mékong (12,3\%).

${ }^{66}$ Il s'agit de Hanoi, de la zone économique du nord-est y compris Quang Ninh, Haiphong et Hai Duong, des Hauts-Plateaux du Centre y compris Gia Lai, Dak Lak, Dak Nong, Lam Dong, d'Hô Chi Minh-Ville et de la zone industrielle Sud-Est, incluant Binh Duong et Dong Nai. Dans chaque région, 1000 migrants et 1000 non-migrants ont été interrogés.
} 
autres. En effet, si les recensements permettent une large couverture du pays, ils comportent plusieurs limites. Ils ne comptent que les personnes qui résident de manière permanente dans les localités enquêtées (celles qui y vivent depuis six mois et plus) sans tenir compte des migrants provisoires (qui y vivent depuis moins de six mois) et des migrants mobiles qui résident tantôt dans une localité, tantôt dans une autre. Ils ne collectent pas non plus d'informations concernant la raison du déplacement ni les difficultés et les défis que rencontrent les migrants sur leurs lieux d'arrivée. Aussi, les résultats d'enquêtes constituent un apport précieux.

La définition de la migration interne dans ce chapitre est celle qui a été utilisée pour les recensements, selon lesquels " une personne est considérée comme migrante si ses lieux de résidence au moment du recensement et cinq ans auparavant ne se situent pas dans la même commune » (unité administrative la plus petite) (Comité de pilotage central du Recensement de la population et du logement de 2009, 2010). Elle permet de répartir les migrations en quatre types selon l'unité administrative concernée, chacune étant comprise dans l'unité de niveau supérieur. Par ordre croissant, on distingue les migrations entre communes, districts et provinces. Les migrations entre communes s'effectuent d'une commune à une autre à l'intérieur d'un même district. Celles entre districts ont lieu au sein d'une même province. Un migrant entre provinces peut également avoir migré entre régions si ces provinces sont situées dans deux régions différentes.

Les résultats montrent que l'on observe une augmentation des migrations internes de 1999 à 2009, notamment au départ du delta du Mékong, de la région Centre Nord ainsi que des Côtes centrales, la principale région d'immigration étant le Sud-est (voir Carte 1 en tête d'ouvrage). Les caractéristiques des migrants, les causes de leurs migrations et les difficultés qu'ils rencontrent sont ensuite analysées.

\section{I- Augmentation des migrations internes de 1999 à 2009}

De 2004 à 2009, près de 7 millions de personnes ont migré, soit une hausse de près de $30 \%$ par rapport à la période 1994-1999. En effet, au cours de la période 2004-2009 on dénombrait un effectif de migrants supérieur à celui de la période 1994-1999 de 2,2 millions de personnes, dont 1,4 million entre provinces (Tableau 1).

Tableau 1: Population selon le statut migratoire en 1999 et 2009

\begin{tabular}{lcccccc} 
Type de migration & \multicolumn{2}{c}{$\begin{array}{c}\text { Effectif des migrants } \\
\text { (milliers) }\end{array}$} & $\begin{array}{c}\text { Effectif des non-migrants } \\
\text { (milliers) }\end{array}$ & \multicolumn{2}{c}{$\begin{array}{c}\text { Taux de migration } \\
\text { (pour mille) }\end{array}$} \\
& $1994-1999$ & $2004-2009$ & $1994-1999$ & $2004-2009$ & $1994-1999$ & $2004-2009$ \\
\hline Même district & 1343 & 1618 & 67808 & 76893 & 19 & 21 \\
Interdistricts & 1138 & 1709 & 68013 & 76802 & 16 & 22 \\
Inter provinces & 2001 & 3398 & 67150 & 75113 & 29 & 43 \\
\hline Ensemble & 4482 & 6725 & 202971 & 228808 & 29 & 43 \\
\hline Inter régions & 1334 & 2361 & 67817 & 76150 & 19 & 30 \\
\hline
\end{tabular}

Note : Le taux de migration correspond au nombre de migrants pour 1000 habitants âgés de 5 ans et plus au moment du recensement. Les migrants sont ceux qui n'étaient pas présents dans le ménage 5 ans avant le recensement.

Source: Comité central de pilotage du recensement de la population et du logement du Viêt Nam de 2009, 2010, p.77 
La migration du milieu rural au milieu urbain est la forme principale de migration entre districts et entre provinces (Tableau 2). En 1999, la migration du milieu rural au milieu rural était la plus fréquente avec cependant un faible écart entre migrations au départ du milieu rural. Ainsi, la tendance à migrer du milieu rural vers le milieu urbain a commencé dès la fin des années 1990 et est devenue de plus en plus fréquente durant la première décennie du $\mathrm{XXI}^{\mathrm{e}}$ siècle. Au sein d'un même district, la migration entre localités rurales est la plus fréquente. Vient ensuite la migration entre localités urbaines.

Tableau 2: Répartition des migrants selon leurs milieux de résidence et leur sexe (\% du total des migrants)

\begin{tabular}{|c|c|c|c|c|c|c|c|c|c|}
\hline & \multicolumn{9}{|c|}{ Recensement de 2009} \\
\hline & \multicolumn{3}{|c|}{ Dans un même district } & \multicolumn{3}{|c|}{ Entre districts } & \multicolumn{3}{|c|}{ Entre provinces } \\
\hline & $\begin{array}{c}\text { Hom- } \\
\text { mes }\end{array}$ & $\begin{array}{r}\text { Fem- } \\
\text { mes }\end{array}$ & Total & $\begin{array}{c}\text { Hom- } \\
\text { mes }\end{array}$ & $\begin{array}{r}\text { Fem- } \\
\text { mes }\end{array}$ & Total & $\begin{array}{c}\text { Hom- } \\
\text { mes }\end{array}$ & $\begin{array}{r}\text { Fem- } \\
\text { mes }\end{array}$ & Total \\
\hline rural-rural & 32.7 & 47.9 & 42.3 & 19.2 & 25 & 22.5 & 34.7 & 36.0 & 35.4 \\
\hline rural-urbain & 11.0 & 11.1 & 11.1 & 23.6 & 25.4 & 24.6 & 44.2 & 44.8 & 44.5 \\
\hline urbain-rural & 7.7 & 6.1 & 6.7 & 13.4 & 11.3 & 12.2 & 8.0 & 6.7 & 7.3 \\
\hline urbain & 48.6 & 35.0 & 40,0 & 43.8 & 38.3 & 40.7 & 13.1 & 12.5 & 12.8 \\
\hline Ensemble & 100.0 & 100.0 & 100.0 & 100.0 & 100.0 & 100.0 & 100.0 & 100.0 & 100.0 \\
\hline Effectif & 563364 & 972602 & 1535966 & 724635 & 940352 & 1664987 & 1565461 & $\begin{array}{r}176687 \\
1 \\
\end{array}$ & 3332331 \\
\hline
\end{tabular}

\begin{tabular}{|c|c|c|c|c|c|c|c|c|c|}
\hline & \multicolumn{9}{|c|}{ Recensement de 1999} \\
\hline & \multicolumn{3}{|c|}{ Dans un même district } & \multicolumn{3}{|c|}{ Entre districts } & \multicolumn{3}{|c|}{ Entre provinces } \\
\hline & $\begin{array}{c}\text { Hom- } \\
\text { mes }\end{array}$ & $\begin{array}{c}\text { Fem- } \\
\text { mes }\end{array}$ & Total & $\begin{array}{c}\text { Hom- } \\
\text { mes }\end{array}$ & $\begin{array}{c}\text { Fem- } \\
\text { mes }\end{array}$ & Total & $\begin{array}{c}\text { Hom- } \\
\text { mes }\end{array}$ & $\begin{array}{c}\text { Fem- } \\
\text { mes }\end{array}$ & Total \\
\hline rural-rural & 36.1 & 45.7 & 41.7 & 26.9 & 28.9 & 28.0 & 39.1 & 39.0 & 39.1 \\
\hline rural-urbain & 21.1 & 17.8 & 19.2 & 20.2 & 20.9 & 20.6 & 34.5 & 37.8 & 36.2 \\
\hline $\begin{array}{l}\text { urbain-rural } \\
\text { urbain- }\end{array}$ & 10.0 & 8.0 & 8.8 & 12.3 & 10.8 & 11.5 & 10.5 & 7.9 & 9.2 \\
\hline urbain & 32.8 & 28.5 & 30.3 & 40.6 & 39.4 & 39.9 & 15.8 & 15.3 & 15.6 \\
\hline Ensemble & 100.0 & 100.0 & 100.0 & 100.0 & 100.0 & 100.0 & 100.0 & 100.0 & 100.0 \\
\hline Effectif & 536805 & 741579 & 1278384 & 506011 & 612430 & 1118441 & 975605 & 978576 & 1954181 \\
\hline
\end{tabular}

Sources : Ministère du Plan et de l'Investissement et Office général des Statistiques, 2010, p. 110.

\section{II- Des régions contrastées}

Les taux d'émigration et d'immigration varient selon les régions (voir Figure 1, Carte 1 en tête d'ouvrage et Tableau 8 en annexe).

Le tableau 4 décrit l'émigration durant les cinq années précédant les recensements de 1999 et 2009. Il montre que le delta du Mékong ainsi que la région Centre Nord et Côtes centrales connaissent la plus forte émigration. Au cours de la période 1994-1999, les émigrés en provenance de ces deux régions représentaient $48 \%$ du total des émigrés. Pour la période 2004-2009, cette proportion s'élève à 64\%. Ainsi, la tendance s'est accentuée. Pour le Centre Nord et Côtes centrales, le taux d'émigration a été multiplié par plus de 3 entre les deux recensements. Bien que l'émigration soit plus faible dans les Hauts-Plateaux du Centre, le taux d'émigration y a été multiplié par 1,5 pendant 
cette période. L'émigration relativement forte du delta du Fleuve Rouge a eu tendance à diminuer ou à ne pas augmenter.

Figure 1: Taux d'émigration selon les régions entre 1994-1999 et 2004-2009

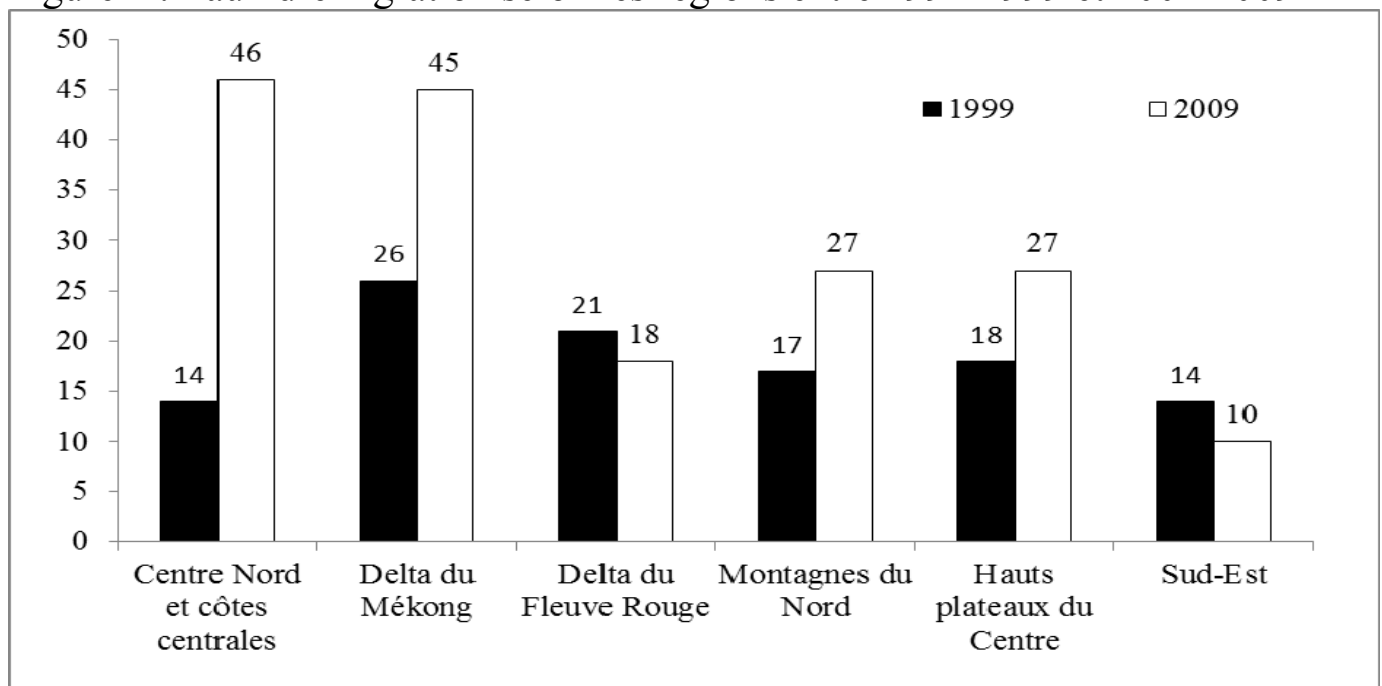

Note : Le taux de migration correspond au nombre de migrants pour 1000 habitants âgés de 5 ans et plus au moment du recensement. Les migrants sont ceux qui n'étaient pas présents dans le ménage 5 ans avant le recensement.

Source: Comité central de pilotage du recensement de la population et du logement du Viêt Nam de 2009, 2010, p.79

Les flux migratoires durant la période 2004-2009 se dirigent principalement vers les provinces hautement urbanisées. La migration du milieu rural au milieu rural qui constituait la tendance principale des périodes précédentes a été remplacée par la migration du milieu rural au milieu urbain. La figure 2 nous montre que le Sud-est est la première zone d'immigration. En cinq ans (2004-2009), 1635000 personnes y ont immigré, soit le triple du nombre atteint en 1994-1999 (tableau 3). À cette même date, le taux d'immigration y est de 127 pour mille (Tableau 4), soit trois fois plus élevé que dans les Hauts Plateaux du centre, huit fois plus élevé que dans le delta du Fleuve Rouge et 32 fois plus élevé que dans le delta du Mékong. Cette région qui selon les résultats du recensement de 2009 compte 14025000 personnes, soit 16,34\% de la population du Viêt Nam, est la plus développée économiquement. Elle a un taux d'urbanisation de 57,1 \% (Office général des Statistiques, 2010, p. 63). Elle contribue à plus de deux tiers des recettes budgétaires annuelles du pays. Les provinces de Hô Chi Minh-Ville, Binh Duong et Dông Nai y forment un quadrilatère de développement économique. Celui-ci, malgré une superficie qui ne représente que $7,1 \%$ de la superficie du pays, joue un rôle décisif pour le rythme de croissance socio-économique nationale. Selon les chiffres de 2004, il génère 37,40\% du PIB national, 47,12\% de la valeur de la production industrielle, et 55,76\% des recettes budgétaires (Official Binh Duong Province Website, 2014). En 2012, la province de Binh Duong a attiré à elle seule $17,1 \%$ du volume total des investissements directs étrangers. Elle connait un rythme de croissance élevé. Son PIB progresse en moyenne de 14,5\% par an (Official Binh Duong Province Website, 2014). En 2010, l'industrie représente 63\% du PIB, les services 32,6\%, l'agriculture et la sylviculture 4,4\% (Official Binh Duong Province Website, 2014). Binh Duong possède 28 zones et groupes industriels sur plus de 8.700 ha, avec plus de 1200 entreprises dont les fonds s'élèvent à plus de 13 milliards de dollars (Official Binh Duong Province Website, 2014). Le taux d'immigration dans cette 
province s'élève à 365,9 pour mille. Durant la période 2004-2009, un tiers des habitants de Binh Duong étaient des immigrants.

Figure 2: Effectifs des immigrés et émigrés durant les cinq années précédant le recensement de 2009 selon les régions

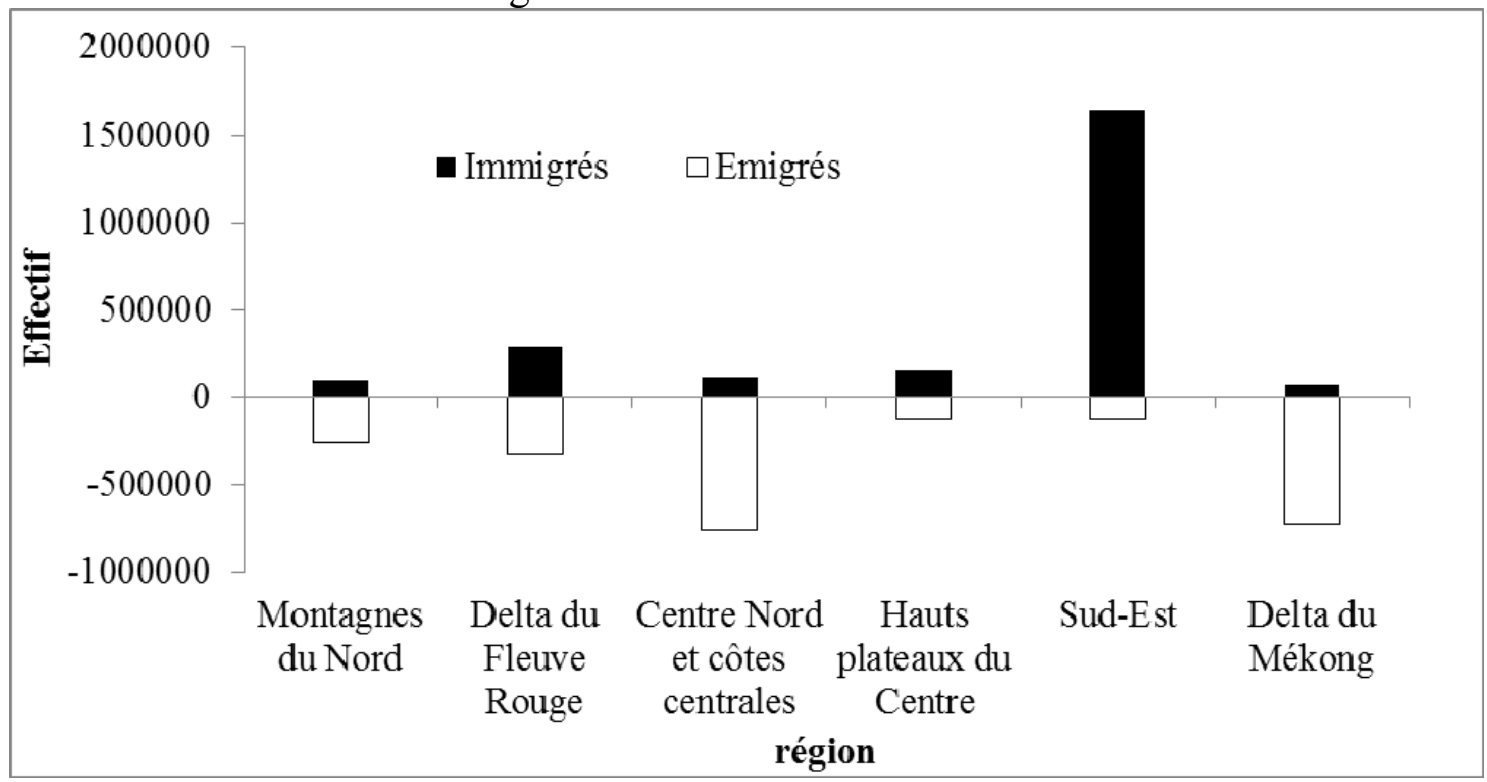

Source: Recensement de la population et du logement, 2010.

Le Fleuve Rouge est la deuxième région d'immigration du pays. Durant la période 2004-2009, elle a attiré près de 290000 migrants. Ainsi, le delta du Fleuve Rouge et le Sud-Est attirent $81,5 \%$ des immigrants. La valeur de la production du delta du Fleuve Rouge a connu une forte hausse passant de 44100 milliards de dôngs en 2000 à 168400 milliards en 2009, soit 25,7\% du PIB industriel du pays. Fin 2009 le delta du Fleuve Rouge comptait 61 zones industrielles occupant 13800 ha dont 9400 ha de terres agricoles louées. À l'échelle nationale, il possédait $26 \%$ des zones industrielles et $23 \%$ des superficies de terrains étaient occupées par ces zones industrielles. Les localités qui regroupaient le plus d'industries étaient Hanoi et Nam Dinh qui constituent également des centres économiques régionaux. Le delta du Fleuve Rouge est également une région à forte émigration. Celle-ci étant plus forte que l'immigration, le taux net migratoire est négatif (-2 pour mille).

Si, durant la période 1994-1999, les Hauts Plateaux du centre avaient le plus fort taux d'immigration (93 pour mille), en 2004-2009 l'attractivité de cette région a fortement baissé. Le nombre d'immigrants y a été réduit de moitié et le taux d'immigration a également été fortement réduit (-57 pour mille) pour atteindre 36 pour mille en 20042009 alors que l'évolution de ce taux est insignifiante dans les autres régions.

À l'exception du sud-est où le taux net de migration est positif en milieu rural, dans toutes les autres régions, ce taux est négatif ou nul (Figure 2). Cela montre que la tendance à migrer du milieu rural vers le milieu urbain a été très forte durant la période 2004-2009.

Le delta du Mékong et la région Centre Nord-côtes centrales ont les taux nets de migration négatifs les plus marqués. Comme nous l'avons analysé auparavant, ces régions sont dynamiques et particulièrement attractives sur le plan économique. Si, pour le recensement de 1999, les Hauts Plateaux du centre constituaient la région la plus attractive pour les migrants, ce n'était plus le cas en 2009, mais il reste tout de même la troisième région en termes d'immigration. 
Tableau 3 : Taux d'immigration selon les recensements de 1999 et 2009

\begin{tabular}{lrrrrr} 
Région & $\begin{array}{c}\text { Effectif des immigrants } \\
\text { (milliers) }\end{array}$ & \multicolumn{2}{c}{ Taux d'immigration } & \multicolumn{2}{c}{$\begin{array}{c}\text { Différence } \\
\text { (pour mille) }\end{array}$} \\
& $1994-$ & $2004-$ & $1994-$ & $2004-$ & $2004-2009$ \\
& 1999 & 2009 & 1999 & 2009 & $1994-1999$ \\
\hline Sud-est & 580 & 1635 & 63 & 127 & 64 \\
Delta du Fleuve Rouge & 163 & 289 & 11 & 16 & 5 \\
Hauts-Plateaux du Centre & 326 & 166 & 93 & 36 & -57 \\
Régions montagneuses du nord & 84 & 91 & 9 & 9 & 0 \\
Centre septentrional, côtes centrales & 114 & 110 & 7 & 6 & -1 \\
Delta du Mékong & 67 & 70 & 5 & 4 & -1 \\
\hline Total du Pays & 1334 & 2361 & 19 & 30 & 11 \\
\hline
\end{tabular}

Source: Recensement de la population et du logement, 2010.

Tableau 4 : Taux moyens d'immigration, d'émigration et taux moyens net de migration selon le type de migration et le milieu de résidence (pour mille) durant les cinq années précédant le recensement de 2009

\begin{tabular}{lrrrrrrrrrrr} 
& \multicolumn{3}{c}{ Taux d'immigration } & \multicolumn{3}{c}{ Taux d'émigration } & \multicolumn{2}{c}{ Taux net de migration } \\
& \multicolumn{4}{c}{$\begin{array}{c}\text { En- } \\
\text { sem- } \\
\text { Région }\end{array}$} & $\begin{array}{c}\text { urbain } \\
\text { rural }\end{array}$ & $\begin{array}{c}\text { brbain } \\
\text { rural }\end{array}$ & $\begin{array}{c}\text { sem- } \\
\text { ble }\end{array}$ & urbain & $\begin{array}{c}\text { rural } \\
\text { En- } \\
\text { sem- } \\
\text { ble }\end{array}$ \\
\hline Montagnes du nord & 30,5 & 5,0 & 9,0 & 22,6 & 24,1 & 27,0 & 7,9 & $-19,1$ & -18.0 \\
Delta du Fleuve Rouge & 25,7 & 10,2 & 16,0 & 12,8 & 18,2 & 18,0 & 12,9 & $-8,0$ & -2.0 \\
Centre Nord, côtes centr. & 11,5 & 4,1 & 6,0 & 19,9 & 47,0 & 45,0 & $-8,3$ & $-42,9$ & -38.0 \\
Hauts plateaux du Centre & 42,4 & 27,2 & 36,0 & 21,5 & 24,9 & 27,0 & 20,9 & 2.2 & 9.0 \\
Sud-Est & 125,3 & 104,2 & 127,0 & 10,5 & 6,3 & 10,0 & 114,8 & 97,9 & 117.0 \\
Delta du Mékong & 6,0 & 3,5 & 4,0 & 16,2 & 49,7 & 46,0 & $-10,2$ & $-46,2$ & -42.0 \\
\hline
\end{tabular}

Sources: Calculs des auteurs à partir des chiffres du Ministère du Plan et de l'Investissement et de l'Office général des Statistiques, 2010, p. 114.

\section{III- Les migrants : caractéristiques et motivations}

Les résultats du recensement de 2009 montrent que les migrants sont pour la plupart jeunes. Leur âge médian n'est que de 25 ans tandis que celui des non-migrants est de 30 ans (Office général des Statistiques, 2011). Ces résultats sont similaires à ceux des enquêtes précédentes. Parmi les migrants enquêtés, la part de ceux âgés de moins de 30 ans est de 50,4\%. Cette proportion est de $73 \%$ à Hô Chi Minh-Ville et de $72 \%$ dans les zones industrielles du Sud-est et du Nord-est » (Office général des Statistiques, 2005). La majorité des migrants de moins de 30 ans sont célibataires. Ceux-ci représentent $51 \%$ des immigrants à Hanoi et 68\% à Hô Chi Minh-Ville (période 1986-1996). L'enquête sur la migration en 2004 montre qu'avant leur première migration, $70 \%$ des hommes et $66 \%$ des femmes sont célibataires (Office général des Statistiques, 2005).

Les résultats du recensement montrent également une particularité intéressante : les femmes migrent de plus en plus. Durant la période 1994-1999, il y avait 105 femmes migrantes pour 100 hommes migrants. Dans le recensement de 2009, ce rapport est de 109 femmes pour 100 hommes (Office général des Statistiques, 2011). Dans l'ensemble, les femmes migrent plus souvent au sein d'un même district que les hommes. Ceux-ci effectuent davantage de migrations de plus longues distances (Tableau 5). Par ailleurs, la migration à l'intérieur du milieu rural est plus fréquente 
chez les femmes tandis que les hommes pratiquent plus la migration entre zones urbaines. Cela est vrai pour les deux recensements de 2009 et 1999.

Tableau 5. Taux moyens nets de migration des hommes et des femmes selon le type de migration durant les cinq années précédant les recensements de 1989, 1999 et 2009 $(\%)$

\begin{tabular}{lrrrrrr} 
& \multicolumn{2}{c}{1989} & \multicolumn{2}{c}{1999} & \multicolumn{2}{c}{2009} \\
& Hommes & \multicolumn{1}{c}{ Femmes } & Hommes & Femmes & Hommes & Femmes \\
\hline Même district & - & - & 41.8 & 58.2 & 36.4 & 63.6 \\
Interdistricts & 48.2 & 51.8 & 45.2 & 54.8 & 43.4 & 56.6 \\
Inter provinces & 57.3 & 42.7 & 50.0 & 50.0 & 46.9 & 53.1
\end{tabular}

Note : Le taux net de migration correspond à la différence des taux d'immigration et d'émigration.

Sources: Calculs des auteurs fondés sur les chiffres du Ministère du Plan et de l'Investissement et de l'Office général des Statistiques, 2010, p. 107.

Les recensements de 1999 et 2009 ne collectant pas d'information sur les motivations de migration, ce chapitre utilise les données de l'enquête nationale sur la migration de 2004 (Office général des Statistiques et UNFPA, 2005, p. 42) et d'autres études telles que : "Migration, pauvreté et environnement urbain » (Gubry et coll., 2007) et «Jeunes migrants travaillant dans le secteur informel à Hanoi», cette deuxième étude portant sur une population âgée de 15 à 24 ans (Nguyen et coll., 2009).

Selon les résultats de l'Enquête nationale sur la migration de 2004, les principales motivations de la migration renvoient à des facteurs économiques et à la situation du lieu d'immigration. Plus de 50\% des migrants déclarent qu'ils avaient trouvé du travail, tandis que $47 \%$ répondent qu'ils voulaient améliorer leurs conditions de vie (Office général des Statistiques et UNFPA, 2005). L'Enquête sur la migration, la pauvreté et l'environnement urbain (Gubry et coll., 2007) donne comme principale raison de la migration de Hanoi vers Hô Chi Minh-Ville le souhait d'améliorer ses conditions de vie : meilleures infrastructures, logement ou location moins chers, emploi, recherche d'un lieu pour le commerce (tableau 6). Enfin, l'Enquête sur les jeunes migrants qui travaillent dans le secteur informel à Hanoi met en avant des revenus plus élevés et des opportunités d'emploi comme principaux facteurs d'attraction des jeunes vers la capitale politico-administrative.

L'augmentation des revenus est le facteur le plus attrayant à Hanoi. Elle est mentionnée par $60,5 \%$ des répondants. Viennent ensuite l'obtention d'un emploi $(43,6 \%)$ et les études $(13,6 \%)$. Cette réalité correspond à la tendance commune au Viêt Nam. En effet, les principales motivations de la migration sont l'augmentation des revenus $(46,3 \%)$, l'obtention d'un emploi $(23,1 \%)$, les études $(10 \%)$, le rapprochement familial $(4,0 \%)$. D'autres raisons telles que le mariage ou le traitement des maladies n'occupent qu'une part très faible (Nguyen et coll., 2009). 
Tableau 6 : Raison du changement de résidence (\%) selon la ville de résidence en 2007 (plusieurs réponses possibles)

\begin{tabular}{lrr}
\multicolumn{1}{c}{ Raison du changement de résidence } & Hanoi & $\begin{array}{c}\text { Hô Chi } \\
\text { Minh-Ville }\end{array}$ \\
\hline Meilleures infrastructures & 33,9 & 18,1 \\
Meilleur logement ou location moins chère & 28,9 & 30,0 \\
Meilleur environnement du milieu d'habitat & 25,2 & 14,8 \\
Propriétaire ne veut plus louer le logement & 18,0 & 4,6 \\
Devenu propriétaire & 15,7 & 19,4 \\
Études & 13,4 & 5,1 \\
Séparation d'un ménage & 11,0 & 9,3 \\
Recherche d'un meilleur travail & 10,9 & 22,4 \\
Rapprochement familial & 10,2 & 6,3 \\
Affaires, artisanat & 7,8 & 11,8 \\
Mariage, divorce & 7,0 & 5,0 \\
Relogement (plan d'urbanisation, décongestionnement) & 3,9 & 9,2 \\
\hline Effectif des ménages interrogés & 128 & 236 \\
\hline
\end{tabular}

Source : Gubry et coll., 2007, Enquête Migration, pauvreté et environnement urbain

\section{IV-Difficultés rencontrées par les migrants sur le lieu d'arrivée}

Les migrants doivent faire face à de nombreuses difficultés. Les plus remarquables concernent le lieu d'habitation, une situation professionnelle instable et un manque d'information. Elles sont liées en partie au statut administratif des migrants.

\section{$\underline{\text { IV.1- Un fonctionnement contraignant du statut de résidence }}$}

Selon la loi sur la résidence de 2006, tous les citoyens doivent s'enregistrer. L'enregistrement de la situation de résidence suit des procédures administratives qui aboutissent à la délivrance d'un permis de résidence dont les modalités sont les suivantes :

- KT1 : résident permanent de la commune avec enregistrement du ménage dans la commune;

- KT2 : résident permanent de la commune avec inscription du ménage dans une autre commune ou résident d'une autre commune (le nom de cette autre commune est indiqué dans une case spécifique) avec inscription du ménage dans cette commune ;

- KT3 : résident temporaire de long terme (6 mois et plus);

- KT4 : résident temporaire de court terme (non permanent), potentiellement mobile.

Avant 2007, les migrants étaient confrontés à de nombreuses difficultés pour leur enregistrement. Par conséquent, beaucoup n'étaient pas inscrits et ne pouvaient donc pas bénéficier de la politique sociale du gouvernement sur le lieu d'arrivée (ils étaient bénéficiaires de cette politique sur leur lieu de départ).

Depuis l'entrée en vigueur de la loi sur la résidence le 1er juillet 2007, l'enregistrement de l'état de résidence est plus facile. Les migrants qui ont été enregistrés, même s'ils relèvent du régime KT4, peuvent bénéficier de la politique sociale sur leur lieu d'arrivée. Cependant, il reste une partie non négligeable de migrants non enregistrés. Les résultats de l'enquête sur les jeunes migrants travaillant dans le secteur informel montrent également que la majorité des jeunes migrants ne sont inscrits 
qu'à titre provisoire et à court terme sur le registre civil local (KT4) : 65,8\% chez les hommes et $59,7 \%$ chez les femmes.

\section{IV.2- Un habitat plus précaire}

Les différentes sources utilisent des questions sur l'état du lieu d'habitation, ce qui nous permet de comparer l'habitat des migrants et des non-migrants.

En $2009^{67}$, la situation de logement des migrants est dans l'ensemble moins bonne que celle des non-migrants. La proportion de personnes vivant dans des maisons simples est inférieure à $22 \%$ chez les non-migrants, alors que chez les migrants elle est toujours supérieure à 26\% (tableau 7).

Tableau 7: Type d'habitat selon le statut migratoire en 2009 (\%)

\begin{tabular}{|c|c|c|c|c|c|c|}
\hline & \multicolumn{2}{|c|}{ Dans un même district } & \multicolumn{2}{|c|}{ Entre districts } & \multicolumn{2}{|c|}{ Entre provinces } \\
\hline & Migrant & $\begin{array}{c}\text { Non } \\
\text { migrant }\end{array}$ & Migrant & $\begin{array}{c}\text { Non } \\
\text { migrant }\end{array}$ & Migrant & $\begin{array}{c}\text { Non } \\
\text { migrant }\end{array}$ \\
\hline Maison en dur & 32,4 & 40,0 & 31,6 & 39,8 & 23,0 & 39,6 \\
\hline Maison en semi-dur & 40,5 & 37,4 & 41,2 & 37,5 & 48,5 & 37,6 \\
\hline $\begin{array}{l}\text { Matériaux non } \\
\text { durables }\end{array}$ & 28,2 & 22,6 & 27,3 & 22,7 & 26,8 & 22,8 \\
\hline Total & 100,0 & 100,0 & 100,0 & 100,0 & 100,0 & 100,0 \\
\hline Effectif & 1618160 & 71686913 & 1708896 & 73305072 & 3397904 & 75013968 \\
\hline
\end{tabular}

Note: la classification des habitations repose sur les matériaux principaux des trois constituants essentiels que sont les murs, le toit et le sol. Les matériaux non durables comprennent les matériaux végétaux, la terre et la tôle ondulée. Les maisons en dur sont composées de béton, ciment, pierre, bois et briques.

Source : Office général des Statistiques 2011, p. 129

Lors de l'enquête sur les jeunes migrants qui travaillent dans le secteur informel à Hanoi, environ $43 \%$ des répondants ont déclaré que leurs conditions d'habitation actuelles sont «pires » que dans le lieu précédent. Plus de $60 \%$ des jeunes migrants vivent dans des auberges de construction simple, près de $30 \%$ dans des maisons à étages. Les $10 \%$ qui restent vivent dans des baraques provisoires. Les jeunes migrants vivent souvent avec d'autres, cinq en moyenne, dans une même chambre, avec une superficie moyenne de $7 \mathrm{~m}^{2}$ par personne. Environ $50 \%$ vivent avec moins de $5 \mathrm{~m}^{2}$ par personne. Cette superficie est bien inférieure à celle de la moyenne nationale. La moyenne était de $16,3 \mathrm{~m}^{2}$ par personne en $2008\left(18,7 \mathrm{~m}^{2}\right.$ dans les zones urbaines et 15,4 $\mathrm{m}^{2}$ à la campagne) et $17,9 \mathrm{~m}^{2}$ par personne en $2010\left(20,7 \mathrm{~m}^{2}\right.$ en ville et $16,7 \mathrm{~m}^{2}$ à la campagne) (Office général des Statistiques, 2011, p. 382). La plupart des jeunes de l'échantillon vivent dans un logement qu'ils louent $(60,0 \%)$ ou chez leur employeur $(34,0 \%)$. Seuls $2,3 \%$ ont leur propre habitation tandis que les autres vivent chez des personnes de leur connaissance. Le reste $(3,7 \%)$ vit sur son lieu de travail tel qu'un chantier ou un restaurant (Nguyên, Bui, 2012).

\section{IV.3- Une situation professionnelle et sociale instable}

Selon la réglementation du Code du Travail, toute activité employant des travailleurs doit faire l'objet d'un contrat. En fonction du temps d'utilisation des travailleurs, le

\footnotetext{
${ }^{67}$ En raison de différences dans l'élaboration des variables sur l'état des habitations entre les deux recensements, la comparaison doit être menée de manière prudente (Office général des Statistiques, 2011). Aussi, cette partie ne s'appuie que sur les données du recensement de 2009.
} 
contrat peut être à durée déterminée, indéterminée ou être saisonnier (article 27, Code du Travail amendé de 2002). Quand il s'agit d'emplois temporaires de moins de trois mois ou de travaux ménagers dans des familles, les parties concernées peuvent s'engager oralement, mais elles sont soumises aux réglementations de la législation du travail (article 27, Code du Travail de 1994). Or, selon les résultats de l'enquête nationale sur la migration de 2004 , seuls $79 \%$ des migrants qui avaient un travail rémunéré avaient signé un contrat. De même, l'enquête sur les jeunes migrants travaillant dans le secteur informel montre que seulement un cinquième des jeunes de l'échantillon $(19,3 \%)$ ont un contrat de travail ou une convention écrite. Ainsi, pour la majorité des jeunes travaillant dans le secteur informel, le contrat de travail prend la forme d'un engagement oral. Cela désavantage les travailleurs sur le plan judiciaire en cas de litige avec les employeurs.

Les résultats d'enquête nationale de migration de 2004 (Ibid., 2005) montrent qu'il existe une différence significative entre les personnes inscrites dans le registre civil local et celles non inscrites. Seulement $68 \%$ de ces dernières ont un contrat de travail, tandis que ce taux chez les premières est de 75\% (Office général des Statistiques, 2005). Parmi les personnes inscrites dans le registre civil local à titre provisoire (KT4), les femmes sont plus nombreuses à avoir un contrat de travail que les hommes. Les personnes non inscrites dans le registre civil local et celles inscrites à titre provisoire pour une courte durée ont des emplois peu rémunérés et peu protégés par un contrat de travail.

Démunis de contrat de travail écrit, les jeunes migrants travaillant dans le secteur informel à Hanoi n'ont pas non plus accès à différents types d'assurance dont la cotisation doit être payée par l'employeur : assurance maladie, assurance sociale et assurance chômage. Dans l'échantillon ( $\mathrm{N}=903)$, seuls $6,2 \%$ des jeunes ont une assurance maladie dont la cotisation est payée par l'employeur, 3,0\% ont une assurance sociale et $0,2 \%$ sont munis d'une assurance chômage (Nguyên, Bui, 2011).

\section{IV.3- Manque d'information}

Les travailleurs migrants manquent d'information et des connaissances nécessaires pour se protéger de risques en matière de santé. En effet, la connaissance des jeunes migrants sur le Code du travail est limitée : 41,5\% des jeunes interviewés ne connaissent aucune disposition de ce Code, $75,3 \%$ de ces jeunes ne savent pas que les employeurs doivent aider les travailleurs à traiter les maladies professionnelles ; 72,3\% ne savent pas qu'il existe un régime allégé de temps de travail pour les femmes enceintes de sept mois et plus et celles qui élèvent des enfants de moins de 12 mois ; $72,4 \%$ ne sont pas au courant de la politique permettant aux travailleurs de faire un bilan de santé périodique (Nguyen et coll. 2011). La proportion des jeunes qui ne connaissent pas les autres politiques (congés payés en cas de maladie, congés maternité pour les femmes, mise en place des cabinets de toilette séparés pour les hommes et les femmes, mise à la disposition des travailleurs de vestiaires, dédommagement en cas d'accident de travail) oscille entre 57,5\% à 68,1\% (Nguyen et coll. 2011).

La connaissance des jeunes travaillant dans le secteur informel sur les soins de la santé reproductive est également très limitée, à l'exception du VIH/sida dont presque tous les répondants ont entendu parler. Seuls $69 \%$ des répondants connaissent le nom de la syphilis, 63\% connaissent la blennorragie, 55\% connaissent 1'hépatite, 27\% sont au courant de la maladie causée par les chlamydiae (Luu et coll. 2013). Quant aux voies de transmission du VIH, 59\% seulement savent qu'il peut se transmettre de la mère à l'enfant, 59\% connaissent la transmission par le partage de seringues et $51 \%$ 
connaissent la transmission par voie sanguine. Sur la prévention des maladies sexuellement transmissibles et du VIH/Sida, seulement 65\% évoquent l'utilisation du préservatif (Luu et coll. 2013). Les connaissances des jeunes migrants sur les mesures contraceptives et les dangers de l'avortement sont très sommaires. De plus, les connaissances des hommes dans ce domaine sont plus limitées que celles des femmes (Luu et coll. 2013).

\section{Conclusion}

Depuis plus de dix ans, les migrations internes connaissent une évolution rapide en raison d'une forte croissance économique sous l'influence de la politique d'ouverture. La croissance économique engendre une forte urbanisation, une intégration internationale de plus en plus large et une différenciation chaque jour plus profonde en termes de répartition de ressources et de niveau de développement entre les régions. Dans ce contexte, la migration interne se développe. Les zones agricoles sont souvent des zones d'émigration tandis que les zones industrialisées et urbanisées accueillent les immigrants. Les migrants sont surtout des jeunes. De plus, avec le développement des industries légères, des services et le besoin grandissant de services à domicile dans les grandes villes, on observe une tendance à la féminisation de la migration. De nombreuses motivations sont données à cette migration. Les principales sont d'ordre économique. Or, les migrants travaillant dans le secteur informel, notamment les jeunes, n'ont souvent pas de contrat de travail et ne bénéficient pas des politiques sociales contrairement à ce que stipule le Code du travail (assurance sociale, assurance maladie, congés payés). Ainsi, malgré une certaine amélioration, la vie des migrants dans leur localité d'accueil reste difficile. Les principales difficultés concernent le logement, l'accès aux services de santé et l'intégration à la communauté locale.

Pour y remédier, il est important de diffuser des connaissances juridiques auprès des jeunes migrants travaillant dans le secteur informel, par le biais de la communauté, des organisations sociales locales et d'autres moyens de communication. Il importe également de vulgariser le Code du travail chez les employeurs, que ce soit des entreprises ou des patrons de petits établissements. Parallèlement, il faut faire des inspections régulières et prendre des mesures rigoureuses à l'encontre des personnes qui violent la législation en la matière afin de défendre les droits et intérêts légitimes des travailleurs.

La migration est un problème de plus en plus complexe sur les plans économique, social et environnemental tant pour les localités de départ que pour celles d'arrivée. Il importe donc de mener des recherches et de perfectionner la politique migratoire pour une répartition raisonnable de la population entre les régions et les unités administratives. Cela permettra afin d'exploiter au mieux les potentialités foncières et les ressources naturelles, de soulager la pression démographique dans les zones à haute densité de population, notamment dans les grandes villes - zones qui connaissent un taux d'immigration en forte augmentation. Il faut également promulguer des politiques de définition des priorités d'investissement et de création d'emploi dans les régions à forte émigration pour que les habitants puissent y trouver du travail dans d'autres domaines que l'agriculture sans avoir à quitter leur région d'origine. Il faut communiquer sur les intérêts et les défis de la migration, notamment en terme de santé en général et de santé reproductive en particulier pour que les migrants se protègent de la prostitution, des stupéfiants et d'autres problèmes sociaux. Il importe également de communiquer sur les droits et devoirs des travailleurs, de même que sur les connaissances 
et savoirs-faire nécessaires à l'accomplissement d'une migration dans de bonnes conditions (inscription au registre civil, recherche d'emploi, signature de contrat de travail, soin de santé, pratique d'épargne). Il est important de citer des exemples de migrants ayant réussi à éradiquer la pauvreté et à s'enrichir de manière honnête.

L'urbanisation sous l'influence du développement économique et de la restructuration de la main d'œuvre se poursuivra fortement. Les régions à forte immigration doivent donc planifier et investir pour accueillir le flux de migrants. Leur aménagement doit tenir compte de l'envergure, la densité et la structure de la population dans l'avenir.

Enfin, les autorités locales doivent mettre l'accent sur les contributions des migrants à leur localité. Il faut séparer les fonctions économiques et sociales du «livret de famille» tout en favorisant l'inscription au registre civil. Ces localités doivent également promouvoir les soins de santé reproductive des migrants, notamment la prévention des maladies sexuellement transmissibles en communiquant les connaissances nécessaires, en fournissant des consultations en la matière et en développant le réseau de soins de santé.

\section{Remerciements}

Cette recherche a bénéficié d'un financement de la Fondation Nationale pour le Développement de la Science et la Technologie du Viêt Nam (Nafosted) relatif au protocole II6.2-2012.01.

\section{Bibliographie}

Comite Central de Pilotage du ReCensement de la population et du logement, Recensement de la population et du logement du Viêt Nam de 2009: Principaux résultats, Hanoi, Office Général des Statistiques, 2010, p. 506. [BAN CHİ DẠO TÔNG DIÊU TRA DAN SỐ VA NHA Ở TRUNG ƯONG. Tổng điều tra Dân số và nhà ở Việt Nam, năm 2009. Các kết quả chủ yếu. Hà Nôi, 6-2010, 506 trang.].

Gubry Patrick, NGUYEN Thi Thieng, LE Thi Huong et coll., 2007. Enquête Migration, pauvreté et environnement urbain, IRD, IPSS-UNE, HIDS-Ho Chi Minh Ville, 2007, (dactyl.).

LOI NUMERO 35/2002/QH10 Code du travail amendé de 2002 adopté le 2 avril 2002 par l'Assemblée nationale de la République socialiste du Viêt Nam, $\mathrm{X}^{\mathrm{e}}$ législature, lors de sa $11^{\mathrm{e}}$ session. [LUậT SỐ 35/2002/QH10 Bộ Luật Lao động sửa đổi 2002 được Quốc hội nước Cộng hoà xã hội chủ nghĩa Việt Nam Khoá X, kỳ họp thứ 11 thông qua ngày 02 tháng 4 năm 2002.]

LOI NUMERO 35-L/CTN Code du travail de 1994 adopté le 23 juin 1994 par l'Assemblée nationale de la République socialiste du Viêt Nam, IX ${ }^{\mathrm{e}}$ législature, lors de sa $5^{\mathrm{e}}$ session. [LUậT SỐ 35-L/CTN Bộ luật Lao động 1994 được Quốc hội nước Cộng hoà xã hội chủ nghĩa Việt Nam khoá IX, kỳ họp thứ 5 thông qua ngày 23 tháng 6 năm 1994.]

LOI NUMERO 74/2006/QH11 Code du travail amendé de 2006 adopté le 29 novembre 2006 par l'Assemblée nationale de la République socialiste du Viêt Nam, $\mathrm{XI}^{\mathrm{e}}$ législature, lors de sa $10^{\mathrm{e}}$ session. [LUậT SỐ 74/2006/QH11 Bộ Luật lao động sửa đổi 2006 được Quốc hội nước Cộng hoà xã hội chủ nghĩa Việt Nam khoá XI, kỳ họp thứ 10 thông qua ngày 29 tháng 11 năm 2006.] 
LOI NUMERO 84/2007/QH11 Code du travail amendé de 2007 adopté le 2 avril 2007 par l'Assemblée nationale de la République socialiste du Viêt Nam, $\mathrm{XI}^{\mathrm{e}}$ législature, lors de sa $11^{\mathrm{e}}$ session. [LUẬT SỐ 84/2007/QH11 Bộ luật lao động sửa đổi 2007 được Quốc hội nước Cộng hòa xã hội chủ nghĩa Việt Nam khoá XI, kỳ họp thứ 11 thông qua ngày 02 tháng 4 năm 2007.]

LuU Bich Ngoc, Nguyen Thi Thieng, BuI Thi Hanh, «Connaissance sur la santé reproductive et les comportements à haut risque des jeunes migrants travaillant dans le secteur informel à Hanoi ». in Colloque Mondialisation, migration interne et risques en terme de santé communautaire au Viêt Nam sur les résultats du programme "Peer-IRD.AIRD", Hanoi, le 9 mai 2013, 48 diapositives. [Lưu Bích Ngọc, Nguyễn Thị Thiềng và Bùi thị Hạnh, 2013, Hiểu biết về sức khoẻ sinh sản và hành vi nguy cơ cao của thanh niên di cư làm việc trong khu vực phí chính thức tại Hà Nội. Bài trình bày tại hội thảo "hội thảo công bố kết quả chương trình "PeerIRD.AIRD" mang tên Toàn cầu hoá, di cư nội địa và nguy cơ về sức khoe cộng đồng ở Việt Nam. Hà nội ngày 9 tháng 5 năm 2013.]

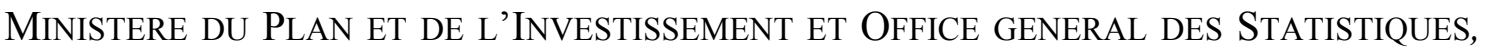
Recensement de la population et des ménages 2009. Migration et Urbanisation au Viêt Nam : état des lieux, tendances et différences, Hanoi, 2010, 142 p. [Tổng điều tra Dân số và Nhà ở Việt Nam năm 2009 - Di cư và đô thị hoá ở Việt Nam: Thực trạng, xu hướng và những khác biệt, Hà nội, năm 2010, 142 p.],

NGuYEn Thị Thiêng, But Thị Hanh, Qualité de vie des jeunes migrants qui travaillent dans le secteur informel à Hanoi, Document présenté au Colloque international de Vietnamologie, Hanoi, novembre 2012, 34 diapositives.

NguYEN Thị Thiêng, LuU Bich Ngoc et coll., Jeunes migrants travaillant dans le secteur informel à Hanoi, Plan du Viêt Nam, Rapport de recherche, Hanoi, Zone de projet de Hanoi et Université nationale d'Économie, Institut de la Population et des Questions Sociales, mai 2009, 166 p. [NGUYen Thi ThIENG, LuU Bich NGOC et coll., Plan tại Việt Nam - Vùng dự án Hà Nội và Trường ĐH Kinh tế quốc dân - Viện Dân số và các vấn đề xã hội, Báo cáo nghiên cứu "Thanh thiếu niên di cư làm việc trong khu vực phi chính thức tại Hà Nội”, Hà Nội - 5/2009, 166 trang.]

Office general des StatistiQues, United Nations Population Fund (UNFPA), The 2004 Viêt Nam Migration Survey: The main results, Statistics Publisher, Hanoi, 2005, p. 196. [TỔNG CụC THỐNG KE, QUỸ DAN SỐ LIEN HIỆP QUỐC (UNFPA), 2005, Điều tra di cư Việt Nam 2004: Những kết quả chủ yếu, NXB Thống kê, Hà Nội, 2005, 196 trang.]

Office general Des StatistiQues, Recensement de la Population et du Logement de 2009 : Migration et urbanisation au Viêt Nam, Hanoi, Office général des Statistiques, 2011a, p. 140. [Bộ KẾ HOẠCH VA ĐẦU TÚ- TổNG CụC THỐNG KE, 2011, Tổng Điều tra Dân số và Nhà ở năm 2009: Di cư và đô thị hoá ở Việt Nam, Hà Nội 2011, 140 trang.]

OfFICE GENERAL DES STATISTIQUES, 2011, Situation socio-économique de 10 ans du Viêt Nam 2001-2010, Office général des Statistiques, Hanoi, 2011b, p. 242. [TổNG CỤC THỐNG KE, 2011, Tình hình kinh tế xã hội Việt Nam 10 năm 2001-2010, Nhà xuất bản Thống kê, Hà Nội, 2011, 242 trang.]

United NATIONS PopUlation Fund UNFPA, Domestic migration: The real situation in Viêt Nam, Hanoi, UNFPA, 6/2007, p. 30. [QUỸ DÂN SỐ LIÊN HIỆP QUỐC (UNFPA), 2007, Di cư trong nước: Hiện trạng ở Việt Nam, Hà Nội, 6/2007, 30 trang.] 
WeBsite BINH DuONG Province, http://eng.binhduong.gov.vn/en/status_pages.php?id= 42\&idcat $=15 \&$ idcat $2=0$ [Consulté le 11 octobre 2014].

\section{Annexe}

Tableau 8: Effectif des émigrants et taux d'émigration selon les régions entre 19941999 et 2004-2009

\begin{tabular}{lcccc} 
Région & $\begin{array}{c}\text { Effectif des émigrants } \\
\text { (milliers) }\end{array}$ & \multicolumn{2}{c}{ Taux d'émigration } \\
& 1999 & 2009 & 1999 & 2009 \\
\hline Delta du Mékong & 211 & 734 & 14 & 46 \\
Centre Nord et côtes centrales & 425 & 775 & 26 & 45 \\
Delta du Fleuve Rouge & 333 & 331 & 21 & 18 \\
Montagnes du nord & 180 & 271 & 17 & 27 \\
Hauts plateaux du Centre & 60 & 125 & 18 & 27 \\
Sud-Est & 125 & 125 & 14 & 10 \\
\hline Total & 1334 & 2361 & 19 & 30 \\
\hline
\end{tabular}

Source : Comité central de pilotage du recensement de la population et du logement du Viêt Nam de 2009, 2010, p. 79 


\title{
L'environnement urbain : Perception des habitants de Hanoi et Hô Chi Minh-Ville
}

\author{
Lê Thi Huong (HIDS) \\ Nguyên Thi Thiêng (UNE IPSS) \\ Patrick Gubry (IRD UMR "Population et sociétés")
}

Les études environnementales sont nombreuses au Viêt Nam, mais il s'agit surtout d'études scientifiques et techniques sur la pollution, la qualité de l'eau, l'air ou le sol, le bruit et le trafic routier, etc. Ces études ne révèlent pas les préoccupations spécifiques de la population en matière d'environnement et il est difficile de mettre en œuvre des politiques pertinentes dans ce domaine, car il est impossible d'identifier les priorités.

Dans ce contexte, nous avons décidé d'aborder la question à partir des ménages et de leurs points de vue sur l'environnement dans lequel ils vivent et les solutions proposées pour résoudre les problèmes identifiés ${ }^{68}$. Pour ce faire, nous avons mené en 2007 une enquête socio-démographique représentative des ménages dans les deux grandes villes du Viêt Nam sur le thème « Migration, pauvreté et environnement urbain : Hanoi et Hô Chi Minh-Ville » de laquelle les présents résultats ont été tirés.

Les villes de Hanoi et Hô Chi Minh-ville constituent les deux principales métropoles du Viêt Nam (voir figure 4 en annexe et Carte 1 en tête d'ouvrage)). Hanoi, capitale politique du pays, se situe au Nord dans le delta du Fleuve Rouge, zone de riziculture à forte densité de population. Au recensement de 2009, cette ville comprenait 6,5 millions d'habitants, dont 2,6 millions en zone urbaine (41,0 \%). Hô Chi Minh-Ville, principale coeur économique du pays, se situe dans le Sud du pays dans le delta du Mekong. Elle comprend 7,2 millions d'habitants dont 6,0 millions en zone urbaine (83,3\%) (Ban Chỉ Dạo Tổng Diều Tra Dân Số và Nhà Ở Trung Ương, 2010). Ces chiffres ne comportent ni les étrangers, ni la « population flottante » (population séjournant de fait en ville pour une longue durée tout en affirmant qu'elle n'est pas résidente et qui n'est donc pas comptée au recensement).

La méthodologie mise en œuvre dans l'étude est novatrice comparée aux enquêtes existantes, avec d'une part un plan d'échantillonnage à deux degrés ( «îlots » et ménages) à partir d'une base de sondage constituée directement par les « îlots » (l'ensemble du territoire étant divisé en îlots), d'autre part, un inventaire des ménages à l'intérieur des îlots tirés au premier degré, indépendant des données disponibles au niveau de l'administration locale. Cette méthodologie a été mise au point par notre équipe lors d'une précédente enquête (Gubry, Lê Van Thanh, Nguyên Thi Thiêng, 2008), elle a été spécifiquement présentée lors d'un atelier de formation à Hanoi (Gubry, Nguyên Thi Thiêng, 2012); on ne la développera pas ici $^{69}$. Les opinions de la population sur l'environnement ainsi que l'environnement de la maison et celui du quartier ont été étudiés.

Nous nous concentrons ici sur l'environnement du quartier, l'environnement autour de la maison, sur lequel les personnes interrogées ont eu l'occasion de donner leur avis plus fréquemment. Les réponses étudiées ici sont celles données personnellement par les répondants à l'enquête (un répondant par ménage).

\footnotetext{
${ }^{68}$ Ce chapitre reprend et met à jour certaines parties d'une étude inédite sur l'environnement urbain rédigée par Patrick Gubry, Lê Thi Huong et Pham Thuy Huong à partir des résultats de l'enquête.

${ }^{69}$ L'enquête a porté sur 1000 ménages (3 983 personnes) à Hanoi et 1500 ménages (6 592 personnes) à Hô Chi Minh Ville. À Hanoi, 583 hommes $(58,3 \%)$ et 497 femmes ont répondu à l'enquête ; à Hô Chi Minh Ville, 642 hommes $(42,8 \%)$ et 858 femmes ont répondu.
} 


\section{I- Un environnement considéré comme plus mauvais à Hanoi}

À la question « Diriez-vous que l'environnement dans votre quartier est excellent, bon, moyen, mauvais, très mauvais ? ", la réponse est très différente dans les deux villes. A Hanoi, $17,1 \%$ des répondants considèrent leur environnement comme excellent ou bon, mais 29,1\% le considèrent comme mauvais ou très mauvais ; à Hô Chi Minh Ville, les chiffres respectifs sont $24,1 \%$ et $16 \%$ (Tableau 1). À l'opposé de ce qu'on pouvait penser, les gens apparaissent donc plus satisfaits de leur environnement dans l'agglomération la plus grande, qui compte aussi le plus grand nombre de personnes considérant l'état de l'environnement comme moyen (mais on serait tenté de mettre aussi les indécis dans cette catégorie). Le rôle de l'éducation -d'un niveau significativement supérieur à Hanoi- a été suggéré comme étant le facteur principal de cette situation (Gubry, Le Thi Huong, Nguyên Thi Thiêng, 2009). On ne sera pas surpris de relever que les ménages riches sont plus satisfaits de leur environnement que les ménages pauvres. En revanche, il n'y a pas de différence selon le sexe du répondant.

Tableau 1- Evaluation de l'environnement dans le quartier selon la ville (\%)

\begin{tabular}{lrc} 
État de l'environnement & Hanoi & $\begin{array}{r}\text { Hô Chi } \\
\text { Minh-Ville }\end{array}$ \\
\hline Excellent & 1,6 & 2,5 \\
Bon & 15,5 & 21,6 \\
Moyen & 53,8 & 59,9 \\
Mauvais & 26,1 & 14,2 \\
Très mauvais & 3,0 & 1,8 \\
\hline Total & 100,0 & 100,0 \\
\hline Nombre de répondants & 1000 & 1500 \\
\hline
\end{tabular}

Source : Enquête migration, pauvreté et environnement urbain, 2007

Ceux qui ont considéré l'environnement de leur quartier comme " mauvais » ou « très mauvais » ont été interrogés sur les types de nuisances observées. L'odeur des décharges d'ordures, des ordures des marchés, des cadavres d'animaux vient largement en tête dans les deux villes (Tableau 2). Les émanations proviennent du stockage permanent ou temporaire des déchets trop près des habitations. Cette nuisance est immédiatement suivie par l'odeur des égouts. Les problèmes du ramassage des ordures et de l'évacuation des eaux usées sont donc certainement ceux à prendre en compte en tout premier lieu dans l'amélioration de l'environnement urbain. La pollution de l'air par la circulation routière figure en bonne place dans les deux villes.

En revanche, certaines nuisances sont observées plus fréquemment dans l'une des villes. À Hanoi, on cite plus souvent la pollution de l'air par la fumée, car on y utilise plus volontiers le charbon pour la cuisine ; la pollution de l'air par des activités de production y est plus fréquente, sans doute parce que celles-ci sont plus insérées dans le tissu urbain; ainsi, on s'y plaint plus de la forte densité de population et des problèmes de promiscuité. À Hô Chi Minh Ville, on cite beaucoup plus fréquemment les inondations ; outre le problème qu'elles posent pour les transports urbains, elles sont elles-mêmes une source importante de pollution et de problèmes sanitaires. L'acuité des inondations à Hô Chi Minh Ville provient du site géographique de la ville : les faibles dénivelés dans la majeure partie de la ville ne favorisent pas l'écoulement des eaux de pluie et lorsqu'une forte averse survient au moment de la marée haute, qui refoule les eaux de la rivière de Saigon vers l'amont, l'écoulement des eaux vers la mer est retardé. 
Tableau 2- Nuisances observées dans le quartier selon la ville (Plusieurs réponses possibles, question ouverte)

\begin{tabular}{|c|c|c|c|c|}
\hline \multirow[b]{2}{*}{ Nuisances } & \multicolumn{2}{|c|}{ Hanoi } & \multicolumn{2}{|c|}{$\begin{array}{c}\text { Hô Chi Minh- } \\
\text { Ville }\end{array}$} \\
\hline & $\begin{array}{c}\text { Nom- } \\
\text { bre }\end{array}$ & $\begin{array}{l}\text { \% des } \\
\text { répon- } \\
\text { dants }\end{array}$ & $\begin{array}{c}\text { Nom- } \\
\text { bre }\end{array}$ & $\begin{array}{l}\% \text { des } \\
\text { répon- } \\
\text { dants }\end{array}$ \\
\hline Odeur d'ordures, déchets de marché, cadavres d'animaux & 77 & 26,6 & 49 & 20,5 \\
\hline Odeur des égouts, du système d'évacuation & 73 & 25,2 & 46 & 19,2 \\
\hline Pollution par gaz d'échappement, poussière de la circulation & 68 & 23,4 & 35 & 14,6 \\
\hline Fumée, poussière, poussière de charbon & 56 & 19,3 & 14 & 5,9 \\
\hline Pollution de l'air par une usine, un atelier de production & 56 & 19,3 & 28 & 11,7 \\
\hline Forte densité de population, atmosphère suffocante, voisinage & 40 & 18,8 & 10 & 4,2 \\
\hline Odeur d'étang à eau stagnante, de rivière polluée & 29 & 10,0 & 20 & 8,4 \\
\hline Pollution de l'eau, présence d'eaux usées & 24 & 8,3 & 13 & 5,4 \\
\hline Bruit de la circulation routière & 24 & 8,3 & 13 & 5,4 \\
\hline Inondations & 16 & 5,5 & 42 & 17,6 \\
\hline Odeur des déjections d'animaux d'élevage & 16 & 5,5 & 7 & 2,9 \\
\hline Odeur des insecticides, des produits phytosanitaires & 12 & 4,1 & 2 & 0,8 \\
\hline Bruit des usines, des ateliers de production & 8 & 2,7 & 8 & 3,3 \\
\hline Occupation des trottoirs & 6 & 2,1 & 6 & 2,5 \\
\hline Divagation des animaux domestiques & 5 & 1,7 & 10 & 4,2 \\
\hline Mauvaises conditions sanitaires & 5 & 1,7 & 18 & 7,5 \\
\hline Insécurité & 2 & 0,7 & 3 & 1,3 \\
\hline Autre & 0 & 0,0 & 10 & 4,2 \\
\hline Nombre de répondants & 290 & - & 239 & - \\
\hline
\end{tabular}

Source : Enquête migration, pauvreté et environnement urbain, 2007

Plusieurs citations sont significatives et intéressantes de par leur caractère synthétique : «Les poubelles ne sont pas sûres, n’ont pas de couvercle, alors que les déchets des activités quotidiennes sont proches des habitations. L'odeur est insupportable » (homme de 41 ans, arrondissement de Dông Da, Hanoi).

«La pollution de l'environnement est de plus en plus grave parce que les déchets des activités quotidiennes sont de plus en plus nombreux. Le manque de système d'évacuation entraîne la pollution de l'eau » (homme de 26 ans, arrondissement de Tu Liêm, Hanoi).

«La maison étant située dans la rue principale, il y a beaucoup de véhicules, de la poussière, de la fumée et des bruits qui provoquent fatigue et maux de tête » (femme de 49 ans, arrondissement de Binh Thanh, HCMV).

On retiendra aussi le commentaire de cet habitant de Hanoi qui exprime humblement le manque de connaissances sur la question :

« Il existe des effets négatifs, mais on ne sait pas encore quelles sont leurs façons de se manifester. Le mauvais environnement influe sur la population, surtout sur les enfants » (homme de 32 ans, arrondissement de Tây Hô, Hanoi).

En conséquence, nous conclurons en reconnaissant la nécessité d'approfondir les recherches dans ce domaine.

À la question de savoir si l'environnement est en train de s'améliorer ou de se détériorer, le nombre de ceux qui considèrent qu'il n'y a pas de changement ou qui sont indécis est beaucoup plus élevé à Hô Chi Minh Ville (Tableau 3) ; ils font peut-être ainsi preuve d'un manque d'information, à l'inverse des habitants de la capitale. 
Tableau 3- Evaluation de l'évolution de l'environnement selon la ville (\%)

\begin{tabular}{lrc} 
Évolution de l'environnement & Hanoi & Hô Chi Minh-Ville \\
\hline Amélioration & 34,4 & 23,8 \\
Détérioration & 38,3 & 14,8 \\
Pas de changement & 27,3 & 61,3 \\
\hline Total & 100,0 & 100,0 \\
\hline
\end{tabular}

Source : Enquête migration, pauvreté et environnement urbain, 2007

Il n'en demeure pas moins que des efforts de sensibilisation à l'environnement sont en cours. C'est d'ailleurs l'amélioration de la prise de conscience des habitants et du niveau d'instruction qui vient largement en tête des raisons invoquées de l'amélioration de l'environnement dans le quartier, dans les deux villes (Tableau 4). Il est vrai que le rôle d'une meilleure prise de conscience des habitants doit être nuancé par la proportion importante de gens qui pensent que cette conscience s'est au contraire détériorée. L'amélioration des routes et de leur entretien vient en seconde position. Celle du paysage est en bonne place dans les deux villes. En revanche, l'amélioration du système d'égouts n'a été citée qu'à Hanoi. L'augmentation de la pollution de l'air vient en tête des raisons citées de la détérioration de l'environnement du quartier, suivie par l'augmentation de la population. Evidemment cette dernière raison est en quelque sorte une raison générique agissant sur les autres.

Tableau 4- Domaines d'amélioration ou de détérioration de l'environnement du quartier invoqués selon la ville : effectif et pourcentage de répondants (Plusieurs réponses possibles)

\begin{tabular}{|c|c|c|c|c|}
\hline \multirow{2}{*}{ Nuisances } & \multicolumn{2}{|c|}{ Hanoi } & \multicolumn{2}{|c|}{$\begin{array}{c}\text { Hô Chi } \\
\text { Minh-Ville }\end{array}$} \\
\hline & $\begin{array}{l}\text { Nom } \\
\text {-bre }\end{array}$ & $\%$ & $\begin{array}{l}\text { Nom } \\
\text {-bre }\end{array}$ & $\%$ \\
\hline \multicolumn{5}{|l|}{ Domaine d'amélioration } \\
\hline Conscience des habitants ou niveau d'instruction & 179 & 51,9 & 131 & 36,8 \\
\hline Les routes et leur entretien & 77 & 22,3 & 109 & 30,6 \\
\hline Extension, élargissement, réparation, nettoyage, couverture des égouts & 76 & 22,0 & 0 & 0,0 \\
\hline Paysage, espace, environnement, augmentation des espaces verts & 71 & 20,6 & 58 & 16,3 \\
\hline Collecte et traitement des ordures & 39 & 11,3 & 32 & 9,0 \\
\hline Habitat & 30 & 8,7 & 16 & 4,5 \\
\hline Approvisionnement en eau (quantité, qualité, réseau) & 23 & 6,7 & 6 & 1,7 \\
\hline Eloignement des usines des habitations & 17 & 4,9 & 6 & 1,7 \\
\hline Nombre de répondants & 343 & - & 356 & - \\
\hline \multicolumn{5}{|l|}{ Domaines de détérioration } \\
\hline Pollution de l'air, poussière & 137 & 35,9 & 70 & 31,5 \\
\hline Augmentation de la population & 101 & 26,4 & 64 & 28,8 \\
\hline Accumulation des déchets & 78 & 20,4 & 20 & 9,0 \\
\hline Détérioration de la conscience des habitants & 77 & 20,2 & 38 & 17,1 \\
\hline Insuffisance du système d'évacuation & 67 & 17,5 & 25 & 11,3 \\
\hline Dégradation des routes & 31 & 8,1 & 27 & 12,2 \\
\hline Augmentation du bruit & 18 & 4,7 & 11 & 5,0 \\
\hline Rôle inadéquat des pouvoirs publics & 13 & 3,4 & 14 & 6,3 \\
\hline Pollution de l'eau & 11 & 2,9 & 14 & 6,3 \\
\hline Diminution des espaces verts & 10 & 2,6 & 9 & 4,1 \\
\hline Encombrement des trottoirs & 8 & 2,1 & 0 & 0,0 \\
\hline Dégradation de l'habitat & 7 & 1,8 & 0 & 0,0 \\
\hline Développement des épidémies & 4 & 1,0 & 0 & 0,0 \\
\hline Nombre de répondants & 382 & - & 222 & \\
\hline
\end{tabular}

Source : Enquête migration, pauvreté et environnement urbain, 2007 
Relevons quelques phrases significatives :

« La population et les moyens de transport plus nombreux créent la poussière et le bruit » (homme de 39 ans, arrondissement de Thanh Xuân, Hanoi).

« D'assez nombreuses jeunes filles de ce quartier restent après leur mariage, entraînant une densité de population plus élevée. Il n'y a pas de cuisine personnelle, les habitants font la cuisine sur la voie de circulation. Ce n'est pas hygiénique » (homme de 45 ans, arrondissement de Hoan Kiêm, Hanoi).

«La densité est de plus en plus forte. Le manque de conscience des habitants crée la pollution de l'environnement. Le nombre des drogués, de plus en plus important, influence la sécurité des quartiers » (femme de 49 ans, arrondissement de Hoang Mai, Hanoi).

« Les ménages avec des chiens laissent leurs animaux déféquer et uriner dans la rue sans nettoyer » (femme de 51 ans, arrondissement de Binh Thanh, HCMV).

«La quantité d'ordures jetée directement dans la canalisation s'accroît » (homme de 45 ans, arrondissement de Binh Thanh, HCMV).

Comme souvent, en cas de problème, et un peu hâtivement, on incrimine volontiers les immigrants, les allogènes ${ }^{70}$ :

«La population est de plus en plus nombreuse, et des gens de plusieurs origines se mêlent et sont bruyants. Le manque de conscience des immigrants influence l'hygiène générale » (homme de 44 ans, arrondissement de Hai Ba Trung, Hanoi).

« La population est plus nombreuse, surtout les immigrants temporaires qui font la cuisine au charbon, entraînant une odeur insupportable » (homme de 70 ans, arrondissement de Hoang Mai, Hanoi.

«De nombreuses personnes venant d'ailleurs arrivent ici; comme les gens ne vivent ici que temporairement, ils ne sont pas informés sur l'environnement » (femme de 38 ans, $6^{\mathrm{e}}$ arrondissement, HCMV).

Enfin, les deux phrases suivantes résument admirablement la situation de l'environnement urbain :

« Avant, il y avait moins de monde, donc il n'y avait pas beaucoup d'ordures. Maintenant, il y a plus de gens et beaucoup d'ordures » (femme de 47 ans, arrondissement de Tân Binh, HCMV).

« Il y a beaucoup de véhicules, beaucoup de monde, beaucoup de bruit » (femme de 58 ans, $5^{\mathrm{e}}$ arrondissement, HCMV).

\section{II- Localisation du logement : l'importance des axes routiers à grande circulation}

La localisation du logement joue un rôle important sur l'environnement et le cadre de vie. $\mathrm{Au}$ cours de l'enquête, les enquêteurs ont relevé les caractéristiques visibles du logement, indépendamment de toute déclaration des interviewés (Tableau 5).

Cette information est difficile à exploiter, car bon nombre de ces caractéristiques sont ambivalentes : par exemple, la présence d'un étang ou d'une rivière peut être une source d'agrément quand ils ne sont pas pollués; elle devient source de nuisance dans le cas contraire. On retiendra cependant la forte fréquence de la proximité d'un axe routier à grande circulation dans les deux villes. On relèvera également l'insertion des activités économiques dans le tissu urbain : dans 31,4\% des cas à Hanoi et 20,7\% à Hô Chi Minh

${ }^{70}$ La stigmatisation des migrants, phénomène assez universellement répandu, est renforcée au Viêt Nam par l'existence du contrôle résidentiel qui génère d'une part des urbains « de droit» et d'autre part des urbains « illégaux » (Pulliat, 2013). 
Ville, le logement est proche d'un atelier, d'une usine ou d'un chantier (évidemment temporaire dans ce dernier cas).

Tableau 5- Caractéristiques visibles de l'environnement du logement selon la ville: effectif et pourcentage de répondants (Plusieurs réponses possibles)

\begin{tabular}{lrrrr} 
Caractéristiques visibles & Hanoi & \multicolumn{3}{c}{ Hô Chi Minh-Ville } \\
& Nombre & $\%$ & Nombre & $\%$ \\
\hline Axe routier à grande circulation & 252 & 25,2 & 341 & 22,7 \\
Etang & 169 & 16,9 & 58 & 3,8 \\
Rivière & 162 & 16,2 & 174 & 11,6 \\
Petits ateliers de fabrication ou de réparation & 134 & 13,4 & 190 & 12,7 \\
Bois & 133 & 13,3 & 100 & 6,6 \\
Grande usine & 113 & 11,3 & 69 & 4,6 \\
Rizières, de champs ou de friches & 88 & 8,8 & 85 & 5,7 \\
Chantier de construction & 70 & 7,0 & 51 & 3,4 \\
Cimetière & 65 & 6,5 & 18 & 1,2 \\
Parc aménagé & 59 & 5,9 & 34 & 2,3 \\
Voie ferroviaire & 58 & 5,8 & 18 & 1,2 \\
Dépôt d'ordures & 54 & 5,4 & 44 & 2,9 \\
Aéroport, passage d'avions & 2 & 0,2 & 20 & 1,3 \\
Rien à signaler & 299 & 29,9 & 699 & 44,6 \\
\hline Nombre de répondants & 1000 & - & 1500 & -
\end{tabular}

Source : Enquête migration, pauvreté et environnement urbain, 2007

La localisation du logement décrit la géographie des villes vietnamiennes avec une majorité de logements situés sur une petite ruelle (Tableau 6); on entend par là la localisation de la porte d'entrée. Ainsi, l'habitat est souvent à l'abri du bruit de la circulation, mais la promiscuité de la population s'en trouve accrue.

Tableau 6- Localisation du logement selon la ville (\%)

\begin{tabular}{lrr} 
Localisation du logement & Hanoi & $\begin{array}{r}\text { Hô Chi } \\
\text { Minh-Ville }\end{array}$ \\
\hline Sur une rue & 11,2 & 19,5 \\
Sur une grande ruelle (largeur supérieure à 4 m) & 21,9 & 24,3 \\
Sur une petite ruelle (largeur inférieure à 4 m) & 62,1 & 52,7 \\
Sur une ruelle et sur la berge d'une rivière ou d'un étang aménagé & 0,5 & 1,8 \\
Sur une ruelle et sur la berge d'une rivière ou d'un étang non aménagé & - & 0,2 \\
Autre & 4,2 & 1,4 \\
\hline Total & 100,0 & 100,0 \\
\hline
\end{tabular}

Source : Enquête migration, pauvreté et environnement urbain, 2007

La ville de Hanoi est sensiblement mieux équipée en matière de revêtement des rues et les voies en dur y sont plus nombreuses (Tableau 7). Il est logique de trouver le plus de rues en gravier ou en terre dans les arrondissements périphériques et ruraux des deux villes. 
Tableau 7- Revêtement de la rue où se situe le logement, selon la zone d'habitation et la ville $(\%)$

\begin{tabular}{|c|c|c|c|c|c|c|c|c|}
\hline \multirow{2}{*}{$\begin{array}{l}\text { Revêtement } \\
\text { de la rue }\end{array}$} & \multirow[b]{2}{*}{ Centraux } & \multicolumn{2}{|c|}{$\begin{array}{c}\text { Hanoi } \\
\text { Arrondissements }\end{array}$} & \multirow[b]{2}{*}{$\begin{array}{l}\text { Ensem- } \\
\text { ble }\end{array}$} & \multicolumn{4}{|c|}{$\begin{array}{l}\text { Hô Chi Minh-Ville } \\
\text { Arrondissements }\end{array}$} \\
\hline & & $\begin{array}{l}\text { Périphéri } \\
\text {-ques }\end{array}$ & Ruraux & & Centraux & $\begin{array}{l}\text { Périphéri- } \\
\text { ques }\end{array}$ & Ruraux & $\begin{array}{l}\text { Ensem- } \\
\text { ble }\end{array}$ \\
\hline Bitume & 18,9 & 26,7 & 12,9 & 19,8 & 44,0 & 29,4 & 22,1 & 37,4 \\
\hline Béton ou ciment & 80,0 & 68,3 & 71,1 & 73,9 & 52,6 & 23,5 & 23,2 & 41,2 \\
\hline Gravier & 0,5 & 2,2 & 12,2 & 4,1 & 1,5 & 12,0 & 36,8 & 8,8 \\
\hline Terre & 0,7 & 2,9 & 3,8 & 2,2 & 1,9 & 35,0 & 17,9 & 12,6 \\
\hline Total & 100 & 100 & 100 & 100 & 100 & 100 & 100 & 100 \\
\hline
\end{tabular}

Source : Enquête migration, pauvreté et environnement urbain, 2007

Dans 33,7 \% des cas à Hanoi et 22,4 \% des cas à Hô Chi Minh Ville, la rue donnant sur le logement est encombrée, ce qui peut être considéré comme une dégradation de l'environnement, puisque l'encombrement entrave la circulation et peut être une source de pollution selon les activités économiques qui y sont exercées (Tableau 8). Les sources d'encombrement sont surtout les petits commerces informels et le stationnement, et plus à Hanoi, où les voies sont étroites, qu'à Hô Chi Minh Ville. Ce phénomène a déjà été relevé par Drummond (2000), Jensen \& Peppard (2003), mais il est vrai qu'il arrive loin derrière d'autres sources de dégradation.

Tableau 8- Encombrement de la rue donnant sur le logement selon la ville : effectif et pourcentage de répondants (Plusieurs réponses possibles)

\begin{tabular}{lrrrr} 
Type d'encombrement & \multicolumn{2}{c}{ Hanoi } & \multicolumn{3}{c}{ Hô Chi Minh-Ville } \\
& Nombre & $\%$ & Nombre & $\%$ \\
\hline Petits commerçants dans la rue ou sur le trottoir & 225 & 22,5 & 215 & 14,3 \\
Stationnement sur le trottoir & 154 & 15,4 & 178 & 11,9 \\
Stationnement dans la rue & 141 & 14,1 & 138 & 9,2 \\
Déchets dans la rue ou sur le trottoir & 116 & 11,6 & 83 & 5,5 \\
Petits ateliers dans la rue ou sur le trottoir & 51 & 5,1 & 48 & 3,2 \\
Pas d'encombrement & 663 & 66,3 & 1164 & 77,6 \\
\hline Nombre de répondants & 1000 & - & 1500 & \\
\hline
\end{tabular}

Source : Enquête migration, pauvreté et environnement urbain, 2007

\section{III- Des inondations périodiques plus fréquentes à Hô Chi Minh Ville}

On a déjà vu que les inondations ont été beaucoup plus souvent citées comme nuisances à Hô Chi Minh Ville et nous en avons évoqué les raisons; on retrouve ici cette plus forte fréquence, mais il n'en demeure pas moins que les inondations touchent les deux villes (tableau 9). Les inondations sont particulièrement aiguës dans le centre-ville du fait de l'imperméabilisation des terrains par la voirie et les constructions. 
Tableau 9- Répartition des maisons selon le type d'inondation, la zone d'habitation et la ville $(\%)$

\begin{tabular}{|c|c|c|c|c|c|c|c|c|}
\hline \multirow{2}{*}{$\begin{array}{l}\text { Source de } \\
\text { de la maison }\end{array}$} & \multicolumn{4}{|c|}{$\begin{array}{c}\text { Hanoi } \\
\text { Arrondissements }\end{array}$} & \multicolumn{4}{|c|}{$\begin{array}{l}\text { Hô Chi Minh Ville } \\
\text { Arrondissements }\end{array}$} \\
\hline & $\begin{array}{l}\text { Cen- } \\
\text { traux }\end{array}$ & $\begin{array}{l}\text { Périphé } \\
\text {-riques }\end{array}$ & Ruraux & $\begin{array}{c}\text { Ensem- } \\
\text { ble }\end{array}$ & $\begin{array}{l}\text { Cen- } \\
\text { traux }\end{array}$ & $\begin{array}{l}\text { Périphé } \\
\text {-riques }\end{array}$ & Ruraux & $\begin{array}{l}\text { Ensem } \\
\text {-ble }\end{array}$ \\
\hline Rivière et/ou pluie & 3,8 & 1,0 & 2,7 & 2,6 & 7,1 & 7,7 & 4,8 & 7,0 \\
\hline Rivière seulement & 2,9 & 0,0 & 5,7 & 2,7 & 0,8 & 6,7 & 3,7 & 2,7 \\
\hline Pluie seulement & 17,7 & 20,6 & 15,6 & 18,1 & 19,6 & 22,6 & 14,3 & 19,7 \\
\hline Jamais & 75,7 & 78,4 & 76,0 & 76,6 & 72,6 & 63,1 & 77,2 & 70,7 \\
\hline Total & 100,0 & 100,0 & 100,0 & 100,0 & 100,0 & 100,0 & 100,0 & 100,0 \\
\hline
\end{tabular}

Source : Enquête migration, pauvreté et environnement urbain, 2007

\section{IV- La pollution de l’air jugée plus forte à Hanoi}

De manière assez inattendue, la qualité de l'air est jugée sensiblement plus mauvaise à Hanoi (Tableau 10). Logiquement, la qualité de l'air apparaît meilleure en périphérie qu'au centre-ville où se concentrent la circulation et les activités.

Tableau 10- Evaluation de la qualité de l'air selon la zone d'habitation et la ville (\%)

\begin{tabular}{|c|c|c|c|c|c|c|c|c|}
\hline \multirow{2}{*}{ Qualité de l'air } & \multicolumn{4}{|c|}{$\begin{array}{c}\text { Hanoi } \\
\text { Arrondissements }\end{array}$} & \multicolumn{4}{|c|}{$\begin{array}{l}\text { Hô Chi Minh-Ville } \\
\text { Arrondissements }\end{array}$} \\
\hline & $\begin{array}{l}\text { Cen- } \\
\text { traux }\end{array}$ & $\begin{array}{l}\text { Périphé- } \\
\text { riques }\end{array}$ & Ruraux & $\begin{array}{l}\text { Ensem } \\
\text {-ble }\end{array}$ & $\begin{array}{l}\text { Cen- } \\
\text { traux }\end{array}$ & $\begin{array}{l}\text { Périphé- } \\
\text { riques }\end{array}$ & Ruraux & $\begin{array}{l}\text { Ensem } \\
\text {-ble }\end{array}$ \\
\hline Excellente & 1,4 & 0,3 & 3,4 & 1,6 & 3,1 & 1,5 & 3,7 & 2,7 \\
\hline Bonne & 9,3 & 14,9 & 26,6 & 15,6 & 16,4 & 23,3 & 26,6 & 19,5 \\
\hline Moyenne & 41,9 & 41,0 & 43,0 & 41,9 & 60,9 & 48,6 & 56,9 & 57,2 \\
\hline Mauvaise & 29,0 & 33,7 & 23,2 & 29,0 & 16,3 & 22,5 & 9,6 & 17,1 \\
\hline Très mauvaise & 18,3 & 10,2 & 3,8 & 11,9 & 3,4 & 4,1 & 3,2 & 3,5 \\
\hline Total & 100,0 & 100,0 & 100,0 & 100,0 & 100,0 & 100,0 & 100,0 & 100,0 \\
\hline
\end{tabular}

Source : Enquête migration, pauvreté et environnement urbain, 2007

La circulation routière, la stagnation des eaux, les émanations des ordures et les fumées d'usine sont considérées comme les principales responsables de la pollution de l'air dans les deux villes avec une acuité différente : la circulation routière arrive en tête à Hanoi et les émanations des ordures à Hô Chi Minh Ville (Tableau 11).

Tableau 11- Raisons de la pollution de l'air selon la ville : effectif et pourcentage de répondants (Plusieurs réponses possibles)

\begin{tabular}{lrrrr}
\multirow{2}{*}{ Raisons de la pollution de l'air } & \multicolumn{3}{c}{ Hanoi } & \multicolumn{3}{c}{ Hô Chi Minh-Ville } \\
& Nombre & $\%$ & Nombre & $\%$ \\
\hline Circulation routière & 167 & 41,1 & 77 & 24,9 \\
Stagnation des eaux, égout bouché & 139 & 34,1 & 82 & 26,5 \\
Ordures & 94 & 23,1 & 91 & 29,4 \\
Fumée d'usine & 88 & 21,6 & 84 & 27,2 \\
Chantier de construction & 34 & 8,3 & 11 & 3,5 \\
Exiguïté du logement & 14 & 3,4 & 9 & 2,9 \\
Manque d'espace vert & 11 & 2,7 & 4 & 1,3 \\
Pesticides, désherbants & 9 & 2,2 & 6 & 1,9 \\
\hline Nombre de répondants & 407 & - & 309 & - \\
\hline
\end{tabular}

Source : Enquête migration, pauvreté et environnement urbain, 2007 
L'eau elle-même contribue à la pollution de l'air et au dégagement d'odeurs nauséabondes ; plusieurs commentaires insistent sur cet aspect :

«Il y a de la poussière en provenance des usines et des moyens de transport. L'odeur de l'eau du fleuve est insupportable, à cause de l'eau pas encore traitée évacuée directement dans le fleuve » (femme de 31 ans, arrondissement de Hai Ba Trung, Hanoi).

«Le milieu d'habitation est moite. À la saison des pluies, l'eau de la rivière déborde dans les maisons en apportant une odeur très désagréable » (homme de 28 ans, arrondissement de Binh Thanh, HCMV).

Une proportion de $21,4 \%$ des répondants à Hanoi, contre seulement 7,7 \% à Hô Chi Minh Ville, considère que la santé d'un membre du ménage a déjà été affectée par la pollution de l'air. En toute logique, les maladies oto-rhino-laryngologiques arrivent largement en tête (Tableau 12) (cf. à ce sujet Mishra 2004, Nguyen Duc Hiep 2000, Pham Le Tuan 2006).

Tableau 12- Affections déjà contractées par un membre du ménage à cause de la pollution de l'air selon la ville effectif et pourcentage de répondants (Plusieurs réponses possibles)

\begin{tabular}{|c|c|c|c|c|}
\hline \multirow[t]{2}{*}{ Nuisances } & \multicolumn{2}{|c|}{ Hanoi } & \multicolumn{2}{|c|}{$\begin{array}{c}\text { Hô Chi Minh- } \\
\text { Ville }\end{array}$} \\
\hline & Nombre & $\%$ & Nombre & $\%$ \\
\hline Maladie respiratoire, du nez, de la gorge & 190 & 89,2 & 106 & 92,2 \\
\hline Maladie oculaire & 8 & 3,7 & 2 & 1,7 \\
\hline Insomnies & 8 & 3,7 & 0 & 0,0 \\
\hline Rhumatismes & 6 & 2,8 & 0 & 0,0 \\
\hline Maladie auditive & 5 & 2,3 & 1 & 0,8 \\
\hline « Maladies à répétition » & 5 & 2,3 & 0 & 0,0 \\
\hline Maladie du système digestif & 4 & 1,9 & 1 & 0,8 \\
\hline Grippe & 4 & 1,9 & 0 & 0,0 \\
\hline Autre & 5 & 2,3 & 4 & 3,5 \\
\hline Nombre de répondants & 213 & - & 115 & - \\
\hline
\end{tabular}

Source : Enquête migration, pauvreté et environnement urbain, 2007

Ces maladies sont exprimées de diverses manières :

«La poussière très importante dans la rue génère des maladies respiratoires. Cela provoque une toux sèche, surtout chez les enfants et les personnes âgées affaiblies » (homme de 37 ans, arrondissement de Dông Da, Hanoi).

« J'ai de la toux, de la bronchite, de la sinusite à cause de la poussière provenant de l'usine de ciment à Tân Thuân Dông " (homme de 38 ans, $7^{\mathrm{e}}$ arrondissement, HCMV).

L'influence sur la santé des erreurs de manipulation techniques ou des accidents n'est pas oubliée :

« Le remplacement des générateurs d'électricité à huile de mazout, à cause de coupures d'électricité, peut entraîner fumées et pollution, affectant la santé des habitants » (homme de 37 ans, arrondissement de Hai Ba Trung, Hanoi). 


\section{V- Une pollution sonore moins bien supportée à Hanoi}

La pollution sonore incommode plus de gens à Hanoi qu'à Hô Chi Minh Ville (Tableau 13). Il est vrai que les activités économiques y sont plus souvent étroitement imbriquées dans les habitations.

Tableau 13- Estimation du bruit dans le quartier selon la zone d'habitation et la ville (\%)

\begin{tabular}{lrrrrrrrr}
$\begin{array}{l}\text { Trop } \\
\text { de bruit } \\
\text { dans le } \\
\text { quartier }\end{array}$ & Centraux & \multicolumn{2}{c}{$\begin{array}{c}\text { Arrondissements } \\
\text { Périphéri }\end{array}$} & \multicolumn{2}{c}{ Ruraux } & \multicolumn{1}{c}{$\begin{array}{c}\text { Ensem- } \\
\text { ble }\end{array}$} & Centraux & \multicolumn{2}{c}{$\begin{array}{c}\text { Hô Chi Minh-Ville } \\
\text { Périphéri } \\
\text {-ques }\end{array}$} & Ruraux & \multicolumn{1}{c}{$\begin{array}{c}\text { Ensem- } \\
\text { ble }\end{array}$} \\
\hline Oui & 53,7 & 53,0 & 39,9 & 49,8 & 33,4 & 33,8 & 11,6 & 30,7 \\
Non & 46,3 & 47,0 & 60,1 & 50,2 & 66,6 & 66,2 & 88,4 & 69,3 \\
\hline Total & 100,0 & 100,0 & 100,0 & 100,0 & 100,0 & 100,0 & 100,0 & 100,0 \\
\hline
\end{tabular}

Source : Enquête migration, pauvreté et environnement urbain, 2007

Ce n'est guère que dans les arrondissements ruraux des deux villes que les gens souffrent moins du bruit. Les personnes qui ont déclaré qu'il y avait trop de bruit dans le quartier ont été sollicitées pour évaluer la gêne causée par ce bruit sur une échelle allant de 0 (pas de gêne) à 10 (gêne très élevée). Les résultats se répartissent en pourcentages selon les différents niveaux ainsi que le montre la figure 1. Le niveau 0 n'a jamais été donné. La moyenne est de 5,39 à Hanoi contre 5,18 à Hô Chi Minh-Ville.

Figure 1- Evaluation du niveau du bruit sur une échelle de 0 à 10 selon la ville (en \% des répondants)

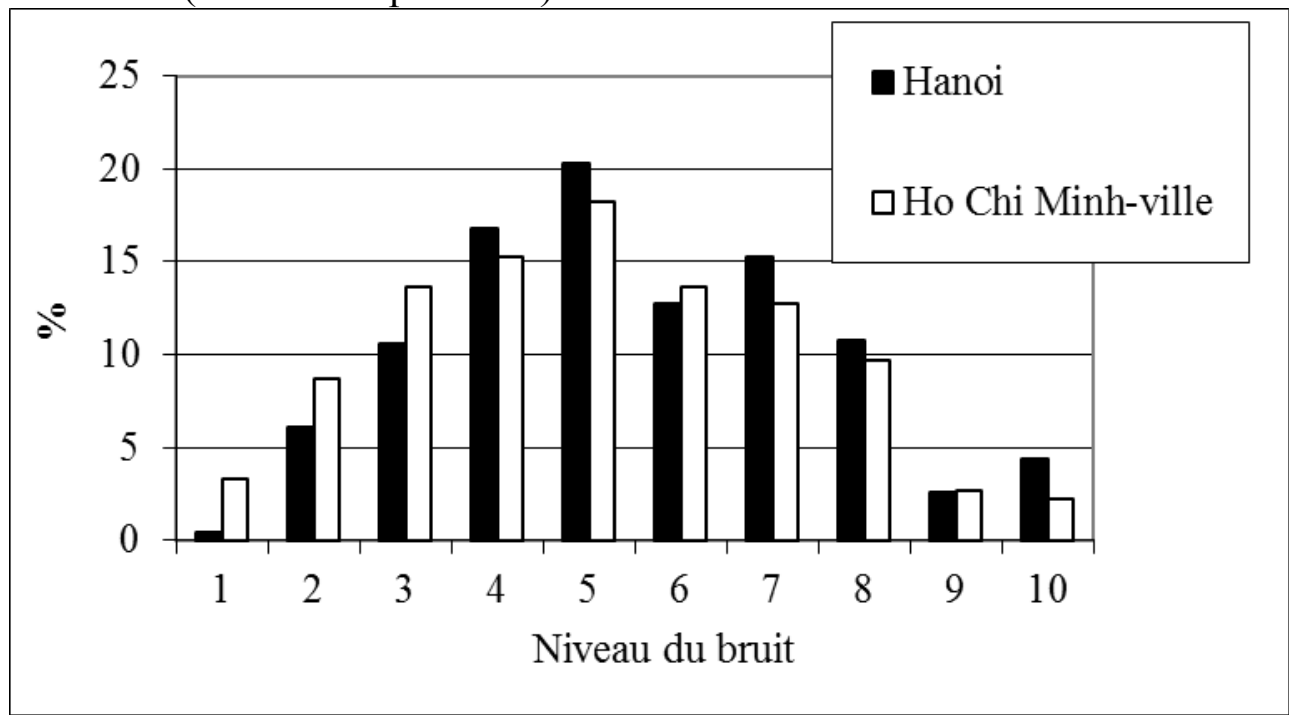

Source : Enquête migration, pauvreté et environnement urbain, 2007

L'origine du bruit est le plus souvent, et de loin, attribuée à la circulation routière dans les deux villes (Tableau 14). À noter que personne ne mentionne l'usage intempestif du klaxon. Les bruits de voisinage suivent ; on rappellera à ce sujet le niveau très élevé des densités de population et donc de la promiscuité dans certains quartiers : par exemple, pour les densités les plus élevées, $92100 \mathrm{hab} . / \mathrm{km}^{2}$ dans le district rural (phuong) Hang Gai, arrondissement Hoan Kiêm à Hanoi ; 112810 hab. $/ \mathrm{km}^{2}$ dans le phuong 7, 10 arrondissement à Hô Chi Minh Ville au recensement de 1999 (Gubry et coll., 2002). Le bruit causé par les ateliers artisanaux et les commerces se détachent également de l'ensemble, les activités économiques restant très imbriquées dans le tissu résidentiel. 
Tableau 14- Origine du bruit selon la ville : effectif et pourcentage de répondants (Plusieurs réponses possibles)

\begin{tabular}{|c|c|c|c|c|}
\hline \multirow{2}{*}{ Origine du bruit } & \multicolumn{2}{|c|}{ Hanoi } & \multicolumn{2}{|c|}{ Hô Chi Minh-Ville } \\
\hline & Nombre & $\%$ & Nombre & $\%$ \\
\hline Circulation routière & 390 & 78,3 & 303 & 66,0 \\
\hline Voisinage & 141 & 28,3 & 158 & 34,4 \\
\hline Atelier artisanal & 58 & 11,6 & 55 & 11,9 \\
\hline Commerce, marché & 43 & 8,6 & 52 & 11,3 \\
\hline Ecole & 27 & 5,4 & 4 & 0,9 \\
\hline Usine & 26 & 5,2 & 23 & 5,0 \\
\hline Karaoké, bar & 19 & 3,8 & 31 & 6,7 \\
\hline Aéroport & 0 & 0,0 & 6 & 1,3 \\
\hline Autre & 55 & 11,0 & 17 & 3,7 \\
\hline Nombre de répondants & 498 & - & 459 & - \\
\hline
\end{tabular}

Source : Enquête migration, pauvreté et environnement urbain, 2007

\section{VI- Opinions des répondants sur la résolution des problèmes de pollution : le rôle prépondérant de l'évacuation des eaux}

La modernisation du système d'évacuation des eaux vient largement en tête dans les deux villes parmi les mesures préconisées pour résoudre les problèmes de pollution (tableau 15). Elle est suivie par la modernisation de la collecte des ordures et les campagnes de sensibilisation à Hanoi ; par l'amélioration de la voirie, les campagnes de sensibilisation et la collecte des ordures à Hô Chi Minh Ville.

Les propositions et commentaires sur ce qu'il convient de faire pour résoudre les problèmes de pollution sont très riches et nombreux; nous n'en citerons ici qu'une sélection :

« Il faut avoir des règles en matière sanitaire sur l'environnement pour les ménages de petits commerçants, surtout les petits marchands d'aliments qui utilisent le charbon, créant une pollution de l'environnement » (homme de 36 ans, arrondissement de Long Biên, Hanoi).

« Les déchets des usines doivent être traités avant d'être évacués à la rivière. Il faut traiter les produits chimiques des usines de production industrielle pour ne pas affecter les habitations » (homme de 48 ans, arrondissement de Gia Lâm, Hanoi).

«Il faut trier les ordures avant de les jeter. Les véhicules de ramassage des ordures doivent venir près des habitations pour éviter aux habitants de se déplacer loin. Il faut recommander aux responsables de demander aux brasseries de traiter leurs ordures pour que ne pas influencer l'environnement » (femme de 54 ans, arrondissement de Ba Dinh, Hanoi).

« Il faut recommander aux autorités de réformer le système d'évacuation d'eau comme promis aux habitants. Il faut réformer le système de ramassage des ordures, régler les horaires de collecte afin qu'ils conviennent aux habitants » (homme de 50 ans, arrondissement de Ba Dinh, Hanoi).

«Il faut déménager les entreprises loin des habitations de la population. Limiter les moyens de transport qui sont trop vieux et rejettent beaucoup de fumée polluant l'air » (femme de 36 ans, arrondissement de Thanh Xuân, Hanoi).

Tableau 15- Mesures préconisées par les répondants ayant relevé un problèmede pollution pour le résoudre selon la ville (Plusieurs réponses possibles) 


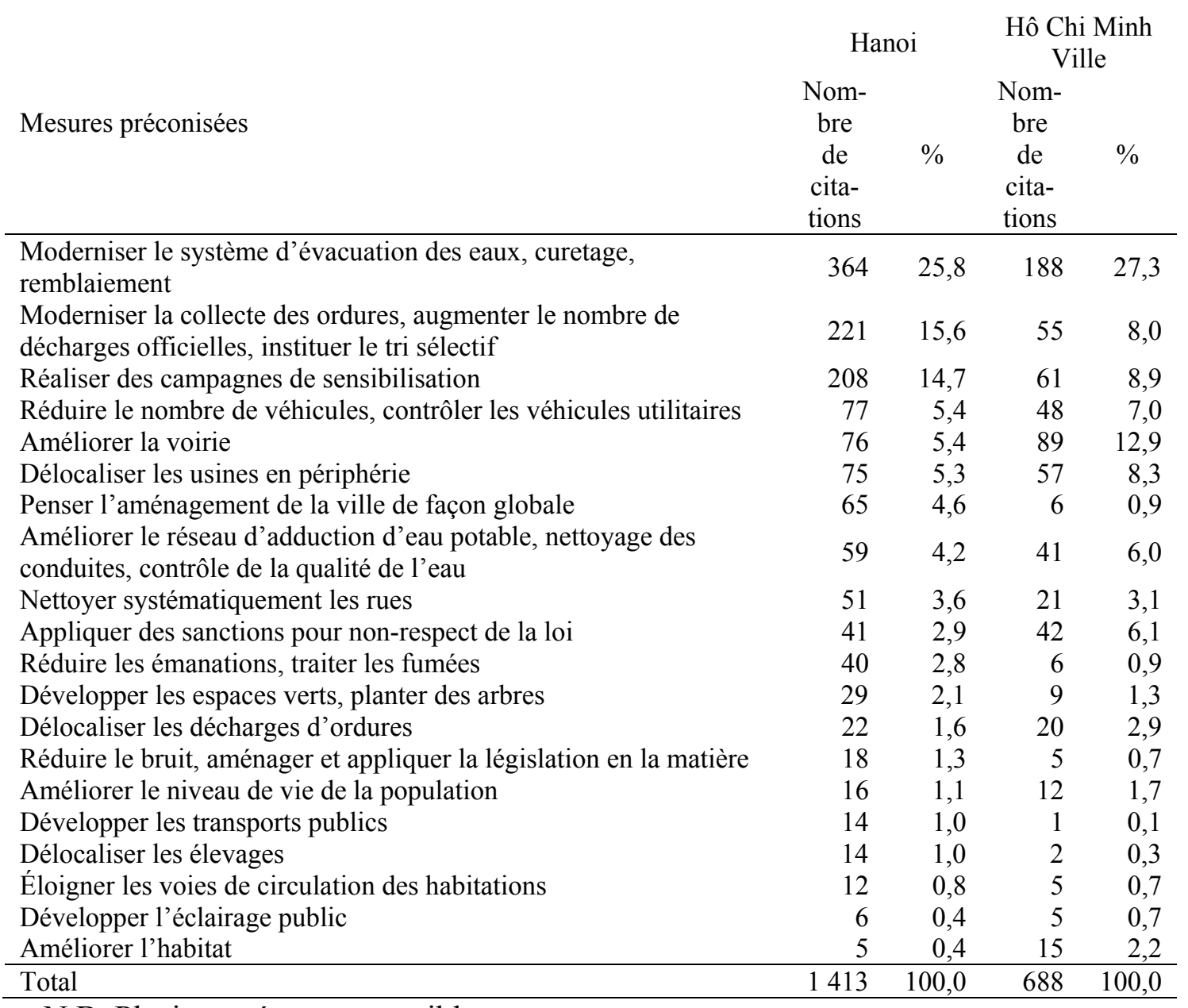

N.B. Plusieurs réponses possibles

Source : Enquête migration, pauvreté et environnement urbain, 2007

« Il faut pulvériser des produits pour tuer les moustiques, les insectes et les rats en permanence. Dans cette zone d'habitation il y a souvent des inondations, c'est pourquoi il y a trop de moustiques et de rats qui affectent la santé de la population » (homme de 53 ans, arrondissement de Hoan Kiêm, Hanoi).

«Il faut avoir un système de règles juridiques sur le problème de l'environnement : il faut avoir une législation pour dire à la population ce qu'elle doit faire pour protéger l'environnement; tous ceux qui ne respectent pas cette législation devraient payer une amende » (homme de 26 ans, arrondissement de Hoang Mai, Hanoi).

«Il faut avoir une politique de protection de l'environnement adaptée à chaque zone. Réaliser le tri des ordures. Elever la sensibilisation des gens à la protection de l'environnement. La campagne sur l'hygiène de l'environnement devrait améliorer le système de collecte des ordures. Par exemple, il ne faut pas laisser les ordures en plein air, il faut avoir une poubelle spéciale pour les ordures, il ne faut pas laisser les gens jeter les ordures directement dans la rue » (homme de 57 ans, arrondissement de Hai Ba Trung, Hanoi).

«Il faut déménager les entreprises loin des zones d’habitations. Interdire aux restaurants karaoké d'exercer au delà de minuit. Répartir la circulation pour éviter les embouteillages. Interdire d'occuper les trottoirs pour des intérêts personnels » (homme de 47 ans, arrondissement de Thanh Tri, Hanoi). 
«Le Gouvernement devrait investir dans les infrastructures, surtout il faut aménager la ville, construire des maisons avec beaucoup d’étages pour avoir plus d'espace vert et éviter la construction sans plan comme maintenant» (homme de 24 ans, arrondissement de Dông Da, Hanoi).

« Il faut imposer une somme à payer par les usines si elles génèrent de la pollution » (homme de 39 ans, $9^{\mathrm{e}}$ arrondissement, HCMV).

«Il faut exiger des zones industrielles qu'elles limitent les déchets qu'elles rejettent dans l'environnement et qu'elles installent leur propre système de traitement » (homme de 42 ans, $9^{\mathrm{e}}$ arrondissement, HCMV).

«Les autorités en charge ont imposé divers moyens pour résoudre le problème comme de réduire et de déplacer les zones polluées, mais sans résultat. Ainsi, je pense que les autorités devraient procéder à des réorganisations internes et ajuster les procédures de travail » (homme de 30 ans, $9^{\mathrm{e}}$ arrondissement, HCMV).

«A la période des inondations, l'eau de la rivière s'étend dans la zone résidentielle des ménages, le niveau de la crue va jusqu'à 0,5 mètre et créé des difficultés aux gens pour se déplacer, ruine la production agricole et affecte sévèrement les conditions économiques des ménages. Les gens sont intervenus plusieurs fois auprès des autorités, mais il n'y a pas de solution » (femme de 48 ans, $12^{\mathrm{e}}$ arrondissement, HCMV).

« Pour l'approvisionnement en eau, il faut demander aux autorités de fournir une eau propre pour l'usage quotidien des citoyens. Pour la question des ordures, il est nécessaire d'avoir des points de collecte des déchets éloignés des zones d'habitation et les citoyens doivent être mieux informés de leur responsabilité » (homme de 54 ans, $12^{\mathrm{e}}$ arrondissement, HCMV).

« Il faudrait démolir et avoir un plan pour les habitations le long de la rivière, car la rivière est surchargée et lourdement polluée. Le Gouvernement doit avoir une politique pour aider la population avec des mesures compensatoires adéquates. Il faut construire de nouvelles maisons, car les maisons occupent le lit de la rivière » (femme de 75 ans, $8^{\mathrm{e}}$ arrondissement, HCMV).

Enfin, certains mettent l'accent sur la nécessité de ne pas tout attendre des pouvoirs publics, mais d'agir immédiatement à titre personnel :

« Il faut soi-même assurer sa protection. Il faut que chacun agisse lui-même, il ne faut pas attendre des mesures du Gouvernement parce que cette attente est trop longue. Nous avons demandé au gouvernement de résoudre nos problèmes depuis longtemps, mais les pouvoirs publics n`ont pas réagi » (femme de 57 ans, arrondissement de Dông Da, Hanoi).

«Les gens doivent avoir davantage conscience de la protection de l’environnement. Ils doivent amender leur propre lieu d'habitation. Il ne faut pas attendre le Gouvernement et se plaindre » (femme de 62 ans, arrondissement de Dông Da, Hanoi). 


\section{VII- Problèmes de sécurité : une préoccupation plus forte à Hanoi}

Une proportion de 33,8\% des répondants à Hanoi et 15,9\% à Hô Chi Minh Ville trouvent qu'il n'y a pas de bonne sécurité dans leur quartier ${ }^{71}$. Le danger a été évalué par ceux qui considéraient que la sécurité n'était pas bonne, en pourcentages, sur une échelle allant de 0 (pas de danger) à 10 (danger très élevé) ainsi qu'il apparaît dans la figure 2 . La plus forte préoccupation des habitants de Hanoi en matière de sécurité peut y être constatée. Personne n'a attribué le niveau 0. La moyenne s'établit à 4,28 à Hanoi contre 3,98 à Hô Chi Minh Ville.

Figure 2- Evaluation du niveau de danger sur une échelle de 0 à 10 selon la ville (en \% des répondants)

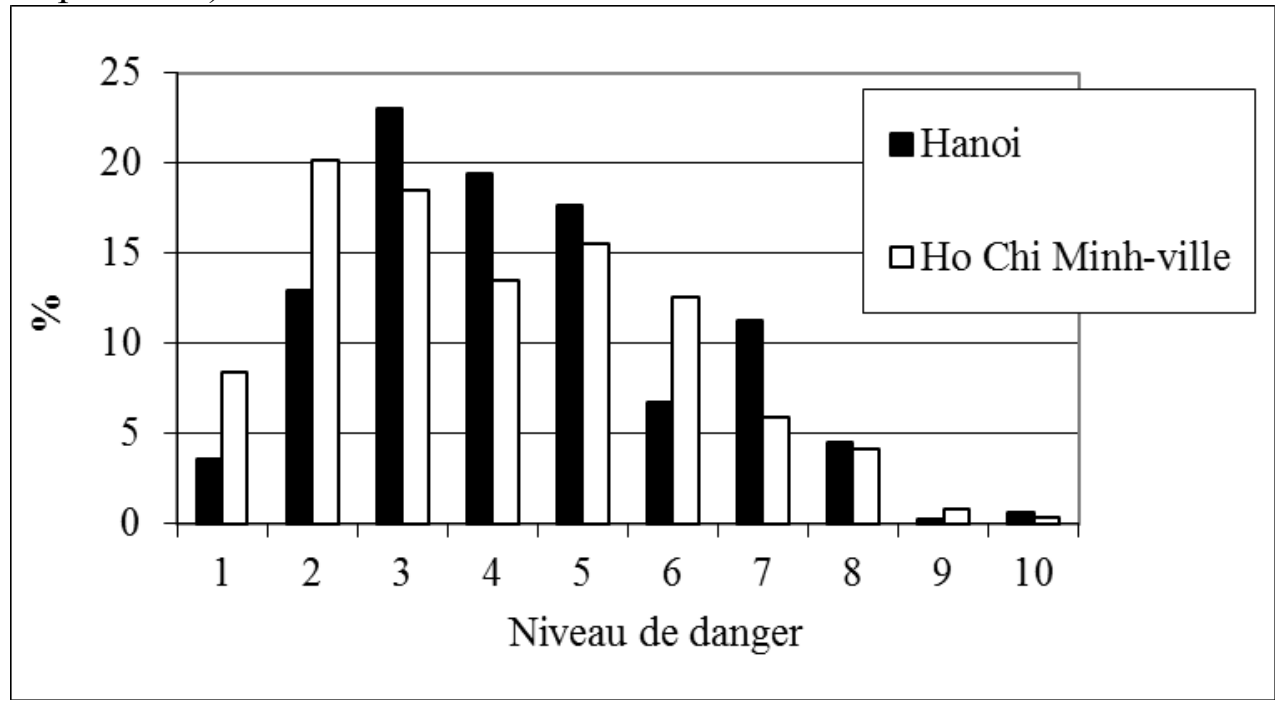

Source : Enquête migration, pauvreté et environnement urbain, 2007

La sécurité apparaît globalement moins bonne dans les arrondissements éloignés du centre-ville (Figures 3 et 4). Cette situation peut être attribuée aux problèmes sociaux qui s'y posent, à la plus faible promiscuité qui fait qu'on peut plus facilement y passer inaperçu, mais aussi sans doute à la densité plus faible des services de police. Des différences apparaissent entre Hanoi et Hô Chi Minh Ville au niveau des arrondissements centraux : la sécurité y semble mieux assurée dans cette dernière ville, ce qui reste à être expliqué.

\footnotetext{
71 Il s'agit ici d'une question spécifique, différente de la question ouverte avec réponses spontanées sur les nuisances traitée dans le tableau 2. On trouve donc ici beaucoup plus de gens préoccupés par la sécurité.
} 
Figure 3- Répartition de la proportion des répondants exprimant un problèmed'insécurité selon l'arrondissement et la ville
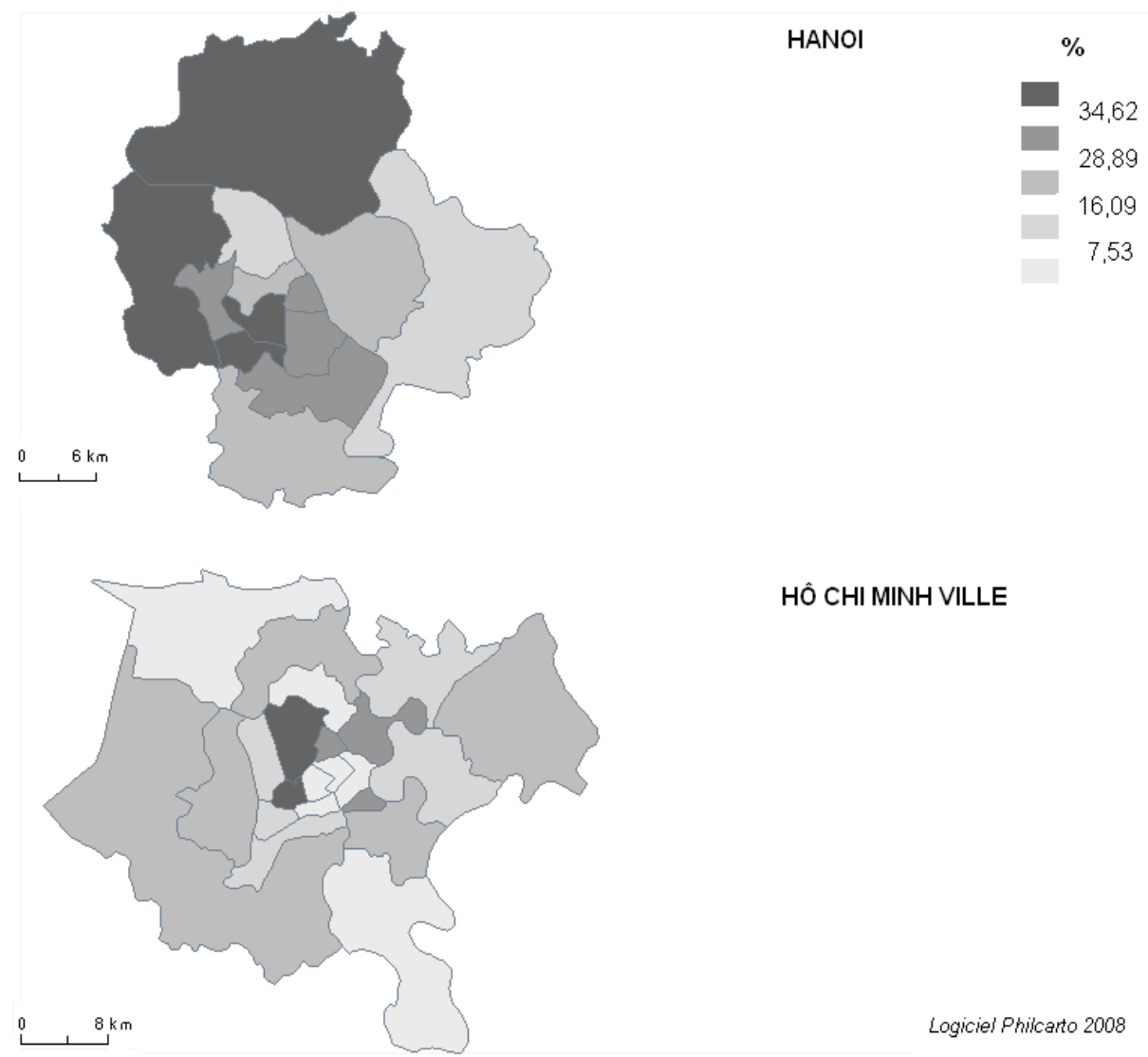

Hô CHI MINH VILLE

Source : Enquête migration, pauvreté et environnement urbain, 2007

Les vols, y compris les vols à la tire, et les cambriolages sont les premiers incriminés dans l'insécurité (Tableau 16). Il est intéressant de relever qu'ils sont directement suivis par la drogue, à un niveau étonnamment élevé à Hanoi, tant il est vrai que la dépendance à la drogue conduit directement au vol pour s'en procurer. On y reviendra au niveau des mesures pour améliorer la sécurité.

Tableau 16- Nature de l'insécurité dans le quartier selon la ville : effectif et pourcentage de répondants (Plusieurs réponses possibles)

\begin{tabular}{lrrrr} 
Nature de l'insécurité & $\begin{array}{c}\text { Hanoi } \\
\text { Nombre de } \\
\text { citations }\end{array}$ & $\%$ & $\begin{array}{c}\text { Hô Chi Minh Ville } \\
\text { Nombre de } \\
\text { citations }\end{array}$ & $\%$ \\
\hline Vols, cambriolages & 295 & 87,5 & 214 & 89,9 \\
Drogue & 114 & 33,8 & 12 & 5,0 \\
Racket, violence & 37 & 11,0 & 6 & 2,5 \\
Meurtres & 10 & 3,0 & 4 & 1,7 \\
Composition de la population & 9 & 2,7 & 9 & 3,8 \\
Alcoolisme & 4 & 1,2 & 2 & 0,8 \\
Jeux d'argent & 3 & 0,9 & 1 & 0,4 \\
\hline Nombre de répondants & 337 & - & 238 & - \\
\hline
\end{tabular}

Source : Enquête migration, pauvreté et environnement urbain, 2007

Les mesures policières sont les plus fréquemment préconisées pour améliorer la sécurité dans le quartier (Tableau 17). On peut y associer l'amélioration de la formation et la sensibilisation des personnels de sécurité, voire le renforcement du contrôle résidentiel. On 
trouve en second lieu l'éducation et l'information de la population en matière de sécurité, mais sans que des mesures très concrètes soient proposées. On retiendra l'importance de la lutte contre la toxicomanie proposée à Hanoi.

Tableau 17- Mesures préconisées par les répondants pour améliorer la sécurité dans le quartier selon la ville : effectif et pourcentage de répondants (Plusieurs réponses possibles)

\begin{tabular}{|c|c|c|c|c|}
\hline \multirow[b]{2}{*}{ Mesures préconisées } & \multicolumn{2}{|c|}{ Hanoi } & \multicolumn{2}{|c|}{$\begin{array}{l}\text { Hô Chi Minh- } \\
\text { Ville }\end{array}$} \\
\hline & $\begin{array}{l}\text { Nom } \\
\text {-bre } \\
\text { de } \\
\text { cita- } \\
\text { tions }\end{array}$ & $\%$ & $\begin{array}{l}\text { Nom } \\
\text {-bre } \\
\text { decita } \\
\text {-tions }\end{array}$ & $\%$ \\
\hline $\begin{array}{l}\text { Développer la police, les milices populaires, les contrôles, la } \\
\text { surveillance de proximité }\end{array}$ & 149 & 44,2 & 141 & 59,2 \\
\hline Éduquer et informer la population & 93 & 27,6 & 23 & 9,7 \\
\hline $\begin{array}{l}\text { Améliorer la formation des policiers, accroître le sentiment de } \\
\text { devoir des gens travaillant dans la sécurité et des responsables } \\
\text { d'îlots }\end{array}$ & 83 & 24,6 & 7 & 2,9 \\
\hline $\begin{array}{l}\text { Gérer la toxicomanie, mener les toxicomanes dans un centre de } \\
\text { cure }\end{array}$ & 39 & 11,6 & 2 & 0,8 \\
\hline Renforcer le contrôle résidentiel & 25 & 7,4 & 6 & 2,5 \\
\hline Punir sévèrement les coupables d'infraction & 19 & 5,6 & 14 & 5,9 \\
\hline Améliorer le niveau de vie de la population & 14 & 4,2 & 3 & 1,3 \\
\hline $\begin{array}{l}\text { Fermer les établissements « louches », les « points de } \\
\text { ralliement» }\end{array}$ & 9 & 2,7 & 0 & 0,0 \\
\hline Développer l'éclairage public & 7 & 2,1 & 1 & 0,4 \\
\hline Nombre de répondants & 337 & - & 238 & \\
\hline
\end{tabular}

Source : Enquête migration, pauvreté et environnement urbain, 2007

Quelques commentaires permettent de préciser les mesures proposées en matière de sécurité ; on y retrouve le souhait d'un contrôle résidentiel renforcé, une certaine méfiance envers les allochtones, mais aussi le souhait d'une solidarité accrue entre les habitants :

«Renforcer la sécurité dans les quartiers d’habitation. Gérer étroitement les personnes logées à titre provisoire pour éviter les mauvaises gens » (homme de 51 ans, arrondissement de Dông Da, Hanoi).

«Gérer étroitement les ménages pour éviter que des gens d'autres endroits viennent ici avec des buts malhonnêtes » (femme de 52 ans, arrondissement de Binh Thanh, HCMV).

« Expliquer aux gens ce qu'est la civilisation urbaine, réduire les querelles entre voisins » (femme de 76 ans, arrondissement de Binh Thanh, HCMV).

«La police du secteur devrait porter l'attention sur les groupes d'individus malfaisants pour éviter l'inquiétude des autochtones » (femme de 60 ans, $12^{\mathrm{e}}$ arrondissement, HCMV).

«Les gens du quartier devraient prendre soin les uns des autres et s'aider mutuellement » (homme de 56 ans, $12^{\mathrm{e}}$ arrondissement, HCMV).

De nombreux intervenants associent l'insécurité à la toxicomanie, la lutte contre celle-ci devant augmenter la sécurité puisque les drogués sont amenés à accomplir des méfaits pour se procurer de la drogue; dans ce domaine, on touche au problème de la corruption, à laquelle le Gouvernement s'est officiellement attaqué depuis plusieurs années :

«Il faut créer les conditions pour que les toxicomanes puissent cesser l'usage de stupéfiants et il faut les stimuler et les aider » (homme de 44 ans, arrondissement de Dông Anh, Hanoi). 
«Il faut mieux gérer la toxicomanie pour que la population ait moins de problèmes » (homme de 50 ans, arrondissement de Hai Ba Trung, Hanoi).

« Il faut que la police et les pouvoirs publics gèrent mieux les toxicomanes et les gens ayant des antécédents judiciaires ou avec la police » (femme de 37 ans, arrondissement de Dông Da, Hanoi).

«La population est très fidèle au parti communiste, mais il y a des gens au pouvoir qui sont corrompus. Même dans les centres de toxicomanie circulent des drogues (héroïne). C'est pourquoi il faut faire en sorte que les gens au pouvoir soient irréprochables, améliorer la manière de gérer; il faut faire et non pas seulement parler » (homme de 70 ans, arrondissement de Hoang Mai, Hanoi).

"Solliciter l'aide de la police dans la poursuite des drogués dépendants » (homme de 55 ans, arrondissement Binh Thanh, HCMV).

\section{Conclusion : de la connaissance à l'action}

Nous avons constaté que l'environnement est considéré presque systématiquement comme plus mauvais par les habitants de Hanoi que par ceux de Hô Chi Minh-Ville. C'est le cas de l'évaluation générale de l'environnement du quartier, de l'évolution de l'environnement, de la pollution de l'air, de la pollution sonore, de l'insécurité... C'est le contraire de ce qu'on pouvait attendre, dans la mesure où les problèmes environnementaux sont probablement plus aigus dans la plus grande des deux villes. Nous avons attribué cela au fait que l'éducation - et la sensibilisation à l'environnement qui va de pair - sont significativement plus élevées à Hanoi, mais il ne faut pas éliminer la possibilité que les répondants aient aussi pris en compte le climat dans leur évaluation de l'environnement. De fait, le climat est objectivement plus difficile à supporter à Hanoi, avec une humidité élevée et des différences de température importantes entre l'été et l'hiver, alors que le climat de Hô Chi Minh Ville, subtropical, est beaucoup plus constant.

Parmi la pollution observée, la pollution de l'air arrive loin en tête dans les deux villes : odeurs dues aux déchets ou aux eaux usées, gaz d'échappement, fumée des usines et combustion du charbon... La pollution de l'air est également mentionnée comme la principale cause de la détérioration de l'environnement du quartier par ceux qui estiment que cet environnement se dégrade. Le trafic routier est le premier mis en cause à Hanoi, la fumée des usines à Hô Chi Minh Ville ; on relève aussi que le quart des maisons sont situées près d'une route à grande circulation. Dans ce contexte, les maladies oto-rhino-laryngologiques sont les maladies les plus courantes.

Les inondations sont un sujet de préoccupation, en particulier à Hô Chi Minh Ville, où un tiers des maisons est concerné, en raison du processus de marée qui a été signalé plus haut, aggravé par la rareté des pentes et l'engorgement du réseau de drainage par les ordures.

Les nuisances sonores sont causées en premier lieu par le trafic routier, suivi des bruits de voisinage en raison de la surpopulation dans les quartiers.

La modernisation du drainage et des égouts, la modernisation de la collecte des ordures et le développement de campagnes de sensibilisation sont les mesures les plus fréquemment proposées pour résoudre ces problèmes de pollution, parmi un large éventail de mesures qui pourraient être envisagées.

Les questions de sécurité préoccupent la population relativement moins que les problèmes de pollution. En fait, la sécurité reste meilleure dans les grandes agglomérations vietnamiennes que dans d'autres villes de taille comparable dans le monde. Il n'en demeure pas moins que le vol et le cambriolage se développent, que les répondants associent clairement à l'augmentation de la toxicomanie. Le développement et l'amélioration des 
services de police, y compris la police de proximité, sont les mesures les plus fréquemment proposées pour améliorer la sécurité. Cela n'est pas indépendant de la lutte contre la toxicomanie, souvent rendue difficile en raison de la corruption de certains fonctionnaires, comme plusieurs répondants l'ont souligné.

La plupart des causes de dégradation de l'environnement et de l'insécurité croissante sont connues, la manière de les combattre l'est moins. La prise en compte des opinions de la population sur ses principales préoccupations en la matière et de ses idées sur la façon de résoudre les problèmes est en tout cas susceptible d'optimiser les actions à mettre en œuvre en les ciblant selon les priorités identifiées sur le terrain.

\section{Bibliographie}

Central Population and Housing Census Steering Committee, The 2009 Vietnam Population and Housing Census: Some key indicators, Hanoi, Office général des Statistiques, 2010, p. 23. [BAN CHİ ĐẠO TỔNG ĐIỀU TRA DÂN SỐ VÀ NHÀ Ở TRUNG ƯOONG, Tồng điều tra dân số và nhà ở Việt Nam năm 2009 : Một số chỉ tiêu chủ yếu. Hà Nội, 2010,23 tr.]

DRUMMOND Lisa B.W. «Street scenes: Practices of public and private space in urban Vietnam », in Urban Studies, Glasgow, n 12/37, 2000, p. 2377-2391.

Gubry Patrick, Lortic Bernard, Greneche Gilles, Le Van Thanh, Le Thi Huong, Tran Thi Thanh Thuy, Nguyen Thi Thiêng, Pham Thuy Huong, V-HoAng Ngân, NGuYen Thê Chinh, "Hô Chi Minh Ville et Hanoi : Population et migrations intra-urbaines », in Séminaire Regards croisés sur Hanoi: Transition, spécificité urbaine et choix de développement, Hanoi, 12-14 novembre 2002, p. 42. [GUBRY Patrick, LORTIC Bernard, Greneche Gilles, LE Văn Thành, LE Thị Hương, TrẦn Thị thanh Thủy, NGUYẾN Thị Thiệng, PHAM Thúy Hương, Vu Hoàng Ngân, NGUYỄN Thế Chính, « Thành Phố Hồ Chí Minh và Hà Nội : Dân số và di dân nội thị. Hội thảo » in Hà Nội thành phố đặc thù và những lựa chọn cho phát triển, Hà Nội, 12-14 tháng 11 năm 2002, 42 tr.]

GubRY Patrick, LE Van Thanh, NGuYen Thi Thiêng, « Méthodologie/ Phương pháp nghiên cứu», in Bouger pour vivre mieux. Les mobilités intra-urbaines à Hô Chi Minh Ville et Hanoi (Viêt-nam) / Di chuyển để sống tốt hơn. Di dân nội thị tại Thành Phố Hồ Chí Minh và Hà Nội (Việt Nam), GuBRY Patrick, LE Thi Huong, NGuYEN Thi Thiêng, PHAM Thuy Huong, TRAN Thi Thanh Thuy, VU Hoàng Ngân (dir.), Hanoi, Université nationale d'Économie, 2008, p. 19-50. / Hà Nội : Nhà Xuất Bản Trường Đại Học Kinh Tế Quốc Dân, 2008, p. 17-46. http://horizon.documentation.ird.fr/exl-doc/pleins_textes/divers0901/010043543.pdf [En ligne, consulté le 10 octobre 2014].

GuBRY Patrick, NGuYÊN Thi Thiêng, LÊ Thi Huong et coll., 2007. Enquête Migration, pauvreté et environnement urbain, IRD, IPSS-UNE, HIDS-Ho Chi Minh Ville, 2007, (dactyl.).

GuBRY Patrick, LÊ Thi Huong, NGUYÊN Thi Thiêng, «Disparities in the city: Poverty and urban environment in Hanoi and Ho Chi Minh City (Vietnam) », in XXVI ${ }^{\text {th }}$ International Population Conference (IUSSP), $27^{\text {th }}$ September-2 ${ }^{\text {nd }}$ October 2009, p. 9.

GubRY Patrick, et NGuYen Thi Thiêng, 2012, « Méthodologie d'enquête en milieu urbain au Viêt Nam : exemple de l'enquête «Migration, pauvreté et environnement urbain : Hanoi et Hô Chi Minh Ville». Atelier de formation aux méthodologies de recherches avancées sur l'analyse des questions démographiques et sociales au Viêt-nam/ Khóa đào 
tạo phương pháp nghiên cứu nâng cao, Phân tích các vấn đề dân số và xã hội ở Việt Nam, Hanoi, ARCUS, IPSS, CERPOS, 19-29 novembre 2012, 28 diapositives.

JENSEN Rolf, PEPPARD Donald, "Hanoi's informal sector and the Vietnamese economy: A case study of roving street vendors ", in Journal of Asian and African Studies, $\mathrm{n}^{\circ} 38$, 2003, p. 71-84.

MisHRA Vinod, «What do we know about health effects of smoke from solid fuel combustion? ", in Population and Health Series, $n^{\circ}$ 117, janvier 2004, p. 43.

NGuYÊN Duc Hiêp, «Air quality in Ho Chi Minh City, Viet Nam », in Proceedings of the Asia-Pacific Conference on Sustainable Energy and Environmental Technology, Lu G.Q., Green P.F., Liu C.Y., Tay J.H., (dir..), Singapore, World Scientific, 2000, p. 67100 .

PHAM Lê Tuân, «Result of survey on asthma in Hanoi pupils», in Journal of Allergy and Clinical Immunology, $\mathrm{n}^{\circ} 27 / 117,2006$, p. 80.

PUlliat Gwenn, «Les migrants à Hanoi : construction politique d'un groupe social dominé», in Espaces et Sociétés, n 154, 2013, p. 87-102. 


\section{Annexe}

Figure 4: Schéma des arrondissements de Hanoi et Hô Chi Minh Ville en 2007
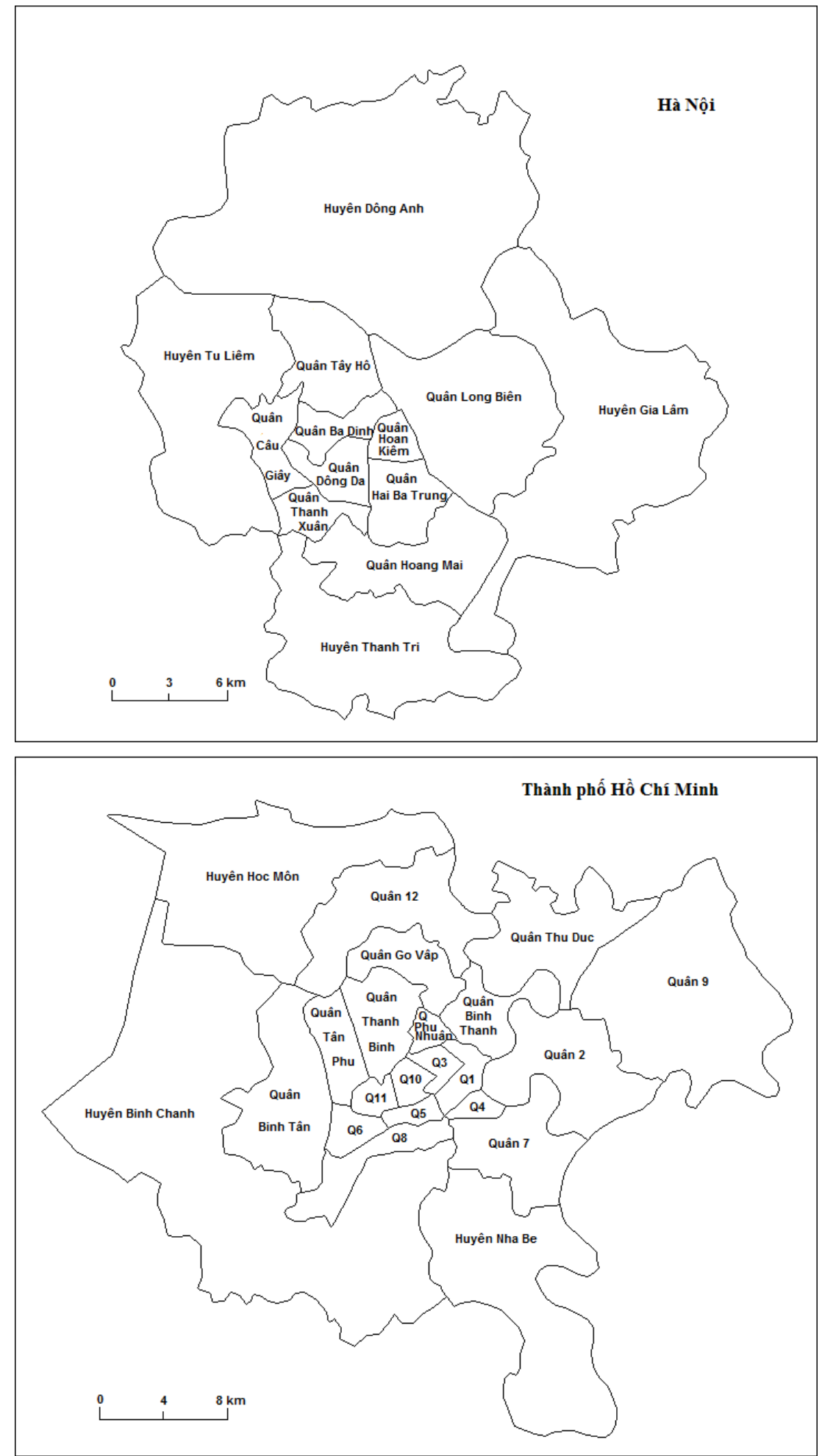

Note : Ne figurent pas sur ce schéma les arrondissements ruraux excentrés de Soc Son (Hanoi), Cu Chi et Cân Gio (Hô Chi Minh Ville)

Source : Enquête migration, pauvreté et environnement urbain, 2007 


\title{
Are non-farm jobs better for rural workers? A Panel Data Analysis of Earnings Gaps in Vietnam
}

\author{
Nguyen Huu Chi (UNE, CEPN Université Paris Nord)
}

In developing countries, agricultural employment accounts for a huge component of rural workforce. Literature have shown that the low agricultural productivity and great dependence of farm production on uncertain natural conditions are driving forces to low labour income in the agricultural sector which in turn results in high poverty incidence in rural area. Thus, a special attention is paid to the identification of rural pathways out of poverty. As summarized by McCulloch et coll. (2007), the main pathway out of poverty would be associated with increases in the productivity of rural poor, whether these increases are realized in farm job, in rural non-farm activity or by rural-urban migration. Traditionally, agricultural growth has been the main ingredient in rural development strategies in many countries. However, it is shown that the reliance on pro-poor agricultural growth as the main path out of poverty today is facing challenges due to a combination of factors leading to increase of risk, uncertainty and raising costs and/or lower return to agricultural investment (Dorward et coll., 2004). Scholars have for long emphasized the need for diversified approaches to fighting rural poverty in order to take the heterogeneity of the rural population into account (Jonasson, 2009). It is widely considered that rural non-farm employment (RNFE) is a potential path out of poverty as it can provide a source of income for rural workers, particularly those who are facing difficulties of securing income due to underemployment, insufficient of cultivable land. Additionally, non-farm employment appears to bring about better earnings than do agriculture jobs. Although this feature of nonfarm employment has long been recognised as being among the most robust findings in comparative international labour economics (Hertz et coll., 2009), not much empirical evidence has been offered on it. This lack of evidence has sometimes been pointed out in the literature. For instance, Lanjouw (2008) argued that even though average earnings in the rural non-farm sector are higher than in agriculture, it is unclear whether income prospects are systematically better in non-farm activities than in agriculture. Winters et coll. (2008) also indicated that while agricultural jobs generally do pay less, there is still a considerable overlap between the farm and non-farm wage distributions in most countries.

To measure farm - non-farm earnings gaps, some studies have been conducted based on both cross-sectional and panel data. For instance, basing on the method of propensity score matching, Dabalen et coll. (2004) estimate return to participation in the rural non-farm sector compared with that in the agricultural sector in Rwanda. They test whether people with similar attributes, but in different sectors, earn different incomes, and find that the selfemployed in the non-farm sector earn significantly more than farm workers do. McCulloch et coll. (2007), in a study of pathways out of rural poverty in Indonesia, use panel data to trace the income changes of people who switch from agriculture to non-farm activities. They find that increased engagement of rural farmers in non-farm businesses has been the most promising way to improve incomes for workers and their household, thus constitute a path out of rural poverty. Jonasson (2009) provides further evidence on this issue in testing for existence of earnings gaps between rural non-farm and farm employment while controlling for skills of workers. His empirical findings indicate that there would be no premium for an unskilled worker who switches from agriculture to rural non-farm employment. By contrast, skilled workers are likely to do better in non-farm than in agricultural jobs since they have higher return to education. However, it is arguable that earnings gaps between non-farm and farm jobs depend not only on individual characteristics but also on the characteristics of the 
non-farm jobs in which workers engage in. So far, there has not been clear evidence on the variation of non-farm - farm earnings gaps by sector as well as status of non-farm employment.

This paper aims at assessing earnings differentials between farm and non-farm jobs with a further attention paid to accounting for heterogeneity among non-farm employment in both formal and informal sector. This analysis is an extension of a work by Nguyen, Nordman and al. (2013) which measures earnings gaps within non-farm sector jobs in Vietnam. We distinguish different types of rural non-farm workers in order to allow earnings gaps to be measured specifically for each type of non-farm jobs compared to agricultural jobs. The main questions to be investigated are: is there a systematic earnings gap between rural nonfarm and agricultural jobs when controlling for factors that potentially determine earnings of individuals, given the heterogeneity among rural non-farm activities as well as employment? Do all non-farm jobs provide pecuniary premiums over agricultural jobs? Do possible gaps vary remarkably along the earnings distributions?

Taking advantage of the rich Vietnam Household Living Standards Survey (VHLSS) dataset in Vietnam (a Living Standard Measurement Study type household survey), in particular its three-wave panel data (2002-2004-2006), we assess the RNFE-agricultural earnings gaps in this country. Our empirical analysis consists of assessing the magnitude of different types of earnings gaps between non-farm and agricultural employment using Ordinary least squares (OLS) and quantile regressions. We use a worker level definition of informality, the so-called informal/formal employment divide ${ }^{72}$ (Hussmanns 2004). Standard earnings equations are estimated at the mean and at various conditional quantiles of the earnings distribution. Furthermore, we estimate fixed effects quantile regressions to control for unobserved individual characteristics, focusing particularly on heterogeneity within both the formal and informal non-farm employment categories. Our purpose is to address the important issue of heterogeneity at two levels: the worker level, taking into account individual unobserved characteristics; the job level, differentiating wage and selfemployed workers. Estimations of earnings gaps from panel data would help preventing the risk of spurious results since we can take into account unobserved heterogeneity as well as, to some extent, the potential endogeneity concerning the selectivity process of job allocation across sectors.

The remainder of this chapter is organized as follows. Section 1 presents the context, the data and some descriptive elements of income dynamics in the recent period, while Section 2 focuses on the econometric approach to assess rural farm - non-farm earnings gaps. Empirical results are presented in section 3 and discussed in Section 4.

\section{I- Context, Labour Market Dynamics in Vietnam and Data}

\section{1- $\quad$ Context}

The growth model embraced by Vietnam during the last two decades, in an urbanization context, has prompted deep social economic transformation. The private sector has been thriving with the transition of a centrally planned economy towards a "socialist-oriented market economy" since the Doi Moi (Renovation) launched in 1986. Economic growth has helped reduce poverty considerably, but in the meantime, sparked increasing social inequality. The gap between regions, areas and social classes has widened (VASS 2010;

\footnotetext{
${ }^{72}$ The definition of this so-called informal/formal employment divide is explained below (see 1.2).
} 
Cling et coll. 2012). Market freedom, meanwhile, paved the way for the development of an informal economy.

In the labour market, two main striking features are at stake: the rising rate of wage and non-farm employment; a sharp increase in real wages and labour incomes in recent years (Cling et coll. 2012). Vietnam's impressive economic growth over the last decade has triggered a sharp increase in the rate of wage employment, which is one of the striking facts of the labour market developments in recent years: the rate rose from 19 percent in 1998 to 33 percent in 2006. Wage employment grew particularly sharply in the industrial sector (including construction) during the last ten years. This spread of wage employment has affected all population categories (urban/rural, male/female, skilled and unskilled), but substantial differences in level subsist. Wage employment is obviously more developed among the most skilled manpower (86 percent among the highly skilled as opposed to barely one-quarter among the unskilled), and it is also more prevalent among urban dwellers and among men (35 percent compared to 25 percent for women).

The spreading of wage employment on the Vietnamese labour market has been accompanied by a steep decline in agricultural employment. From 1998 to 2006, the share of agricultural jobs has been reduced by 18 percentage points, from 67 to 49 percent (Table 1). This trend is due to a vibrant urbanization process (according to the latest population census conducted in 2009, the population has been growing by 3.4 percent annually in urban areas over the last decade, compared to 0.4 percent per year in rural areas; General Statistics Office and UNFPA 2009). But, at the same time, in all kinds of geographic areas, the proportion of out farm jobs has been on the rise, a shift particularly important in suburban areas (Cling et coll., 2010b). For instance, in the rural surroundings of the two biggest cities (Hanoi and Ho Chi Minh), agricultural employment has fallen down from 58 percent to 22 percent during the period. Despite an important rate of underemployment, wages gradually rose from 1998 to 2006. Sharp economic growth prompted a 56 percent increase in wage earners' average annual remuneration over the period observed, which works out at an average annual growth rate of 5.7 percent.

Table 1- Changes in labour structure and earnings in Vietnam 1998-2006

\begin{tabular}{|c|c|c|c|c|c|c|c|c|}
\hline & \multicolumn{4}{|c|}{ Jobs (percent) } & \multicolumn{4}{|c|}{ Real income $(100=1998$; wage only $)$} \\
\hline Sector & 1998 & 2002 & 2004 & 2006 & 1998 & 2002 & 2004 & 2006 \\
\hline Agriculture & 67.1 & 56.5 & 52.0 & 49.2 & 100 & 96.2 & 107.4 & 128.3 \\
\hline Secondary sector & 13.9 & 19.7 & 21.7 & 23.0 & 100 & 109.4 & 119.6 & 134.3 \\
\hline Services & 19.0 & 23.8 & 26.3 & 27.8 & 100 & 146.1 & 158.3 & 177.7 \\
\hline Total & 100 & 100 & 100 & 100 & 100 & 121.2 & 137.1 & 155.7 \\
\hline Wage workers & 17.5 & 28.6 & 31.0 & 33.1 & - & - & - & - \\
\hline
\end{tabular}

1998, Viet Nam Households Living Standards Survey (VHLSS) 2002, 2004, 2006; Cling et coll. 2012.

Real wages grew at a slower pace in agriculture than in other sectors ( 28 percent vs. 34 percent and 78 percent for secondary sector and services) over the period. Wage dynamics was higher for the semi-skilled and high skilled workers than for unskilled workers (67 percent, 62 percent and 36 percent respectively). At the same time, the increase was lower for men than for women (respectively +51 percent and +60 percent from 1998 to 2006), mainly given the changes of the labour market structure (more in favour of female workers). This leads to a reduction in gender inequalities to some extent (Cling et coll., 2012). 
This context has brought a very optimistic view on the dynamism of the economy and of the labour market in Vietnam. Still, this analysis focuses only on the global trends and fails to take into consideration the informal economy. The on-going restructuring on the labour market clearly benefited the non-farm private sector: the formal sector (both domestic and foreign enterprises) but also the household businesses, of which the informal sector is the main part. The share of large enterprises in total labour force doubled, from a very low 4 percent in 1998 to 8 percent in 2006. At the same time, non-farm household businesses employment increased from 20 percent to 35 percent during the same period. In parallel, the informal jobs (not covered by social insurance scheme), still represent a massive component of the economy. The Labour Force Survey (LFS) conducted in 2007 gives a precise picture of these two dimensions of the informal economy (Cling et coll., 2010a). Informal sector jobs represent 23 percent of total jobs and nearly a half of non-farm jobs; informal jobs account for 82 percent of total jobs and two-thirds of non-farm jobs.

Whatever the growth hypotheses in the years to come, the employment in the informal sector and its share in the total employment will increase even without the economic downturn of 2008-2009. This phenomenon is due to the limited capacity of the private formal sector (even if it continues to grow with the same frantic rhythm as prior to the crisis) to absorb the new entrants in the labour market and the workers who move from agricultural to non-farm activities. Therefore, understanding better the informal employment dynamics is a key challenge to design policies aiming at protecting its workers, improving the labour conditions and increasing productivity, keeping with it intrinsic flexibility.

\section{2- $\quad \underline{\text { Data }}$}

The data used in this paper are drawn from three successive rounds of the Vietnam Household Living Standards Surveys (VHLSS 2002, 2004 and 2006). These surveys are Living Standard Measurement Study (LSMS) type surveys, probably one of the most popular household surveys in developing countries. Initially designed by the World Bank to measure and monitor poverty and inequality, LSMS became multi-purpose studies, covering almost all aspects of the economic and domestic activities of households.

In terms of sample design, the VHLSS are a classical three-stage stratified random survey, covering the ordinary households at the national level. ${ }^{73}$ The sample size is quite large even if it has been progressively reduced, from 75,000 in 2002 to 45,000 in 2004 and 2006 (Table 2). A detailed questionnaire (including expenditures and other subject specific modules) has been applied to a random sub-sample of 30,000 and around 9,000 households respectively. To track individual changes over time, a panel component has been implemented, selected among the three sub-samples. As in other studies, individuals have been matched between the three surveys using the common individual identifier across years, cross-checked with gender, age and other individual information. After undertaking thorough data cleaning including checking consistency of time-invariant variables between the three survey rounds, we have been able to recover a substantial number of new individuals, and to correct misclassified ones. In the end, our balanced panel includes 7,408 individuals matched between all the three rounds of VHLSS (Table 2); 10,891 individuals observed only in 2002 and 2004; and 9,529 individuals observed only in 2004 and 2006.

As the major objective of our study is to investigate the question of earnings of rural workers, we retained only those individuals who are occupied workers aged 15 or more,

\footnotetext{
${ }^{73}$ The primary sample units are the communes/wards, the secondary sample units are the census enumeration areas or villages and the tertiary sample units correspond to households. For more details, see Phung and Nguyen (2006).
} 
residing in rural area. ${ }^{74}$ Finally, our empirical analysis is based on a panel of rural workers including 2,718 individuals observed in all three years (balanced part). In the unbalanced parts, there remain 3,820 individuals observed in both 2002 and 2004 but not in 2006, and 3,928 individuals who are observed as non-farm workers in both 2004 and 2006, but were not surveyed in 2002 .

Table 2- Building the panel of individuals with VHLSS 2002, 2004 and 2006

\begin{tabular}{lccc} 
& 2002 & 2004 & 2006 \\
\hline Full sample (household) & 75,000 & 45,000 & 45,000 \\
Detailed sample (household) & 30,000 & 9,000 & 9,000 \\
All individuals & & & \\
- Unbalanced Panel & 18,299 & 27,828 & 16,937 \\
- Balanced panel & 7,408 & 7,408 & 7,408 \\
Rural workers aged 15 years or over & & & \\
- Unbalanced Panel & 6,538 & 10466 & 6,646 \\
- Balanced Panel & 2,718 & 2,718 & 2,718 \\
- Observed in 2002 and 2004 & 3,820 & 3,820 & - \\
- Observed in 2004 and 2006 & - & 3,928 & 3,928 \\
\hline
\end{tabular}

Source: GSO VHLSS 2002, 2004, 2006; author's calculations.

In term of content, from an informal sector and informal employment perspective, the VHLSS does not allow to capture precisely these two concepts according to the international definitions (International Labour Organization, 2003; European Commission, et coll. 2008), as the survey has not been designed for such a purpose. In Vietnam, the informal sector is defined as all private unincorporated enterprises that produce at least some of their goods and services for sale or barter, are not registered (no business licence) and are engaged in non-farm activities. The informal employment corresponds to employment with no social security insurance. On the job side in the VHLSS, the formal/informal divide can only be computed for the wage workers. On the firm side, household businesses can be split between registered and not registered ones, but no information is available on the jobs generated by these businesses. Therefore, we created an informality proxy combining both job and firm approaches. Among non-farm workers, four main groups are distinguished, taking into account their job status. Among wage workers, informal ones are those who do not benefit from social security insurance. Among employers and self-employed, informal workers are those whose business is not registered. This classification provides the best available measures of informality in Vietnam, prior to the LFS 2007 (which unfortunately does not provide any panel component; Cling et coll., 2010a). Finally, our typology in four groups of non-farm workers is perfectly consistent with the International Labour Organization (ILO) definition of informal employment, but not with the informal sector one, as we are not able to distinguish among informal wage workers those who are working in the informal sector from those who are informally employed in the formal sector.

Information on informality can be tracked in the questionnaire by the "Employment" and "Non-Farm Household Business" (NFHB) modules. Apart from our formal/informal variable, we compute the labour income associated with each remunerated job. For wage workers, earnings are obtained by summing the direct wage with all the supplementary benefits perceived in cash or in kind and converted into pecuniary equivalent (public holidays, bonuses, social allowance, etc.). For the self-employed, we compute their annual net income by subtracting all the expenses engaged (intermediary consumption, labour costs,

\footnotetext{
${ }^{74} \mathrm{We}$ should be cautious to consider that all the jobs held by rural workers are rural employment since there should be a considerable number of rural workers undertaking jobs based on temporary and circular movement to cities. As a consequence, we consider in the analyzed sample the rural workers rather than rural employment.
} 
taxes, etc.) to the production generated by the household business. Hourly earnings used in the estimations are deduced using the total number of hours worked during the year. Additionally, all the classical individual and household based socio-demographic variables are appended to our database.

Finally, regional and time deflators have been elaborated to compute real earnings. As the regional deflators (16 locations, i.e. 8 regions in two areas, urban and rural) included in the VHLSS databases have been criticized for not being consistent over time (Mc Caig et coll., 2009), we combined the VHLSS 2006 regional deflators (supposed to be the most reliable one) with the provincial Consumer price indexes (CPIs) (63 provinces) provided by the General Statistics Office aggregated at the regional level. This adjustment is quite substantial, given the high differences in price levels and inflation: a difference of more than 77 percent in prices is observed between the lowest price level (rural North-East region, 2002) and highest one (urban South-East region, 2006), showing that markets are far from being fully integrated in Vietnam.

\section{II- Econometric Approach to Measuring Earnings Gaps between different groups of workers}

The empirical analysis consists of assessing the magnitude of different types of farm non-farm earnings gaps using OLS and quantile regressions with log hourly earnings from principal employment of occupied workers as dependent variable. Standard earnings equations are thus estimated at the mean and at various conditional quantiles of the earnings distribution. The models are regressed on a pooled sample of workers over years employed in the non-farm and agricultural sectors. The different covariates introduced into the regressions are the completed years of education, the years of potential experience (with quadratic profiles for these two regressors), a dummy for being married, a dummy for being a woman, seven regional dummies (in models estimated for the whole Vietnam), and two time dummies to control for macroeconomic trend effects on earnings.

A number of studies based on data on African manufacturing firms have shown that wages are positively correlated to firm size, conditional on standard human capital variables (Strobl and Thornton 2002, Magda 2008, Söderbom, Teal and Wambugu 2005). In this paper, due to lack of information on the demand side characteristics, we cannot control for the size of the wage workers' firms. The literature discusses numerous reasons why wages are positively correlated with firm size. One of the frequently made arguments is that firm size is correlated with omitted worker quality because large firms usually attract more productive workers. In this paper, we control for both observed human capital and timeinvariant unobserved characteristics, thus mitigating the drawback of not accounting for firm size in the regressions.

To account for non-farm vs. farm differences in earnings at the mean earnings level, we turn to Pooled OLS regressions across years and Fixed Effects OLS regressions (FEOLS), the latter accounting for time-invariant unobserved heterogeneity. The FE model can be written as

$$
y_{i t}-x_{t i}^{b} \beta+\gamma E_{i t}+\tau F_{i t}+\alpha_{t}+c_{t t}
$$

Where $x_{i z}$ denotes the vector of characteristics of individual $i$ observed at time $t$ (which includes a constant term), $I_{i t}$ and $F_{i t}$ represent dummies taking value one if person $i$ observed at time $t$ is a non-farm informal and formal worker respectively. $\kappa_{i}$ is the timeinvariant individual heterogeneity (or the individual fixed effect) and $\sigma_{e}$ is an i.i.d. normally distributed stochastic term absorbing measurement error. 
The estimated coefficient $\hat{\gamma}$ and $\hat{\tau}$ are interpreted as measures of the conditional earnings premium/penalty experienced by non-farm workers (informal or formal) compared to agricultural workers. However, as mentioned previously, the informal sector is extremely heterogeneous and a finer job divide should be considered. We then define four categories of rural non-farm workers split by job status (wage workers vs. self-employed workers) and institutional sector (formal vs. informal) and create four dummies taking value one if the individual $i$ at time $t$ is a non-farm informal wage worker $\left(J W_{k}\right)$, a non-farm formal wage worker $\left(F W_{t}\right)$, a non-farm informal self-employed worker $\left(I S_{i t}\right)$ and a non-farm formal self-employed worker $\left(F S_{t}\right)$.

Taking the agricultural workers $\left(A W_{i \mathrm{r}}\right)$ as the reference category, the model we estimate can be written as:

$$
y_{t t}=x_{t t}^{t} \beta+\sigma I W_{t t}+\theta I S_{t t}+\beta F W+\lambda F S_{t t}+\alpha_{t}+a_{t t}
$$

The estimated coefficients $\hat{\delta}, \hat{\theta}, \hat{h}$ and $\hat{\lambda}$ are interpreted, respectively, as the $I W-A W, I S-$ $A W, F W-A W$ and $F S-A W$ conditional earnings gaps. Identification of these conditional earnings gaps relies on the presence in the sample of movers between employment states over time. Those movers can be compared to the stayers in terms of earnings.

As an illustration, we consider a simple two-period example and ten cases of transitions out of the various possibilities of professional trajectories (which are 25 in a two-period example):

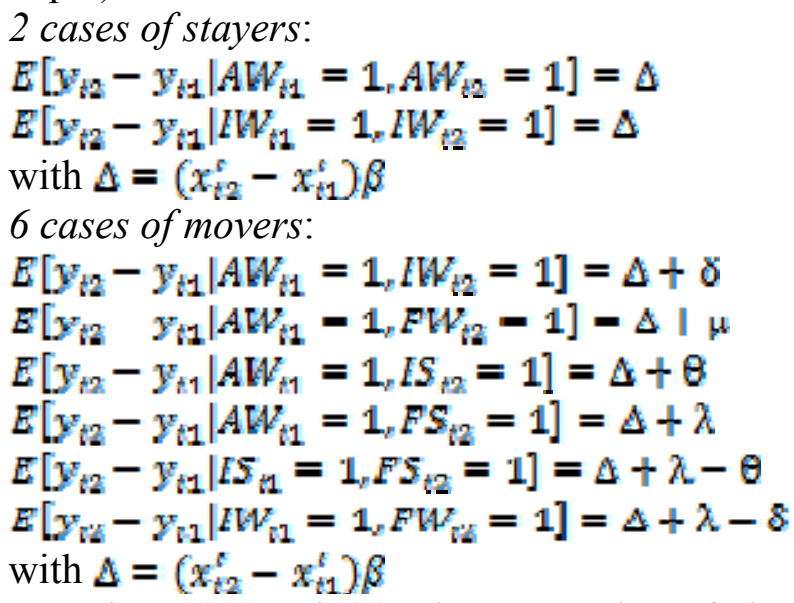

Equations (3) and (4) give examples of the changes in earnings for stayers, i.e. for workers that do not change their employment state between the two periods. Equations (5), (6), (7), and (8) illustrate the changes in earnings for those workers coming from an agricultural job and moving, respectively, into an informal wage job, a formal wage job, an informal self-employed job, and a formal self-employed job; equations (9) and (10) represent these earnings differentials for those coming from an informal self-employed/wage job and moving, respectively, into a formal self-employed/wage job.

The identification strategy of FE on movers is quite standard but, in practice, one should verify that the number of moves across sectors is sufficient for a valid use of this estimator; which is the case, as shown in Table 4 in the next section.

Finally, to allow the earnings gaps between job statuses to differ along the earnings distribution, we rely on Quantile Regressions (QR). Quantile earnings regressions consider specific parts of the conditional distribution of the hourly earnings and indicate the influence of the different explanatory variables on conditional earnings respectively at the bottom, at the median and at the top of the distribution.

Using our previous notation, the model that we seek to estimate is:

$$
q_{Q}\left(y_{t i}\right)=x_{t i}^{t} \beta(Q)+\delta(\theta) I W_{t i}+\mu(\theta) F W_{t i}+\theta(\theta) I S_{t i}+\lambda(\theta) F S_{t i}+\alpha_{t}, \forall Q \in[0,1]
$$


where $q_{e}\left(y_{t t}\right)$ is the $e^{\text {th }}$ conditional quantile of the log hourly earnings. The set of coefficients $\beta(\varrho)$ provide the estimated rates of return to the different covariates at the $\varrho^{\text {th }}$ quantile of the log earnings distribution and the coefficients $\delta(\theta), \mu(\theta)$,

$\theta(\rho)$ and $\lambda(g)$ measure the parts of the earnings differentials that are due to non-farm-farming job differences at the various quantiles. In a quantile regression, the distribution of the error term is left unspecified. The quantile regression method provides robust estimates, particularly for misspecification errors related to non-normality and heteroskedasticity.

We then turn to Fixed Effects Quantile Regressions (FEQR). The extension of the standard QR model to longitudinal data has been originally developed by Koenker (2004). More recently, Canay (2011) proposed an alternative and simpler approach which assumes that the unobserved heterogeneity terms have a pure location shift effect on the conditional quantiles of the dependent variable. In other words, they are assumed to affect all quantiles in the same way. It follows that these unobserved terms can be estimated in a first step by traditional mean estimations (for instance by FE OLS). Then, the predicted $\widehat{\alpha}_{\imath}$ are used to correct earnings, such as $\hat{y}_{i}-y_{i}-\hat{a}_{i}$, which are regressed on the other regressors by traditional QR.

When running the regressions (2) and (11), we always provide robust standard errors using bootstrap replications. To reduce a possible bias due to measurement and reporting errors in the earnings and independent variables, we trim the data and drop influential outliers and observations with high leverage points from our sample that we identify by the DFFITS-statistic. As suggested by Belsley, Kuh and Welsch (1980), we use a cutoff-value $\mid$ IRFFTS $\mid>2 \sqrt{k / N}$ with $k$, the degrees of freedom (plus 1 ) and $N$ the number of observations. This procedure removes 658 observations from our initial unbalanced panel sample.

\section{III- Descriptive statistics and validity checks for earnings gaps measurement}

Table 3 presents some basic summary statistics of the main characteristics of the panel data used in our analysis. These descriptive statistics are reported for sub-samples of rural workers in the whole country. The results obtained for average earnings as well as individual characteristics are in line with common findings in the literature.

Firstly, compared to non-farm workers, those rural workers holding farm jobs earn on average significantly less. Considering, for instance, the relative earning difference between farm and non-farm informal jobs, one can realize that on average farm workers earn 12 percent less than those informally employed in rural non-farm sector. Farm workers appear to have longer potential experience (which is calculated as age minus years of reported schooling minus five) than their counterparts working in the non-farm sector. Women have higher propensity to engage in farm and non-farm informal self-employed jobs. Workers having agricultural job tend to have also a lower level of education compared to those participating in non-farm job. For instance, 29 percent among those workers engaging in farm jobs has no degree at all, whereas, on the other extreme, 35 percent of non-farm formal wage workers are those who obtained undergraduate education.

Secondly, regarding non-farm employment, the results show that workers holding formal jobs earn more on average than those engaged in informal jobs. Among each group of formal and informal workers, self-employed workers are those with higher earnings in comparison with wage earners. Informal workers tend to be younger than their formal worker counterparts, especially for wage workers. Self-employed workers exhibit on average longer potential experience than other non-farm workers on the labour market. As 
expected, workers having higher level of education are less likely to be engaged in informal employment and vice versa.

Table 3- Summary Statistics (pooled waves 2002-2004-2006)

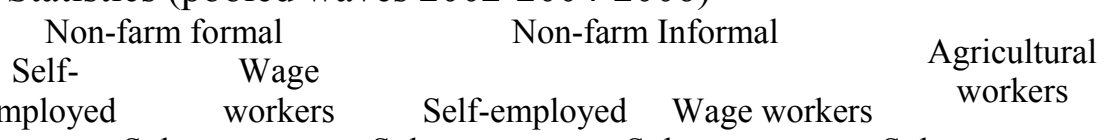

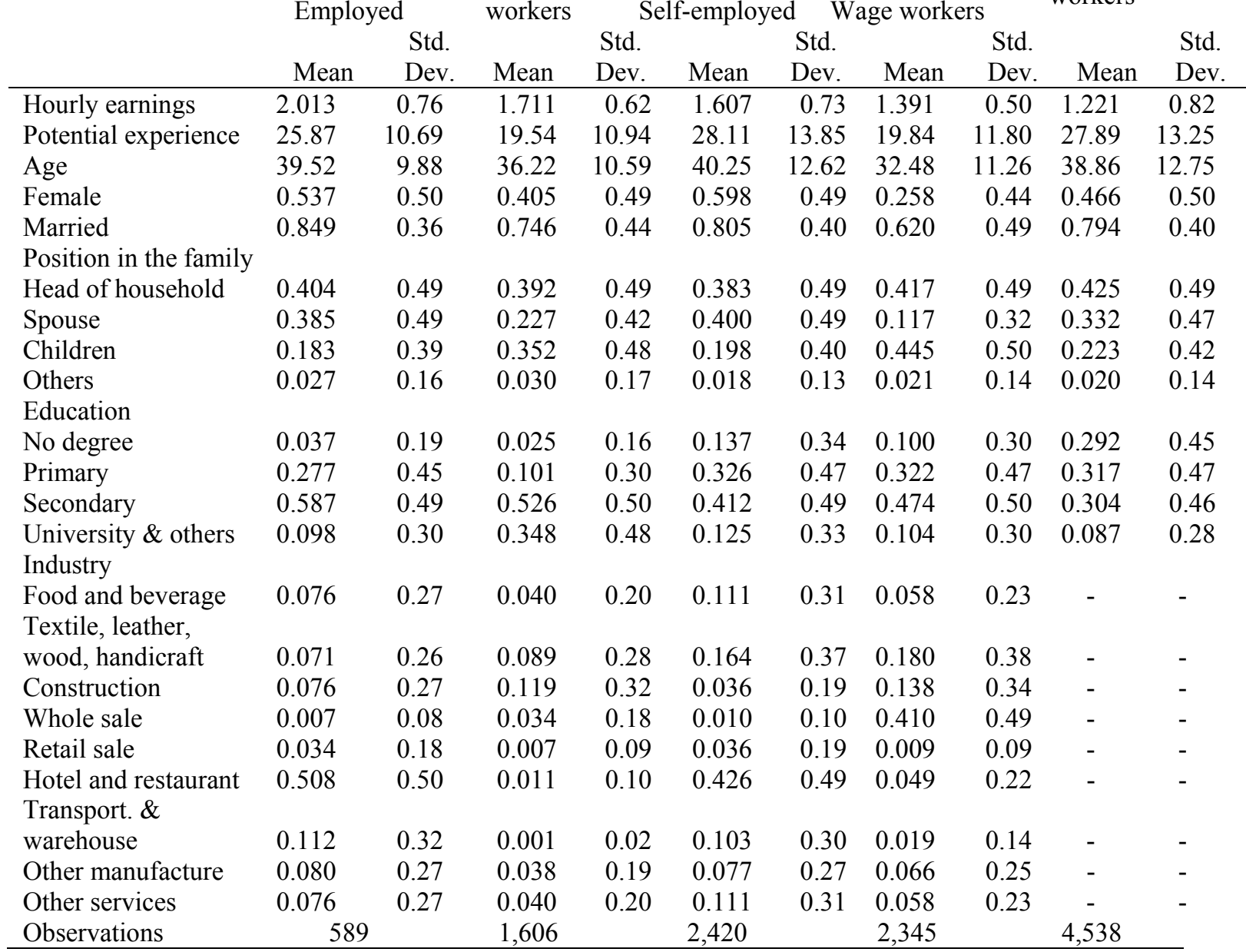

Source: GSO VHLSS 2002, 2004, 2006; author's calculations.

At the aggregate level, the gender ratio does not vary between formal and informal jobs. However, when considering wage jobs, female workers represent more in formal jobs than informal ones or, in other words, male workers engage more in informal wage jobs. Finally, formal and informal workers are differently allocated across branches of activity. Specifically, informal employment is found more in trade, restaurants and transportation, while formal jobs are more concentrated in services. Interestingly, the share of manufacture is much higher for informal jobs than for formal ones (31 percent vs. 18 percent). Within institutional sectors, the distribution is also fairly unbalanced: formal wage workers are stubbornly engaged in services (60 percent), whereas formal self-employed workers hold transportation and hotel $\&$ restaurant jobs (12 percent and 52 percent respectively). Informal wage workers engaged prominently in construction ( 13 percent) and trade ( 35 percent) while informal self-employed job's structure looks like the formal self-employed one. These significant differences in the distribution of job structure underline the importance of controlling for sectors of activity in our earnings gaps estimations.

As presented in the previous section, the presence of movement between employment states overtime is important for the identification of conditional earnings gaps. Therefore, it is useful to have a first look at descriptive figures showing the movement on the rural labour 
market between the years studied. Table 4 reports the transition matrices of employment status between 2002-2004, 2004-2006 and 2002-2006 obtained from our unbalanced panel dataset. In order to provide a more general picture of the dynamics of switching between employment statuses, we present the results obtained from the panel of all individuals aged 15 or more. The categories shown in the matrices include then not only the four non-farm employment statuses and agricultural category, but also "not-working" (the latter category includes, to simplify the notation, those who are inactive or unemployed). ${ }^{75}$ This presentation allows identification of both transition flows between farm and non-farm jobs, as well as those within the non-farm sector employment. The figures in the second row and column of each matrix reveal that the former are not negligible. It is notable that among all transition flows, the most important ones are those between informal non-farm and farm jobs. These patterns of mobility would partly reflect the low entry barriers to both sectors as well as the fact that the majority of the workforce in Vietnam is still predominantly employed in agriculture. Another striking evidence on the flows of transition from non-farm employment is the rather high probability of becoming inactive (or unemployed) for those who were previously self-employed.

Apart from revealing the movement between farm and non-farm jobs, the transition matrices show also that, on average, movement flows between non-farm job's categories are not negligible. For the two-time periods, around one quarter of workers changed position from one of our four job's status to another. Around 20 percent of the total sample moved from non-farm informal to formal jobs and the rates of formal-informal transition are about 40 percent. However, the flows are balanced in absolute terms. The fluidity between wage and non-wage jobs is smaller, but is far from negligible (from 13 percent to 15 percent of the total sample, depending on the years). Here again, the movements to and from non-farm wage jobs are relatively symmetrical. At a more disaggregated level, job mobility is at its highest for formal self-employed workers, where less than two thirds keep the same status in our different panels. Formal wage workers are the most stable ( 82 percent to 74 percent of stayers), while informal workers (wage and non-wage) are in between with a proportion of stayers ranging from 55 percent to 62 percent. Formal wage workers mainly move to informal wage jobs. When moving, non-farm informal wage workers tend to privilege selfemployed positions, and secondarily formal wage jobs. Formal self-employed movers mainly get their business informalized (probably due to adverse conditions). A lower share of informal self-employed workers makes the inverse move, by formalizing their business. However, a substantial proportion also closes their business to become informal wage workers.

All in all, the high consistency between our transitions matrices over different periods is a sound indicator of data quality. We would claim that the observed changes reflect real phenomena and do not mainly capture measurement errors. Furthermore, on the methodological side, the substantial numbers of movers is essential for our estimation strategy.

To end this section on descriptive analysis, let us have look at the income dynamics by employment status of rural workers on the period 2004-2006. The first panel of Table 5 shows the level of real earning in 2006 by transition status, agricultural stayers being our basis. Consistently with Table 4, formal self-employed workers get the highest pay, while

\footnotetext{
${ }^{75}$ It is important to note that unemployment rate is stubbornly low in Vietnam's rural area and this phenomenon is less prevalent in rural areas than in urban areas.
} 
informal wage workers in non-farm sector together with those having farm jobs are at the lowest end of the earnings ladder. ${ }^{76}$

Table 4- Transition matrix of employment status between 2002, 2004 and 2006

2004

\begin{tabular}{|c|c|c|c|c|c|c|c|}
\hline 2002 & $\begin{array}{c}\text { Not- } \\
\text { workin } \\
\mathrm{g}\end{array}$ & $\begin{array}{l}\text { Agri- } \\
\text { cultural } \\
\text { emp. }\end{array}$ & $\begin{array}{l}\text { Formal } \\
\text { Wage }\end{array}$ & $\begin{array}{l}\text { Infor } \\
\text { mal } \\
\text { Wage }\end{array}$ & $\begin{array}{c}\text { Formal } \\
\text { Self- } \\
\text { employed }\end{array}$ & $\begin{array}{c}\text { Informal } \\
\text { Self- } \\
\text { employed }\end{array}$ & Total \\
\hline Not-working & 58.8 & 28.4 & 1.3 & 5.0 & 1.3 & 5.3 & $100(16.7)$ \\
\hline Agricultural emp. & 6.5 & 81.7 & 1.6 & 4.6 & 0.8 & 4.8 & $100(62.5)$ \\
\hline Formal Wage worker & 0.6 & 11.3 & 72.3 & 13.2 & 0.6 & 1.9 & $100(4.4)$ \\
\hline Informal Wage worker & 1.4 & 20.6 & 3.8 & 66.0 & 3.4 & 4.8 & $100 \quad(5.7)$ \\
\hline Formal Self-empl. work. & 4.1 & 12.2 & 4.1 & 2.7 & 51.4 & 25.7 & $100(2.1)$ \\
\hline Informal Self-empl. w. & 4.6 & 21.6 & 0.3 & 6.9 & 8.9 & 57.7 & $100 \quad(8.4)$ \\
\hline Total & 14.5 & 59.6 & 4.8 & 8.8 & 2.8 & 9.6 & $100(100)$ \\
\hline \multirow[b]{2}{*}{2004} & \multicolumn{6}{|c|}{2006} & \multirow[b]{2}{*}{ Total } \\
\hline & $\begin{array}{l}\text { Not- } \\
\text { wor- } \\
\text { king }\end{array}$ & $\begin{array}{l}\text { Agri- } \\
\text { cultural } \\
\text { emp. }\end{array}$ & $\begin{array}{l}\text { Formal } \\
\text { Wage }\end{array}$ & $\begin{array}{l}\text { Infor } \\
\text { mal } \\
\text { Wage }\end{array}$ & $\begin{array}{c}\text { Formal } \\
\text { Self- } \\
\text { employed }\end{array}$ & $\begin{array}{c}\text { Informal } \\
\text { Self- } \\
\text { employed }\end{array}$ & \\
\hline \multirow{6}{*}{$\begin{array}{l}\text { Not-working } \\
\text { Agricultural emp. } \\
\text { Formal Wage worker } \\
\text { Informal Wage worker } \\
\text { Formal Self-empl. work. } \\
\text { Informal Self-empl. w. }\end{array}$} & 63.3 & 23.3 & 3.1 & 5.5 & 1.0 & 3.8 & $100(14.5)$ \\
\hline & 7.1 & 82.0 & 1.7 & 4.4 & 0.9 & 3.9 & $100(59.6)$ \\
\hline & 0.0 & 10.5 & 79.1 & 8.1 & 0.6 & 1.7 & $100(4.8)$ \\
\hline & 1.9 & 23.3 & 7.3 & 61.2 & 1.9 & 4.4 & $100(8.8)$ \\
\hline & 11.0 & 14.0 & 2.0 & 1.0 & 46.0 & 26.0 & $100(2.8)$ \\
\hline & 6.3 & 21.3 & 1.7 & 3.5 & 5.8 & 61.5 & $100 \quad(9.6)$ \\
\hline Total & 14.49 & 57.26 & 6.08 & 9.54 & 2.68 & 9.95 & $100(100)$ \\
\hline \multirow[b]{2}{*}{2006} & \multicolumn{6}{|c|}{2006} & \multirow[b]{2}{*}{ Total } \\
\hline & $\begin{array}{c}\text { Not- } \\
\text { workin } \\
\mathrm{g}\end{array}$ & $\begin{array}{l}\text { Agri- } \\
\text { cultural } \\
\text { emp. }\end{array}$ & $\begin{array}{l}\text { Formal } \\
\text { Wage }\end{array}$ & $\begin{array}{l}\text { Infor } \\
\text { mal } \\
\text { Wage }\end{array}$ & $\begin{array}{c}\text { Formal } \\
\text { Self- } \\
\text { employed }\end{array}$ & $\begin{array}{c}\text { Informal } \\
\text { Self- } \\
\text { employed }\end{array}$ & \\
\hline \multirow{2}{*}{$\begin{array}{l}\text { Not-working } \\
\text { Agricultural emp. }\end{array}$} & 53.5 & 24.4 & 4.8 & 9.4 & 1.2 & 6.8 & $100(16.7)$ \\
\hline & 7.0 & 77.7 & 2.7 & 5.8 & 1.0 & 5.8 & $100(62.5)$ \\
\hline Formal Wage worker & 1.3 & 10.1 & 73.0 & 12.6 & 0.6 & 2.5 & $100(4.4)$ \\
\hline Informal Wage worker & 2.4 & 24.4 & 2.9 & 56.5 & 2.9 & 11.0 & $100 \quad(5.7)$ \\
\hline Formal Self-empl. work. & 10.8 & 13.5 & 2.7 & 1.4 & 44.6 & 27.0 & $100(2.1)$ \\
\hline Informal Self-empl. w. & 8.9 & 28.2 & 2.0 & 5.6 & 9.2 & 46.2 & $100 \quad(8.4)$ \\
\hline Total & 14.49 & 57.26 & 6.08 & 9.54 & 2.68 & 9.95 & $100(100)$ \\
\hline
\end{tabular}

Source: GSO VHLSS 2002, 2006, GSO; author's calculations.

Compared to the pooled sample, in 2006, informal non-farm self-employed workers reversed their position with formal non-farm wage workers, meaning that the earnings hierarchy between these two categories of workers is not fixed, but may vary over time. Furthermore, income levels are highly dependent on transitions. For instance, and as expected, whatever their job status in 2004, those who moved to informal wage jobs earn the less. Conversely, the workers who got the opportunity to open a formal business earn the

\footnotetext{
${ }^{76}$ We observe a slight difference between these results and those presented in Table 3 concerning the earning hierarchy of different groups. This is due to the different samples used: estimated average earnings in this table concern earnings in 2006, whereas the figures in Table 3 come from pooled sample of all the three waves.
} 
most. The results are quite similar in terms of earning growth (second panel of Table 5). Systematically, moving to informal wage jobs is associated with the lowest increase in earnings over the period, whereas being able to change to a formal self-employed job is associated with the highest earnings growth. Of course, these unconditional averages should be controlled for observed and unobserved characteristics, which is the purpose of the following sections.

Table 5- Income dynamics by employment status between 2004 and 2006

\begin{tabular}{|c|c|c|c|c|c|c|}
\hline & \multicolumn{6}{|c|}{ Real income levels in 2006} \\
\hline $2004 \backslash 2006$ & $\begin{array}{l}\text { Agri- } \\
\text { cultural } \\
\text { emp. }\end{array}$ & $\begin{array}{c}\text { Formal } \\
\text { Wage }\end{array}$ & $\begin{array}{c}\text { Informal } \\
\text { Wage }\end{array}$ & $\begin{array}{c}\text { Formal } \\
\text { Self- } \\
\text { employed }\end{array}$ & $\begin{array}{l}\text { Informal } \\
\text { Self- } \\
\text { employed }\end{array}$ & Total \\
\hline Not-working & 100 & 82.7 & 69.8 & 143.4 & 96.5 & 97.0 \\
\hline Agricultural emp. & 92.3 & 131.8 & 95.0 & 207.4 & 96.8 & 126.9 \\
\hline Formal Wage worker & 79.4 & 74.8 & 84.1 & 213.3 & 117.0 & 87.0 \\
\hline Informal Wage worker & 123.6 & 187.7 & 120.5 & 191.9 & 137.4 & 160.9 \\
\hline Formal Self-empl. work. & 104.9 & 84.8 & 83.9 & 152.7 & 114.2 & 113.5 \\
\hline Total & 99.4 & 118.4 & 80.4 & 143.4 & 96.5 & 103.7 \\
\hline \multirow[b]{2}{*}{$2004 \backslash 2006$} & \multicolumn{6}{|c|}{ Real hourly income growth 2004-2006 } \\
\hline & $\begin{array}{l}\text { Agri- } \\
\text { cultural } \\
\text { emp. }\end{array}$ & $\begin{array}{c}\text { Formal } \\
\text { Wage }\end{array}$ & $\begin{array}{c}\text { Informal } \\
\text { Wage }\end{array}$ & $\begin{array}{c}\text { Formal } \\
\text { Self- } \\
\text { employed }\end{array}$ & $\begin{array}{l}\text { Informal } \\
\text { Self- } \\
\text { employed }\end{array}$ & Total \\
\hline Not-working & 100 & 115.4 & 85.2 & 399.8 & 102.0 & 99.8 \\
\hline Agricultural emp. & 87.1 & 116.1 & 126.8 & 240.1 & 211.4 & 117.9 \\
\hline Formal Wage worker & 130.4 & 102.6 & 112.3 & 235.8 & 180.2 & 120.7 \\
\hline Informal Wage worker & 156.5 & 439.4 & 57.0 & 111.5 & 129.8 & 123.2 \\
\hline Formal Self-empl. work. & 157.4 & 109.7 & 90.5 & 235.8 & 120.0 & 125.9 \\
\hline Total & 116.8 & 115.6 & 108.8 & 143.0 & 126.5 & 118.9 \\
\hline
\end{tabular}

Note: base $100=$ Income level and income growth compared to agricultural workers' stayers between 2004 and 2006.

Source: GSO VHLSS 2002, 2004, 2006, author's calculations.

\section{IV- Earnings gaps analysis}

In this section we discuss the earning gaps between rural non-farm jobs (formal and informal) and farm jobs at the aggregate level, estimated using the four estimations procedures presented in Section 3. As discussed earlier, the non-farm sector, and more broadly non-farm employment, is immensely heterogeneous. The theoretical literature suggests that a key divide should be considered between the informal and formal jobs, between wage workers and self-employed. If the point is now well established in the literature, formal job heterogeneity is rarely acknowledged. Therefore, to go beyond previous studies on this issue, we distinguish all rural non-farm workers between four groups, split by job status (wage workers vs. self-employed) and institutional sector (formal vs. informal). In the following discussion, we compare the four non-farm work status with farm workers, as our benchmark. We also investigate the gender issue. 


\section{IV.1- Earnings gaps at the aggregate level}

\section{IV.1.1- Non-farm Formal vs. Agricultural Workers}

At the aggregate level, the OLS estimate of the earning gap between formal non-farm and agricultural workers is near 50 percent (Figure 1, and Table 6). Taking into account the (time invariant) unobservable individual characteristics (UICs) through fixed effect OLS estimation (FEOLS) reduces the earnings penalty significantly, down to 31 percent. Thus, a remarkable proportion of the gap can be explained by unobservable characteristics, the most productive workers privileging the formal non-farm employment. As always, this standard feature does not tell us much about what specific factors are really at play. On the one hand, the innate ability or the "talent parabola" is commonly stressed in the literature. On the other hand, many other explanations can be put forward. For instance, UICs may have to do with more efficient social networks which help getting a formal non-farm job.

Figure 1- Earnings gaps between Non-farm Formal and Agricultural Workers
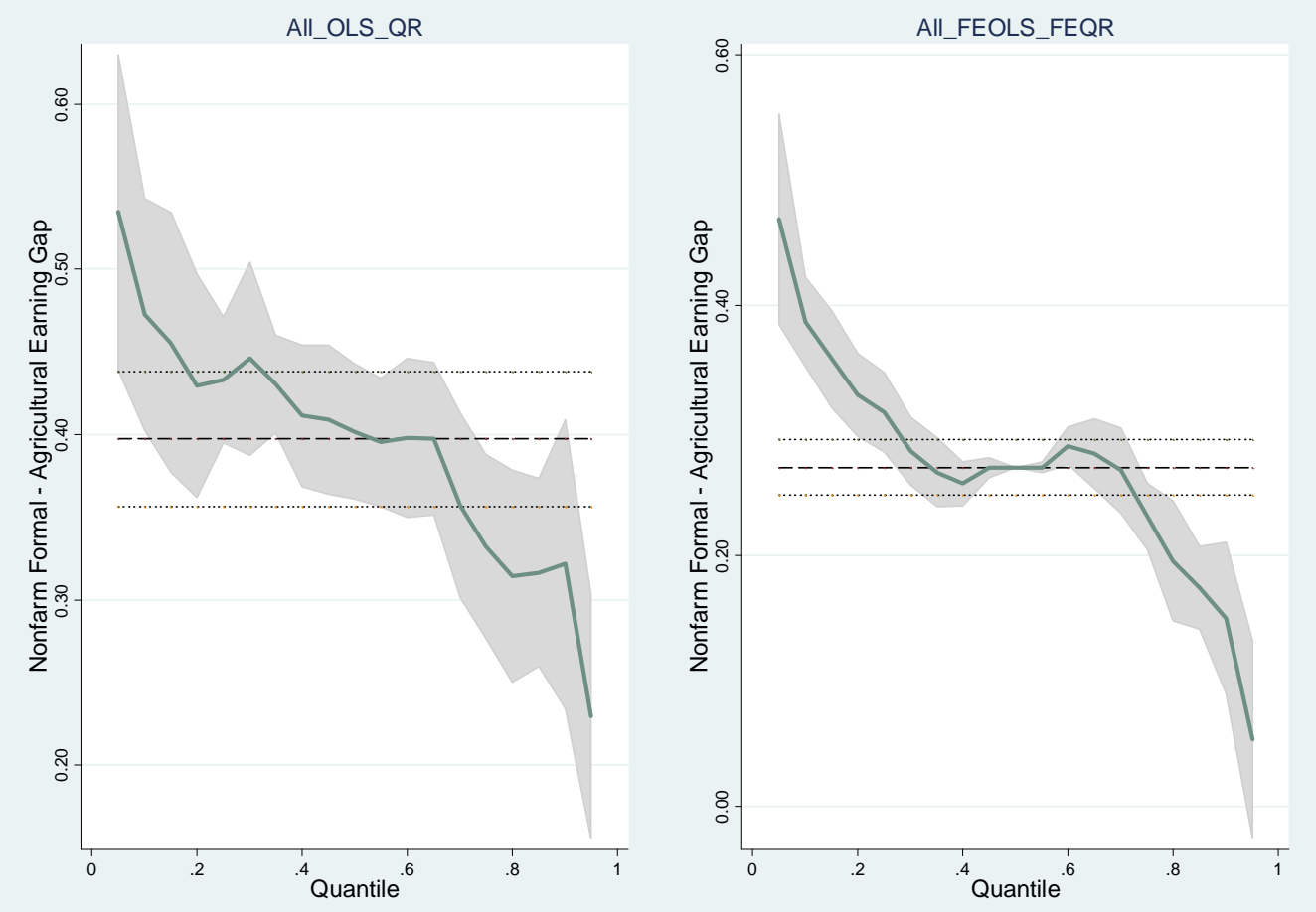

Note: Fixed Effects (FE) OLS are denoted by FEOLS and Fixed Effects Quantile Regressions (QR) by FEQR. Bootstrapped 95 percent confidence intervals are represented by the grey surface for QR and by dashed lines for the OLS.

Source: GSO VHLSS 2002, 2004, 2006, author's calculations.

However, the remaining 31 percent gap, once we control for UICs, highlights that formal non-farm jobs are able to bring about higher earnings per se. Here again, this result can be due to various factors which end up, at the firm level, in a higher productivity or market power, and/or, at the worker level, in a stronger bargaining power of formal workers to negotiate higher earnings.

To go beyond average, we ran quantile regressions (QR). Results obtained show that agricultural workers are suffering earnings penalties at all levels of the conditional distribution with a larger gap at the bottom part. Concretely, from around 60 percent for the first two quartiles of income, the gap reduces to 38 percent at the upper-tier of the 
distribution (quantile q.90). The Fixed Effects Quantile Regression (FEQR) gap confirms the key role of UICs in reducing the "true" gap. The FEQR gaps are decreasing continuously along the earnings distribution for rural workers, from 47 percent for the bottom quantile to around only16 percent for the upper one. This would imply no much premium of moving out of farming employment for those having already high agricultural productivity.

\section{IV.1.2- Non-farm Informal vs. Agricultural Workers}

As expected at aggregate level, those employed informally in rural non-farm sector are on average better-off than those engaging in farm jobs, but the gap is considerably lower than that observed between non-farm formal and agricultural workers (Figure 2 and Table 6). The OLS gap is significantly reduced to 17 percent when individual fixed effects are introduced. These results suggest that informal non-farm workers may have a disadvantage in terms of their unobserved productive attributes.

Figure 2- Earnings gaps between Non-farm Informal and Agricultural Workers
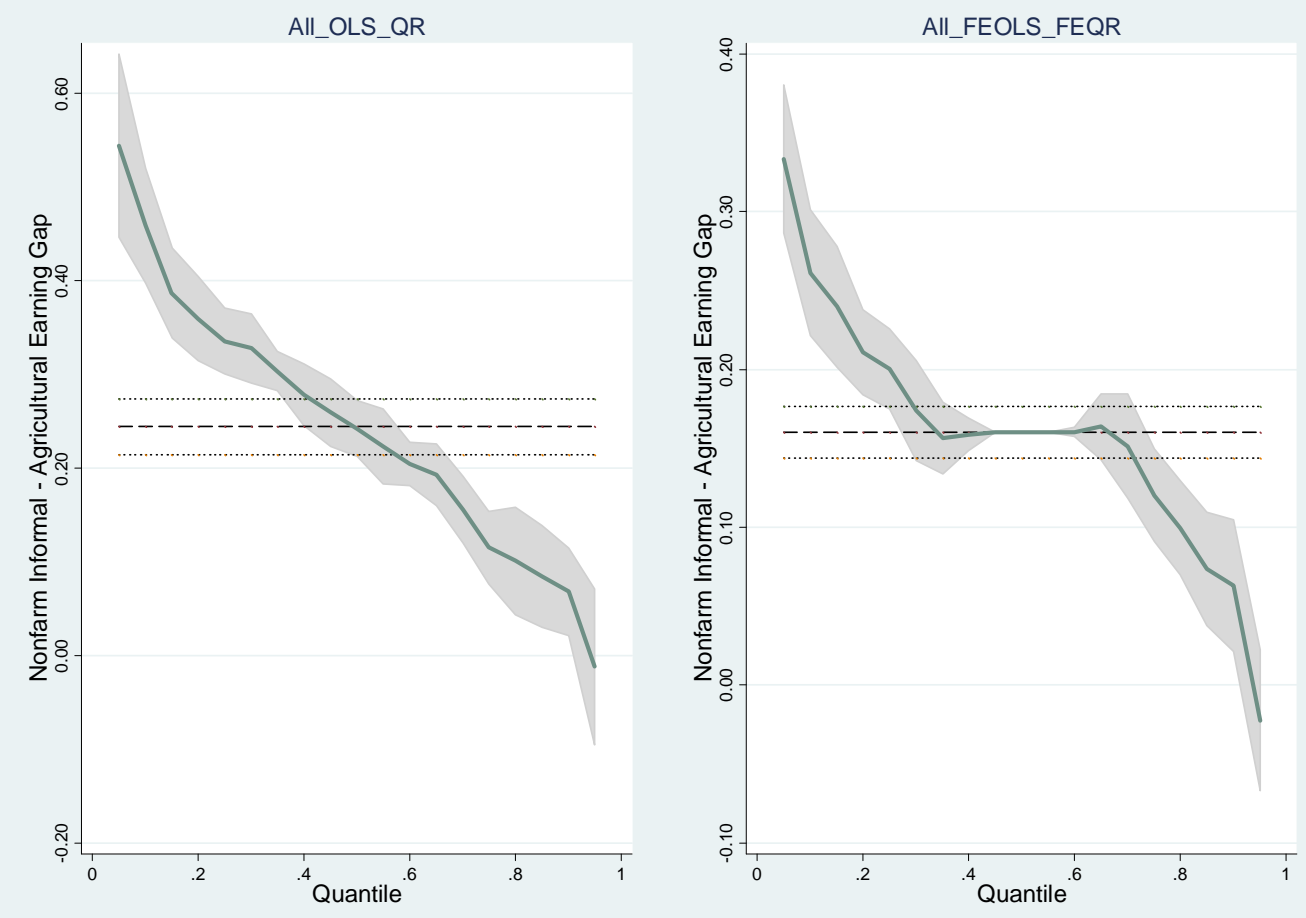

Note: Fixed Effects (FE) OLS are denoted by FEOLS and Fixed Effects Quantile Regressions (QR) by FEQR. Bootstrapped 95 percent confidence intervals are represented by the grey surface for QR and by dashed lines for the OLS.

Source: GSO VHLSS 2002, 2004, 2006, GSO author's calculations.

Considering results obtained from quantile regressions, distributional pattern can be clearly identified no matter whether the UICs are controlled for or not (Figures 2, Tables 7, 8). The rural non-farm informal - agricultural earnings gap is decreasing continuously and significantly from 29.5 percent (quantile q.10) to 6.8 percent (quantile q.90), implying that agricultural workers conserve an earnings disadvantage in comparison with non-farm informal workers at any point in the pay ladder. 
IV.2- Heterogeneity among rural non-farm employment and the variation of farm - nonfarm earnings gap regarding informality.

Given the great heterogeneity among rural non-farm jobs as well as activities stressed in the literature (Foster and Rosenzweig 2004, Lanjouw and Murgai 2009), our analysis in this sub-section relies on results obtained from models in which non-farm workers are disaggregated by employment status (hired and self-employed) and informality

\section{IV.2.1- Non-farm Wage Workers (formal or informal) vs. Farm Workers}

Engaging in wage employment in rural non-farm sector appears to be the main trade-off against the traditional way of making a living in agriculture for rural workers. Whether rural non-farm wage employment can be distinguishable from agricultural jobs in terms of remuneration remains a question drawing considerable attention in the literature on rural economy in developing countries. Based on comparative analysis of wage distribution between rural agricultural and non-farm workers in various countries of different continents, Winters et coll. (2008) have pointed to a lower position of agricultural earnings distribution compared to that of rural non-farm wage workers in all of the African and Latin American countries. They have also mentioned the special case of Vietnam where it is harder to observe clear differences between the two earnings distributions. In our analysis, a disaggregation of rural non-farm wage workers into two groups (formal and informal) allows a finer reassessment of the earnings differentials between non-farm wage workers and agricultural workers.

\section{IV.2.2- Non-farm informal wage vs. farm earnings gaps}

As can be seen from Figures 3 and Tables A1, the conditional OLS gap is positive, with a significant premium of 12 percent for the non-farm informal wage workers. Nevertheless, the FEOLS models reduce the premium further to 8 percent for informal wage workers. Non-farm informal wage workers do have general disadvantage in terms of their unobserved productive attributes, which produce an overestimation of the premium associated with being a wage worker in rural non-farm sector compared to engaging as farm worker if this individual heterogeneity is not accounted for. When turning to quantile regressions (Tables $7,8)$, the distributional profile of the gap presents a clear pattern, showing both positive and negative earnings gaps. The gap steeply decreases with income level, and is less and less in favour of the informal wage workers. In absolute terms, agricultural labourers suffer a penalty at almost but not entire points of the distribution. Positive earnings gap between informal wage workers and agricultural workers is observed of important magnitude at the lower quantiles (up to quantile q.80 in the pooled QR model, and quantile q.70 in the FEQR model). Afterwards, the gap is reversed into a significant premium, growing continuously up to around 39 percent for the richest decile. All in all, and given the size of the premium, we can conclude that rural informal wage employment may be less lucrative that agricultural alternatives, especially for the richest ones who have relatively high farming productivity. As a matter of consequence, we have good presumptions to assert that, in rural Vietnam, engaging in rural non-farm employment is not always a better way for workers to improve earnings. 
Figure 3- Earnings gaps between Non-farm Informal Waged and Farm Workers
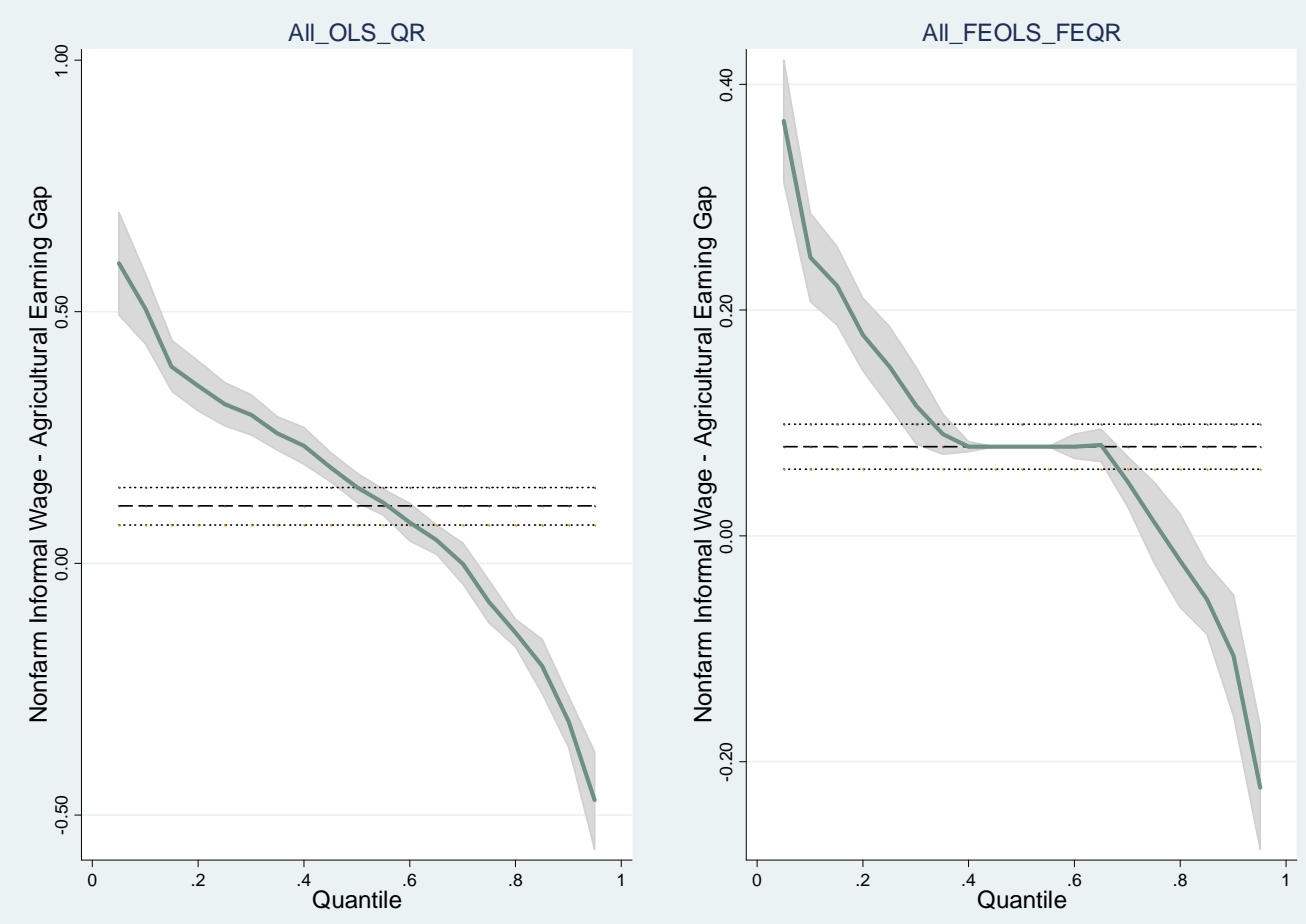

Note: Fixed Effects (FE) OLS are denoted by FEOLS and Fixed Effects Quantile Regressions (QR) by FEQR. Bootstrapped 95 percent confidence intervals are represented by the grey surface for QR and by dashed lines for the OLS.

Source: GSO VHLSS 2002, 2004, 2006, author's calculations.

\section{IV.2.3- Non-farm formal wage vs. farm earnings gaps}

The results shown in Figure 4 and Tables A1, confirm what has been found for the raw earnings differentials reported in Table 4.

Concretely, the conditional OLS earnings gap is positive, with a premium of more than twice that of the premium informal wage workers can have (28.5 percent vs. 12 percent), over farm jobs. When the individual unobserved heterogeneity is controlled for, the earnings premium to formal wage workers reduces to 20 percent. When turning to quantile earnings gaps, the results reveal some interesting points to be discussed. The FEQR gaps are decreasing steeply along the pay scale and turning to negative value from around the upper quantile (q.90), indicating that the farm workers do not appear to earn less than non-farm formal wage workers at these upper positions of the earning distribution.

\section{Non-farm Self-employed vs. Farm Workers}

The earnings comparison of non-farm informal self-employed workers and farm workers is clearly in favour of the former, whatever the model chosen (Figure 5, and Tables 6, columns (3) and (5), Tables 7, 8). 
Figure 4- Earnings gaps between Non-farm Formal Wage and Farm Workers
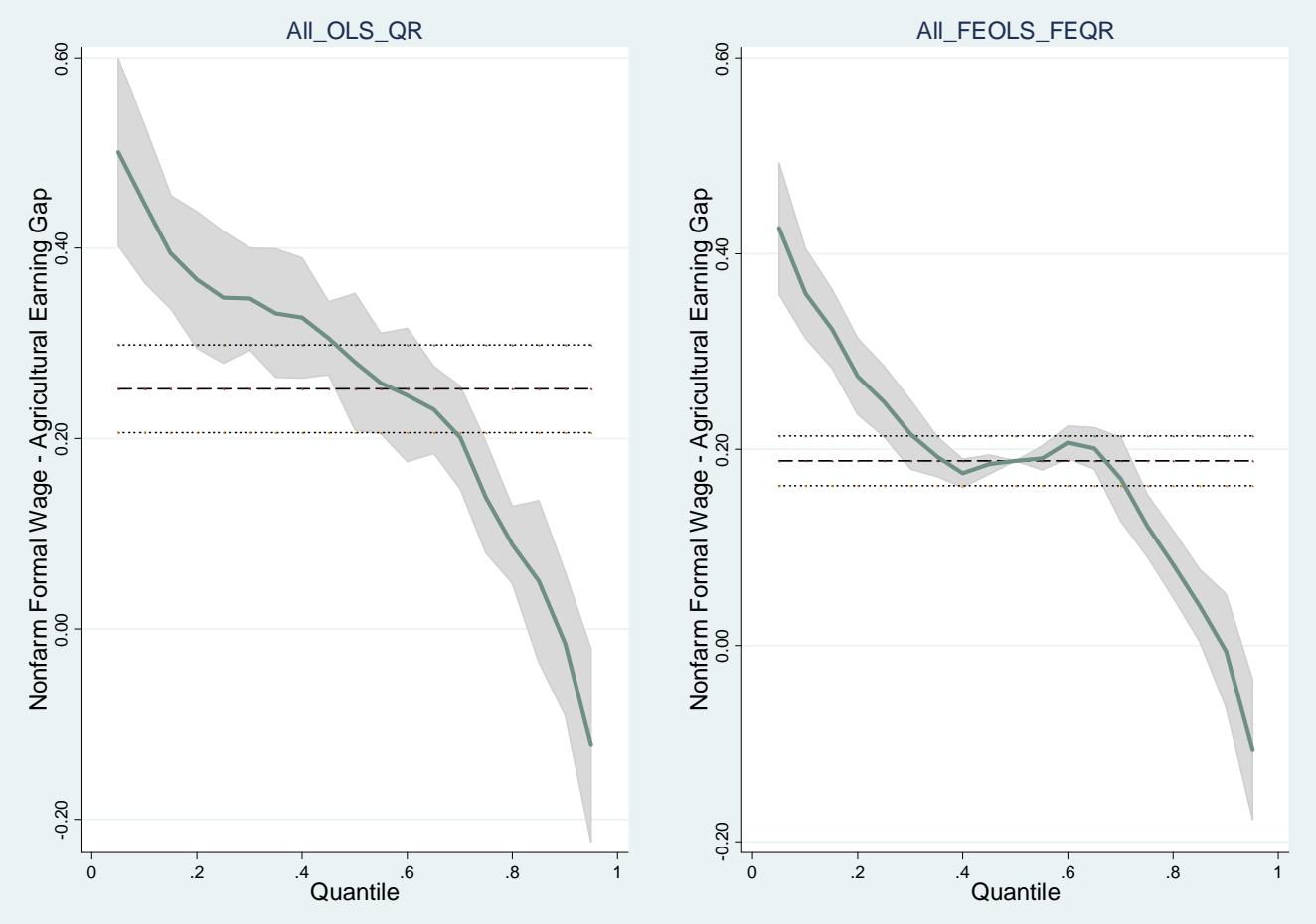

Note: Fixed Effects (FE) OLS are denoted by FEOLS and Fixed Effects Quantile Regressions (QR) by FEQR. Bootstrapped 95 percent confidence intervals are represented by the grey surface for QR and by dashed lines for the OLS.

Source: GSO VHLSS 2002, 2004, 2006, author's calculations.

Compared to agricultural workers, earnings of informal self-employed workers are considerably higher (OLS gap of 42 percent, reduced slightly to 31 percent in FE model at the national level). The reduction of the earnings gaps between non-farm informal selfemployed and agricultural jobs, when unobserved individual characteristics are controlled for, may reflect, to some extent, the disadvantages related to entrepreneurial skills. This indicates generally higher return to self-employment, even for those operating informally, in rural non-farm sector.

The estimate of earnings gaps between non-farm formal self-employed and farm workers presents a 92 percent premium for former, reduced with fixed effects to 50 percent (Figure 6 and Tables 6, columns (3) and (5), and Tables 7, 8). Nevertheless, we should be cautious when interpreting these results, since the self-employed income may be overestimated for at least two reasons. First, the measure of income we computed remunerates both labour and capital factors, the latter being far from negligible in the informal sector (Cling et coll., 2010a). Second, the self-employed income includes the share that should be attributed to the productive contribution of unpaid family workers. As we do not have any order of magnitude of these two phenomena, it is difficult to exclude the possibility that such high premium we obtain may not be substantially reduced, once these two factors are taken into account. $^{77}$

\footnotetext{
${ }^{77}$ The definitive assessment is even more complex as measurement errors in incomes are usually considered as more important for self-employed than for wage workers, the former not knowing their precise level of income (especially informal workers who do not have book accounts), and the richest ones tending to understate their level of activity.
} 
Figure 5- Earnings gaps (Non-farm Informal Self-employed vs. Farm Workers)
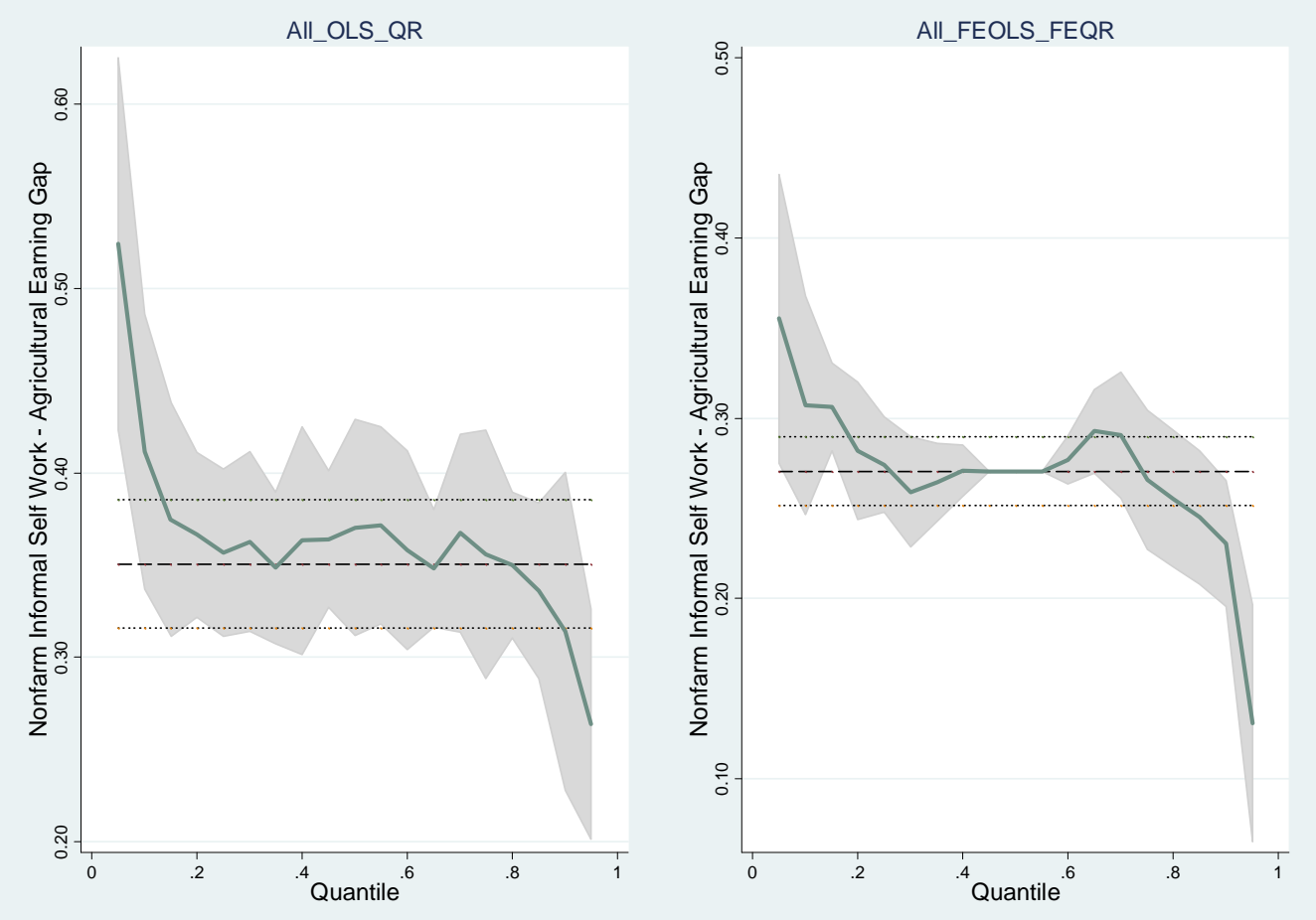

Note: Fixed Effects (FE) OLS are denoted by FEOLS and Fixed Effects Quantile Regressions (QR) by FEQR. Bootstrapped 95 percent confidence intervals are represented by the grey surface for QR and by dashed lines for the OLS.

Source: GSO VHLSS 2002, 2004, 2006, author's calculations.

Turning to quantile regression, the results show that both types of self-employed workers are systematically in a better position than agricultural workers, all along the pay scale. Pooled quantile earnings gap is always positive in favour of formal self-employed workers (at least 88 percent). No distributional effects can be seen in the variation of earnings gap between non-farm formal self-employed and agricultural workers along the pay scale. The FEQR confirm this trend, the only difference is that the range of variation of the gap along the distribution is attenuated. Compared to the earnings gaps between formal wage and agricultural workers, the premium associated with working as self-employment (even for those operating informally) versus agricultural workers appear to be much higher. This implies that their unobserved productive attributes are better than those of the formal wage workers. As for the case of informal self-employed workers, their premium over agricultural workers is always higher than that of the formal wage workers no matter whether unobserved individual characteristics are controlled for or not. Overall, it seems that the Vietnamese labour market functions under a regime of wage repression. Whatever the reasons - macro pressures of international integration or deliberate policies to control inflation, or weak bargaining power of the wage workers, it seems globally preferable to work as an independent (even in the informal sector) than as a wage worker (at least in nonfarm activities) when engaging in rural non-farm sector.

Lastly, it is important to note the difference in the magnitude of earnings premium between two types of self-employment. Non-farm formal self-employed workers have systematically higher earnings premium than their informal counterparts when leaving agricultural employment, all along the pay scale (Tables 7,8 ). This advantage of formal 
household businesses may be due to higher initial level of capital or more productive combination of factors (our models do not provide elements on this point), but it is compatible with the potential intrinsic benefits of getting formal (access to credit and markets) as found by Rand and Torm (2012) in the case of Vietnam.

Figure 6- Earnings gaps (Non-farm Formal Self-employed vs. Farm Workers)
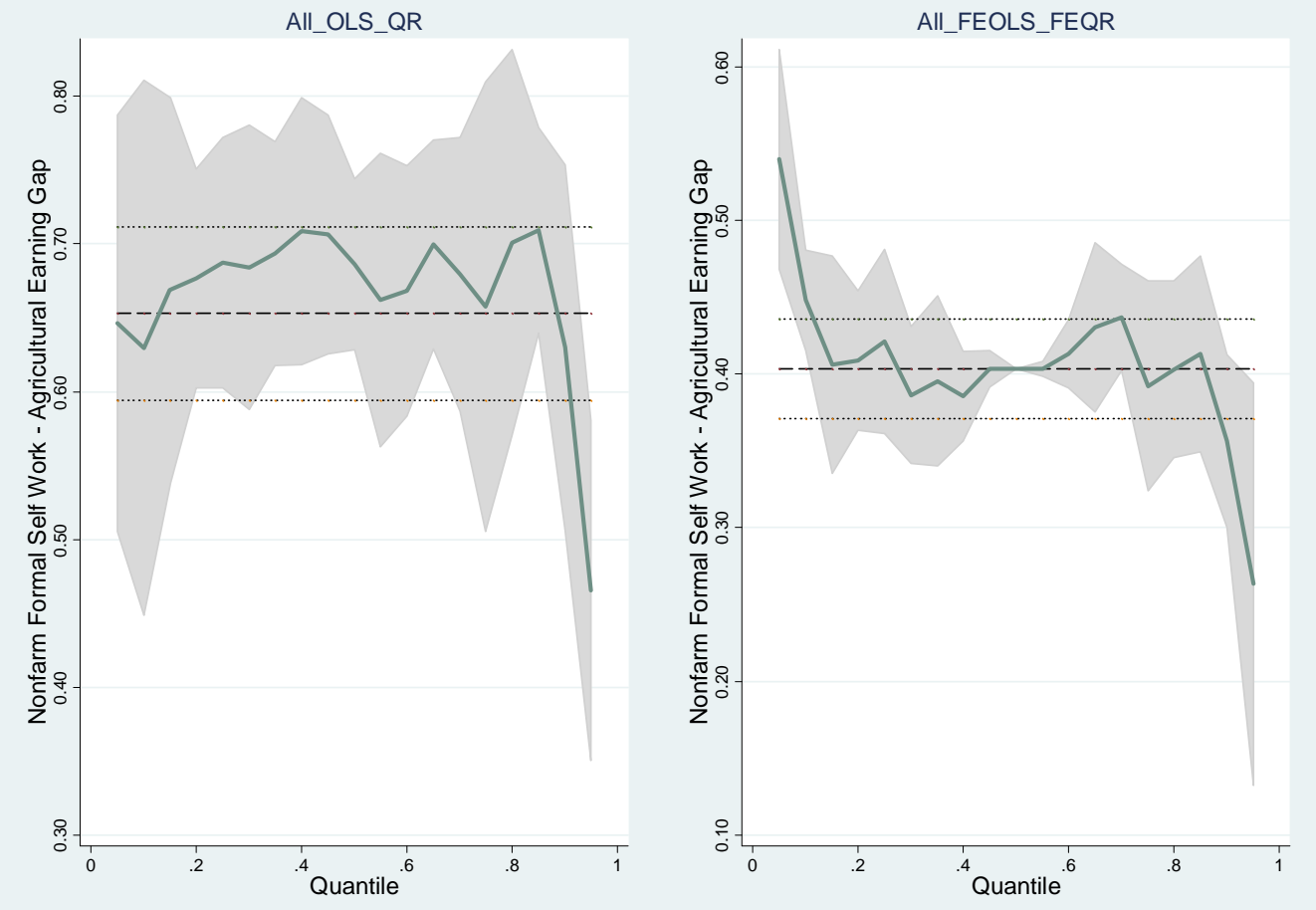

Note: Fixed Effects (FE) OLS are denoted by FEOLS and Fixed Effects Quantile Regressions (QR) by FEQR. Bootstrapped 95 percent confidence intervals are represented by the grey surface for QR and by dashed lines for the OLS.

Source: VHLSS 2002, 2004, 2006, GSO author's calculations.

\section{$\underline{\text { IV.3- A gender perspective }}$}

Taking into account the gender dimension in studying rural non-farm employment and informality is important for various reasons. First, labour market literature has generally shownstrong imbalances in the job structure regarding this dimension. Rural females are less likely to engage in non-farm employment, but when doing so, they are more prone to hold informal jobs than their male counterparts. Second, the raw gender earnings gap is in general significantly higher in the non-farm informal sector. ${ }^{78}$ Finally, and more importantly, the motivation to hold non-farm informal jobs is highly dependent on gender. Women may have a welfare function which is less dependent on income incentives, as they take more care of extra professional activities (as family life, children care, social relations, etc.), where informal jobs could be a more satisfying option. Without going into details, we highlight here the main findings displayed in Figures 7.

\footnotetext{
${ }^{78}$ For Africa, see Nordman, Robilliard and Roubaud (2010) for estimates of the gender earnings gap in the formal and informal sectors of different West African capital cities using household surveys, and Nordman and Wolff (2010) for formal sector gender earnings gaps using matched worker-firm datasets for seven African countries.
} 
Figure 7- Estimated Earnings Gaps for Women and Men Separately by Fixed Effects OLS and QR (with reference to agricultural workers) Source: GSO VHLSS 2002, 2004, 2006, author's calculations

(a) Non-farm Formal - Agricultural Earnings gap
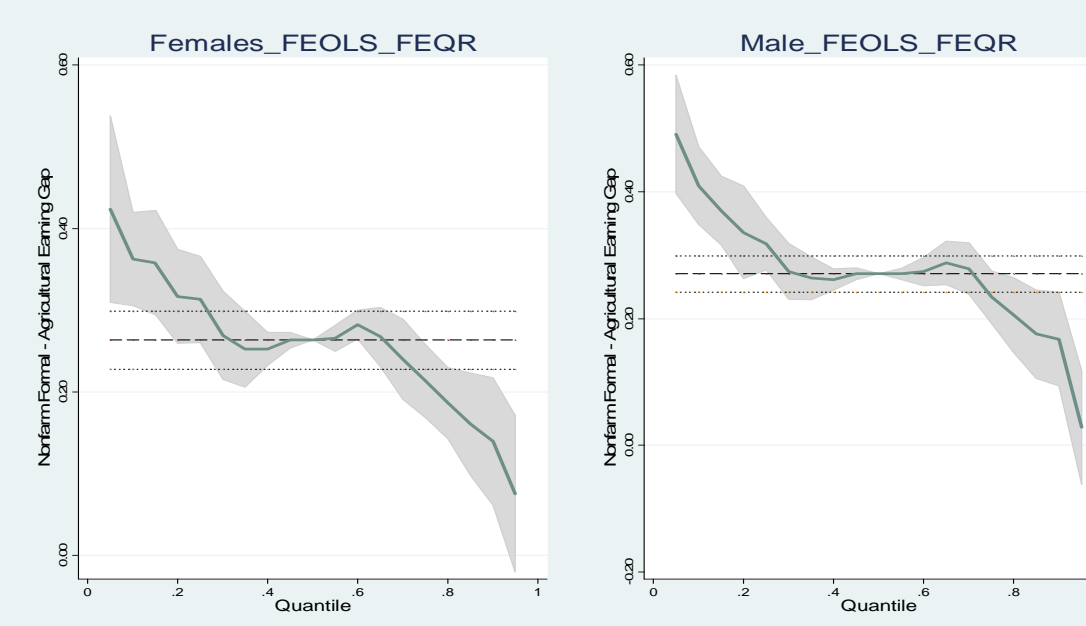

(c) Non-farm Informal Wage - Agricultural Earnings gap
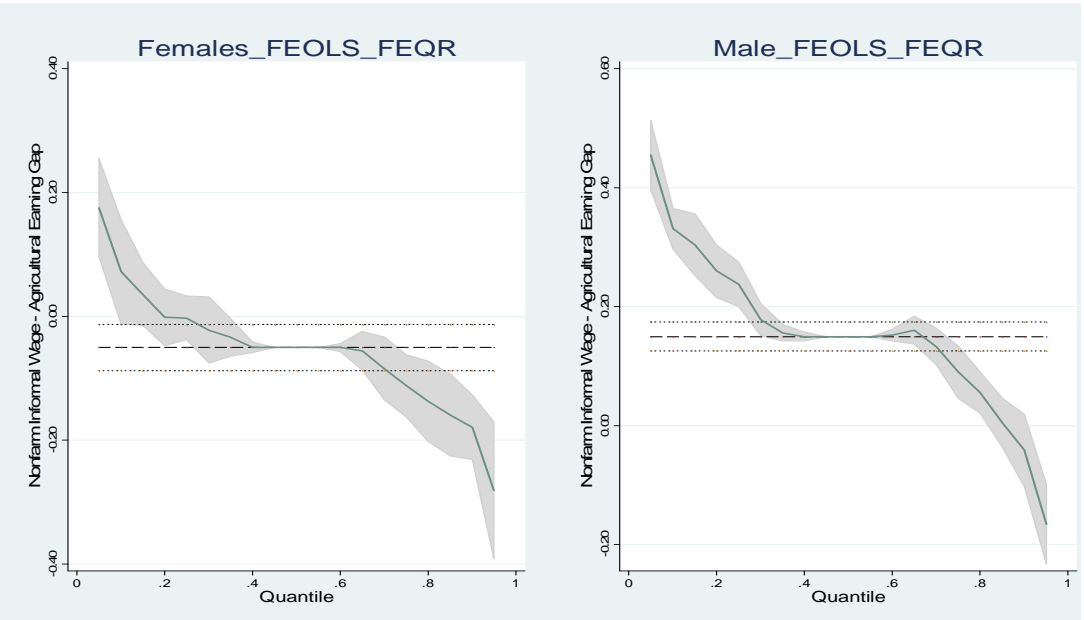

(b) Non-farm Informal - Agricultural Earnings gap
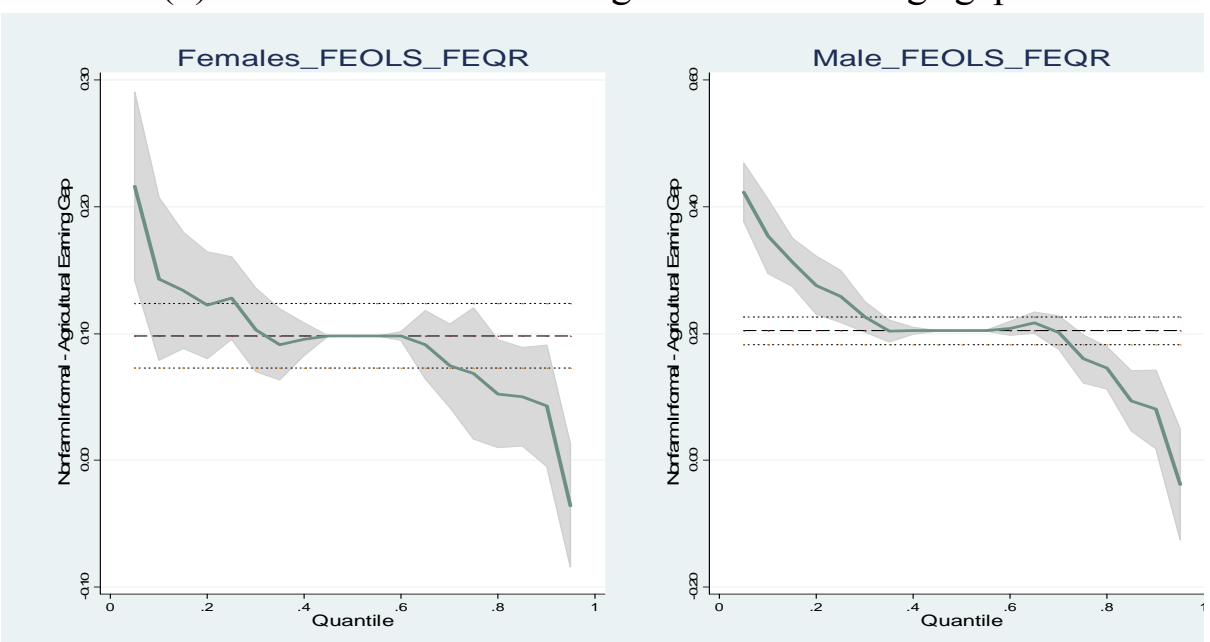

(d) Non-farm Formal Wage - Agricultural Earnings gap

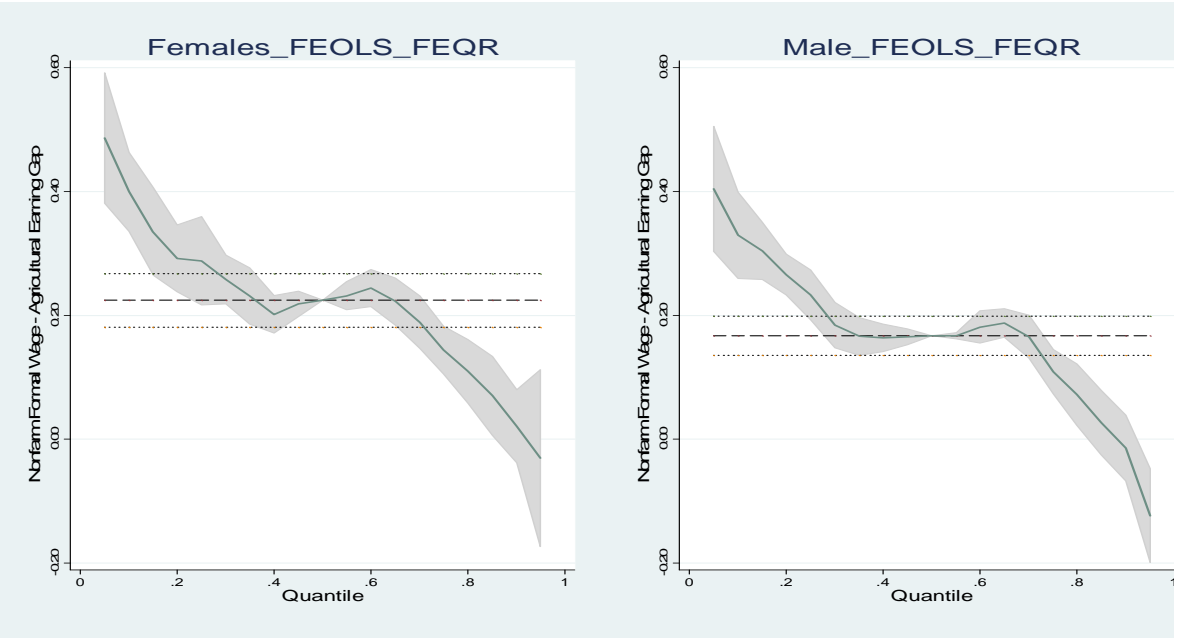


Figure 7- Estimated Earnings Gaps for Women and Men Separately by Fixed Effects OLS and QR (with reference to agricultural workers) Source: GSO VHLSS 2002, 2004, 2006, author's calculations

(e) Non-farm Informal Self-employed - Agricultural Earnings gap
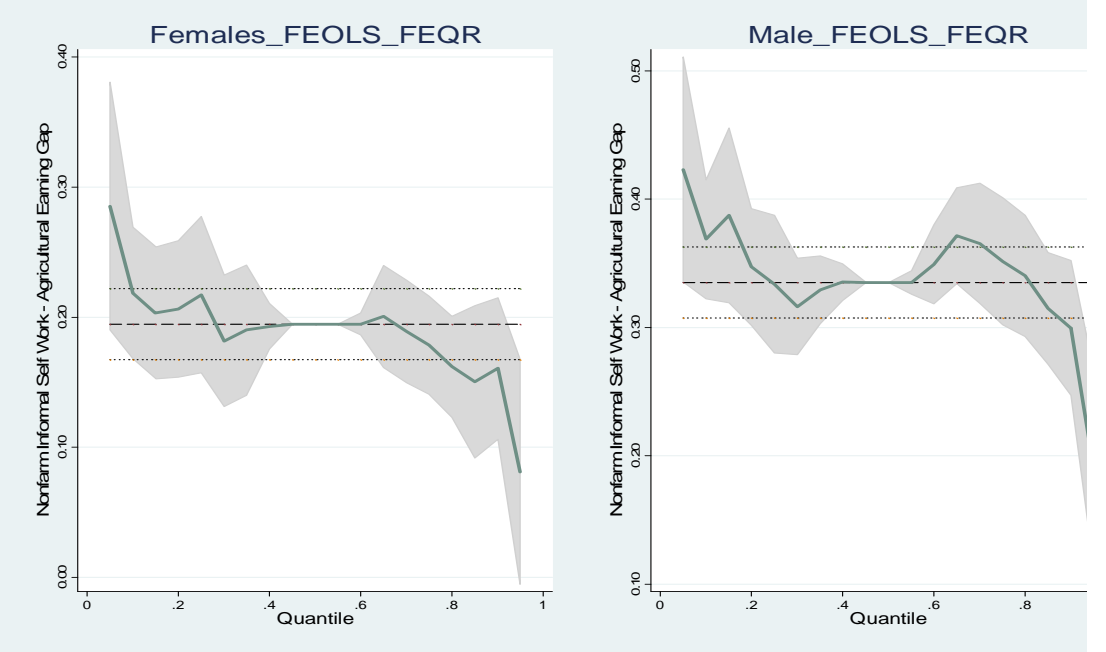

(f) Non-farm Formal Self-employed - Agricultural Earnings gap
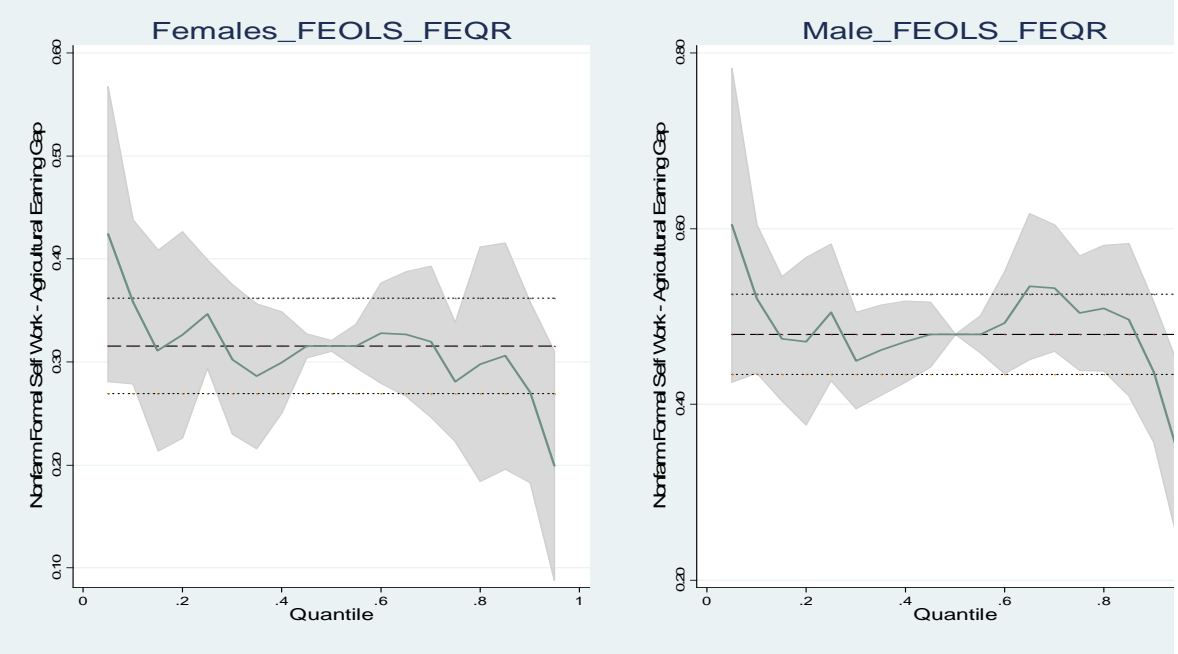
Firstly, whatever the models' specifications and the category of workers considered, rural females are always benefiting less premium when participating in non-farm jobs. For instance, at the aggregate non-farm informal/farm level, the estimated FEOLS earnings gap is 10 percent for females whereas the corresponding figure obtained is 22 percent for males. Such a feature is compatible with the idea mentioned above, that women may accept lower wages in the informal sector because it provides other non-pecuniary advantages, relatively more valuable to them. However, it can also reveal barriers or labour market segmentation, which would be more pronounced for women competing for non-farm salaried jobs.

Secondly, in spite of differences in absolute levels, the distributional profile of the earnings gaps is quite similar across gender: no noticeable effect for non-farm formal self-employed workers, a decreasing premium for all types of non-farm workers, both formal and informal.

Lastly, the sorting process in the allocation of men and women across sectors (which is partly revealed by the effect of controlling for unobserved individual characteristics) does not differ substantially across gender: informal wage workers have negative unobserved individual characteristics (in order to get a better income) vis-à-vis agricultural workers, while the unobserved skills are higher for non-farm self-employed workers (whether formal or informal). The only exception is for male wage workers, who have comparable unobserved individual characteristics along the formal/informal divide.

\section{Conclusion}

This paper focuses on measuring and analyzing earnings gap between non-farm and farm workers in rural area of Vietnam in order to assess if the participation in non-farm employment is really able to improve earnings for rural workers, thus a path out of poverty for rural population. Taking advantage of the rich VHLSS datasets, the three rounds of panel surveys (2002, 2004 and 2006) give the unique opportunity to control for time invariant unobserved individual characteristics. Using both standard and fixed effects earnings equations estimated at the mean and at various conditional quintiles of the earnings distribution, we address the key issue of heterogeneity, at three different levels: at the worker level, taking into account individual unobserved characteristics; at the job level, comparing different types of non-farm workers with agricultural workers; at the distributional level. Gender issues are also examined.

Our results suggest that the non-farm - agricultural earnings gap highly depends on the nonfarm workers' job status (wage employment vs. self-employment) and on their relative position in the earnings distribution. Rural workers can earn more when engaging in non-farm activities instead of keeping agricultural jobs, but this is not always the case for all types of non-farm jobs. In many cases, non-farm jobs (such as informal wage employment and even formal wage jobs at upper quintiles) are not as rewarding as agricultural jobs. In other words, engaging in non-farm jobs is generally more rewarding than agricultural jobs at lower quintiles. This feature is due to the relatively low wages of both formal and informal wage jobs in rural non-farm sector. However, the reason for such results should be investigated further by undertaking some case studies for refining non-farm job earnings by industry or in specific geographical areas. Second, females are always financially benefiting less when they are employed in non-farm employment, particularly when engaging in informal jobs. This feature opens the space for specific policies to align women's functioning of rural labour market with men's one (reduction in the barriers of entry to formal jobs, etc.).

Our analysis raises further promising prospects, and could be extended in various directions. A first extension would be to better control for individual unobserved characteristics, by purging our earning estimations of differences in the amount of physical capital (for selfemployed workers) and social networks. A firm based panel approach may be an interesting 
alternative entry in this respect. The purpose would be to focus on the adverse (or positive) effect of family and kinship ties and identifies the key determinants of potential redistributive pressure exerted by the kinship ties, extended family, and network capital on the performance of non-farm household businesses (NFHBs). A first draft of a paper on this issue has been undertaken (Nguyen and al. 2011). Preliminary results show that, when controlling for timeinvariant unobserved heterogeneity of the NFHBs, being an informal NFHB corresponds to a penalty in annual value added of about 30 percent. Also for this type of household firms, we find evidence of a productivity differential between family and hired labour, with a gap of roughly 15 percentage points in favour of the latter. Using information on the entrepreneurs' social capital, we obtain some results confirming the importance of unlocking financial constraints (on physical capital) and improving access to professional support for successful household entrepreneurship. First, we always find a positive effect on the informal business' technical efficiency of benefiting from a loan from the kin. Second, for these informal entrepreneurs, being a member of a business association or having a friend producing the same product is beneficial in terms of efficiency, perhaps thanks to knowledge spill-over and/or shared clienteles. Professional network capital thus appears to be one important ingredient of informal business performance. Another potential extension would be to exploit further the nature of our data (three point panel) by estimating dynamic earnings equations. Lastly, our work could be usefully complemented by investigating the determinants of job's satisfaction, to enlarge the perspective which relies exclusively on the earning outputs and to check for the robustness of our conclusions.

\section{References}

BARGAIn Olivier, Kwenda Prudence, Is Informality Bad? Evidence from Brazil, Mexico and South Africa, IZA Discussion Paper, n 4711, January 2010, p. 30.

Belsley David, KuH Edwin, RoY Welsch, Regression Diagnostics: Identifying Influential Data and Sources of Collinearity, New York, John Wiley, 1980, 292 p.

CANAY, Ivan A., "A simple approach to Quantile Regression for Panel Data" in The Econometrics Journal, 14, 2011, p. 368-386.

Cling Jean-Pierre, NGuyen Huu Chi, Razafindrakoto Mireille, Roubaud François, "Urbanisation et Insertion sur le Marché du Travail au Vietnam : Poids et Caractéristiques du Secteur Informel", communication at the Regional Conference Trends in urbanization and peri-urbanization in South-East Asia, CEFURDS/IRD, Ho Chi Minh City, December, 9-11, 2012, p. 205-226.

Cling, Jean-Pierre, Nguyen Thi Thu Huyen, Nguyen Huu Chi, Phan Thi Ngọc Tram, RAZAFINDRAKOTO Mireille, Roubaud François, The Informal Sector in Vietnam: A focus on Hanoi and Ho Chi Minh City, Hanoi, The Gioi Edition, 2010a, 248 p.

Cling, Jean-Pierre, RaZAFindrakoto Mireille, Roubaud François, « Assessing the Potential Impact of the Global Crisis on the Labour Market and the Informal Sector in Vietnam », in Journal of Economics \& Development, $\mathrm{n}^{\circ} 38$, June 2010b, p. 16-25.

Dickens William et LANG Kevin, «A Test of Dual Labour Market Theory », in The American Economic Review, n 4/75, p. 792-805.

DORWARD Andrew, KYDD Jonathan, MORRISON Jamie, UREY Ian, « A Policy Agenda for ProPoor Agricultural Growth », in World Development, $\mathrm{n}^{\circ}$ 1/32, 2004, p. 73-89 
Dabalen Andrew, Paternostro Stefano et GaËlle Pierre, The Returns to Participation in the Non-farm Sector in Rural Rwanda, World Bank Policy Research Working Paper WPS3462, 2004, 33 p.

European COMMISSION, InTERnATIONAL MONETARy Fund, Organization FOR ECONOMIC Co-operation and Development, United Nations, World Bank, The System of National Accounts 2008 (2008 SNA), New York, A Handbook, 2009, p. 722.

Falco Paolo, Kerr Andrew, Rankin Neil, SAndefur Justin, and Teal Francis, « The Returns to Formality and Informality in Urban Africa, CSAE WPS.2010-03, University of Oxford, 2010, 27 p. + appendices.

Fernández Rosa, Nordman Christophe, « Are There Pecuniary Compensations for Working Conditions? », in Labour Economics, n 2/16, april 2009, p. 194-207.

Foster, Andew D.; RosenzWeIG, Mark R.; 2004. Agricultural development, industrialization and rural inequality, 37 p. + appendices (dact.) accessed at http://www.ccpr.ucla.edu/ events/ccpr-seminars-previous-years/Sem04F\%20Rosenzweig\%20Agricultural\%20Deve lopment\%20Industrialization\%20and\%20Rural\%20Inequality.pdf on 11/12/2014 15:33.

General Statistics Office, UNFPA, The 2009 Vietnam Population and Housing Census. Implementation and Preliminary Results, Hanoi, August 2009, 48 p.

Hertz Tome, Winters Paul, QuiÑones Esteban, AZZARi Carlo, DAvis Benjamin, Wage Inequality in International Perspective: Effects of Location, Sector, and Gender, Paper presented at the FAO-IFAD-ILO Workshop on Gaps, trends and current research in gender dimensions of agricultural and rural employment: differentiated pathways out of poverty, Rome, 31 March - 2 April 2009.

HuSSMANNS Ralf, Measuring the informal economy: from employment in the informal sector to informal employment, Working Paper, $\mathrm{n}^{\circ}$ 53, December 2004, p. 42.

InTERNATIONAL LABOUR ORgANiZATION, « Guidelines Concerning a Statistical Definition of Informal Employment », in Seventeenth International Conference of Labour Statisticians, Geneva, 2003, 3 p. + appendices.

Jonasson, Erik, Earnings Differentials in the Rural Labour Market: Does Non -Agricultural Employment Pay Better? Working Papers, $\mathrm{n}^{\circ}$ 7, 2008, 21 p.

KOENKER Roger, «Quantile Regression for Longitudinal Data », in Journal of Multivariate Analysis, $\mathrm{n}^{\circ}$ 91, 2004, p. 74-89.

LANJOUw Peter, « Does the Rural Non-farm Economy Contribute to Poverty Reduction? », in Transforming the Rural Non-farm Economy: Opportunities and Threats in the Developing World, Haggblade Steven, Hazell Peter et Reardon Thomas (Éds), Baltimore, John Hopkins University Press, 2008, p. 55-80

LANJOUw, Peter, Murgai, Rinku, « http://ideas.repec.org/p/wbk/wbrwps/4858.html », Agricultural Economics, 40, 2009, p. 243-263.

LEWIS Artur, « Economic Development with Unlimited Supplies of Labour », in Manchester School, n² 2/28, Mai 1954, p. 139-191.

Magda, Iga, Wage Mobility in Times of Higher Earnings Disparities: It is Easier to Climb the Ladder?, ISER Working Paper Series, Institute for Social and Economic Research, 2008, 25 p. 
MALONEY William, « Does Informality Imply Segmentation in Urban Labor Markets? Evidence from Sectoral Transitions in Mexico », in World Bank Economic Review, $\mathrm{n}^{\circ}$ 2/13, 1999, p. 275-302.

MALONEY William, «Informality Revisited », in World Development, ${ }^{\circ}$ 7/32, 2004, p. 11591178.

MCCAIG Brian, BENJAMIn Dwayne, BRANDT Loren, The Evolution of Income Inequality in Vietnam, 1993-2006, unpublished manuscript, Australian National University and University of Toronto, 2009, 67 p.

McCulloch Neil, Weisbrod Julian, Timmer Petter, Pathways out of Poverty During an Economic Crisis: An Empirical Assessment of Rural Indonesia, Washington, World Bank, 2007, p. 50.

Nguyen, Huu Chi, Nordman, Christophe J., Household Entrepreneurship and Social Networks in Vietnam: Evidence from Panel Data, DIAL Research Paper DT/2014/22, 2014.

Nguyen Huu Chi, Nordman Christophe, Roubaud François, Who Suffers the Penalty? A Panel Data Analysis of Earnings Gaps in Vietnam, IZA Discussion Paper Series, $\mathrm{n}^{\circ} 7149$, Janvier 2013, p. 47.

Nordman Christophe, Robilliard Anne Sophie et Roubaud François, «Decomposing Gender and Ethnic Earnings Gaps in Seven cities in West Africa ", in Urban Labour Markets in Sub-Saharan Africa, Philippe De Vreyer et RoubAUD François (éd.), Africa Development Forum, 2013, p. 271-295.

NORDMAN Christophe, WolfF François-Charles, "Gender Differences in Pay in African Manufacturing Firms », in Gender Disparities in Africa's Labor Market, François-Charles Wolff, Christophe Jalil NOrdman ed., Washington D.C., World Bank, 2010, pp. 155-192.

PhUng Du et NGUYen Phong, Vietnam Household Living Standards. Survey (VHLSS) 2002 and 2004 Basic Information, Vietnam General Statistics Office, unpublished manuscript, Hanoi, 2006, 47 p.

RAND John, ToRM Nina, "The Benefits of Formalization: Evidence from Vietnamese SMEs», in World Development, vol. 40, n5, 2012, pp. 983-998.

RoUBAUD François, L'économie informelle au Mexique : de la sphère domestique à la dynamique macro-économique, Paris, Karthala/Orstom, 1994, 453 p.

SöDerbom Mans, Teal Francis, Wambugu Anthony, « Unobserved Heterogeneity and the Relation between Earnings and Firm Size: Evidence from Two Developing Countries », in Economics Letters, $\mathrm{n}^{\circ}$ 2/87, 2005, p. 153-159.

Strobl Eric, ThORNTON Robert, Do Large Employers Pay More in Developing Countries? The Case of Five African Countries, IZA Discussion Paper, $\mathrm{n}^{\circ} 660,2002,35$ p. + appendices.

Vietnam ACADEmy of Social ScIEnCes, "Rapid Assessment of the Social Impacts of the Global Economic Crisis in Vietnam: Summary of First Round Research", Oxfam Discussion Paper, July, 2010, 12 p.

Winters Paul, De la O Ana Paula, QuiÑones Esteban, Hertz Thomas, DAVIS Benjamin, ZEZZA, Alberto, Covarrubias Katia, K Carletto, Stamoulis Kosta, Rural Wage Employment and Household Livelihood Strategies: A Multi-Country Analysis, ESA Working paper, unpublished paper, 2008, 36 p. + appendices. 


\section{Appendix}

Table 6- Mean Earnings Regressions for Rural Workers in Vietnam Dependent Variable: Log Hourly Real Earnings - VHLSS 2002-2004-2006

\begin{tabular}{|c|c|c|c|c|c|}
\hline VARIABLES & $\begin{array}{c}\text { (1) } \\
\text { Pooled OLS }\end{array}$ & $\begin{array}{c}(2) \\
\text { Pooled OLS }\end{array}$ & $\begin{array}{c}(3) \\
\text { Pooled OLS }\end{array}$ & $\begin{array}{c}\text { (4) } \\
\text { Fixed Effects }\end{array}$ & $\begin{array}{c}(5) \\
\text { Fixed Effects }\end{array}$ \\
\hline Formal & & $\begin{array}{c}0.397 * * * \\
(0.021)\end{array}$ & & $\begin{array}{c}0.270 * * * \\
(0.043)\end{array}$ & \\
\hline Informal & & $\begin{array}{c}0.244 * * * \\
(0.015)\end{array}$ & & $\begin{array}{c}0.160 * * * \\
(0.034)\end{array}$ & \\
\hline Wage_inf & & & $\begin{array}{c}0.114 * * * \\
(0.016)\end{array}$ & & $\begin{array}{l}0.079 * * \\
(0.036)\end{array}$ \\
\hline Wage_for & & & $\begin{array}{c}0.252 * * * \\
(0.022)\end{array}$ & & $\begin{array}{c}0.189 * * * \\
(0.050)\end{array}$ \\
\hline Self_inf & & & $\begin{array}{c}0.351 * * * \\
(0.019)\end{array}$ & & $\begin{array}{c}0.271 * * * \\
(0.045)\end{array}$ \\
\hline Self_for & & & $\begin{array}{c}0.653 * * * \\
(0.032)\end{array}$ & & $\begin{array}{c}0.403 * * * \\
(0.060)\end{array}$ \\
\hline Yearsch & $\begin{array}{c}0.021 * * * \\
(0.006)\end{array}$ & $\begin{array}{c}0.019 * * * \\
(0.006)\end{array}$ & $\begin{array}{c}0.006 \\
(0.006)\end{array}$ & $\begin{array}{c}0.027 \\
(0.017)\end{array}$ & $\begin{array}{c}0.027 \\
(0.017)\end{array}$ \\
\hline Yearsch2 & $\begin{array}{c}0.002 * * * \\
(0.000)\end{array}$ & $\begin{array}{c}0.001 * * * \\
(0.000)\end{array}$ & $\begin{array}{c}0.002 * * * \\
(0.000)\end{array}$ & $\begin{array}{l}-0.001 * \\
(0.001)\end{array}$ & $\begin{array}{l}-0.001 \\
(0.001)\end{array}$ \\
\hline Expe & $\begin{array}{c}0.014 * * * \\
(0.002)\end{array}$ & $\begin{array}{c}0.015 * * * \\
(0.002)\end{array}$ & $\begin{array}{c}0.013 * * * \\
(0.002)\end{array}$ & $\begin{array}{c}0.001 \\
(0.012)\end{array}$ & $\begin{array}{c}0.001 \\
(0.012)\end{array}$ \\
\hline Expe2 & $\begin{array}{c}-0.000 * * * \\
(0.000)\end{array}$ & $\begin{array}{c}-0.000 * * * \\
(0.000)\end{array}$ & $\begin{array}{c}-0.000 * * * \\
(0.000)\end{array}$ & $\begin{array}{c}0.000 \\
(0.000)\end{array}$ & $\begin{array}{c}0.000 \\
(0.000)\end{array}$ \\
\hline Female & $\begin{array}{c}-0.104 * * * \\
(0.013)\end{array}$ & $\begin{array}{c}-0.109 * * * \\
(0.013)\end{array}$ & $\begin{array}{c}-0.149 * * * \\
(0.013)\end{array}$ & & \\
\hline Married & $\begin{array}{c}0.128 * * * \\
(0.018)\end{array}$ & $\begin{array}{c}0.133 * * * \\
(0.018)\end{array}$ & $\begin{array}{c}0.117 * * * \\
(0.018)\end{array}$ & $\begin{array}{l}-0.024 \\
(0.049)\end{array}$ & $\begin{array}{l}-0.029 \\
(0.049)\end{array}$ \\
\hline Year 2004 & $\begin{array}{c}0.328 * * * \\
(0.016)\end{array}$ & $\begin{array}{c}0.241 * * * \\
(0.015)\end{array}$ & $\begin{array}{c}0.241 * * * \\
(0.015)\end{array}$ & $\begin{array}{c}0.262 * * * \\
(0.020)\end{array}$ & $\begin{array}{c}0.265 * * * \\
(0.021)\end{array}$ \\
\hline Year 2006 & $\begin{array}{c}0.421 * * * \\
(0.016)\end{array}$ & $\begin{array}{c}0.407 * * * \\
(0.016)\end{array}$ & $\begin{array}{c}0.411 * * * \\
(0.016)\end{array}$ & $\begin{array}{c}0.430 * * * \\
(0.034)\end{array}$ & $\begin{array}{c}0.434 * * * \\
(0.034)\end{array}$ \\
\hline Constant & $\begin{array}{c}0.497 * * * \\
(0.037)\end{array}$ & $\begin{array}{c}0.399 * * * \\
(0.038)\end{array}$ & $\begin{array}{c}0.514 * * * \\
(0.038)\end{array}$ & $\begin{array}{c}0.968 * * * \\
(0.266)\end{array}$ & $\begin{array}{c}0.977 * * * \\
(0.266)\end{array}$ \\
\hline $\begin{array}{l}\text { Observations } \\
\text { R-squared } \\
\text { Number of id }\end{array}$ & $\begin{array}{c}11498 \\
0.181\end{array}$ & $\begin{array}{c}11498 \\
0.211\end{array}$ & $\begin{array}{l}11498 \\
0.229\end{array}$ & $\begin{array}{l}11498 \\
0.151 \\
6425\end{array}$ & $\begin{array}{c}11498 \\
0.155 \\
6425\end{array}$ \\
\hline
\end{tabular}

Robust standard errors in parentheses

$* * * \mathrm{p}<0.01, * * \mathrm{p}<0.05, * \mathrm{p}<0.1$

Source: GSO VHLSS 2002, 2004, 2006, author's calculations 
Table 7- Pooled Quantile Earnings Regressions for Rural Workers in Vietnam. Dependent Variable: Log Hourly Real Earnings - Vietnam VHLSS 2002-2004-2006. Source: GSO VHLSS 2002, 2004, 2006, author's calculations

\begin{tabular}{|c|c|c|c|c|c|c|c|c|c|c|}
\hline VARIABLES & $\begin{array}{c}(1) \\
\text { Pooled .10 }\end{array}$ & $\begin{array}{c}(2) \\
\text { Pooled } .25\end{array}$ & $\begin{array}{c}(3) \\
\text { Pooled } .50\end{array}$ & $\begin{array}{c}(4) \\
\text { Pooled .75 }\end{array}$ & $\begin{array}{c}(5) \\
\text { Pooled .90 }\end{array}$ & $\begin{array}{c}(6) \\
\text { Pooled } .10\end{array}$ & $\begin{array}{c}(7) \\
\text { Pooled } .25\end{array}$ & $\begin{array}{c}(8) \\
\text { Pooled } .50\end{array}$ & $\begin{array}{c}(9) \\
\text { Pooled .75 }\end{array}$ & $\begin{array}{c}(10) \\
\text { Pooled } .90\end{array}$ \\
\hline Formal & $\begin{array}{c}0.472 * * * \\
(0.041)\end{array}$ & $\begin{array}{c}0.433 * * * \\
(0.027)\end{array}$ & $\begin{array}{c}0.402 * * * \\
(0.025)\end{array}$ & $\begin{array}{c}0.332 * * * \\
(0.031)\end{array}$ & $\begin{array}{c}0.322 * * * \\
(0.042)\end{array}$ & & & & & \\
\hline Informal & $\begin{array}{c}0.459 * * * \\
(0.032)\end{array}$ & $\begin{array}{c}0.335 * * * \\
(0.020)\end{array}$ & $\begin{array}{c}0.242 * * * \\
(0.016)\end{array}$ & $\begin{array}{c}0.115 * * * \\
(0.021)\end{array}$ & $\begin{array}{c}0.068 * * \\
(0.030)\end{array}$ & & & & & \\
\hline Wage_inf & & & & & & $\begin{array}{c}0.505 * * * \\
(0.041)\end{array}$ & $\begin{array}{c}0.316 * * * \\
(0.022)\end{array}$ & $\begin{array}{c}0.150 * * * \\
(0.020)\end{array}$ & $\begin{array}{c}-0.076^{* * *} \\
(0.023)\end{array}$ & $\begin{array}{c}-0.315 * * * \\
(0.031)\end{array}$ \\
\hline Wage_for & & & & & & $\begin{array}{c}0.446 * * * \\
(0.040)\end{array}$ & $\begin{array}{c}0.348 * * * \\
(0.029)\end{array}$ & $\begin{array}{c}0.280 * * * \\
(0.025)\end{array}$ & $\begin{array}{c}0.139 * * * \\
(0.029)\end{array}$ & $\begin{array}{c}-0.016 \\
(0.043)\end{array}$ \\
\hline Self_inf & & & & & & $\begin{array}{c}0.412 * * * \\
(0.038)\end{array}$ & $\begin{array}{c}0.357 * * * \\
(0.028)\end{array}$ & $\begin{array}{c}0.370 * * * \\
(0.024)\end{array}$ & $\begin{array}{c}0.356 * * * \\
(0.031)\end{array}$ & $\begin{array}{c}0.314 * * * \\
(0.032)\end{array}$ \\
\hline Self_for & & & & & & $\begin{array}{c}0.630 * * * \\
(0.092)\end{array}$ & $\begin{array}{c}0.687 * * * \\
(0.049)\end{array}$ & $\begin{array}{c}0.686^{* * * *} \\
(0.032)\end{array}$ & $\begin{array}{c}0.658 * * * \\
(0.057)\end{array}$ & $\begin{array}{c}0.630 * * * \\
(0.045)\end{array}$ \\
\hline Yearsch & $\begin{array}{l}-0.015 \\
(0.011)\end{array}$ & $\begin{array}{l}-0.012 \\
(0.008)\end{array}$ & $\begin{array}{c}0.005 \\
(0.006)\end{array}$ & $\begin{array}{c}0.045^{* * *} \\
(0.008)\end{array}$ & $\begin{array}{c}0.078 * * * \\
(0.012)\end{array}$ & $\begin{array}{l}-0.018^{*} \\
(0.010)\end{array}$ & $\begin{array}{c}-0.020 * * \\
(0.008)\end{array}$ & $\begin{array}{l}-0.007 \\
(0.005)\end{array}$ & $\begin{array}{c}0.022 * * * \\
(0.008)\end{array}$ & $\begin{array}{c}0.033 * * * \\
(0.010)\end{array}$ \\
\hline Yearsch2 & $\begin{array}{c}0.003 * * * \\
(0.001)\end{array}$ & $\begin{array}{c}0.003 * * * \\
(0.000)\end{array}$ & $\begin{array}{c}0.002 * * * \\
(0.000)\end{array}$ & $\begin{array}{l}-0.000 \\
(0.000)\end{array}$ & $\begin{array}{c}-0.002 * * * \\
(0.001)\end{array}$ & $\begin{array}{c}0.003 * * * \\
(0.001)\end{array}$ & $\begin{array}{c}0.003 * * * \\
(0.001)\end{array}$ & $\begin{array}{c}0.003 * * * \\
(0.000)\end{array}$ & $\begin{array}{c}0.002 * * * \\
(0.000)\end{array}$ & $\begin{array}{l}0.001 * \\
(0.001)\end{array}$ \\
\hline pe & $\begin{array}{c}0.014 * * * \\
(0.004)\end{array}$ & $\begin{array}{c}0.017 * * * \\
(0.003)\end{array}$ & $\begin{array}{c}0.018 * * * \\
(0.002)\end{array}$ & $\begin{array}{c}0.018 * * * \\
(0.002)\end{array}$ & $\begin{array}{c}0.017 * * * \\
(0.003)\end{array}$ & $\begin{array}{c}0.013 * * * \\
(0.003)\end{array}$ & $\begin{array}{c}0.016^{* * * *} \\
(0.003)\end{array}$ & $\begin{array}{c}0.016^{* * *} \\
(0.002)\end{array}$ & $\begin{array}{c}0.016 * * * \\
(0.003)\end{array}$ & $\begin{array}{c}0.015^{* * *} * \\
(0.004)\end{array}$ \\
\hline Expe2 & $\begin{array}{c}-0.000 * * * \\
(0.000)\end{array}$ & $\begin{array}{c}-0.000 * * * \\
(0.000)\end{array}$ & $\begin{array}{c}-0.000 * * * \\
(0.000)\end{array}$ & $\begin{array}{c}-0.000 * * * \\
(0.000)\end{array}$ & $\begin{array}{c}-0.000 * * * \\
(0.000)\end{array}$ & $\begin{array}{c}-0.000 * * * \\
(0.000)\end{array}$ & $\begin{array}{c}-0.000 * * * \\
(0.000)\end{array}$ & $\begin{array}{c}-0.000 * * * \\
(0.000)\end{array}$ & $\begin{array}{c}-0.000 * * * \\
(0.000)\end{array}$ & $\begin{array}{c}-0.000 * * * \\
(0.000)\end{array}$ \\
\hline Female & $\begin{array}{c}-0.207 * * * \\
(0.024)\end{array}$ & $\begin{array}{c}-0.168 * * * \\
(0.015)\end{array}$ & $\begin{array}{c}-0.121 * * * \\
(0.013)\end{array}$ & $\begin{array}{c}-0.058 * * * \\
(0.019)\end{array}$ & $\begin{array}{l}-0.035 \\
(0.026)\end{array}$ & $\begin{array}{c}-0.203 * * * \\
(0.026)\end{array}$ & $\begin{array}{c}-0.184 * * * \\
(0.019)\end{array}$ & $\begin{array}{c}-0.166^{* * * *} \\
(0.015)\end{array}$ & $\begin{array}{c}-0.148 * * * \\
(0.018)\end{array}$ & $\begin{array}{c}-0.148 * * * \\
(0.024)\end{array}$ \\
\hline Married & $\begin{array}{c}0.141^{* * *} \\
(0.034)\end{array}$ & $\begin{array}{c}0.097 * * * \\
(0.029)\end{array}$ & $\begin{array}{c}0.085^{* * *} \\
(0.019)\end{array}$ & $\begin{array}{c}0.136^{* * *} \\
(0.024)\end{array}$ & $\begin{array}{c}0.195^{* * *} \\
(0.033)\end{array}$ & $\begin{array}{c}0.158^{* * * *} \\
(0.036)\end{array}$ & $\begin{array}{c}0.100^{* * * *} \\
(0.028)\end{array}$ & $\begin{array}{c}0.083 * * * \\
(0.021)\end{array}$ & $\begin{array}{c}0.093 * * * \\
(0.025)\end{array}$ & $\begin{array}{c}0.118^{* * *} \\
(0.038)\end{array}$ \\
\hline Year 2004 & $\begin{array}{c}0.367 * * * \\
(0.031)\end{array}$ & $\begin{array}{c}0.303 * * * \\
(0.022)\end{array}$ & $\begin{array}{c}0.202 * * * \\
(0.017)\end{array}$ & $\begin{array}{c}0.142 * * * \\
(0.021)\end{array}$ & $\begin{array}{c}0.146^{* * *} \\
(0.031)\end{array}$ & $\begin{array}{c}0.357 * * * \\
(0.032)\end{array}$ & $\begin{array}{c}0.296 * * * \\
(0.021)\end{array}$ & $\begin{array}{c}0.201 * * * \\
(0.017)\end{array}$ & $\begin{array}{c}0.137 * * * \\
(0.018)\end{array}$ & $\begin{array}{c}0.150 * * * \\
(0.025)\end{array}$ \\
\hline Year 2006 & $\begin{array}{c}0.468 * * * \\
(0.030)\end{array}$ & $\begin{array}{c}0.423 * * * \\
(0.022)\end{array}$ & $\begin{array}{c}0.336^{* * * *} \\
(0.018)\end{array}$ & $\begin{array}{c}0.347 * * * \\
(0.021)\end{array}$ & $\begin{array}{c}0.381 * * * \\
(0.030)\end{array}$ & $\begin{array}{c}0.475 * * * \\
(0.028)\end{array}$ & $\begin{array}{c}0.427 * * * \\
(0.024)\end{array}$ & $\begin{array}{c}0.342 * * * \\
(0.020)\end{array}$ & $\begin{array}{c}0.321 * * * \\
(0.021)\end{array}$ & $\begin{array}{c}0.358 * * * \\
(0.027)\end{array}$ \\
\hline Constant & $\begin{array}{c}-0.352 * * * \\
(0.072)\end{array}$ & $\begin{array}{c}0.082 \\
(0.050)\end{array}$ & $\begin{array}{c}0.450 * * * \\
(0.037)\end{array}$ & $\begin{array}{c}0.715 * * * \\
(0.048)\end{array}$ & $\begin{array}{c}1.075 * * * \\
(0.066)\end{array}$ & $\begin{array}{c}-0.357 * * * \\
(0.075)\end{array}$ & $\begin{array}{c}0.135^{* *} \\
(0.055)\end{array}$ & $\begin{array}{c}0.568 * * * \\
(0.042)\end{array}$ & $\begin{array}{c}0.911 * * * \\
(0.049)\end{array}$ & $\begin{array}{c}1.346 * * * \\
(0.066)\end{array}$ \\
\hline Observations & 11498 & 11498 & 11498 & 11498 & 11498 & 11498 & 11498 & 11498 & 11498 & 11498 \\
\hline
\end{tabular}


Table 8- Fixed Effects Quantile Earnings Regressions for Rural Workers in Vietnam Dependent Variable: Log Hourly Real Earnings Vietnam VHLSS 2002-2004-2006. Source: GSO VHLSS 2002, 2004, 2006, author's calculations

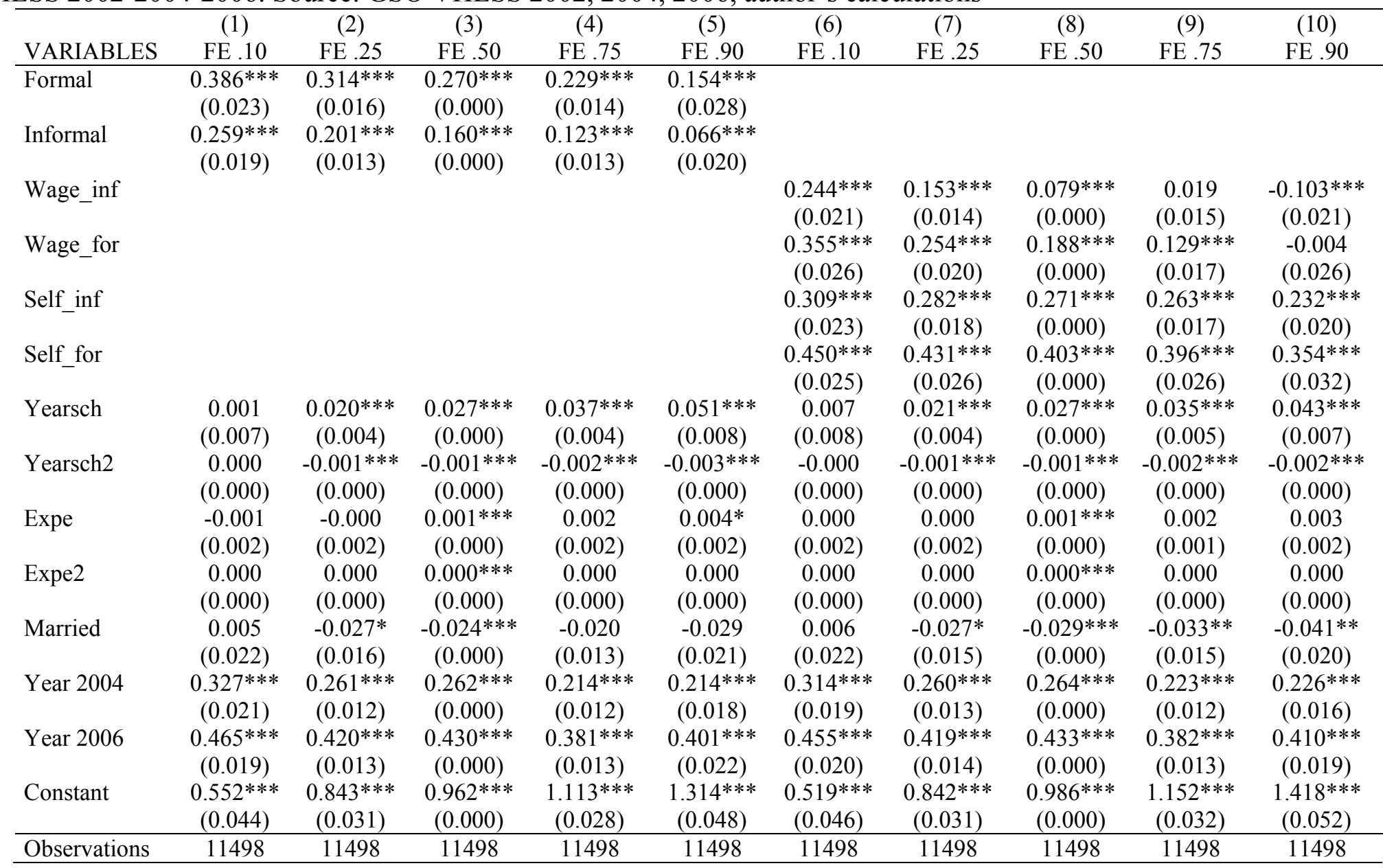

Standard errors in parentheses

$* * * \mathrm{p}<0.01, * * \mathrm{p}<0.05, * \mathrm{p}<0.1$ 


\title{
Conclusion- La population au centre des transitions en cours au Viêt Nam
}

\author{
Myriam de LOENZIEN (IRD-CEPED)
}

Cet ouvrage propose une analyse des transformations qu'a connues la population du Viêt Nam au cours des dernières décennies, ainsi que de quelques changements économiques et socioculturels qui les ont accompagnées. Il parait au moment où ce pays dresse un bilan de 30 années de politique du Renouveau (Dôi Moi), point de départ des problématiques exposées ici. Caractérisées par l'adoption et la mise en œuvre dans les années 1980 d'une série de mesures consistant principalement en une ouverture à l'économie de marché et un développement des échanges internationaux, ces réformes ont été marquées par l'adhésion du Viêt Nam à l'Association des Nations du Sud-est asiatique (ASEAN), à l'Organisation internationale de la Francophonie (OIF) puis à l'Organisation mondiale du Commerce (OMC). Elles ont eu pour effet de lancer le pays dans un processus long et complexe de restructurations économiques et politicoadministratives en réponse aux graves crises survenues pendant la période collectiviste. Elles ont conduit à des transitions qui se sont traduites par une industrialisation, une urbanisation, une métropolisation accrues, ainsi qu'une décentralisation (de Terssac et coll. 2012).

Les chapitres de ce livre soulignent la dimension démographique de ces transformations. Ils suggèrent quelques pistes de réflexion et perspectives en termes d'évolution du pays et de recherches à venir dans le domaine des sciences sociales sur le Viêt Nam contemporain. Celles-ci s'articulent autour de plusieurs paradoxes. Tout d'abord, la rapidité des changements observés contraste avec l'inertie qui caractérise habituellement les phénomènes démographiques. L'amélioration très significative des conditions de vie de la population s'est aussi accompagnée d'une montée de différents types d'inégalités. Les liens de solidarité se développent parallèlement à une plus grande individualisation des parcours socio-économiques. Ces transformations posent des questions de régulation sociale locale dans un contexte de mondialisation. Ainsi, ces analyses actualisent les interrogations sur la notion de transition, au cœur des problématiques sur le Viêt Nam contemporain en sciences sociales.

Le contraste entre l'inertie des phénomènes démographiques et la rapidité conjuguée à l'ampleur des changements survenus est présent dans la plupart des chapitres de cet ouvrage. La vitesse des transformations concerne le déroulement de la transition démographique et plus largement l'évolution de l'ensemble du «paysage démographique » (chapitres 1, 2, 6 et 7), mais aussi technologique, social et économique. C'est le cas des nouvelles technologies relatives à la santé de la reproduction (chapitre 2) et des normes de comportements sexuels et reproductifs (chapitre 6). Cette rapidité est également mentionnée pour l'accélération de la croissance économique, le développement du secteur formel et de l'urbanisation (chapitre 3), mais aussi l'adoption de nouvelles orientations politiques, notamment pour la lutte contre le VIH/sida (chapitre 4). Elle contredit le principe souvent affirmé en démographie suivant lequel les configurations relatives à la façon dont les individus d'une collectivité naissent, se marient, donnent naissance à des enfants ou meurent sont dotées d'une inertie relativement forte. Ce paradoxe s'explique de plusieurs manières: le Viêt Nam a bénéficié d'innovations initiées ailleurs, les différents types d'effets entrent en synergie les unes avec les autres, et ces phénomènes nouveaux constituent l'expression de processus amorcés longtemps avant qu'ils n'acquièrent une visibilité. 
Le déséquilibre des rapports de masculinité à la naissance en fournit un exemple éclairant (chapitre 2). Ce phénomène n'est véritablement devenu l'objet de mesures statistiquement significatives à l'échelle du pays qu'au moment du recensement de 2009. Or, il résulte en partie de l'utilisation d'innovations biomédicales dont les effets ont été décuplés dans un contexte de baisse rapide de la fécondité, commencée dès les années 1970 (Haughton, 1997; Guilmoto, 2012). Il s'est ensuite accentué et est considéré aujourd'hui comme susceptible de provoquer de grandes perturbations en raison d'un déficit de femmes sur le marché matrimonial au Viêt Nam, mais également dans quelques pays voisins tels que la Chine à l'horizon d'une vingtaine d'années (Bongaarts et coll. 2015). L'inertie qui a contribué à ralentir l'expression du phénomène participe maintenant à sa pérennité.

Si les conditions de vie se sont considérablement améliorées au Viêt Nam au cours des dernières décennies, les indicateurs démographiques font aussi apparaître des inégalités croissantes (chapitre 1). Ce parallèle entre d'une part un rythme accéléré de croissance économique, de réduction de la pauvreté, d'amélioration de la santé et de l'éducation et d'autre part l'augmentation d'inégalités a été mis en avant dans plusieurs travaux (Litchfield et coll., 2004 ; Phan et coll. 2010). Le diagnostic d'une montée des inégalités est cependant nuancé, voire remis en cause par ailleurs (OECD ; 2014). Ces inégalités sont souvent mesurées au niveau des ménages (Rolleston \& Krutikova, 2014). Elles se creusent au détriment des populations les plus pauvres et des membres des minorités ethniques (Axelson et coll. 2012). Elles sont accentuées par la vulnérabilité des plus défavorisés face aux problèmes socio-économiques (Imai et coll. 2011). Entre régions géographiques du pays, les indicateurs sont particulièrement défavorables aux zones isolées, montagneuses ou agricoles, par contraste avec les deux premières métropoles, Hô Chi Minh-Ville et Hanoi (Litchfield et coll., 2004). Dans le domaine de la santé, plusieurs auteurs soulignent l'aggravation des écarts chez les mères, les jeunes enfants et les nouveau-nés, notamment en termes d'accès aux infrastructures sanitaires (Goland et coll. 2012 ; Granlund et coll. 2010 ; Axelson et coll. 2012).

Les difficultés sont en partie liées à la remise en cause de l'Etat providence. Les réformes adoptées dans les années 1980 ont en effet conduit à l'abandon du système de subventions au profit du paiement des prestations par les utilisateurs, tout en gardant des objectifs de lutte contre les inégalités (London, 2011; London, 2008 ; Bélanger et coll. 2004). Les responsables communautaires ont ainsi développé une approche gestionnaire orientée vers une recherche d'autonomie financière (London, 2014). Les nouveaux dispositifs de redistribution ont des effets contrastés selon les mécanismes sollicités. Par exemple, les pensions et prestations d'assurance sociale contribuent à diminuer certaines inégalités entre les foyers, mais en accentuent d'autres (Nguyen, 2013).

Devenus des acteurs essentiels de la lutte contre les inégalités, les ménages sont fortement engagés dans un secteur de production économique informel dont nous avons pu explorer l'ampleur et l'hétérogénéité (chapitre 3). En prenant en charge les frais de santé et d'éducation, ainsi qu'en mettant en œuvre des stratégies éducatives et sanitaires, les individus tentent de pallier les lacunes du système public. Ils renforcent donc des liens d'entraide et de solidarité au sein desquels la famille occupe une place prépondérante. Comme nous l'avons vu, les réponses à l'épidémie VIH/sida constituent de ce point de vue le lieu d'un important engagement des membres de la famille, qui s'exprime dans la prise en charge d'un enfant ou d'un adulte séropositif (chapitres 4 et 5). Les études actuelles et à venir pourront certainement apporter une connaissance plus précise de ce que l'on qualifie d'entrepreneuriat social, et de ses conséquences socioéconomiques, notamment dans les domaines de la santé et de l'éducation. 
La mobilité géographique de la population dont nous avons mesuré l'ampleur croissante (chapitre 7) constitue un élément de stratégie des ménages pour lutter contre la pauvreté. A l'échelle macrosociale, il a été établi que les migrations contribuent à réduire les inégalités lorsqu'elles ont pour destination les grands centres industriels (Phan et coll. 2010). Il pourra être intéressant d'étudier à l'avenir dans quelle mesure l'assouplissement des règles de séjour des migrants conditionne des changements de répartition de la population et conduira à une réduction des écarts, dans un contexte où le statut de migrant peut être associé à différents types de vulnérabilité, comme l'a montré une étude réalisée auprès des jeunes femmes (Guilmoto et coll., 2014).

La manifestation et la gestion des problèmes sociaux locaux sont fortement dépendantes d'enjeux internationaux. Dans le domaine de la santé et de l'éducation, les priorités fixées par les grandes agences multilatérales déterminent l'attribution d'aides qui jouent un rôle déterminant dans les programmes locaux. L'exemple des problèmes de santé est probant. La dimension internationale de ceux-ci concerne non seulement la façon dont se propagent les pathologies, mais aussi les conditions de lutte contre leurs conséquences. Cet aspect est apparu très clairement dans le cas de la survenue d'épidémies au cours des dernières décennies (SARS, grippe aviaire, VIH/sida, Zika). L'épidémie VIH/sida au Viêt Nam est à cet égard exemplaire, avec son détachement de la politique des fléaux sociaux en 2004, et plus récemment le développement d'actions de réhabilitation des personnes qui exercent une activité de commerce sexuel, ainsi que la mise en place de programmes d'utilisation de la méthadone comme produit de substitution à la drogue (Reid et coll. 2011; Tomori et coll. 2014). Aujourd'hui, l'épidémie est considérée comme stabilisée, mais deux types de difficultés se manifestent. D'une part, le tarissement des sources de financement pose la question de la pérennité des interventions, notamment en matière de prise en charge des personnes séropositives, qu'elles soient adultes (chapitre 4) ou qu'il s'agisse d'enfants dont nous avons pu évaluer l'ampleur des besoins (chapitre 5). D'autre part, l'épidémie reste très présente dans certains groupes de population, au premier rang desquels les consommateurs de drogue, les hommes ayant des rapports sexuels avec d'autres hommes et les travailleurs et travailleuses du sexe (Socialist Republic of Vietnam, 2014). Ainsi, la mise en place de programmes de réduction des dommages nécessite des approches spécifiques.

Les problèmes sociodémographiques associés aux enjeux environnementaux constituent un autre domaine en plein essor en sciences sociales. Ils mettent en avant la confrontation de situations individuelles au sein de systèmes de contraintes collectives, comme nous l'avons vu avec l'exemple de l'étude menée à Hanoi et Hô Chi Minh-Ville (chapitre 8). Le développement de l'industrialisation et de l'urbanisation, avec leurs conséquences en termes d'épuisement des ressources et de pollution environnementale, mais aussi les effets de plus en plus sensibles du changement climatique, dans un pays où une grande partie des terres émergées se situe à des altitudes très faibles, vont constituer des défis d'une importance croissante dans les décennies à venir concernant le déplacement des populations et l'adéquation du milieu de vie à des conditions viables et stables.

Pour rendre compte de la complexité des enjeux que traverse le Viêt Nam, de nombreux auteurs évoquent la pluralité des transitions et suggèrent le caractère systémique des transformations. En effet, comme nous l'avons vu, la notion de transition est fréquemment utilisée par les démographes, sociologues, économistes et autres chercheurs en sciences sociales. Elle a été reprise par plusieurs auteurs de cet ouvrage pour évoquer la transition politico-économique d'une économie planifiée à une économie de marché. Elle rend compte de la transition démographique caractérisée par 
les baisses successives de la mortalité et la fécondité. Plus spécifiquement, la notion de transition des rapports de masculinité est mobilisée pour l'étude de la sélection sexuelle à la naissance (chapitre 2). De même, le passage de la prégnance de hiérarchies familiales et conjugales dites verticales vers un fonctionnement plus individualisé voire individualiste dit horizontal est décrit comme un ensemble de transitions sexuelles (chapitre 6). Enfin, ce terme de transition est également utilisé dans le cadre d'études économiques pour faire référence à l'évolution d'une organisation économique majoritairement informelle vers la mise en place d'un système formel d'organisation du travail, ou encore le passage d'une situation où les activités agricoles sont prépondérantes vers le développement d'autres formes d'emplois, manifeste dans la répartition de la population selon les statuts professionnels (chapitre 9).

Il est à cet égard significatif que dans ces exemples le concept de transition soit appliqué à des phénomènes clairement ancrés dans une discipline. En effet, si la transition démographique met en relation des grandeurs spécifiquement démographiques, la fécondité et la mortalité, les autres sciences considèrent dans leurs modèles les aspects démographiques comme exogènes, tenant peu compte des interactions que la population entretient avec les systèmes et environnements politiques, économiques, sociaux ou géographiques. Or, des approches pluridisciplinaires, alliant l'économique, le socioculturel et la dynamique démographique, voire les conditions biomédicales, ont montré comment le fait d'intégrer ces différents types de transitions conduit à mieux comprendre les changements en cours. De même, quelques modèles de transition pourraient être mis à l'épreuve dans le cadre des études sur le Viêt Nam. Par exemple, il pourrait être intéressant d'étudier dans quelle mesure la seconde révolution démographique pourrait constituer une référence pertinente pour appréhender les évolutions démographiques futures de ce pays. De telles réflexions permettront peutêtre de proposer des modèles innovants comme cela est actuellement le cas de travaux sur la fin de l'Etat providence en Asie (Ochiai et coll. 2013), qui remettent profondément en cause les cadres de pensée utilisés en sciences sociales généralement issus d'écoles occidentales, fournissant des outils conceptuels heuristiques inédits pour la compréhension de la situation et du devenir des sociétés situées en Asie, voire dans d'autres continents.

\section{Bibliographie}

Axelson, Henrik, Ulf-G. Gerdtham, Björn Ekman, Dinh Thi Phuong Hoa, and Tobias Alfvén. "Inequalities in Reproductive, Maternal, Newborn and Child Health in Vietnam: A Retrospective Study of Survey Data for 1997-2006." BMC Health Services Research 12, no. 1, 2012: 456.

Bélanger Danièle, and Jianye Liu. "Social Policy Reforms and Daughters' Schooling in Vietnam." International Journal of Educational Development 24, no. 1, January 2004: 23-38.

Bongaarts, John, and Christophe Z. Guilmoto. "How Many More Missing Women? Excess Female Mortality and Prenatal Sex Selection, 1970-2050." Population and Development Review 41, no. 2 (June 2015): 241-69.

de Terssac, Gilbert, An Quoc Truong, and Michel Catlla. "Le Viêt Nam Demain? Tendances émergentes et Voies Possibles." In Viêt Nam En Transitions, Sous la direction de Gilbert de Terssac, Truong An Quoc, Michel Catlla., collection De l'Orient à l'Occident. Lyon: ENS éditions, 2014: 283-290. 
Goland, Emilia, Dinh Thi Hoa, and Mats Målqvist. "Inequity in Maternal Health Care Utilization in Vietnam." International Journal for Equity in Health 11, no. 1, 2012: 24.

Granlund, David, Nguyen T. Chuc, Ho D. Phuc, and Lars Lindholm. "Inequality in Mortality in Vietnam during a Period of Rapid Transition." Social Science \& Medicine 70, no. 2, January 2010: 232-39.

Guilmoto, Christophe Z. "Son Preference, Sex Selection, and Kinship in Vietnam." Population and Development Review 38, no. 1, 2012: 31-54.

Guilmoto, Christophe Z., and Myriam de Loenzien. "Shifts in Vulnerability Landscapes: Young Women and Internal Migration in Vietnam." Genus 70, no. 1, 2014: 27-56.

Haughton, Jonathan. "Falling Fertility in Vietnam.” Population Studies 51, no. 2, 1997: 203-11.

Imai, Katsushi S., Raghav Gaiha, and Woojin Kang. "Poverty, Inequality and Ethnic Minorities in Vietnam." International Review of Applied Economics 25, no. 3, May, 2011: 249-82.

Litchfield, Julie, and Patricia Justino. "Welfare in Vietnam during the 1990s: Poverty, Inequality and Poverty Dynamics." Journal of the Asia Pacific Economy 9, no. 2, 2004: 145-69.

London, Jonathan D. "Reasserting the State in Viet Nam Health Care and the Logics of Market-Leninism." Policy and Society 27, no. 2, 2008: 115-28.

London, J. D., “Contemporary Vietnam's education system: Historical roots, current trends" in D. Jonathan London Ed., Education in Vietnam, Singapore: ISEAS, 2011, pp. 1-56.

London, Jonathan D. "Welfare Regimes in China and Vietnam." Journal of Contemporary Asia 44, no. 1, 2014: 84-107.

Nguyen, Viet Cuong. "The Impact of Social Security on Household Welfare: Evidence from a Transition Country." European Journal of Development Research 25, no. 5, April 18, 2013: 737-57.

Ochiai, Emiko, and Hosoya Leo Aoi. Transformations of the Intimate and the Public in Asia Modernity, Brill, Vol. 5, Boston, 2013, 314 p.

OECD, Social Cohesion Policy Review of Viet Nam, Development Centre Studies, OECD Publishing, Paris, 2014, 204 p.

Phan, Diep, and Ian Coxhead. "Inter-Provincial Migration and Inequality during Vietnam's Transition." Journal of Development Economics 91, no. 1, January 2010: $100-112$.

Reid, G., and Peter Higgs. "Vietnam Moves Forward with Harm Reduction: An Assessment of Progress." Global Public Health 6, no. 2, March 2011: 168-80.

Rolleston, Caine, and Sofya Krutikova. "Equalising Opportunity? School Quality and Home Disadvantage in Vietnam." Oxford Review of Education 40, no. 1, January 2, 2014: 112-31.

Socialist Republic of Vietnam. "Vietnam AIDS Response Progress Report 2014. Following up the 2011 Political Declaration on HIV/AIDS." National Committee for AIDS, Drug and Prostitution Prevention and Control, 2014.

Tomori, Cecilia, Vivian F. Go, Le Nhan Tuan, Nguyen Mai Huong, Nguyen Thanh Binh, Carla E. Zelaya, David D. Celentano, Do Tuan Dat, and Vu Minh Quan. "'In Their Perception We Are Addicts': Social Vulnerabilities and Sources of Support for Men Released from Drug Treatment Centers in Vietnam." International Journal of Drug Policy 25, no. 5, September 2014: 897-904. 